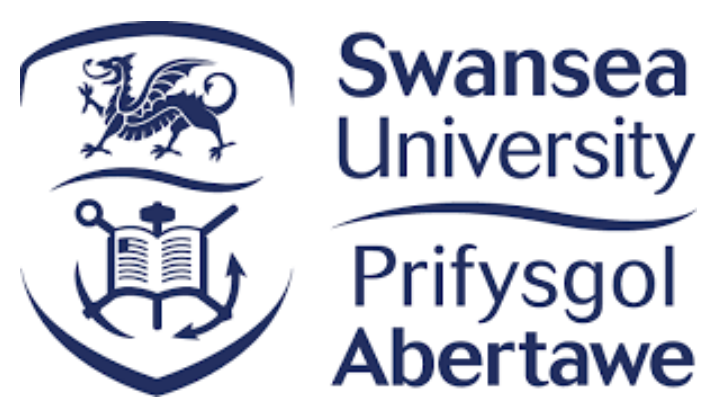

\title{
An Investigation of Quality of Urban Life: A Case Study of Quba Municipality, Medina City, Saudi Arabia
}

\section{A thesis submitted to Swansea University, Geography Department in fulfilment of the} requirements for the Degree of Doctor of Philosophy

By

Abdulaziz R. Alrashidi

Swansea University

January 2022 


\section{Declarations}

This work has not previously been accepted in substance for any degree and is not being concurrently submitted in candidature for any degree.

Signed.

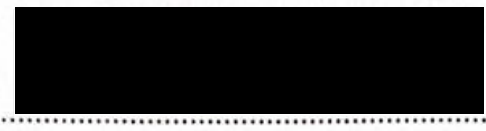

Date..25:01.2022

This thesis is the result of my own investigations, except where otherwise stated.Other sources are acknowledged by footnotes givingexplicitreferences. A bibliography isappended.

Signed.......

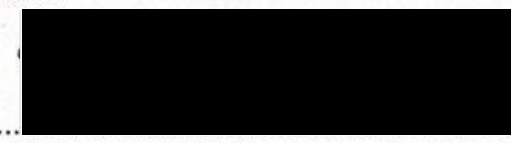

Date. $25 \cdot 01 \cdot 2022$

I hereby give consent for my thesis, if accepted, to be available for photocopying and for inter-library loan, and for the title and summary to be made available to outside organisation Signed.......

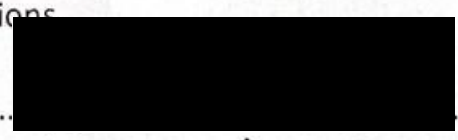

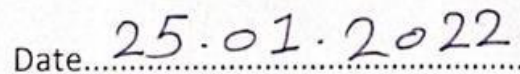

The University's ethical procedures have been followed and, where appropriate, that ethical approval has been granted.

Signed.

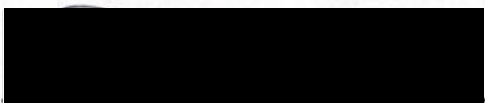

Date. $25 \cdot 01 \cdot 2022$ 


\section{Acknowledgments}

In the Name of ALLAH (GOD), the Most Gracious and the Most Merciful. All the praises and thanks be to ALLAH Almighty for giving me his blessing and the strength to complete this thesis.

First of all, I would like to express my sincere thanks to my supervisor Prof. Sergei Shubin who spared no effort in supporting me during my $\mathrm{PhD}$ study. I thank him for his generous support in providing advice that had an impact on completing this thesis. Likewise, I would like to express my gratefulness to my second supervisor Dr Richard Smith for his support in providing useful advice and feedback.

I would also extend my thanks to my sponsors: Taibah University and the Saudi Cultural Bureau in London for their support. I would also express my gratefulness to the staff in the Geography Department in particular, and Swansea University in general, for their generous support during my $\mathrm{PhD}$. Finally, I would like to thank all those who participated in this research during the fieldwork through the survey, photo diaries study and the expert interviews. 


\begin{abstract}
The term quality of urban life (QoUL) usually refers to the well-being of people and the quality of the environment in which they live. Over the past three decades, there has been an increasing interest in the study and an investigation of QoUL, which has attracted the attention of researchers in several fields, such as geography, psychology, economics, politics and sociology. In general, outputs of QoUL investigation can be used to diagnose previous policy strategies and to design future planning policies. The investigation of QoUL provides a basis of data that helps identify issues concerning the urban environment, causes of dissatisfaction, people's priorities in their daily lives, as well as monitoring and assessment of the efficiency of policies and strategies related to QoUL. Despite the large body of QoUL literature, there is still no agreed definition, or agreement over the way in which QoUL is investigated. However, there is wide consensus that QoUL has both subjective and objective dimensions. Although QoUL has been of interest in many disciplines around the world during the past three decades, it is relatively unexplored in Saudi Arabia. Therefore, this research aims to investigate the QoUL in the city of Medina, the case of Quba Municipality. To this end, this research uses different conceptual approaches, namely functionalist and postmodernity approaches, in order to provide an overarching understanding of the QoUL characteristics in the study area. Such understanding involves identifying the most influential aspects of the physical, economic, social and cultural domains, and exploring the impact of these aspects on QoUL in the study area. In so doing, this thesis tries to address some key challenges related to the current approaches to QoUL and provides a research framework of investigation that may lead to rethinking about QoUL.
\end{abstract}




\section{Abbreviations}

CPI: City Prosperity Index

FSCP: Future Saudi Cities Program

GASTAT: General Authority for Statistics

GDE: General Directorate of Education

GDHA: General Directorate of Health Affairs

MDA: Medina Development Authority

MRM: Medina Regional Municipality

MRSC: Medina Research and Studies Centre

OECD: Organization for Economic Co-operation and Development

QMO: Quba Municipality Office

QoL: Quality of Life

QoUL: Quality of Urban Life

SCTNH: Saudi Commission for Tourism and National Heritage

SMMRA: Saudi Ministry of Municipal and Rural Affairs

SWB: Subjective Well-Being

WHO: World Health Organisation

WHOQOL: World Health Organisation Quality of Life Assessment 


\section{Table of Contents}

An Investigation of Quality of Urban Life: A Case Study of Quba Municipality, Medina City, Saudi Arabia...............................................................................................i

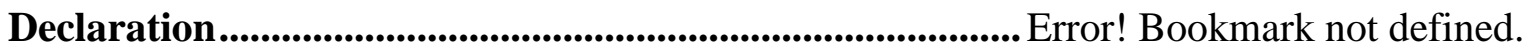

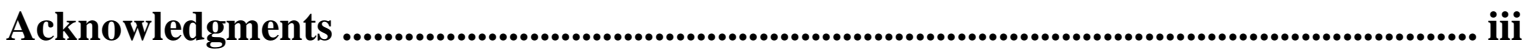

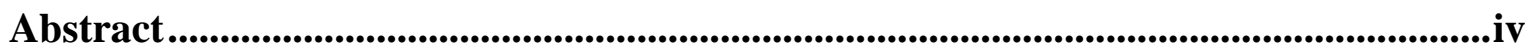

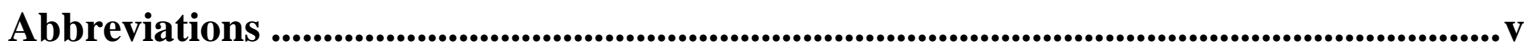

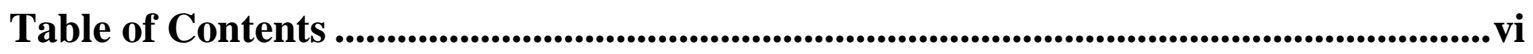

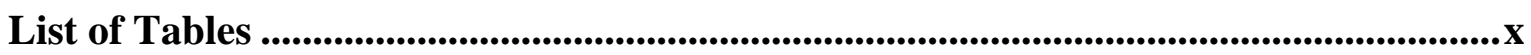

List of Figures ............................................................................................................................................

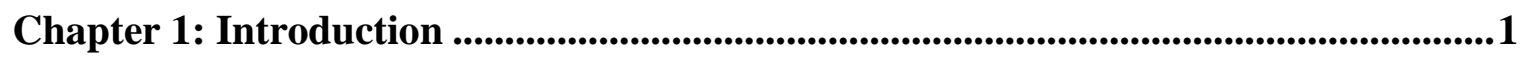

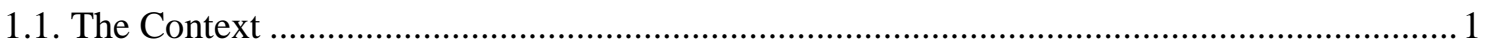

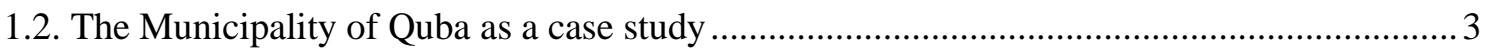

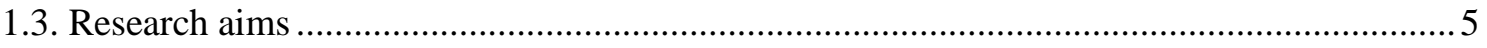

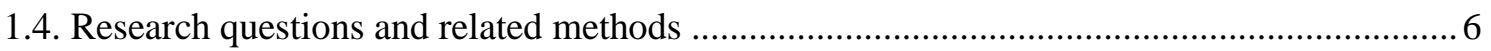

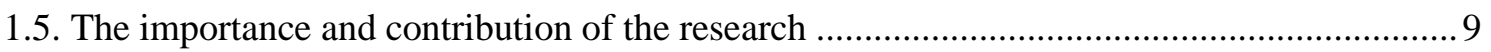

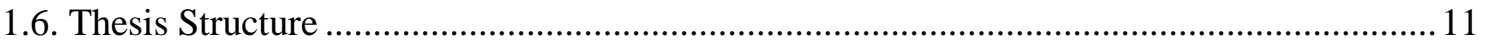

Chapter 2: Literature Review ...............................................................................................15

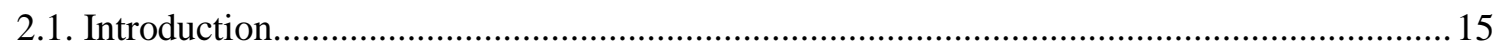

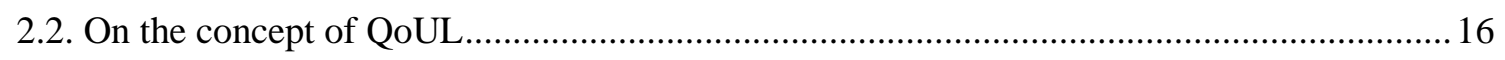

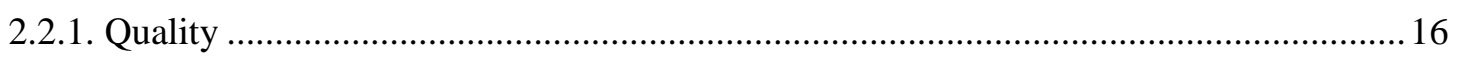

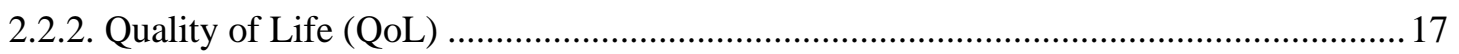

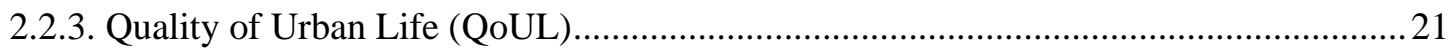

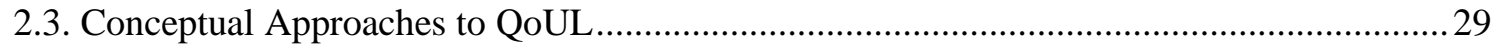

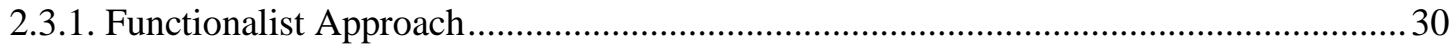

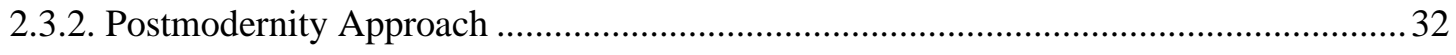

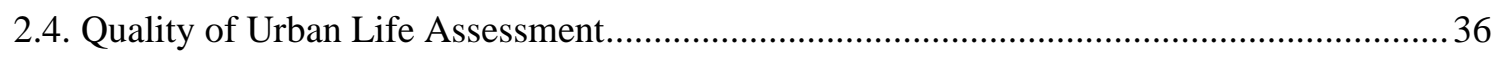

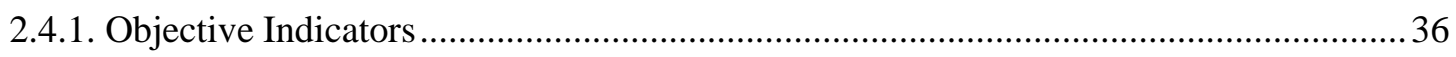


2.4.2. Subjective Indicators .37

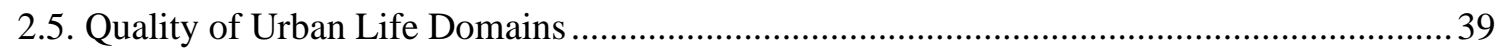

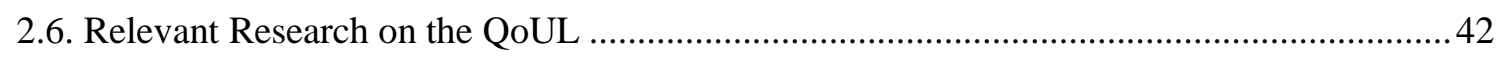

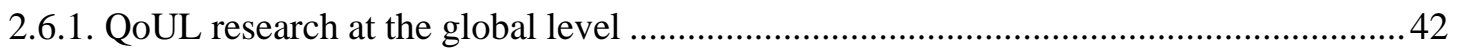

2.6.2. QoUL research in Saudi Arabia .................................................................................. 49

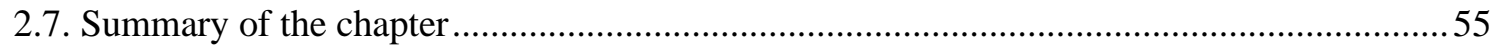

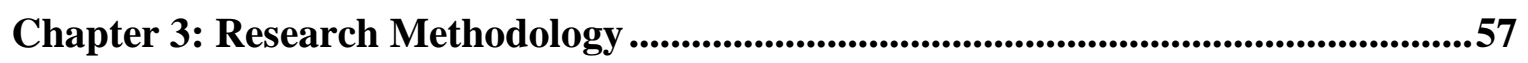

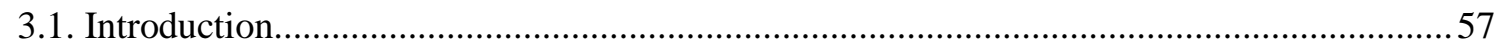

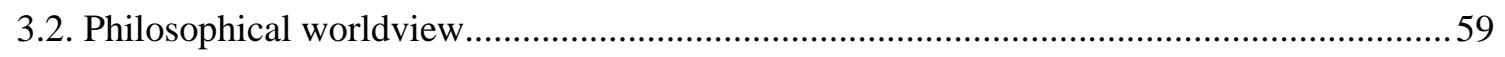

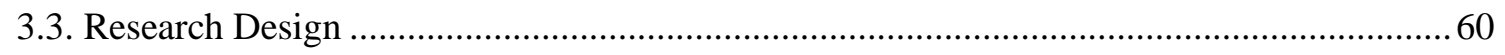

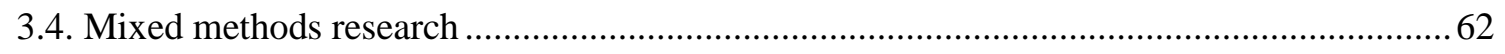

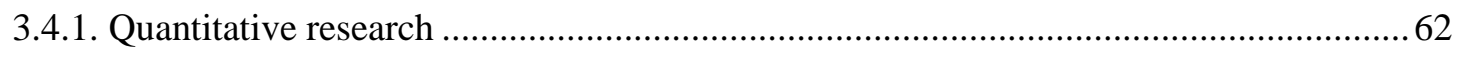

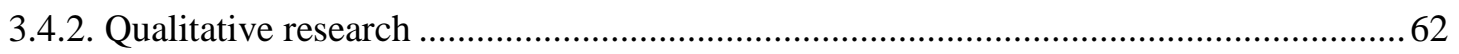

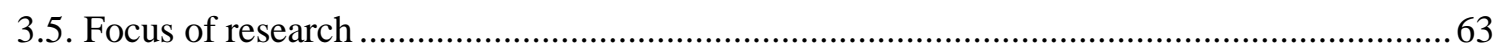

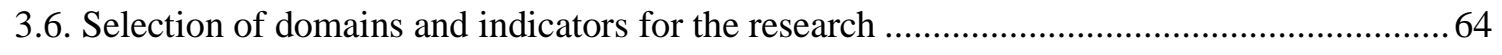

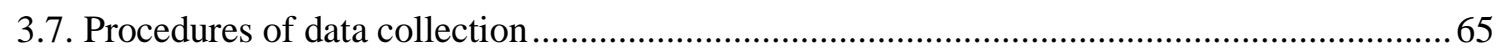

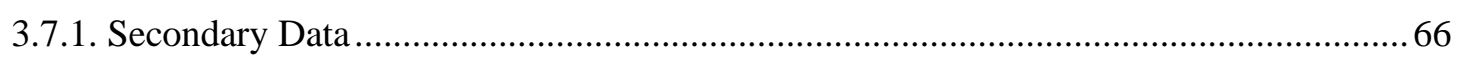

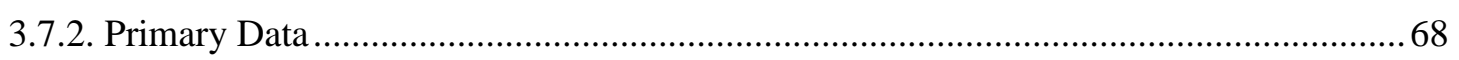

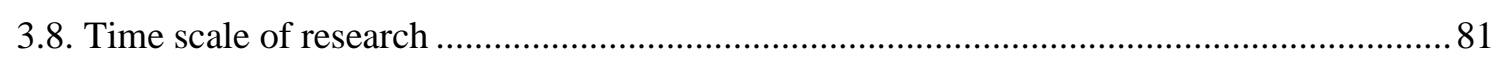

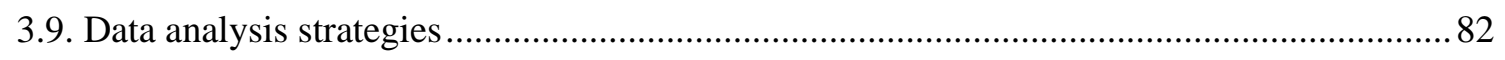

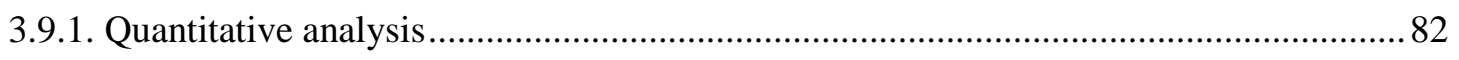

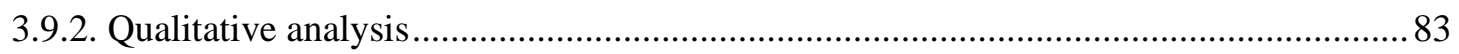

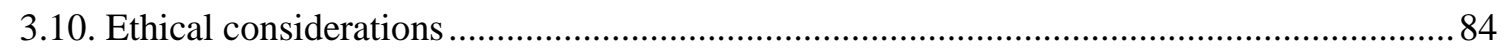

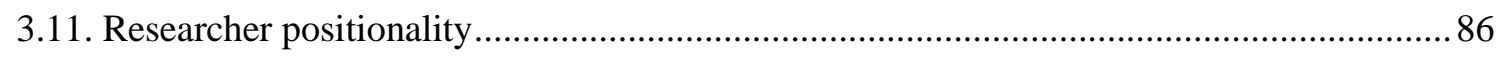

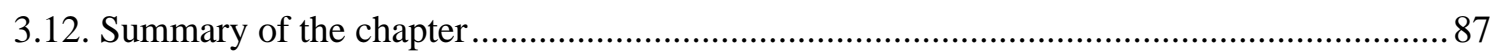

Chapter 4: General Reflection on QoUL in Quba ...............................................................88

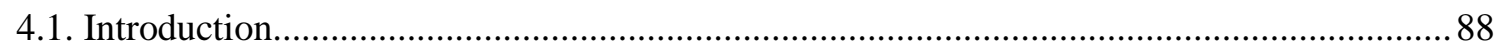

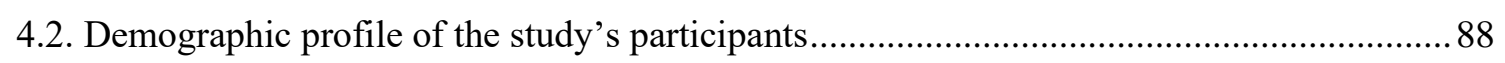

4.3. General perception of QoUL (status of QoUL) .............................................................. 91

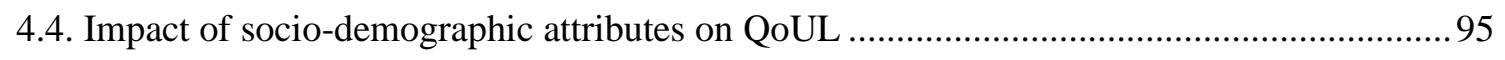

4.5. Ranking aspects of life in relation to their impact on the QoUL ......................................... 103

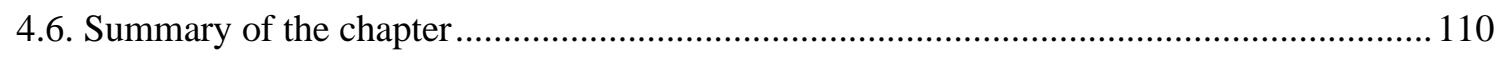

Chapter 5: Housing Quality and QoUL ..................................................................112 
5.1. Introduction 112

5.2. Satisfaction level with the accommodation ...

5.3. Satisfaction with the accommodation and QoUL

5.4. Type of accommodation and satisfaction with housing............................................ 122

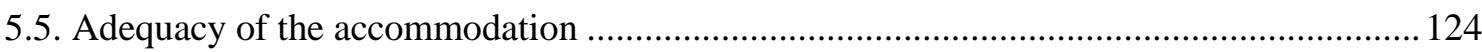

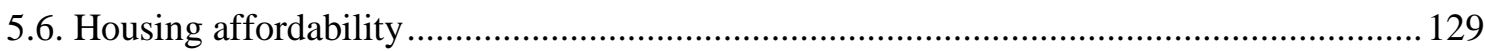

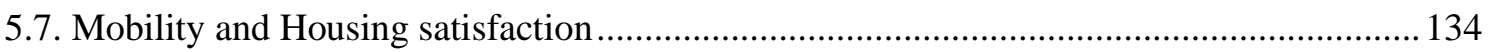

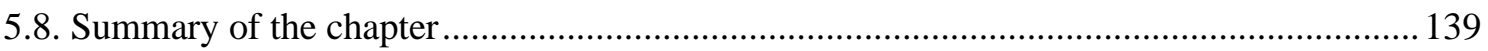

Chapter 6: Neighbourhood Quality and QoUL .................................................144

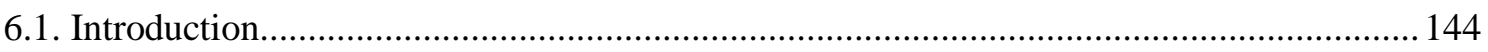

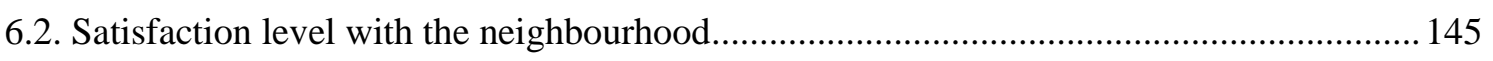

6.3. Satisfaction with the neighbourhood and QoUL ................................................... 149

6.4. Neighborhood components and QoUL .................................................................. 151

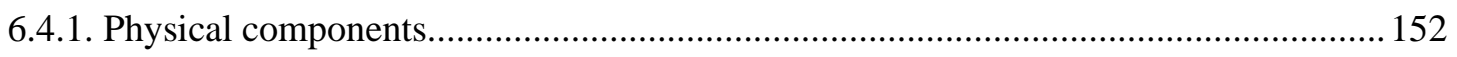

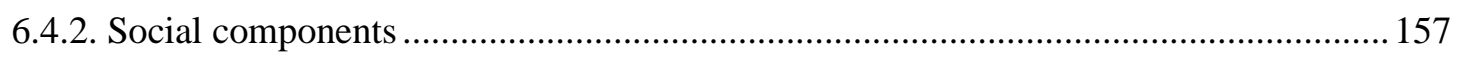

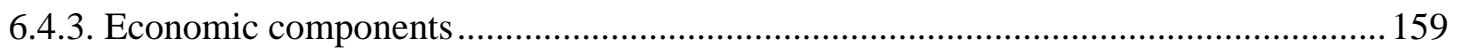

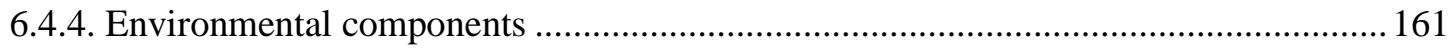

6.5. Quality of the neighbourhood's infrastructure .................................................... 164

6.6. Aesthetics and attractiveness of the neighbourhood .............................................. 167

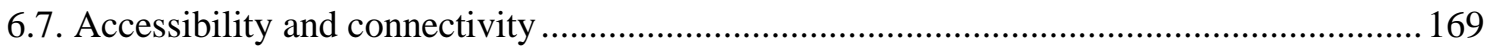

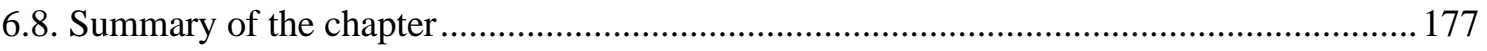

Chapter 7: Urban Services Quality and QoUL ...................................................................181

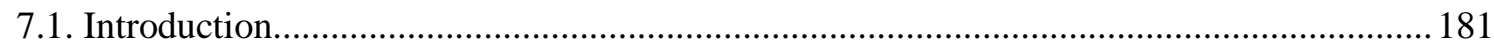

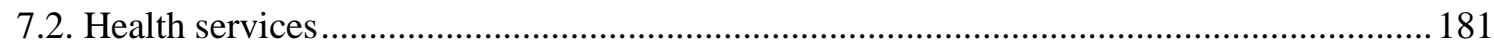

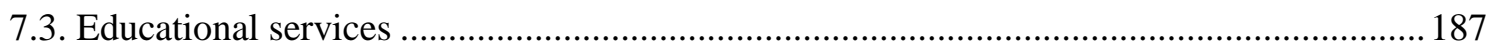

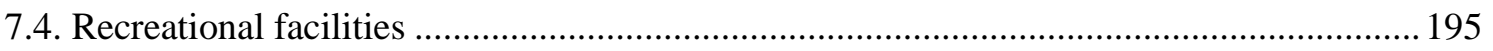

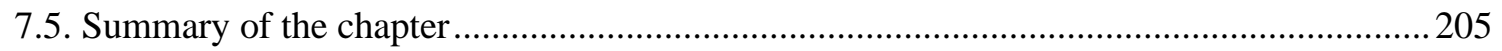

Chapter 8: Social and Cultural Domains of QoUL.........................................................208

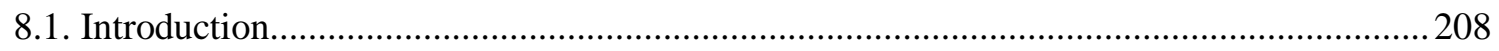

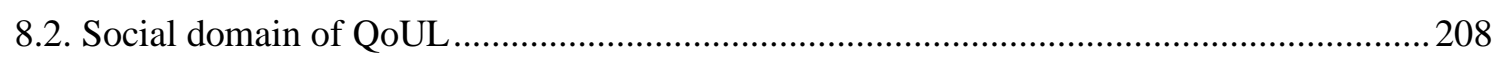

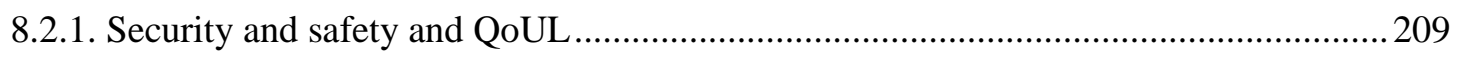

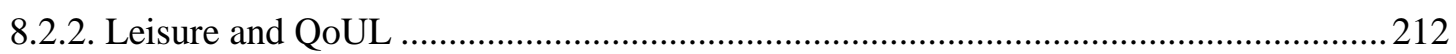

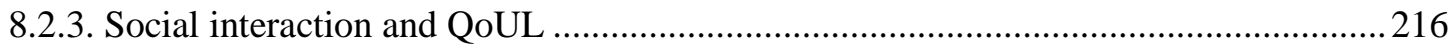




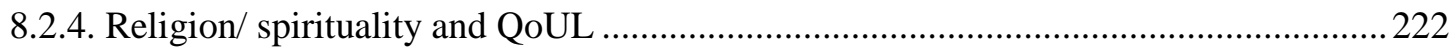

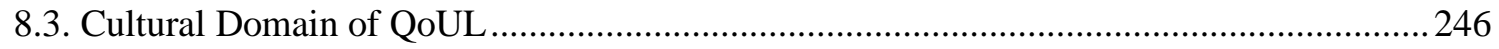

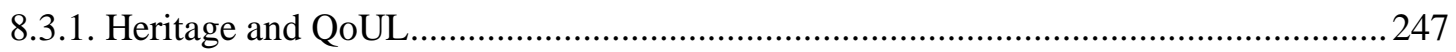

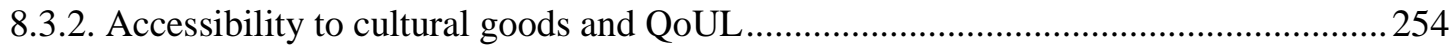

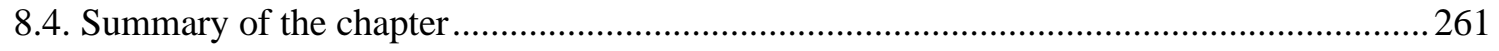

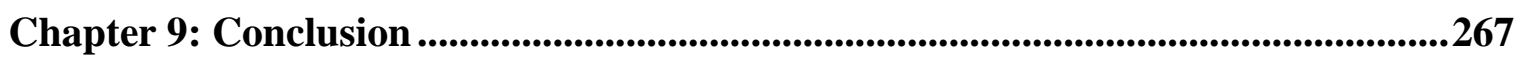

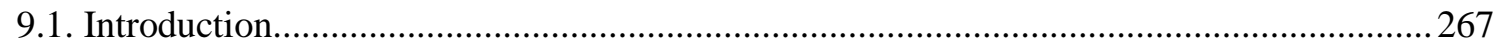

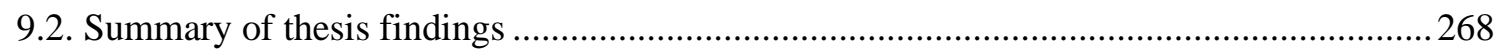

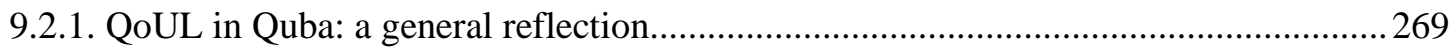

9.2.2. Perceived physical attributes of the urban environment in Quba ............................... 270

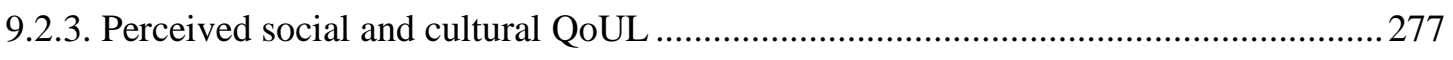

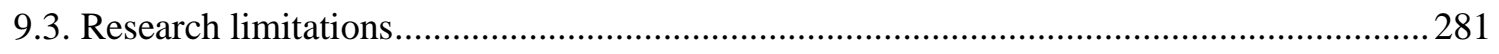

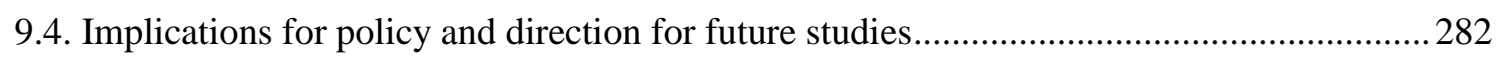

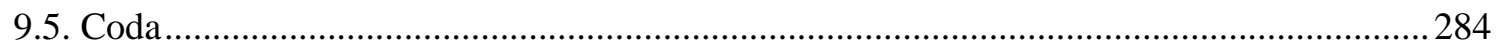

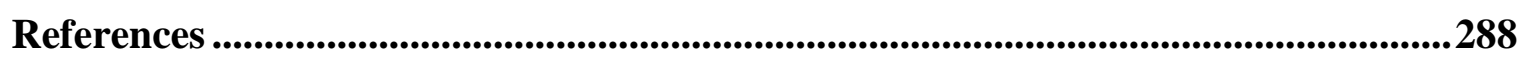

Appendix 1: Questionnaire (English version). ........................................................327

Appendix 2: Questionnaire (Arabic version)..........................................................341

Appendix 3: Photo diaries instructions sheet (English version). ..................................356

Appendix 4: Photo diaries instructions sheet (Arabic version). .................................358

Appendix 5: Participants Consent Form (English version). ........................................360

Appendix 6: Participants Consent Form (Arabic version). ............................................362

Appendix 7: Information Sheet (English version). ................................................364

Appendix 8: Information Sheet (Arabic version)....................................................367 


\section{List of Tables}

Table 2.1: QoUL domains employed in a range of selected studies 41

Table 3.1: Domains and indicators selected for the study .65

Table 3.2: Sample of the secondary data 68

Table 3.3: Sample detail of the photo diaries study .79

Table 3.4: Sample detail of the expert interviews .81

Table 3.5: Timetable for the data collection 82

Table 4.1: Socio-demographic attributes of the study's participants .90

Table 4.2: Significant values obtained from testing the relationship between QoUL and socio-demographic attributes.

Table 4.3: Association between gender and QoUL Level. Cross-tabulation. .96

Table 4.4: Association between gender and employment status. Cross-tabulation .98

Table 4.5: Correlation between residential status and QoUL level: Spearman's rho .98

Table 4.6: Association between residential status and QoUL Level. Cross-tabulation..... . .99

Table 4.7: Association between monthly income and QoUL level. Cross-tabulation ....... 101

Table 4.8: Correlation between household monthly income and QoUL level 101

Table 4.9: Spearman's rho result of examining the correlation between aspects of life ranking and QoUL level.

Table 4.10: Mean, standard deviation and variance of aspects of life 105

Table 5.1: Relationship between age and satisfaction with accommodation. Crosstabulation.

Table 5.2: The relationship between residential status and satisfaction with accommodation. Cross-tabulation.

Table 5.3: Correlation between satisfaction with the accommodation quality and QoUL. Spearman's rho 120 
Table 5.4: The relationship between satisfaction with accommodation quality and QoUL.

Cross-tabulation

Table 5.5: The relationship between type of accommodation and satisfaction with the accommodation. Cross-tabulation

Table: 5.6: Relationship between agreement degree that accommodation is adequate to live in and satisfaction with the accommodation. Cross-tabulation

Table 5.7: Relationship between type of accommodation and the agreement degree that accommodation is adequate to live in. Cross-tabulation.

Table 5.8: Relationship between gender and the agreement degree that accommodation is adequate to live in. Cross-tabulation.

Table 5.9: Relationship between residential status and the agreement degree that accommodation is adequate to live in. Cross-tabulation.

Table 5.10: Relationship between accommodation satisfaction and percentage of household income spent on housing. Cross-tabulation....

Table 5.11: Frequency distribution about the agreement degree that it is affordable to buy or rent a home in the municipality of Quba

Table 5.12: Relationship between agreement degree about housing affordability and QoUL. Cross-tabulation

Table 5.13: Relationship between likelihood to move to other accommodation and satisfaction with the accommodation. Cross-tabulation

Table 5.14: Relationship between likelihood to move to other accommodation and QoUL.

Cross-tabulation 136

Table 6.1: Relationship between residential status and satisfaction with the neighbourhood. Cross-tabulation

Table 6.2: Relationship between income and satisfaction with the neighbourhood. Crosstabulation.

Table 6.3: The correlation between neighbourhood satisfaction and QoUL. Spearman's correlation test.

Table 6.4: The relationship between neighbourhood satisfaction and QoUL. Crosstabulation.

Table 6.5: Relationship between satisfaction with the neighbourhood and satisfaction with the accommodation. Cross-tabulation. 
Table 6.6: Frequency measurement of the availability of facilities 155

Table 6.7: The relationship between satisfaction with the neighbourhood and issues with the facilities. Cross-tabulation.

Table 6.8: The correlation between neighbourhood satisfaction and social components. Spearman's correlation 158

Table 6.9: Relationship between satisfaction with the neighbourhood and job opportunities. Cross-tabulation

Table 6.10: Frequency measurement of suffering from environmental issues

Table 6.11: The relationship between satisfaction with neighbourhood and environmental issues. Cross-tabulation.

Table 6.12: Correlation between neighbourhood satisfaction and the agreement degree that the neighbourhoods have a good infrastructure. Spearman's correlation test

Table 6.13: Relationship between neighbourhood satisfaction and the agreement degree that the neighbourhood has a good basic infrastructure. Cross-tabulation

Table 6.14: Relationship between neighbourhood satisfaction and the agreement degree that the neighbourhood is an attractive and well-organised place to live. Cross-tabulation

Table 6.15: Frequency measurement of accessibility and connectivity issues 170

Table 6.16: The relationship between satisfaction with neighbourhood and accessibility and connectivity issues. Cross-tabulation

Table 6.17: Relationship between neighbourhood satisfaction and other accessibility and connectivity indicators. Spearman's correlation test.

Table 7.1: Objective indicators of the health services in Quba municipality. Source: GDHA (2018)

Table 7.2: Correlation between overall satisfaction with quality of healthcare services and other health service variables. Spearman's correlation test

Table 7.3: Relationship between QoUL and overall satisfaction with the quality of health care services

Table 7.4: Objective indicators of the educational services in Quba municipality. Source: GAE (2018).

Table 7.6: The association between and the overall satisfaction level with the quality of educational services and other educational variables using Spearman's correlation..... 
Table 7.7: Relationship between overall satisfaction with the quality of educational services QoUL. Cross-tabulation

Table 7.8: Parks within the municipality of Quba. Source: MRM, 2018 196

Table 7.9: Correlation between the overall satisfaction with the quality of recreational facilities and other recreational facilities variables using Spearman's correlation.... 201

Table 7.10: Relationship between QoUL and overall satisfaction with the quality of recreational facilities

Table 8.1: Relationship between security situation evaluation and QoUL level using Kruskal Wallis test

Table 8.2: Evaluation of security situation and gender. Cross-tabulation

Table 8.3: The association between agreement degree there is good accessibility to leisure activities that are suitable for all groups of people and QoUL level.

Table 8.4: Association between amount of social interaction with neighbours and QoUL level

Table 8.5: Correlation between social interaction and social support. Spearman's rho ....219

Table 8.6: The association between satisfaction with neighbours and QoUL level 222

Table 8.7: Relationship between the extent to which spiritual/religious beliefs can help to improve QoUL and QoUL level.

Table 8.8: The relationship between the sense of the spirituality of place and QoUL level

Table 8.9: Agreement level that religious practices and beliefs have an impact on improving QoUL and QoUL.

Table 8.10: Correlation between frequent attendance at the Mosque and QoUL.

Spearman's rho.....

Table 8.11: The association between the amount of social interaction with neighbours and the frequency of attendance at the mosque

Table 8.12: Relationship between age and a sense of spirituality of place. Cross-tabulation

Table 8.13: Relationship between marital status and a sense of spirituality of place. Crosstabulation.

Table 8.14: Frequent attendance at the mosque and age. Cross-tabulation .244 
Table 8.15: Relationship between QoUL level and cultural domain indicators 246

Table 8.16: The relationship between the extent to which heritage and religious landmarks affect QoUL and QoUL level. Cross-tabulation

Table 8.17: The relationship between the extent to which religious landmarks and heritage enhance QoUL and QoUL level. Cross-tabulation

Table 8.18: The relationship between accessibility to the libraries and museums and QoUL. Cross-tabulation

Table 8.19: Relationship between accessibility to cultural activities such as conferences and exhibitions of hobbies, arts, crafts, and traditional food and QoUL. Cross-tabulation 


\section{List of Figures}

Fig 1.1: Neighbourhoods of Quba Municipality, modified by the researcher based on the MapInfo's maps from MRM (2018).

Fig 1.2: Thesis structure.

Fig 2.1: Conceptual model of factors that contribute to community QoL from a human ecological perspective (Shafer et al. 2000, p. 166)

Fig 2.2: Components of QoL (Baycan Levent \& Nijkamp, 2006, p. 272).

Fig 2.3: Relationship between domain satisfactions and life satisfaction (Campbell et al., 1976, p. 16).

Fig 2.4: Relationships between residential domain satisfactions and QoL (Marans, 2012, p. 14).

Fig 2.5: Model showing relationships between objective conditions, subjective responses, and neighbourhood satisfaction. (Marans, 2012, p. 15).

Fig 2.6: Conceptual framework showing relation between environment and QoL (Das, 2008, p. 301). .28

Fig 2.7: The wheel of cities' prosperity (UN-Habitat, 2013, p. 12) ..................................51

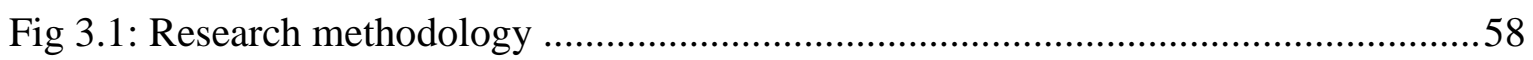

Fig 3.2: Convergent parallel design for the study based on Creswell and Plano-Clark (2011).

Fig 3.3: Study Area. Source: The researcher based on Google Earth and the MRM's website (2018)

Fig 3.4: Data resources used for the research .66

Fig 4.1: QoUL level on a scale 1-10 91

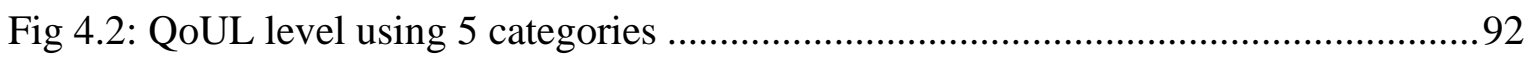

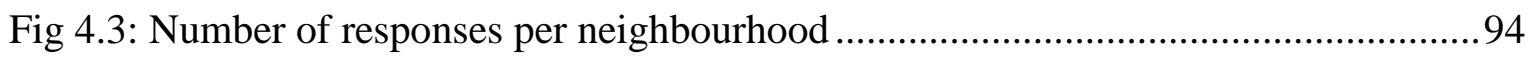

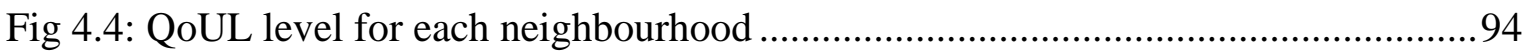




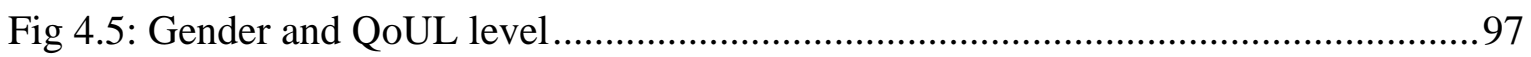

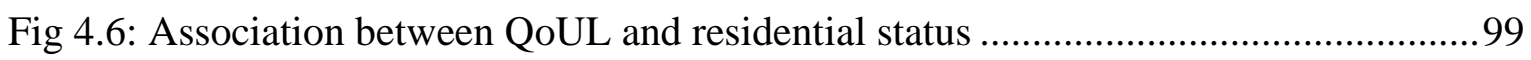

Fig 4.7: Relationship between monthly income and QoUL.......................................... 101

Fig 4.8: Health rank. Note: where 1 is the highest ranking and 6 the lowest ................... 106

Fig 4.9: Security rank. Note: where 1 is the highest ranking and 6 the lowest.................. 106

Fig 4.10: Recreation rank. Note: where 1 is the highest ranking and 6 the lowest........... 107

Fig 5.1: Satisfaction level with accommodation ........................................................... 113

Fig 5.2: Association between residential status and satisfaction with accommodation.... 116

Fig 5.3: A photograph representing home ownership taken by participant $\mathrm{PH} 23 \ldots \ldots \ldots . .118$

Fig 5.4: Relationship between satisfaction with accommodation quality and QoUL....... 121

Fig 5.5: Relationship between type of accommodation and satisfaction with the accommodation

Fig 5.6: Relationship between agreement degree that accommodation is adequate to live in and satisfaction with the accommodation

Fig 5.7: Relationship between type of accommodation and the agreement degree that

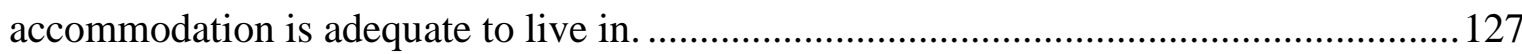

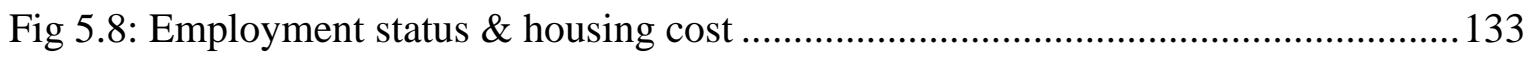

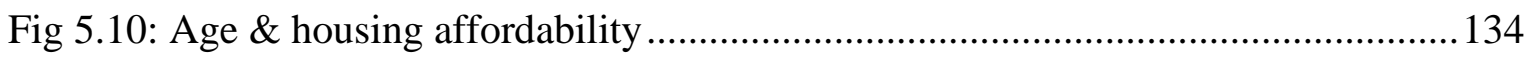

Fig 5.12: Relationship between likelihood to move to other accommodation and satisfaction with the accommodation

Fig 5.13: Relationship between likelihood to move to other accommodation and the three

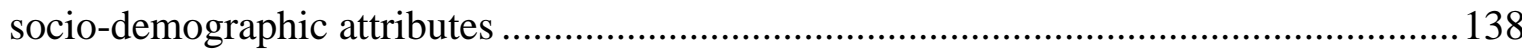

Fig 6.1: Satisfaction with the neighbourhood using two forms of scales ........................ 146

Fig 6.2: Association between neighbourhood satisfaction and QoUL............................ 150

Fig 6.3: Relationship between satisfaction with the neighbourhood and satisfaction with the accommodation

Fig 6.4: Relationship between neighbourhood satisfaction and the availability of four physical components 
Fig 6.5: Relationship between environmental components and satisfaction with the neighbourhood

Fig 6.6: Relationship between neighbourhood satisfaction and the agreement degree that the neighbourhood has a good basic infrastructure. 166

Fig 6.7: Relationship between neighbourhood satisfaction and the agreement degree that the neighbourhood is an attractive and well-organised place to live. 168

Fig 6.8: The relationship between satisfaction with the neighbourhood and accessibility and connectivity issues

Fig 6.9: Relationship between neighbourhood satisfaction and accessibility to the main road, sidewalks and situation with traffic jams

Fig 6.10: Sidewalk within Quba's neighbourhoods taken by participants PH 6 and PH 1 respectively

Fig 7.1: Association between satisfaction with quality of health services and health services variables

Fig 7.2: Overall satisfaction with healthcare services

Fig 7.3: Association between satisfaction with the quality of health services and QoUL.

Fig 7.4: Correlation between the overall satisfaction level with the quality of educational services and other educational variables

Fig 7.5: Overall satisfaction with the quality of the educational services

Fig.7.6: Relationship between overall satisfaction with the quality of educational services and QoUL

Fig 7.7: Some of the public parks within Quba municipality (MRM, 2018)..... 196

Fig 7.8: Sports areas within some of Quba's neighbourhoods.

Fig 7.9: Park near the old railway station. Photo diary taken by participant $\mathrm{PH} 9$. 198

Fig 7.10: Children's playground within the central park in Quba 199

Fig 7.11: Overall satisfaction with recreational facilities 200

Fig 7.12: Correlation between the overall satisfaction with the quality of recreational facilities and other recreational facilities variables 201 
Fig 7.13: Relationship between QoUL and overall satisfaction with the quality of recreational facilities .203

Fig 8.1: Security evaluation of the study's respondents .210

Fig 8.2: Relationship between security situation evaluation and QoUL level. 211

Fig 8.3: Agreement degree that there is good accessibility to leisure activities 213

Fig 8.4: The relationship between accessibility to leisure activities and QoUL .214

Fig 8.5: Association between amount of social interaction with neighbours and QoUL .217

Fig 8.6: One of the social interaction pictures. Photo diary by participant PH 1. 218

Fig 8.7: Relationship between social interaction and social support 220

Fig 8.8: Education level vs. social interaction .221

Fig 8.10: Relationship between satisfaction with neighbours and QoUL .222

Fig 8.11: Quba square and the beginning of the Quba route. This photo shows a complete Qur'an verse written on a public building. Photo diary by participant PH11. 226

Fig 8.12: Al Jum'ah Mosque, one of the historical mosques in Quba, where the first Jumu'ah prayer (Friday prayer) was held. Photo diary by participant PH 19. 228

Fig 8.13: Relationship between QoUL and the agreement level that religious practices and beliefs have an impact on improving QoUL 230

Fig 8.14: People in the squares surrounding Quba Mosque, talking to each other and taking pictures. Photo diary taken by participant PH 2.

Fig 8.15: People break their fast in the mosque. Photo diary by participant $\mathrm{PH} 23$ 238

Fig 8.16: Age and frequent attendance at the mosque 244

Fig 8.17: Gender and frequent attendance at the mosque

Fig 8.18: The relationship between the extent to which heritage and religious landmarks affect QoUL and QoUL level.

Fig 8.19: The relationship between the extent to which religious landmarks and heritage enhance QoUL and QoUL level.

Fig 8.20: An archaeological farm containing some traditional tools that were used in the past, especially in agriculture. Photo diary by participant $\mathrm{PH} 9$. 
Fig 8.21: A set of historical sites that represent heritage in the study area. Photo diaries by participants PH 6, PH 5 and PH 25 respectively.

Fig 8.22: The relationship between accessibility to the libraries and museums and QoUL 256

Fig 8.23: Agreement that there is accessibility to cultural activities ..............................258

Fig 8.24: Relationship between accessibility to cultural activities and QoUL 259 


\section{Chapter One}

\section{Introduction}

\subsection{The Context}

The term quality of urban life (QoUL) usually refers to the well-being of people and the quality of the environment in which they live. Over the past three decades, there has been an increasing interest in the study and investigation of QoUL which has attracted the attention of researchers within different disciplines, such as geography, psychology, architecture, sociology, politics to name a few. The increased interest in this field is largely related to the fact that about half the of world's population now live in cities, with an expected increase in urbanisation. In this context, the world's cities are considered as centres for many aspects of life including economic, social and cultural activity. However, due to the rapid increase of urbanisation within these sites, several issues such as unemployment, housing shortages, insecurity and social deprivation have been identified, which reflect the negative aspects of urban development (Rezvani et al., 2012). Since these urban issues primarily relate to peoples' daily life, they have aroused the interest of both scholars and policymakers, and QoUL has become more closely associated with these issues. QoUL has therefore been considered as one of the most important topics to have significant implications for policies of urban development.

QoUL has been described as a multidimensional concept because it involves focusing on several dimensions of the urban sphere including physical, social, economic and environmental quality (Marans \& Stimson, 2011). It has also been interchangeably used with several other concepts such as life satisfaction, happiness, subjective well-being (SWB), welfare, liveability and so forth, which make it more complex and multifaceted. Yet, it is in urban development that QoUL has been affirmed as a particularly important tool. In this respect, outputs of QoUL investigations are used both in the evaluation of past and current planning strategies, and in developing plans for the future. Moreover, QoUL 
study allows for the identification of urban issues, and priorities, and for the detection of causes of dissatisfaction on the part of citizens with aspects of life in the environment in which they live. In addition, it helps to evaluate the efficiency of these services and develop strategies in order to improve the liveability of the cities (Rezvani et al., 2012).

Within the majority of the literature, the most widely accepted and agreed approach in measuring QoUL is the multi-dimensional approach, which includes both objective and subjective approaches (e.g., Andrews \& Withey, 1976; McCrea et al., 2006; Yinshe, 2005; Marans \& Stimson, 2011; Felce \& Perry 1995). The majority of these studies have stressed that QoUL depends not only on the objectively measured condition of the urban environment, but also on people's perception of the environment in which they live, their experiences and the extent of their needs and expectations. However, it is widely reported that the relationship between those two approaches is still weak (e.g., McCrea et al., 2006; Das, 2008; McCrea, et al., 2011; Mohit, 2013; Soleimani et al., 2014). This, therefore, poses a challenge for the majority of investigators, especially with regard to the findings obtained from using these two approaches.

While QoUL has been investigated during the past three decades in different parts of the world, and is a growing field of research, it is still relatively unexplored in Saudi Arabia, which indicates a visible gap that needs to be addressed, particularly in the context of Medina. This thesis therefore represents an initial attempt to discover the QoUL in a new spatial context that presents different social, culture, environmental and geographical characteristics. In this context, Medina city is one of the most important cities in terms of social, cultural and special characteristics. It also represents the second holy city for Muslims after Makkah, which makes it more important than other Saudi cities. These were, therefore, key motivating factors in selecting Medina as a case study as a contribution to the global literature.

The interest of this thesis in examining the QoUL arose from the existence of key challenges related to the current approaches to QoUL investigation in general and in the Saudi context in particular. Firstly, social and cultural elements tend to be overlooked, where the focus is more on economic and physical elements of QoUL. Secondly, elements of QoUL such as heritage, spirituality and religion, are almost always less obvious and perhaps have even been disregarded. Thirdly, there is a lack of complexity and critical approaches to QoUL in 
relation to (a) scale, where research has focused on city and regional levels, while scales such as dwelling and neighbourhood have been ignored in the context of the study area. And (b), most research has ignored different experiences of QoUL that are based on a social group - the elderly, young, men and women, for example. An additional challenge is the way in which the impact of a range of indicators of QoUL are identified and assessed.

Accordingly, this thesis aims to investigate the QoUL in the city of Medina, specifically the case of Quba Municipality. In doing so, this research uses different conceptual approaches, namely functionalist and postmodernity approaches, in order to provide an overarching understanding of the QoUL characteristics in the study area. Such understanding involves identifying the most influential aspects of physical, economic, social and cultural domains, and exploring the impact of these aspects on QoUL in the study area. In so doing, this thesis not only addresses the QoUL in a new spatial context, but also provides a research framework of investigation that may lead to rethinking QoUL by using these conceptual approaches. It also provides a critical approach by highlighting the impact of various domains on the QoUL.

In order to set the thesis more fully in context, the next section presents the background to the study area itself, followed in section three by elaborating the research aims and objectives. The research questions and the related methods are then discussed in section four and the important contributions of the research are presented in section five. The chapter ends with outlining the thesis structure in section six.

\subsection{The Municipality of Quba as a case study}

The municipality of Quba is one of the most important municipalities of Medina, associated with both ancient and modern history. The importance of this municipality lies in its long history, represented by many historical events related to the biography of the Prophet Muhammad, peace be upon him (PBUH), and in the establishment of Quba Mosque. This mosque has given the Quba area its importance both in the past and the present, where it is visited by millions of pilgrims during the year, as well as accounting for the preference for housing in the nearby areas on the part of many of the city's residents. Furthermore, the municipality of Quba contains many historical mosques such as Al Jumaah Mosque, as 
along with wells and other historical places (SCTNH, 2018). The municipality of Quba is one of the largest municipalities of Medina, located to the south, covering an area of 348.57 square kilometres, with a population of 337,124 inhabitants. The municipality encompasses 30 neighbourhoods (see Figure 1.1) namely, Qurban, Albahr, Al Mughaisilah, Al Duwaimah, Prince Nayef, Al Qaswa, Abu Buraiqa, Al Hijrah, Al-Ehn, Jubour, Al Ranuna, Shoran, Al Jumaah, Al Ausba, Al Khatim, Al Dhahirahh, Al Hadeqah Al Sacab, Rahat, Al Jabirah, As sad, Aljussah Bani Baiadhah, Al- Rawabi, Shaibiyah, Nubala'a, Al Rummana, Al Hasa, Al Mazayean and Al Sharayea (QMO, 2018).

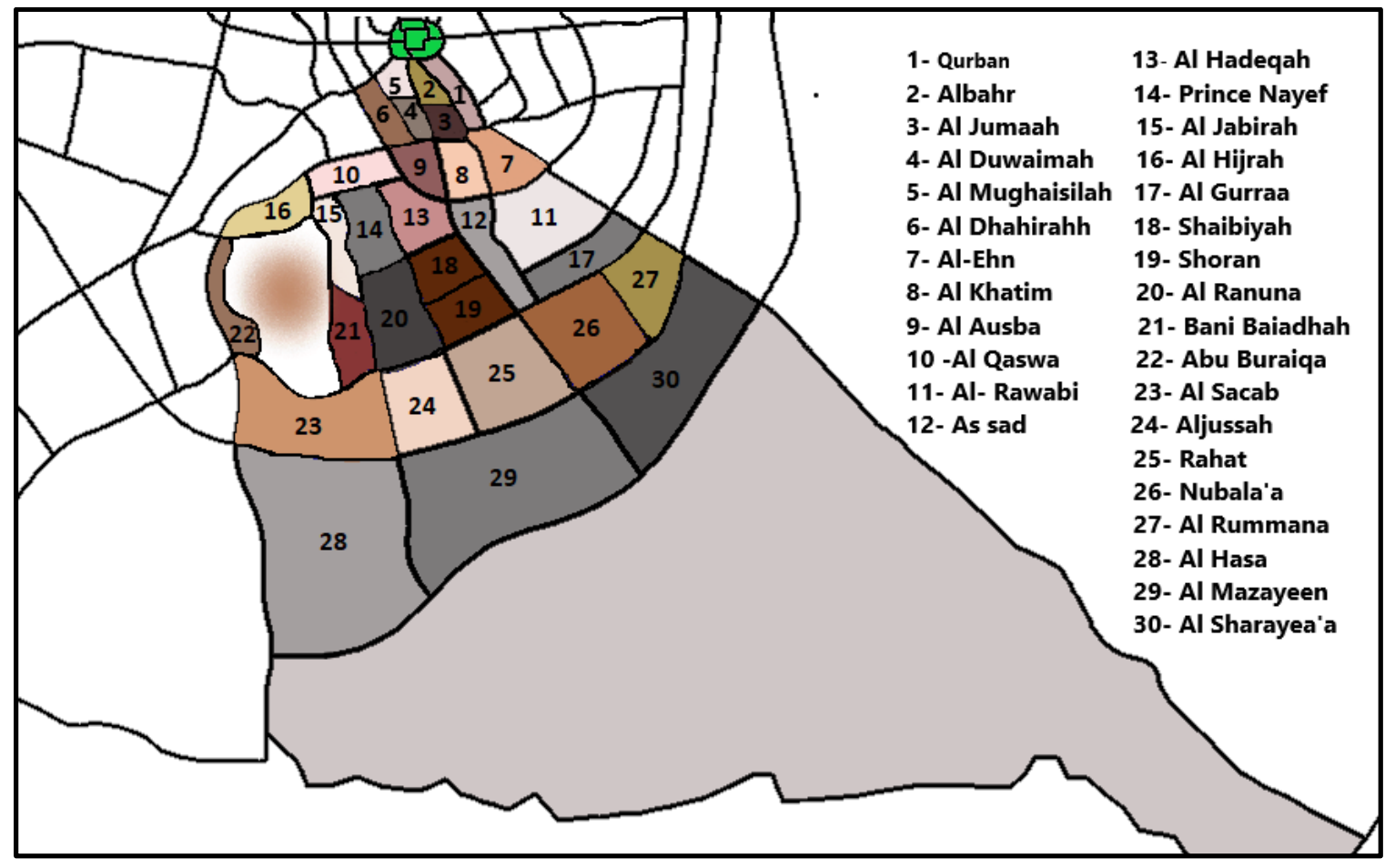

Fig 1.1: Neighbourhoods of Quba Municipality, modified by the researcher based on the MapInfo's maps from MRM (2018)

The reason behind the choice of Quba municipality as a case study is that it is linked to the old urban bloc of Medina which includes a number of the oldest neighbourhoods, such as Qurban, Quba, Al-Mughaisilah, Al Jubour and Al-Duwaimah, while at the same time, including new neighbourhoods that have recently emerged such as Prince Nayef, Al-Qaswa, Al- Rawabi, Al-Ghurraa, Al-Ranuna and Shoran. In this context, the mixture of old and new neighbourhoods contributes to the diversity of the urban environment attributes (historical and contemporary) which may result in a variety of social, cultural and economic life 
experiences. At the same time, however, this diversity of neighbourhoods may lead to variation in QoUL, which makes it difficult to identify outcomes about the overall QoUL. In addition, this municipality is considered as a vital location in Medina because it is situated in the area that connects two important religious landmarks, namely the Prophet's Mosque and the Quba Mosque. Thus, the choice of Quba municipality, with these two religious landmarks, allows for a better exploration of the religious dimensions of QoUL, such as spirituality for example. Alongside the famous Mosque of Quba, which is considered the first mosque to be established in the world (Ayyad, 2017), the municipality encompasses many historical sites, the presence of which is important in the consideration of heritage as a key element of QoUL in this thesis.

The study area was also chosen as it is undergoing an important development with regard to Medina city, which could lead to changes in the understanding of QoUL. One of the most important features of this developmental stage is the project of "Durb Al-Sunnah" which the government has recently decided to establish. It is a walking route of three kilometres (about 1.8 mile) linking the Prophet's Mosque and the Quba Mosque (Al Suqairi, 2015; Al Harthi, 2015). According to the local decision-makers, this project is likely to be one of the most important economic components to Medina and the municipality of Quba in particular, especially in terms of tourism, where it is likely to attract investment and many tourists, along with large numbers of pilgrims and visitors. Moreover, the historical features that distinguish the municipality of Quba hold significant religious dimensions not only for the city's local residents but also for the millions of visitors who come from all over the world every year.

\subsection{Research aims}

In investigating QoUL in Medina, specifically with regard to Quba municipality, this thesis uses functionalist and postmodernity approaches to highlight the physical, social and cultural aspect of the urban environment and the extent to which they contribute to the QoUL of those living in Quba. In doing so, this research aims to,

- evaluate the status of urban quality of life in Quba, taking into consideration the impact of the socio-demographic attributes. 
- determine the most influential aspects on the QoUL of those living in Quba.

- assess the quality of physical and functional attributes of the built environment in Quba.

- examine the influence of social and cultural domains on the QoUL of those living in Quba.

- provide a critical complex approach in relation to the impact of physical, social and cultural domains on QoUL.

\subsection{Research questions and related methods}

In line with the conceptual approaches used in this thesis, a mixture of quantitative and qualitative methods was adopted to answer research questions. The research questions and the associated methods and techniques are given below.

\section{RQ 1: What is the relationship between socio-demographic attributes and perceived QoUL of Quba's residents?}

In general, measuring the QoUL depends on how people perceive it. However, this perception is often influenced by the demographic characteristics of the residents such as gender, age, education level, income, marital status, and so forth. In this way, residents view the objective features of the environment in which they live at different levels of perception according to their particular demographic characteristics. Consequently, subjective evaluations are considerably varied from one person to another. In this regard, a questionnaire is employed to answer this research question in order to gain a better understanding of the impact of the diverse socio-demographic attributes on perceived QoUL in the study area. To this end, the association between socio-demographic attributes and the QoUL domains selected for the study area is examined, where the significant values likely to be obtained from this association might help to shed light on the ways in which those demographic attributes impact QoUL perception. 


\section{RQ 2: How do Quba's residents perceive their QoUL?}

What emerges strongly from QoUL literature is that the overall QoUL is derived from the interaction between people and the components of the urban environment in which they live. Thus, perception of the overall QoUL is based on how people rate their QoUL in terms of these various components. In this context, perception of QoUL is based on how people rate their QoUL. Therefore, the most appropriate method to examine this is to directly ask people about their feelings based on their experiences in their daily lives. In so doing, the overall QoUL level can then be linked to the socio-demographic attributes of the residents, as well as to their perception regarding specified QoUL domains of the study area, in order to obtain the significant values from testing the association between QoUL level and those domains. To this end, this thesis relies on the questionnaires to answer this question, where a rating scale is used to obtain the overall QoUL level in the study area.

\section{RQ 3: How do functionalist and postmodernity approaches re-define the QoUL characteristics?}

The QoUL has several dimensions, such as the social, cultural, physical and economic. Each of those dimensions is an integral part of the process of measuring the QoUL in a specific place. Therefore, this thesis looks at the QoUL from several aspects of functionalist thinking and postmodernity. 'Functionalist' refers to the integration of social groups or the society in terms of the function of its constituent elements, while a postmodern perspective focuses on differences, uniqueness and individuality to the needs and situations of all members of society. These two approaches are extensively reviewed in Chapter Two. Generally, the thesis relies on the literature to answer this question, where the components of the QoUL that have been emphasised by the majority of studies (e.g., functional aspects, cultural aspects, economic aspects etc.) are identified. Then, those key elements of QoUL are grouped into each conceptual approach in accordance with the perspective or point of view of each of those conceptual approaches. In light of the broadness of this research question, two sub-questions are further asked in order to address it in a meaningful way. These subquestions and the associated methods and techniques are set out below. 
- What are the key elements of a postmodern approach that lead to thinking about QoUL differently?

As pointed out in Chapter Two, postmodernity symbolises the transformations in culture and the social realm that have a clear impact on the QoUL of a society. Accordingly, this thesis focuses on the extent to which social and cultural aspects influence QoUL with more concentration on religion and heritage and how these key elements contribute to the QoUL in the study area. This concentration is in response to the lack of research on the impact of these two key elements on the QoUL. To answer this sub-question, quantitative research using questionnaires is adopted to assess the contribution of such cultural and social elements by measuring residents' level of satisfaction with their interaction with them. In addition, qualitative research using photo diaries and follow-up interviews is adopted. Photo diaries were adopted as a tool to help gain insightful information about the ways in which participants perceive their surroundings. More specifically, since religion and beliefs are often referenced through the practices and performance, photo dairies assist in observing and recording participants' performances as well as their interactions with those around them (Schultze, 2012). Moreover, interviews with experts are also undertaken to provide different perspectives in order to support data obtained from the above methods. In so doing, triangulated methods are used to get better results.

- What are the key elements of the functionalist approach that lead to think about QoUL differently?

In this thesis, the key elements of the functionalist approach with respect to the functional aspects of QoUL, refer to the physical domain in terms of indicators such health, education, recreation, housing and the condition of the neighbourhood. In the majority of QoUL literature, these functional attributes are often referred to as the objective indicators prevailing where people live, all contributing to QoUL, especially in terms of residents' feelings and levels of satisfaction as they interact with them. Therefore, this thesis relies fundamentally on the use of the questionnaires to assess the quality of these indicators in order to gain a better understanding of how residents of Quba view those components of the urban environment. In addition to that, official statistical data are used to gain more information about the availability and accessibility of those attributes. 


\subsection{The importance and contribution of the research}

Since the nature of the study of QoUL is relatively unexplored in the context of Saudi Arabia and in the city of Medina in particular, this thesis is considered as a contribution to the QoUL research by highlighting the experience of QoUL in a new research context. Therefore, conducting a research study in a such a cultural context has the potential to provide influential guidance for QoUL research, and in offering fresh perspectives towards improving urban development, which in turn contributes to a better QoUL. Moreover, this research uses different approaches that address some of the issues inherent in the concept of QoUL in terms of broadening the scope of examining functional, economic, social and cultural aspects, as well as intentionally combining objective and subjective indicators of QoUL. The contribution of this research can be explained in the following four specific areas:

- Firstly, this study is the first on QoUL in Saudi Arabia and in the city of Medina in particular. In so doing, this study will open the door to more studies on the investigation of QoUL in the context of Saudi Arabia. Such study provides guidelines for policy-makers to improve the standard of physical, social, cultural and economic life of Medina's community.

- Secondly, this study provides an additional database regarding the profile of the urban environment in Medina, specifically in the municipality of Quba, that can offer an evaluation of QoUL in the study area that might be useful for decision-makers in terms of identification of the factors that affect QoUL.

- Thirdly, engaging people in the decision-making process is one of the most important elements of the success of urban development. This can be achieved by involving residents in evaluating the urban environment in which they live by taking into consideration their opinions and concerns regarding their urban environment through, for example, social surveys. In the case of Medina, this phenomenon is somewhat new because of the scarcity of such surveys. Therefore, this research may contribute to the development of the culture of participation of citizens in the local development of Medina and the municipality of Quba in particular. In addition, it might contribute to raising awareness among citizens as well as a sense of responsibility towards their 
urban environment. Thus, the subjective indicators adopted in this research are key elements in achieving this.

- Finally, this study employs different approaches to the QoUL. These approaches facilitate discerning which characteristics of QoUL can be understood as key elements. The postmodern approach looks at the QoUL in terms of diversity, which is considered as a unique feature of QoUL, as well as in terms of some as yet undervalued and relatively underexplored urban features like heritage and religion that might influence the urban community. The functionalist approach discusses the functional system of the city, including the basic services provided, which reflect the extent to which citizens are satisfied with their urban environment. By using these approaches, this research not only examines how satisfied people are with their urban environment, but also considers how this environment can be attractive by achieving the maximum criteria of QoUL and therefore, enhancing its competitiveness.

The aim of this thesis, as reflected in the above areas of contribution, is to highlight the QoUL as one of the most important tools of regional planning and urban development. This study seeks to build comprehensive knowledge about the QoUL experience in its physical, social, cultural, economic and environmental dimensions in order to begin to fill the gap in the QoUL research in Saudi Arabia and Medina in particular. Additionally, this thesis can make a contribution to improving the QoUL of Medina's residents by highlighting the strengths and weaknesses of the urban environment in which they currently live, potentially providing important results for decision-makers in Medina. Furthermore, the social participation represented by the opinions of the residents can be a crucial supporter of the development of policies and the establishment of long-term goals (Santos and Martins, 2006).

For the field of QoUL and urban development, this thesis provides some key conceptual and methodological contributions. As indicated above, conceptually, it brings two approaches into the field that offer alternative and different perspectives regarding the urban environment itself, thus offering a deeper/broader understanding of QoUL. Methodologically, this thesis presents an additional qualitative method in the form of photo diaries in order to obtain more insight into how people experience their QoUL. In so doing, 
this research reveals some of the aspects of the urban environment that have been merely hinted at in the QoUL literature. Thus, this research is innovative in using these different conceptual approaches to contribute to QoUL research by rethinking the underlying aspects of life.

\subsection{Thesis Structure}

In order to provide a coherent narrative consistent with the nature of the research, this thesis will be structured into nine chapters, covering the five main parts of the research, namely, introduction, literature review, methodology, the empirical work and conclusions (see Figure 1.2). Following on from the overview of the study, its context and aims presented here, Chapter 2 moves to the literature review. This chapter sets out the foundation of knowledge concerning QoUL in order to gain an understanding regarding the existing research and debates relevant to this concept, and to set a theoretical reference for the thesis. The chapter starts with discussing the concepts of the QoUL including its definitions and conceptualisation in section two. It then discusses the conceptual approaches adopted for this thesis in section three. Section four discusses the assessment of the QoUL, while in section five, domains of QoUL are highlighted. Finally, in section six, related works on the investigation of QoUL in some selected world cities, as well as the related work to QoUL in Saudi Arabia and Medina in particular, are reviewed.

Chapter 3 presents the methodological framework of the research. It discusses the philosophical worldview of this research (section two) and sets out the research design of the thesis (section three). Research methods are discussed in section four, and in section five, the conceptual approaches and related methods. The chapter moves on to set out the focus of research (section six); the selection of domains and indicators for the research (section seven); procedures of data collection (section eight); and the time scale of research (section nine). Data analysis strategies are discussed in section ten and the chapter ends by discussing the ethical consideration of this research in section eleven.

Chapter 4 provides the broader analysis of the QoUL in the study area in order to set up the foundation for the empirical work of the research. It presents the demographic profile of the participants in section two. Then, it looks at the general perception of QoUL (status of 
QoUL) in section three. In section four, it discusses the impact of socio-demographic attributes on QoUL. Finally, it discusses the influence of various aspects of life on the QoUL in section five.

Chapters 5 to 8 examine the detail of the empirical research itself in terms of particular identifiable elements. Chapter 5 begins the discussion of QoUL at the narrowest scale dwelling (i.e., housing). It analyses the satisfaction level with accommodation taking into account the impact of the socio-demographic attributes of the participants. Section three then discusses the relationship between satisfaction with the accommodation and QoUL. In sections four, five and six, the relationship between issues of housing, including tenure type, type of accommodation, adequacy of the accommodation, and fiscal burden and affordability and satisfaction with housing as well as QoUL is highlighted. Finally, mobility as an indication of housing satisfaction is highlighted in section seven.

Chapter 6 examines the QoUL at the broader scale of the neighbourhood. As with the previous chapter, it starts with discussing the satisfaction level with neighbourhood before moving on in sections two and three to discuss the relationship between satisfaction with the neighbourhood and QoUL, taking into consideration the influence of the sociodemographic attributes of the participants. The chapter continues by highlighting the relationship between neighbourhood components, including physical, social, economic and environmental components, and satisfaction with the neighbourhood as well as overall QoUL in section four. The quality of the neighbourhood's infrastructure is discussed in section five, while aesthetics and the attractiveness of the neighbourhood is discussed in section six. Finally, issues of accessibility and connectivity are highlighted in section seven.

Chapter 7 discusses the QoUL at the municipal level, where it assesses the satisfaction with the functional attributes within the municipality of Quba and the impact they have on the overall QoUL. Within this discussion, the use of subjective and objective indicators is taken into account. In section two, health services and their objective attributes alongside subjective evaluation and the relation to the QoUL are presented. Likewise, educational and recreational services are respectively discussed in sections three and four.

Chapter 8 discusses the social and cultural aspects of the urban environment in the study area in two main sections. Within the social domain, security and safety, leisure activities 
social interaction, religion and spirituality are the focus of attention. The relationship between these indicators and the QoUL is examined, taking into account the impact of the socio-demographic attributes. Within the section two, cultural domains including heritage, accessibility to the libraries and museums, accessibility to the cultural activities such as conferences and exhibitions of hobbies, arts and so forth are highlighted. The relationship between QoUL and these aspects is examined, taking into consideration the influence of the socio-demographic attributes.

The concluding Chapter (Chapter 9), presents a summary of the findings related to the main objectives of this thesis that have been obtained in the analytical chapters, drawing these together to consider the final outcomes of this thesis. After outlining the limitations of the research, suggestions are made as to the implications for policy raised by the research and directions for future studies are discussed. 


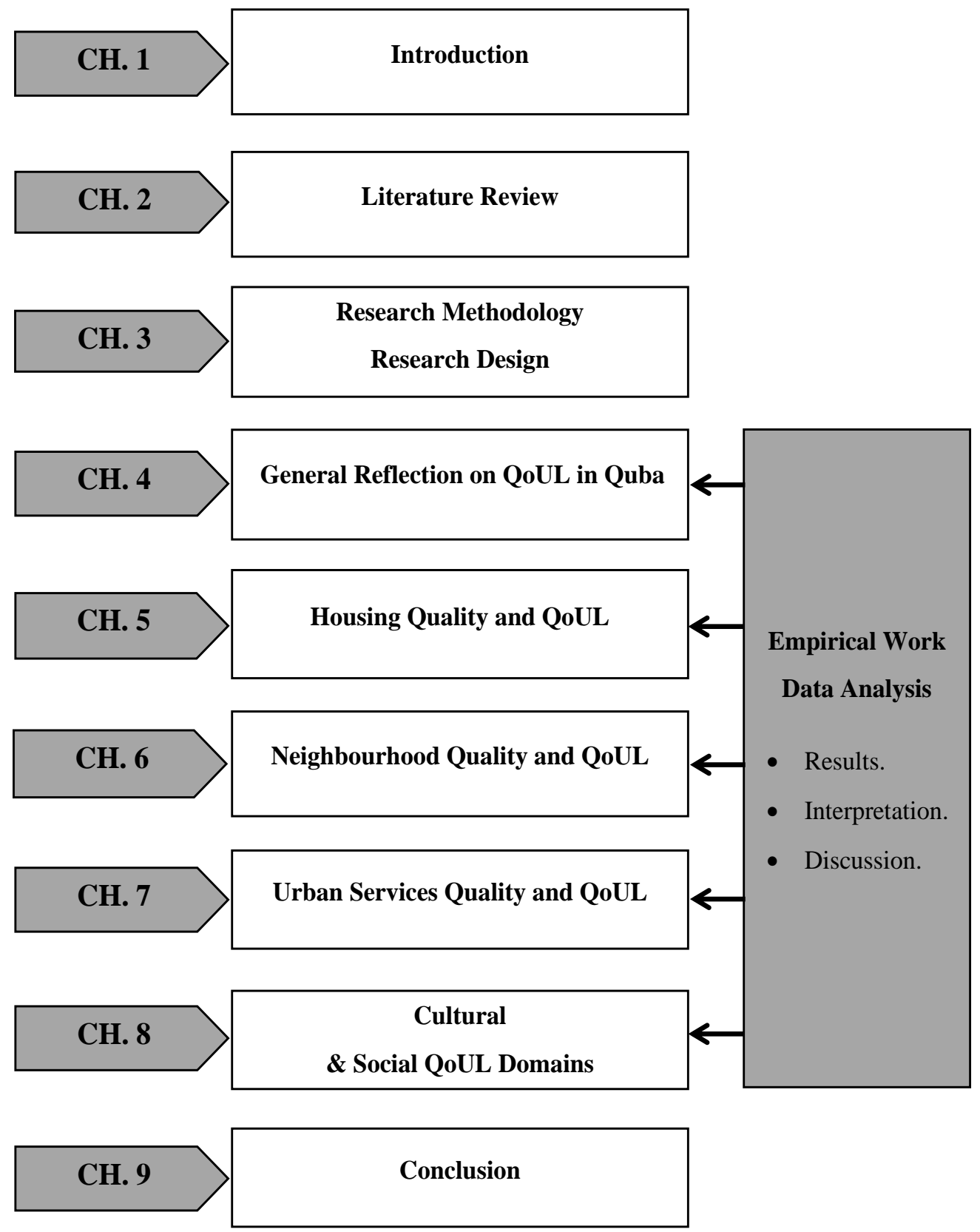

Fig 1.2: Thesis structure 


\section{Chapter Two}

\section{Literature Review}

\subsection{Introduction}

As noted in Chapter One, investigating the notion of QoUL has been a growing area of research across a range of disciplines, particularly over the past three decades. This growing interest in QoUL study was prompted by a range of factors, and perhaps most particularly, by uncontrolled urban growth in many countries of the world, especially the Global South, with the attendant economic and social issues that emerged alongside such rapid urban growth. In this context, and against the background of the urban growth witnessed in many cities in the modern era, QoUL has become an important tool in the field of appropriate planning for liveable and sustainable cities. Marans and Stimson (2011) point out that the importance of the investigation into the QoUL is not only in looking at how people behave in their environment, but also in arriving at the degree of satisfaction and happiness they have within that environment, which has seen broad effects in the research and urban policies in many regions of the world. Hence, investigation into this field helps to identify issues related to the social, cultural, physical and economic aspects of urban life, as well as identifying the causes of life dissatisfaction. This, in turn, can help to monitor and evaluate the strategies and policies related to QoUL, which further contributes to improving the overall QoUL.

This chapter reviews the notion of the QoUL by providing a deep understanding of the relevant terms and exploring the literature that deals with its definitions, domains and assessment. In line with the conceptual approaches used in this research, the chapter focuses primarily on addressing the conceptual and theoretical aspects of this notion. By highlighting a number of issues related to the social, cultural, physical and economic aspects, this research seeks to build knowledge about the QoUL more broadly in an attempt to fill the gap in the QoUL literature in Saudi Arabia. The chapter therefore firstly focuses on the definition and characteristics of QoUL, as well as some terms associated with this 
notion such as happiness, satisfaction, well-being and liveability. Secondly, it reviews the assessment and domains of QoUL. Within these stages of the review, related works on the investigation of QoUL in some selected world cities are reviewed, along with the related work to QoUL in Saudi Arabia and Medina in particular. In so doing, this chapter seeks to set out the theoretical and conceptual framework on which the analytical work of this research will be built. However, due to the multifaceted nature and complexity that distinguishes the notion of the QoUL, as well as the abundance of related works on this field, this literature review is not exhaustive.

\subsection{On the concept of QoUL}

There have been several attempts to develop a specific definition of QoUL by researchers interested in this term from many fields. Yet, there is no agreed definition of the QoUL due to the multiplicity and complexity of the aspects associated with it. Liu (1976) suggested that there are as many definitions of the quality of life as there are people. Baker and Intagliata (1982) likewise point out that there are as many definitions as the number of people studying the phenomenon, referring to the lack of agreement between those who are trying to activate the concept. In order to gain a better understanding of this notion, this section breaks down the phrase into its constituent parts, looking at the concepts of quality, quality of life (QoL) and quality of urban life (QoUL). In addition, terms associated with the notions QoL and QoUL, such as happiness, satisfaction, well-being, urban environmental, liveability, residential perception and sustainability, will be reviewed. Thus, different perspectives on the meaning of the QoUL are explored to gain more insight into this concept.

\subsubsection{Quality}

Chandrupatla (2009) defines the term 'quality' in the following statement: "quality denotes an excellence in goods and services, especially to the degree they conform to requirements and satisfy customers" (p. 2). From the perspective of customer-oriented quality, Drucker (1985) states that "quality in a product or service is not what the supplier puts in. It is what 
the customer gets out and is willing to pay for. Customers pay only for what is of use to them and gives them value. Nothing else constitutes quality" (p. 228). This user-driven definition of quality refers to service based upon that person's evaluation of their complete experience, or to the perception that the customer has of the product. What connects "quality" to "QoL", is the degree of attractiveness and ability to meet needs. In other words, the link between "quality" and "QoL" is the degree of excellence of a set of characteristics and features that should be available in the product or service (i.e., urban environment, QoUL components) that make it fully functional and thus satisfying the consumer (i.e., individuals, citizens).

Although the quality of the service or product can be easily measured in terms of functional and physical characteristics, there is a need to evaluate the perceptions of people regarding their experience. It has been argued that "quality" of any entity has a subjective dimension that is perceptual as well as having an objective reality. This applies to the environment where it can be defined as having built, natural and socio-cultural dimensions. Each environment will have specific characteristics with respect to those dimensions. However, the urban places where people live consist of all three of these dimensions. Thus, these dimensions are important components of the QoL (Marans \& Stimson, 2011). Therefore, this interaction between subjective and objective quality is one of the implicit aspects of QoUL research.

\subsubsection{Quality of Life (QoL)}

A wide range of QoL definitions have been found in the literature. However, there is no consensus on a specific definition of the term. It has been described in literature as a complex concept due to its multidimensionality. Dissart and Deller (2000) suggest that "QoL can mean different things to different people, encompassing such notions as well-being centred on the individual to good place centred on the location" (pp. 135-136). While Cutter (1985) defines the QoL "as the individual's happiness or satisfaction with life and environment including needs and desires, aspirations, lifestyle preferences, and other factors that determine overall well-being" (p.1). Another definition was determined by Pacione (2003) who states, "QoL refers to either the conditions of the environment in which people live, 
(air and water pollution, or poor housing, for example), or to some attribute of people themselves such as health or educational achievement" (p.19). According to Diener (2006, p.4), QoL usually refers to the degree to which a person's life is desirable versus undesirable, often with an emphasis on external components, such as environmental factors and income. In contrast to SWB, which is based on subjective experience, QoL is often expressed as more "objective" and describes the circumstances of a person's life rather than his or her reaction to those circumstances.

From the definitions above, it can be noted that most have a focus on well-being, which is considered, from the researcher's point of view, the primary objective of QoL research. However, some of those definitions do not include the main components of the work field, the QoL itself. For example, such definitions sometimes focus on psychological aspects of an individual (internal), while neglecting the physical aspects (external). Thus, the definition of QoL should not only be limited to environmental circumstances or objective aspects, but it should be broadly defined to include the relationship between individuals and the environment in which they live. In other words, the definition of QoL should include the circumstances of the environment as well as the human perceptions, feelings and thoughts towards that environment.

In the 1970s, pioneering work in the field of QoL research was conducted by a group of social science researchers, notably Campbell et al. (1976) and Andrews and Withey (1976). From the perspective of a sense of well-being, happiness and life satisfaction, Campbell et al. (1976) suggested that QoL is people's perception of and satisfaction with various domains of their life. Andrews and Withey (1976) described the QoL from a global perspective of well-being which is based on the individuals' thinking and feeling regarding their life concerns. Those researchers pointed out that the affective assessment can play an important role in an individual's perception of their life experiences.

Other researchers have utilised definitions of satisfaction and happiness to assess QoL. The Index of Well-Being developed by Campbell et al. (1976) for example, assesses satisfaction and happiness as a measure of QoL. Nevertheless, despite the relationship between life satisfaction and happiness, they are not synonymous (Holthus \& Manzenreiter, 2017). According to Kozma, et al. (1991) happiness is "a transitory mood brought about by the 
relative weights of positive and negative feelings" (p. 22), while Veenhoven (2001) suggests that "happiness is the degree to which a person evaluates the overall quality of his present life-as-a-whole positively. In other words, how much one likes the life one leads" (p.4). Life satisfaction on the other hand refers to the cognitive judgemental evaluation of an individual's life (Diener,1984). Shin and Johnson (1978) define life satisfaction as "a global assessment of a person's QoL according to his chosen criteria', (p. 478). Satisfaction judgements are based on a comparison of one's circumstances with what is thought to be an appropriate criterion. Therefore, it is important to note that the judgement of how satisfied individuals are with the current state of their lives depends on a comparison with a criterion which each person sets for themselves and it is not imposed externally (Diener et al., 1985). Life satisfaction is stable over age and across age groups even though there is a decline in some objective respects such as income and marriage that occur with respect to age (Diener et al., 1999).

From the above, it should be noted that happiness and life satisfaction are closely related to each other. Yet, they may still differ from each other ( $\mathrm{Ng}$, 2014). Happiness is considered as a reflection of one's present state of well-being, while life satisfaction is a response to external circumstances, and is influenced by personal background and environmental features. The measurement of happiness is an effective assessment that reflects how the respondent feels and it might be a transitory affective state. Life satisfaction is more than just an emotional state, it is a cognitive assessment based on the facts of the circumstances of the individual and derives from the comparison of the aspirations of that individual to their actual achievement (Zhan, 1992). Nevertheless, in short, happiness and life satisfaction are both considered as subjective dimensions of QoL. Thus, as a result, as they are often based on the attitudes and feelings of the individuals, they may be viewed as indicators that do not necessarily reflect the actual reality of QoL.

Looking more closely at the term "wellbeing," many social researchers have defined this as the subjective component of QoL. Indeed, several researchers evidence that wellbeing is the main indicator of QoL. It is generally understood that well-being is the emotional and cognitive assessments of individuals for their lives whether at a specific moment or for longer periods. Commonly, every person's emotions, moods and subjective evaluative judgements are known to fluctuate over time. Therefore, researchers investigate both these 
fluctuations and examine the longer-term mean level differences that exist between societies and individuals (Diener al., 2003). According to Haas (1999), the term well-being is often interchangeably used with QoL. However, it is not clear how well-being differs from QoL which needs to be clarified. As with life satisfaction, well-being relies on subjective evaluation. Therefore, well-being is often embodied in QoL definitions along with life satisfaction, as the components of the broader concept of QoL. However, well-being and QoL cannot be considered as synonymous because well-being is often purely subjective, while QoL has both objective and subjective aspects.

In summary, the concept of QoL has been discussed by many researchers in various fields (e.g., geography, health, economics, architecture and psychology). However, despite the intense interest in this field since the 1970s, there is still no consensus related to the definition of QoL, its assessment and components. In addition, there is still no consensus on what the best methods are to measure the QoL in a given place. This is due to the complexity and multidimensionality of this concept, as well as the weak relationship between subjective and objective indicators, which form the basis of QoL measurement. Furthermore, the concept of QoL is related to many concepts such as well-being, life satisfaction, and happiness that often overlap.

Whichever approach is considered, the relationship between people and the environment is an important consideration when QoL is assessed. As mentioned previously, this concept has been defined and discussed by many studies in various fields, each of which has discussed the concept from a particular perspective, with particular objectives for the research. For example, researchers in the field of health examine QoL from a purely health perspective, while economists discuss QoL from a purely economic perspective and so on. In terms of geography, a geographical perspective on QoL describes the relationship between man and the environment, as well as focusing on the spatial variation and locational advantages and disadvantages of QoL levels (Randall \& Morton, 2003). More importantly, the investigation of QoL which is conducted by geographers is often linked to the place (urban environment), which will be discussed in the next section (2.2.3). 


\subsubsection{Quality of Urban Life (QoUL)}

The term QoUL is not a simple term. It is a complex and multidimensional term which has been defined by various disciplines (Serag El Din et al. 2013). The multidimensional nature of this term reflects the variety and complexity of the examined dimensions in a given place (Psatha et al., 2011). While QoL as a concept is related only to human beings, QoUL is related to places, specifically a geographical aspect of QoL. Thus, the term QoUL usually refers to the well-being of people and the quality of the environment in which they live. Predominantly, it refers to both objective and subjective assessments (Dissart and Deller, 2000). Building on the literature, the term QoUL can be defined as "a reflection of the interaction between people and the urban environment in which they live. This interaction includes the satisfaction with many dimensions of life in the urban environment, such as the physical, economic, social and cultural dimensions, and the extent to which these dimensions affect daily lives of the residents."

Just as QoL is associated with terms such as happiness, satisfaction and wellbeing, other terms and concepts are also associated with QoUL as well. The literature identifies ideas such as urban environmental quality, liveability, quality of place, residential perception/ satisfaction and sustainability. Veenhoven (1996) defined liveability "as the degree to which the provisions and requirements of a given environment fit the needs and capacities of its citizens" (p. 8). According to this author, liveability is the extent to which nations meet the minimum food and safety needs. Thus, liveability embraces the QoUL, especially with regard to the ability of the urban environment to meet the needs of its citizens. With respect to sustainability, the UN World Commission on Environment and Development Report (1987) defines it as "the effective use of natural, human, and technological resources to meet today's community needs without compromising the ability of future generations to meet their needs" (p. 43).

In his conceptual framework, Shafer et al. (2000) shows how the concepts of QoL, liveability and sustainability relates to each other. In this way, liveability is considered as a result of the interactions between the economic and physical domains. This interaction between these three domains is defined as QoUL (see Figure 2.1). This conceptual model was developed to recognise the basic relationship between component parts of a place in terms of the social, economic and physical realms. In addition, the model highlights how 
QoL is created by the interaction between economic, community and environmental quality. Despite its broadness, the main feature of this integrated approach is that it provides points of departure for both assessment purposes and policy tools. A fundamental implication of this approach is that social, environmental, spatial, economic and land-use planning cannot be separated from each other. For instance, in order to maintain the cohesion of society, economic benefits must be equally distributed to all groups of the community as well as taking into account economic sustainability.

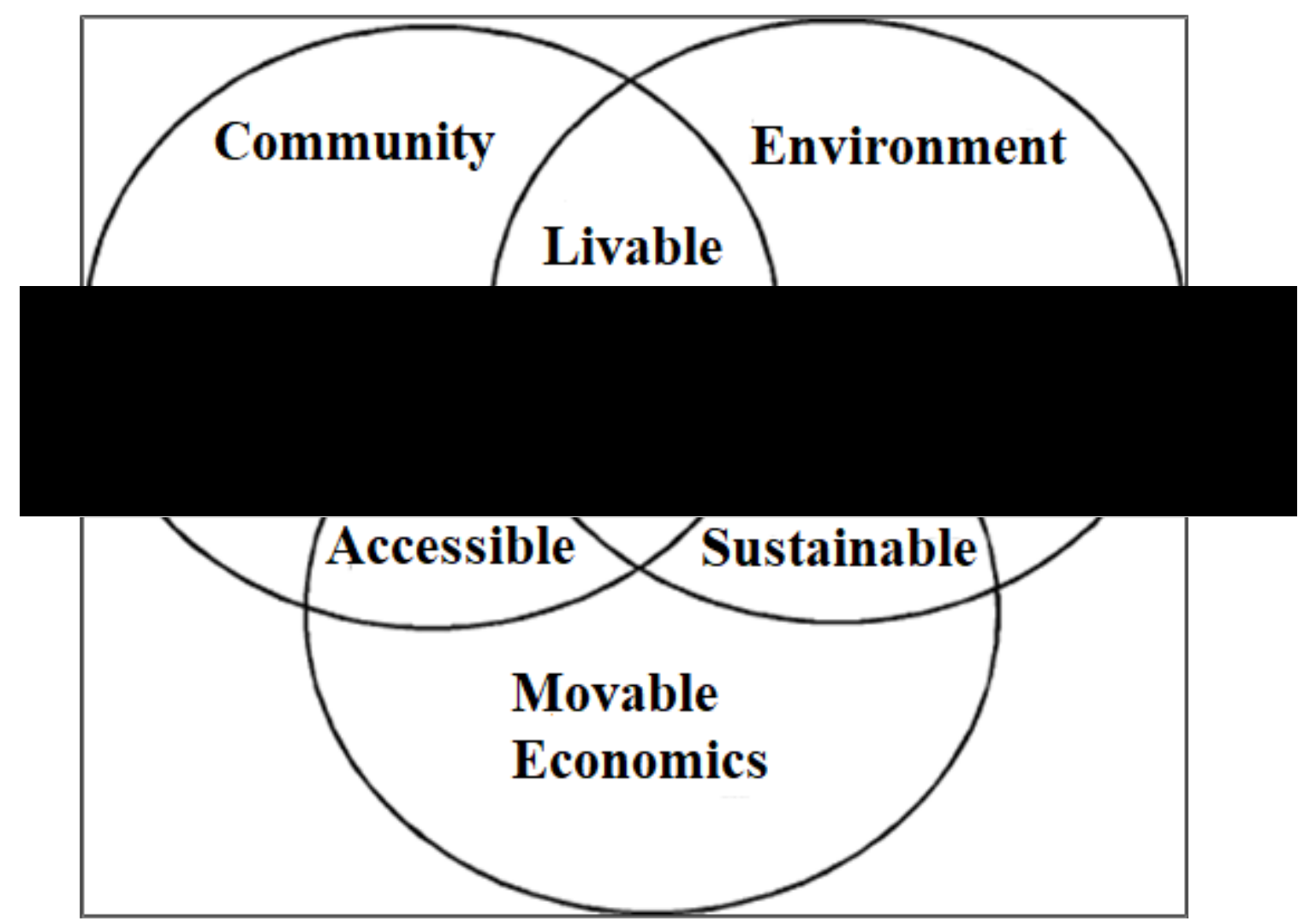

Fig 2.1: Conceptual model of factors that contribute to community QoL from a human ecological perspective (Shafer et al., 2000, p. 166)

Baycan-Levent and Nijkamp (2006) suggested that QoUL refers to the degree of satisfaction or the excellence of urban life. They also look at this term as the performance level of urban life towards the community's needs, evaluating the term from a taxonomic perspective, looking at how it addresses many dimensions of QoUL that range from environmental to economic and social components (Figure 2.2). The authors point to the concepts of quality, 
QoL, quality of environment, measurement of QoL, liveability, and sustainability, referring in addition to associated concepts such as satisfaction well-being and performance. In their evaluation of the multidimensional aspects of QoUL, the authors have tried to address the implications of urban and spatial planning with respect to the dimensions associated with QoUL.

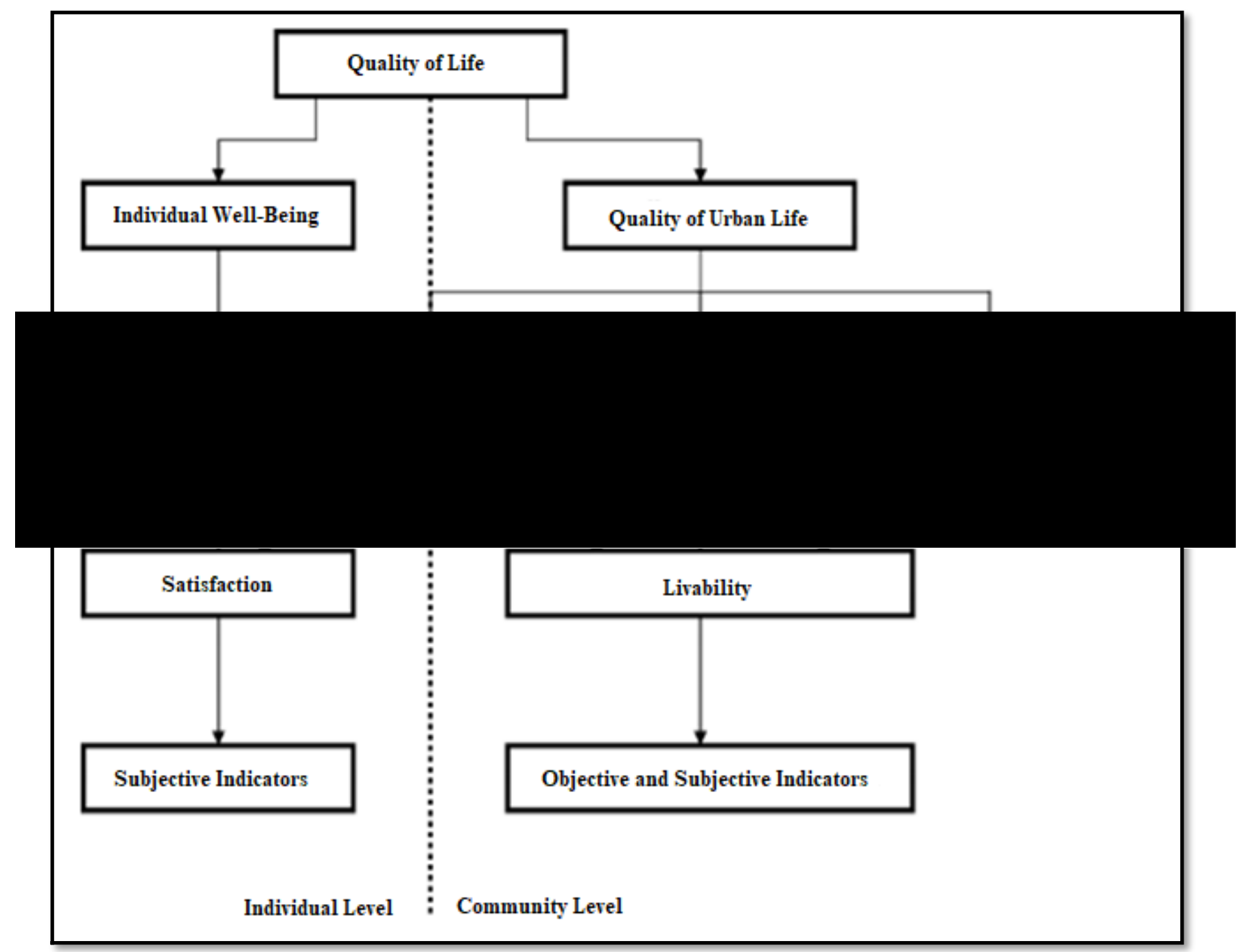

Fig 2.2: Components of QoL (Baycan-Levent \& Nijkamp, 2006, p. 272)

Campbell et al.'s (1976) research, explaining the relationship between various domains of satisfaction, is considered to be one of the most highly referenced conceptual frameworks in the literature on QoL. The authors regard the evaluator or features of the person and the context is key importance in understanding QoL. The context refers to the objective 
attributes and is considered to be the actual status. The authors compared the assessments of individuals and measured many domains of their lives, and then determined the way the degree of each domain explicated the experience of QoL. They found that the domain of satisfaction is a reflection of individuals' perceptions and the way they make assessments of domain attributes, which are often influenced by objective characteristics. (Campbell et al., 1976). It has been emphasised that the geographical setting or quality of place (city, neighbourhood or housing) is a subjective phenomenon. Thus, a person who lives in that place may have different opinions about it, which in turn are likely to reflect assessments and perceptions affected by their own individual characteristics, desires and past experiences.

The model created by Campbell et al. (1976) (see Figure 2.3) clarifies the relationship between various residential domains and shows how these domains combine with other satisfaction domains contribute to QoL. The model is based on four main principles: first, the experience of people is derived from their interaction with the associated objective attributes; second, the subjective experiences of people are different from the objective attributes; third, people respond to their experiences with the environment; fourth, the level of satisfaction in various life domains contributes to the overall QoL experience. It can be noted that this model identifies a series of different objective features of each life domain and satisfaction assessment of these domains, which in turn is affected by individual standards of comparison and the characteristics of the individual. 


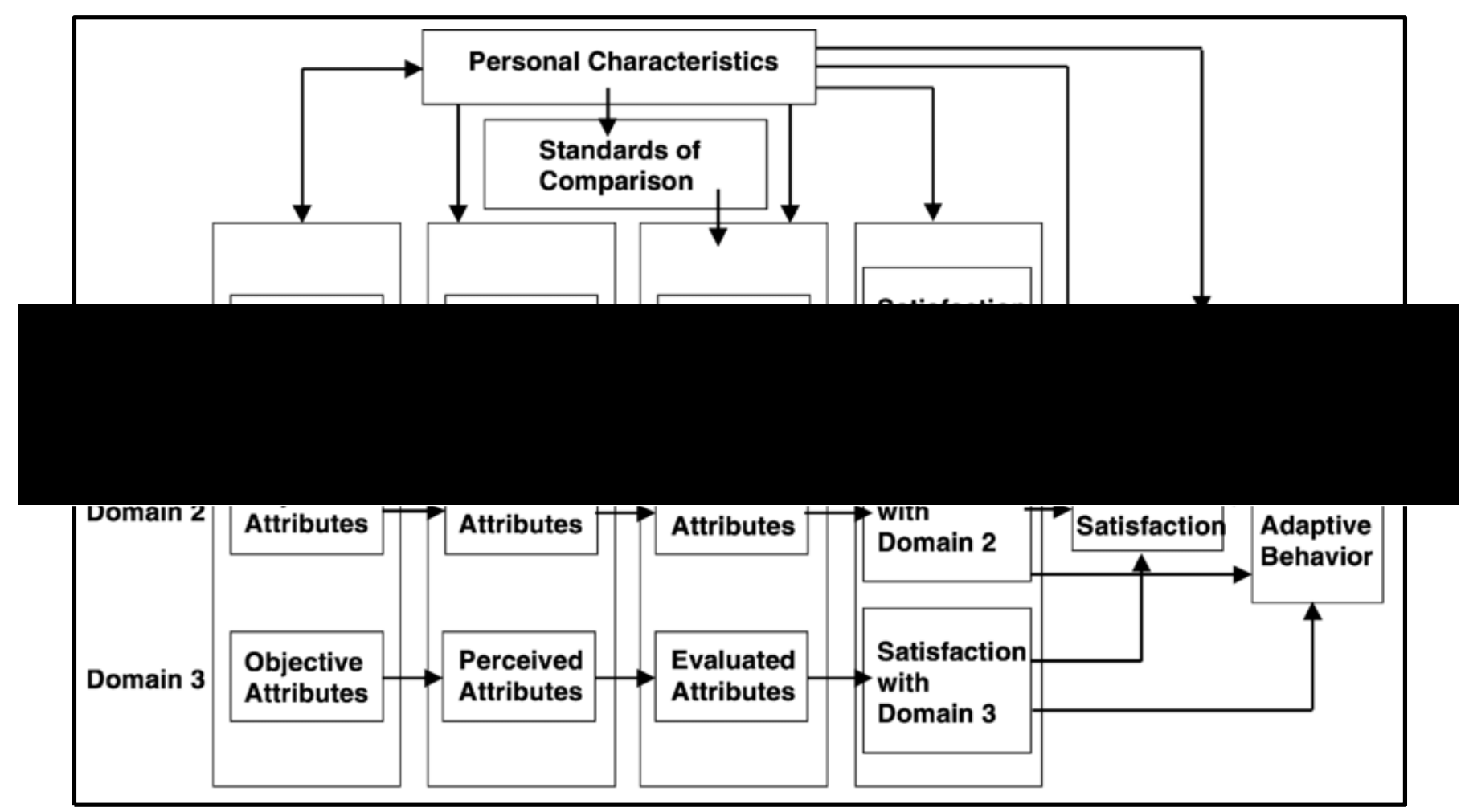

Fig 2.3: Relationship between domain satisfactions and life satisfaction (Campbell et al., 1976, p. 16)

Marans and Rodgers (1975), cited by Marans (2012) have proposed a similar model to show the relationships between the domain of residential satisfaction and QoL, and how those domains along with other domains contribute to QoUL (Figure 2.4). The model reflects the following assumptions: (1) that the quality of the environmental or geographical setting (region, city, or neighbourhood) cannot be captured through a single measure, it requires the assessment of the multiple features of those settings; and (2) the QoL is a subjective phenomenon which reflects the life experiences of the residents where the objective conditions of the environment themselves do not reflect the true QoL of that setting (Marans, 2012, p.14). 


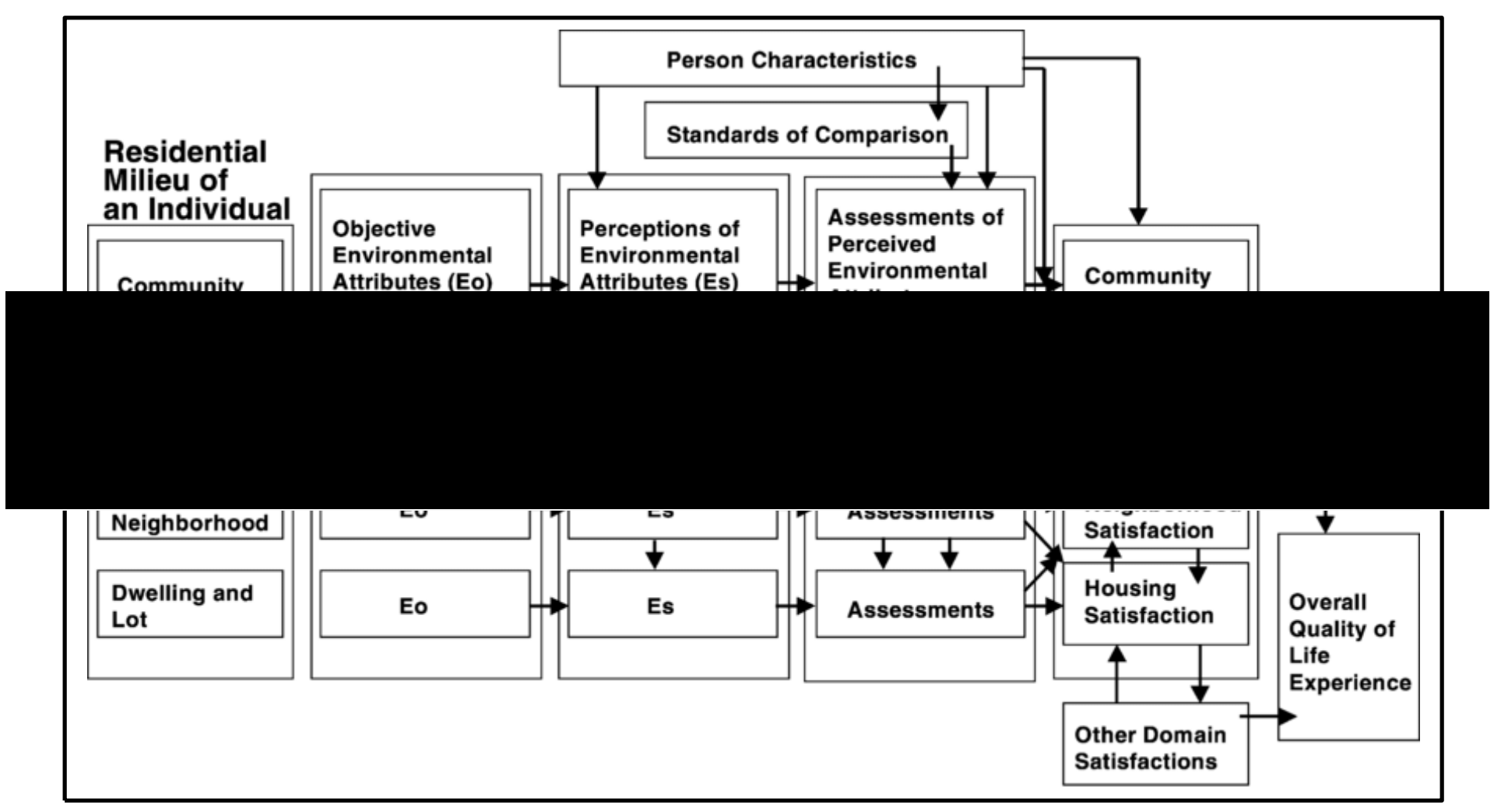

Fig 2.4: Relationships between residential domain satisfactions and QoL (Marans, 2012, p. 14)

Another key model is that of Marans (2012), illustrating the relationships between objective conditions, subjective responses, and neighbourhood satisfaction (Figure 2.5). In this, different satisfaction domains, including satisfaction with the place, are considered to be important results worthy of research, whether from a policy or theoretical perspective. Policymakers are often looking for the most effective means of enhancing satisfaction with the areas within their remit, and there is, therefore, broad agreement that satisfaction as an individual well-being indicator is one of the important outcomes of QoUL research. However, there are other results of significance in terms of well-being that may be tested in QoL research (Marans, 2012). 


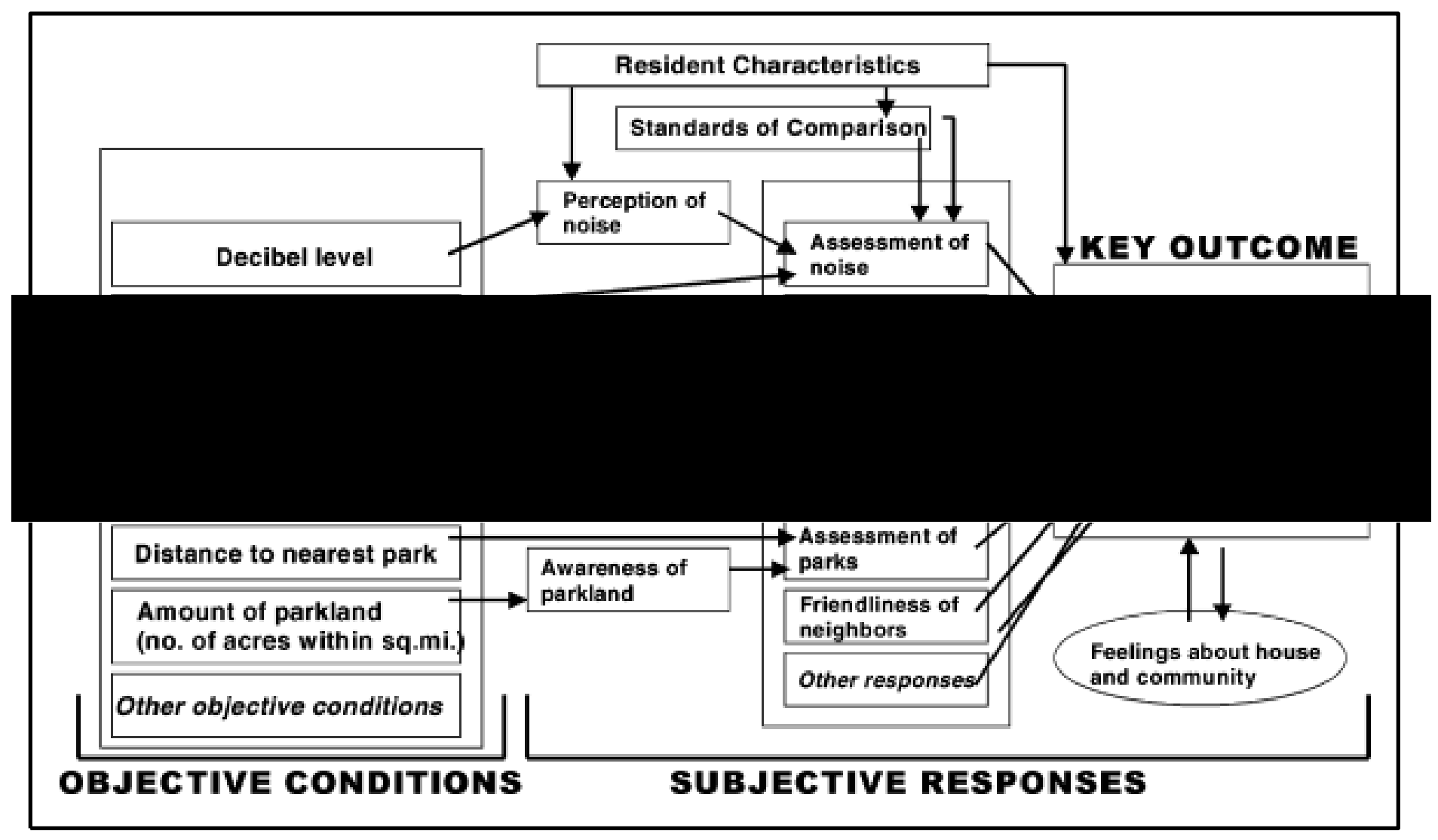

Fig 2.5: Model showing relationships between objective conditions, subjective responses, and neighbourhood satisfaction. (Marans, 2012, p. 15)

McCrea et al. (2005) suggest that the QoUL can be defined in two broad ways of traditional measurements. One of those traditional measurements is subjective measurement where QoUL is conceptualised as a satisfaction in a set of urban domains, such as neighbourhood and housing, which, along with satisfaction within other domains, may contribute to the general satisfaction of the individuals' lives. Another traditional measurement is objective measurement where QoUL is conceptualised as an average of different objective measures of the urban environment such as housing costs, crime rates, pollution levels and so on. To explore QoL in the living environment, Das (2008) has created a conceptual framework illustrating the relationship between the urban environment and the QoL (Figure 2.6), based on the combination of objective circumstances of living conditions and the subjective evaluation of those living conditions by individuals themselves. In this conceptual framework, the external condition of the social, economic and physical environment 
comprises the objective QoL, and satisfaction with that condition comprises the subjective QoL. An important aspect to note from Das's conceptual framework is the interactions between people and their urban environment which contribute to the overall QoL.

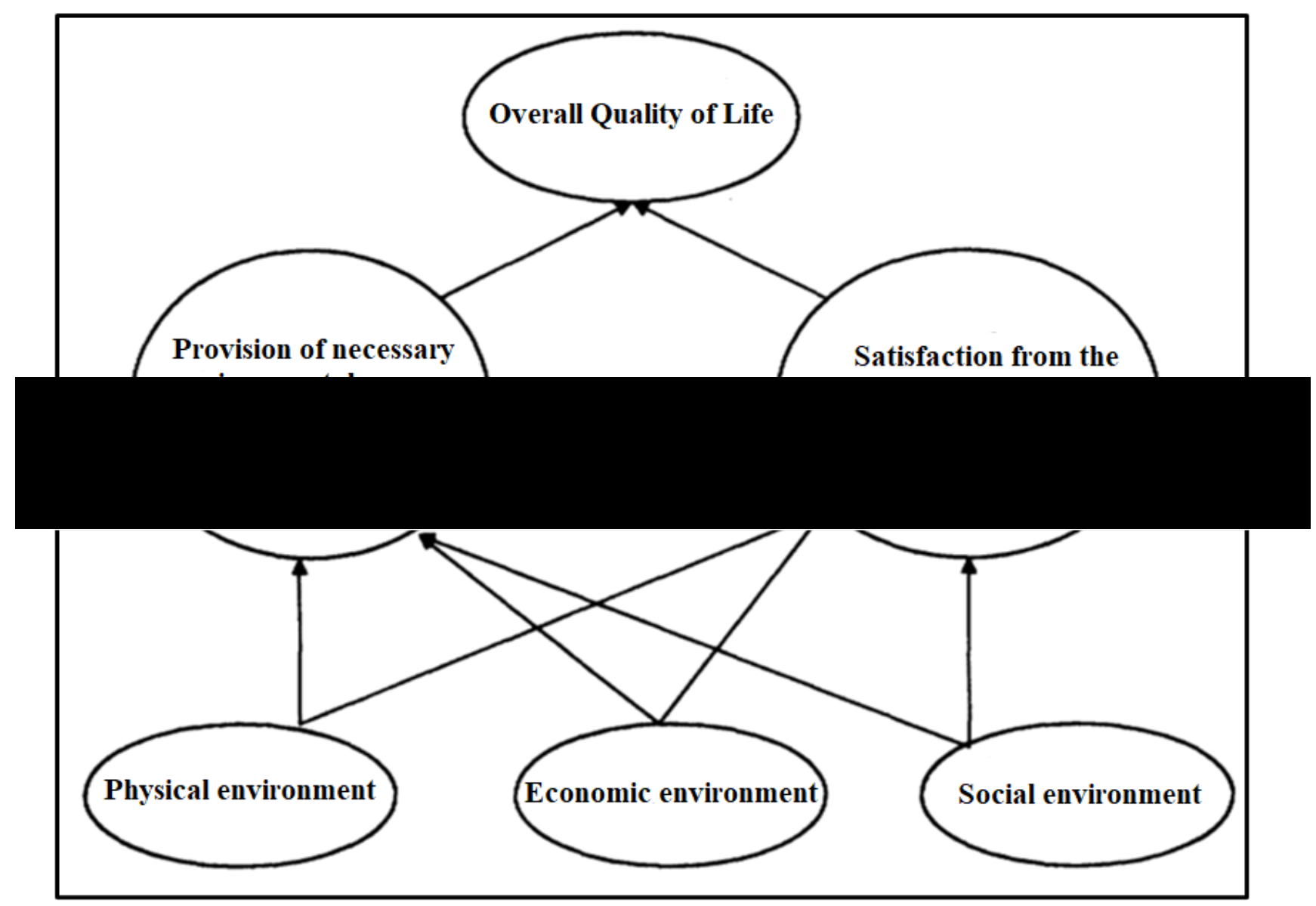

Fig 2.6: Conceptual framework showing relation between environment and QoL (Das, 2008, p. 301)

In contrast to many of the studies reviewed above, Richard Florida uses the term "quality of place" rather than the traditional concept of QoL (e.g., Florida, 2005; Florida, 2014; Florida \& Small, 2016), maintaining that the concept of "place quality" refers to the unique characteristics which determine the place and make it more attractive. In this regard, Florida divided the quality of place into three key dimensions in the form of three questions. (1) What's there: the combinations of the built environment and the natural environment; (2) who's there: the diverse kinds of people; and (3) what's going on: the vibrancy of street life, 
arts, music and people engaging in outdoor activities (Florida, 2012, pp. 280-281). Thus, in terms of understanding the characteristics of the urban environment, the focus emerging from these three questions falls on (1) the built environment, which refers to the objective indicators of QoUL (uses), (2) people who live in that environment (users) and (3) life experience resulting from the interaction between people and that built environment. Accordingly, those key aspects of the urban environment can shape the overall QoUL.

Although in most of his works on quality of place Florida has focused on certain categories of people, such as creatives, talented, bohemians (Florida, 2003), he does refer to some key indicators that can be used as QoUL indicators such as social interaction and diversity. In addition, he set out a number of markers for a city's quality of place that can be considered as factors that can enhance QoUL. One marker concerns the support of diversity within the built environment, where such diversity has the potential to make a city a cauldron of creativity. Other markers include among their key elements a number of indicators of social life such as leisure, security and health as well as economic support (Mail, 2014).

Despite the many conceptual frameworks presented in the literature, there is no agreed single/overarching conceptual framework, perhaps due to the multiplicity and complexity of the concept, resulting in differing points of view among researchers. However, it can be concluded from the studies reviewed above that at its basis, the concept of the QoUL symbolises the relationship between people and their surrounding environment. In that sense, QoUL from the geographical perspective refers to the interaction between the components of the urban environment, which consists of a variety of complex indicators.

\subsection{Conceptual Approaches to QoUL}

Having explored the terminology behind QoUL and the varied approaches taken within the literature to determine how QoUL might be constituted, the following section turns to examining the two particular conceptual approaches adopted by this thesis that seek to offer an alternative avenue for expanding or deepening understanding of the QoUL. The conceptual approaches this thesis focuses on are those of functionalist and postmodernity, 
and each of these are discussed at length below with the aim of eliciting key ideas embodied within them to aid QoUL investigation.

\subsubsection{Functionalist Approach}

Before taking up the functionalist approach, the notion of functionalism needs to be briefly highlighted. According to Harvey and Holly (2014) functionalism is the notion that is concerned with functions and the function analysis of particular customs, acts and artefacts within the community (p. 73). In another definition, functionalism is a term associated with the social sciences, used to explain the behavioural and social phenomena in terms of the function and the role played by those phenomena in maintaining the general order of which they are considers a part (Gregory, 2009). In the late nineteenth century, functionalism become one of the most important perspectives on human affairs (Benko, 2004). As with any concept within the conceptual frameworks of social sciences, however, functionalism definitions have differed across disciplines. For the geographic field, Harvey and Holly (2014) note that several geographic issues can be studied and understood within the functionalism framework where that might provide a methodological and philosophical umbrella for various subjects in this field.

Generally, the main development of functionalism is associated with the work of sociologists such as Emile Durkheim, Alfred Radcliffe-Brown, and Bronislaw Malinowski among others (Harvey and Holly, 2014). For example, Emile Durkheim concentrated on the order issues and the positive effects of social institutions and explained their presence in terms of their necessary functional contribution (Pope, 1975). From this point of view, social institutions are considered as one of the most important structural components of the societies that address a specific function or basic activity such as education, economy, religion, and so on. From the functionalist perspective, these specific activities are considered to be all related to each other within the social institution's framework (Verwiebe, 2014). By looking at those social institutions and their importance for enhancing social life, it should be noted that the relationship between the functionalism and QoUL lies in the functional necessities of the community system, including the environment and the requirements of human life. Thus, the functional contribution of social patterns to meet the requirements of the social system would maximise the QoUL (Ferriss, 2004). 
QoUL study is concerned with places and the relationships resulting from the interaction between these settings and those who occupying them. In the modern era, more people live in the urban setting, that is, in cities and metropolitan areas (Stoltman, 2012). Therefore, the functionalism perspective focuses on the advantages offered by those cities to the community, where the assumption is that cities serve several significant functions for society. In the field of QoUL, these functions refer to the physical attributes that distinguish each city, including housing, neighbourhood and city services such as transportation, health care, recreation and other basic urban services, which are classified as objective indicators of QoUL (Delsante, 2016). Thus, the functionalist approach to QoUL involves thinking about functional attributes and the extent of their contribution to the quality of the urban environmental.

The idea of functionalism as described by Durkheim is an integration of social groups or the society as a whole, and to achieve this, the constituent parts must contribute to their comprehensive process, and these contributions are necessary (Benko, 2004). This point of view could be applied to QoUL. To illustrate this, when objective indicators of QoUL that represent the patterns of the urban environment are being aggregated as basic uses and functions, then the needs of people are likely to be met, thus enhancing the QoUL (Saeidi \& Oktay, 2012). Based on functionalism perspective, however, failure to provide consistency between those functional attributes in the framework of the urban environment may result in a defect in the overall QoUL. In a very narrow sense, the functionalist approach focuses on the role of housing, neighbourhood and other support services as basic functions that reproduce specific spatial frameworks. In this context, the primary task of any city is considered to be the provision of quality basic services that are in line with the needs and aspirations of its residents. 'Functionality' of the city is explored in relation to a system of concrete choices and actions that make everyday life smoother for the residents. In terms of the QoUL, the functional aspects are often represented by the services provided by the city, such as health, education, recreation and other services. In this regard, the study of QoUL is mostly concerned with the analysis of the satisfaction the residents demonstrate with these services and potential ways of improving this satisfaction.

However, the broader focus of the functional perspective extends beyond the analysis of the mechanisms that recreate and reproduce the city's built environment and includes the 
exploration of the importance of social institutions. Given the recent changes in the urban and social environment in Saudi Arabia, there are a number of areas that illustrate how the functional approach can re-evaluate the QoUL. For example, recent changes in transportation and mobility, which are linked to the transformation of the built environment, also have an impact on the development of social and cultural aspects of urban life. In the study area, the transformation of Quba Street into a walking route has led to the development of social aspects of urban life including increased social interaction, intensified use of community spaces, and the development of diverse relations between different social groups. From the functionalist perspective, such developments are seen as contributing to the increase in the accessibility and "publicness" of urban space (Zukin, 1995, p.11). Moreover, the change in these functional attributes has also led to the increased sense of symbolic control and stronger spiritual connections, as this route links two holy landmarks, the Prophet's Mosque and Quba Mosque, the most important Islamic landmarks (WAS, 2015). In this context, the Quba route not only improves transportation options and residents' mobility, but it also affects social and cultural aspects of urban life. Creation of this walkway also increased user diversity and public participation in creating more liveable urban spaces (Carr and Lynch, 1981), with a more balanced mix of activities and user groups (particularly women) occupying local streets. The resultant improved gender mix in the area has also led to a re-thinking of the recreational strategies developed in Quba municipality and the re-evaluation of the meaning of this type of functional attribute. As this example demonstrates, the functionalist approach can help to evaluate not only changes in the urban infrastructure, but also in understanding the impact of these changes on people's perceptions of the urban environment and in the re-evaluation of the QoUL. However, the functionalist approach does not fully take account of broader cultural diversity and creation of difference, which are reflective of the recent social changes in Saudi Arabia.

\subsubsection{Postmodernity Approach}

To address some of the limitations of the functionalist approach, this thesis attempts to engage with the ideas of difference producing values (high/low, aesthetic/useful) that exceed the existing measurements of QoUL and consider often overlooked meanings attached to cultural attitudes and spiritual engagements with urban environments. At its simplest level, postmodernism came as reaction to modernism and a movement of thinking 
(Harvey, 1990, p. 7). According to Unwin (1992) it is concerned both with a critique of modernism, and more importantly with a search for difference. As such, it has at its basis a rejection of grand theory, and a desire to create a new intellectual and physical environment for human existence (p.178). Gregory (1989), as cited by Unwin (1992) sees postmodernism as combination of three main features: it is suspicious of systems of thought that claim to be complete and comprehensive; it is hostile to the totalising ambitions of the conventional social sciences (and, for that matter, those of the humanities) and one of its key characteristics is its accent on heterogeneity and difference. Postmodernism can therefore be seen as encompassing a range of arguments critical of the all-embracing theoretical certainty associated with logical positivism and capitalism (Unwin, 1992).

One key feature of postmodernism is the focus on difference. For QoL, the difference lies in ordinary individuals' everyday lives, including differential practices, perceptions and experiences of the city. In this context, the organisation of both time and space has become more flexible, with individuals dismantling and rebuilding traditional patterns of individual pathways and paths of life. Thus, the traditional lifestyles of individuals have become more differentiated, whether in terms of ethnicity, recreational taste, sexuality, mealtimes, patterns of work and lifestyles (Bramham, 1997). Moreover, postmodernism refers to a new condition that modern society and culture enters. Thus, in this new condition, the forms of technology and information have been changed (Oktay, 2016). This may be due to the shift in culture, society, technology and subsequent transformations in transportation and communication.

As a historical moment, postmodernism has grown out of modernity and, despite some distinguishing markers of difference with the modernist age, it is important not to overexaggerate this fissure (Nayak \& Jeffrey, 2011). "The idea of linear chronology is itself modern. Modernity and postmodernity then link and intersect each other as part of a continuum, as a subtly illustrated in Warhol manufacturing plant of pop art." (Lyotard, as cited by Nayak and Jeffrey, 2011, p. 205) In his book Postmodern Geography, Minca (2001) suggests that there are three major references to postmodern thought: namely, (1) a series of distinctive and cultural stylistic practices that are in and of themselves intrinsically interesting; (2) the totality of such practices, viewed as a cultural ensemble characteristic of the temporary epoch of capitalism (usually distinguished by the term post modernity); and 
(3) a philosophical and methodological discourse antagonistic to the precepts of Enlightenment thought, most especially the hegemony of any single intellectual persuasion (p. 1).

For urban geography, Pacione (2005) pointed out that postmodernism explores the place of different social groups in the residential mosaic of the city by focusing on the lifestyle and residential experience of various populations such as ethnic minorities, affluent groups, the elderly, disabled, and the poor. According to Dear (1986, as cited by Peet, 1998, p.218) postmodern geography first emerged in 1986 with publications on postmodern urbanism and planning. Postmodern conscious human geography derives from diverse debates on perspectives on social theory, work on the cultural landscapes of modernity, and the notion of flexible specialisation. For Dear, geography has been most strongly influenced by postmodernism in the following aspects:

- Cultural landscapes and place making; with an increasing emphasis on the urban.

- The economic landscapes of post-Fordism; with particular interest in global-local connections and the spatial division of labour.

- Philosophical and theoretical disputes related to space and problems of language.

- Problems of representation in geographical writing and cartography.

- Politics of postmodernity, feminist geography's discontent with postmodernism, questions of post-colonialism.

- The construction of the individual and boundaries of the self (i.e., the issue of identity).

- Reassertions of natural and environmental issues, which has taken many forms, including a fresh look at the relationships between place and health.

Postmodern theory began to influence urban geography between 1980 and 1990. The postmodern perspective is certainly characterised by an emphasis on human difference and a rejection of the grand theory. In the field of social geography of the city, the most important contribution of the postmodern perspective is the way in which it places the focus on the different and unique needs and situations of all individual members of society. Such emphasis has been reflected in gender difference studies in urban labour markets, and exclusion areas occupied by minority groups, in sexuality, social status, disability, age and 
race. There is considerable criticism directed at the postmodern approach to the city in that relativism seems unlimited. Because it privileges the views of all individuals, there appears to be no limit to the range of possible interpretations of any situation, there is "no real world" (Aguilar, 2009).

According to Pacione (2009) the study of urban phenomena from multiple perspectives of diverse individuals and groups is considered as an integral part of cultural transformation in urban geography. In addition to fundamental societal divisions based on class and ethnicity, postmodernism has helped to focus particular attention on social divides based on gender and sexuality as presented in feminist geography and in queer theory. Men and women not only use cityscapes in different ways but experience and perceive them differently. City environments both create and reflect gender roles in society.

Postmodernism refers to the postmodern condition of a society, a consequence of globalisation and information technology, and the differentiation between lifestyles and public benefit. Postmodernism is an intellectual and cultural phenomenon. Alongside the term/phrase postmodernist society, which refers to the measurement of the expression of the phenomenon within the social realm, economics, and politics, there is postmodernism, which also refers to many of the approaches that emerged as a response to modernism in architecture and other forms of art, as well as within religion, philosophy, culture and society (Oktay, 2016). For the QoL, the real importance of postmodernism lies in the way in which it represents a shift in thinking about style and aesthetics. Postmodernism not only searches for specific patterns, but also presents some alternative values of QoL. These alternative values challenge many of the normative values and principles that urban planning has developed in modern times. From that point of view, postmodernism presents an issue for the serious reconsideration of urban planning purposes (Taylor, 2010).

The central values of postmodernism are a celebration of the city's greater life because of its diversity and pluralism, and the freedom of choice that results from this diversity. However, these values link postmodernisms with liberalism, where liberals celebrate the opportunity through which individuals can experience their freedom of choice (Taylor, 2010 , p. 166). This may be familiar in some societies, especially in the advanced world, while it is undesirable in other societies, a situation that may apply to the Medina society, which is often subject to culture and religion as one of the main tools of state policy. 
Nevertheless, when diversity is seen as one of the positive aspects that enhance QoUL, diversity of uses is taken into consideration. This diversity of uses covers a range of functions and activities such as educational facilities, recreation, playgrounds, cultural institutions as well as accommodation types. (Saeidi \& Oktay, 2012)

Social and cultural changes can be noted in the physical reality of urban spaces. Urban areas reflect those changes, and therefore the postmodern city emphasises the QoL experiences and the importance of the city. Heritage features as an example of one of the most important aspects of QoUL, being developed in an attempt to produce an attractive environment for tourism and trade (Bramham, 1997). Medina city, as one of the world's historic cities for example, includes a number of sites and historical monuments such as mosques and religious shrines which are visited by millions of visitors annually from around the world. Along with the religious importance of Medina, religious heritage is considered one of the most important elements of QoL in Medina. At the global level, by improving historic neighbourhoods, gentrification of certain residential areas, or transforming waterfront areas, the city reflects the image and expectations of a postmodern audience.

\subsection{Quality of Urban Life Assessment}

In order to understand the QoUL in a geographical setting, there is a need to measure conditions using sets of domains and indicators (Marans \& Stimson, 2011). Commonly, QoUL can be measured either objectively or subjectively (Yinshe, 2005; McCrea et al., 2006; Marans \& Stimson, 2011). However, there is a debate about which approach is better. Despite the weakness of the relationship between objective and subjective indicators, most researchers would agree that objective as well as subjective indicators are necessary to measure QoUL. These two indicators are discussed below.

\subsubsection{Objective Indicators}

Objective indicators are mostly available through using official sources such as statistical data and censuses (Marans, 2012). These indicators have obvious advantages including data availability, comprehensiveness, and inter-community comparability. Nevertheless, using such indicators is considered an indirect measuring method and, therefore, the results 
depend on the interpretation of these indicators (Yinshe, 2005). According to Van Kamp et al. (2003), objective indicators are necessary for aspects of the urban environment which are hard to assess. In addition, they shape the departure point for environmental policy and enable the validation of subjective measures.

Early studies into QoL were dominated by the Social Indicators Movement, where there was an attempt to model QoL in terms of social or demographic variables which are objectively defined. Dale (1980) described objective indicators of QoL as "counts of various phenomena, such as level of income and education, residential densities and unemployment figures" (p. 504). This information was easily available, but it was soon recognised that objective measures revealed little regarding individuals' experience of life. Instead, further research was needed on subjective measures of satisfaction and happiness to deal directly with the individual's sense of well-being (Smith \& Clay, 2010).

\subsubsection{Subjective Indicators}

Subjective indicators are based on direct reports from people that contain their feelings and perceptions which are often contrasted with the objective indicators (Andrews, 1974; Haslauer et al. 2014; Yinshe, 2005). In other words, subjective indicators refer to the opinions of the individuals regarding the environment in which they live. These indicators are obtained through surveys and interviews and often assessed on a Likert-scale (Marans \& Stimson, 2011; Marans, 2012; Haslauer et al., 2014; Soleimani et al., 2014). Subjective indicators of QoUL facilitate the gaining of insight into the satisfaction/well-being of an individual, and into what individuals consider important. In addition, they contribute to the commitment of those individuals to their environment, and to the creation of public support (Van Kamp et al., 2003). In this context, Abrams suggests that "claims for the utility of perceptual indicators resides not in the argument that by themselves they can provide policy guidance and measures of policy evaluation, but that they are a necessary part, in conjunction with objective indicators in any adequate process of societal decision making" (Abrams 1976, as cited by Noll, 2013, p. 8). Thus, it can be noted that most researchers in the literature claim that subjective evaluations which contain the sense of individuals are necessary for obtaining the direct information about their evaluation regarding the objective domains of the environment in which they live. 
However, the sole use of subjective indicators as a way to measure QoL has been criticised by many social researchers. Many of these critics contend that subjective reports of wellbeing might be measuring internal dispositions more than external conditions related to QoL (Felce \& Perry, 1995). For instance, similar living conditions can, and have been, assessed differently where the individuals who live in substandard conditions may report being satisfied, while individuals living in appropriate conditions may report their dissatisfaction (Christoph \& Noll, 2003). The main difference between the two types of indicators is that objective indicators seek to assess the material living conditions of the urban environment, while subjective indicators measure individuals' perceptions of these conditions. Although researchers may have a favoured approach, the majority accept the basic assumption of behaviourism that individuals' perceptions and their material conditions normally match each other.

Nevertheless, some researchers argue that QoL is a subjective phenomenon and may or may not be related to desirable objective characteristics of the urban environment (Grayson, 1998). They emphasise that QoL is more appropriately measured by individuals' evaluation in the physical and social realms of the urban environments in which they live. However, an approach using just subjective indicators is imperfect, because people being interviewed may have their own interpretations about a matter and assess it according to different considerations. In fact, most researchers in the field of QoL provide evidence that the two types of indicators are complementary to each other, and therefore they can compensate for the defects of the other (Mohit, 2013). Thus, the study of the QoUL is appropriate to combine subjective evaluation of the residents about their environment with objective domains (Cobb, 2000).

Andrews and Withey (1976) are amongst those who suggest that both subjective and objective indicators are important for measuring QoL. According to these authors, the relationship between the conditions of the urban environment and the sense or perceptions of people about their environment, can only be shown when subjective and objective indicators are measured at the same time. In addition to the relationship between subjective and objective indicators, Dale (1980) confirms that most researchers in the field of social well-being consider the 'softer' subjective measures as a beneficial and necessary supplement to the 'harder' objective measures. 
To sum up, the correspondence between objective and subjective QoUL has consistently been shown to be weak or limited, indicating that an individual's perception of satisfaction and happiness is not necessarily strongly linked to the actual status of their lives at any given time (Abbey \& Andrews, 1985). Moreover, the influence of personality traits might serve to weaken the correlation between subjective and objective indicators (McCrea et al., 2006).

\subsection{Quality of Urban Life Domains}

The urban environment in which people live can be depicted from different perspectives, each representing a specific facet of life. In this way, urban environmental quality can be measured by using a set of domains or components followed by a number of sub-domains or indicators (Shoeibi et al., 2015). According to Yinshe (2005), these domains can usually be determined through a logical process of deconstructing the generic goal of QoUL. Frequently, each domain of QoUL can be broken down into measurable indicators that depict the overall status of QoUL. This section reviews the QoUL domains and their indicators that have been discussed by a number of previous studies in order to identify the most common domains of QoUL, which then can help to propose preferred candidate domains and indicators for this research.

From an urban social geographical perspective, Smith (1973) identified six domains of QoL, namely economic status, environment, health, education, social disorganisation, and participation and equality. He then broke those domains down into forty-eight QoL indicators (cited in Yinshe, 2005). By summarising QoL domains from those six selected prototype studies, Yinshe (2005) selected eight domains for the study of Saskatoon neighbourhoods. These are, housing, health, employment and income, land-use and environment, crime and safety, education, social environment and services, and community participation. Shucksmith et al. (2009), on the other hand, have determined seven life domains to investigate QoL in 25 member states of Europe. These domains are income and deprivation; housing; educational and internet use; employment and working condition; work-life balance; access to work; school; family; friends; services and SWB. By comparing these two studies, it can be noted that the most common indicators are housing, education, services, income and employment. While a health indicator has been included in Yinshe's criteria, it is missing in the study conducted by Shucksmith et al. (2009). 
In another study based in Saskatoon, Randall and Morton (2003) established a model to investigate QoL in the city, determining eleven life domains, namely, health, housing, employment, education, finances, security, leisure, social opportunities, accessibility, environment quality and social environment/stability. Compared with the previous work, this study attempted to expand QoL indicators by adding leisure, stability and environmental quality indicators, while McMahon (2002) attempted to divide QoL indicators broadly to include waste management, energy, transport, environmental protection, biodiversity, housing and shelter, sustainable business, health and well-being, community safety, social economy, leisure, culture and tourism, land use and development, education, poverty and social exclusion. More recently, Shoeibi et al. (2015) have used nine subjective indicators including 37 components for each indicator to investigate QoL in Sonqor city. These nine indicators are economic status, housing, education, healthcare, environment, transportation, infrastructure, recreation and security. The study conducted by the World Health Organisation Quality of Life Assessment (WHOQOL) (1998) took a slightly different approach, focusing on six, broad QoL domains including the physical, psychological, level of independence, social relationships, environment and spirituality/religion/personal beliefs. Table 2.1. presents all the various domains determined by the above studies.

By reviewing the QoUL indicators that have been selected in the previous studies, it can be noted that the most common indicators employed are health, education, economic status, housing, environment, safety and security, leisure and recreation, transportation. These indicators seem to be important QoUL components due to their frequency in the studies. The functional system of each indicator, however, may vary according to the culture, policy and characteristics of the community under investigation as well as the research objectives (Shoeibi et al., 2015). The educational system, for example, differs from one society to another in terms of structure and organisation, especially with regard to gender regulation. In the case of Saudi Arabia, the educational system is incompatible with the mixture of gender seen in the educational institutions of other contexts. 
Table 2.1: QoUL domains employed in a range of selected studies

\begin{tabular}{ll}
\hline Source/ Study & Domains/ Indicators \\
\hline Smith (1973) & $\begin{array}{l}\text { Economic status, environment, health, education, social disorganisation, and } \\
\text { participation and equality }\end{array}$ \\
\hline Felce \& Perry (1995) & $\begin{array}{l}\text { Physical well-being, emotional well-being, material well-being, social well- } \\
\text { being, productive well-being. }\end{array}$ \\
\hline WHOQOL (1998) & $\begin{array}{l}\text { Physical, psychological, level of independence, social relationships, } \\
\text { environment and spirituality/religion/personal beliefs. }\end{array}$ \\
\hline McMahon (2002) & $\begin{array}{l}\text { Waste management, energy, transport, environmental protection, } \\
\text { biodiversity, housing and shelter, sustainable business, health and well-being, } \\
\text { community safety, social economy, leisure, culture and tourism, land use and } \\
\text { development, education, poverty and social exclusion. }\end{array}$ \\
\hline Randall \& Morton (2003) & $\begin{array}{l}\text { Health, housing, employment, education, finances, security, leisure, social } \\
\text { opportunities, accessibility, environment quality and social environment/ } \\
\text { stability. }\end{array}$ \\
\hline Yinshe (2005) & $\begin{array}{l}\text { Housing, health, employment and income, land-use and environment, crime } \\
\text { and safety, education, social environment and services, and community } \\
\text { participation. }\end{array}$ \\
\hline Carver County Quality of & $\begin{array}{l}\text { Economic, education, environment, growth \& housing, health, mobility, } \\
\text { leisure \& recreation, social, public safety. }\end{array}$ \\
\hline Sife Report (2006) & $\begin{array}{l}\text { Income and deprivation, housing, educational and internet use, employment } \\
\text { and working condition, work-life balance, access to work, school, family, } \\
\text { friends and services, subjective well-being. }\end{array}$ \\
\hline Shoeibi et al. (2015) & $\begin{array}{l}\text { Economic status, housing, education, healthcare, environment, transportation, } \\
\text { infrastructure, recreation and security. }\end{array}$ \\
\hline $\begin{array}{l}\text { OECD Better Life Index } \\
\text { (2015) }\end{array}$ & $\begin{array}{l}\text { Housing, income, jobs, community, education, environment, civic, } \\
\text { engagement, health, life satisfaction, safety, work-life balance. }\end{array}$ \\
\hline $\begin{array}{l}\text { Material living conditions, productive or main activity, health, education, } \\
\text { leisure and social interactions, economic security and physical safety, } \\
\text { governance and basic rights, natural and living environment, overall } \\
\text { experience of life. }\end{array}$ \\
\hline (20015)
\end{tabular}

In general, it is difficult to include all QoUL indicators as discussed in the literature in one model. In most cases, the determination of these indicators tends to vary according to the nature and approach as well as the objectives of the study. As a result, there is no standard way of selecting and classifying life quality domains and indicators; they are often chosen and classified intuitively (Diener, 1995). Hence, these domains should be divided as broadly as possible to give scope to the study (Yinshe, 2005). In this research, the QoUL indicators (i.e., health, education, economic status, housing, environment, safety and security, leisure and recreation) that have been frequently mentioned in the previous studies will be considered. However, many of them will be considered as indicators of the general domains 
adopted in this research, namely the physical, social and cultural domains, which will be discussed in detail in Chapter 3.

\subsection{Relevant Research on the QoUL}

The burgeoning of interest in measuring/evaluating QoUL since the 1960s across a wide range of disciplines has yielded a large number of case studies within the literature. Reviewing all of these falls outside the scope of this thesis, thus, to provide context for this study, a selected review of research undertaken on the QoUL at a global level is offered in this section. In addition, relevant research within the context of Saudi Arabia is reviewed. Through this review, some of the general findings obtained in those studies will be presented here in this chapter, while some of these findings are presented more extensively within the empirical chapters.

\subsubsection{QoUL research at the global level}

From the literature, it is apparent that QoUL is most commonly investigated at various geographic scales, ranging from dwelling, neighbourhood to community or city and regional level. At the city level, there are some studies that have been conducted to explore the overall QoUL. These include, for example, the work of Das (2008) who investigated the underlying dimensions of overall QoL in the city of Guwahati; Shoja et al. (2014) who assessed the subjective urban QoL in the city of Yazd in Iran; and Rezvani, et al. (2012) who measured the QoUL in the town of Noorabad. A range of other studies concentrate on studying QoUL at the neighbourhood level, such as the study conducted by Soleimani et al. (2014) to assess QoL in transitional neighbourhoods. Another study by Stancu et al. (2016) attempted to identify the level of QoL in two districts of Timișoara city. Oktay and Marans (2010) assessed the satisfaction of the residents within the neighbourhoods of the walled city of Famagusta. In the city of Doha, Qawasmeh (2014) has investigated the QoUL in residential neighbourhoods.

As noted above, the studies on QoUL investigation ranged from neighbourhood to city level as geographical units or scales. Therefore, the QoUL investigation within these studies is considered to be scale-dependent (i.e., city or neighbourhood). Although the investigation 
of QoUL at a specific geographic scale (e.g., neighbourhood) could offer certain patterns of QoUL measures (Walsh et al., 2004), it is important to investigate QoUL at various scales simultaneously (e.g., city and neighbourhood) in order to explore different urban environmental patterns and therefore understand the overall QoUL (Kweon \& Marans, 2011).

It should be noted, however, that those geographical scales refer to the spatial domains of distinguishable social or physical features of an urban arena (Reardon et al., 2008). Several of these studies tend to rank geographical scales according to these features. In this context, there are assumptions that objective indicators of the urban environment can affect people's assessment of that urban environment (Marans \& Stimson, 2011). From this, it can be deduced how QoUL would vary at different scales. It should therefore be taken into account that people's satisfaction may occur at different levels based on the social and physical characteristics of each geographical scale. Furthermore, several studies highlight that QoUL at various geographic scales is in fact a subjective phenomenon, where each person occupying these scales might differ in their view about them (Marans \& Stimson, 2011).

As already established from the literature, QoUL domains are key elements of the QoUL investigation. Within the empirical studies reviewed above, researchers differed in identifying and dividing these dimensions. However, most of them were in agreement that the physical, social, economic comprised the main QoUL dimensions. For example, Das (2008) separated the urban environment into three domains: the physical, social and economic environments. Following Das (2008), Rezvani et al. (2012) created a conceptual framework that combines those three domains to evaluate QoL in urban areas of Noorabad. Those two studies consider that the external condition of those dimensions is involved in the objective QoL, whereas the satisfaction from such conditions encompasses subjective QoL. The study conducted by Qawasmeh (2014) however, ignores the important economic dimension to focus on the two dimensions of the physical and social.

In light of what has been presented above, it can be noted that each focused on specific dimensions of the urban environment, whether that was in terms of the three dimensions of the physical, social and economic (e.g., Srivastava, 2005; Mridha \& Moore, 2011; Marans, 2012; Azad et al., 2015), or only two of those dimensions, or indeed, chose to concentrate 
on slightly different dimensions. This may have been due to the variation in the objectives as well as the differentiation in the nature of these studies, nevertheless, there seems to be a general agreement that those dimensions all contribute to the QoUL. Whatever domains and indicators are selected, the overall life satisfaction of residents is mostly reflected by satisfaction with numerous different life domains (Christoph \& Noll, 2003). Thus, QoUL life domains should be broadly defined to include the most important aspects of the living environment (Yinshe, 2005).

A number of other studies (e.g., Marans and Rodgers 1975; Campbell et al., 1976; Gruber \& Shelton, 1987; Lu, 1999; Parkes et al., 2002; Sirgy and Cornwell, 2002; Grzeskowiak et al., 2003; Hur and Morrow-Jones 2008) more specifically focused on the satisfaction with urban living at different scales including dwelling, neighbourhood and local community. At the dwelling level, studies such as Lu (1999) and Campbell et al. (1976) found that home features like house structure, tenure, age and size are significant predictors of housing satisfaction. Within several studies on housing satisfaction, home ownership was found to be a key element in perceiving home (e.g., Lu, 1999; Elsinga and Hoekstra, 2005; Diaz-Serrano, 2009; Zhang et al., 2018). Home ownership was found to be not only a key element in predicting housing satisfaction, but also with respect to neighbourhood satisfaction (e.g., Lu, 1999; Parkes et al., 2002; Grinstein-Weiss et al., 2011). Another important issue regarding housing is the affordability, which was found to be important in predicting housing satisfaction. However, the high cost of housing was found to be related to a better quality of the accommodation (Lu 1999; Vera-Toscano \& Ateca-Amestoy, 2007).

Bringing all these housing aspects together, several studies, including the work of Andrews and Withey (1976), Peck \& Kay Stewart (1985), Kahlmeier et al. (2001), Das (2008) and Zhang et al. (2018), have emphasised that housing satisfaction mediated by these aspects contribute to the overall QoUL. However, satisfaction with housing is influenced by features of the surrounding environment such as neighbours, housing characteristics and community size (Campbell et al., 1976; Lu, 1999; Parkes et al., 2002). Moreover, satisfaction with housing can also be predicted by community satisfaction (Campbell et al., 1976). In this context, housing satisfaction is connected with other indicators of the urban environment at different scales. In this sense, some studies have shown that home satisfaction has a strong positive effect on neighbourhood satisfaction (e.g., Lu, 1999; Mohan \& Twigg, 2007). On 
the relationship between those two physical indicators of home and community, there is evidence from several studies in the literature (e.g., Morris and Winter, 1978; Gruber \& Shelton, 1987; Lu, 1999; Sirgy and Cornwell, 2002; Grzeskowiak et al., 2003) to suggest that feelings towards the home are more likely to spill over into feelings toward the neighbourhood and vice versa.

As for the neighbourhood scale, there are several studies that have dealt with neighbourhood satisfaction. In this regard, Sirgy and Cornwell (2002) have conducted a literature review of the various characteristics of the neighbourhood that affect satisfaction with it. These characteristics were grouped into physical, social and economic attributes. Physical attributes were represented by the upkeep of homes and yards, landscape in the neighbourhood, crowding and noise level, nearness of neighbourhood to facilities and quality of the environment. Social attributes included social interactions with neighbours, outdoor play space, the people who lived in the neighbourhood, ties of community, crime, race relations in the community and the sense of privacy at home. Economic attributes included home value in the neighbourhood, cost of living, socio-economic status of the neighbourhood and neighbourhood improvement. While neighbourhood satisfaction is related to these features, it also linked to other urban attributes including housing and community. That is, satisfaction with neighbourhood is related to the satisfaction at different scales (i.e., dwelling and community). In this context, Sirgy and Cornwell (2002) found that satisfaction with the economic features of neighbourhoods was a good indicator of housing satisfaction, and that satisfaction with the social features of the neighbourhood was a good indicator of community satisfaction.

Perceiving aesthetics as one of the physical characteristics of the neighbourhood was found by many studies to be a key element in increasing satisfaction with neighbourhood (e.g., Parkes et al., 2002; Sirgy \& Cornwell, 2002; Hur \& Morrow-Jones, 2008). Gruber and Shelton (1987) moreover, stated that the neighbourhood's attractiveness is the most important determinant of neighbourhood satisfaction. Sirgy \& Cornwell (2002) would argue that it is a range of important factors in the neighbourhood that contributes to QoL of the residents in terms of neighbourhood satisfaction, including environmental adequacy, local safety, public school adequacy, and housing satisfaction. In terms of the neighbourhood scale then, what is emphasised through all these studies is that overall 
satisfaction with the neighbourhood is closely related to satisfaction with a range of characteristics of that neighbourhood (Permentier, et al., 2010). The combination of these functions and characteristics as a whole tend to contributes to the QoUL, which is consistent with the idea of functionalism discussed earlier in this chapter.

At the community level, satisfaction is often predicted by the provision of urban services such as health, education, parks and green areas, recreation opportunities, transportation, and so on (e.g., Campbell et al., 1976; Sirgy and Cornwell, 2002). Access to these services has been shown to be an important component of QoUL (Rogerson et al., 1989; Glaeser et al., 2000). In this context, Grzeskowiak et al., (2003) suggests the greater the satisfaction with these services, the greater the satisfaction with community. By reviewing the literature, it has been found that most studies have focused on the degree of satisfaction with those services and the extent to which this affects the QoUL, but predominantly in terms of subjective evaluation. The actual status of these services, however, has been not adequately highlighted. That is, some indicators that could give a comprehensive picture of the urban services provided have been barely highlighted. For example, there was a notable lack in exploring indicators such as the waiting time to see a doctor as an indicator of health provision. Similarly, educational provision indicators such as the average density of classes and student-teacher ratio were barely highlighted or explored in the QoUL literature. Considering these sub-indicators of the urban services domain perhaps gives a clearer picture regarding the overall quality of these services.

Another aspect arising from this review, is that the studies mentioned still suffer from some limitations, such as the absence of an investigation into the overall quality of the neighbourhood. In this regard, research has often overlooked some characteristics of the urban environment that may affect people's satisfaction with neighbourhoods, which in turn affects the overall QoUL. For example, most of the studies have focused heavily on the functional and physical aspects of housing, while few of them have paid attention to social aspects. That is, studying the quality of the neighbourhood should include the physical, social, economic, and environmental aspects in order to attain a comprehensive picture of the quality of the neighbourhood as one of the most important of the QoUL indicators. 
Although the literature has highlighted most of the aspects of life in relation to the QoUL, a remarkable gap can be noticed in terms of the cultural and social domains of the QoUL. There is little focus on social indicators such as social interaction with neighbours and social support, nor on cultural aspects of the urban environment, and more specifically on heritage. With regard to the social domain of the QoUL, there are a few studies that have covered social interaction with neighbours and social support, for example the work of Datta et al. (2015) - who found that transformed grades of QoL improved significantly in all domains after social interaction compared to the degree at the beginning of the study - Van den Berg and Timmermans (2015), Helgeson (2003) and Yarcheski et al. (1994). However, these studies were conducted in western countries, which indicates a weakness within the QoUL literature.

Another important social indicator that has been found to be inadequately explored in the QoUL literature is religion. Although there is a widespread recognition by several studies regarding the importance of the religion in people's daily lives (e.g., Peterson \& Webb, 2006; Lun \& Bond, 2013), there are few studies that have addressed this indicator and its contribution to QoUL. Research carried out by Ferriss (2002), Diener et al. (1999), Sawatzky, et al. (2005) and Magalhaes, et al. (2015) does suggest that religion and spiritualty contribute to a higher of QoL, and that religious beliefs and practices contribute to the improvement of QoUL, especially with regard to the physical and mental health aspects (e.g., Hadaway, 1978; Ellison, 1991; Levin \& Chatters, 1998; Siegel \& Schrimshaw, 2002; Helliwell \& Putnam, 2005; Lim and Putnam, 2010; Sinnewe et al., 2015). Other studies have challenged this, contending that religion may promote anxiety and depression (e.g., Kennedy, 1998; El-Jamil, 2003; Van de Velde et al., 2017). These studies, however, were conducted in western countries, which are mostly Christian, therefore indicating a gap within the QoUL literature in relation to the impact of religion more widely on daily life.

Within the Islamic context, a limited number of studies have been conducted on the relationship between religiosity and SWB. These include the work of Al-Kandari, 2003; Baroun, 2006; Abdel-Khalek and Lester, 2007; Tiliouine and Belgoumidi, 2009; Al-Naaimi \& Younis, 2010; Abdel-Khalek, 2011; Abdel-Khalek \& Eid, 2011; and Hossain \& Rizvi 2016. For the Saudi context, only one study (Abdel-Khalek, 2008) has been conducted regarding the religion and SWB. In general, these studies found a similar result to those 
conducted in the western countries, where a presence of a positive relationship between religion and SWB enhancing happiness and life satisfaction was confirmed. However, some of these studies, namely Abdel-Khalek (2002), Baroun, (2006), Abdel-Khalek and Lester (2007) and Abdel-Khalek (2008), did find a negative impact of religion, including anxiety and depression. In reviewing these studies, however, some limitations can be observed. One of these limitations is that most of those studies used limited samples, where, apart from the two studies of Hossain \& Rizvi (2016) and Al-Kandari (2003), they focused on participants who were adolescents and undergraduate students. In addition, these studies have not adequately highlighted the religious practices and beliefs as key elements in the relationship between religion and SWB.

Another key element of QoUL is the cultural domain including heritage and other cultural goods such libraries, museums, and engagement in cultural activities. Yet, it appears from the literature that few studies have highlighted these indicators. Among those that have, Agisilaos, (2012), Logan (2016), and Hayes (2017) pointed out that heritage promotes the economic development of the urban environment, while Bramham (1997) evidenced that heritage features are being developed in an attempt to produce an attractive environment for tourism and trade, and Evan (2014) referred to many aspects of heritage in terms of providing fulfilment and pleasure, meaning and identity, social capital, community cohesion and economic benefits such as tourism and job creation. Although these studies have indicated the benefits that can be gained from heritage, they have not shown how heritage contributes to the overall QoUL. Very few studies have linked heritage to the QoL and SWB. Fujiwara et al. (2014), Massey, 2012, Grazuleviciute, 2006 have touched on this, where, for example, Fujiwara et al. (2014) found that heritage positively affects life satisfaction. Massey (2012) has linked QoL issues such as health and well-being, citizenship and social cohesion, and education and employment to living heritage, while Ander et al. (2012) mainly focused on the health benefits of heritage. However, with the exception of Fujiwara et al. (2014) and Ander et al. (2012), most of these works were reports which did not obtain enough evidence regarding the extent to which heritage affected wellbeing.

Other cultural goods such libraries and museums, as well as engagement in cultural activities like conferences and exhibitions, have long be considered as key elements of the urban 
environment. For the field of QoUL, however, those indicators are still rather unexplored. What is missing to some extent is how such cultural goods contribute to the quality of people's lives. A limited number of studies have investigated the relationship between cultural goods and SWB (e.g., Packer, 2008; Fujiwara, 2013; Fujiwara et al., 2014; and Wheatley \& Bickerton, 2016). Their findings indicate that cultural goods and the engagement in cultural activities are positively associated with well-being, including happiness, and more specifically stress the economic value of visiting those cultural goods.

\subsubsection{QoUL research in Saudi Arabia}

Although investigation into the QoUL has attracted the attention of researchers since the 1960s, comprehensive research into this field has not yet been addressed in the context of Saudi Arabia. The studies on QoL that have been conducted in Saudi Arabia, namely the work of Bin Saif et al. (2013), Almutairi et al. (2015) and Alsamghan et al. (2016), do focus on QoL in terms of the relationship between people and their environment but address that relationship from a purely health perspective. The notion of QoL is much broader than this. On the other hand, the study conducted by Khizindar (2012) did attempt a broader investigation into the direct effects of the cultural, social, economic and environmental impacts of tourism on the perceptions of Saudi residents' overall QoL. This study found that the economic aspect of tourism negatively affects the QoL, while the other three dimensions of social, cultural and environmental impacts have a positive impact on the overall QoL. What distinguishes this study is that it highlights the important dimensions of QoL, namely, cultural, socio-economic and environmental aspects which are known to significantly contribute to people's perception of their QoL. However, this study suffers from limitations including those related to the sample selection, where the study indicates that $85.7 \%$ of participants were male, which may not adequately reflect a good understanding of residents' perceptions due to the lack of female representation.

Recently, the Saudi Ministry of Municipal and Rural Affairs (SMMRA) in cooperation with the UN-Habitat has established the Future Saudi Cities Program (FSCP) in response to the rapid growth of population and urban areas, and the expansion of social and economic realms in the country. The programme aims to achieve sustainable urban growth by providing a better urban environment for 17 Saudi cities including Medina, in accordance 
with the standards set out for the prosperity and well-being of the city (Al-shaikh, 2016). The FSCP emphasises the principles of equality and social integration as a fulcrum for sustainable urbanisation by focusing on mainstreaming the benefits of development. Accordingly, UN-Habitat has created a tool called the City Prosperity Index (CPI) in order to measure the current and future progress of cities toward prosperity. The CPI is a new method to measure the comprehensive prosperity indexes of cities where it measures prosperity levels according to six fundamental dimensions, namely productivity, QoL, infrastructure, justice and social integration, governance and legislation, and environmental sustainability (Al-shaikh, 2016).

These dimensions are considered by UN-Habitat as the wheel of cities' prosperity, as illustrated in Figure 2.7. First, productivity refers to economic growth and development, income generation, employment and equal opportunities further provide adequate living standards. Second, QoL refers to meeting the needs of the citizens in terms of providing a better environment for living by providing social services such as education, health, recreation, safety and security to improve living standards. Third, infrastructure includes the physical assets, amenities, road network, adequate water, sanitation, power supply and so on that enhance the QoL. Fourth, equality and social integration is where the city is described as prosperous when it achieves the maximum of equality by reducing extreme poverty and deprivation as well as the incidence of slums and marginalisation. Fifth, governance and legislation indicate the extent to which the city can combine sustainability and shared prosperity through effective urban governance and the dissemination of appropriate policies, laws and regulations. Finally, environmental sustainability requires that city growth and economic development does not destroy or degrade the environment, and therefore, the prosperous city maintains natural assets for the sake of sustainable urbanisation (UN-Habitat, 2015). 


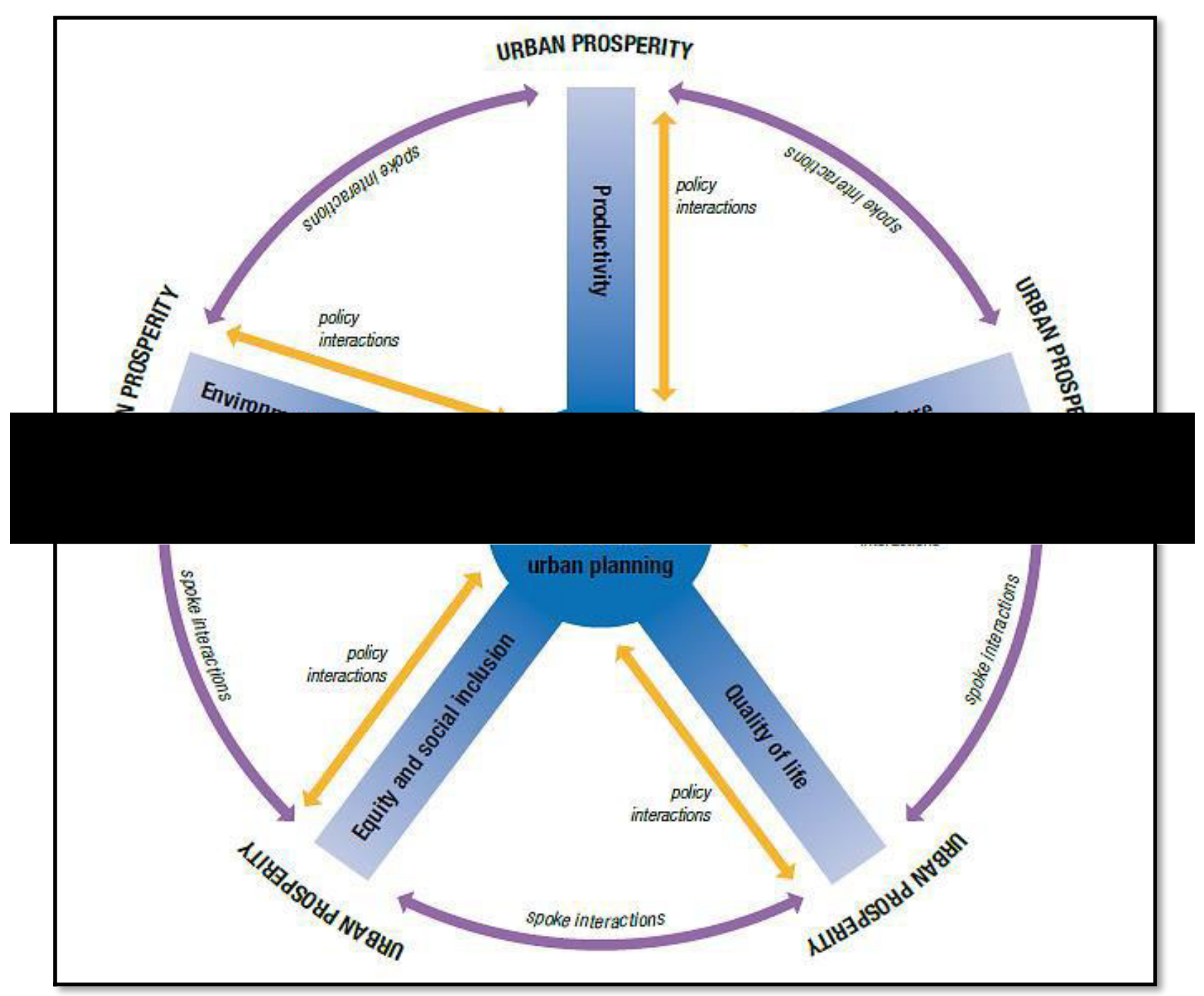

Fig 2.7: The wheel of cities' prosperity (UN-Habitat, 2013, p. 12)

This conceptualisation of prosperity provides a unique and holistic vision of sustainable urban development, illustrating the different dimensions of city growth. From this standpoint, a prosperous, sustainable and equitable city is considered to be a well-planned, safe, healthy, green and comprehensive city for the most important elements of life (UNHabitat, 2013). This conceptualisation provides a new model of urbanisation in the global scale, and therefore, giving the policymakers the opportunity to compare the results of their cities' prosperity surveys with other global cities to find, if at all possible, solutions to urban issues. However, it seems that the adopted measurement of the dimensions mentioned in this framework follows the objective approach, which needs to be integrated with the 
subjective approach in order to give a more comprehensive picture of prosperity especially when measuring a dimension like QoUL.

Under the umbrella of FSCP, the six prosperity dimensions were investigated with respect to Medina city as one of the seventeen Saudi cities targeted in this programme. The CPI Profile Report of Medina has provided a summary of the basic information and urban statistics about the city of Medina and has given an overview of its achievements that may contribute to its prosperity. Accordingly, the objective of this investigation is to identify the strengths and weaknesses of the factors of prosperity in Medina as well as the level of achievement towards the fulfilment of prosperity objectives. In addition, it highlights the contrast in the six dimensions of prosperity. The results show that the general index of prosperity in Medina is $48.8 \%$, which gives Medina a weak rating according to the global scale of urban prosperity. In spite of that, the QoL dimension was rated moderately strong, where it achieved 65.1\% (UN-Habitat \& SMMRA, 2016).

The QoL dimension in the CPI Profile of Medina includes the indicators of health, education, safety and security and public spaces. The question here is whether this gives us a comprehensive image of the QoUL in Medina? As argued in this chapter, the QoUL is a multidimensional and comprehensive concept that includes all aspects of everyday life in the city. Thus, to reiterate Yinshe's (2005) assertion, QoUL life domains should be defined broadly enough to include the most important aspects of the living environment. Despite the fact that the CPI used to investigate prosperity in Medina comprises dimensions that may represent the main domains of QoUL, the QoL dimension itself only included the four indicators mentioned above. More importantly, the CPI survey conducted with regard to Medina seems to refer only to one approach to the whole investigation, that is an objective approach, which is primarily based on official sources such as statistical data and censuses. However, as the above discussion of the chapter has again shown, the sole use of the objective indicators to measure QoL has been widely criticised in the literature. In this regard, several studies indicate that QoL is a subjective phenomenon which reflects the life experiences of the residents where the objective conditions of the environment themselves do not reflect the true QoUL of that setting (van Kamp et al., 2003; Marans, 2012). 
Building on what has been presented from reviewing the relevant research on the QoUL, a number of significant factors can be noted. Firstly, most studies in this field have focused mainly on the built environment and the extent of its contribution to the QoUL with little focus on the social aspects of the urban environment. That is, the focus was more on the physical aspects of the urban environment, while social and cultural aspects, especially religion and heritage, have been hardly highlighted. In addition, it can be seen that there is a lack of coverage of both the positive and negative aspects of the urban environment. That is, most of these studies have shown the positive side of the effects of the various indicators of the urban environment, while few of those studies have drawn out the other side with regard to the effect of these indicators on the QoUL, which indicates the lack of a critical approach. Regarding the Saudi context, it can be noted that the QoUL was not addressed in a manner commensurate with its multiplicity of dimensions, especially in its subjective dimension, which has led to the absence of this concept within the Saudi literature. Accordingly, this research will critically investigate the QoUL using the conceptual approaches discussed earlier in this chapter. In so doing, this research explores the physical social cultural domains of the QoUL in an attempt to fill the gap in the field of the QoUL as well as in the Saudi literature.

Within the review of those studies, key issues with regard to the methodological approaches can also be identified. The methodologies used in QoUL studies are often focused on either quantitative or qualitative research methodology, whereas, relatively few of these studies combine these two methods of research or what is known as the mixed methods. It may be helpful, as a means of highlighting this, to categorise the empirical studies reviewed according to each type of research method. First, the quantitative method, which is based on the use of questionnaires (Bamberger, 2000; Philip, 1998), was adopted by studies including the work of Randall and Morton (2003), Senlier, et al. (2008), Rezvani, et al. (2012), Shoja et al. (2014), Soleimani et al. (2014) and Stancu et al. (2016). These studies were varied in terms of the data collection. For example, Senlier, et al. (2008) Rezvani, et al. (2012), Shoja et al. (2014) and Stancu et al. (2016) used primary data, whereas Randall and Morton (2003) and Soleimani et al. (2014) used both primary and secondary data. Second, the qualitative method, which is based on the data collected by either interview, focus groups, discussions recorded by video or audio (Philip, 1998; Bengtsson, 2016) was adopted by studies including the work of Oktay and Marans (2010) and Qawasmeh (2014). 
These studies differed in terms of the utilisation of the tools. While Oktay and Marans (2010) focus only on the interview, Qawasmeh (2014) combined the interview and observation as tools of the data collection. In the third category, Das (2008) used a mixed method that combined quantitative and qualitative research methods.

It is important to note that the differences in choosing the method, whether quantitative, qualitative or both, does depend on the research questions that need to be answered (Katsirikou \& Skiadas, 2010). An additional factor that may affect the choice of the method is the availability of the data sources, such as secondary data (Watzlawik \& Born, 2007). This in turn may lead to relying on only one source for data collection. For example, the study conducted by Rezvani et al. (2012) was limited to the sole use of subjective indicators using social surveys, due to the lack of data available. It is, therefore, useful to combine quantitative and qualitative as a mixed method in order to collect a greater amount of data (Tonon, 2015). Additionally, the mixed method provides a comparison of qualitative and quantitative data, which in turn provides an understanding of the inconsistencies between qualitative and quantitative results as well as the understanding and analysis of the research problem (Tonon, 2015; Wisdom \& Creswell, 2013). Accordingly, this study will adopt a mixture of quantitative and qualitative methods to collect and analyse data in order to understand the overall QoUL in the municipality of Quba. 


\subsection{Summary of the chapter}

QoUL is a concept that has been the interest of many disciplines since it emerged with the Social Indicators Movement in the late 1960s and early 1970s. Despite the huge amount of literature on the QoUL, there is no specific definition nor a unified conceptual framework due to the nature of this concept, which is characterised by multiplicity and complexity. The study of QoUL in general includes the complexity of the individual, family and community, and, moreover, QoUL comprises multiple dimensions including social, physical and economic which are measured at different scales including dwelling, neighbourhood, city or state scale. As a result, the QoUL becomes increasingly multifaceted and complicated. On the investigation of QoUL in general, components of an urban environment and the methods to measure these components have differed according to the interests and goals of the studies, and in line with the differences of disciplines. However, there is general agreement that the investigation has two main aspects: objective and subjective dimensions (Diener \& Suh, 1997; Van Kamp et al., 2003; Das, 2008; Sirgy, 2012; Mohit, 2013; Khaef $\&$ Zebardast, 2015). In this context, most studies assert that both objective and subjective approaches are necessary to investigate QoUL. Integration of these dimensions, would, therefore, help to capture the broad nature that characterises the concept QoUL.

Since QoUL has been used to measure the dimensions of the urban environment, a wide range of domains and sub-domains have been employed. Most of those domains are related to the physical, social, and economic domains. However, there is still no agreement regarding specific domains of QoUL, where determination of those domains depends on the objectives of the study investigating the QoUL in a geographic setting. The choices and determination of these domains may vary according to the differences of disciplines and perspectives from which the topic is approached. (Van Kamp et al., 2003). As result, domains and indicators to be used in the investigation of QoUL are varied from one study to another.

The objective and subjective indicators of the urban environment are commonly measured at different levels. These levels are represented by spatial or geographical scales such as community, neighbourhood and an individual's dwelling. Thus, the satisfaction degree of individuals with each of these scales is captured in a composite QoUL measure 
(Guhathakurta \& Cao, 2011). From this review, it can be noted that most of the studies in the literature have focused on the investigation of QoUL at the neighbourhood and the dwelling level. Perhaps this indicates that the investigation of QoUL at neighbourhood level in particular could be important in the exploration of the patterns of the urban environment and the allocation of resources. However, one of the limitations of these studies is that they have largely relied on only one geographic scale. This is due to the fact that certain patterns of QoUL measures can only be found at specific geographic scales (Kweon \& Marans, 2011). In sum, the investigation of QoUL at the neighbourhood level as a geographical scale which is frequently referred to by many investigators is an important method through which to compare different neighbourhoods in the level of QoUL. Kweon and Marans (2011) go on to comment that this comparison could be in terms of the spatial variation of QoUL across neighbourhoods, which in turn reveals some significant QoUL indicators such as that of inequality, and, importantly, how open spaces of the neighbourhood play an important role in enhancing QoUL (Kweon \& Marans, 2011).

In light of what has been presented in this chapter, it can be said that most studies concerning the QoUL have focused more on the physical conditions of the urban environment with little focus on the social and cultural aspects. Therefore, the review of this literature has opened the horizons for a new direction for this research to measure the QoUL by focusing more on the social and cultural aspects and the extent of their contribution to the urban quality of life of residents in a cultural context that is far different from other cultural contexts. For Quba municipality and Saudi Arabia in general, investigation into the QoUL offers a new case study linked to a culture that is sharply different to many cultures around the world, providing an additional model for urban quality study. By doing so, the investigation of QoUL in Medina is likely to open up new prospects for many researchers interested in urban environmental studies, whether academics or local decision-makers.

In light of the material set out in this chapter, Chapter 3 turns to a discussion of the methodological framework for this thesis. 


\section{Chapter Three}

\section{Research Methodology}

\subsection{Introduction}

Since the QoUL has been relatively under-explored in the context of Saudi Arabia and in the city of Medina in particular, this research examines the notion of QoUL that has been determined as the level of urban life performance with respect to the residents' needs, aspirations, expectations and overall QoUL experience. The aim of this thesis is to investigate the QoUL in the city of Medina with the focus on the municipality of Quba as a case study. In doing so, the research uses different conceptual approaches namely, functionalist and postmodern approaches in an attempt to identify the key elements of QoUL in the study area.

In order to understand the general framework that includes the associated concepts, characteristics and the ways in which QoUL can be measured, a comprehensive literature review has been conducted. As a result, research methods and instruments as well as domains and indicators of QoUL have been identified. Therefore, this chapter presents the research methodology in relation to philosophical worldview, research design and research approach. In addition, the focus of research and the domains and indicators for the study area are specified. Finally, the chapter discusses the procedures of data collection, time scale of research, data analysis strategies and ethical considerations. Additionally, justifications in relation to the research methodology have been made. An overview of the flow of the research methodology for this thesis is presented in Figure 3.1. 


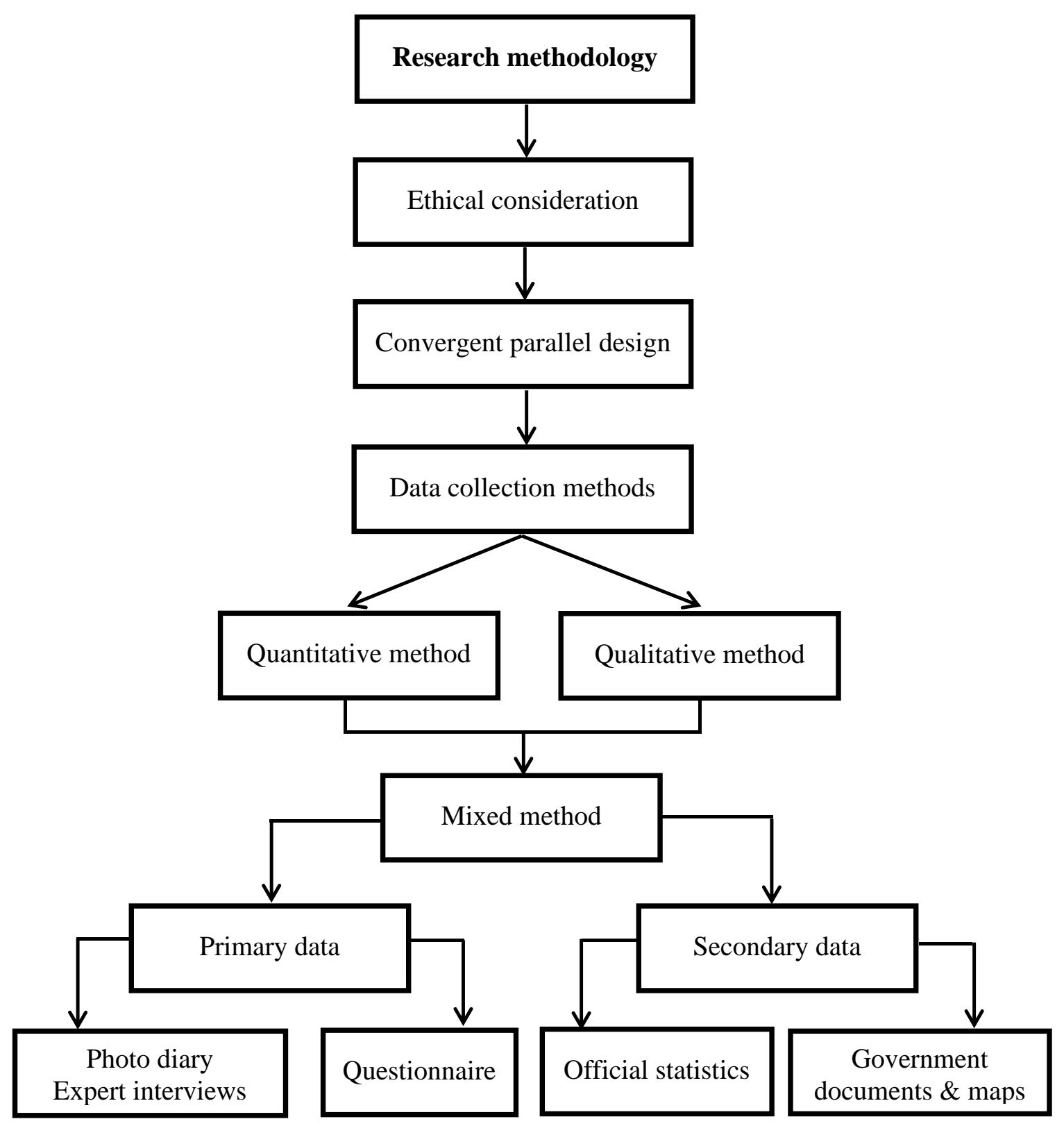

Fig 3.1: Research methodology 


\subsection{Philosophical worldview}

As mentioned earlier, the QoUL is a highly complex and multifaceted concept, as it includes subjective and objective features that need to be viewed as a whole in a particular study. Although the relationship between those traits has been described as weak, they are considered as complementary to each other. Therefore, QoUL can be fully comprehended only if these indicators are integrated and understood within a study, which requires multiple approaches (Diener \& Suh 1997). The approach using the combination of objective and subjective indicators and the use of more than one methodology is preferred by many investigators in the QoUL field. In this context, the question of the philosophical worldview framing this research arises, which is mostly 'pragmatism,' which supports multiple views and practices.

Pragmatism is derived from the work of James, Mead, Peires and Dewey (Creswell, 2009). It is a philosophical tradition centred on the linking of practice and theory (Zupan, 2012). Pragmatism arises out of situation, actions and consequences. It is concerned with applications and solutions that emphasise research problems and with the use of several approaches available to understand the problem (Creswell, 2009). In the field of QoUL therefore, pragmatism enables researchers to choose the research strategy, methods, tools and procedures that meet the purpose of the research. Moreover, Creswell (2009) and other authors evidence that research as underpinned by pragmatism occurs in historical, social, political and a range of other circumstances.

In light of these features, pragmatism opens the door to research for different worldviews, a variety of research tools and procedures as well as different methods of data collection and analysis. This is consistent with the QoUL research that often relies on a mixture of methods. In this respect, QoUL research is associated with what is called the 'pluralistic approach', which has recently become one of the accepted strategies in the field of QoUL. The key feature of this approach is that methodological pluralism comprises finding value in a variety of sources of information (May et al., 2016). Therefore, this approach is becoming one of the recommended methods in many of QoL research in the literature (e.g., Bonham et al., 2000; Schalock 2004; Dunning et al., 2008). For QoUL study and particularly this thesis, the pluralistic approach focuses on personal appraisals and functional assessments. In this context, personal appraisals refer to the subjective assessment of people 
regarding their urban environment, while functional assessments address the objective aspects of the urban environment (Schalock, 2004).

\subsection{Research Design}

According to Creswell (2009), research design is the proposal or plan of procedures to conduct a piece of research. It is the framework that involves the intersection of the purpose of the research, philosophy, the research strategies, analytical procedures, and the specific methods for use in the research study. The considerations that have prompted the researcher to discover an appropriate design for the present study relate firstly to the multiplicity and complexity of the QoUL domains and indicators that have been drawn for the study area. Second, the conceptual approaches to be applied in this research need more than a single tool to collect data. Thirdly, consideration needs to be given as to finding an optimal way to interpret the results of the research. The final consideration relates to collecting the data for the research concurrently (one phase), where the fieldwork is conducted outside the UK, to save time and costs. Therefore, a convergent parallel design has been chosen for this thesis.

The convergent parallel design has emerged from the multitrait-multimethod matrix developed by Campbell and Fiske (1959) who evidence that the best way to understand the psychological trait is by collecting different forms of information about it in different ways (Creswell \& Alquhtani Trans, 2018). This design entails collecting and analysing both qualitative and quantitative data of a single phenomenon separately and then, mixing the two analysed data by merging the results during the interpretation (Creswell \& Plano Clark, 2011). Accordingly, this research uses a convergent parallel design to collect quantitative and qualitative data concurrently. In so doing, both quantitative and qualitative results will be converged in the interpretation phase, to compare them, support and relate findings, which in turn leads to a holistic interpretation (Creswell \& Plano Clark, 2011).

The reason behind choosing this design is firstly, to obtain a more complete understanding from the two databases regarding the experience of QoUL in the study area. The purpose of combining qualitative and quantitative data is the "enriching" that involves identifying issues or acquiring information that may not obtained by quantitative surveys (Carvalho \& White, 1997). For instance, some information about the QoUL domains to be examined may 
not be obtained through using a questionnaire (i.e., close-ended questions) and which need more investigation by means of an in-depth interview. This is likely to facilitate gaining more insight into how people experience their daily lives. Secondly, as the convergent parallel design involves collecting data in one phase, it is an appropriate design for this research where the collection of data needs to be carried out in one visit. Finally, this design is in line with the philosophy of this research that involves the use of several methods to gain a deeper understanding of the phenomenon, as discussed in section 3.2 above concerning philosophical worldview. The mixed methods design for this research is illustrated in Figure 3.2.

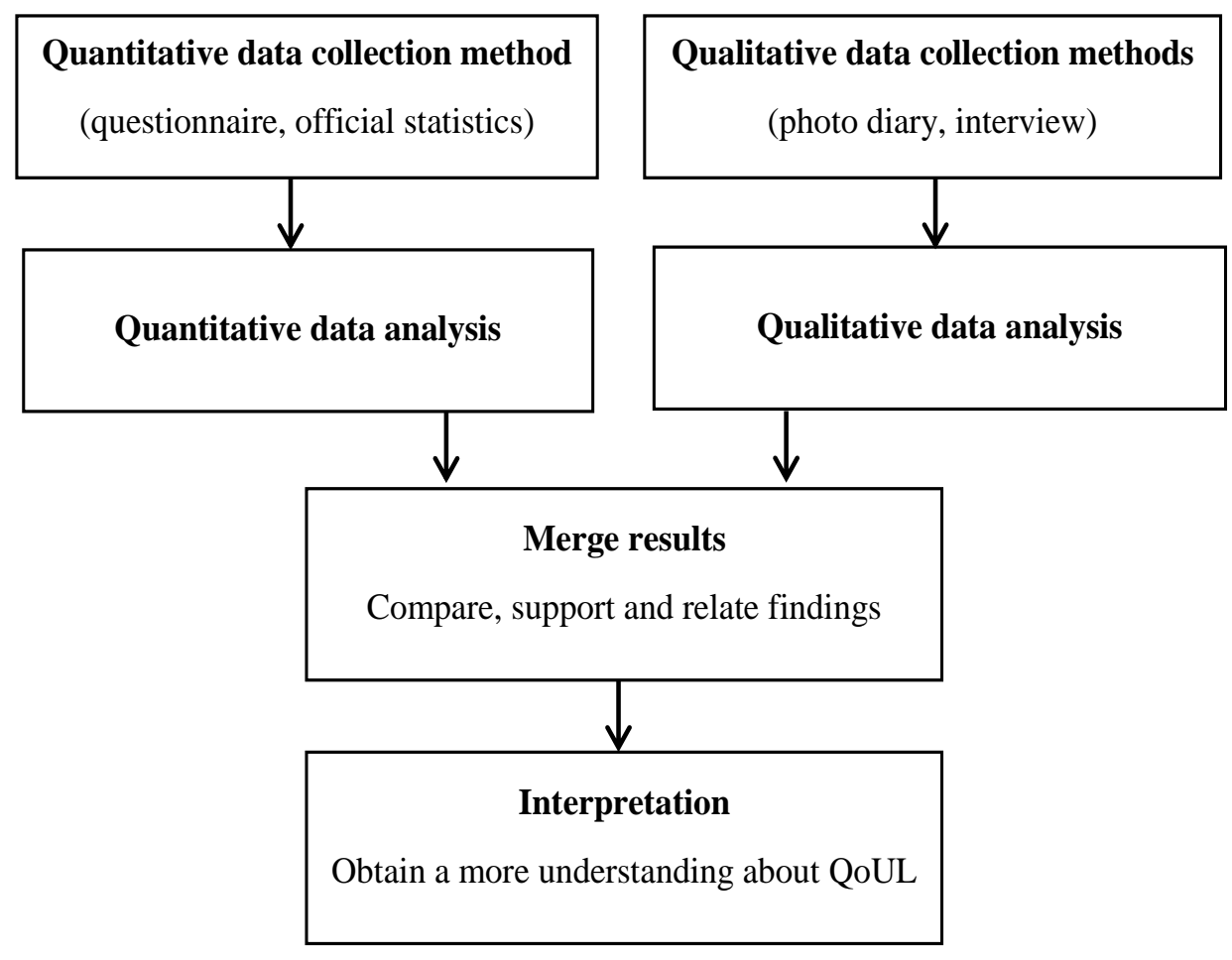

Fig 3.2: Convergent parallel design for the study based on Creswell and Plano-Clark (2011)

As illustrated in the Figure 3.2 above, the use of convergent parallel design involves the integration of both quantitative and qualitative data as two separate sets of data. The first quantitative strand collects data using questionnaires and official statistics. The second qualitative strand collects data using photo diaries and follow-up interviews, as well as semi- 
structured interviews with experts. These two sets of data are collected and analysed independently, while the results from the analyses of both sets of data are merged during the interpretation stage to compare, relate findings and support each other's findings. In so doing, a more holistic interpretation of the QoUL in the study area is likely to be gained.

\subsection{Mixed methods research}

Due to the multidimensional nature of the QoUL that has been emphasised in the literature, a mixture of quantitative and qualitative methods to collect and analyse data has been adopted for this research, which provides a comprehensive and more complete understanding of a research problem, and therefore producing useful information (Muijs, 2004).

\subsubsection{Quantitative research}

Quantitative research is one of the most utilised researches in the social sciences, as can be widely observed in the QoUL literature. According to Creswell (2013) "quantitative research involves the collection of data so that information can be quantified and subjected to statistical treatment in order to support or refute 'alternate knowledge claims"' (p. 153). It also comprises data collection that is typically numeric, where researchers use mathematical models as the methodology to analyse data (Muijs, 2004; Williams, 2007). In that regard, quantitative research allows for the gaining of data that can be analysed statistically (Hittleman and Simon, 1997). Quantitative research in this methodology, therefore, refers to the strategies of inquiry that are based on official statistics and documents as well as household surveys (i.e., questionnaires), to obtain information regarding the physical, economic, social and cultural domains, including information regarding the perception and judgement of the population regarding these domains.

\subsubsection{Qualitative research}

Qualitative research on the other hand, is designed to help researchers understand the social and cultural contexts in which people live, by discovering what these people say and do (Myers, 2009). In that sense, qualitative research focuses on words in order to express reality as well as describing people and phenomena (Amaratunga et al., 2002). In many disciplines, 
especially human geography, qualitative data can be obtained by adopting a variety of methods such as observation, focus groups, interviews, visual methods using media such as photographs, film and so on (Philip, 1998). Accordingly, photo diaries and follow-up interviews as well as interviews with experts have been adopted as qualitative methods for this thesis. The quantitative and qualitative methods will be discussed in detail in section 3.7 concerning the data collection procedures.

With reference to the literature review, it can be said that most of the studies on the QoUL emphasise the combination of objective and subjective aspects in measuring the QoUL (e.g., Das, 2008; Marans \& Stimson, 2011; Mohit, 2013; Khaef \& Zebardast, 2015), which supports the use of the pluralistic approach in QoUL research (Schalock, 2004). In this context, the measurement of the QoUL needs more than one approach and data collection tool, also in line with the nature of this concept as multi-dimensional and complex. Thus, pragmatism as the framework philosophy of this thesis offers the researcher the freedom to use multiple tools to collect data such as questionnaires, interviews and photo diaries as a combination of mixed methods.

\subsection{Focus of research}

The primary focus of this research is the municipality of Quba as a case study, which is located in the south west of Medina, Saudi Arabia (see Figure 3.3). This municipality is the largest municipality of Medina's seven municipalities in terms of population and area size, where it occupies about $23.9 \%$ of Medina's area (QMO, 2018). The municipality encompasses 30 neighbourhoods, namely Qurban, Albahr, Al Mughaisilah, Al Duwaimah, Prince Nayef, Al Qaswa, Abu Buraiqa, Al Hijrah, Al-Ehn, Jubour, Al Ranuna, Shoran, Al Jumaah, Al Ausba, Al Khatim, Al Dhahirahh, Al Hadeqah Al Sacab, Rahat, Al Jabirah, As sad, Aljussah Bani Baiadhah, Al- Rawabi, Shaibiyah, Nubala'a, Al Rummana, Al Hasa, Al Mazayean and Al Sharayea (QMO, 2018). 


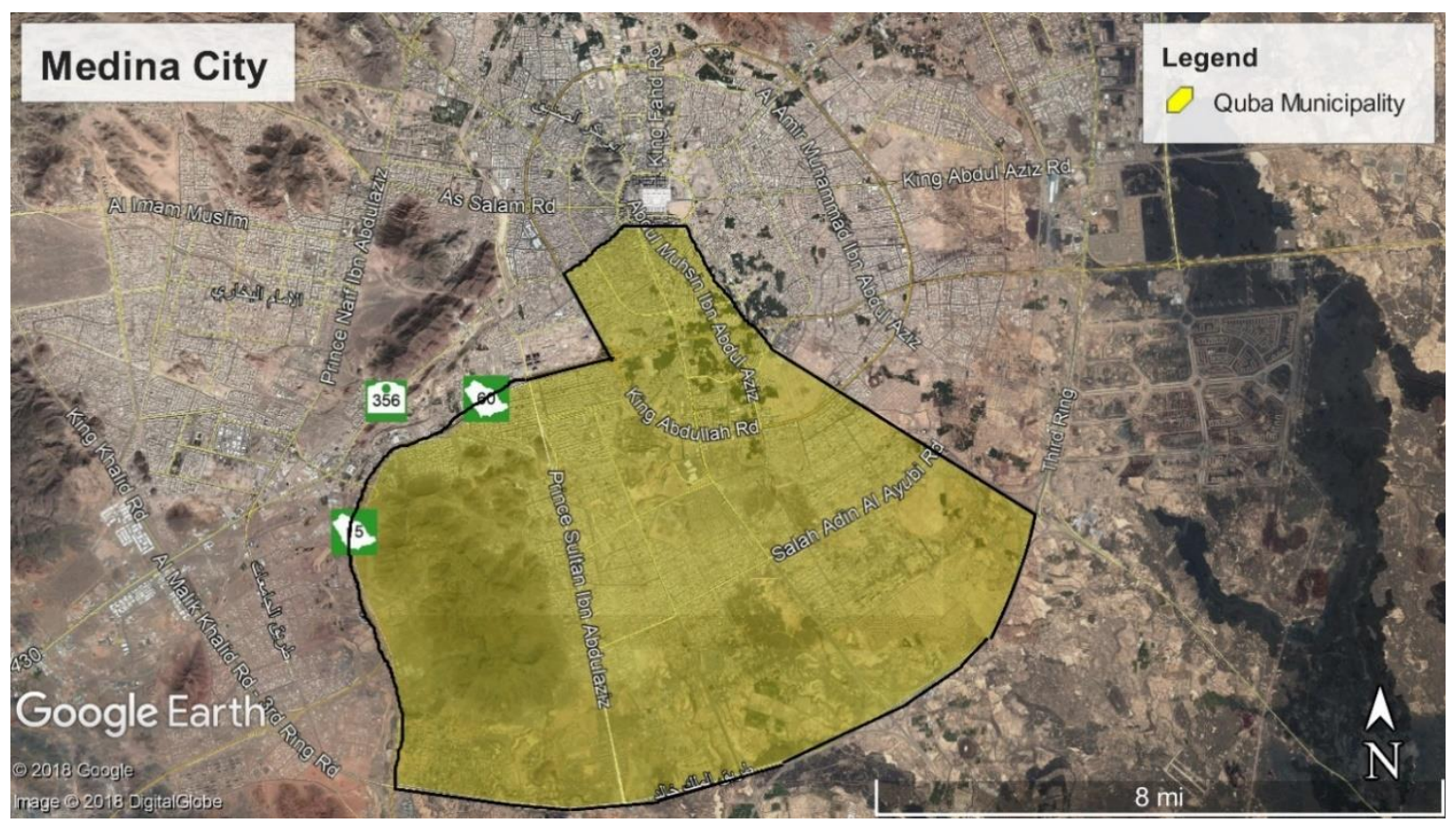

Fig 3.3: Study Area. Source: The researcher, based on Google Earth and the MRM's website (2018).

\subsection{Selection of domains and indicators for the research}

The QoUL in an urban setting can be understood by measuring a set of domains and indicators that reflect the urban environmental quality in that setting. Accordingly, to understand the experience of QoUL in the municipality of Quba, a set of domains followed by a range of indicators and variables have been determined. In this regard, three main domains of QoUL were identified. Each of these domains comprise a set of indicators. The physical domain of the urban environment is represented by the sub-domains of housing, neighbourhood and urban services. In addition to that, social and cultural domains, followed by sub-domains, were adopted. The selection of these domains is based on the review of a range of studies in the QoUL literature. The domains and indicators adopted for this research are shown in Table 3.1. 
Table 3.1: Domains and indicators selected for the study

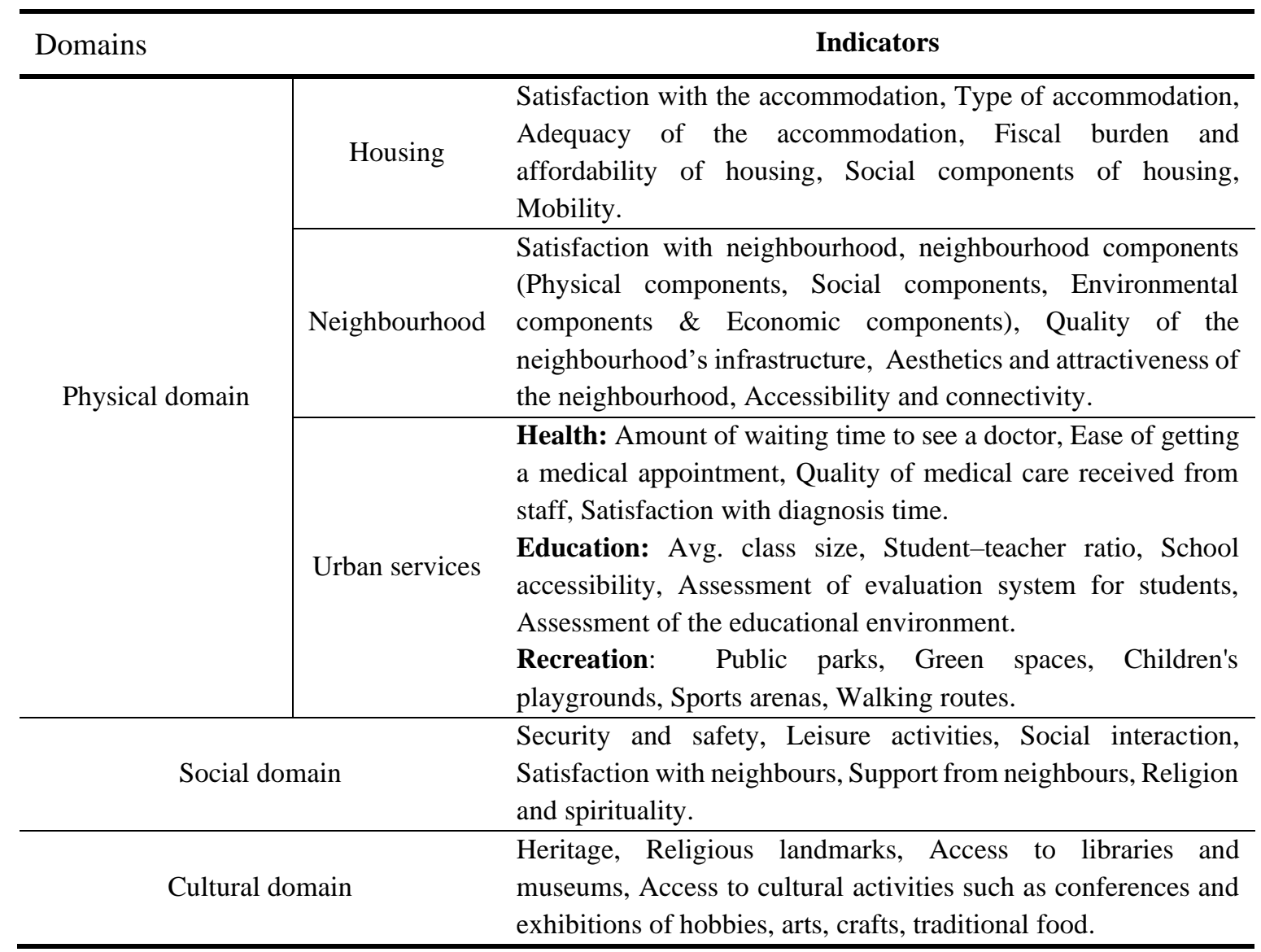

\subsection{Procedures of data collection}

The process of data collection in this research is based on the QoUL literature, and particularly the work of Myers (1988), who suggested different stages of data collection for an effective and comprehensive QoL research. These stages comprise firstly reviewing the literature, including the scholarly literature, on QoL; secondly, the collection of data by investigators that represent the indicators of different domains of the QoL; thirdly, conducting a survey in order to identify citizens' perceptions regarding QoL in their urban environment. According to Myers (1988), such a survey should cover all subjects measured by objective indicators, as well as topics too subjective to measure with objective data. Along with focusing on citizens' perceptions of trends, the survey should also inquire about the priorities that these citizens place on various aspects of the QoUL (Myers, 1988). 
Following Myers, building on the literature review, data were collected using both secondary and primary data sources (see Figure 3.4), where the secondary data supported one stage of collection, namely the official statistics and documents, while the primary data supported three different stages of data collection, namely questionnaires, photo diaries and follow-up interviews, and expert interviews. Each type of data including the stages of data collection are presented in Figure 3.4 below.

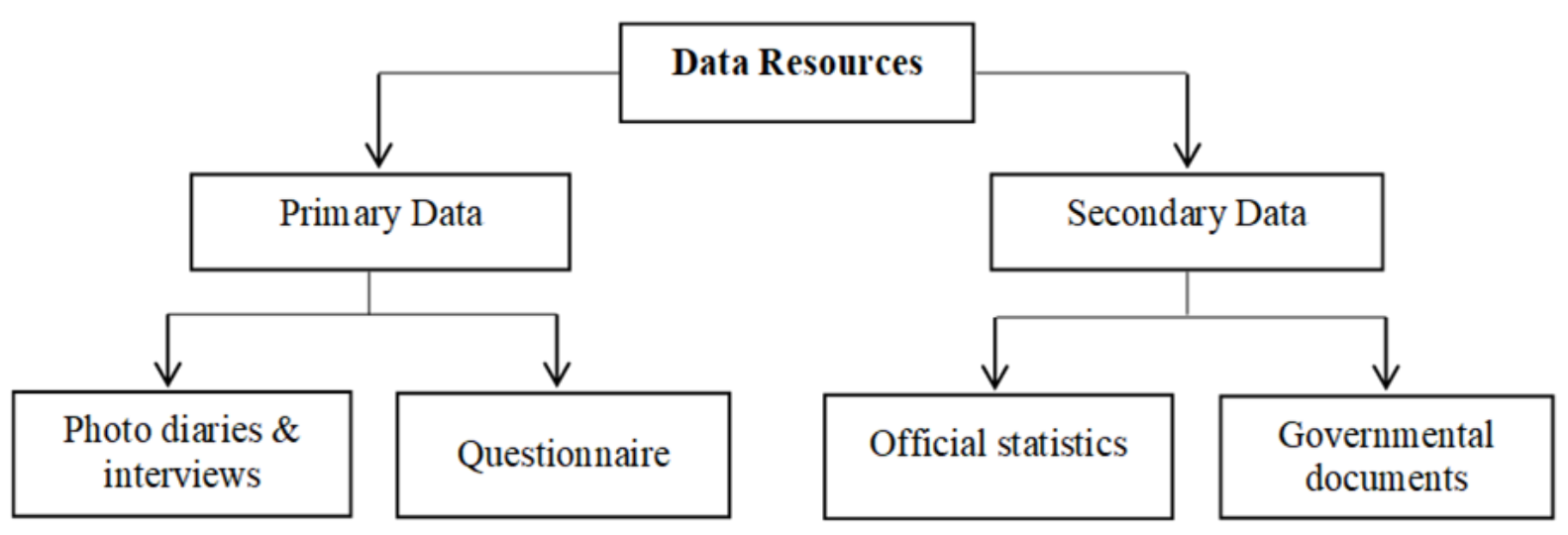

Fig 3.4: Data resources used for the research

\subsubsection{Secondary Data}

Secondary data is a set of reports, statistics, governmental documents and censuses collected by governments, research institutes, and so forth (Flowerdew \& Martin, 2005; Vartanian, 2011; Rezvani et al., 2012). For the QoUL research, secondary data includes statistical, and reports related to aspects of the urban environment identified as an important part of QoUL research. In most QoUL studies reviewed in the literature, the information collected from secondary data resources are related to the objective indicators of QoUL (e.g., Marans \& Stimson, 2011; Mulligan \& Carruthers, 2011; Stimson et al., 2011; Rezvani et al., 2012). As discussed in Chapter 2, this information often centres on aspects of health, education, recreation, housing, and crime rates among others. For this thesis, therefore, secondary data were chosen in order to provide the main source of data related to the objective indicators 
of QoUL study area, including censuses, statistical data and documents relating to the aspects of health, education, recreation, public spaces and heritage.

To collect the official data related to the aspects of QoUL mentioned above, several related governmental agencies in Medina were visited during the fieldwork. During these visits, a letter written by the project supervisor was presented to facilitate the task of the data collection relevant to the research project. This letter contained a description of the research project, including the title of the research, the main objective, data collection instruments and the duration of the collection. This step helped to clarify the main purpose of the researcher's visit to those governmental agencies, which are often cautious in giving any official information. The stage of collecting the official data was designed to obtain the following information:

- Official census data, which includes population and demographic information at the city level were collected from the General Authority for Statistics (GaStat).

- Reports and official statistics issued by government agencies such as the Medina Regional Municipality (MRM), Saudi Commission for Tourism and National Heritage (SCTNH), General Directorate of Health Affairs (GDHA), General Directorate of Education (GDE), Quba Municipality Office (QMO), Medina Development Authority (MDA) and Medina Research and Studies Centre (MRSC). These resources were selected to provide some objective indicators of QoUL in the municipality of Quba, including data regarding the government facilities such as health and education facilities, public spaces such as parks and recreation facilities, as well as cultural heritage indicators such as places, monuments and museums. The sample of the secondary data used in this research including the name, and type of documents is presented in Table 3.2. 
Table 3.2: Sample of the secondary data

\begin{tabular}{lcccc}
\hline No. & Name of the documents & $\begin{array}{l}\text { Type of the } \\
\text { documents }\end{array}$ & Source & $\begin{array}{c}\text { Date of } \\
\text { Publishing }\end{array}$ \\
\hline 1 & Cultural heritage indicators & Statistics & SCTNH & 2018 \\
\hline 2 & Health indicators & Statistics & GDHA & 2018 \\
\hline 3 & Educational indicators & Statistics & GAE & 2018 \\
\hline 4 & Geographical Background of Quba Municipality & Report & QMO & 2018 \\
\hline 5 & Comprehensive Plan for Medina & Report & MDA & 2012 \\
\hline 6 & Urban indicators of Medina & Report & MRM & 2017 \\
\hline 9 & The 16th Directory of Services in Medina & Report & GaStat & 2017 \\
\hline 10 & Humanising cities program for Medina & Report & MDA & 2018 \\
\hline
\end{tabular}

\subsubsection{Primary Data}

Primary data is the original data collected by researchers for a particular research or project. This type of data can be collected by many tools in different ways, most commonly questionnaires, interviews and observations (Salkind, 2010). The QoUL literature supports information collected by questionnaires and interviews as primary data, as illustrated in the works of Kerce, (1992), McCrea et al. (2011), Rezvani et al. (2012), Marans, (2014) and Soleimani et al. (2014). For this thesis, primary data are used to produce the subjective indicators of the QoUL in the study area, which refer to the judgements and perceptions of people about their urban environment. In addition to that, the judgement of experts regarding several QoUL domains in the study area was obtained. To collect the primary data, therefore, the research used three stages alongside the first stage of the secondary data collection. These three stages encompass conducting household surveys using an online questionnaire, photo diaries and follow-up interviews, and interviews with experts, which are discussed in turn below.

\subsubsection{Household Survey}

The household survey was the first stage of data collection, representing the quantitative part of this thesis. In this regard, a self-administered online questionnaire was employed using a survey platform operated by SurveyMonkey to collect the data from the participants living in the study area. Based on the QoUL literature, this data collection method is the 
most common tool used, especially in gathering subjective evaluation of QoUL (Türksever $\&$ Atalik, 2001). In this thesis, an online questionnaire using survey platform operated by SurveyMonkey was utilised to collect the data from the participants who live in the study area. The reasons for the choice of this type of questionnaire were as follows. Firstly, the online questionnaire saves time and cost (Bell, 2010), especially since this fieldwork was conducted outside the UK. Secondly, it offered the possibility of collecting data and reaching as many participants as possible in a relatively short time (Howard, 2009). Thirdly, online questionnaires tend to be returned considerably more quickly than other versions (Bryman, 2012). Fourthly, an online survey, especially a web survey, facilitates better accuracy of data entered (Bryman, 2012). Finally, and the most importantly, there is the possibility of including diverse participants (i.e., gender diversity) in a situation where data collection from diverse participants is often considered as one of the research limitations in Saudi Arabia (e.g., Khizindar, 2012), due the prevailing culture that often prevents the meeting between males and females. This tool, therefore, could help to gain a better understand regarding the perceptions of the residents about the urban environment in light of the adequacy of representation of the participants in the study area.

The questionnaire was disseminated to the residents within the sample frame during the fieldwork, by means of a web address (link) to which respondents could be directed in order to complete it (Bryman, 2012) The dissemination of the questionnaire to the targeted population was firstly carried out using social media; the Saudi population in general has one of the highest per capita rates of using social media platforms (Sanchez, 2017), where the use of social media in general has become increasingly popular (Efthymiou \& Antoniou, 2012). For the process of disseminating this questionnaire, the researcher relied on assistants who were distributed in the neighbourhoods of Quba municipality, along with the additional support of the Media Centre at Taibah University in Medina, where the questionnaire was sent to the e-mail lists available in the database there.

\section{- Questionnaire Sampling}

In order to gain a better understanding of how people experience their QoUL in Quba, the aim was to have as many respondents as possible. In this research, the household survey comprised in general the population living in the municipality of Quba. The sample is represented by any available adult (18 years old or older) willing to take part in this study 
and share their experience. The diversity of participants in terms of gender and ages was of interest. It therefore became important to apply an appropriate sampling technique to this end, since the researcher encountered some difficulties during the data collection phase related to the time as well as the extent to which respondents could be reached.

In light of the circumstances mentioned above, a snowball sampling technique was applied in order to reach the participants. This technique is one of the most common forms of nonprobability sampling. It is particularly used when the researcher finds the population of interest hard to reach, perhaps because it is difficult to communicate with them (Etikan, et al., 2016; Dhivyadeepa, 2015; Macnee \& McCabe, 2008). For this thesis, this sampling technique is appropriate to create a balance within the respondents in terms of gender diversity, where it has been difficult to reach the female community in the study area, helping to obtain a range of experiences and views regarding the QoUL in the study area. However, there are weaknesses of adopting this approach to sampling. One such weakness is that this technique may generate a bias which may affect the validity of the sample, since items are not drawn randomly, but depend on the subjective choices of respondents who are reached first. Thus most snowball samples tend to exhibit a level of bias and so do not allow researchers to make claims of generality from a particular sample (Atkinson \& Flint, 2001). Despite that, this technique significantly helped in reaching a large number of participants and a greater sample diversity in record time, especially with regard to the access to the female community in the study area.

Alongside helping to reach a certain segment of society in an area, the snowball technique was also employed to facilitate obtaining a large number of respondents. To this end, the questionnaire was sent to an initial group of respondents who then sent it to further respondents and so on. This supported the process of the dissemination of the questionnaire in terms of gaining a higher rate of respondents within the respondents own social networks. As a result, 837 respondents were reached. The completion rate of this questionnaire is $65.47 \%$, representing 548 respondents who fully completed the form. In terms of gender diversity, which was a key element of the limitations of this thesis, an appropriate balance was achieved, where 440 respondents were males, constituting $52.57 \%$, while the number of the female respondents is 397 , constituting $47.43 \%$. However, 289 respondents who did 
not complete the questionnaire were excluded in order to not affect the analysis process of this thesis.

\section{- Questionnaire design}

In this research, questionnaires are used to evaluate QoUL domains and indicators in the study area, in which residents who live in Quba municipality are asked to evaluate those domains in accordance with their needs and experience. As this type of data collection method is widely used in the QoUL literature, the questionnaire used in this thesis was designed based on the literature (i.e., WHOQOL Group, 1998; Christakopoulou et al., 2001; WHOQOL SRPB Group, 2006). This questionnaire mostly included closed questions and a few open-ended questions to measure the citizens' satisfaction with those domains and indicators. Hence, it includes both quantitative and qualitative data. Most of items in the tool were created based on the Likert scale using a 5-point scale, typically multiple-choice options ranging from 1 very unsatisfied, to 2 unsatisfied, 3 neutral, 4 satisfied and 5 very satisfied. However, a 3-point Likert Scale has been used in some cases. This is because results in some tables (i.e., cells) have not met the minimum requirements of Chi-squared tests. Therefore, columns in some tables have been aggregated to be a 3-point Likert Scale instead of a 5-point scale. As evidenced from the literature, the Likert scale is one of the most used options within such questionnaires (e.g., McCrea, et al., 2005; Keul \& Prinz, 2011; Salleh \& Badarulzaman, 2012; Shuja et al., 2014; Stasson et al., 2016). In addition to that, ranking scales, rating scales and dropdown questions were utilised.

In this questionnaire, the aim was to include most of the QoUL domains and indicators to gain a better understanding of the overall QoUL in the study area. Therefore, the questionnaire was presented in six parts. Except for the introduction, each part addressed a particular topic related to the research. First, the introduction gave a welcome and brief overview of the study aims, along with instructions and brief information about the researcher and supervisors (i.e., names and contact details). The second part included sociodemographic attributes including gender, age, marital status, educational level, employment status, income and residential status. The third part encompassed statements regarding perception about QoUL. The fourth part covered statements regarding the physical domain of the QoUL, which included the domains of housing, neighbourhood and urban services. 
Each of these domains was followed by several variables. The fifth part included statements regarding the social aspects, while the sixth part covered statements about the cultural domains. Finally, it concluded with any added comments or opinions on the part of the respondents. In short, the first part dealt with introductory material, the second part with demographic information, the third part perception about QoUL), and the three following parts encompassed the main domains of the QoUL selected for this thesis. The questionnaire was initially designed in the English language (see Appendix 1), then, was translated into Arabic (see Appendix 2) in order to be understood by the local community in the study area. After completing the questionnaire in a written version, it was loaded onto the software to send it to the residents in the study area using the web address that held the questionnaire in an online version.

\subsubsection{Photo Diary Study}

For measuring QoUL experience in a specific place, the perceptions of residents about the urban environment in which the live, as well as how they experience their daily life, need to be identified. In this thesis, a photo diary study is the second stage of data collection within primary data and represents the qualitative part of this thesis. This method is considered in the literature as a useful way of gaining insight into how people view their urban environment. The photo diary is known as a visual method in qualitative research, especially in the field of human geography (Philip, 1998; Learning Space Toolkit, 2018). This method entails participants taking a number of photographs regarding their daily lives (DeLyser et al., 2010; Hay, 2016). After taking these photographs within a specific period of time (i.e., a short period of time), the researcher conducts an in-depth interview with the participants to discuss the photographs in order gain more information about them (Latham, 2003; Learning Space Toolkit, 2018). The photo diary is thus used to explore the meaning of captured photographs (Carter \& Mankoff, 2005). In this context, participants could be asked about where they took the pictures, why they chose these specific pictures, how important these pictures are to them, and what they feel towards those pictures (Radley, 2010).

In this thesis, therefore, the photo diary study has been adopted as a qualitative method in order to explore further the QoUL domains that have been selected for this research, and because it is likely to give an insightful understanding about the real context in which the participant lives. Furthermore, it allows the researcher to gain rich information from the 
residents while they are experiencing their day-to-day lives. In terms of this thesis, the photo diary study is based on taking -one to two photos every day for two weeks, taking into account the components of the urban environment in the municipality of Quba which are based on prespecified themes related to the QoUL domains selected for this thesis. After the process of taking pictures, the participants are required to take part in an in-depth, face-toface interview.

To apply this method for the case of Quba municipality, 25 photo diaries and follow-up interviews were conducted. The process was firstly to determine the participating sample, then determine an appointment for the initial interview. Secondly, initial interviews were conducted with participants to explain the themes of the photo diaries, with more focus on the social and cultural themes. Before beginning those interviews, an instruction sheet, including the targeted themes for the photo diaries and the study procedures, were given to the participants (see Appendices $3 \& 4$ ). The duration of these initial interviews was between 15-20 minutes, where some participants met together in groups. Thirdly, follow-up interviews were conducted after the participants had taken their photos for the purpose of the study. These interviews were conducted separately to discuss the photographs taken. The duration of these follow-up interviews was between 20-30 minutes. In this thesis, ethnic background as well as diversity of age was considered, with participants from multicultural and different age groups taking part.

\subsubsection{Expert interviews}

Interviews with experts is the third stage within the primary data, representing the second qualitative part in this thesis. This method is based on face-to-face interview technique, which, according to the literature, is the most utilised instrument in empirical social researches (Mikkelsen \& Di Nucci, 2015). In this thesis, the main objective of the expert interviews is to obtain further explanations and details regarding QoUL in Medina and the municipality of Quba in particular, and to support the findings of the research especially in the interpretation phase. In choosing this method, a number of considerations were taken into account. Firstly, interviews with experts are likely to provide some effective information, which may contribute to the richness of this thesis by maintaining a consistency that may help in comparisons and assessments of the results from both qualitative and quantitative research. Secondly, expert interviews are likely to help capture more 
information about the priorities that the citizens place on different aspects of QoUL as well as about the urban issues that might affect the overall QoUL of Medina's citizens. Finally, the experts may offer different views regarding the experience of QoUL in the city of Medina and the municipality of Quba in particular. In line with the multidimensional and complex nature of the QoUL, it was important to conduct interviews with experts from different institutions and organisations in various disciplines in Medina. That is, a mix of interviews was needed in order to obtain different opinions in accordance with the dimensions of QoUL, as well as to get a better understating regarding both the explicit and implicit links between the various aspects related to urban environment in the study area. Therefore, a number of organisations in Medina, from the academic field and professional arena, both governmental and non-governmental agencies, were selected. This determination of these organisations was based on considerations such as involvement in urban development, namely contribution within education, research planning and so on, and in this regard, the targeted organisations were Taibah University, DAM, MRSC and other non-governmental agency. However, due to some recommendations from the experts being interviewed, one of the interviews was conducted with an expert in an organisation outside Medina.

At this stage of qualitative data collection, semi-structured interviews were conducted with 10 experts from different disciplines. The first step in this phase was to identify experts who had experience or relevance to the field of research, and whom the researcher was confident had a degree of knowledge and an appropriate experience within the organisations mentioned earlier. To this end, these organisations were visited to identify the potential interviewees. However, some of the organisations asked the researcher to apply for a permission to conduct the interviews. Therefore, a request was made in the form of a written letter explaining the research project and the purpose of the interview. In addition to that, the recommendation from the research supervisor for conducting these interviews was attached. Although this procedure took some time, it helped to reach the experts who are interested in research area.

The second step was to meet the experts who had been identified. Therefore, an initial meeting with each expert was carried out in which a set format was followed. An introduction about the researcher and the nature of the research project, including its 
objectives and importance as well as the purpose of the interview was given. In addition to that, the reason behind choosing the interviewees, as well as the extent of the contribution of this interview to enrich the research were presented. In most of these initial meetings, an appointment was arranged for the interview, including date, time and location, while a few experts requested the contact details to contact the researcher later to arrange an appropriate date for the interview.

The final step of this stage of collecting the primary data is to conduct the actual interviews for the purposes of this thesis. After the initial meeting with identified experts, subsequent meetings were arranged to conduct the interviews at various times during the fieldwork. These interviews were conducted in the places where the participants worked, in both governmental and non-governmental organisations. Most of these participants were located in a separate office, which enabled the interview to take place in a quiet atmosphere. Before the start of these interviews, participants were given the opportunity to ask questions about the research topic if they had any. Furthermore, participants' consent was obtained for an audio digital recorder to be used to record the interview; all participants accepted this step. In this regard, the participants were informed that the recording would be kept confidential, and only used for later reference. In addition to that, notes were written down in a notebook during the discussion. After finishing the interview, the participants were thanked for their acceptance and willingness to conduct this interview.

Within these interviews, which took a minimum of 38 minutes and a maximum of 58 minutes, a series of topics related to the focus of research were discussed in an attempt to highlight aspects affecting the QoUL. Through these discussions, there was an opportunity to obtain a set of ideas and views that reflected the impact of these aspects on the QoUL in the study area. In order to conserve the consistency in those interviews, an interview schedule was prepared in advance. In this schedule, the queries attempted to cover the topics in relation to the research focus, especially the main domains of the QoUL selected for this research. Moreover, a greater generality in addressing the ideas of the research was considered in an attempt to get a comprehensive view of the QoUL components in the study area. The questions covered in the interviews were as follows: 
- What is your expertise about the QoUL?

- According to the literature, urban environmental quality can be measured by using set of domains, namely, urban, economic, social and cultural domains followed by number of sub-domains or indicators. In your opinion, what is the key indicators of QoUL in the city of Medina?

- Could you please rank those indicators?

- In your opinion, what are the main concerns of people regarding the quality of their urban environment?

- To what extent do physical indicators including urban aspects such as health, education, housing and security services and others urban aspects such as public spaces and the overall appearance of the neighbourhoods contribute to the overall satisfaction of the residents in the Quba municipality?

- In your opinion, to what extent do social aspects such as security, leisure/ recreation activities and social interaction affect the QoUL?

- In what way does spirituality/religiosity affect the QoUL of the residents in Quba municipality?

- To what extent does the presence of the historical landmarks including the heritage contribute to the QoUL in Quba?

- To what extent do cultural monuments such as museums and libraries as well as cultural events such as exhibitions and conferences contribute to QoUL experience in the study area?

- In general, what are the urban issues that need to be considered in the neighbourhoods of Quba municipality?

- Finally, is there anything would you like to add to this discussion?

\section{- Qualitative Sampling (photo diaries \& expert interviews)}

In general, qualitative research is characterised by the sample size, which has been often described in many qualitative studies as small (e.g., Creswell, 2013; Vasileiou et al., 2018; Dworkin, 2012; Gentles et al., 2015). In this regard, many of those studies advocate using smaller samples when doing qualitative research. For example, Mason (2012) suggests that it is better in qualitative research to conduct a smaller number of interviews that can be 
analysed creatively and interpretively, rather than identifying samples of larger sizes, where the time for doing the analysis might run out. Regardless of the limited size of the sample, the general objective of qualitative research is to obtain useful information, which is often characterised by depth and complexity as well as the context surrounding the phenomenon under investigation (Dworkin, 2012; Gentles, et al. 2015).

Since the qualitative methods in this thesis have tended to be mostly based on interviewing, a sampling approach was adopted for both photo diaries and expert interviews. The aim of using these approaches is to gain a deeper understanding of the QoUL in the study area, including its characteristics, circumstances and the factors contributing to its overall state. To this end, rich data containing useful information that can answer the research questions were needed. Therefore, access to a sample representing the population in the study area first, and then an appropriate level of education with respect to these populations was one of the most important considerations in the selection of the sample for photo diaries. In addition, participants with a deeper knowledge and proper experience were needed for the expert interviews. Accordingly, a purposive sampling technique was used to select the participants for both qualitative methods used in this thesis.

Purposive sampling is one of the sampling techniques frequently used in qualitative researches (Polit \& Beck, 2008). Purposive sampling is known as judgemental, selective or subjective sampling, where sampling depends on the researcher's judgement when it comes to selecting the units to be investigated (Johnson \& Lavrakas, 2008; Christensen, 2014; Dhivyadeepa, 2015). This technique of sampling has been criticised as reflecting the bias of the researcher, as well as not being representative of the population. However, the use of this technique in the research that employ a mixed methods design is not a weakness (Dhivyadeepa, 2015). Based on the consideration of the qualitative samples mentioned earlier, as well as what distinguishes purposive sampling discussed above, this thesis used this technique of sampling for both qualitative methods, as discussed below.

\section{Photo Diaries Sampling}

For this method of data collection, a purposive sampling technique was employed to select 25 participants within in the municipality of Quba. Although this size of the sample is considered in many qualitative researches as an acceptable size, it is assumed from the point 
of view of this thesis as relatively large. This assumption is based on the process involved in conducting this study, which requires conducting two interviews: one as an initial interview to explain the themes related to the study and the instructions for the photo diary, and the second is a follow-up interview to discuss the photographs that have been taken by the participants.

The sample for the photo diaries was purposefully selected to meet a number of criteria. Firstly, in order to gain insight into how people experience their daily lives with regard to the QoUL, there was a need to reach participants with an appropriate level of education as the researcher had faced a negative research experience when attempting research with a resident with a low level of education. It was important to reach those who had a high level of education in order to obtain sufficient information regarding the experience of the QoUL in the study area, as participants with a good level of education are more likely to express their experience in such a way that the researcher can obtain data that enriches research. Secondly, there was a need to find participants from different cultural backgrounds as diversity is a key element for selection. In this context, a purposive sample entails obtaining participants with certain characteristics that include as much diversity as possible among them (Macnee \& McCabe, 2008).

To select this sample, the aim was to identify participants from different neighbourhoods in the study area in accordance with the criteria mentioned above. Therefore, a snowball approach was employed to reach the targeted size of the sample, as well as diversity within the sample in terms of gender. To this end, an initial group of participants was identified who then have given support in finding further participants until the target sample was reached. As a result, a relatively appropriate balance among the participants in terms of gender was met, where the sample of the photo diaries is represented by 15 males and 10 females. In addition, a diversity of culture among participants was also met, with participants from different cultural backgrounds were obtained. The demographic information (age, gender, educational level, ethnic background etc.) of these participants is shown in Table 3.3 . 
Table 3.3: Sample detail of the photo diaries study

\begin{tabular}{|c|c|c|c|c|c|c|}
\hline $\begin{array}{l}\text { Code of } \\
\text { the interviewee }\end{array}$ & Gender & Age & $\begin{array}{c}\text { Marital } \\
\text { status }\end{array}$ & Education & $\begin{array}{c}\text { Employment } \\
\text { status }\end{array}$ & Nationality \\
\hline PH 1 & Male & 43 & Married & Master & Employee & Saudi \\
\hline PH 2 & Male & 34 & Married & Bachelor & Employee & Saudi \\
\hline PH 3 & Male & 38 & Married & Master & Employee & Saudi \\
\hline PH 4 & Male & 43 & Married & Bachelor & Employee & Saudi \\
\hline PH 5 & Male & 28 & Married & Bachelor & Student & Yemeni \\
\hline PH 6 & Male & 30 & Single & Bachelor & Employee & Saudi \\
\hline PH 7 & Male & 35 & Married & Bachelor & Employee & Saudi \\
\hline PH 8 & Male & 45 & Married & Bachelor & Employee & Saudi \\
\hline PH 9 & Male & 37 & Married & Bachelor & Employee & Saudi \\
\hline PH 10 & Male & 34 & Married & Bachelor & Unemployed & French \\
\hline PH 11 & Female & 41 & Married & $\mathrm{PhD}$ & Employee & Egyptian \\
\hline PH 12 & Female & 52 & Married & $\mathrm{PhD}$ & Employee & Egyptian \\
\hline PH 13 & Female & 46 & Widow & $\mathrm{PhD}$ & Employee & Saudi \\
\hline $\mathrm{PH} 14$ & Female & 42 & Married & $\mathrm{PhD}$ & Employee & Egyptian \\
\hline PH 15 & Female & 41 & Married & $\mathrm{PhD}$ & Employee & Saudi \\
\hline PH 16 & Female & 39 & Married & Master & Employee & Saudi \\
\hline PH 17 & Female & 42 & Married & $\mathrm{PhD}$ & Employee & Saudi \\
\hline PH 18 & Female & 38 & Married & $\mathrm{PhD}$ & Employee & Saudi \\
\hline PH 19 & Female & 45 & Married & $\mathrm{PhD}$ & Employee & Saudi \\
\hline PH 20 & Female & 40 & Married & Master & Employee & Egyptian \\
\hline PH 21 & Male & 34 & Married & Master & Student & Indonesian \\
\hline PH 22 & Male & 30 & Married & Bachelor & Student & Nigerian \\
\hline PH 23 & Male & 42 & Married & Bachelor & Employee & Algerian \\
\hline PH 24 & Male & 27 & Married & Bachelor & Student & Sri Lankan \\
\hline PH 25 & Male & 30 & Married & Master & Student & Guinean \\
\hline
\end{tabular}




\section{Expert Interview Sampling}

As this method aims to generate a richness of data, purposive sampling was used to select potential experts or informants who have knowledge relevant to the research focus, and who the researcher understands to have proper experience. That is, for the purpose of gaining insightful information that can contribute to the richness of research, 10 experts within various disciplines in Medina were selected to be interviewed face-to-face. However, one interview was conducted by phone with an expert who was highly recommended by one of other experts. Alongside the consideration of selecting experts with relevant knowledge, there was a need for diversity among those experts in terms of the disciplines, to offer different views that could enrich the research, in line with the multidimensional nature of the research focus as represented by the QoUL domains identified for this study.

This size of sample was determined largely by the fact that these types of interviewees (i.e., experts) are often harder to access, which could be a significant issue with regard to the timing of data collection and the overall schedule of the research. In light of this, a snowball approach was used to help reach the sample size, taking into account the criteria for selecting participants as well as the time determined for this stage within the time frame of the data collection. This approach entailed interviewing an initial group of experts. In so doing, there was an opportunity to obtain recommendations for further interviews with relevant experts.

Within this sample, 4 of the interviews were carried out with university professors, while 3 interviews were conducted with urban planners from MDA, and 2 interviews were conducted with former architects at MRM, and 1 interview was conducted with a professor at MRSC. The sample detail of these interviewees (name, job position, organisation etc.) can be seen in Table 3.4. 
Table 3.4: Sample detail of the expert interviews

\begin{tabular}{|c|c|c|c|c|c|}
\hline No. & Name of interviewee & Job position & Organisation & Date & Duration \\
\hline 1 & Osama Nassar & $\begin{array}{c}\text { University professor/ } \\
\text { Architecture }\end{array}$ & Taibah University & 12.11.2018 & $55 \mathrm{~min}$ \\
\hline 2 & Khaled Alsaidalani & $\begin{array}{c}\text { University professor/ } \\
\text { Human Geography }\end{array}$ & Taibah University & 15.11.2018 & $55 \mathrm{~min}$ \\
\hline 3 & Khaled Hakim & Urban planner/ Architecture & MDA & 28.11 .2018 & $53 \mathrm{~min}$ \\
\hline 4 & Jalal Abadah & Urban Adviser/ Architecture & MDA & 05.12 .2018 & $47 \mathrm{~min}$ \\
\hline 5 & Salah Salamah & $\begin{array}{c}\text { University professor/ } \\
\text { Medina history }\end{array}$ & Taibah University & 10.12.2018 & $58 \mathrm{~min}$ \\
\hline 6 & $\begin{array}{l}\text { Mohammed } \\
\text { Al-Shaibani }\end{array}$ & $\begin{array}{c}\text { Scientific Department } \\
\text { Supervisor/ } \\
\text { Islamic history }\end{array}$ & MRSC & 15.12.2018 & $49 \min$ \\
\hline 7 & Saeed Jood & $\begin{array}{l}\text { Former Urban Planner/ } \\
\text { Engineering consultant }\end{array}$ & $\begin{array}{l}\text { Private Engineering } \\
\text { Consulting Office }\end{array}$ & 25.12.2018 & $39 \mathrm{~min}$ \\
\hline 8 & Abdullah Alhusyn & $\begin{array}{c}\text { University } \\
\text { professor/Architecture }\end{array}$ & $\begin{array}{l}\text { King Saud } \\
\text { University }\end{array}$ & 02.01.2019 & $40 \mathrm{~min}$ \\
\hline 9 & Abdulaziz Kaaki & $\begin{array}{c}\text { Author specialising in urban } \\
\text { studies of Medina/ } \\
\text { Architecture }\end{array}$ & $\begin{array}{l}\text { Dar Al-Madinah } \\
\text { Museum }\end{array}$ & 09.01.2019 & $38 \mathrm{~min}$ \\
\hline 10 & Abdullah Kabir & $\begin{array}{l}\text { Researcher in the history } \\
\text { and landmarks of Medina }\end{array}$ & MDA & 15.01.2019 & $46 \min$ \\
\hline
\end{tabular}

\subsection{Time scale of research}

In line with the research design of the study, namely a convergent parallel design, the survey followed the 'cross-sectional study' type which has been defined by Sekaran (2016) as "a study in which data are gathered just once, perhaps over a period of days, weeks or months, in order to answer a research question" (p. 104). Accordingly, the data collection was carried out in one phase, over three months from 23 October 2018 to 23 January 2019. A crosssectional study is the appropriate technique for this research in terms of time and costs, especially since this study was conducted outside the UK.

This period was chosen as an appropriate period to collect the data for a number of reasons. Firstly, the beginning of this period is the end of the Umrah and Hajj seasons when the city is crowded for about four months. Secondly, there is an educational holiday of up to four months prior to the period of the data collection, as well as some public holidays, which may have made it difficult to reach a sufficient number of participants. In addition, the 
closure of most governmental facilities at separate periods of times within those same four months might also have made it difficult to collect the objective data of the study area. Therefore, this specific time for the data collection was designed to facilitate the accessibility of both subjective and objective data. The details of the data collection timeline are given in Table 3.5.

Table 3.5: Timetable for the data collection

\begin{tabular}{cccc}
\hline Data Collection Method & Type of Data & Start Date & End Date \\
\hline Questionnaire (Subjective Indicators) & Primary data & 23.10 .2018 & 03.12 .2018 \\
\hline Photo Diary (Subjective Indicators) & Primary data & 04.12 .2018 & 18.12 .2018 \\
\hline Follow up interview (Subjective Indicators) & Primary data & 19.12 .2018 & 02.01 .2019 \\
\hline Expert interview & Primary data & 03.01 .2019 & 10.01 .2019 \\
\hline Survey for the Official data (Objective Indicators) & Secondary data & 11.01 .2019 & 22.01 .2019 \\
\hline End of the data collection 23.01.2019 & & \\
\hline
\end{tabular}

\subsection{Data analysis strategies}

As this research uses a mixture of methods (i.e., quantitative and qualitative research), analysis of the data is divided into two main sections: quantitative analysis and qualitative analysis.

\subsubsection{Quantitative analysis}

Quantitative analysis was mainly based on the use of SPSS statistics 25 software. The SPSS is the most common statistical data analysis software package (Muijs, 2004). Before the start of the data analysis, numerical data were recoded into ordinal form to make it possible to examine the relationship between variables, including the variables related to the sociodemographic attributes such as age, number of people living at home, as well as other variables such as the period of time living at the accommodation, number of visits to the doctor and waiting times to see doctors. In addition, as some data including those related the QoUL level, satisfaction with accommodation and satisfaction with the neighbourhood were collected in their original form of a 1-10 scale, transformation was needed to convert those variables into ordinal data of five categories to make investigating the relationship 
between these variables and others practically possible when using cross-tabulation. The original form of these data (i.e., 1-10 scale) was also used in order to provide higher levels of confidence.

As the underlying data were ordinal, based on the five-point Likert scale, as well as in some cases nominal, non-parametric tests were used because the obtained data are not normally distributed. These tests included Spearman's correlation, Chi-square, Kruskal Wallis ANOVA and Mann-Whitney tests. To measure the strength of association between two ordinal variables, Spearman's correlation was used. The Chi-square test using crosstabulation, was used to test if there is a significant association between two variables (either ordinal or nominal). The Kruskal-Wallis or one-way ANOVA test was used to examine statistical difference in domains between demographic variables that appear in more than two categories. The Mann-Whitney tests was used examine statistical difference in domains between demographic variables that appear only in two categories. In addition, measures of central tendency as well as descriptive univariate analysis comprising frequencies were used in some cases. Bar charts were also used for additional illustration regarding the associations found between different variables.

\subsubsection{Qualitative analysis}

According to Bogdan and Biklen (2007), qualitative analysis is defined as "working with the data, organising them, breaking them into manageable units, coding them, synthesising them, and searching for patterns" (p. 3). Qualitative data analysis aims to discover concepts, patterns, themes and meanings. This thesis employs the two approaches of word frequencies and similarities using NVivo, and thematic analysis to analyse the data. The process of qualitative data analysis entails sequential procedures. First, interviews were transcripted in their original form (i.e., in the Arabic language), then they were translated from Arabic to English before being imported to the software. NVivo 12 software was used for ease of management. Through the process of coding and thematic analysis of the data, it became clear that there were several key themes arising, which have been considered to be key elements of the QoUL, particularly those related to the social and cultural domains. 


\subsection{Ethical considerations}

The importance of the ethical considerations is evident in the fact that the individuals involved in the study express themselves and their experiences in their daily life. Moreover, this importance is also evident in the role played by the researcher in different contexts, and in the personal privacy of individuals during the data collection. Thus, ethical considerations have become paramount in the research process (Creswell \& Alquhtani Trans, 2018). As this thesis involves human subjects by using both qualitative and quantitative methods, ethical issues have been taken into account. Thus, an ethics application was appended with the research proposal, and research tools to be used in the field work along with an ethics declaration were submitted to the Ethics Committee in the College of Science at Swansea University. The application was approved prior to fieldwork commencing.

Concerns about research ethics are a matter of ongoing debate. Most discussions on ethical principles, however, tend to revolve around particular ethical issues (Bryman, 2012). This thesis thus considers the following ethical issues.

- Informed consent forms

According to Kumar (2011) informed consent implies that the participants are well-aware of the information that will be elicited from them and of the reasons for searching for that information. In addition, other aspects such as the purpose of the research, the expectations concerning their participation in the research, and the impact of participation on them should be clarified. That is, the respondents must be given the opportunity to be fully informed about the nature of the research at the outset (Bryman, 2012). In this thesis, the participants were informed about the main objective of this study and about the circumstances of their participation, including voluntariness and the confidentiality of the information they provide, as well as their contribution to this research. Thus, the participants' informed consent was obtained in a written format (see Appendices 5 \& 6).

For the surveys (i.e., questionnaires), participants were informed that the questionnaire was for research purposes only and any information they provided would be treated in strictest confidence. This was clearly mentioned on the first page of the questionnaire. For the qualitative methods, namely, the photo diaries study and expert interviews, before the start 
of interviews all participants were informed about the purpose of the study, voluntary participation and opportunities to withdraw at any time, and their consent was audiorecorded. For the photo diaries, participants' consent was obtained for an audio digital recorder to be used to record the interview, with all participants agreeing to this. In this regard, the participants were informed that the recording would be kept confidential, and only used for the purpose of this specific study. In addition, all participants were anonymised (given codes instead of their actual names). For the expert interviews, participants agreed to the interviews to be recorded and they were assured that their responses would be used solely for the purpose of the research. All interviewed experts agreed to their names being mentioned in this thesis. In this regard, the waiving of the right to anonymity by each expert was audio-recorded at the beginning of each interview.

\section{- Anonymity and confidentiality}

This principle involves to not revealing the identity of the participants and maintaining the confidentiality of the information provided by them (Akaranga \& Makau, 2016). In this thesis, the participants were assured that the information they provided within their participation would be kept confidential and would only be used for research purposes. In addition, non-disclosure of any personal information related to them was assured, and that the information they provided would be anonymous. In this regard, participants in this thesis are referred to by using codes rather than mentioning their names.

\section{- Harm and Risk}

This principle implies that the research does not harm participants in relation to some harms such as loss of self-esteem, stress, and exposure of their privacy (Bryman, 2012). In this thesis, participants were assured that their participation would not cause any harm. To this end, participants were interviewed in comfortable situations both in terms of time and place. In addition, participants were treated in accordance with the principles of research ethics, which emphasise avoiding the use of an inappropriate manner of conversation. 


\subsection{Researcher positionality}

This section reviews and discusses the positionality of the researcher during the data collection process. As a male researcher, it was expected that speaking to females in the gender-segregated society of Saudi Arabia would be difficult and require special efforts to connect with this category of participant. Despite the fact that the new Saudi Labour Code, adopted in 2005, "no longer requires sex segregation" (Ertürk, 2009, p.11) in universities and other workplaces, according to the country's Islamic law and traditions men are not expected to interview women directly. As a result, I had to undertake several steps in order to reach the female participants in the academic community at Taibah University. One of these steps was the use of the group audio communication at the university, where during the first meeting the themes and instructions for the photo diaries were explained to the whole group at one time. Then, each participant was interviewed separately on the phone. In my approach, I drew on theoretical orientation that viewed cross-gender interviewing as an intersubjective practice (Maynard and Purvis, 1994) requiring additional reflexivity when raising questions and letting respondents speak for themselves. I was particularly aware that my background, attributes and values could have affected some of the answers provided by female interviewees, so I was used sensitive language and reflexive strategies to avoid reproducing the gender-specific norms dominating the public discourse in Saudi Arabia. Many of the women I spoke to had a high level of education, which helped me to find "an opening, a way of gaining access to sources for understanding through growing involvement" (Lave \& Wenger, 1991, p. 37) and co-construct the narratives in my study. Moreover, the recent social changes in Saudi Arabia have started to affect the ways in which gender roles are perceived, particularly in relation to the meeting/ speaking of men with women, which made my fieldwork a bit easier. 


\subsection{Summary of the chapter}

Despite the existence of a wealth of literature in the urban field, QoUL has been relatively unexplored in the context of Saudi Arabia in general, and Medina in particular. In the field of QoUL in general, some indicators of QoUL such as religion and heritage and their influence on the QoUL have not been adequately addressed. Moreover, most of the methodologies adopted in these studies have not adequately examined how people experience their daily lives. As result, it is still somewhat unclear how those indicators influence the QoUL. Therefore, this thesis has adopted two conceptual approaches in an attempt to gain a comprehensive understanding of the urban environment by dividing its characteristics and highlighting the most important aspects affecting the QoUL. Hence, there is a need to identify different methods that are in line with conceptual approaches adopted. Thus, a mixture of quantitative and qualitative research and the associated tools have been adopted.

This chapter has presented the methodological framework for this thesis, including the philosophical worldview, research design, research approach, focus of research, selection of domains and indicators for the research, procedures of data collection, time scale of research, data analysis, the ethical issues which need to be considered and finally discussion of the researcher positionality during the data collection prosses. In so doing, this chapter has set out the justification for and the usefulness of the methodological approach of this thesis. For example, the philosophical worldview of this research (i.e., pragmatism) has led to the identification the research strategy, methods, tools and procedures that are in line with the nature of the QoUL. As a result, quantitative research, represented by the use of the administered questionnaire, and qualitative research, especially the photo diaries study, have facilitated the data collection that indeed reflects the experience of the QoUL in the study area. However, a number of challenges have been faced in terms of the data collection procedures, most notably the extent to which participants can be reached as well as the time frame for collecting and translating the data, since fieldwork has been done outside the UK. 


\section{Chapter Four}

\section{General Reflection on QoUL in Quba}

\subsection{Introduction}

While Chapter 3 presented the research methodology by identifying the research methods, instruments and strategies of the analysis, this chapter represents the start of the analytical part of this thesis, where it sets out a general overview of and reflection on various indicators on the QoUL in Quba municipality. Firstly, it presents the demographic profile of the participants followed by a discussion as to the impact of the socio-demographic attributes on the QoUL in the study area. Secondly, the chapter provides an analysis of the current state of the QoUL in the study area, including the general perception of the residents about their QoUL as well as the most influential aspects of life that have impact on the QoUL. In so doing, this chapter represents the broader analysis of the QoUL in the study area, which will be relied upon for the further analysis of the more specific parts within this thesis. That is, the analysis here sets the foundation for the rest of the empirical work of this thesis.

\subsection{Demographic profile of the study's participants}

The underlying sample comprised 548 participants out of the total population of the municipality of Quba, which stands at 337,124 inhabitants (QMO, 2018). (See Table 4.1) Within this sample, $50.4 \%$ of the participants were males, and $49.6 \%$ females, which represents almost a balance between participants in terms of gender. About $40.7 \%$ of participants were aged 30 years old or less, while $33.8 \%$ of participants fell into the $31-40$ age group, and $25.5 \%$ of participants were over 40 years of age. In terms of marital status, the highest percentage of participants (69.7\%) is in the married category, followed by the singles category $(27.4 \%)$, while the lowest proportion of participants is in the category of divorcees $(2.9 \%)$. With regard to the educational level, more than half of participants have bachelor's degrees $(59.1 \%)$, representing the highest number of the participates, while the 
lowest percentage $(2.7 \%)$ of the participants have only primary and secondary school level education. In terms of employment status, the highest percentage of participants (50\%) fall into the category of employees, while the lowest percentage of participants (2.4\%) fall into the category of self-employed. Regarding residential status, the percentages of home ownership and rental were very close, where $45.1 \%$ own a home and $46.9 \%$ rent a home, whereas only $8.0 \%$ of participants still live in the family home. In terms of number of people living within a home, the size of families ranges from 1-5 persons (43.5\%) and 6-10 persons (48.3\%). In terms of monthly income, $2.4 \%$ of the participants earn less than 1,500 SAR (400 USD), while the highest percentage of the participants (28.1\%) earn between 5,00010,000 SAR (1,333-2,666 USD). 
Table 4.1: Socio-demographic attributes of the study's participants

\begin{tabular}{|c|c|c|c|}
\hline & & No. & $\%$ \\
\hline \multirow{2}{*}{ Gender } & Female & 272 & $49.6 \%$ \\
\hline & Male & 276 & $50.4 \%$ \\
\hline \multirow{3}{*}{ Age groups } & $<=30$ & 223 & $40.7 \%$ \\
\hline & $31-40$ & 185 & $33.8 \%$ \\
\hline & $>40$ & 140 & $25.5 \%$ \\
\hline \multirow{3}{*}{ Marital status } & Divorced & 16 & $2.9 \%$ \\
\hline & Married & 382 & $69.7 \%$ \\
\hline & Single & 150 & $27.4 \%$ \\
\hline \multirow{5}{*}{ Educational level } & Primary \& Secondary school & 15 & $2.7 \%$ \\
\hline & High School & 83 & $15.1 \%$ \\
\hline & Diploma & 50 & $9.1 \%$ \\
\hline & Bachelor's & 324 & $59.1 \%$ \\
\hline & Master's or PhD & 76 & $13.9 \%$ \\
\hline \multirow{5}{*}{ Employment status } & Employed & 274 & $50.0 \%$ \\
\hline & Other (students) & 44 & $8.0 \%$ \\
\hline & Retired & 33 & $6.0 \%$ \\
\hline & Self-employed & 13 & $2.4 \%$ \\
\hline & Unemployed & 184 & $33.6 \%$ \\
\hline \multirow{3}{*}{ Residential status } & Other & 44 & $8.0 \%$ \\
\hline & Own home & 247 & $45.1 \%$ \\
\hline & Rent home & 257 & $46.9 \%$ \\
\hline \multirow{3}{*}{ No. of people living at home } & $1-5$ persons & 238 & $43.5 \%$ \\
\hline & $6-10$ persons & 264 & $48.3 \%$ \\
\hline & $>=11$ persons & 45 & $8.2 \%$ \\
\hline \multirow{6}{*}{ Monthly Income } & $<1500$ (400 USD) & 13 & $2.4 \%$ \\
\hline & $1,500-3,000$ (400-800 USD) & 35 & $6.4 \%$ \\
\hline & 3,000-5,000 (800-1,333 USD) & 65 & $11.9 \%$ \\
\hline & $5,000-10,000(1,333-2,666$ USD) & 154 & $28.1 \%$ \\
\hline & $10,000-15,000(2,666-4,000$ USD $)$ & 129 & $23.5 \%$ \\
\hline & $>15,000(4,000$ USD $)$ & 152 & $27.7 \%$ \\
\hline
\end{tabular}




\subsection{General perception of QoUL (status of QoUL)}

Figures 4.1 and 4.2 illustrate the results obtained from survey in relation to the general perception of QoUL. Using a scale 1-10, Figure 4.1 shows the frequency distribution of data in its original form, while Figure 4.2 shows the frequency distribution of data in transformed form of five categories ranging from very low to very high. Each of the two scales of the original form are represented by one category of the converted form. Using the scale 1-10 where 1 indicates very poor and 10 indicates excellent, the average score was found to be 6.77. It can be noted from Figure 4.1 that the majority of participants tended to rank their QoUL between 5 (moderate) and 8 (high). Figure 4.2 represents the QoUL level in categorical form, where it can be seen that about $56.2 \%$ of the participants considered their QoUL as being high or very high, while only $13.5 \%$ of the participants considered their QoUL as being low or very low.

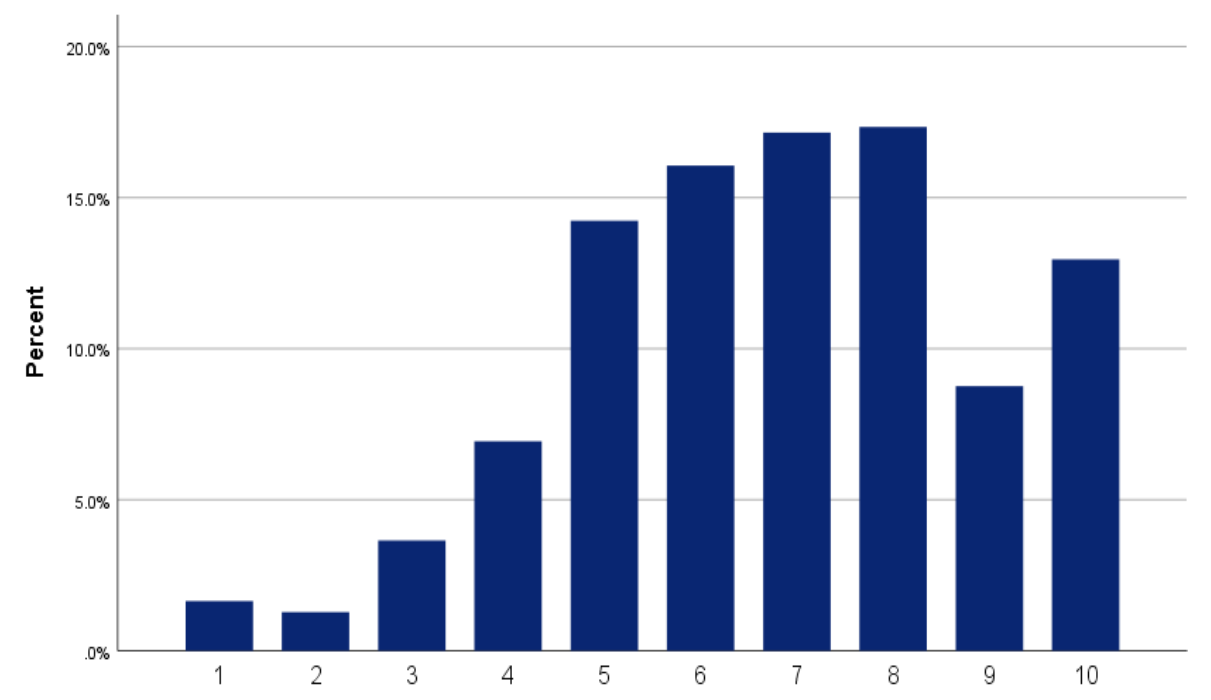

Fig 4.1: QoUL level on a scale 1-10 


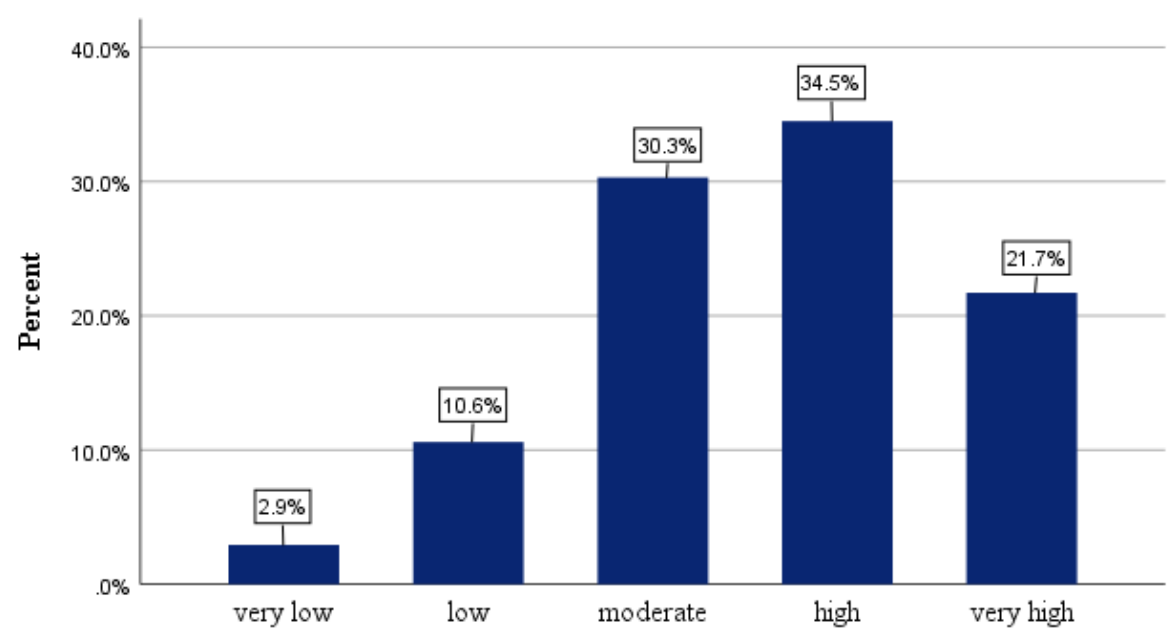

Fig 4.2: QoUL level using 5 categories

These results seem to be consistent with the results of the UN-Habitat report for City Prosperity (Medina CP Profile) in the case of Medina (UN-Habitat \& SMMRA, 2016). In this report, the QoL was determined to be moderately high (65.1\%). However, the QoL index in that study was limited to a few indicators concerning health, education, security, and public places. In this thesis, the positive results of the general QoUL level may be attributed to a number of factors that have led the residents of the municipality of Quba to positively perceive their environment. In general terms, these might be determined as firstly, the quality of the urban environment in the municipality of Quba, at least in some objective indicators. Secondly, the role spirituality may play in producing participants' positive responses, which will be discussed in detail in the following chapters of the thesis. Finally, socio-demographic attributes may play a role in people's perception regarding the environment in which they live. These circumstances will also be discussed in detail in the following chapters.

Within the investigation of the general QoUL perception, different geographical locations were considered. In this respect, the Chi Square test was applied in order to find any sort of association between QoUL level and the location of different neighbourhoods, the results indicated that there is a significant association between QoUL level and the neighbourhoods' location. It can be noted from Figure 4.4 that there are some variations in the perception of the QoUL within the neighbourhoods of Quba municipality. It can be seen 
that the highest QoUL value has been achieved in neighbourhoods such as Al Ranuna, Al Bahr and Al Jumaah. By referring to the sites of those neighbourhoods on the map of Quba, interpretations can begin to be made regarding the relationship between the QoUL level and neighbourhoods' location.

The neighbourhoods that achieved high and very high QoUL, such as Al Bahr and Al Jumaah, are located in the area between the Prophet Mosque (Al Masjid Al Nabawi) and Quba Mosque as well as being in proximity to Al Jumaah Mosque. These mosques represent the most famous holy sites in Medina. Therefore, one possible interpretation with regard to the relationship between the place and the QoUL values scored is that residents seem to have a positive QoUL level when they live close to those holy sites. This interpretation is supported by the answers of the residents from the qualitative question (open-ended question). In this question the residents were asked what they most like regarding their neighbourhood. The most frequent answer is that their neighbourhoods are close to the holy sites mentioned above. This suggests that residents may prefer to live near holy sites because the spirituality related to those holy sites may make them feel comfortable and reassured.

In addition, social relationships might be a factor in the relationship between the location of the neighbourhood and the QoUL. Some participants' responses from the qualitative results support this idea, where they referred to the importance of proximity to parents, relatives and neighbours. In this regard, a number of participants mentioned that the neighbourhoods where they live are characterised by houses that are close to each other. They therefore pointed out that this kind of neighbourhood form supports social relations with relatives or neighbours. In this context, the neighbourhoods that have achieved higher QoUL scores are characterised by the old urban fabric, which is distinguished by the closeness of buildings to each other as well as the proximity to the holy sites, which, according to many participants, adds to the spirituality of the place. Although such neighbourhoods may lack modernity and may suffer from urban issues such as a lack of services as well as narrow spaces and streets, they seem to be preferable to residents of the municipality of Quba than other modern neighbourhoods. This is supported by arguments made by interviewees, both experts and residents, who pointed out that old neighbourhoods are characterised by the close relationship between their residents, respect for the right of the neighbours, help for the needy and participation with others whether in their joys or sorrows. 


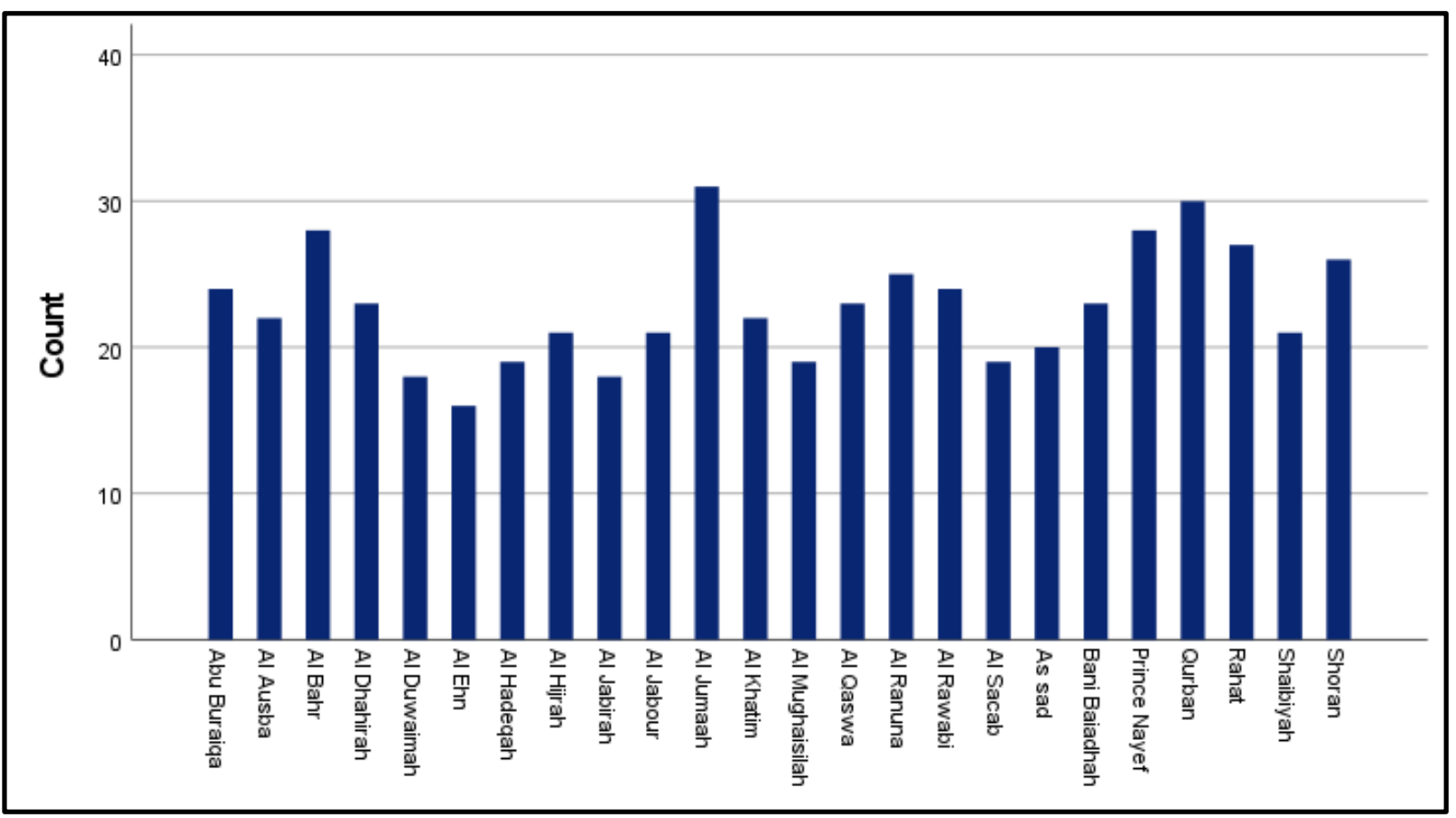

Fig 4.3: Number of responses per neighbourhood

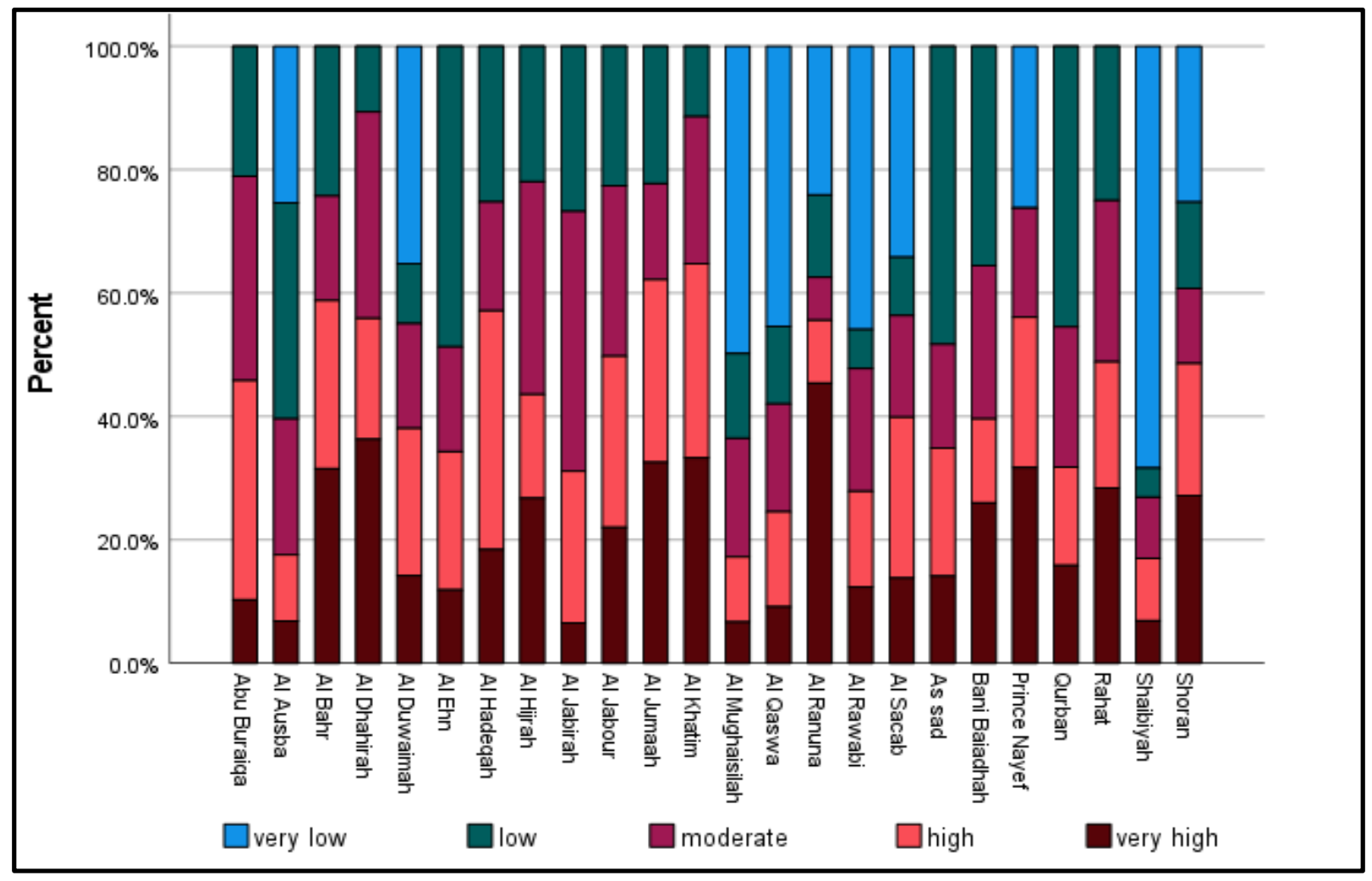

Fig 4.4: QoUL level for each neighbourhood 


\subsection{Impact of socio-demographic attributes on QoUL}

In order to gain more insight into the impact of the socio-demographic attributes on the QoUL of Quba residents, the relationship between each socio-demographic attribute and QoUL was tested using the Kruskal-Wallis H Test or Mann-Whitney Test. These tests were used to examine statistical difference in domains between demographic variables that appear in more than two categories. In addition, a cross-tabulation was applied for further exploration. In this regard, results have indicated significant associations between some socio-demographic attributes and QoUL. Table 4.2 shows significant values obtained from testing the relationship between QoUL level and socio-demographic attributes. The results illustrated that no significant association was found in relation to the impact of age, marital status, level of education and employment status or number of people living at home. Nevertheless, the results have indicated the presence of a significant relationship between QoUL and other socio-demographic attributes of residential status, household monthly income and gender. In order to obtain a better understanding concerning the associations between QoUL and these particular socio-demographic attributes, a cross-tabulation was applied.

Table 4.2: Significant values obtained from testing the relationship between QoUL and sociodemographic attributes

\begin{tabular}{ccc}
\hline Socio- demographic attributes & \multicolumn{2}{l}{ Significance value obtained using Chi-Square Test } \\
\hline Gender & $0.05 \leq 0.05$ & Significant \\
\hline Age category & $0.76>0.05$ & Insignificant \\
\hline Marital status & $0.60>0.05$ & Insignificant \\
\hline Level of education & $0.50>0.05$ & Insignificant \\
\hline Employment status & $0.13>0.05$ & Insignificant \\
\hline Residential status & $0.01<0.05$ & Significant \\
\hline No. of people living at home & $0.36>0.05$ & Insignificant \\
\hline Household monthly income & $0.03<0.05$ & Significant \\
\hline
\end{tabular}

The relationship between gender and people's perception is one of the crucial aspects of the QoUL investigation. Therefore, it was important to focus on this relationship, and crosstabulation was applied for this purpose. Table 4.3 shows that there are slight differences 
among participants in terms of reporting the QoUL level. By looking at Table 4.3, it can be seen that females are more positive than males in terms of reporting QoUL level, where $56.6 \%$ of them stated that their QoUL is high or very high. On the other hand, the ratio of positive report of QoUL is slightly lower in the case of males, where 55.8\% of them stated that their QoUL is high or very high. Moreover, females are lesser in reporting negative QoUL level, where only $10.7 \%$ stated that their QoUL is low or very low. In contrast, there is a slightly higher percentage of reporting a negative QoUL level in the case of males, where $16.3 \%$ of them stated that their QoUL is low or very low.

Table 4.3: Association between gender and QoUL Level. Cross-tabulation

\begin{tabular}{|c|c|c|c|c|c|c|c|c|}
\hline & & & \multicolumn{5}{|c|}{ QoUL level } & \multirow[b]{2}{*}{ Total } \\
\hline & & & $\begin{array}{l}\text { Very } \\
\text { Low }\end{array}$ & Low & Moderate & High & $\begin{array}{l}\text { Very } \\
\text { High }\end{array}$ & \\
\hline \multirow{4}{*}{ Gender } & \multirow{2}{*}{ Female } & Count & 3 & 26 & 89 & 89 & 65 & 272 \\
\hline & & $\%$ & $1.1 \%$ & $9.6 \%$ & $32.7 \%$ & $32.7 \%$ & $23.9 \%$ & $100.0 \%$ \\
\hline & \multirow{2}{*}{ Male } & Count & 13 & 32 & 77 & 100 & 54 & 276 \\
\hline & & $\%$ & $4.7 \%$ & $11.6 \%$ & $27.9 \%$ & $36.2 \%$ & $19.6 \%$ & $100.0 \%$ \\
\hline \multirow{2}{*}{\multicolumn{2}{|c|}{ Total }} & Count & 16 & 58 & 166 & 189 & 119 & 548 \\
\hline & & $\%$ & $2.9 \%$ & $10.6 \%$ & $30.3 \%$ & $34.5 \%$ & $21.7 \%$ & $100.0 \%$ \\
\hline
\end{tabular}

Chi-square $=9.36$ p-value $<0.05$

This disparity in reporting the QoUL level (see Figure 4.4) may be due to some aspects of the local culture. In Saudi culture in particular, the responsibilities of the household mostly lie with the man (the head of household), including in general household responsibilities such as the cost of living and taking care of family, and so on. Therefore, this may be one of the interpretations for female prevalence in terms of reporting positive QoUL level, suggesting that females may be happier than men. This, however, contradicts some studies including the work of Hating et al. (1984) and Diener and Diener, (2009) who found that men may be happier than women. For instance, Diener and Diener evidence that women have a lower level of satisfaction with life than men, because women usually have less power and resources than men. 


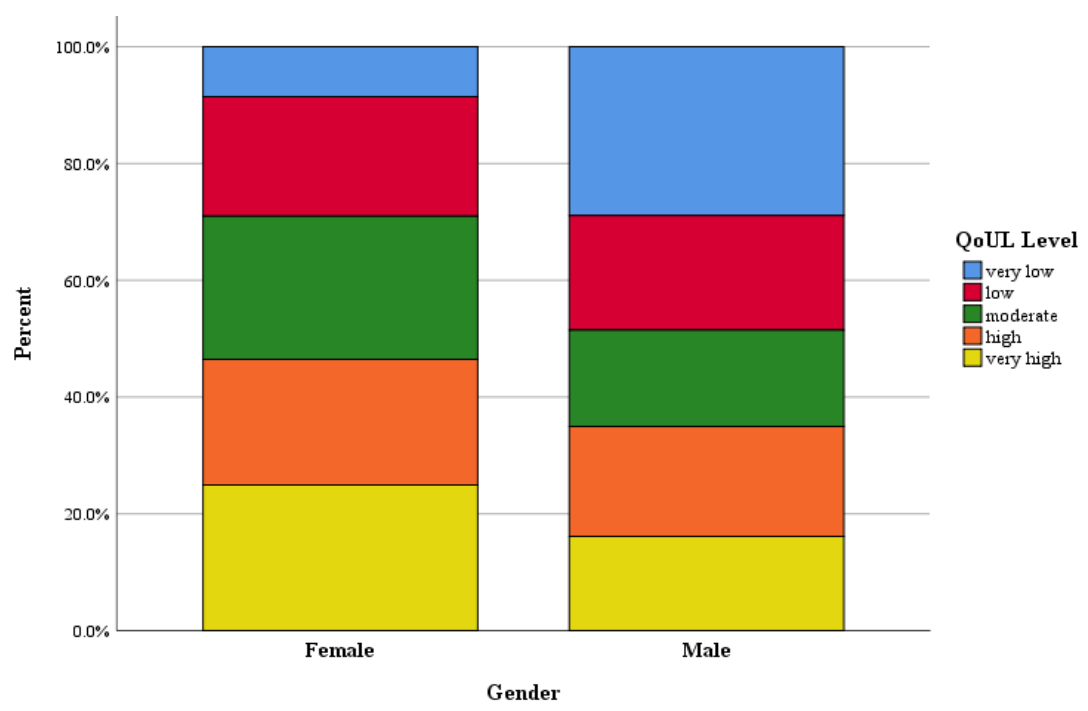

Fig 4.5: Gender and QoUL level

In this thesis, as suggested above, the reason women rate as happier than men may be that most women rely heavily on men for many aspects of daily life. This may be subject to the role of culture that is generated by religion. In this regard, religion urges men to take care of women, considering that this is one of the duties incumbent upon the man as head of the household, and protecting the vulnerability of women should be seen as one of the most important aspects of the relationship between men and their family, not only between a man and his wife, but also with other family members such as daughters and sisters. By referring to Islamic legislation in this regard, it was found that taking care of women, including the fulfilment of material and immaterial aspects, should be observed as a religious duty (Al Khayat, 2003; Bani \& Pate, 2015). However, such findings need further investigation where taking care of women is translated into practice.

The association between gender and employment status was also tested for further exploration, in order to see whether employment status does matter in relation to life satisfaction for females in particular, in line with the findings in the wider literature where employment status was widely found to play an important role in life satisfaction determination. A cross-tabulation was applied for this purpose. The result indicated that there is a significant association between gender and employment status using the ChiSquare $(p$-value $=0.000<0.05)$. It can be noted from Table 4.4 that more than half of females (59.2\%) are unemployed, while only $8.3 \%$ of males are. From this result, as well as the result from testing the relationship between gender and QoUL, it can be concluded that 
although most of the females are unemployed, they are, according to their own evaluation, happier than the males.

Table 4.4: Association between gender and employment status. Cross-tabulation.

\begin{tabular}{|c|c|c|c|c|c|c|c|c|}
\hline & & & \multicolumn{5}{|c|}{ Employment status } & \multirow{2}{*}{ Total } \\
\hline & & & Employed & Other & Retired & Self-employed & Unemployed & \\
\hline \multirow{4}{*}{ Gender } & \multirow{2}{*}{ Female } & Count & 71 & 31 & 5 & 4 & 161 & 272 \\
\hline & & $\%$ & $26.1 \%$ & $11.4 \%$ & $1.8 \%$ & $1.5 \%$ & $59.2 \%$ & $100.0 \%$ \\
\hline & \multirow{2}{*}{ Male } & Count & 203 & 13 & 28 & 9 & 23 & 276 \\
\hline & & $\%$ & $73.6 \%$ & $4.7 \%$ & $10.1 \%$ & $3.3 \%$ & $8.3 \%$ & $100.0 \%$ \\
\hline \multirow{2}{*}{\multicolumn{2}{|c|}{ Total }} & Count & 274 & 44 & 33 & 13 & 184 & 548 \\
\hline & & $\%$ & $50.0 \%$ & $8.0 \%$ & $6.0 \%$ & $2.4 \%$ & $33.6 \%$ & $100.0 \%$ \\
\hline
\end{tabular}

Chi-square $=192.38$ p-value $<.000$

For the relationship between QoUL Level and residential status, a negative correlation was found $\left(\mathrm{r}=-.158^{* *}, \mathrm{p}\right.$-value<.000) using Spearman's Rho test. Table 4.5 shows the results of testing this relation. For additional detail, a cross-tabulation was also applied to get a more in-depth understanding of this relationship.

Table 4.5: Correlation between residential status and QoUL level: Spearman's rho.

\begin{tabular}{llrr}
\hline & & \multicolumn{2}{c}{ QoUL level } \\
\hline Spearman's rho & Residential status & Correlation Coefficient & $-.158^{* *}$ \\
\cline { 3 - 4 } & & Sig. (2-tailed) & .000 \\
\cline { 2 - 3 } & & $\mathrm{N}$ & 548 \\
\hline
\end{tabular}

Table 4.6 represents the relationship between residential status and QoUL level, which is found to be statistically significant. It seems that participants who own their home are more likely to report a positive QoUL level, where 65.6\% of them stated that their QoUL is high or even very high compared to those who live in a rented home, where $46.3 \%$ of them stated that their QoUL is high or very high. Others who live within the family home seemed to report a high ratio of positive of QoUL, where $61.4 \%$ of them stated that their QoUL is high or very high. In terms of reporting better QoUL levels, this category seems to be a closer to the category of home ownership. However, the number of participants in this category (i.e., living in the family home) is very small compared to other categories. From cross-tabulating residential status and QoUL, it can be noted that home ownership is positively reflected in 
the QoUL, where it is clear that the residents of Quba who own a home are the most satisfied participants in comparison to those who rent their house. Although participants who live in a family home represent a high percentage in reporting a positive QoUL level, they still represent a small number among the total number of the participants. Figure 4.5 clearly shows the association between QoUL and residential status.

Table 4.6: Association between residential status and QoUL Level. Cross-tabulation.

\begin{tabular}{|c|c|c|c|c|c|c|c|c|}
\hline & & & \multicolumn{5}{|c|}{ QoUL level } & \multirow[b]{2}{*}{ Total } \\
\hline & & & $\begin{array}{l}\text { Very } \\
\text { Low }\end{array}$ & Low & Moderate & High & $\begin{array}{l}\text { Very } \\
\text { High }\end{array}$ & \\
\hline \multirow{6}{*}{$\begin{array}{c}\text { Residential } \\
\text { Status }\end{array}$} & \multirow{2}{*}{ Other } & Count & 1 & 3 & 13 & 18 & 9 & 44 \\
\hline & & $\%$ & $2.3 \%$ & $6.8 \%$ & $29.5 \%$ & $40.9 \%$ & $20.5 \%$ & $100.0 \%$ \\
\hline & \multirow{2}{*}{$\begin{array}{l}\text { Own } \\
\text { home }\end{array}$} & Count & 4 & 23 & 58 & 98 & 64 & 247 \\
\hline & & $\%$ & $1.6 \%$ & $9.3 \%$ & $23.5 \%$ & $39.7 \%$ & $25.9 \%$ & $100.0 \%$ \\
\hline & \multirow{2}{*}{$\begin{array}{l}\text { Rent } \\
\text { home }\end{array}$} & Count & 11 & 32 & 95 & 73 & 46 & 257 \\
\hline & & $\%$ & $4.3 \%$ & $12.5 \%$ & $37.0 \%$ & $28.4 \%$ & $17.9 \%$ & $100.0 \%$ \\
\hline \multirow{2}{*}{\multicolumn{2}{|c|}{ Total }} & Count & 16 & 58 & 166 & 189 & 119 & 548 \\
\hline & & $\%$ & $2.9 \%$ & $10.6 \%$ & $30.3 \%$ & $34.5 \%$ & $21.7 \%$ & $100.0 \%$ \\
\hline
\end{tabular}

Chi-square $=21.47$ p-value $<.006$

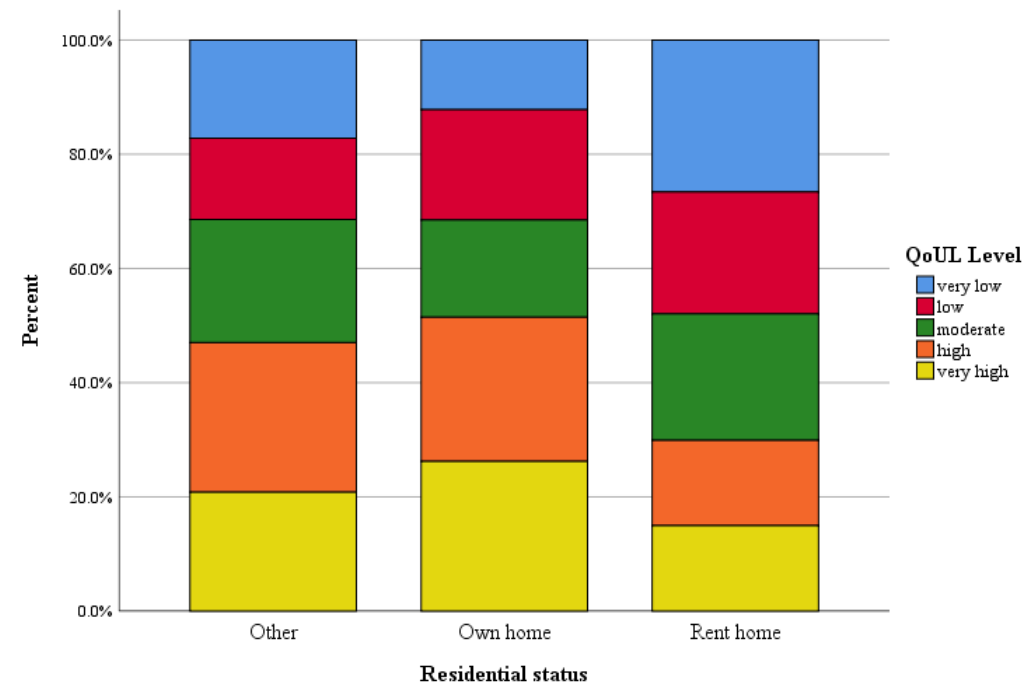

Fig 4.6: Association between QoUL and residential status

In light of those results, it can be said that home ownership in general plays an important role in enhancing QoUL. This assumption is supported by the literature where many studies have confirmed that the best form of residential status is home ownership, which is likely to help people to achieve a better QoUL including in terms of life satisfaction (e.g., Rohe \& 
Stegman, 1994; Rohe \& Basolo, 1997; Scanlon, 1999; Rohe et al., 2001; Ren et al., 2016). It is argued that home ownership can contribute to the QoUL in several ways. It can promote neighbourhood health, finance, security and stability (Retsinas \& Belsky, 2002). Moreover, home ownership may associate with social status and personal freedom which might lead to a higher level of self-esteem and influence the way in which the homeowner is viewed by others, which in turn influences self-esteem (Rohe et al., 2001).

In addition, privacy is another benefit of home ownership, especially in the case of Medina, and more specifically, within the Saudi norms. In this context, privacy is an important aspect of the residential status for people in Medina and Saudi Arabia in general and is more likely to be met in the case of home ownership. This assumption is based on the fact that home ownership gives freedom and greater control over property, something which might not be available in the case of rented homes (Ariff \& Hilary, 2009). Thus, this may be one of the reasons why the highest ratio of positive QoUL level was associated with those who own a home. In the context of this study, residents may prefer owned homes over other residential forms, because they can provide more privacy, in line with local culture and norms. Furthermore, as noted above, there are some aspects of home ownership mentioned in the literature (e.g., Elsinga \& Hoekstra, 2005; Rohe \& Lindblad, 2013; Yun \& Evangelou, 2016) such as security and social stability that are likely to enhance the QoUL.

With respect to monthly income and its impact on the QoUL, there was no significant association between the two variables. However, a noticeable positive relationship has been found between the two variables, where it can be noted from table 4.8 that percentages of the positive report of QoUL levels tend to increase steadily with the increase of monthly income. Figure 4.6 clearly illustrates this relationship. For more exploration with regard to the relationship between income and QoUL, a Spearman's Rho test was employed. Results in table 4.7 show that there a significant, but low positive correlation between the two variables. 
Table 4.7: Association between monthly income and QoUL level. Cross-tabulation.

\begin{tabular}{|c|c|c|c|c|c|c|}
\hline & & \multicolumn{5}{|c|}{ QoUL level } \\
\hline & & & Low & Moderate & High & Total \\
\hline \multirow{10}{*}{$\begin{array}{l}\text { Monthly } \\
\text { Income }\end{array}$} & \multirow{2}{*}{$<3000$} & Count & 12 & 17 & 19 & 48 \\
\hline & & $\%$ & $25.0 \%$ & $35.4 \%$ & $39.6 \%$ & $100.0 \%$ \\
\hline & \multirow{2}{*}{$3000-5000$} & Count & 9 & 23 & 33 & 65 \\
\hline & & $\%$ & $13.8 \%$ & $35.4 \%$ & $50.8 \%$ & $100.0 \%$ \\
\hline & \multirow{2}{*}{$5000-10000$} & Count & 20 & 48 & 86 & 154 \\
\hline & & $\%$ & $13.0 \%$ & $31.2 \%$ & $55.8 \%$ & $100.0 \%$ \\
\hline & \multirow{2}{*}{$10000-15000$} & Count & 16 & 42 & 71 & 129 \\
\hline & & $\%$ & $12.4 \%$ & $32.6 \%$ & $55.0 \%$ & $100.0 \%$ \\
\hline & \multirow{2}{*}{$>15000$} & Count & 17 & 36 & 99 & 152 \\
\hline & & $\%$ & $11.2 \%$ & $23.7 \%$ & $65.1 \%$ & $100.0 \%$ \\
\hline & \multirow{2}{*}{ Total } & Count & 74 & 166 & 308 & 548 \\
\hline & & $\%$ & $13.5 \%$ & $30.3 \%$ & $56.2 \%$ & $100.0 \%$ \\
\hline
\end{tabular}

Chi-square $=13.68$ p-value $<.088$

Table 4.8: Correlation between household monthly income and QoUL level

\begin{tabular}{lllr}
\hline & & \multicolumn{1}{c}{ QoUL Level. } \\
\hline Spearman's rho & $\begin{array}{l}\text { Household Monthly income } \\
\text { in SAR }\end{array}$ & Correlation Coefficient & $.105^{*}$ \\
\cline { 2 - 4 } & & Sig. (2-tailed) & .014 \\
\cline { 2 - 3 } & $\mathrm{N}$ & 548 \\
\hline
\end{tabular}

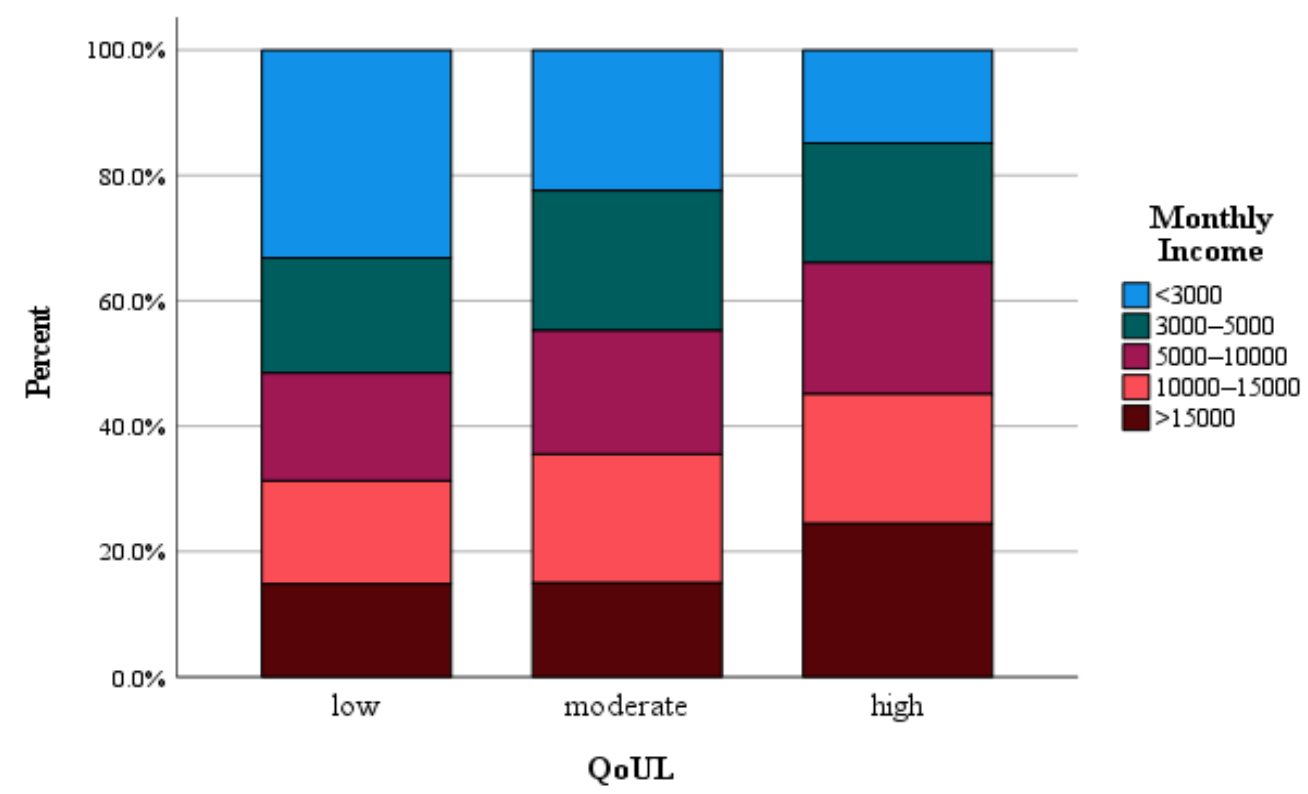

Fig 4.7: Relationship between monthly income and QoUL 
In light of these results, it can be said that monthly income has an impact on the QoUL of those living in Quba. In this context, the relationship between monthly income and QoUL, namely in terms of aspects such as SWB, life satisfaction and happiness, many studies in literature found a positive relationship (e.g., Diener, et al., 1993; Diener \& Diener, 1995; Veenhoven, 2001; Diener et al., 2009). Many of these studies pointed out that the positive relationship between income and QoUL relies on the fact that income helps people meeting human basic needs such as food, health, security, comfortable housing, and so forth (Diener, et al., 1993). It may be obvious that income is a critical aspect of QoUL because it meets the basic needs of people and many other aspects of their lives. However, by referring to the qualitative findings from the photo diaries in the current study, some social aspects including religion may affect the relationship between monthly income and QoUL. In this context, spirituality may play an important role in people's satisfaction with aspects of their lives, including income. It has been argued by some participants in photo diary interviews that earning money does not necessarily lead to achieving a higher level of QoUL, compared to some spiritual aspects associated with religious commitment including performance of religious duties and feeling attached to God. In addition, the sanctity of the place that provides more spirituality may mean much to those who live in Medina, where the impact of this on the QoUL has been discussed in depth, including aspects related to the financial side. The following quote was made by one of the participants who stated,

This city is the best place to live, even if I did not have the appropriate means of living here, I just want to live in this place (PH 25, Male, 30 years old).

The relationship of finances with the QoUL was also discussed with many interviewees, many of whom pointed out that the money that is earned while living in Medina, even if it a little, was considered a blessing (called Baraka in Arabic) ${ }^{1}$ and it lasts longer, compared to any other place. This is part of the religious beliefs of the local population, where many

\footnotetext{
${ }^{1}$ The word barakah is one of the concepts on which human prosperity is based. It increases in quality and quantity in accordance with the grace of God. In addition, barakah means steadiness and permanence in many aspects of life including happiness (Demirel \& Sahib, 2015).
} 
of them believe that the Prophet Muhammad (PBUH) prayed for this city by blessing it in all aspects of life, including health, food and money.

\subsection{Ranking aspects of life in relation to their impact on the QoUL}

The impact of various aspects of life on the QoUL was measured using a scale from 1-6 according to their influence, where 1 represents the most influential aspect, while 6 is the least influential. The ranking of aspects of life was analysed using multiple modes of analysis in order to get a better understanding concerning the most influential aspects of life on the QoUL, as well as to gain insightful information regarding the aspects of life that people place at the centre of their priorities. The methods employed included Spearman's correlation test as well as measures of spread such as standard deviation and variance.

Spearman's correlation test was applied in order to examine any sort of correlation between QoUL and the ranking of aspects of life, where both aspects of life and QoUL were measured on an ordinal scale. Table 4.9 presents the results of testing the correlation between aspects of life ranking and QoUL level. It should be noted that in terms of these correlations, it is the level of influence that is reflected in a negative or positive correlation. The more influential aspects are, the higher QoUL will be in the case of the negative correlations, whereas the less influential aspects are, the higher QoUL will be in the case of the positive correlations. It can be noted from the results set out in the table that health, and employment and finance are significantly correlated with QoUL. While health negatively correlated with QoUL, employment and finance are positively correlated with QoUL. One possible explanation regarding the positive relationship between the QoUL and employment and finance is that participants look at the negative sides of employment and finance such as income and unemployment. Thus, it was found that this aspect of life was placed at a high rank because it negatively affects the QoUL of the participants. On the contrary, the negative relationship between the QoUL and health suggests that participants look at the positive sides of this aspect of life, considering that health positively affects the quality of their urban lives. For other aspects of life including education, recreation, security and housing, no correlations were found between them and the QoUL. 
Table 4.9: Spearman's rho result of examining the correlation between aspects of life ranking and QoUL level

\begin{tabular}{cccc}
\hline Aspects of Life & $\begin{array}{c}\text { Spearman's correlation } \\
\text { coefficient } \mathbf{s}(\mathbf{R s})\end{array}$ & Results & $\begin{array}{c}\text { Type of } \\
\text { correlation }\end{array}$ \\
\hline Health & -0.116 & Significant & Negative \\
\hline Education & 0.08 & insignificant & Positive \\
\hline Security & -0.07 & insignificant & Negative \\
\hline Employment and Finance & 0.102 & Significant & Positive \\
\hline Housing & -0.05 & insignificant & Negative \\
\hline Recreation & 0.03 & insignificant & Positive \\
\hline
\end{tabular}

An additional analysis was conducted in order to gain more insight into the ranking given by participants to each aspect of life and the values of QoUL level. Table 4.10 presents results using mean, standard deviation and variance for each of aspect of life. It can be noted from the results that security and health are the aspects of life with the highest standard deviation and variance, followed by employment and finance, then housing with the same standard deviation $(\mathrm{SD}=1.3)$ and a similar variance. In contrast, education and recreation were the lowest standard deviation and variance among the other aspects of life. In this regard, it can be said that the aspects of life with higher standard deviation and variances refer to a large variation in people's views on the impact of these aspects on their urban quality of life, and thus less agreement. In this regard, higher standard deviation and variation indicate that the values are more widely distributed, signifying that participants' views are widely dispersed and do not agree on a particular value. This means that these aspects of life were disputed by the participants due to the diversity of views regarding the importance each aspect held. On the contrary, the aspects with the lowest values of standard deviation and variances represent a smaller and narrower divergence in the opinions regarding the impact of these aspects, and therefore, more agreement. That means that participants generally tended to agree on the importance of education and entertainment for the quality of their urban lives. 
Table 4.10: Mean, standard deviation and variance of aspects of life

\begin{tabular}{cccc}
\hline Aspects of Life & Mean & Standard deviation & Variance \\
\hline Health & 2.19 & 1.34 & 1.8 \\
\hline Education & 3.20 & 1.25 & 1.6 \\
\hline Security & 2.44 & 1.50 & 2.2 \\
\hline Employment and Finance & 3.86 & 1.3 & 1.7 \\
\hline Housing & 3.8 & 1.3 & 1.8 \\
\hline Recreation & 5.5 & 1.15 & 1.32 \\
\hline
\end{tabular}

In reference to Table 4.10, the mean values indicate the degree to which the aspects of life that have been ranked by the participants influence their QoUL. Here, the lower the mean of values, the higher the rank of aspects of life. For instance, health (mean value $=2.19)$ and security (mean value $=2.44$ ) obtained the lowest mean of values. This therefore indicates that these aspects of life are the most influential among the other aspects. In contrast, the higher the mean values, the lower the rank of aspect of life. For example, recreation obtained the highest mean of values (5.5), which indicates that this aspect of life is the least influential among other aspects. For additional clarity, the results of ranking aspects of life are presented as bar charts in Figures 4.7, 4.8 and 4.9 using the examples of health, security and recreation. Health, for instance, obtained a high rank, where about $40.69 \%$ of the participants placed it in the top rank among the other aspects of life as the most influential aspect. Similarly, security was placed at high rank, which came after health in terms of its influence on the QoUL, where $35.58 \%$ of the participants placed it in the top rank among the other aspects of life. In contrast, recreation obtained a lower rank among other aspects of life, where $79.1 \%$ of the participants placed it on the lowest rank. 


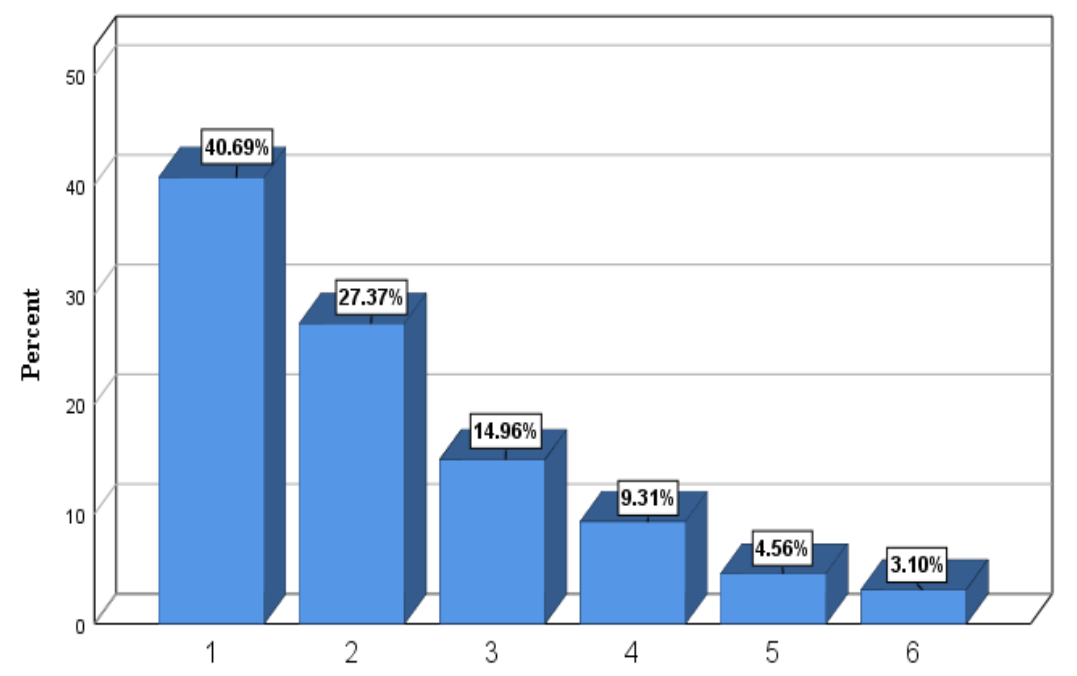

Fig 4.8: Health rank. Note: where 1 is the highest ranking and 6 the lowest

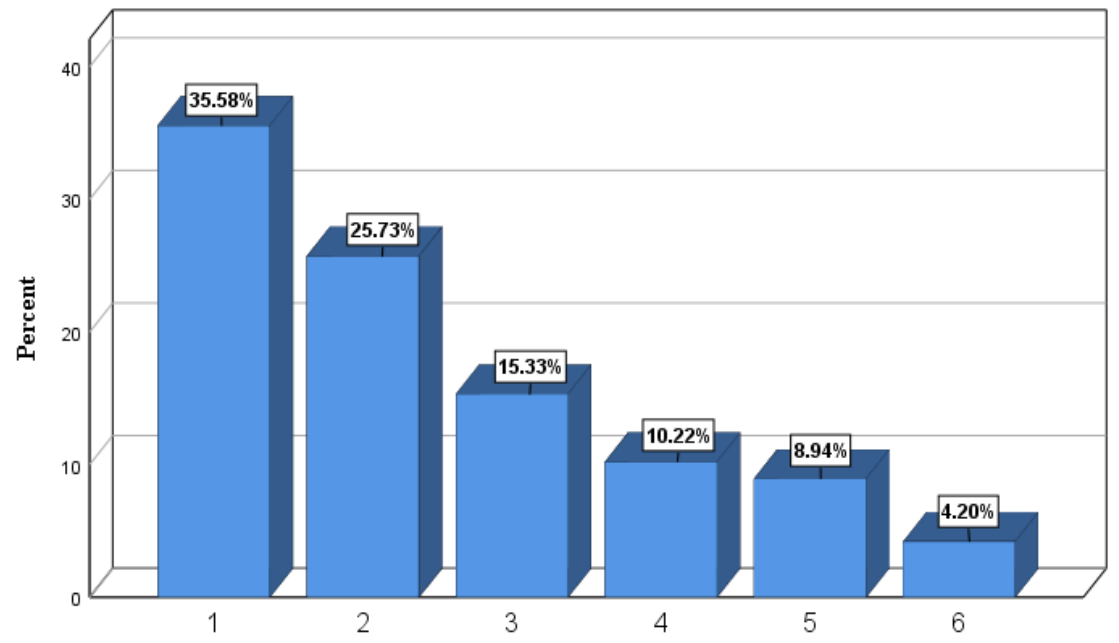

Fig 4.9: Security rank. Note: where 1 is the highest ranking and 6 the lowest 


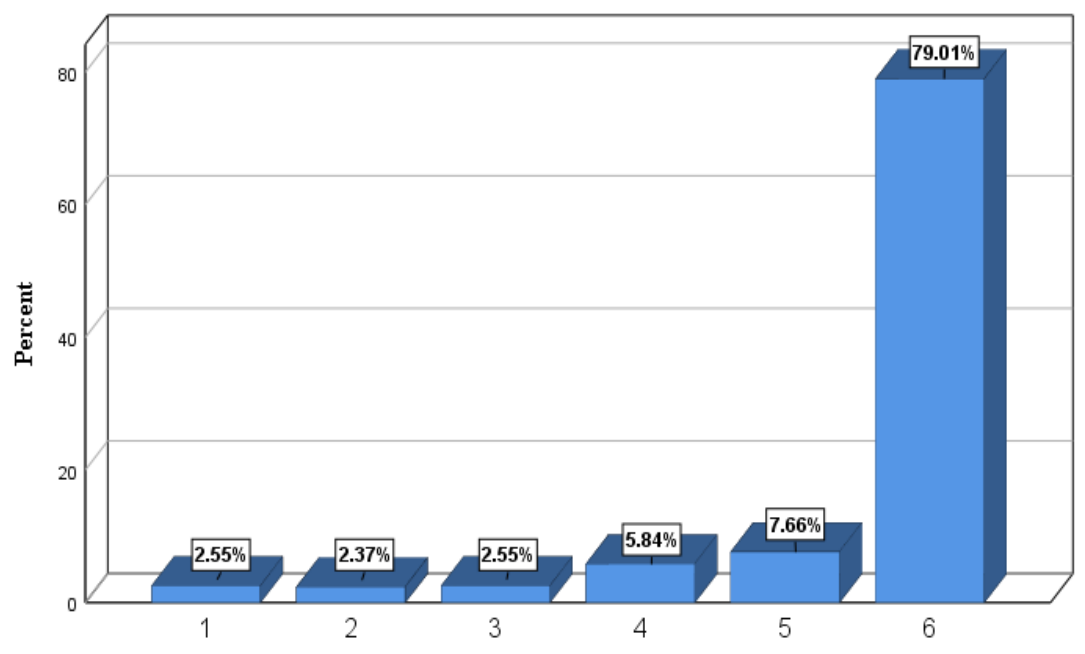

Fig 4.10: Recreation rank. Note: where 1 is the highest ranking and 6 the lowest

Given these findings, it is worth making further reflection on what may lie behind the strong emergence of health and security as important factors, and similarly of recreation as the least. One view is that such determination of importance reflects the needs that people place at the centre of their priorities. The levels of priorities given to these aspects of life indicate the extent to which people need these aspects in their daily lives. This might be thought of in global terms, linked to general human nature, where health and security are essential physiological necessities that need to be met, regardless of the culture of society. In contrast, recreation, generally agreed in this study to be the least influential, may reflect a lower level of perceived need, representing instead aspects of collective interest.

These findings with regard to health and security are in line with many studies within the literature that have addressed them as key indicators of QoUL and as the most significant elements of SWB. These studies include the work of Palmore and Luikart (1972), Campbell et al. (1976), Diener (1984) and Hsieh (2005). For example, Campbell et al. (1976, as cited in Diener et al., 2009) note that when participants were asked to rank the importance of several aspects of life, health was at the top of rank, and likewise, Hsieh (2005) found that the participants considered health as the most important among other aspects of life. Security also affects SWB in a similar way to health, and in some studies is deemed to be at the top of people's priorities and one of the most influential aspects of life (e.g., Milbrath, 1978; Santos and Martins, 2006; Senlier et al., 2008). Moreover, the issue of security comes first 
in the context of human needs according to Maslow's hierarchy of needs (Maslow, 1943). In the context of Islamic culture, especially in Saudi Arabia, people may believe that achieving security and health will fulfil the other life requirements. In this regard, Aba AlKhail pointed out that security as well as health precede the other aspects, because meeting these two aspects will lead to meeting many other aspects of life (Aba Al-Khail, 2019).

Being healthy and secure are one of the main aspects of daily life. This is what has been found in the expert interviews which discussed the most important aspects that enhance the QoUL. In this context, one of the experts referred to the importance of health and security to meet the basic needs of human beings to ensure a decent life:

What a person needs in his daily life is to be healthy in his/ her body and a secure in his/ her society. Therefore, meeting these two aspects can lead to meeting most aspects of life (Khaled Alsaidalani, an expert).

This expert pointed out that the interest in meeting these two things stems from the religious teachings that the community adhere to in Medina. According to Alsaidalani, this assumption is based on what the Prophet Muhammad (PBUH) referred to in his hadith ${ }^{2}$ about the proper perspective of the essential things in life, where he is quoted as saying "whoever of you gets up in the morning feeling physically healthy, enjoying security and having his food for the day is like one who has the world at his fingertips". (Related by Ibn Majah, cited in Al-Khayat, 2004, p.14) Thus, hadith such as this and others are often referred to when talking about aspects of life, which may affect people's perception of those aspects.

These findings concerning the importance of health in human life prompted the search for the relationship between religion and health. Since the 1960s, the number of western studies examining the relationship between "prayer" and health has been increasing. Despite the multiplicity of the methodologies of these studies, they often emphasise the importance of prayer in health and its role in the healing of physical and psychological diseases, specifically, with the religious revival in the era of 'Reagan' (Al Samaan, 2006). At that time, the study of Bayard and his colleagues became known as the guiding light for those

\footnotetext{
${ }^{2}$ The word hadith refers to the record of the speeches and actions made by the Prophet Muhammad (PBUH) regarding certain issues (Batyrzhan, et al., 2014).
} 
who followed this path. In this regard, Bayard divided intensive care heart patients into two groups. The first group asked the families of the patients and the church to pray for them to be healed, while the second group did not. This study found that the first group (which performed the prayer) recovered faster and more efficiently than the second group (Al Samaan, 2006). In this context, spirituality may play a role in health improvement including the enhancement of mental health and general healthcare (Simao et al., 2016).

In addition, religion strongly affects people in the Eastern Mediterranean Region; it is a part of everyday life for individuals. In this context, all religions call for health care and avoidance of risks to human health. For example, dealing with tobacco use from a religious point of view is a key component of the WHO's Tobacco Free Initiative program. In 1996, these efforts began with the publication of the first edition of the health promotion, which aims to promote health education through religious teachings (namely the ruling on smoking in Islam whether at home or in public). This ruling is to prohibit the smoking or consider it as an abhorrent to such a degree as to be prohibited. In 2000, another edition was published with both Christian and Islamic views regarding smoking, where the Vatican has issued legislation for tobacco bans in 2002 (WHO, n.d.). Garrusi and Nakhaee (2012) evidence that most religions prohibit smoking from the point of view that smoking is harmful to health. In this regard, many studies have pointed out that smokers have a lower QoL than others (Rezaei et al., 2017), while quitting the smoking habit enhances QoL in all its aspects including the reduction of anxiety and symptoms of depression (Despoina et al., 2017). 


\subsection{Summary of the chapter}

This chapter explored the general reflection of various indicators on the QoUL in the municipality of Quba by highlighting the impact of the socio-demographic attributes on the QoUL, general perception of the QoUL, and the most influential aspects of life on the QoUL of those living in Quba. With regard to the impact of socio-demographic attributes on the QoUL of the residents of Quba, the results indicated a presence of significant association between some socio-demographic attributes and the level of QoUL. Those attributes were residential status and household monthly income, as well as gender. In contrast, no significant association was found between QoUL and other socio-demographic attributes including age, marital status, level of education, employment status, and number of people living at home. Those findings reflect the extent to which socio-demographic attributes contribute to the QoUL. By referring to the literature, there are some assumptions about the impact of certain socio-demographic attributes, with many studies suggesting that there is a positive relationship between monthly income and QoUL. However, such assumptions may not be consistent with studies across cultural contexts, including this study. In this thesis, the results with regard to the influence of socio-demographic attributes on QoUL may indicate that monthly income as an example may not be as important to the residents of Quba as much as other social environmental elements such as spirituality and religion. Nevertheless, the results of the impact of residential status in this thesis are consistent with the assumptions regarding the benefits of home ownership, where many studies assert its positive impact on the QoUL in many aspects, including security, social stability, and selfesteem.

With regard to the analysis of the evaluation of the overall level of QoUL, the results showed that the residents of Quba evaluated their QoUL as a moderate, where the average score was found to be 6.77 using a scale of 1-10. From this result, it can be said that there seems to be a variation in the opinions of the residents of Quba regarding their QoUL, which may be affected by a number of socio-demographic attributes such as their gender, household income and residential status. In addition to the evaluation of the QoUL level, the participants were asked to rank some life aspects according to their influence on the QoUL of these participants. In this regard, results showed that health and security are the most influential aspects of life, whereas the least influential aspect of life was the recreation 
indicator. In this regard, people seem to prioritise these aspects because they primarily affect their personal lives. Moreover, religion highlights the importance of these two aspects in people's lives, which makes or directs them to pay more attention to those aspects over others. Within these findings, it has also been noted that religion has a role in people's health, as Christianity and Islam in particular, forbid dealing with certain behaviours, including smoking as an example.

Overall, it is striking from the findings obtained from the analysis within this chapter that the characteristics of Medina in general and the Quba municipality in particular are reflected in the level of QoUL. These characteristics include the functional attributes (i.e., physical attributes) as well as the social and cultural aspects. Therefore, the level of QoUL can be considered somewhat as a positive, which may be due to the positive view of the urban environment in which Quba residents live. This is perhaps due to the improvement in the quality of the physical characteristics of the urban environment in the municipality of Quba, at least in some places within the municipality, as well as the impact of some social and cultural factors such as religion and spirituality. The integration of such indicators may enhance the QoUL including the increase of life satisfaction. In the case of Quba, however, it is noted that people may perceive these indicators of the urban environment from different perspectives. For example, some people may be happy even in the worst conditions of the urban environment, while others may not be happy even in the best conditions in the urban environment in which they live. This may be due to the fact that people vary in terms of age, gender, and possibly the degree of religiosity (Fadda \& Jirón, 1999).

The findings of this chapter, starting from the impact of socio-demographic attributes and evaluation of the overall level of QoUL to the findings of ranking the aspects of life, could be considered as a basis for the investigation of the particular contribution of social, physical and economic indicators which will be discussed in the following chapters. While this chapter has set the foundation for the rest of the QoUL investigation in this research, Chapter 5 marks the start of the empirical work into the selected domains of QoUL by highlighting the quality of housing as one of the physical attributes of QoUL, where QoUL at the narrowest scale is examined. 


\section{Chapter Five}

\section{Housing Quality and QoUL}

\subsection{Introduction}

Following on from the groundwork laid in Chapter 4 as to the general overview of the empirical work regarding the status and perception of QoUL in the municipality of Quba, and examination of the initial findings as to the aspects of life that residents identify as most influential in impacting that QoUL, the aim of this chapter is to deepen the analysis by narrowing the focus to the narrowest scale of the study area, namely housing/ dwelling.

Using a functionalist approach, the current chapter highlights the aspects that influence QoUL at the narrowest scale: dwelling (i.e., housing). In this respect, the physical attributes of housing that influence satisfaction with housing, and in turn the overall QoUL, are highlighted, taking into account the social, cultural factors that distinguish the study area. Thus, this chapter investigates the housing issue and its impact on the QoUL for Quba's residents. The investigation encompasses measuring the general satisfaction with accommodation and the influence it has on the QoUL and bringing to the fore the issues of housing that influence housing satisfaction, including, tenure type, type of accommodation, adequacy of the accommodation and fiscal burden and affordability. In addition, mobility as an indication of housing satisfaction is highlighted. Taking into account the fact that people perceive their urban environment in different ways, the relationship between sociodemographic attributes of the participant and how they perceive housing quality is examined. 


\subsection{Satisfaction level with the accommodation}

Among other domains of QoUL, housing is considered as an important element that contribute the overall QoUL. In this context, several studies, including for example Peck \& Kay Stewart (1985), Kahlmeier et al. (2001), Das (2008) and Zhang et al. (2018) have stressed that satisfaction with housing is strongly associated with a significant increase in overall satisfaction with life. Since housing is an important aspect of life, satisfaction with it is a strong indication of life satisfaction judgements. Accordingly, in this thesis, satisfaction with accommodation on the part of residents of Quba was assessed, and the outcome of the housing satisfaction subsequently used as a foundation for the rest of the analytical work within this chapter. Participants were asked how satisfied they are with the quality of accommodation in which they live, and Figure 5.1 presents the findings using two forms of measurement: first, outcomes of the satisfaction using a scale of 1-10; second, outcomes of the satisfaction using a scale of five categories (i.e., the Likert Scale). Using the 1-10 scale, where 1 represents very dissatisfied and 10 represents very satisfied, results indicated a relatively high level of satisfaction with accommodation with an average score of 6.39. Within these outcomes, values of 8 and 10 have the highest frequencies, indicating a positive impression regarding satisfaction with the accommodation. Using the second form of measurement, results also indicated a positive satisfaction with the accommodation, where $52.2 \%$ of the respondents stated that they are satisfied with their accommodation compared to $21.8 \%$ of those who were not satisfied.
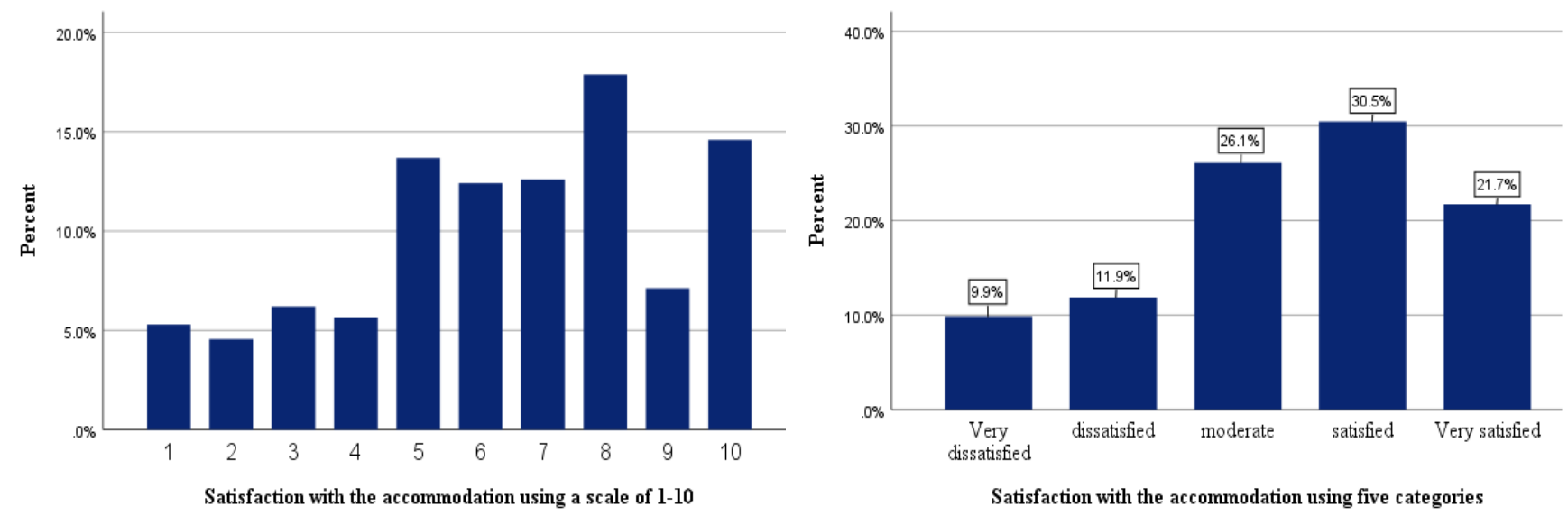

Fig 5.1: Satisfaction level with accommodation 
The results obtained above are very close to those obtained from measuring the general level of QoUL in Chapter 4, where findings also indicated a relatively high level of QoUL with an average score of 6.77 using a scale of 1-10, and by the five categories form of measurement $56.2 \%$ of the participants stated that their QoUL is high, compared to $13.5 \%$ of those who stated that their QoUL is low. These outcomes indicate the presence of a strong relationship between satisfaction with QoUL in general and satisfaction with an accommodation, where it appears from these two findings that there is a kind of consistency and compatibility between the degree of satisfaction with residence and satisfaction with the QoUL. This indicates that satisfaction with accommodation predicts the satisfaction with QoUL in general. That is, the relationship between these two variables implies that lower satisfaction with accommodation may adversely influence the perceived QoL. Therefore, if people's housing preferences cannot be met, satisfaction with their housing as well as their overall QoL may decrease (Tremblay \& Dillman, 1983), as supported by a number of studies focused on this relationship between housing satisfaction and QoUL, for example, Andrews and Withey (1976), Peck \& Kay Stewart (1985). However, satisfaction with the home may vary over time. In this context, Valentine (2001) pointed out that meaning of home as well as satisfaction with it change during the course of life. That is, satisfaction with the home is influenced by the characteristics of those living in it. For example, Valentine suggested that the importance of home increases as people get older.

\section{- Impact of socio-demographic attributes}

With regard to the impact of socio-demographic attributes of the participants on housing satisfaction, only age and residential status were found to be significantly correlated with housing satisfaction. A cross-tabulation was applied for further exploration regarding those relationships. Table 5.1 presents outcomes from cross-tabulating age and satisfaction with housing. It should be noted that the highest percentage of the positive report of accommodation satisfaction in found in the group aged 40 years and older, where $60.7 \%$ of them stated that they are satisfied or very satisfied with their accommodation. One of the possible explanations regarding this result is that the elderly, especially those of advanced age, may be more satisfied with their housing, perhaps because their aspirations regarding housing have become less, which leads them to be content with their current accommodation. Another possible explanation is that the elderly may have a higher income 
level as well as a smaller family size in some cases which might make them more satisfied with their current accommodation, as pointed out by Campbell et al. (1976).

Table 5.1: Relationship between age and satisfaction with accommodation. Cross-tabulation.

\begin{tabular}{|c|c|c|c|c|c|c|c|c|}
\hline & & & \multicolumn{5}{|c|}{ Satisfaction with the accommodation } & \multirow[b]{2}{*}{ Total } \\
\hline & & & $\begin{array}{c}\text { Very } \\
\text { dissatisfied }\end{array}$ & dissatisfied & $\begin{array}{c}\text { Moderately } \\
\text { satisfied }\end{array}$ & satisfied & $\begin{array}{c}\text { Very } \\
\text { satisfied }\end{array}$ & \\
\hline \multirow{6}{*}{ Age } & \multirow{2}{*}{$<=30$} & Count & 20 & 23 & 61 & 61 & 58 & 223 \\
\hline & & $\%$ & $9.0 \%$ & $10.3 \%$ & $27.4 \%$ & $27.4 \%$ & $26.0 \%$ & $100.0 \%$ \\
\hline & \multirow{2}{*}{$31-40$} & Count & 19 & 30 & 54 & 51 & 31 & 185 \\
\hline & & $\%$ & $10.3 \%$ & $16.2 \%$ & $29.2 \%$ & $27.6 \%$ & $16.8 \%$ & $100.0 \%$ \\
\hline & \multirow{2}{*}{$>40$} & Count & 15 & 12 & 28 & 55 & 30 & 140 \\
\hline & & $\%$ & $10.7 \%$ & $8.6 \%$ & $20.0 \%$ & $39.3 \%$ & $21.4 \%$ & $100.0 \%$ \\
\hline \multirow{2}{*}{\multicolumn{2}{|c|}{ Total }} & Count & 54 & 65 & 143 & 167 & 119 & 548 \\
\hline & & $\%$ & $9.9 \%$ & $11.9 \%$ & $26.1 \%$ & $30.5 \%$ & $21.7 \%$ & $100.0 \%$ \\
\hline
\end{tabular}

Chi-square $=16.593, \mathrm{p}$-value $<.035$.

In terms of the influence of residential status (tenure type) on the accommodation satisfaction, results obtained from cross-tabulating the two variables indicated that home ownership is positively correlated with the satisfaction with accommodation. It can be seen from Table 5.2 that $67.2 \%$ among those who owned homes stated that they are satisfied or very satisfied with their accommodation. Almost the same relationship can be seen in the case of those who have other residential statuses, ${ }^{3}$ though they represent a relatively small number of the respondents. In contrast, respondents who rented their homes are less likely to be satisfied, where $35.8 \%$ of them stated that they are satisfied or very satisfied with their accommodation. Figure 5.2 shows that higher satisfaction with accommodation is associated with both those who own a home and those who live in an owned home, whereas lower accommodation satisfaction is associated with renters.

3 'Other residential status' was interpreted by the participants as a 'family owned home'. 
Table 5.2: The relationship between residential status and satisfaction with accommodation. Crosstabulation.

\begin{tabular}{|c|c|c|c|c|c|c|c|c|}
\hline & & & \multicolumn{5}{|c|}{ Satisfaction with the accommodation } & \multirow[b]{2}{*}{ Total } \\
\hline & & & $\begin{array}{c}\text { Very } \\
\text { dissatisfied }\end{array}$ & dissatisfied & $\begin{array}{c}\text { Moderately } \\
\text { satisfied }\end{array}$ & satisfied & $\begin{array}{c}\text { Very } \\
\text { satisfied }\end{array}$ & \\
\hline \multirow{6}{*}{$\begin{array}{l}\text { Residential } \\
\text { status }\end{array}$} & \multirow{2}{*}{ Other } & Count & 4 & 3 & 9 & 14 & 14 & 44 \\
\hline & & $\%$ & $9.1 \%$ & $6.8 \%$ & $20.5 \%$ & $31.8 \%$ & $31.8 \%$ & $100.0 \%$ \\
\hline & \multirow{2}{*}{ Own home } & Count & 12 & 23 & 46 & 92 & 74 & 247 \\
\hline & & $\%$ & $4.9 \%$ & $9.3 \%$ & $18.6 \%$ & $37.2 \%$ & $30.0 \%$ & $100.0 \%$ \\
\hline & \multirow{2}{*}{ Rent home } & Count & 38 & 39 & 88 & 61 & 31 & 257 \\
\hline & & $\%$ & $14.8 \%$ & $15.2 \%$ & $34.2 \%$ & $23.7 \%$ & $12.1 \%$ & $100.0 \%$ \\
\hline \multirow{2}{*}{\multicolumn{2}{|c|}{ Total }} & Count & 54 & 65 & 143 & 167 & 119 & 548 \\
\hline & & $\%$ & $9.9 \%$ & $11.9 \%$ & $26.1 \%$ & $30.5 \%$ & $21.7 \%$ & $100.0 \%$ \\
\hline
\end{tabular}

Chi-square $=58.059, \mathrm{p}$-value $<.000$.

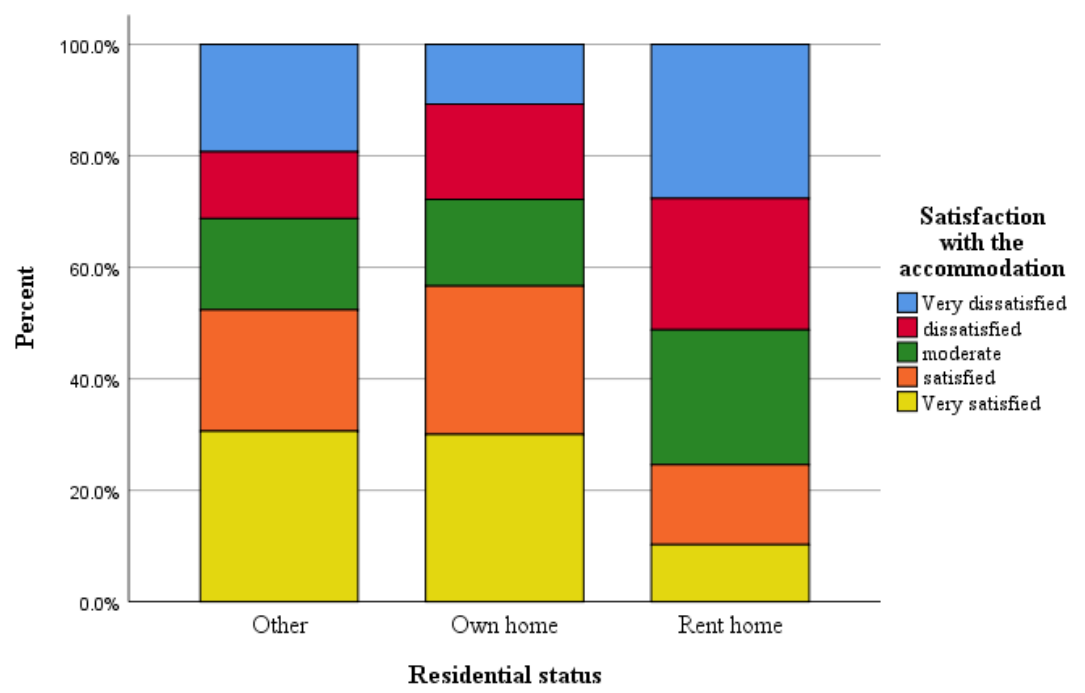

Fig 5.2: Association between residential status and satisfaction with accommodation

In light of the above results, it is clear that home ownership plays an important role in residential satisfaction, which in turn leads to an increase in satisfaction with the overall QoUL. This result is in line with findings obtained from a large body of research, where it has been found that home ownership strongly increases satisfaction with housing. Those researches including the work of Elsinga and Hoekstra (2005), Diaz-Serrano (2009), Hu (2011) and Zhang et al. (2018). For example, Diaz-Serrano (2009) who investigated housing satisfaction across European countries, asserts that home ownership is the most important 
way of wealth accumulation and personal success. While Elsinga and Hoekstra (2005) who investigated the relationship between home ownership and housing satisfaction in eight European countries, point out that home ownership ensures security, self-esteem, freedom and financial advantage, and thus satisfaction with housing. These authors mention some reasons why people prefer home ownership over other residential statuses, among them that home ownership is more financially attractive, and that home ownership provides personal identity and autonomy. In this context, home ownership may allow owners to control their homes in line with their needs and desires.

Qualitative findings of this thesis, namely the photo dairies, emphasised these features of home ownership. It has been found that this type of tenure to be a key element of housing satisfaction, which in turn benefits other aspects of life including mental health and emotional well-being, that may contribute to the overall QoUL. In this regard, one of the participants in the photo diaries who had just bought his own home, referred to home ownership in the following way:

Owning a home is one of the greatest possessions that an individual may acquire. Since we owned our home after a long time, we have experienced a feeling of psychological comfort and a sense of belonging to the place in which we live. More importantly, we had the freedom to act in our house as we like without any restrictions as before. I will never forget the joy and happiness of my family the first time we entered our own home, which was the most wonderful moment for me. ( $\mathrm{PH} \mathrm{23,} \mathrm{male,} 42$ years old) 


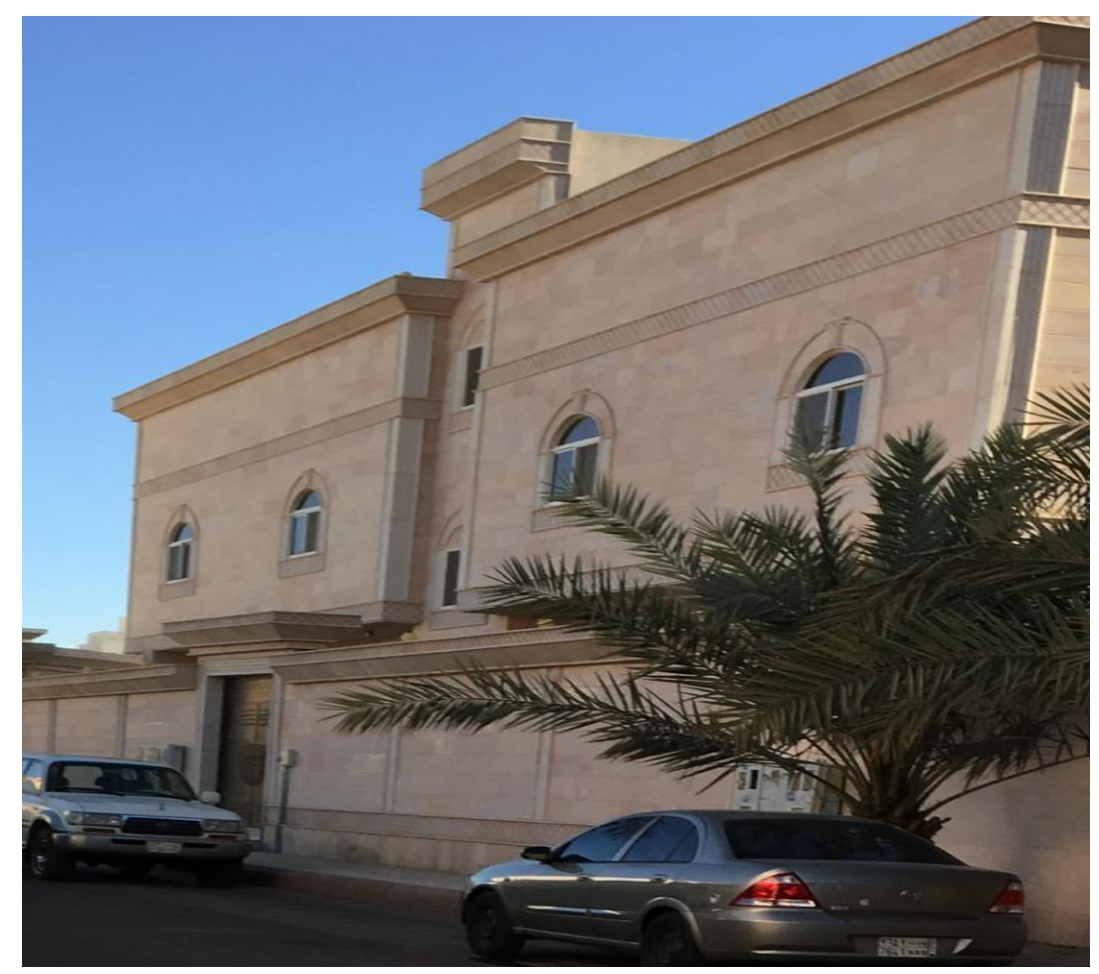

Fig 5.3: A photograph representing home ownership taken by participant PH 23

Figure 5.3 presents one of the photographs that was discussed with participant $\mathrm{PH} 23$, where he linked this photo to the possession of his new home. From the above quotation, it is clear how important home ownership is in meeting and promoting aspects of life including psychological aspects, happiness and life satisfaction. These positive aspects are likely to enhance the relationship between family members within the home, which in turn may serve to promote other aspects of life that may contribute to improving overall QoUL. Referring to the results obtained from assessing the relationship between housing and QoUL in Chapter 4, a similar positive relationship was found between the general level of QoUL and home ownership, indicating the important role of home ownership in promoting benefits for many aspects of life including privacy, security, self-esteem and stability. Within these results, privacy may be considered one of the most important outcomes of home ownership for the residents of Quba and the Saudi community in general. In that respect, it is likely that home ownership means freedom and greater control over the property, which allows residents to act in their homes according to the prevailing culture.

On the other hand, home ownership may not always be the preferred residential status. In this regard, people's perception of home ownership may vary according to their 
personalities, and particularly with respect to age. In this context, the meaning of the home, especially regarding tenure, may vary according to age difference. Older people may be more concerned with the use or quality of their homes rather than in monetary value, regarding it as a long-term home. In contrast, the home can be just a commodity for youth, and a trading point in a housing trajectory (Valentine, 2001). Young people may prefer to rent a home rather than own it, due to the fact that they are most likely to be more mobile and less stable in one place in terms of the dynamic changes in their lifestyle such as increasing family size or having job opportunities elsewhere. Moreover, home ownership may cause a fear regarding keeping up mortgage payments ae well as the costs of maintaining the property (Valentine, 2001).

\subsection{Satisfaction with the accommodation and QoUL}

Housing is an important aspect for most people, as it meets the primary need for shelter. For the QoUL, satisfaction with housing is a critical aspect. In that sense, satisfaction with housing is one of the factors that affects the achievement of other aspects of life (Joyce et al., 1999). In all parts of the world, housing is not only just a place for accommodation, but also a place where a person feels safe and enjoys privacy and belonging. It is a key element of people's daily lives, as it the place where one finds refuge, comfort and life satisfaction (Sirgy and Cornwell, 2002). From this point of view, housing is likely to play an important role in one's QoUL, in that better housing facilities lead to community growth and stability, as well as improving health conditions, and increasing safety and the level of education among the residents (Assaf et al., 2010). These benefits of housing are one of the main components of the QoUL that have a direct impact on the socio-economic aspects of people.

In light of the early findings from this chapter which indicate that the increase of housing satisfaction is associated with the increase in life satisfaction, the relationship between housing satisfaction and the QoUL was examined. In order to this, the Chi-square test was used to assess the relationship between the variables. Results revealed a significant relationship between satisfaction with accommodation and the QoUL. In addition, Spearman's rho test was also used to assess the strength of that relationship, where a positive moderated correlation between the two variables is revealed, as illustrated in Table 5.3. For 
further exploration regarding the relationship between housing satisfaction and QoUL, a cross-tabulation was applied. Cross-tabulating the two variables in Table 5.4 shows that a higher positive report of housing satisfaction is associated with a higher positive report of QoUL level, where $74.1 \%$ of the respondents who reported high levels of satisfaction with their accommodation stated that their QoUL is high. It is clear from this result that the degree of satisfaction with the accommodation is positively correlated with the QoUL, where the increase in the level of satisfaction with accommodation contributes to a higher level of QoUL, illustrated in Figure 5.4. Referring to the findings obtained from assessing the general QoUL level in Chapter 4, a strong relationship between housing and QoUL can be noticed where there appears to be some consistency between these two levels of satisfaction.

Table 5.3: Correlation between satisfaction with the accommodation quality and QoUL.

Spearman's rho.

\begin{tabular}{lllr}
\hline & & QoUL Level \\
\hline Spearman's & $\begin{array}{l}\text { Satisfaction with the } \\
\text { rho }\end{array}$ & Correlation Coefficient & $.416^{* *}$ \\
\cline { 2 - 4 } & & Sig. (2-tailed) & .000 \\
\cline { 2 - 4 } & $\mathrm{N}$ & 548 \\
\hline
\end{tabular}

**. Correlation is significant at the 0.01 level (2-tailed).

Table 5.4: The relationship between satisfaction with accommodation quality and QoUL. Crosstabulation.

\begin{tabular}{|c|c|c|c|c|c|c|}
\hline & & & \multicolumn{4}{|c|}{ QoUL Level } \\
\hline & & & Low & Moderate & High & Total \\
\hline \multirow{6}{*}{$\begin{array}{l}\text { Satisfaction with } \\
\text { accommodation } \\
\text { quality }\end{array}$} & \multirow{2}{*}{ Dissatisfied } & Count & 35 & 48 & 36 & 119 \\
\hline & & $\%$ & $29.4 \%$ & $40.3 \%$ & $30.3 \%$ & $100.0 \%$ \\
\hline & \multirow{2}{*}{$\begin{array}{c}\text { Moderately } \\
\text { satisfied }\end{array}$} & Count & 16 & 67 & 60 & 143 \\
\hline & & $\%$ & $11.2 \%$ & $46.9 \%$ & $42.0 \%$ & $100.0 \%$ \\
\hline & \multirow{2}{*}{ Satisfied } & Count & 23 & 51 & 212 & 286 \\
\hline & & & $8.0 \%$ & $17.8 \%$ & $74.1 \%$ & $100.0 \%$ \\
\hline \multirow{2}{*}{\multicolumn{2}{|c|}{ Total }} & Count & 74 & 166 & 308 & 548 \\
\hline & & $\%$ & $13.5 \%$ & $30.3 \%$ & $56.2 \%$ & $100.0 \%$ \\
\hline
\end{tabular}

Chi-square $=96.523, \mathrm{p}$-value $<.001$. 


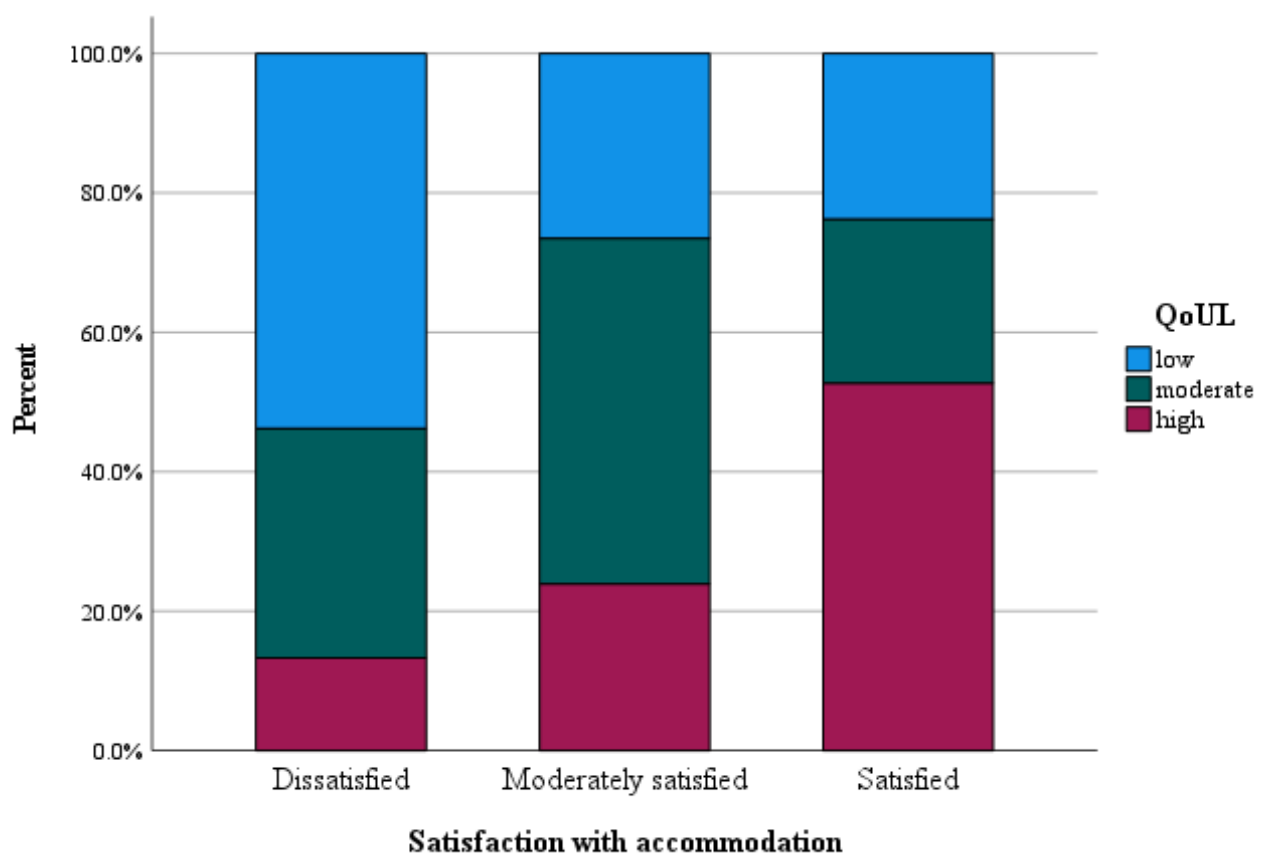

Fig 5.4: Relationship between satisfaction with accommodation quality and QoUL

The current findings support several studies in the literature including the work of Peck and Kay Stewart (1985), Das (2008), Zhang et al. (2018), Kahlmeier et al. (2001), Vera-Toscano and Ateca-Amestoy (2007). For example, Zhang et al. (2018) maintain that housing satisfaction increases people's sense of belonging to a community and promotes social stability. Moreover, Vera-Toscano and Ateca-Amestoy (2007) argue that housing, from a social point of view, is more than just a residential unit, it is a place where safety and privacy are provided. Tremblay \& Dillman (1983) aptly comment, "The fact that housing is an important component of life should not be surprising inasmuch as the home is the most immediate aspect of our living experiences and as such shapes our lives” (p. 20).

Qualitative finding from the expert interviews in this thesis emphasised the important role of housing in building family. One of the experts stressed this importance as follows:

Peace of mind, happiness and stability are one of the most important outcomes of housing satisfaction, as for the most of Saudi communities, a home is considered the key element in building a family in an effective and right manner. Consequently, the success of each member of families is 
related to how satisfied they are with their home, which is likely to result in the successes in other aspects of their lives. (Saeed Jood, an urban planner).

The effect of housing satisfaction may differ from person to person and from one culture to another. In general, satisfaction with housing is likely to have positive effects on family members regardless of their culture. For Saudi societies, however, the impact of residential satisfaction may be somewhat different, especially when it comes to the culture and norms that characterise these societies, and particularly those related to gender. In this context, many people in Saudi society may see the home as an important element in preserving the family from moral issues. Hence, it can be concluded from the above quote that the home is the main component of family formation in accordance with the culture and religious legislation followed by Saudi societies, where in most cases the roles of household members are governed by these legislations. In this context, satisfaction with housing as a key component in promoting many aspects, including social as well as economic life, may lead to satisfaction with life, and in turn, a better QoUL.

\subsection{Type of accommodation and satisfaction with housing}

Satisfaction with accommodation was found to be affected by the type of accommodation involved. Within this investigation, results have revealed a significant association between satisfaction with accommodation and the type of accommodation. It can be seen from Table 5.5 that higher levels of satisfaction with accommodation is reported by those living in villas and houses. Among those who live in villas, $83.3 \%$ stated that they are satisfied or very satisfied with their accommodation, while $61.3 \%$ among those who live in houses did the same. In contrast, a slightly lower level of satisfaction was reported by those living in flats, where $44.7 \%$ of them stated that they are satisfied or very satisfied with their accommodation. Figure 5.5 clearly illustrates the positive effect of detached accommodations on household satisfaction, where high satisfaction with accommodation is associated with houses and villas. 
Table 5.5: The relationship between type of accommodation and satisfaction with the accommodation. Cross-tabulation.

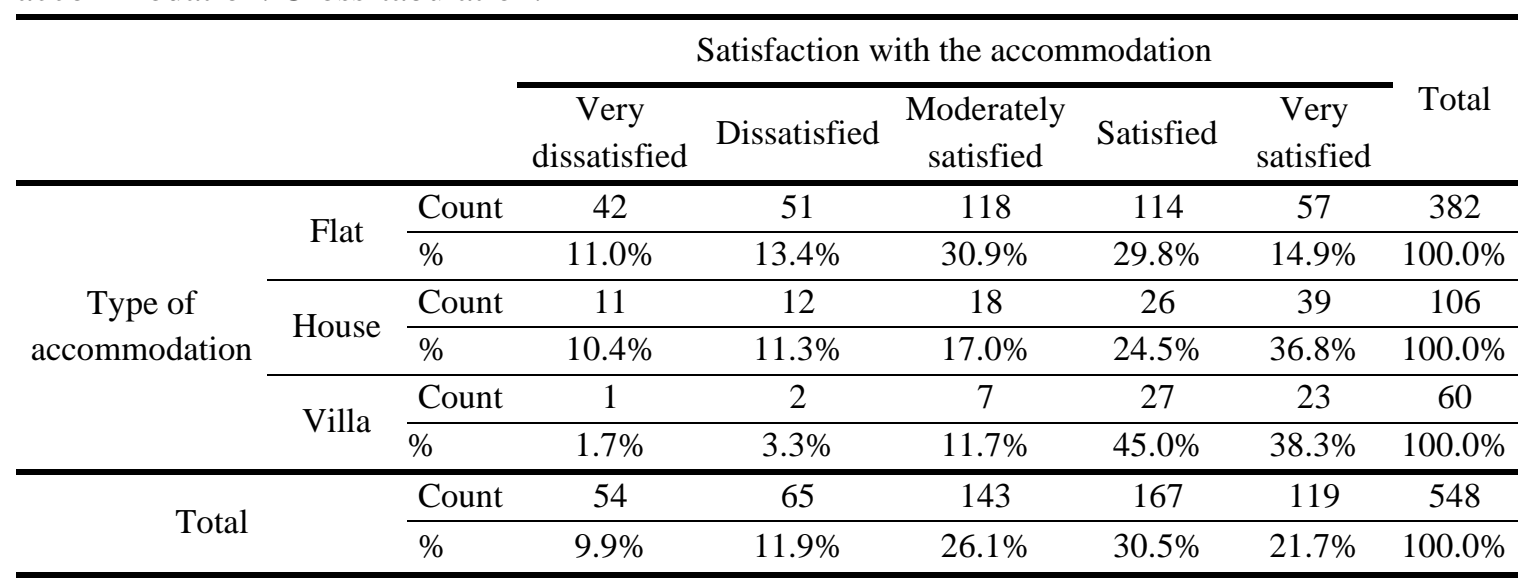

Chi-square $=52.841, \mathrm{p}$-value $<.000$.

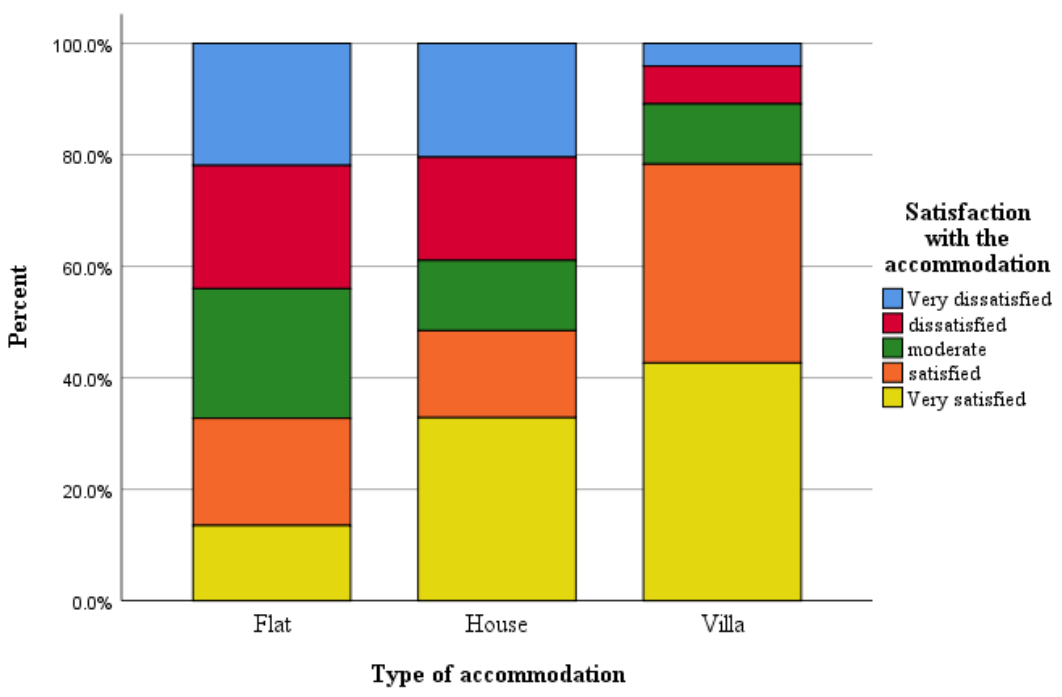

Fig 5.5: Relationship between type of accommodation and satisfaction with the accommodation

Possible interpretations regarding those results can be made. One interpretation is that detached accommodation, including houses and villas, may offer more space and therefore, more facilities and services. Such advantages may afford other social aspects such as privacy. In this context, Abdul Salam et al. (2014), who conducted a study on population distribution and household conditions in Saudi Arabia, pointed out that detached houses provide a privacy feature because they contain greater number of rooms, which in turn provides a segregation of genders, practically in terms of hospitality, which is considered to be one of the most important features of culture and norms that determine housing situation. 
In that sense, housing satisfaction is not only dependent on the physical features of a house, but also on the social and cultural characteristics of its occupiers (Oladapo, 2006).

Despite the high cost that may be associated with this type of residence, the assumption might be made that living in a villa leads to an increase in residential satisfaction and thus a higher QoUL, and this is supported in this case study. At the same time, however, living in a villa may be associated with lower QoUL, as the physical appearance that characterises this type of accommodation does not necessarily meet the needs of many in Saudi society in particular. From this standpoint, the villa may not be a desirable form of residency in Saudi society for several reasons, the most important of which are related to the design that in most cases relies on open spaces, which may in fact contribute to reduced privacy. Saudi cultural standards often require a gender segregation in times of meeting together, consequently, an open plan design is likely to curtail meetings between relatives, friends and neighbours if those standards cannot be met. Therefore, this may influence the social networks within the society which can cause some of those who live in these types of homes to find themselves in social isolation, so to speak, because they are unable to host anyone other than relatives, and this may thus extend to affect their overall QoUL.

\subsection{Adequacy of the accommodation}

The adequacy of the accommodation indicator is used to measure the residential environment as an indication of housing satisfaction. In this regard, the relationship between adequacy of the accommodation and accommodation satisfaction was examined. Results indicated a significant association between the two variables. It can be noted from Table 5.6 that higher satisfaction with the accommodation is associated with higher agreement level that the accommodation is adequate to live in, where $70.1 \%$ of those who strongly agreed that their accommodation is adequate to live in stated that they are satisfied or very satisfied with their accommodation. Nevertheless, disagreement that accommodation is adequate to live in was found to be associated with higher dissatisfaction with the accommodation, where $65.4 \%$ of those who strongly disagreed that their accommodation is adequate to live in stated that they are dissatisfied with their accommodation. Figure 5.6 presents this type 
of relationship, clearly showing that satisfaction with accommodation increases when people report a higher agreement that accommodation is adequate to live in, and vice versa.

Table: 5.6: Relationship between agreement degree that accommodation is adequate to live in and satisfaction with the accommodation. Cross-tabulation.

\begin{tabular}{|c|c|c|c|c|c|c|}
\hline & & & \multicolumn{4}{|c|}{ Satisfaction with the accommodation } \\
\hline & & & Dissatisfied & $\begin{array}{c}\text { Moderately } \\
\text { satisfied }\end{array}$ & Satisfied & Total \\
\hline \multirow{6}{*}{$\begin{array}{l}\text { To what extent } \\
\text { do you agree or } \\
\text { disagree that your } \\
\text { accommodation } \\
\text { is adequate to } \\
\text { live in? }\end{array}$} & \multirow{2}{*}{ Disagree } & Count & 68 & 27 & 9 & 104 \\
\hline & & $\%$ & $65.4 \%$ & $26.0 \%$ & $8.7 \%$ & $100.0 \%$ \\
\hline & \multirow{2}{*}{ Neutral } & Count & 21 & 39 & 26 & 86 \\
\hline & & $\%$ & $24.4 \%$ & $45.3 \%$ & $30.2 \%$ & $100.0 \%$ \\
\hline & \multirow{2}{*}{ Agree } & Count & 30 & 77 & 251 & 358 \\
\hline & & $\%$ & $8.4 \%$ & $21.5 \%$ & $70.1 \%$ & $100.0 \%$ \\
\hline \multirow{2}{*}{\multicolumn{2}{|c|}{ Total }} & Count & 119 & 143 & 286 & 548 \\
\hline & & $\%$ & $21.7 \%$ & $26.1 \%$ & $52.2 \%$ & $100.0 \%$ \\
\hline
\end{tabular}

Chi-square=203.790, p-value $<.001$.

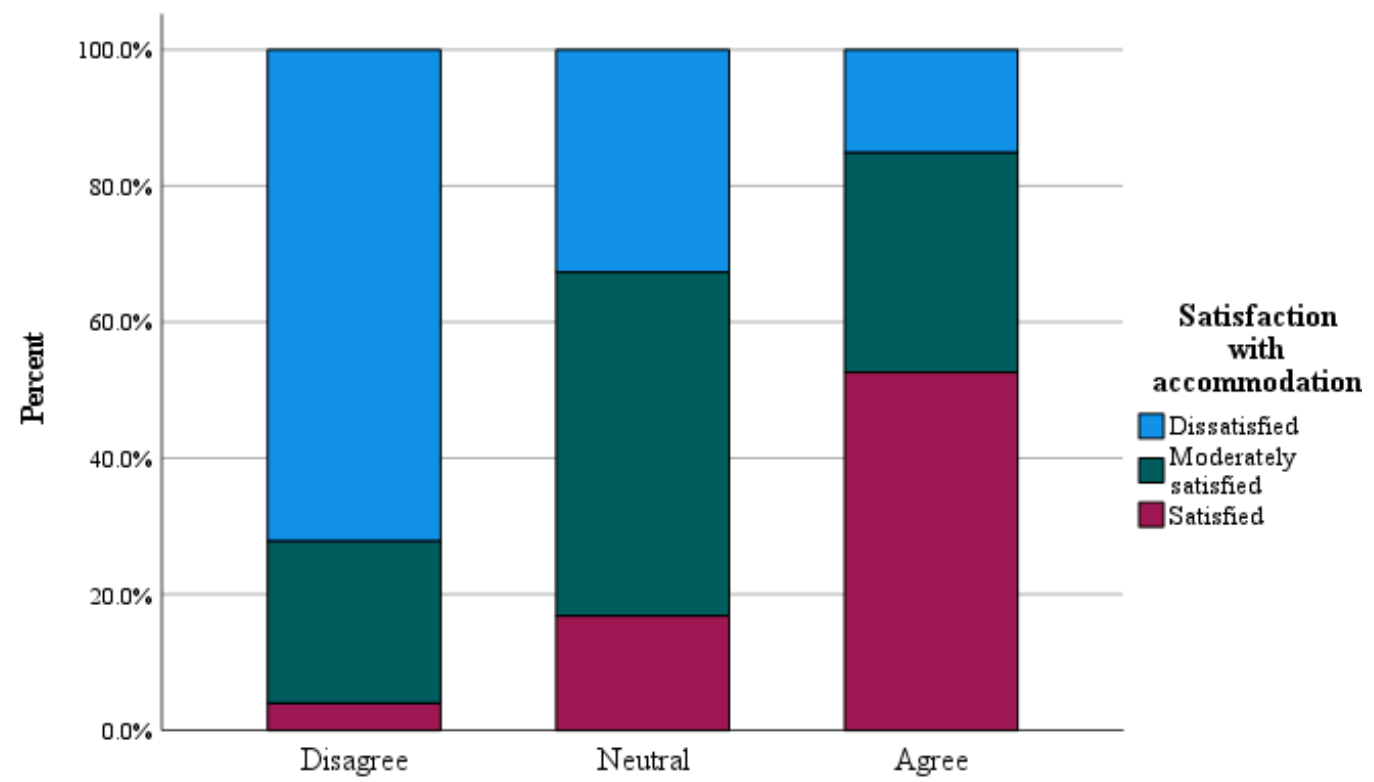

To what extent do you agree or disagree that your accommodation is adequate to live in?

Fig 5.6: Relationship between agreement degree that accommodation is adequate to live in and satisfaction with the accommodation 
The influence of accommodation type on how participants perceive the adequacy of their accommodation was assessed using the Chi-square test. The results indicated a significant association between these two variables. It can be noted from Table 5.7 that those living in a villa reported high agreement regarding the adequacy of the accommodation, where $86.7 \%$ of them agreed or strongly agreed that their accommodation is adequate to live in. This percentage decreases in the case of those living in houses and flats. In terms of those living in a house, $70.8 \%$ agreed that their accommodation was adequate, while this percentage dropped to $60.5 \%$ in the case of those living in flats. Figure 5.7 clearly shows this relationship, where a positive report of accommodation adequacy is associated with houses and villas. This result supports findings obtained from examining the relationship between type of accommodation and satisfaction with the accommodation in Table 5.5, where it is very clear that the type of accommodation plays an important role in how people perceive their accommodation.

Table 5.7: Relationship between type of accommodation and the agreement degree that accommodation is adequate to live in. Cross-tabulation.

\begin{tabular}{|c|c|c|c|c|c|c|c|c|}
\hline & & & \multicolumn{5}{|c|}{$\begin{array}{c}\text { To what extent do you agree or disagree that your } \\
\text { accommodation is adequate to live in? }\end{array}$} & \multirow{2}{*}{ Total } \\
\hline & & & $\begin{array}{l}\text { Strongly } \\
\text { disagree }\end{array}$ & Disagree & Neutral & Agree & $\begin{array}{l}\text { Strongly } \\
\text { agree }\end{array}$ & \\
\hline \multirow{6}{*}{$\begin{array}{c}\text { Type of } \\
\text { accommodation }\end{array}$} & \multirow{2}{*}{ Flat } & Count & 28 & 55 & 68 & 170 & 61 & 382 \\
\hline & & $\%$ & $7.3 \%$ & $14.4 \%$ & $17.8 \%$ & $44.5 \%$ & $16.0 \%$ & $100.0 \%$ \\
\hline & \multirow{2}{*}{ House } & Count & 4 & 13 & 14 & 32 & 43 & 106 \\
\hline & & $\%$ & $3.8 \%$ & $12.3 \%$ & $13.2 \%$ & $30.2 \%$ & $40.6 \%$ & $100.0 \%$ \\
\hline & \multirow{2}{*}{ Villa } & Count & 1 & 3 & 4 & 22 & 30 & 60 \\
\hline & & $\%$ & $1.7 \%$ & $5.0 \%$ & $6.7 \%$ & $36.7 \%$ & $50.0 \%$ & $100.0 \%$ \\
\hline \multirow{2}{*}{ Total } & & Count & 33 & 71 & 86 & 224 & 134 & 548 \\
\hline & & $\%$ & $6.0 \%$ & $13.0 \%$ & $15.7 \%$ & $40.9 \%$ & $24.5 \%$ & $100.0 \%$ \\
\hline
\end{tabular}

Chi-square $=55.028, \mathrm{p}$-value $<.000$. 


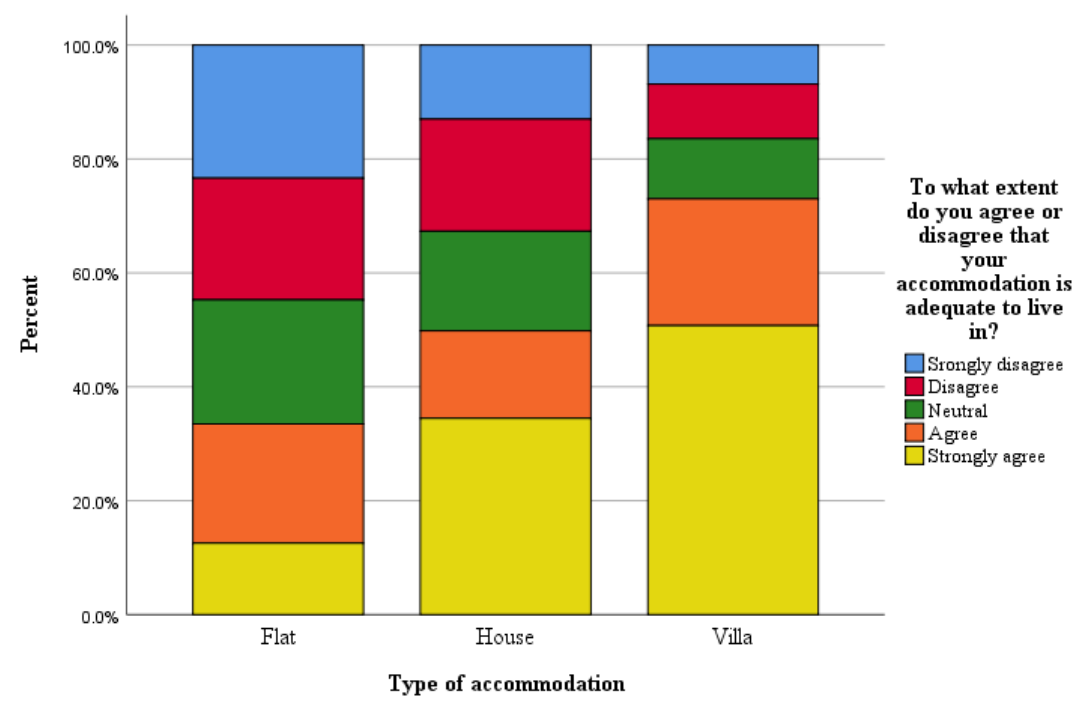

Fig 5.7: Relationship between type of accommodation and the agreement degree that accommodation is adequate to live in

\section{- Impact of socio-demographic attributes}

In relation to the impact of socio-demographic attributes on the perception of the accommodation's adequacy, only gender and residential status was significantly associated. With regard to the impact of gender on the perception of accommodation adequacy, within the overall tendency towards reporting that accommodation is adequate to live in, there is a slight gender difference, as presented in Table 5.8. Females are more likely than males to disagree with the adequacy of the accommodation, where $60.3 \%$ of them stated that they agree or strongly agree that the accommodation they live in is adequate. This ratio increases in the case of males, where $70.3 \%$ of them state that they agree or strongly agree that the accommodation they live in is adequate. As noted above, an agreement whether accommodation is adequate to live or not could be an indication of housing satisfaction. Therefore, females may be less agreed with adequacy of the accommodation because they are dissatisfied with the accommodation itself. Linking this to cultural context of Quba, it could be said that women, especially those who are married, are generally more responsible for household chores including keeping house up and looking after children. Differences in class and age between the women may affect this, but, regardless of these differences, most women in Saudi society are likely to be responsible for those tasks. In addition, women at 
home may be subject to repression which may change the meaning of the home. Hence, these considerations may cause the differences between females and males.

Table 5.8: Relationship between gender and the agreement degree that accommodation is adequate to live in. Cross-tabulation.

\begin{tabular}{|c|c|c|c|c|c|c|c|c|}
\hline & & & \multicolumn{5}{|c|}{ Agreement degree that accommodation is adequate to live in } & \multirow[b]{2}{*}{ Total } \\
\hline & & & $\begin{array}{l}\text { Strongly } \\
\text { disagree }\end{array}$ & Disagree & Neutral & Agree & $\begin{array}{c}\text { Strongly } \\
\text { agree }\end{array}$ & \\
\hline \multirow{4}{*}{ Gender } & \multirow{2}{*}{ Females } & Count & 21 & 43 & 44 & 98 & 66 & 272 \\
\hline & & $\%$ & $7.7 \%$ & $15.8 \%$ & $16.2 \%$ & $36.0 \%$ & $24.3 \%$ & $100.0 \%$ \\
\hline & \multirow{2}{*}{ Males } & Count & 12 & 28 & 42 & 126 & 68 & 276 \\
\hline & & $\%$ & $4.3 \%$ & $10.1 \%$ & $15.2 \%$ & $45.7 \%$ & $24.6 \%$ & $100.0 \%$ \\
\hline \multirow{2}{*}{\multicolumn{2}{|c|}{ Total }} & Count & 33 & 71 & 86 & 224 & 134 & 548 \\
\hline & & $\%$ & $6.0 \%$ & $13.0 \%$ & $15.7 \%$ & $40.9 \%$ & $24.5 \%$ & $100.0 \%$ \\
\hline
\end{tabular}

Chi-square $=9.171$, p-value $<0.05$.

For the impact of residential status, Table 5.9 presents the cross-tabulation of the residential status and the agreement degree that accommodation is adequate to live in. Results show that a higher degree of agreement that accommodation is adequate to live is associated with those who own their home as well as with those who live in owned family homes. In this respect, $76.1 \%$ of those who own their home stated that they agree or strongly agree that their accommodation is adequate to live in. This percentage tends to decrease in the case of other residential statuses, where $63.6 \%$ of those living in an owned family home stated that they agree or strongly agree that their accommodation is adequate to live in, while this was $55.3 \%$ of those who rented their home. This may be because owning a house may provide an opportunity to make any adjustments such as expanding or adding facilities when needed. In this context, Altas and Ozsoy (1998) argue that users can achieve satisfaction with the place where they live by changing the physical characteristics of that place to create more flexible and adaptable spaces. That is, the more changes in the places, the more suitable the places will be, and hence, the more satisfaction with residency. This option might be available only in the case of home ownership, which makes occupiers the ones who are able to change the places in which they live in accordance with their needs and aspirations. This result confirms the extent to which home ownership contributes to residential satisfaction that was obtained from assessing the relationship between residential status and satisfaction with accommodation. 
Table 5.9: Relationship between residential status and the agreement degree that accommodation is adequate to live in. Cross-tabulation.

\begin{tabular}{|c|c|c|c|c|c|c|c|c|}
\hline & & & \multicolumn{5}{|c|}{ Agreement degree that accommodation is adequate to live in } & \multirow[b]{2}{*}{ Total } \\
\hline & & & $\begin{array}{l}\text { Strongly } \\
\text { disagree }\end{array}$ & Disagree & Neutral & Agree & $\begin{array}{c}\text { Strongly } \\
\text { agree }\end{array}$ & \\
\hline \multirow{6}{*}{$\begin{array}{l}\text { Residential } \\
\text { status }\end{array}$} & \multirow{2}{*}{ Other } & Count & 2 & 5 & 9 & 15 & 13 & 44 \\
\hline & & $\%$ & $4.5 \%$ & $11.4 \%$ & $20.5 \%$ & $34.1 \%$ & $29.5 \%$ & $100.0 \%$ \\
\hline & \multirow{2}{*}{ Own home } & Count & 5 & 23 & 31 & 97 & 91 & 247 \\
\hline & & $\%$ & $2.0 \%$ & $9.3 \%$ & $12.6 \%$ & $39.3 \%$ & $36.8 \%$ & $100.0 \%$ \\
\hline & \multirow{2}{*}{ Rent home } & Count & 26 & 43 & 46 & 112 & 30 & 257 \\
\hline & & $\%$ & $10.1 \%$ & $16.7 \%$ & $17.9 \%$ & $43.6 \%$ & $11.7 \%$ & $100.0 \%$ \\
\hline \multirow{2}{*}{\multicolumn{2}{|c|}{ Total }} & Count & 33 & 71 & 86 & 224 & 134 & 548 \\
\hline & & $\%$ & $6.0 \%$ & $13.0 \%$ & $15.7 \%$ & $40.9 \%$ & $24.5 \%$ & $100.0 \%$ \\
\hline
\end{tabular}

Chi-square $=56.550, \mathrm{p}$-value $<.000$.

\subsection{Housing affordability}

Housing affordability generally refers to the cost of housing either for tenants or the owneroccupiers and the extent to which this cost is compatible with the financial capacity of the households. The cost of housing represented by the percentages of income spent on housing was found to be an important factor influencing housing satisfaction and in turn overall satisfaction with life. Florida, et al. (2013) argue that happiness is expected to be in the places where housing is less expensive and more affordable. This is because housing is the biggest cost factor for most individuals and families. In this thesis, statistical analysis using cross-tabulation revealed a significant association between satisfaction with accommodation and the percentages of household income spent on housing costs. It can be seen from Table 5.10 that nearly $62 \%$ of those who spend $10 \%$ or less are satisfied or very satisfied with their accommodation. However, nearly $49 \%$ among those who spend more than $50 \%$ of their income reported a higher satisfaction with their accommodation. This result is noteworthy as this seems to be something of an anomaly. That is, satisfaction with housing is usually expected to be associated with lower cost, and not vice versa as found in this result. 
Table 5.10: Relationship between accommodation satisfaction and percentage of household income spent on housing. Cross-tabulation.

\begin{tabular}{|c|c|c|c|c|c|c|c|c|}
\hline & & & \multicolumn{5}{|c|}{ Satisfaction with the accommodation } & \multirow[b]{2}{*}{ Total } \\
\hline & & & $\begin{array}{c}\text { very } \\
\text { dissatisfied }\end{array}$ & dissatisfied & moderate & satisfied & $\begin{array}{c}\text { Very } \\
\text { satisfied }\end{array}$ & \\
\hline \multirow{12}{*}{$\begin{array}{l}\text { Percentage of } \\
\text { household } \\
\text { income spent on } \\
\text { housing }\end{array}$} & \multirow{2}{*}{$\begin{array}{c}\text { Less than } \\
10 \%\end{array}$} & Count & 4 & 9 & 19 & 25 & 27 & 84 \\
\hline & & $\%$ & $4.8 \%$ & $10.7 \%$ & $22.6 \%$ & $29.8 \%$ & $32.1 \%$ & $100.0 \%$ \\
\hline & \multirow{2}{*}{$10 \%-19 \%$} & Count & 9 & 9 & 29 & 24 & 22 & 93 \\
\hline & & $\%$ & $9.7 \%$ & $9.7 \%$ & $31.2 \%$ & $25.8 \%$ & $23.7 \%$ & $100.0 \%$ \\
\hline & \multirow{2}{*}{$20 \%-29 \%$} & Count & 7 & 14 & 29 & 40 & 18 & 108 \\
\hline & & $\%$ & $6.5 \%$ & $13.0 \%$ & $26.9 \%$ & $37.0 \%$ & $16.7 \%$ & $100.0 \%$ \\
\hline & \multirow{2}{*}{$30 \%-39 \%$} & Count & 7 & 12 & 34 & 38 & 18 & 109 \\
\hline & & $\%$ & $6.4 \%$ & $11.0 \%$ & $31.2 \%$ & $34.9 \%$ & $16.5 \%$ & $100.0 \%$ \\
\hline & \multirow{2}{*}{$40 \%-49 \%$} & Count & 12 & 8 & 13 & 18 & 11 & 62 \\
\hline & & $\%$ & $19.4 \%$ & $12.9 \%$ & $21.0 \%$ & $29.0 \%$ & $17.7 \%$ & $100.0 \%$ \\
\hline & \multirow{2}{*}{$\begin{array}{c}\text { more than } \\
50 \%\end{array}$} & Count & 15 & 13 & 19 & 22 & 23 & 92 \\
\hline & & $\%$ & $16.3 \%$ & $14.1 \%$ & $20.7 \%$ & $23.9 \%$ & $25.0 \%$ & $100.0 \%$ \\
\hline \multirow{2}{*}{\multicolumn{2}{|c|}{ Total }} & Count & 54 & 65 & 143 & 167 & 119 & 548 \\
\hline & & $\%$ & $9.9 \%$ & $11.9 \%$ & $26.1 \%$ & $30.5 \%$ & $21.7 \%$ & $100.0 \%$ \\
\hline
\end{tabular}

Chi-square $=58.059$, p-value $<.000$.

A possible interpretation regarding these results could be made. Those who spend high percentages of their income on their accommodation may believe that high costs of housing are often associated with better quality of housing, and thus, report a satisfaction with housing. This is supported by the argument made by Lu (1999) who pointed out that housing costs are positively related to housing satisfaction. That is, the higher the costs spent on housing, the more satisfaction with housing, where this leads to a better quality of housing and thus higher satisfaction with it. Furthermore, Vera-Toscano and Ateca-Amestoy (2007) found that the higher the value of the home, the more likely an individual would be satisfied with it. These authors argued that naturally individuals do not prefer to spend more on housing, but this willingness may reflect the fact that higher rent is generally associated with better quality housing.

To further explore the economic aspect of housing, participants were asked about the extent to which housing is affordable. Frequency distribution indicated that $47.4 \%$ of the respondents disagreed that it is affordable to buy or rent a home in the municipality of Quba, compared to $21.7 \%$ of those who agreed that it was (see Table 5.11). The high ratio of disagreement regarding housing affordability may reflect how difficult it is for households 
to own or rent a home. This finding also suggests that affordability is subjective; it depends on a person's income, class and social capital.

Table 5.11: Frequency distribution about the agreement degree that it is affordable to buy or rent a home in the municipality of Quba

\begin{tabular}{llcccc}
\hline & & Frequency & Percent & Valid Percent & Cumulative Percent \\
\hline Valid & Strongly disagree & 113 & 20.6 & 20.6 & 20.6 \\
\cline { 2 - 6 } & Disagree & 147 & 26.8 & 26.8 & 47.4 \\
\cline { 2 - 6 } & Neutral & 169 & 30.8 & 30.8 & 78.3 \\
\cline { 2 - 6 } & Agree & 97 & 17.7 & 17.7 & 96.0 \\
\cline { 2 - 6 } & Strongly agree & 22 & 4.0 & 4.0 & 100.0 \\
\cline { 2 - 6 } & Total & 548 & 100.0 & 100.0 & \\
\hline
\end{tabular}

In order to gain insight into how affordability of housing affects the QoUL of those living in Quba, the relationship between the agreement degree that it is affordable to buy or rent a home and QoUL was examined using the Chi-Square test. Results revealed a significant association between the two variables. Table 5.12 presents cross-tabulating those two variables, where it can be seen that higher dissatisfaction is associated with lower QoUL. From this Table, it can be noted that $62.2 \%$ among those who stated that their QoUL low stated that they disagree that it is affordable to buy or rent a home in the municipality of Quba. Such higher ratios reflect the negative impact of housing economics on the QoUL of those living in Quba. 
Table 5.12: Relationship between agreement degree about housing affordability and QoUL. Crosstabulation.

\begin{tabular}{|c|c|c|c|c|c|c|}
\hline & & & \multicolumn{4}{|c|}{$\begin{array}{l}\text { To what extent do you agree or disagree that it is affordable } \\
\text { to buy or rent a home in the municipality of Quba? }\end{array}$} \\
\hline & & & Disagree & Neutral & Agree & Total \\
\hline \multirow{6}{*}{ QoUL } & \multirow{2}{*}{ Low } & Count & 46 & 21 & 7 & 74 \\
\hline & & $\%$ & $62.2 \%$ & $28.4 \%$ & $9.5 \%$ & $100.0 \%$ \\
\hline & \multirow{2}{*}{ Moderate } & Count & 84 & 53 & 29 & 166 \\
\hline & & $\%$ & $50.6 \%$ & $31.9 \%$ & $17.5 \%$ & $100.0 \%$ \\
\hline & \multirow{2}{*}{ High } & Count & 130 & 95 & 83 & 308 \\
\hline & & $\%$ & $42.2 \%$ & $30.8 \%$ & $26.9 \%$ & $100.0 \%$ \\
\hline \multirow{2}{*}{\multicolumn{2}{|c|}{ Total }} & Count & 260 & 169 & 119 & 548 \\
\hline & & $\%$ & $47.4 \%$ & $30.8 \%$ & $21.7 \%$ & $100.0 \%$ \\
\hline
\end{tabular}

Chi-square $=16.097, \mathrm{p}$-value $<.003$.

- Impact of socio-demographic attributes

With regard to the impact of socio-demographic attributes on housing affordability perception, employment and residential status were found to be significantly associated with the cost of housing represented by the percentages of income spent on housing. In terms of the impact of employment, retirees and self-employees were likely to spend more on housing (see Figure 5.8). This may be because these groups often have a better financial position which gives them the opportunity to spend more on housing. In relation to the impact of the residential status, homeowners where less likely to spend on housing, whereas those who rented their homes are most likely to spend more on housing (see Figure 5.9). This may reflect the difference between being renter and owner a home in terms of financial status. In this context, home ownership may enhance the financial situation over time and thus a better QoUL. This assumption is supported by findings obtained earlier in this chapter, where it was noted that homeownership may help accumulate wealth, indicating that the home is not only a place to live, but also a place to invest. 


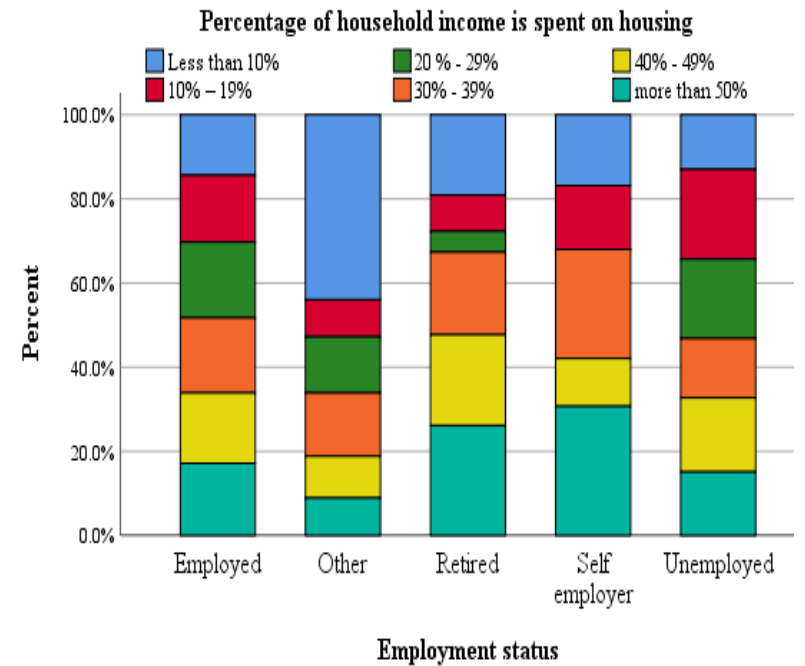

Fig 5.8: Employment status \& housing cost

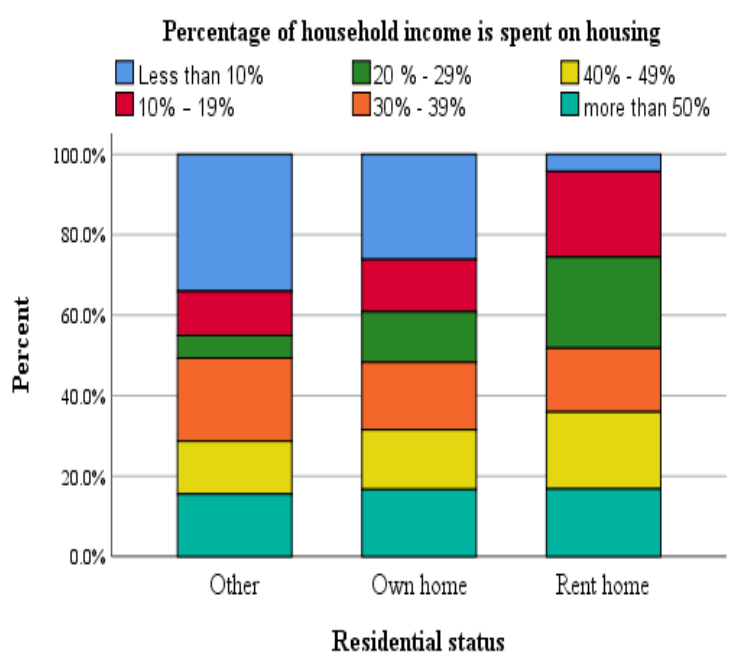

Fig 5.9: Residential status \& housing cost

The extent to which housing is affordable was also affected by socio-demographic attributes. In this respect, only age and residential status were found to be significantly associated with the agreement degree that housing is affordable. A cross-tabulation was applied for further exploration. The impact of age indicated that the highest disagreement was reported by those who were in middle age. In this respect, $54 \%$ of this age group disagree and strongly disagree that it is affordable to buy or rent a home in the municipality of Quba. Figure 5.10 illustrated this relationship. A possible explanation is that this age group may be about to start renting or buy a home, and therefore may be the most impacted/influenced by housing affordability. Another possible explanation is that for this age group, the household at this point has become larger, and therefore there may be a need to expand the home, which requires purchasing or renting a home commensurate with the size of that household. With respect to the impact of residential status, higher disagreement that housing is affordable is associated with those who live in a family-owned home or rent, as shown in Figure 5.11. Such a degree of disagreement about the extent to which housing is affordable may be due to the thought or intention to own a home or move to another home, given that these groups of occupiers do not own the house in which they currently live, and therefore they seem to disagree. 


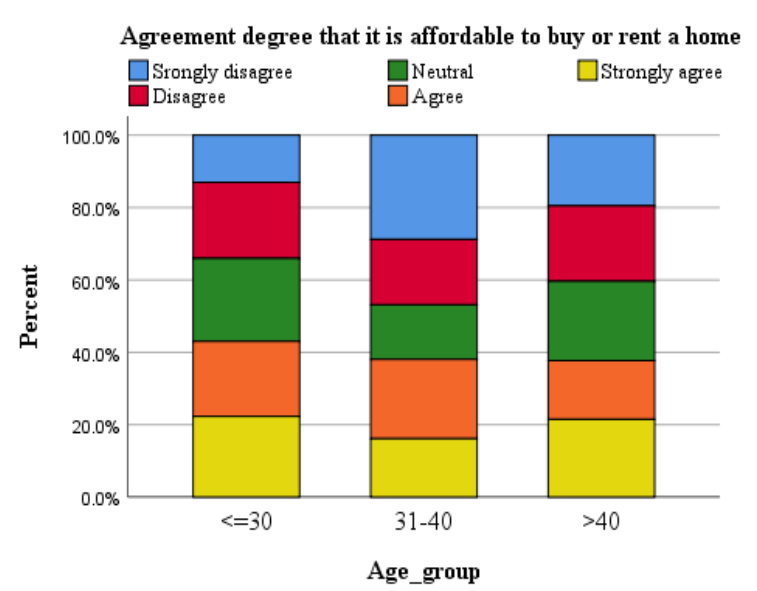

Fig 5.10: Age \& housing affordability

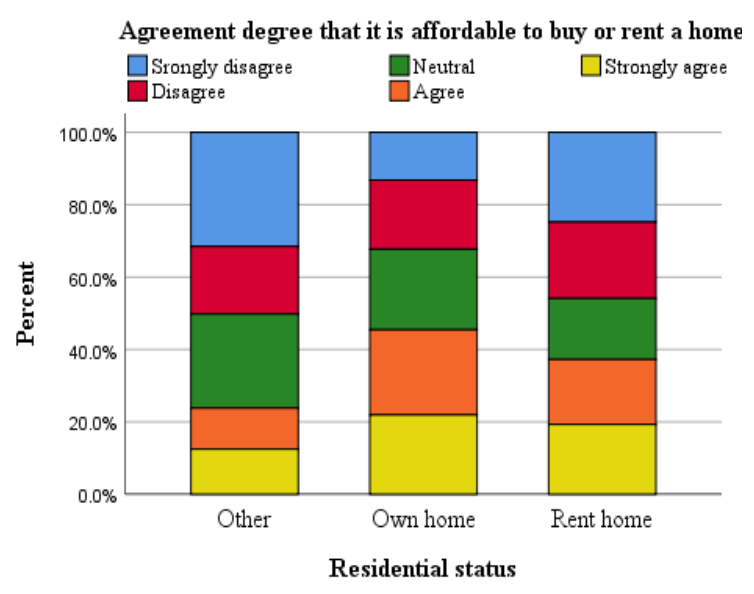

Fig 5.11: Residential status \& housing affordability

\subsection{Mobility and Housing satisfaction}

Satisfaction with housing determines whether residents stay or move out (Hur \& MorrowJones, 2008, Mohit \& Azim, 2012). Accordingly, intention to move was highlighted as an indication of housing satisfaction. In this respect, for further exploration regarding the satisfaction of Quba's residents with their accommodation, participants were specifically asked about how likely they are to move to another accommodation. In doing so, crosstabulation was applied to assess the relationship between likelihood to move to another accommodation and satisfaction with the accommodation. Results revealed an inverse significant relationship between the two variables, where the level of satisfaction with accommodation increases as the likelihood to move to another accommodation decreases. That is, respondents reporting higher satisfaction with accommodation are the least likely to move to another accommodation. It can be seen from Table 5.13 that $77.4 \%$ among those who have no intention of moving to another accommodation stated that they are satisfied with their accommodation compared to $39.5 \%$ among those who likely to move to another accommodation. This result reflects the extent to which satisfaction with the accommodation affects residential mobility. Figure 5.12 illustrates this relationship, where higher satisfaction with the accommodation is associated with the unlikelihood of moving out. In the context of Quba, historical and religious significance may play an important role in this relationship, where residents might be spiritually attached to their homes. Hence, they might not be eager to move to another accommodation. Furthermore, intentions to 
move out or stay may be affected not only by how people are satisfied with the accommodation in which they live, but also by the extent to which housing is accessible. Therefore, availability of housing stock as well as its affordability may also determine whether residents stay or move out. That is, if residents perceive that there is a shortage in housing stock or housing is unaffordable, their mobility propensity may decrease.

Table 5.13: Relationship between likelihood to move to other accommodation and satisfaction with the accommodation. Cross-tabulation.

\begin{tabular}{|c|c|c|c|c|c|c|}
\hline & & & \multicolumn{4}{|c|}{ Satisfaction with the accommodation } \\
\hline & & & Dissatisfied & Moderate & Satisfied & Total \\
\hline \multirow{6}{*}{$\begin{array}{c}\text { How likely are } \\
\text { you to move to } \\
\text { other } \\
\text { accommodation } \\
\text { ? }\end{array}$} & \multirow{2}{*}{ Unlikely } & Count & 15 & 21 & 123 & 159 \\
\hline & & $\%$ & $9.4 \%$ & $13.2 \%$ & $77.4 \%$ & $100.0 \%$ \\
\hline & \multirow{2}{*}{ Neutral } & Count & 12 & 30 & 43 & 85 \\
\hline & & $\%$ & $14.1 \%$ & $35.3 \%$ & $50.6 \%$ & $100.0 \%$ \\
\hline & \multirow{2}{*}{ Likely } & Count & 92 & 92 & 120 & 304 \\
\hline & & $\%$ & $30.3 \%$ & $30.3 \%$ & $39.5 \%$ & $100.0 \%$ \\
\hline \multirow{2}{*}{\multicolumn{2}{|c|}{ Total }} & Count & 119 & 143 & 286 & 548 \\
\hline & & $\%$ & $21.7 \%$ & $26.1 \%$ & $52.2 \%$ & $100.0 \%$ \\
\hline
\end{tabular}

Chi-square=67.192, p-value $<.001$.

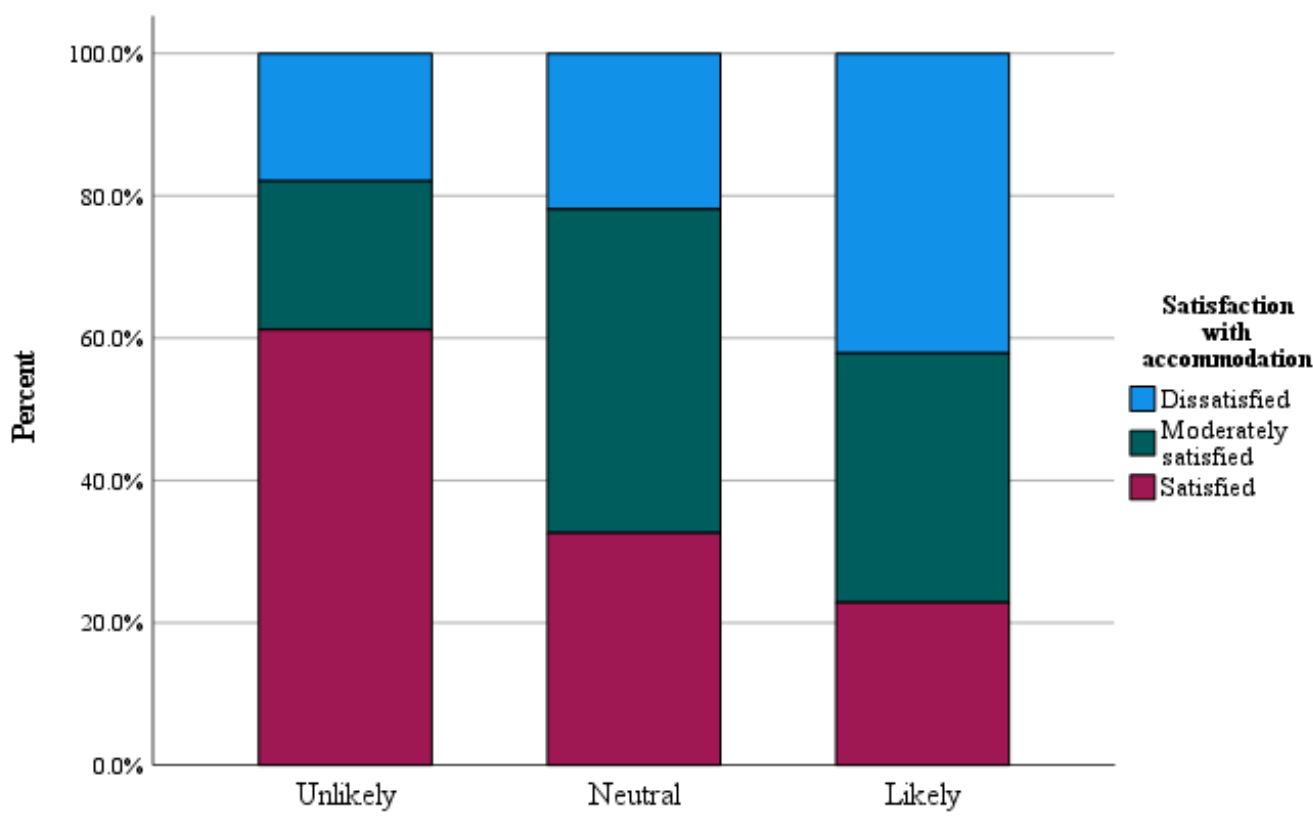

How likely are you to move to other accommodation?

Fig 5.12: Relationship between likelihood to move to other accommodation and satisfaction with the accommodation 
Several studies evidence that satisfaction with the residence affects the desire of residents to relocate, where low satisfaction with the residence leads people to move out, and mobility in this context may influence QoL (Hur \& Morrow-Jones, 2008). Therefore, relationship between likelihood to move to another accommodation and the QoUL level was examined in order to assess the effect of this variable on the QoUL of Quba's residents. Table 5.14 presents outcomes from cross-tabulating likelihood to move to another accommodation and QoUL. Results revealed an inverse significant association between the two variables, where QoUL increases with a decrease of the likelihood to move to another accommodation. In this respect, $67.3 \%$ among those who have no intention to move to another accommodation stated that their QoUL is high, compared to $52.3 \%$ among those who have the intention to move to another accommodation. Although the percentage of the positive report of the QoUL that is associated with the likelihood to move to another accommodation is relatively high, it is still clear that QoUL is affected by being likely to move to another accommodation. Key findings from this relationship can be identified. That is, when people are not happy or satisfied with their lives, they want to move to another place of residence. However, if they cannot move out, their QoUL might be negatively affected.

Table 5.14: Relationship between likelihood to move to other accommodation and QoUL. Crosstabulation.

\begin{tabular}{|c|c|c|c|c|c|c|}
\hline & & & \multicolumn{4}{|c|}{ QoUL level } \\
\hline & & & Low & Moderate & High & Total \\
\hline \multirow{6}{*}{$\begin{array}{l}\text { How likely are you } \\
\text { to move to other } \\
\text { accommodation? }\end{array}$} & \multirow{2}{*}{ Unlikely } & Count & 13 & 39 & 107 & 159 \\
\hline & & $\%$ & $8.2 \%$ & $24.5 \%$ & $67.3 \%$ & $100.0 \%$ \\
\hline & \multirow{2}{*}{ Neutral } & Count & 13 & 30 & 42 & 85 \\
\hline & & $\%$ & $15.3 \%$ & $35.3 \%$ & $49.4 \%$ & $100.0 \%$ \\
\hline & \multirow{2}{*}{ Likely } & Count & 48 & 97 & 159 & 304 \\
\hline & & $\%$ & $15.8 \%$ & $31.9 \%$ & $52.3 \%$ & $100.0 \%$ \\
\hline \multirow{2}{*}{\multicolumn{2}{|c|}{ Total }} & Count & 74 & 166 & 308 & 548 \\
\hline & & $\%$ & $13.5 \%$ & $30.3 \%$ & $56.2 \%$ & $100.0 \%$ \\
\hline
\end{tabular}

Chi-square $=12.429, \mathrm{p}$-value $<.014$. 
- Impact of socio-demographic attributes

The intention to move to another accommodation was found to be affected by some of the socio-demographic attributes of the participants. In this respect, residential status, number of people living at home and age were significantly associated with the likelihood to move to another accommodation. For the impact of residential status, cross-tabulation indicated that $68.4 \%$ among those who rent their homes reported being likely or extremely likely to move to another accommodation. This percentage tended to decrease in the case of those who own their homes as well as with those who live in family-owned homes, which is clearly illustrated in Figure 5.13. This result indicates that home ownership is a predominant factor that affects many aspects of housing discussed above in this chapter. In a challenge to the findings of this thesis, Peter Saunders (1989), as cited in Valentine (2001) argued however that renters are more likely to live in one neighbourhood longer than owners and therefore their sense of home is often associated with the neighbourhood community. In this respect, this thesis would respond that findings obtained from Chapter 4 have revealed a significant association between QoUL and residential status, where it has been found that renters report the lowest QoUL level among other residential statuses (i.e., homeownership and family-owned home).

As for the impact of the number of people living at the accommodation, households with a large number of members reported a high degree of intent to move out, where $71.2 \%$ of them reported being likely or extremely likely to move to another accommodation. This ratio tends to decrease with the decrease of the household number as is clearly shown in Figure 5.13. That is, the increase in the number of family members increases the likelihood of the family moving to another accommodation. In this case, therefore, mobility may become a need rather than a desire, and this may affect the QoUL, especially in its economic dimension, where mobility may entail high costs. Mobility in this context may thus reflect the nature of residential mobility that is often associated with the growth of family size, where more spaces and facilities are needed (Clark et al., 2006).

Another socio-demographic factor is age. This factor was found to be significantly associated with the intention to move to another accommodation. Cross-tabulating these two variables indicated that younger people are most likely to move to another accommodation. 
For example, $58.3 \%$ of those who are 30 years old or younger stated that are likely or very likely to move. The likelihood to move to another accommodation decreases in the case of those aged 40 years or older, as illustrated in Figure 5.13. Generally, the relationship between those two variables indicated that intention to move out decrease as people get older. A possible interpretation regarding this relationship is that older people are most likely to be attached to their homes, and thus, they do not want to move to another place. Those findings are in line with the findings from the study of Saunders (1989), as cited in Valentine, 2001), where he found that desire of people to move out of the home decreases dramatically after the age of 45 . In this context, he argued that older people are more attached to their homes because they often see the home as the place where they put down roots, and thus it is the embodiment of the past.
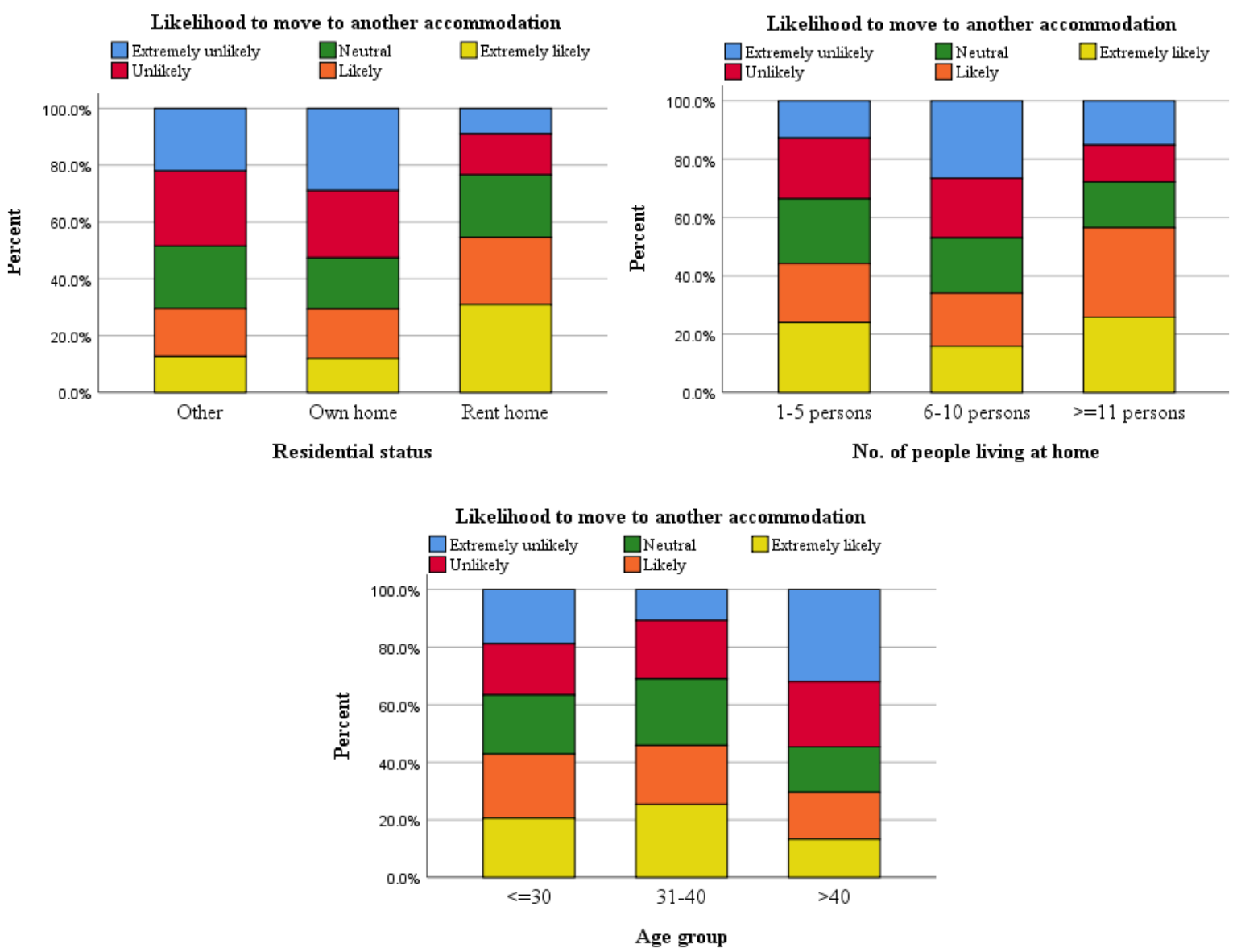

Fig 5.13: Relationship between likelihood to move to other accommodation and the three sociodemographic attributes 


\subsection{Summary of the chapter}

This chapter has discussed the housing circumstances in relation to the QoUL. Several dimensions of housing, including satisfaction level with the accommodation, QoUL and satisfaction with the accommodation, type of accommodation and satisfaction with the housing, adequacy of the accommodation, affordability of housing and mobility and housing satisfaction were highlighted. Assessment of the general satisfaction with housing showed a relatively high level of satisfaction with the accommodation within the municipality of Quba. However, this degree of satisfaction was influenced by a number of sociodemographic attributes including age and residential status. Older people were more likely to be satisfied with their accommodation. This may be due to a number of factors including socio-economic characteristics of this age group of age, and that the importance of the home increases as people get older, which may have an influence on their perception regarding the accommodation they live in. Residential status clearly influenced the satisfaction with accommodation. Those who owned their home were more likely to be satisfied with their homes. The most notable reason behind this relationship is that home ownership is associated with social and economic benefits including security, self-esteem, autonomy, privacy and freedom, as well as the economic benefits including financial and wealth accumulation. Findings from the qualitative research, namely the photo diaries emphasised these benefits of home ownership. Nevertheless, the perception of home ownership may vary according to the characteristics of the people such as age, which may influence preferences for certain residential statuses. In addition, the preference for home ownership might be changed as a result of the dynamic growth of the households as well as due to the economic concern related to this type of tenure. In this thesis, social aspects may be more important than economic aspects. That is, the investment advantage of home ownership may not matter as much as privacy, which maybe the fundamental concern to many of those who live in Quba.

In order to gain insight into the contribution of housing satisfaction to the QoUL in Quba, the relationship between these variables was examined. Higher satisfaction with the accommodation was associated with higher levels of QoUL. Other findings in this thesis indicated a strong relationship between housing and QoUL, where there appears to be some consistency between these two levels of satisfaction. Therefore, it can be said that 
satisfaction with housing determines overall satisfaction with the QoUL, as the increase in satisfaction with this indicator significantly contributes to satisfaction with the overall QoUL. These findings are supported by several studies in the literature that emphasise the importance of housing, where it has been stressed that this physical attribute has a strong positive correlation with overall life satisfaction and QoUL. From increasing people's sense of belonging to a community and promoting social stability to safety and privacy and other personal benefits, housing is considered to be the most influential factor that promotes QoUL. In this thesis, housing represented by home ownership was not only found to promote these benefits, but also psychological aspects, happiness and life satisfaction, as well as enhancing relationship between family members, which in turn all contribute to improving the overall QoUL. In that sense, it can be said that the home is not only the physical object/building/arena in which people experience their daily lives but is the aspect that combines several benefits that together contribute to a better QoUL.

Housing satisfaction was found to be affected by several dimensions. For example, type of the accommodation was found to have a strong influence on housing satisfaction. In this regard, detached homes, including houses and villas, were associated with a higher satisfaction with the accommodation, although the proportion of homeowners of these types of accommodation is $30.28 \%$, which is considered to be low compared to those who live in flats $(69.70 \%)$. The higher level of satisfaction associated with detached homes may be due to the fact that detached houses offer more space, and thus more facilities and services. Or perhaps more importantly, that they meet a number of social aspects such as privacy. That is, more facilities at home enable/facilitate more privacy, which is especially relevant/ significant in a community like Saudi Arabia, where culture and norms impose segregation between genders, particularly in the case of visiting non-relatives. Although a higher satisfaction with accommodation is linked to villas, only a limited number live in this type of home, and in the general Saudi context, this type of residence may not meet the needs of the people due to cultural norms that have a significant impact in determining the type of appropriate accommodation. Therefore, the villa may not be the desired residential form for the majority of the people in Quba in particular and Saudi Arabia in general.

Adequacy of the accommodation was highlighted as an indication of housing satisfaction. Statistical testing revealed a significant association between perceiving the adequacy of the accommodation and satisfaction with it. In this respect, a strong positive relationship 
between the two variables has been indicated, where satisfaction with accommodation increases when participants tend to agree that the accommodation, they live in is adequate. In this thesis, adequacy as one of the indicators of housing quality is affected by the type of accommodation, which in turn affects the QoUL of those living in Quba. What matters in this relationship is how the home meets the cultural norms of the urban environment in Quba. In most studies on housing quality, the focus on the adequacy of housing has been mostly on the crowdedness of the home and the quality of its facilities, while cultural norms may not be acknowledged as important. However, the meanings of housing differ from one culture to another. That is, what is considered adequate in a specific cultural context may not be adequate in another context, as meanings of home are relative in terms of their relevance to local norms and circumstances. In addition, socio-demographics affected the perception of accommodation adequacy. In this regard, gender and residential status was significantly associated with this indicator of housing. Females were likely to disagree that the accommodation they live in is inadequate, while in terms of residential status homeowners were the most likely to agree that their accommodation is adequate to live, where the ability to modify houses is the key relationship between these two variables.

Housing affordability as an economic indicator of housing was highlighted. This indicator was found to be significantly associated with housing satisfaction. Statistical tests indicated that relationship between the cost of housing represented by the percentages of income spent on housing and satisfaction with accommodation is inverse. That is, those who spend less were the most satisfied with their accommodation. At the same time, however, a direct relationship could be noticed: those who spent half of their income on their homes reported higher levels of satisfaction. This has been justified by the assumption that increased costs are often associated with the higher quality of a home, and thus, higher satisfaction with it. In this respect, no statistical differences were found in relation to the impact of the sociodemographic attributes, except for employment and residential status. The extent to which housing is affordable was also affected by socio-demographic attributes including age and residential status.

In order to gain more insight into the housing satisfaction and the affect it has on QoUL, residential mobility represented by the intention to move was highlighted. An inverse significant relationship between likelihood to move to another accommodation and satisfaction with the accommodation was found. In this respect, residents who reported 
higher satisfaction with the accommodation they live in, reported an unlikelihood to move to another accommodation, which at the same time reflected on their overall QoUL, where a higher degree of unlikelihood to move to another accommodation was associated with a higher level of QoUL. These findings indicate the extent to which residential mobility as an outcome of housing satisfaction affects the QoUL of those living in Quba. In addition, access to housing with respect to the extent to which housing is affordable, also affects the propensity to move. That is, housing accessibility also determines whether residents stay or move out.

Residential mobility, however, was found to be affected by some of the socio-demographic attributes including residential status, number of people living at home and age. For residential status, those who rented their homes are most likely to move to another accommodation. In terms of the impact of the household size, the more household members there are, the more the likelihood to move to another accommodation. That is expected, because residential mobility is often associated with the growth of family size, where more spaces and facilities are needed. The residential status represented by home ownership, as well as the type of residence, not only affects the current state of QoUL but also affects a life path. That is, home ownership may contribute alongside the benefits mentioned earlier to the economic aspect, where owners accumulate more wealth over time. For the type of residence, however, residential mobility associated with household growth may be become a need over time, which may affect the QoUL. In addition to these socio-demographic attributes, age clearly affected the perception of residential mobility. Young people were found to be most likely to move out, whereas mobility decreases in the case of the elderly. While housing dimensions including home ownership, type of accommodation, adequacy of the homes and housing affordability influenced housing satisfaction, residential mobility was affected by the level of the satisfaction with accommodation. Generally speaking, these dimensions were found to indirectly influence QoUL. That is, those dimensions affect satisfaction with the accommodation which in turn influence the overall QoUL. In light of these results, it can be said that housing is a notable factor that indirectly affects the QoUL by means of a number of dimensions that have negative and positive repercussions. Home ownership and type of accommodation were the most influential factors in housing satisfaction, which in turn affected the QoUL for those living in Quba. In this context, the type of accommodation is considered as a prominent dimension in the context of Quba, as 
cultural norms control the housing preferences, which distinguishes the study area from other cultural contexts around the world.

Drawing from the perspective of functionalism discussed in the literature, it can be said that housing is the core of the functional attributes of the urban environment, where many aspects of life, such as the social and economic dimensions that affect the QoUL of those who inhabit it, are combined. Therefore, failure to provide housing that contributes to enhancing an individual's ability to meet their needs is likely to lead to failure to achieve other dimensions, which ultimately is a cause of lower QoUL. That is, housing is a key part of the urban environment system that unites with other aspects of that environment in a comprehensive process that aims to achieve the necessary aspects of life according to the functionalist approach used in this thesis (as discussed in Chapter 2). Consequently, meeting this material aspect is likely to contribute to meeting a series of other aspects of life that ultimately contribute to a better urban quality of life.

While this chapter has highlighted the QoUL at the narrowest scale by discussing the quality of housing in relation to the QoUL in Quba, the next step is to highlight it at the broader scale (i.e., the neighbourhood), and it is to this that Chapter 6 now turns. 


\section{Chapter Six}

\section{Neighbourhood Quality and QoUL}

\subsection{Introduction}

Having explored QoUL at the narrowest scale in the previous chapter by highlighting the quality of housing primarily in terms of satisfaction with housing and its impact on the QoUL of Quba's residents, the current chapter continues in using a functionalist approach to highlight the QoUL at the broader scale: neighbourhood. The term 'neighbourhood' is often used to describe the sub-divisions of urban or rural areas such as cities, towns and villages (Berk, 2005). In that sense, neighbourhood is often a part of a larger whole and at the same time a system in itself, with specific mechanisms and functions (Kallus et al., 2000), and is considered as one of the most important urban units in which interactions occur between people themselves on the one hand, and between those occupying the neighbourhoods and the built environment qualities on other hand. Consequently, those interactions shape the QoUL, and have an inevitable impact on the perception of the QoUL in those neighbourhoods.

For many, the attractiveness of the neighbourhood as well as its friendliness are the most important determinants of acceptance and satisfaction of the neighbourhood (Gruber \& Shelton, 1987). In this respect, a large body of research indicates that satisfaction with neighbourhood is an important predictor of satisfaction with life (e.g., Campbell et al., 1976; Andrews and Withey,1976; Morris and Winter, 1978; Sirgy and Cornwell, 2002). Sirgy and Cornwell (2002) posit that "satisfaction with the physical, social, and economic neighborhood characteristics affects life satisfaction through the mediation effect of one's overall feelings toward the neighborhood" (p. 79). That is, satisfaction with those features of the neighbourhood can contribute to the overall satisfaction with neighbourhood, which in turn plays a positive role in the general feelings towards life and the overall QoUL. 
In this thesis, neighbourhood is represented by the urban setting in which dwellings and the built environment are located. It refers to the social, physical and economic environment that surrounds the dwelling unit. Accordingly, this chapter explores the neighbourhood as one of the most important physical indicators of the QoUL, as the place where people live and interact. The exploration is based on the investigation of the neighbourhood quality and its impact on the overall QoUL of Quba residents. Satisfaction with the neighbourhood is assessed as a foundation for the rest of the empirical work within this chapter taking into account the impact of the socio-demographic attributes: that is, the result of the overall satisfaction with neighbourhood is used to examine the relationship with the QoUL level that has been obtained in Chapter 4 in order to get insight into the contribution of the neighbourhood to the QoUL of those living in Quba. Neighbourhood components including the physical, social, economic and environmental are highlighted in order to get more insight into the roles of these components in the neighbourhood satisfaction and overall QoUL, while the quality of neighbourhood infrastructure, alongside aesthetics and the attractiveness of the neighbourhood are discussed for further exploration as to the contribution of the neighbourhood in the QoUL in the study area. Finally, accessibility and connectivity issues including availability of parking spaces, sidewalks, traffic and the distance to the mosques as well as the distance to work are discussed.

\subsection{Satisfaction level with the neighbourhood}

In this chapter, neighbourhood satisfaction was measured using two forms of measures (see Figure 6.1). The first is based on measuring neighbourhood satisfaction using a scale of 110 in order to obtain the average neighbourhood satisfaction score, where values 1 and 2 represent very dissatisfied, the values of 9 and 10 donate very satisfied. The second is a converted form using the five-point Likert scale ranging from very dissatisfied to very satisfied. Using the original 1-10 scale, results show an average score of 5.7, where the values 5 and 6 are the highest frequencies. In using the converted form of five categories, results show that $39.4 \%$ of respondents stated that they are satisfied with their neighbourhoods, whereas $26.8 \%$ of the respondents were not satisfied. 


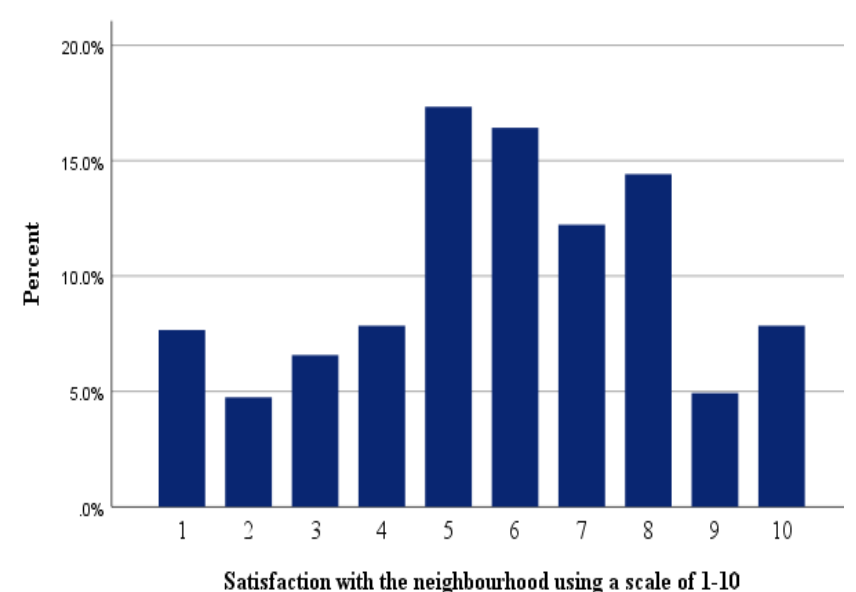

Satisfaction with the neighbourhood using a scale of $1-10$

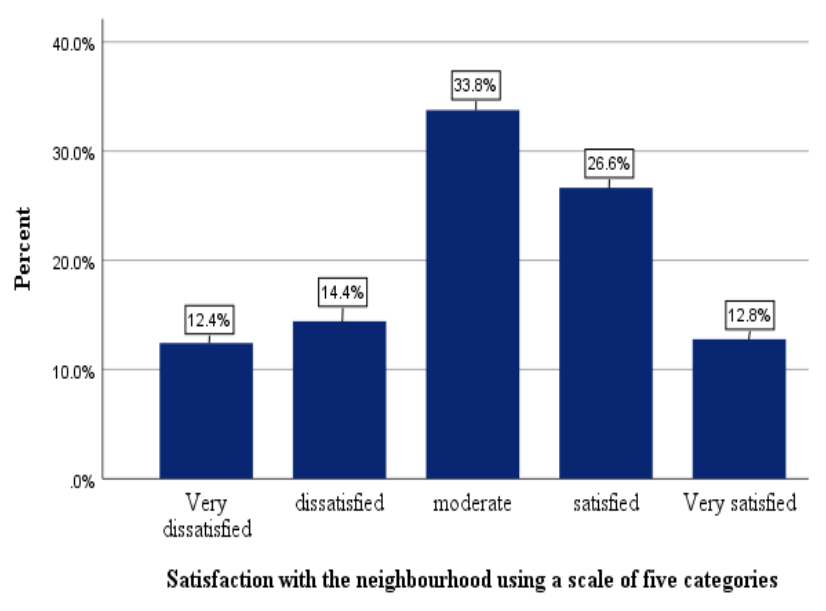

Fig 6.1: Satisfaction with the neighbourhood using two forms of scales

The results above indicate a moderate satisfaction with the neighbourhoods in the study area. Referring to the results obtained from measuring the satisfaction with housing in Chapter 5, it has been found that the level of satisfaction with the neighbourhood (an average score of 5.7) is somewhat close to the level of housing satisfaction (an average score of 6.40) using a scale of 1-10. As mentioned within the investigation of the housing indicator, satisfaction with residency contributes to satisfaction with life, which is in turn contributes to neighbourhood satisfaction, since feelings towards the house spill over to feelings towards the neighbourhood, and vice versa. This type of relationship has been emphasised by several studies in the literature including the work of Sirgy \& Cornwell (2002), Grzeskowiak et al. (2003) and Hur \& Morrow-Jones (2008). However, in this thesis, when using the converted form of measure (i.e., the Likert Scale) there seems to be noticeable differences between housing satisfaction and neighbourhood satisfaction, where $52 \%$ of respondents who were satisfied with their accommodation compared to $39.4 \%$ of those who were satisfied with their neighbourhoods. Despite that, an influence of housing satisfaction on neighbourhood satisfaction can be noticed. That is, satisfaction with the housing as a physical component affected the degree of satisfaction with the neighbourhood, which supports the assumption of the studies above with regard to the relationship between housing satisfaction and neighbourhood satisfaction. The relationship between neighbourhood satisfaction and other components of the neighbourhood including physical, social and economic will be explored in more detail later within this chapter. 


\section{- Impact of socio-demographic attributes}

With regard to the impact of socio-demographic attributes on neighbourhood satisfaction, no proof was found in relation to the impact of age, marital status, education, employment, gender and number of people living at home. At the same time, residential status and income were found to influence the satisfaction with the neighbourhood. Table 6.1 shows that $44.1 \%$ among those who own homes stated that they are satisfied or very satisfied with neighbourhood in which they live. A fairly similar positive report of neighbourhood satisfaction can be seen in the case of those who live in a family-owned home, where $41 \%$ of them stated being satisfied or very satisfied. In contrast, this percentage seems to be lower in the case of those who rent their homes, where $34.6 \%$ reported being satisfied or very satisfied. Although the percentages of satisfaction level with the neighbourhood across residential statuses is somewhat close to each other, it is still be observed that homeownership is a key influence on the satisfaction with neighbourhood. This indicates that housing represented by home ownership predicts satisfaction with the neighbourhood. This is supported by several studies including Lu, (1999) Parkes et al. (2002) and GrinsteinWeiss et al. (2011). For example, Lu (1999) and Parkes et al. (2002) indicated that home ownership may allow residents to choose the dwelling unit in the neighbourhood they prefer, and thus, have higher satisfaction with the neighbourhood. Likewise, Grinstein-Weiss et al. (2011) pointed out that when people want to own a home, they are more careful to choose the neighbourhood, which in turn affects their perception regarding that neighbourhood. 
Table 6.1: Relationship between residential status and satisfaction with the neighbourhood. Crosstabulation.

\begin{tabular}{|c|c|c|c|c|c|c|c|c|}
\hline & & & \multicolumn{5}{|c|}{ Satisfaction with the neighbourhood } & \multirow[b]{2}{*}{ Total } \\
\hline & & & $\begin{array}{c}\text { Very } \\
\text { dissatisfied }\end{array}$ & Dissatisfied & Moderate & Satisfied & $\begin{array}{c}\text { Very } \\
\text { satisfied }\end{array}$ & \\
\hline \multirow{6}{*}{$\begin{array}{l}\text { Residential } \\
\text { status }\end{array}$} & \multirow{2}{*}{ Other } & Count & 7 & 5 & 14 & 9 & 9 & 44 \\
\hline & & $\%$ & $15.9 \%$ & $11.4 \%$ & $31.8 \%$ & $20.5 \%$ & $20.5 \%$ & $100.0 \%$ \\
\hline & \multirow{2}{*}{ Own home } & Count & 24 & 27 & 87 & 71 & 38 & 247 \\
\hline & & $\%$ & $9.7 \%$ & $10.9 \%$ & $35.2 \%$ & $28.7 \%$ & $15.4 \%$ & $100.0 \%$ \\
\hline & \multirow{2}{*}{ Rent home } & Count & 37 & 47 & 84 & 66 & 23 & 257 \\
\hline & & $\%$ & $14.4 \%$ & $18.3 \%$ & $32.7 \%$ & $25.7 \%$ & $8.9 \%$ & $100.0 \%$ \\
\hline \multirow{2}{*}{\multicolumn{2}{|c|}{ Total }} & Count & 68 & 79 & 185 & 146 & 70 & 548 \\
\hline & & $\%$ & $12.4 \%$ & $14.4 \%$ & $33.8 \%$ & $26.6 \%$ & $12.8 \%$ & $100.0 \%$ \\
\hline
\end{tabular}

Chi-square $=15.451, \mathrm{p}$-value $<0.05$

For the impact of income on neighbourhood satisfaction, results revealed a significant association between the two variables. It can be noted from Table 6.2 that the highest positive report of neighbourhood satisfaction was stated by those who earn 1,500 SR or less (about 400 USD). However, this group represents a very small percentage of the total number of the sample. For other groupings of monthly income, neighbourhood satisfaction steadily increases with the increase of monthly income. Apart from those who earn 1,500 SA or less, respondents earning more than 15,000 SR reported the highest positive report of neighbourhood satisfaction, where $39.6 \%$ stated that they are satisfied and very satisfied. From this result, it can be said that income positively contributes to neighbourhood satisfaction. That is, the higher household income, the greater the satisfaction with the neighbourhood will be. This is supported by finding from several studies including the work of Mohan and Twigg (2007), Lu (1999) and Permentier et al. (2010). Within the findings of these studies, the main reason beyond this positive relationship is that the higher the income, the more opportunities there are for the residents to select the neighbourhood they prefer, which ultimately makes them satisfied. 
Table 6.2: Relationship between income and satisfaction with the neighbourhood. Crosstabulation.

\begin{tabular}{|c|c|c|c|c|c|c|c|c|}
\hline & & & \multicolumn{5}{|c|}{ Satisfaction with the neighbourhood } & \multirow{2}{*}{ Total } \\
\hline & & & $\begin{array}{c}\text { Very } \\
\text { dissatisfied }\end{array}$ & Dissatisfied & Moderate & Satisfied & $\begin{array}{c}\text { Very } \\
\text { satisfied }\end{array}$ & \\
\hline \multirow{12}{*}{$\begin{array}{c}\text { Monthly } \\
\text { income of the } \\
\text { households }\end{array}$} & \multirow{2}{*}{$<1500$} & Count & 2 & 1 & 3 & 2 & 5 & 13 \\
\hline & & $\%$ & $15.4 \%$ & $7.7 \%$ & $23.1 \%$ & $15.4 \%$ & $38.5 \%$ & $100.0 \%$ \\
\hline & \multirow{2}{*}{$1500-3000$} & Count & 12 & 5 & 11 & 3 & 4 & 35 \\
\hline & & $\%$ & $34.3 \%$ & $14.3 \%$ & $31.4 \%$ & $8.6 \%$ & $11.4 \%$ & $100.0 \%$ \\
\hline & \multirow{2}{*}{$3000-5000$} & Count & 7 & 12 & 25 & 15 & 6 & 65 \\
\hline & & $\%$ & $10.8 \%$ & $18.5 \%$ & $38.5 \%$ & $23.1 \%$ & $9.2 \%$ & $100.0 \%$ \\
\hline & \multirow{2}{*}{$5000-10000$} & Count & 23 & 24 & 49 & 43 & 15 & 154 \\
\hline & & $\%$ & $14.9 \%$ & $15.6 \%$ & $31.8 \%$ & $27.9 \%$ & $9.7 \%$ & $100.0 \%$ \\
\hline & \multirow{2}{*}{$10000-15000$} & Count & 16 & 16 & 46 & 33 & 18 & 129 \\
\hline & & $\%$ & $12.4 \%$ & $12.4 \%$ & $35.7 \%$ & $25.6 \%$ & $14.0 \%$ & $100.0 \%$ \\
\hline & \multirow{2}{*}{$>15000$} & Count & 8 & 21 & 51 & 50 & 22 & 152 \\
\hline & & $\%$ & $5.3 \%$ & $13.8 \%$ & $33.6 \%$ & $32.9 \%$ & $14.5 \%$ & $100.0 \%$ \\
\hline \multirow{2}{*}{\multicolumn{2}{|c|}{ Total }} & Count & 68 & 79 & 185 & 146 & 70 & 548 \\
\hline & & $\%$ & $12.4 \%$ & $14.4 \%$ & $33.8 \%$ & $26.6 \%$ & $12.8 \%$ & $100.0 \%$ \\
\hline
\end{tabular}

Chi-square $=40.301, \mathrm{p}$-value $<0.005$

\subsection{Satisfaction with the neighbourhood and QoUL}

With regards to the contribution of the neighbourhood to the QoUL, association between satisfaction with the neighbuorhood and QoUL level was examined using Spearman's Correlation Test in order to assess the strength of the association between these two variables. Results indicated a moderate positive relationship between QoUL and satisfaction with the neighbourhood as illustrated in Table 6.3. Cross-tabulation was also applied for further exploration regarding that relationship. Results indicated a significant association between satisfaction with the neighbourhood and QoUL. It can be seen from Table 6.4 that a higher level of QoUL is associated with greater satisfaction with the neighbourhood, where $78.2 \%$ of the respondents who reported that they are satisfied with their neighbourhood stated that their QoUL is high. This percentage gradually decreases with the decrease in the satisfaction with neighbourhood, indicating that neighbourhood plays an important role in predicting QoUL. Figure 6.2 clearly illustrates this type of relationship, where QoUL increases with the increase of neighbourhood satisfaction. 
Table 6.3: The correlation between neighbourhood satisfaction and QoUL. Spearman's correlation test .

\begin{tabular}{llr|r}
\hline & & \multicolumn{1}{c}{$\begin{array}{c}\text { Satisfaction with the } \\
\text { neighbourhood }\end{array}$} \\
\hline QoUL level & Correlation & 1.000 & $.423^{* *}$ \\
\cline { 2 - 4 } & Sig. (2-tailed) & 548 & .000 \\
\cline { 2 - 4 } & $\mathrm{N}$ & 548 \\
\hline
\end{tabular}

**. Correlation is significant at the 0.01 level (2-tailed).

Table 6.4: The relationship between neighbourhood satisfaction and QoUL. Cross-tabulation.

\begin{tabular}{|c|c|c|c|c|c|c|}
\hline & & & \multicolumn{4}{|c|}{ QoUL level } \\
\hline & & & Low & Moderate & High & Total \\
\hline \multirow{6}{*}{$\begin{array}{l}\text { Satisfaction with } \\
\text { the neighbourhood }\end{array}$} & \multirow{2}{*}{ Dissatisfied } & Count & 43 & 59 & 45 & 147 \\
\hline & & $\%$ & $29.3 \%$ & $40.1 \%$ & $30.6 \%$ & $100.0 \%$ \\
\hline & \multirow{2}{*}{ Moderate } & Count & 25 & 66 & 94 & 185 \\
\hline & & $\%$ & $13.5 \%$ & $35.7 \%$ & $50.8 \%$ & $100.0 \%$ \\
\hline & \multirow{2}{*}{ Satisfied } & Count & 6 & 41 & 169 & 216 \\
\hline & & $\%$ & $2.8 \%$ & $19.0 \%$ & $78.2 \%$ & $100.0 \%$ \\
\hline \multirow{2}{*}{\multicolumn{2}{|c|}{ Total }} & Count & 74 & 166 & 308 & 548 \\
\hline & & $\%$ & $13.5 \%$ & $30.3 \%$ & $56.2 \%$ & $100.0 \%$ \\
\hline
\end{tabular}

Chi-square $=13.084, \mathrm{p}$-value $<.011$.

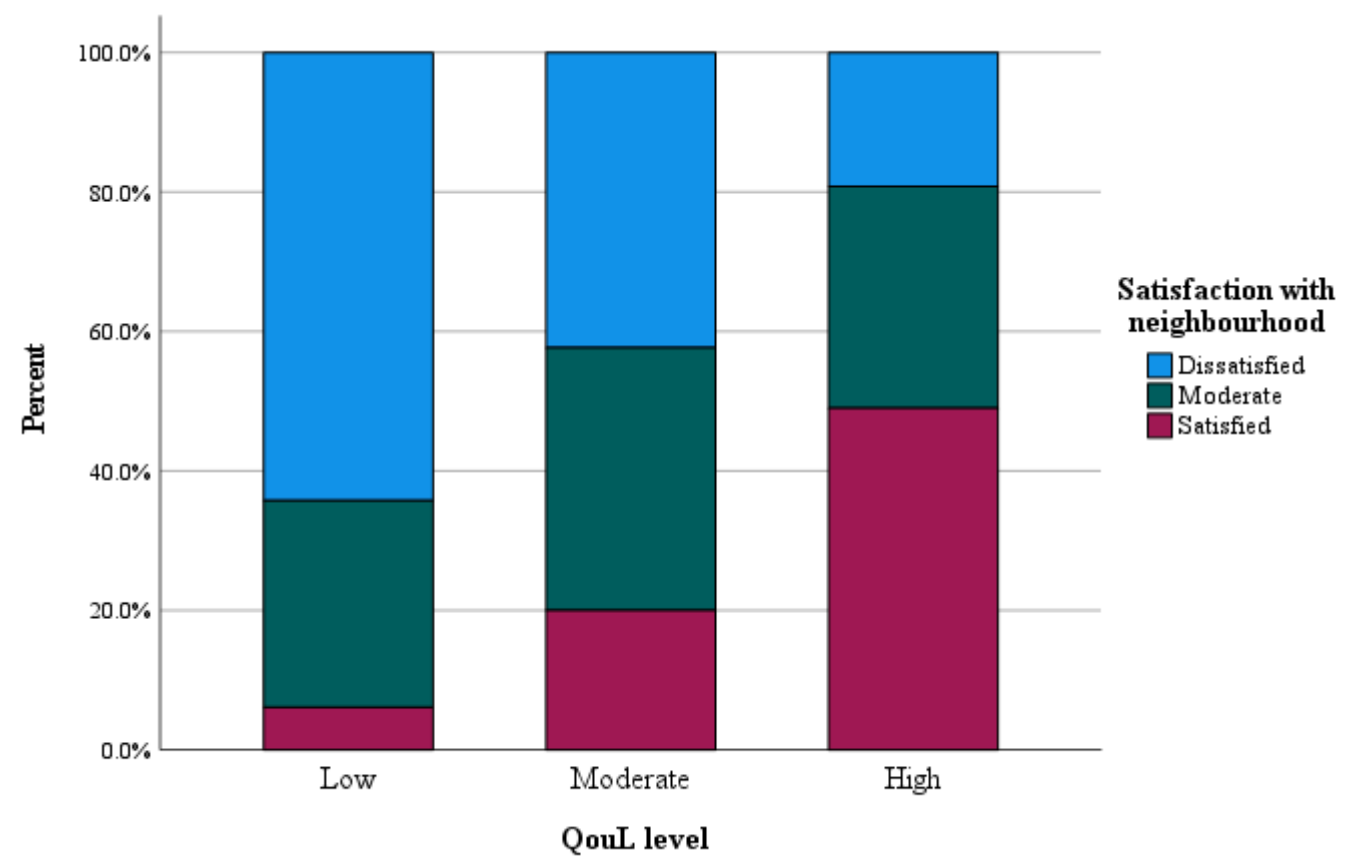

Fig 6.2: Association between neighbourhood satisfaction and QoUL 
One possible explanation for the relationship between neighbourhood satisfaction and the QoUL is that Quba residents may be satisfied with the neighbourhoods in which they live because of satisfaction with some particular aspects of those neighbourhoods. In this respect, Permentier et al. (2010) stressed that satisfaction with the characteristics of a neighbourhood is closely related to the overall satisfaction with the neighbourhood itself. As already noted above, the overall satisfaction with the neighbourhood, in turn, contributes to the overall life satisfaction (Sirgy and Cornwell, 2002). That is, life satisfaction meditated by the satisfaction with neighbourhood contributes to the overall QoUL. In this context, the importance of the neighbourhood satisfaction is based on the fact that neighbourhood is most likely to be the primary urban environment unit in which social life occurs, thus affecting the overall QoUL of the residents (Mridha \& Moore, 2011).

However, it may not always be the positive side of neighbourhoods that contributes to the QoUL. The meaning of the neighbourhood may vary according to what happens in its surroundings. Accordingly, the importance of the neighbourhood to the QoUL may decrease or increase according to the perception of the residents of that neighbourhood. For example, residents of a neighbourhood may have social relationships outside it, such as with the extended family or even with co-workers. In this context, traditional social ties within the Saudi society are based on the blood relationship in the same tribe (Achoui, 2006). Therefore, such ties have led to the emergence of societies outside the residential neighbourhood, whether at the regional level or even at the national level. Consequently, the emergence of these communities may reduce the importance of the neighbourhood. In this regard, Valentine (2001) pointed out that communities which are not predicated on space have been named by some writers as "communities without propinquity" (Webber, 1953) or "place-free" or "stretched-out" communities as suggested by John Silk (1999).

\subsection{Neighborhood components and QoUL}

For further exploration regarding the satisfaction with the quality of the neighbourhood, a set of various components and issues of the urban environment and the extent in which they influence neighbourhood satisfaction is highlighted. These components comprise the physical, social, economic, environmental and issues of accessibility. The physical aspects 
of the neighbourhood include housing and the availability of basic services such as health, education and recreation facilities. Security, social interactions, support from neighbours and sense of spirituality were considered as social components. Economic components include cost of living in the neighbourhood and job opportunities. Noise and pollution were considered as the environmental factors. Those components are discussed below.

\subsubsection{Physical components}

It has been argued that the housing unit cannot be separated from the surrounding environment, namely the neighbourhood (Gruber \& Shelton, 1987), and thus housing is considered to be one of the most important physical components of the urban environment that is likely to contribute the neighbourhood satisfaction and overall QoUL. The relationship between housing and neighbourhood, however, seems to be a two-way relationship. In this regard, several studies (e.g., Mohan \& Twigg, 2007; Lu, 1999) on the one hand emphasised that housing satisfaction contributes to neighbourhood satisfaction. While on the other hand, as noted by Varady and Preiser (1998) and Parkes et al. (2002) housing satisfaction is affected by neighbourhood attributes including physical and social attributes. Since satisfaction with neighbourhood is linked to satisfaction with the housing, the relationship between housing and neighbourhood is explored, in order to gain understanding of the impact of the housing on neighbourhood satisfaction, and consequently on the QoUL. In this regard, a significant association was found between satisfaction with the neighbourhood and satisfaction with housing. It can be noted from Table 6.5 that $66.2 \%$ of the respondents who reported dissatisfaction with neighbourhood stated that they are dissatisfied very dissatisfied with their accommodation. On the other hand, $85.7 \%$ of the respondents who reported satisfaction with neighbourhood stated that they are satisfied or very satisfied with their accommodation. Figure 6.3 illustrates this relationship, where it is clearly shown that satisfaction with the neighbourhood sharply increases with the increase of accommodation satisfaction. 
Table 6.5: Relationship between satisfaction with the neighbourhood and satisfaction with the accommodation. Cross-tabulation.

\begin{tabular}{|c|c|c|c|c|c|c|c|c|}
\hline & & & \multicolumn{5}{|c|}{ Satisfaction with accommodation } & \multirow[b]{2}{*}{ Total } \\
\hline & & & $\begin{array}{c}\text { Very } \\
\text { dissatisfied }\end{array}$ & Dissatisfied & Moderate & Satisfied & $\begin{array}{c}\text { Very } \\
\text { satisfied }\end{array}$ & \\
\hline \multirow{10}{*}{$\begin{array}{l}\text { Satisfaction } \\
\text { with the } \\
\text { neighbourhood }\end{array}$} & \multirow{2}{*}{$\begin{array}{c}\text { Very } \\
\text { dissatisfied }\end{array}$} & Count & 31 & 14 & 11 & 11 & 1 & 68 \\
\hline & & $\%$ & $45.6 \%$ & $20.6 \%$ & $16.2 \%$ & $16.2 \%$ & $1.5 \%$ & $100.0 \%$ \\
\hline & \multirow{2}{*}{ dissatisfied } & Count & 12 & 29 & 24 & 9 & 5 & 79 \\
\hline & & $\%$ & $15.2 \%$ & $36.7 \%$ & $30.4 \%$ & $11.4 \%$ & $6.3 \%$ & $100.0 \%$ \\
\hline & \multirow{2}{*}{ moderate } & Count & 5 & 16 & 82 & 59 & 23 & 185 \\
\hline & & $\%$ & $2.7 \%$ & $8.6 \%$ & $44.3 \%$ & $31.9 \%$ & $12.4 \%$ & $100.0 \%$ \\
\hline & \multirow{2}{*}{ satisfied } & Count & 3 & 4 & 21 & 77 & 41 & 146 \\
\hline & & $\%$ & $2.1 \%$ & $2.7 \%$ & $14.4 \%$ & $52.7 \%$ & $28.1 \%$ & $100.0 \%$ \\
\hline & \multirow{2}{*}{$\begin{array}{c}\text { Very } \\
\text { satisfied }\end{array}$} & Count & 3 & 2 & 5 & 11 & 49 & 70 \\
\hline & & $\%$ & $4.3 \%$ & $2.9 \%$ & $7.1 \%$ & $15.7 \%$ & $70.0 \%$ & $100.0 \%$ \\
\hline \multirow{2}{*}{\multicolumn{2}{|c|}{ Total }} & Count & 54 & 65 & 143 & 167 & 119 & 548 \\
\hline & & $\%$ & $9.9 \%$ & $11.9 \%$ & $26.1 \%$ & $30.5 \%$ & $21.7 \%$ & $100.0 \%$ \\
\hline
\end{tabular}

Chi-square $=366.872, \mathrm{p}$-value $<.000$.

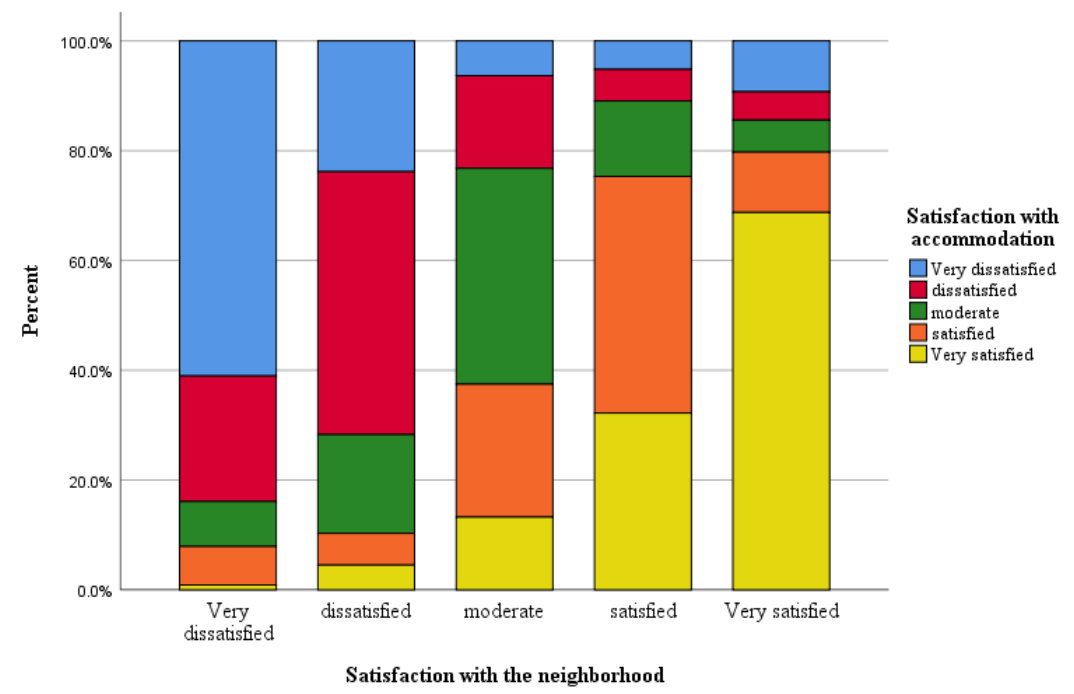

Fig 6.3: Relationship between satisfaction with the neighbourhood and satisfaction with the accommodation

From these results, it can be said that the relationship between satisfaction with housing and with the neighbourhood is a direct relationship, as is demonstrated in Figure 6.3 above. That is, the more satisfaction with the accommodation, the greater the satisfaction with the neighbourhood and vice versa. This indicates the important role of housing in neighbourhood satisfaction. As noted earlier in the chapter, studies such as those conducted 
by Sirgy \& Cornwell (2002), Grzeskowiak et al. (2003) and Hur \& Morrow-Jones (2008) have confirmed this, pointing out that it is perhaps not surprising that feelings of satisfaction towards dwelling will spill over into feelings towards the neighbourhood, since the dwelling is part of that neighbourhood. What should be noted again here, in line with findings in the socio-demographic aspects, is the factor of housing ownership; as one of the important indicators of housing, it appears to be a key element in predicting neighbourhood satisfaction, since residential status was found to be significantly associated with neighbourhood satisfaction. Accordingly, ownership may make residents more attached to their neighbourhood and thus, more satisfied with it. In this context, Oktay, et al. (2009) evidenced that home ownership is positively associated with feelings of attachment, where homeowners are more attached to their neighbourhoods than tenants.

Another physical component is that represented by basic services such as health, education and recreation facilities including children's playgrounds and green spaces. The availability and proximity to such facilities is one of the aspects that often concerns residents, and consequently affects the satisfaction with their neighbourhood (Ahlbrandt, 1984; Permentier et al., 2010; Basolo \& Strong, 2002). These physical attributes will be highlighted in relation to their association with neighbourhood satisfaction as a direct relationship, and with the QoUL as an indirect relationship. The direct relationship between these physical attributes and QoUL will be discussed within the assessments of urban services (i.e., functional attributes) in Chapter 7 following, where satisfaction of the Quba residents with regard to these attributes will be extensively discussed.

For the purposes of this chapter, in terms of the general findings, participants were asked about the lack of these facilities as an indication of the satisfaction with the neighbourhood in which they live. Table 6.6 presents analysis for the frequency distribution of the facilities availability measurement within the neighbourhoods. Results indicate that the respondents do not see that there is a lack in health facilities and schools within the neighbourhood in which they live, where $70.8 \%$ of them stated that there is no lack in health facilities, and $61.3 \%$ stated that there is no lack in schools, indicating satisfactory responses from the majority of the participants regarding those two physical components. For the playground and green spaces, however, respondents seem to be unsatisfied with these two components, where $72.8 \%$ of them stated that there is a lack in playgrounds, while $80.8 \%$ stated there is 
a lack of the green spaces. These high percentages of negative reporting probably indicate that the lack of green spaces and children's playgrounds is considered as one of the issues that residents suffer from, which may affect their satisfaction with the neighbourhood, and in turn affects the overall QoUL. In the big picture of QoUL, green spaces as an example promote many aspects of life including health and general well-being.

Table 6.6: Frequency measurement of the availability of facilities

\begin{tabular}{cccccc}
\hline \multirow{2}{*}{ Measurement of lack facilities } & \multicolumn{5}{c}{ Frequencies distribution } \\
\cline { 2 - 6 } & Yes & Frequency & No & Frequency & Total \\
\hline Lack of Schools & $29.2 \%$ & 160 & $70.8 \%$ & 388 & 548 \\
\hline Lack of Health Facilities & $38.7 \%$ & 212 & $61.3 \%$ & 336 & 548 \\
\hline Lack of Playground & $72.8 \%$ & 399 & $27.2 \%$ & 149 & 548 \\
\hline Lack of Green Spaces & $80.8 \%$ & 443 & $19.2 \%$ & 105 & 548 \\
\hline
\end{tabular}

An additional statistical procedure was conducted to examine the relationship between the four physical components and satisfaction with the neighbourhood in order to gain more insight into the effect of those components on the residents' perception regarding the neighbourhood in which they live. Using cross-tabulation, results have indicated a significant association between neighbourhood satisfaction and all of the physical components. It can be noted from Table 6.7 that reporting a lack of those facilities is linked to lower satisfaction with the neighbourhood and vice versa. Figure 6.4 clearly illustrates this type of relationship, where it can be noted that satisfaction with the neighbourhood tends to be lower when reporting a lack of those components and vice versa. These results support findings from several studies in the literature including the work of Ahlbrandt (1984) Permentier et al. (2010) Basolo and Strong (2002) who emphasised the role of these physical components in the prediction of neighbourhood satisfaction and consequently the QoUL, where satisfaction with those physical components leads to satisfaction with the neighbourhood. This reflects how important these components are to the residents in meeting their daily needs, and that meeting those needs through those aspects is likely to contribute to a higher QoUL. 
Table 6.7: The relationship between satisfaction with the neighbourhood and issues with the facilities. Cross-tabulation.

\begin{tabular}{|c|c|c|c|c|c|c|c|c|}
\hline & & & \multicolumn{5}{|c|}{ Satisfaction with neighbourhood } & \multirow[b]{2}{*}{ Total } \\
\hline & & & $\begin{array}{c}\text { Very } \\
\text { dissatisfied }\end{array}$ & Dissatisfied & Moderate & Satisfied & $\begin{array}{c}\text { Very } \\
\text { satisfied }\end{array}$ & \\
\hline \multirow{4}{*}{$\begin{array}{l}\text { Lack of } \\
\text { Schools }\end{array}$} & \multirow{2}{*}{ No } & Count & 40 & 44 & 126 & 115 & 63 & 388 \\
\hline & & $\%$ & $10.3 \%$ & $11.3 \%$ & $32.5 \%$ & $29.6 \%$ & $16.2 \%$ & $100.0 \%$ \\
\hline & \multirow{2}{*}{ Yes } & Count & 28 & 35 & 59 & 31 & 7 & 160 \\
\hline & & $\%$ & $17.5 \%$ & $21.9 \%$ & $36.9 \%$ & $19.4 \%$ & $4.4 \%$ & $100.0 \%$ \\
\hline \multirow{2}{*}{ Total } & & Count & 68 & 79 & 185 & 146 & 70 & 548 \\
\hline & & $\%$ & $12.4 \%$ & $14.4 \%$ & $33.8 \%$ & $26.6 \%$ & $12.8 \%$ & $100.0 \%$ \\
\hline \multicolumn{9}{|c|}{ Chi-square $=31.05, \mathrm{p}$-value $<.001$} \\
\hline \multirow{4}{*}{$\begin{array}{l}\text { Lack of } \\
\text { Health } \\
\text { Facilities }\end{array}$} & \multirow{2}{*}{ No } & Count & 28 & 37 & 108 & 101 & 62 & 336 \\
\hline & & $\%$ & $8.3 \%$ & $11.0 \%$ & $32.1 \%$ & $30.1 \%$ & $18.5 \%$ & $100.0 \%$ \\
\hline & \multirow{2}{*}{ Yes } & Count & 40 & 42 & 77 & 45 & 8 & 212 \\
\hline & & $\%$ & $18.9 \%$ & $19.8 \%$ & $36.3 \%$ & $21.2 \%$ & $3.8 \%$ & $100.0 \%$ \\
\hline \multirow{2}{*}{ Total } & & Count & 68 & 79 & 185 & 146 & 70 & 548 \\
\hline & & $\%$ & $12.4 \%$ & $14.4 \%$ & $33.8 \%$ & $26.6 \%$ & $12.8 \%$ & $100.0 \%$ \\
\hline \multicolumn{9}{|c|}{ Chi-square $=45.02, \mathrm{p}$-value $<.001$} \\
\hline \multirow{4}{*}{$\begin{array}{l}\text { Lack of } \\
\text { Playground }\end{array}$} & \multirow{2}{*}{ No } & Count & 12 & 14 & 42 & 46 & 35 & 149 \\
\hline & & $\%$ & $8.1 \%$ & $9.4 \%$ & $28.2 \%$ & $30.9 \%$ & $23.5 \%$ & $100.0 \%$ \\
\hline & \multirow{2}{*}{ Yes } & Count & 56 & 65 & 143 & 100 & 35 & 399 \\
\hline & & $\%$ & $14.0 \%$ & $16.3 \%$ & $35.8 \%$ & $25.1 \%$ & $8.8 \%$ & $100.0 \%$ \\
\hline \multirow{2}{*}{ Total } & & Count & 68 & 79 & 185 & 146 & 70 & 548 \\
\hline & & $\%$ & $12.4 \%$ & $14.4 \%$ & $33.8 \%$ & $26.6 \%$ & $12.8 \%$ & $100.0 \%$ \\
\hline \multicolumn{9}{|c|}{ Chi-square $=28.35, \mathrm{p}$-value $<.001$} \\
\hline \multirow{4}{*}{$\begin{array}{l}\text { Lack of } \\
\text { Green } \\
\text { Spaces }\end{array}$} & \multirow{2}{*}{ No } & Count & 11 & 9 & 30 & 27 & 28 & 105 \\
\hline & & $\%$ & $10.5 \%$ & $8.6 \%$ & $28.6 \%$ & $25.7 \%$ & $26.7 \%$ & $100.0 \%$ \\
\hline & \multirow{2}{*}{ Yes } & Count & 57 & 70 & 155 & 119 & 42 & 443 \\
\hline & & $\%$ & $12.9 \%$ & $15.8 \%$ & $35.0 \%$ & $26.9 \%$ & $9.5 \%$ & $100.0 \%$ \\
\hline \multirow{2}{*}{\multicolumn{2}{|c|}{ Total }} & Count & 68 & 79 & 185 & 146 & 70 & 548 \\
\hline & & $\%$ & $12.4 \%$ & $14.4 \%$ & $33.8 \%$ & $26.6 \%$ & $12.8 \%$ & $100.0 \%$ \\
\hline
\end{tabular}

Chi-square $=24.17, \mathrm{p}$-value $<.001$ 

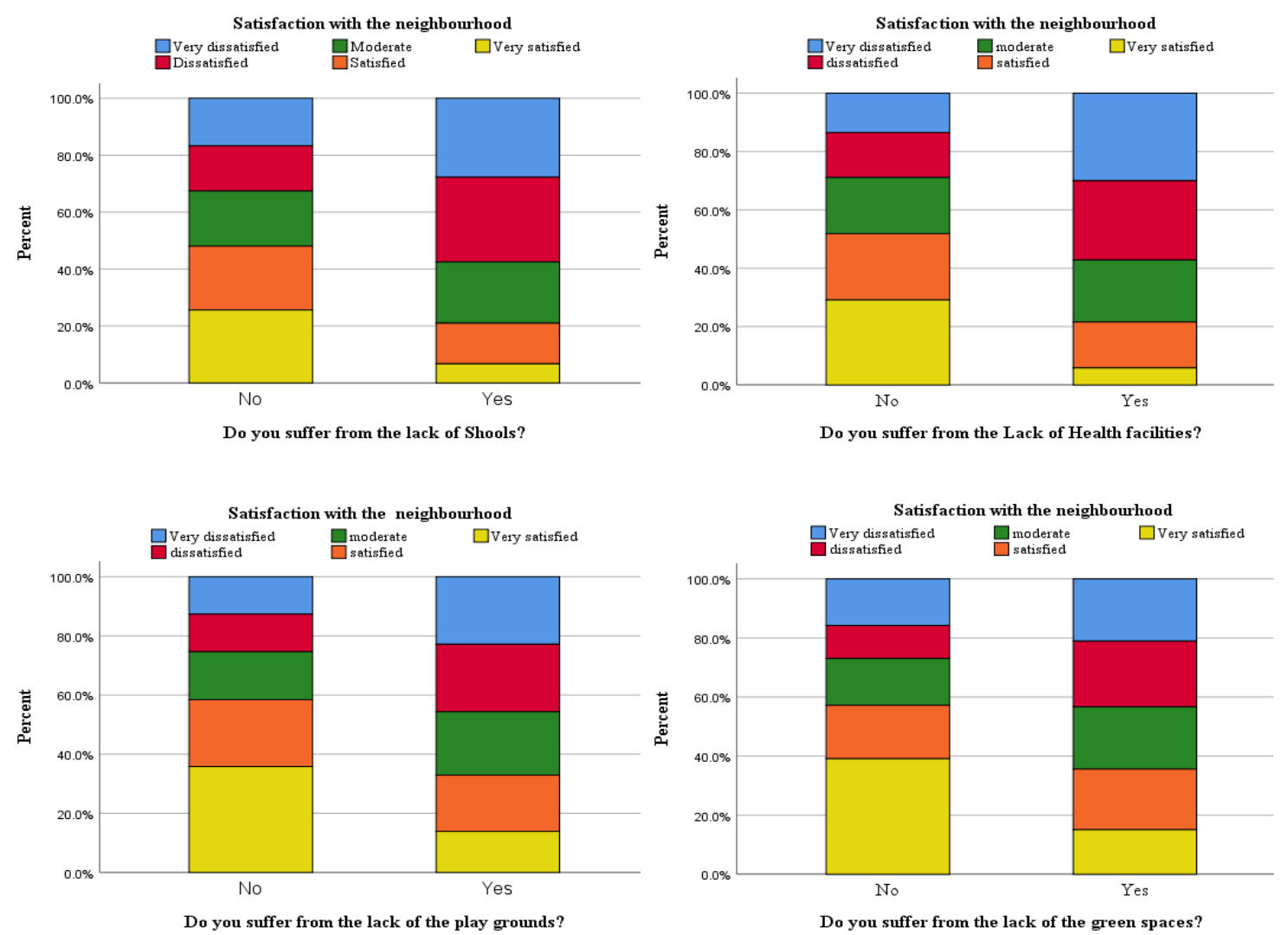

Fig 6.4: Relationship between neighbourhood satisfaction and the availability of four physical components

\subsubsection{Social components}

Findings from Chapter 4 indicated that social components of the urban environment including social relations, religion and spirituality play an important role in the overall QoUL. With respect to the neighbourhood, it has been argued by a large body of research that social components are among the significant factors that predict neighbourhood satisfaction (e.g., Marans and Rodgers, 1975; Gruber and Shelton, 1987; Ahlbrandt, 1984; Sirgy and Cornwell, 2002; Hur and Morrow-Jones, 2008). In this thesis, the social variables including security, social interactions, support from neighbours, satisfaction with neighbours and sense of spirituality and their impact on neighbourhood satisfaction were highlighted. Results from the statistical analysis using Spearman's correlation test have revealed a significant positive correlation between neighbourhood satisfaction and all these social variables (see Table 6.8). That is, that these variables are positively contributing to the neighbourhood satisfaction for those living in Quba. 
Table 6.8: The correlation between neighbourhood satisfaction and social components. Spearman's correlation.

\begin{tabular}{lccccc}
\hline & Security & $\begin{array}{c}\text { Social } \\
\text { interactions }\end{array}$ & $\begin{array}{c}\text { Support from } \\
\text { neighbours }\end{array}$ & $\begin{array}{c}\text { Satisfaction } \\
\text { with neighbours }\end{array}$ & $\begin{array}{c}\text { Sense of } \\
\text { spirituality }\end{array}$ \\
\hline $\begin{array}{l}\text { Correlation } \\
\text { Coefficient }\end{array}$ & .347 & .163 & .227 & .231 & .193 \\
\hline Results & Significant & Significant & Significant & Significant & Significant \\
\hline
\end{tabular}

These findings are in line with several studies in the literature that assert the important role of social components in the residential satisfaction. For example, but not limited to, Marans and Rodgers (1975) and Hur \& Morrow-Jones (2008) found that perception of crime significantly influences neighbourhood satisfaction. For the other social variables, findings from several studies indicate that that neighbourly relationships, including social interactions with neighbours, plays an important role in neighbourhood satisfaction (e.g., Sirgy and Cornwell, 2002; Ahlbrandt, 1984; Marans and Rodgers, 1975). The current findings obtained from measuring the relationship between neighbourhood satisfaction and social variables also support findings from Chapter 4 , where those variables were found to significantly contribute to the overall satisfaction with life. This indicates that the relationship between social life and neighbourhood satisfaction is a sequential process, where social components predict neighbourhood satisfaction, and neighbourhood satisfaction predicts overall satisfaction with life. What matters in this kind of relationship is that the neighbourhood is the key element through which people perceive their QoUL; it is the urban unit through which people experience their daily lives. In this context, social life including social relations and the perception of security is the most important component that predicts satisfaction with the neighbourhood. In light of this, the first relationship (i.e., social life versus neighbourhood satisfaction) is the most important through which the sequence of relationship takes place. This is because satisfaction with the overall QoUL is an outcome of the relationship between neighbourhood satisfaction and perceived social life. That is, social components predict neighbourhood satisfaction, and satisfaction with neighbourhood predicts overall QoUL. 


\subsubsection{Economic components}

Economic features including cost of living and job opportunities tend to contribute to the satisfaction with the neighbourhood and ultimately the overall QoUL (Sirgy and Cornwell, 2002). In order to investigate the role of the economic attributes to the neighbourhood satisfaction as well as the QoUL within Quba, the relationship between the cost of living and job opportunities and satisfaction with the neighbourhood, as well as the QoUL, is examined. Statistical analysis indicated that no significant associations were found between cost of living and neighbourhood satisfaction and QoUL. However, a significant association was found between satisfaction with the neighbourhood and job opportunities using the Chi-square Test. Table 6.9 presents a cross-tabulation of the two variables. It can be noted that lower satisfaction with the neighbourhood is associated with reporting disagreement that there are plenty of opportunities to find an appropriate job, where $55.9 \%$ and 56.9\% among those who reported disagreement regarding job availability stated that they are dissatisfied or very dissatisfied with the neighbourhood. Such higher ratios indicate the negative impact of this economic indicator on the neighbourhood satisfaction of those living in Quba. That is, when people are not satisfied with the availability of the jobs within their area, they are likely to be dissatisfied with the neighbourhood in which they live. At the same time, the tendency is for respondents $u$ to be satisfied with their neighbourhood, although they are not satisfied with the availability of jobs. These results indicate that this economic indicator is directly and inversely associated with neighbourhood satisfaction. 
Table 6.9: Relationship between satisfaction with the neighbourhood and job opportunities. Cross-tabulation.

\begin{tabular}{|c|c|c|c|c|c|c|c|c|}
\hline & & & \multicolumn{5}{|c|}{$\begin{array}{l}\text { To what extent do you agree or disagree there are } \\
\text { plenty of opportunities to find an appropriate job? }\end{array}$} & \multirow{2}{*}{ Total } \\
\hline & & & $\begin{array}{l}\text { Strongly } \\
\text { disagree }\end{array}$ & Disagree & Neutral & Agree & $\begin{array}{c}\text { Strongly } \\
\text { agree }\end{array}$ & \\
\hline \multirow{10}{*}{$\begin{array}{l}\text { Satisfaction with the } \\
\text { neighbourhood }\end{array}$} & \multirow{2}{*}{$\begin{array}{c}\text { Very } \\
\text { dissatisfied }\end{array}$} & Count & 23 & 15 & 18 & 3 & 9 & 68 \\
\hline & & $\%$ & $33.8 \%$ & $22.1 \%$ & $26.5 \%$ & $4.4 \%$ & $13.2 \%$ & $100.0 \%$ \\
\hline & \multirow{2}{*}{ dissatisfied } & Count & 17 & 28 & 14 & 12 & 8 & 79 \\
\hline & & $\%$ & $21.5 \%$ & $35.4 \%$ & $17.7 \%$ & $15.2 \%$ & $10.1 \%$ & $100.0 \%$ \\
\hline & \multirow{2}{*}{$\begin{array}{l}\text { Moderately } \\
\text { satisfied }\end{array}$} & Count & 42 & 48 & 58 & 27 & 10 & 185 \\
\hline & & $\%$ & $22.7 \%$ & $25.9 \%$ & $31.4 \%$ & $14.6 \%$ & $5.4 \%$ & $100.0 \%$ \\
\hline & \multirow{2}{*}{ satisfied } & Count & 25 & 47 & 38 & 25 & 11 & 146 \\
\hline & & $\%$ & $17.1 \%$ & $32.2 \%$ & $26.0 \%$ & $17.1 \%$ & $7.5 \%$ & $100.0 \%$ \\
\hline & \multirow{2}{*}{$\begin{array}{c}\text { Very } \\
\text { satisfied }\end{array}$} & Count & 12 & 12 & 19 & 13 & 14 & 70 \\
\hline & & $\%$ & $17.1 \%$ & $17.1 \%$ & $27.1 \%$ & $18.6 \%$ & $20.0 \%$ & $100.0 \%$ \\
\hline \multirow{2}{*}{ Total } & & Count & 119 & 150 & 147 & 80 & 52 & 548 \\
\hline & & $\%$ & $21.7 \%$ & $27.4 \%$ & $26.8 \%$ & $14.6 \%$ & $9.5 \%$ & $100.0 \%$ \\
\hline
\end{tabular}

Chi-square $=36.571, \mathrm{p}$-value $<.002$.

From the results of measuring the relationship between economic components and neighbourhood satisfaction particularly with regard to the availability of the jobs opportunities, it can be said that this economic component has a negative and positive effect on the neighbourhood satisfaction. In this regard, disagreement regarding job availability was associated with lower satisfaction with the neighbourhood, while at the same time being associated with a higher satisfaction with the neighbourhood. This result may appear somewhat odd, but possible interpretations regarding those relationships can be made. One of these interpretations is that people may dissatisfied with their neighbourhood because they perceive that there is no availability of jobs opportunities, while others are satisfied because they prefer to live in that neighbourhood regardless of the lack of the opportunities of the jobs because factors such as living in a good house or having a good relationship with neighbours may outweigh job concerns.

Other possible explanations indicate that some social and cultural factors may play an important role in this relationship. Referring to the results obtained in Chapter 4 on the extent to which social and cultural components affect the QoUL in the municipality of Quba, it can be said that people's sense of spirituality of the place may play a role in the relationship between satisfaction with the economic aspects and satisfaction with the neighbourhood as well as the QoUL. In addition, the social components of the 
neighbourhood as discussed in this chapter, including security, social relations and sense of spirituality, may affect people's perception regarding the economic components particularly with regard to the availability of the job's opportunities in relation to the neighbourhood satisfaction. Further research may be required for deeper exploration regarding the interaction of these economic components in predicting neighbourhood satisfaction.

\subsubsection{Environmental components}

Environmental issues, and especially air pollution, have been widely seen as significant public health problems, particularly within the larger cities in the developing world (McMichael, 2000). Many studies consider these environmental issues as factors affecting residential well-being and QoL (e.g., Lansing and Maran, 1969; Jacobs et al., 1984; Davis \& Fine-Davis, 1991; van Praag \& Baarsma, 2004; Welsch, 2006; Marques \& Lima, 2011; $\mathrm{Xu}$ et al., 2016). In this thesis, environmental issues including noise, air pollution, and rubbish/ litter are highlighted as some of the key environmental issues that may affect neighbourhood satisfaction. In this respect, participants were asked about the issues that face them in the neighbourhood in which they live specifically in terms of noise, air pollution and rubbish in the streets. Table 6.13 presents frequencies distribution of those issues. It can be noted from this Table that there is a general tendency in seeing those issues as not a problem, where $67.5 \%, 76.6 \%$ and $61.1 \%$ of the participants, respectively, reported that they do not suffer from those issues. Despite these high percentages of reporting a negative response with respect to those issues, the positive response can be still observed as relatively high, where $32.5 \%, 23.4 \%$ and $38.9 \%$ of the respondents, respectively, stated that they are suffering from those issues. It can be said, therefore, that these issues still exist as areas of concern, and they might affect neighbourhood satisfaction.

Table 6.10: Frequency measurement of suffering from environmental issues

\begin{tabular}{cccccc}
\hline \multirow{2}{*}{ Environmental issues } & \multicolumn{5}{c}{ Frequencies distribution } \\
\cline { 2 - 6 } & Yes & Frequency & No & Frequency & \multirow{2}{*}{ Total } \\
\hline Noise & $32.5 \%$ & 178 & $67.5 \%$ & 370 & 548 \\
\hline Air pollution & $23.4 \%$ & 128 & $76.6 \%$ & 420 & 548 \\
\hline Rubbish in the street & $38.9 \%$ & 213 & $61.1 \%$ & 335 & 548 \\
\hline
\end{tabular}


In order to gain insight into the impact of these environmental issues on neighbourhood satisfaction, a cross-tabulation was applied for this purpose. Results revealed a significant association between the three measures and satisfaction with the neighbourhood. It can be seen from Table 6.14 that noise, air pollution and rubbish in the street were mostly linked to dissatisfaction with the neighbourhood, where $44.9 \%, 50.7 \%$ and $45.6 \%$ of the respondents who reported that they are suffering from those issues, respectively, stated that they are dissatisfied or very dissatisfied with neighbourhood in which they live. Figure 6.5 clearly illustrates this relationship, where it is obvious that satisfaction with neighbourhood is low when reporting a positive response of suffering from these environmental issues. More importantly, air pollution in particular was found to be the most influential issue on neighbourhood satisfaction for those who live in Quba. One possible interpretation regarding this may be due to the increase in car ownership in Medina, where statistics indicate that the average car ownership is 0.32 car per individual (Urban Observatory of Medina, 2016). This high rate in car ownership may itself be due to a lack of public transportation in the study area. Consequently, this may lead to more air pollution and, in turn, negatively affect satisfaction with neighbourhood, and thus overall QoUL. In this context, numerous studies have pointed that air pollution negatively and strongly affects health and a sense of personal well-being as well as causing anxiety and depression (e.g., Marques \& Lima, 2011; Xu et al., 2016; and Darcin, 2017). This indicates the important role of this factor in affecting the QoUL which needs to be addressed. 
Table 6.11: The relationship between satisfaction with neighbourhood and environmental issues. Cross-tabulation.

\begin{tabular}{|c|c|c|c|c|c|c|c|c|}
\hline & & & \multicolumn{5}{|c|}{ Satisfaction with neighbourhood } & \multirow[b]{2}{*}{ Total } \\
\hline & & & $\begin{array}{c}\text { Very } \\
\text { dissatisfied }\end{array}$ & Dissatisfied & Moderate & Satisfied & $\begin{array}{c}\text { Very } \\
\text { satisfied }\end{array}$ & \\
\hline \multirow{4}{*}{ Noise } & \multirow{2}{*}{ No } & Count & 29 & 38 & 125 & 120 & 58 & 370 \\
\hline & & $\%$ & $7.8 \%$ & $10.3 \%$ & $33.8 \%$ & $32.4 \%$ & $15.7 \%$ & $100.0 \%$ \\
\hline & \multirow{2}{*}{ Yes } & Count & 39 & 41 & 60 & 26 & 12 & 178 \\
\hline & & $\%$ & $21.9 \%$ & $23.0 \%$ & $33.7 \%$ & $14.6 \%$ & $6.7 \%$ & $100.0 \%$ \\
\hline \multirow{2}{*}{ Total } & & Count & 68 & 79 & 185 & 146 & 70 & 548 \\
\hline & & $\%$ & $12.4 \%$ & $14.4 \%$ & $33.8 \%$ & $26.6 \%$ & $12.8 \%$ & $100.0 \%$ \\
\hline \multicolumn{9}{|c|}{ Chi-square $=54.60, \mathrm{p}$-value $<.001$} \\
\hline \multirow{4}{*}{$\begin{array}{l}\text { Air } \\
\text { pollution }\end{array}$} & \multirow{2}{*}{ No } & Count & 33 & 49 & 141 & 133 & 64 & 420 \\
\hline & & $\%$ & $7.9 \%$ & $11.7 \%$ & $33.6 \%$ & $31.7 \%$ & $15.2 \%$ & $100.0 \%$ \\
\hline & \multirow{2}{*}{ Yes } & Count & 35 & 30 & 44 & 13 & 6 & 128 \\
\hline & & $\%$ & $27.3 \%$ & $23.4 \%$ & $34.4 \%$ & $10.2 \%$ & $4.7 \%$ & $100.0 \%$ \\
\hline \multirow{2}{*}{ Total } & & Count & 68 & 79 & 185 & 146 & 70 & 548 \\
\hline & & $\%$ & $12.4 \%$ & $14.4 \%$ & $33.8 \%$ & $26.6 \%$ & $12.8 \%$ & $100.0 \%$ \\
\hline \multicolumn{9}{|c|}{ Chi-square $=56.05, \mathrm{p}$-value $<.001$} \\
\hline \multirow{4}{*}{$\begin{array}{l}\text { Rubbish in } \\
\text { the street }\end{array}$} & \multirow{2}{*}{ No } & Count & 18 & 32 & 106 & 121 & 58 & 335 \\
\hline & & $\%$ & $5.4 \%$ & $9.6 \%$ & $31.6 \%$ & $36.1 \%$ & $17.3 \%$ & $100.0 \%$ \\
\hline & \multirow{2}{*}{ Yes } & Count & 50 & 47 & 79 & 25 & 12 & 213 \\
\hline & & $\%$ & $23.5 \%$ & $22.1 \%$ & $37.1 \%$ & $11.7 \%$ & $5.6 \%$ & $100.0 \%$ \\
\hline \multirow{2}{*}{ Total } & & Count & 68 & 79 & 185 & 146 & 70 & 548 \\
\hline & & $\%$ & $12.4 \%$ & $14.4 \%$ & $33.8 \%$ & $26.6 \%$ & $12.8 \%$ & $100.0 \%$ \\
\hline
\end{tabular}

Chi-square $=92.63, \mathrm{p}$-value $<.001$ 

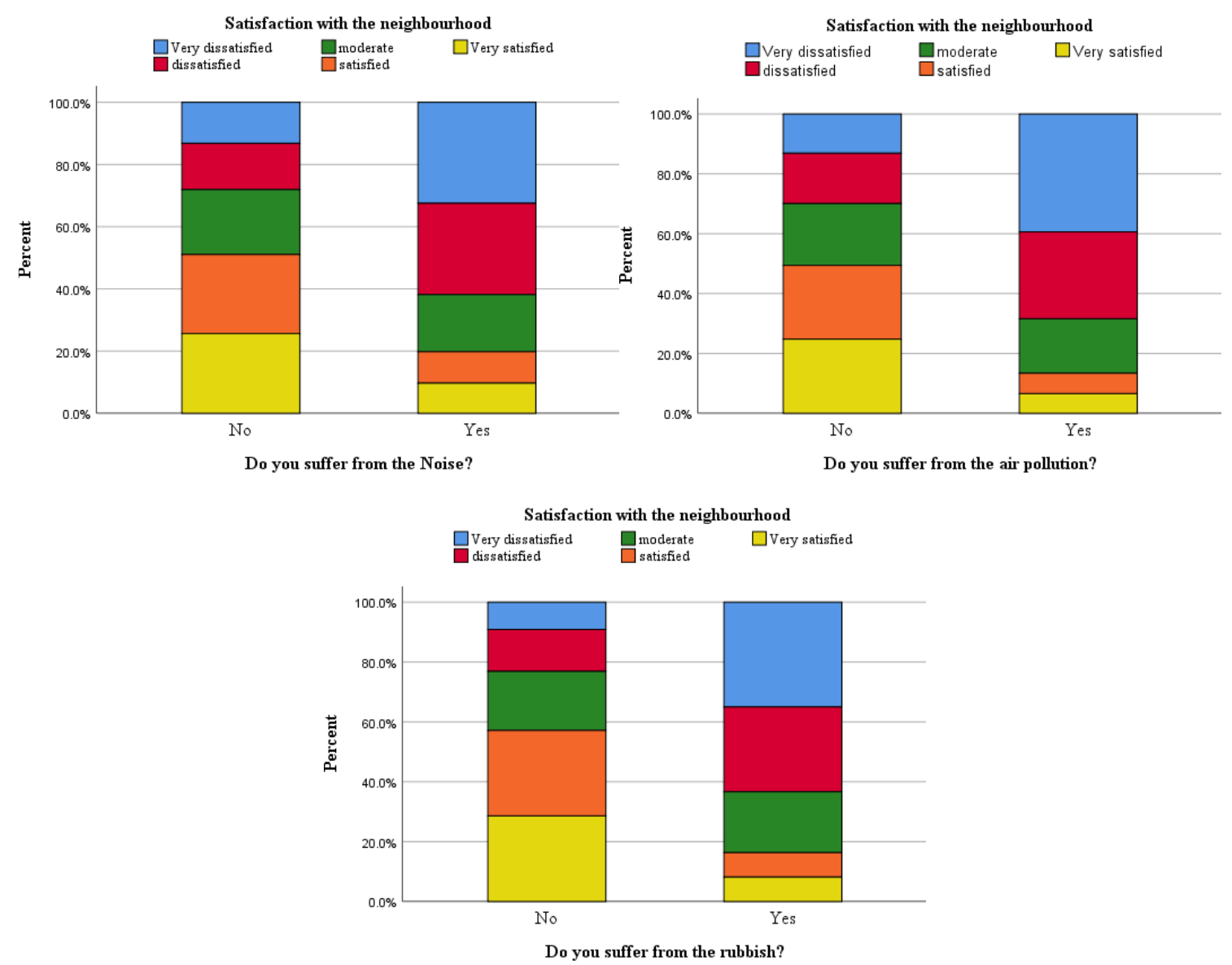

Fig 6.5: Relationship between environmental components and satisfaction with the neighbourhood

\subsection{Quality of the neighbourhood's infrastructure}

For the quality of basic infrastructure, including streets, pavements, lighting and so forth, and its influence on neighbourhood satisfaction, participants were asked to report the extent of their agreement that the neighbourhood in which they live has a good infrastructure. Using Spearman's correlation test, results have indicated a moderate strong correlation between neighbourhood satisfaction and the agreement degree that the neighbourhoods have a good infrastructure in terms of the above-mentioned aspects. Table 6.15 presents this type of relationship. 
Table 6.12: Correlation between neighbourhood satisfaction and the agreement degree that the neighbourhoods have a good infrastructure. Spearman's correlation test.

\begin{tabular}{lllr|r}
\hline & & & \multicolumn{1}{c}{$\begin{array}{c}\text { Satisfaction with the } \\
\text { neighbourhood }\end{array}$} \\
\hline Spearman's rho & $\begin{array}{l}\text { Agreement degree that } \\
\text { neighbourhoods have a good } \\
\text { basic infrastructure such as } \\
\text { streets, pavements, lighting ...etc. }\end{array}$ & $\begin{array}{l}\text { Correlation } \\
\text { Coefficient }\end{array}$ & 1.000 & $.590^{* *}$ \\
\cline { 3 - 5 } & Sig. (2-tailed) & .000 \\
\hline
\end{tabular}

**. Correlation is significant at the 0.01 level (2-tailed).

For further exploration, an additional analysis regarding the relationship between neighbourhood satisfaction and the degree of agreement that the neighbourhoods have a good infrastructure was conducted using cross-tabulation. Results revealed a significant association between neighbourhood satisfaction and the extent to which respondents agreed that it has a good infrastructure. It can be seen from Table 6.16 that $80.1 \%$ of the respondents who agreed that the neighbourhood they live in has a good infrastructure stated that they are satisfied with their neighbourhood. On the other hand, $50.0 \%$ of the respondents who disagreed stated that they are dissatisfied. These two ratios seem fairly close which indicates that satisfaction with the neighbourhood increases with the increase of the resident's positive perception regarding the infrastructure of the neighbourhoods and vice versa. This may be due to the variation in the condition of the infrastructures across the neighbourhoods. Despite that, an apparent association between having a good infrastructure and neighbourhood satisfaction can be still observed, which is clearly illustrated in Figure 6.6, where the satisfaction with the neighbourhood sharply increases with the increase of the degree of agreement with having a good infrastructure. 
Table 6.13: Relationship between neighbourhood satisfaction and the agreement degree that the neighbourhood has a good basic infrastructure. Cross-tabulation.

\begin{tabular}{|c|c|c|c|c|c|c|}
\hline & & & \multicolumn{3}{|c|}{ Satisfaction with neighbourhood } & \multirow[b]{2}{*}{ Total } \\
\hline & & & Dissatisfied & Moderate & Satisfied & \\
\hline \multirow{6}{*}{$\begin{array}{c}\text { Agreement degree } \\
\text { neighbourhoods have a } \\
\text { good basic infrastructure } \\
\text { such as streets, pavements, } \\
\text { lighting ...etc. }\end{array}$} & \multirow{2}{*}{ Disagree } & Count & 118 & 79 & 39 & 236 \\
\hline & & $\%$ & $50.0 \%$ & $33.5 \%$ & $16.5 \%$ & $100.0 \%$ \\
\hline & \multirow{2}{*}{ Neutral } & Count & 21 & 84 & 56 & 161 \\
\hline & & $\%$ & $13.0 \%$ & $52.2 \%$ & $34.8 \%$ & $100.0 \%$ \\
\hline & \multirow{2}{*}{ Agree } & Count & 8 & 22 & 121 & $\overline{151}$ \\
\hline & & $\%$ & $5.3 \%$ & $14.6 \%$ & $80.1 \%$ & $100.0 \%$ \\
\hline \multirow{2}{*}{ Total } & & Count & 147 & 185 & 216 & 548 \\
\hline & & $\%$ & $26.8 \%$ & $33.8 \%$ & $39.4 \%$ & $100.0 \%$ \\
\hline
\end{tabular}

Chi-square $=213.145, \mathrm{p}$-value $<.001$

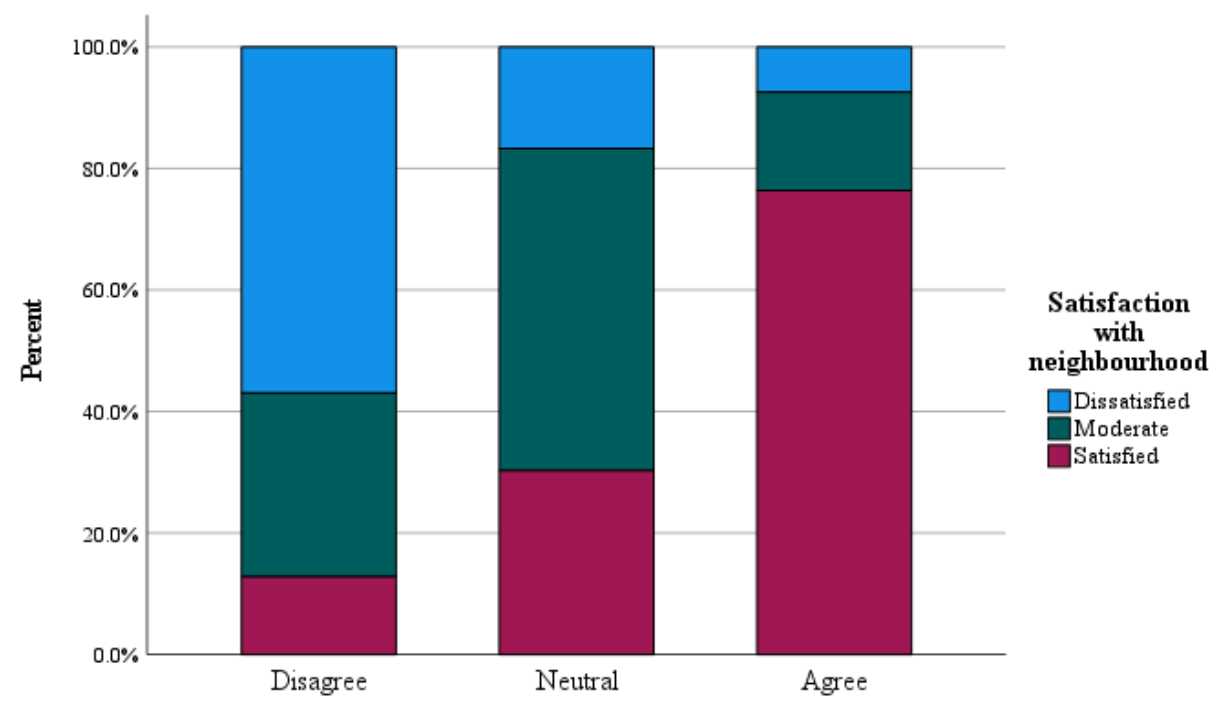

Agreement degree neighbourhoods have a good basic infrastructure

Fig 6.6: Relationship between neighbourhood satisfaction and the agreement degree that the neighbourhood has a good basic infrastructure

The degree of agreement about having a good infrastructure, however, may vary from person to person depending on the needs and aspirations of particular residents. For example, some may be content with what is available in terms of the infrastructure in the area in which they live, such as the presence of a park or children's playground, while others may see the necessity for the presence of other physical components. Therefore, evaluation of a good or bad infrastructure depends on how people perceive it, as with the evaluation of the overall QoUL, where people assess their QoUL based on their own 
perception. In addition, some socio-demographics were found to influence the relationship between having a good infrastructure and satisfaction with the neighbourhood. In this respect, only employment was found to be significantly associated with the agreement degree that there is a good infrastructure. With regard to the influence of employment, the self-employed seem to be most dissatisfied with the infrastructure, where $61.6 \%$ of them disagree or strongly disagree that there is a good infrastructure. However, this group represents a small percentage of the total number of the sample. Yet, within the much larger group of employees, a relatively high ratio of negative response was also found, where $48.5 \%$ expressed disagreement. For other groups, no clear picture can be seen with regard the perception of other groups.

\subsection{Aesthetics and attractiveness of the neighbourhood}

The aesthetics and attractiveness of the residential context were highlighted as one of the key elements of the neighbourhood that might influence satisfaction with it. In this respect, the participants were asked to report the extent of their agreement that the neighbourhood in which they live is an attractive and well-organised place to live. Cross-tabulation revealed similar results that were obtained from cross-tabulating neighbourhood satisfaction with the agreement degree that the neighbourhood has a good basic infrastructure as illustrated in Table 6.16. However, the percentage of those reporting being dissatisfied with the neighbourhood and the agreement degree that the neighbourhood is an attractive and well-organised place to live is a bit higher, where $54.2 \%$ among those who reported disagreeing that neighbourhood is an attractive and well-organised place to live stated that they are dissatisfied or very dissatisfied with their neighbourhoods. On the other hand, 71.6\% among those who agreed that the neighbourhood they live in is an attractive and well-organised place to live reported a higher level of satisfaction with their neighbourhoods (see Table 6.17). From these results, it can be said that a greater degree of agreement that the neighbourhood is an attractive and well-organised place to live, the higher level of satisfaction with neighbourhoods and vice versa, which is clearly illustrated in Figure 6.7, and which indicates the influence of the appearance of the neighbourhood on how the residents regard the residential context. These findings are reflected in several studies in the literature, including the work of Lee et al. (2016), Parkes et al. (2002), Sirgy 
and Cornwell (2002), Hur and Morrow-Jones (2008) and Gruber and Shelton (1987), which have emphasised the influence of the appearance and attractiveness of the neighbourhood on people's satisfaction with the residential context in which they live. That is, those who perceive that the appearance of their neighbourhood is bad are likely to report dissatisfaction with it, and vice versa.

Table 6.14: Relationship between neighbourhood satisfaction and the agreement degree that the neighbourhood is an attractive and well-organised place to live. Cross-tabulation.

\begin{tabular}{|c|c|c|c|c|c|c|}
\hline & & & \multicolumn{4}{|c|}{ Satisfaction with neighbourhood } \\
\hline & & & Dissatisfied & Moderate & Satisfied & Total \\
\hline \multirow{6}{*}{$\begin{array}{l}\text { Agreement degree that } \\
\text { neighbourhood is an } \\
\text { attractive and well- } \\
\text { organised place to live }\end{array}$} & \multirow{2}{*}{ Disagree } & Count & 96 & 64 & 17 & 177 \\
\hline & & $\%$ & $54.2 \%$ & $36.2 \%$ & $9.6 \%$ & $100.0 \%$ \\
\hline & \multirow{2}{*}{ Neutral } & Count & 32 & 80 & 48 & 160 \\
\hline & & $\%$ & $20.0 \%$ & $50.0 \%$ & $30.0 \%$ & $100.0 \%$ \\
\hline & \multirow{2}{*}{ Agree } & Count & 19 & 41 & 151 & 211 \\
\hline & & $\%$ & $9.0 \%$ & $19.4 \%$ & $71.6 \%$ & $100.0 \%$ \\
\hline \multirow{2}{*}{ Total } & & Count & 147 & 185 & 216 & 548 \\
\hline & & $\%$ & $26.8 \%$ & $33.8 \%$ & $39.4 \%$ & $100.0 \%$ \\
\hline
\end{tabular}

Chi-square $=201.805, \mathrm{p}$-value $<.001$

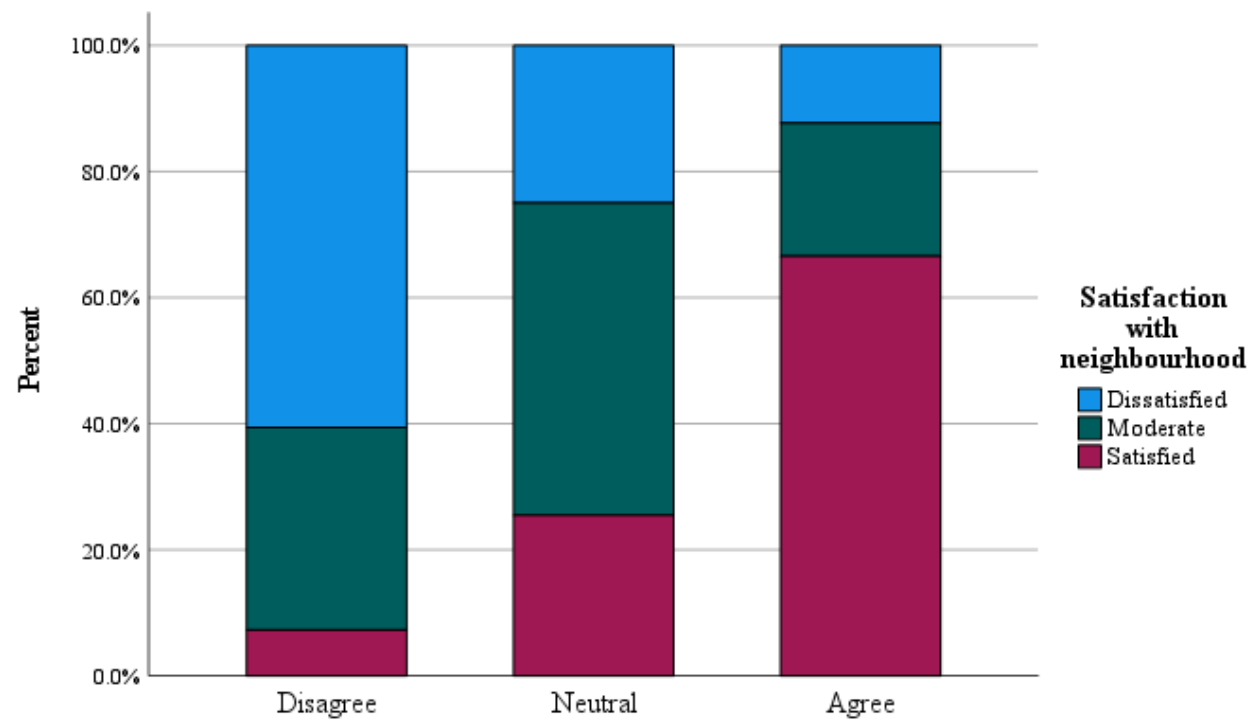

Agreement degree that neighbourhood is an attractive and wellorganised place to live

Fig 6.7: Relationship between neighbourhood satisfaction and the agreement degree that the neighbourhood is an attractive and well-organised place to live 


\subsection{Accessibility and connectivity}

Accessibility and connectivity have been often addressed as two of the transportation indicators. In this thesis, however, transportation is not included in the indicators that have been adopted for the investigation of the QoUL. This is due to the lack of an integrated system for public transport in the study area that could be evaluated within the time of the fieldwork of this thesis. Therefore, accessibility and connectivity in this thesis refer to the ease of access and connectivity in terms of commuting between the neighbourhood and public facilities, namely work and the mosque, car parking and basic shopping centres. ${ }^{4}$ In addition, other indicators such as walkability, ease of access to the main road and traffic jams are highlighted. These aspects of accessibility and connectivity are likely to affect travel time, which in turn affects neighbourhood satisfaction and overall QoUL. In regard to this, Yuan et al. (2009) noted that increased travel time or a long commute may negatively affect personal life, including health, spending less time with families, as well as affecting worker productivity that all result from lost time in transit. The accessibility of the public facilities such as mosques, car parking, shopping centres, and pedestrian walkways are all considered to be factors that predict residential satisfaction (Mohit et al., 2010).

As stated above, for the purposes of this thesis, accessibility and connectivity indicators including distance to the work, distance to the mosque, lack of car parking and lack of basic shopping centres were considered as issues that might affect residents within their neighbourhoods. Hence, respondents were asked whether they suffer from those issues in the neighbourhood in which they live. Table 6.18 presents frequency distribution responses for the four measures. Generally, a high percentage of respondents report not suffering from these accessibility and connectivity issues. Within those frequencies, a long distance to the mosque recorded the highest percentage of not being an issue, where $90.3 \%$ of the respondents stated that this is not a difficulty, indicating the ease of access to this public facility. In contrast, lack of car parking recorded a relatively high percentage of being an issue, where $37.6 \%$ of the respondents stated that they suffer from the lack of car parking.

\footnotetext{
${ }^{4}$ In this thesis, basic shopping centres refer to the local supermarkets that cater for a household's basic needs, such as food and other basic goods.
} 
Such findings reflect the prevailing culture of car ownership in Quba communities, and perhaps in the Saudi society in general.

Table 6.15: Frequency measurement of accessibility and connectivity issues

\begin{tabular}{cccccc}
\hline \multirow{2}{*}{ Accessibility and connectivity issues } & \multicolumn{5}{c}{ Frequencies distribution } \\
\cline { 2 - 6 } & Yes & Frequency & No & Frequency & Total \\
\hline Long distance to mosque & $9.7 \%$ & 53 & $90.3 \%$ & 495 & 548 \\
\hline Long distance to work & $23.4 \%$ & 128 & $76.6 \%$ & 420 & 548 \\
\hline Lack of car parking & $37.6 \%$ & 206 & $62.4 \%$ & 342 & 548 \\
\hline Lack of basic shopping centres & $18.4 \%$ & 101 & $81.6 \%$ & 447 & 548 \\
\hline
\end{tabular}

The relationship between the accessibility and connectivity indicators and satisfaction with neighbourhood was examined using cross-tabulation in order to gain more insight into the impact of those indicators on the perception of Quba's residents regarding their neighbourhood. Results in Table 6.19 indicate that all measures, except for long distance to work, are significantly associated with the satisfaction with the neighbourhood. Figure 6.8 shows the relationship between satisfaction with the neighbourhood and the four measures, where higher satisfaction with neighbourhood is associated with reporting in terms of the lack of car parking issues and vice versa. In this context, it is logical to presume that the convenient location of these local facilities affects the residents' satisfaction with the neighbourhood (Djebarni \& Al-Abed, 2000). 
Table 6.16: The relationship between satisfaction with neighbourhood and accessibility and connectivity issues. Cross-tabulation.

\begin{tabular}{|c|c|c|c|c|c|c|c|c|}
\hline & & & \multicolumn{5}{|c|}{ Satisfaction with neighbourhood } & \multirow[b]{2}{*}{ Total } \\
\hline & & & $\begin{array}{c}\text { Very } \\
\text { dissatisfied }\end{array}$ & Dissatisfied & Moderate & Satisfied & $\begin{array}{c}\text { Very } \\
\text { satisfied }\end{array}$ & \\
\hline \multirow{4}{*}{$\begin{array}{l}\text { Long } \\
\text { distance to } \\
\text { mosque }\end{array}$} & \multirow{2}{*}{ No } & Count & 54 & 70 & 167 & 139 & 65 & 495 \\
\hline & & $\%$ & $10.9 \%$ & $14.1 \%$ & $33.7 \%$ & $28.1 \%$ & $13.1 \%$ & $100.0 \%$ \\
\hline & \multirow{2}{*}{ Yes } & Count & 14 & 9 & 18 & 7 & 5 & 53 \\
\hline & & $\%$ & $26.4 \%$ & $17.0 \%$ & $34.0 \%$ & $13.2 \%$ & $9.4 \%$ & $100.0 \%$ \\
\hline \multirow{2}{*}{ Total } & & Count & 68 & 79 & 185 & 146 & 70 & 548 \\
\hline & & $\%$ & $12.4 \%$ & $14.4 \%$ & $33.8 \%$ & $26.6 \%$ & $12.8 \%$ & $100.0 \%$ \\
\hline \multicolumn{9}{|c|}{ Chi-square $=14.03, \mathrm{p}$-value $=.007$} \\
\hline \multirow{4}{*}{$\begin{array}{l}\text { Long } \\
\text { distance to } \\
\text { work }\end{array}$} & \multirow{2}{*}{ No } & Count & 44 & 60 & 144 & 114 & 58 & 420 \\
\hline & & $\%$ & $10.5 \%$ & $14.3 \%$ & $34.3 \%$ & $27.1 \%$ & $13.8 \%$ & $100.0 \%$ \\
\hline & \multirow{2}{*}{ Yes } & Count & 24 & 19 & 41 & 32 & 12 & 128 \\
\hline & & $\%$ & $18.8 \%$ & $14.8 \%$ & $32.0 \%$ & $25.0 \%$ & $9.4 \%$ & $100.0 \%$ \\
\hline \multirow{2}{*}{\multicolumn{2}{|c|}{ Total }} & Count & 68 & 79 & 185 & 146 & 70 & 548 \\
\hline & & $\%$ & $12.4 \%$ & $14.4 \%$ & $33.8 \%$ & $26.6 \%$ & $12.8 \%$ & $100.0 \%$ \\
\hline \multicolumn{9}{|c|}{ Chi-square $=7.26, p$-value $=.123$} \\
\hline \multirow{4}{*}{$\begin{array}{l}\text { Lack of car } \\
\text { parking }\end{array}$} & \multirow{2}{*}{ No } & Count & 33 & 39 & 107 & 108 & 55 & 342 \\
\hline & & $\%$ & $9.6 \%$ & $11.4 \%$ & $31.3 \%$ & $31.6 \%$ & $16.1 \%$ & $100.0 \%$ \\
\hline & \multirow{2}{*}{ Yes } & Count & 35 & 40 & 78 & 38 & 15 & 206 \\
\hline & & $\%$ & $17.0 \%$ & $19.4 \%$ & $37.9 \%$ & $18.4 \%$ & $7.3 \%$ & $100.0 \%$ \\
\hline \multirow{2}{*}{ Total } & & Count & 68 & 79 & 185 & 146 & 70 & 548 \\
\hline & & $\%$ & $12.4 \%$ & $14.4 \%$ & $33.8 \%$ & $26.6 \%$ & $12.8 \%$ & $100.0 \%$ \\
\hline \multicolumn{9}{|c|}{ Chi-square $=29.07, \mathrm{p}$-value $<.001$} \\
\hline \multirow{4}{*}{$\begin{array}{l}\text { Lack of } \\
\text { basic } \\
\text { shopping } \\
\text { centres }\end{array}$} & \multirow{2}{*}{ No } & Count & 37 & 60 & 152 & 135 & 63 & 447 \\
\hline & & $\%$ & $8.3 \%$ & $13.4 \%$ & $34.0 \%$ & $30.2 \%$ & $14.1 \%$ & $100.0 \%$ \\
\hline & \multirow{2}{*}{ Yes } & Count & 31 & 19 & 33 & 11 & 7 & 101 \\
\hline & & $\%$ & $30.7 \%$ & $18.8 \%$ & $32.7 \%$ & $10.9 \%$ & $6.9 \%$ & $100.0 \%$ \\
\hline \multirow{2}{*}{ Total } & & Count & 68 & 79 & 185 & 146 & 70 & 548 \\
\hline & & $\%$ & $12.4 \%$ & $14.4 \%$ & $33.8 \%$ & $26.6 \%$ & $12.8 \%$ & $100.0 \%$ \\
\hline
\end{tabular}

Chi-square $=49.90, \mathrm{p}$-value $<.001$ 

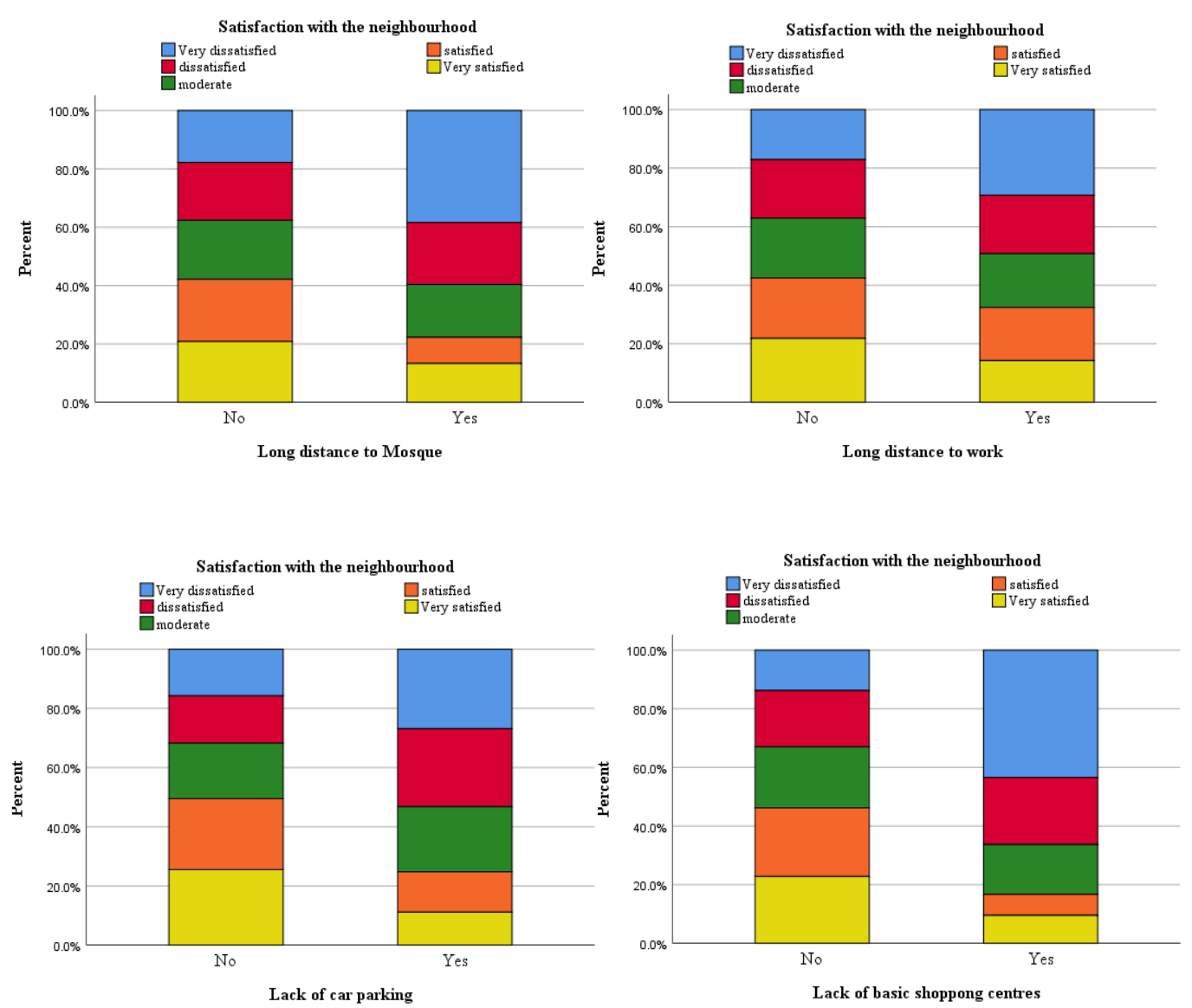

Fig 6.8: The relationship between satisfaction with the neighbourhood and accessibility and connectivity issues

As for distance to the mosque, $41.2 \%$ among those who do not consider distance to the mosque as a problem stated that they are satisfied or very satisfied with the neighbourhood compared to $22.6 \%$ of those who suffer from the long distance to the mosque. Proximity to this public facility may be one of the most important factors that predict satisfaction with the neighbourhood. This assumption is supported by the fact that many people in the study area go more frequently to the mosques, according to the findings obtained from Chapter 8. Thus, the distance between home and the mosque may be of interest to many of those who live in Quba's neighbourhoods. Referring to the qualitative findings, specifically the photo diaries and follow-up interviews, it has been found that proximity to the mosque is a key element in selecting a neighbourhood. In this respect, participant PH 7 pointed out that 'the main factor in choosing the neighbourhood is the presence of the mosque within walking distance from home' (Male, $37 \mathrm{yrs}$ ). Therefore, distance to the mosque may 
influence residents' satisfaction with neighbourhood. In the best knowledge of this thesis, no study has been found that supports these findings.

In terms of the lack of car parking, 36.4\% among those who suffer from this issue stated that they are dissatisfied or very dissatisfied with the neighbourhood compared to $21 \%$ of those who have the same satisfaction degree but report lack of car parking as not a problem. Although there is no significant gap between the percentages of reporting suffering from of lack of car parking and the degree of satisfaction with the neighbourhood, the relationship between these two variables can still be observed. In this context, Stubbs (2002) pointed out that residents often attribute a distinctive and positive value of parking provision to satisfaction with their neighbourhoods.

As for the lack of basic shopping centres, $49.5 \%$ among those who suffer from this issue stated that they are dissatisfied or very dissatisfied compared to $21.7 \%$ of those with the same satisfaction degree but who report not suffering from the lack basic shopping centres. From this result, it can be observed how important the basic shopping centres are in residents' perception regarding the neighbourhood in which they live, where reporting not suffering from the lack of this facility is associated with higher satisfaction with the neighbourhood. Since these shopping centres are frequently visited to buy basic goods that households need such as food and other essential items, the inaccessibility of those shopping centres may lead to longer travel time, which may lead to dissatisfaction with the neighbourhood. According to the report of the Quality of Life Program 2020, ${ }^{5}$ shopping and dining are the most popular activities in Saudi society, indicating the importance of the accessibility of shopping, at least for the majority of that society.

The relationship between satisfaction with the neighbourhood and other accessibility and connectivity indicators was examined for further exploration regarding the impact of these indicators on the perception of Quba's residents concerning their neighbourhood. Those included the ease of access to the main road, walkability (i.e., suitable sidewalks for the streets) and traffic jams. To do this, Spearman's rho correlation test was applied to assess the relationship between neighbourhood satisfaction and these variables. Results revealed

\footnotetext{
${ }^{5}$ Quality of Life Program 2020 is a delivery plane, one of the Saudi Vision 2030 realisation programmes.
} 
a positive significant correlation between these indicators and satisfaction with the neighbourhood (see Table 6.20). Although the correlations appeared to be weak, the impact of these indicators on the residents' satisfaction with their neighbourhood is still observable. For instance, it can be seen from Figure 6.9 that satisfaction with the neighbourhood rises with the rise of the agreement degree that it is easy to access to the main road as well as the accessibility of sidewalks. Similar results can be identified in the case of satisfaction with respect to traffic jams, where satisfaction with this variable contribute to higher satisfaction with the neighbourhood.

Table 6.17: Relationship between neighbourhood satisfaction and other accessibility and connectivity indicators. Spearman's correlation test.

\begin{tabular}{lccc}
\hline & $\begin{array}{c}\text { Access the main road in } \\
\text { the neighbourhood }\end{array}$ & $\begin{array}{c}\text { Suitable sidewalks to walk } \\
\text { along the streets }\end{array}$ & $\begin{array}{c}\text { Satisfaction concerning } \\
\text { traffic jams in the } \\
\text { municipality of Quba }\end{array}$ \\
\hline $\begin{array}{l}\text { Correlation } \\
\text { Coefficient }\end{array}$ & .352 & .248 & .323 \\
\hline Result & Significant & Significant & Significant \\
\hline
\end{tabular}
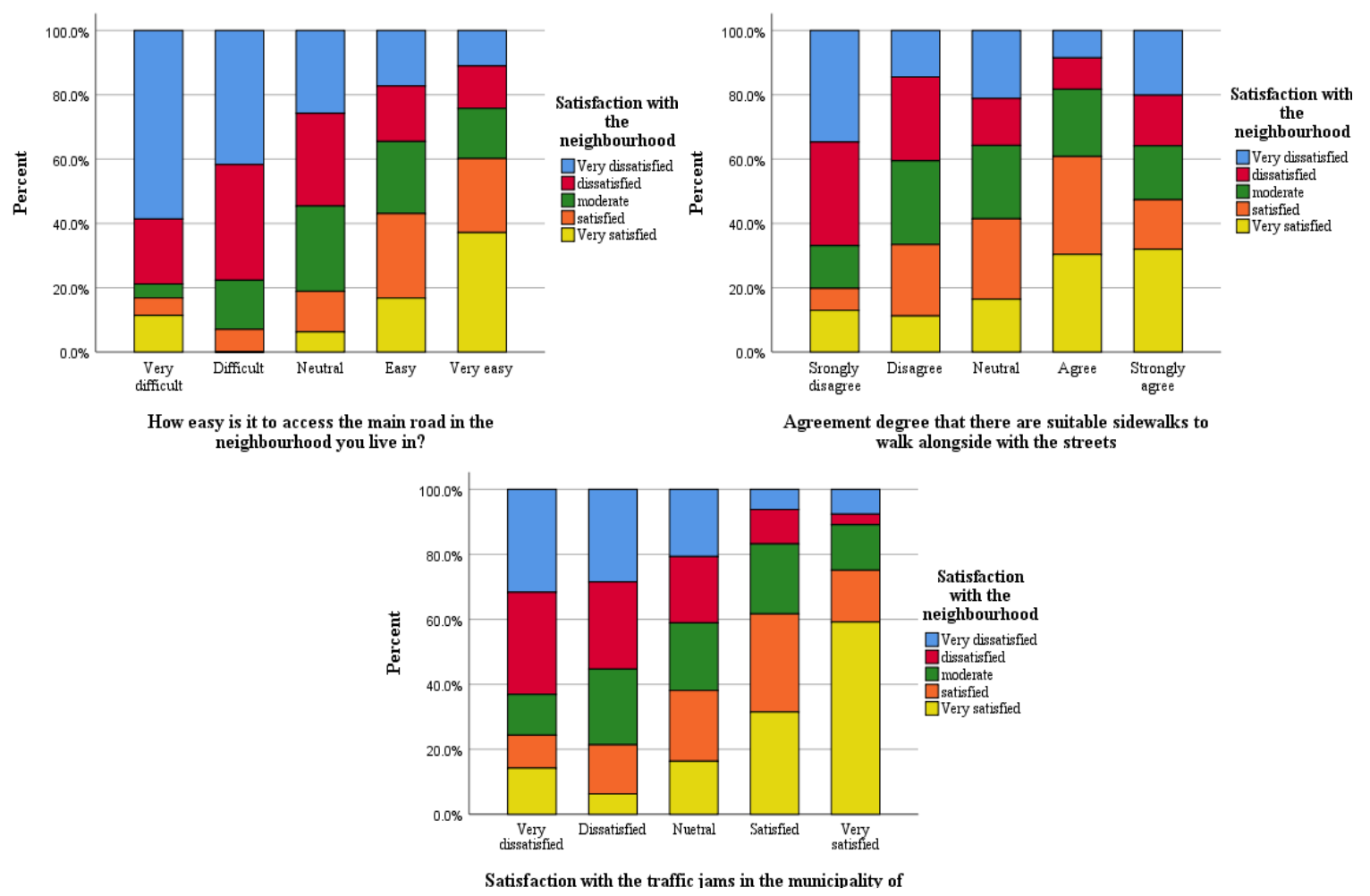

Fig 6.9: Relationship between neighbourhood satisfaction and accessibility to the main road, sidewalks and situation with traffic jams 
Although the relationships obtained from the results above are considered to be weak, it is still obvious that accessibility and connectivity indicators are significantly correlated with the satisfaction with Quba's neighbourhoods, indicating the important role of those indicators in the perception of Quba's residents of their neighbourhood. Easy access to the main road as an example, could lead to easy access and connection with public facilities within the city, such as health, education, and workplaces, leading to a shorter travel distance between home and those facilities. However, accessibility to these kinds of main roads may have negative consequences, including such issues as noise, air pollution, along with health considerations such as anxiety and traffic accidents (Hamersma et al., 2014; Kwan, 2019; Carrier et al., 2016; Michaud et al., 2008). Living near the main roads may depend on the expectations of the residents; what is positive for some may be negative for others. In the case of Quba, further research may be required for deeper exploration regarding the extent to which living near a main road affects residential quality.

Accessibility to sidewalks is also one of the more important aspects that might affect residents' satisfaction with the neighbourhood. Despite the fact that Quba is characterised by a car-dominant culture, there are groups within society, such as children and those who do not have car access, who need this kind of infrastructure. In this thesis, it has been found that this component is important for those living in Quba, where frequency distribution indicates that nearly $65 \%$ are interested in the sidewalks. In this context, walkable neighbourhoods can encourage residents to walk more, and perhaps also enhance physical activity and social connection, which in turn have positive effects on health and well-being, especially for the elderly, and in turn again on satisfaction with overall QoUL (Yu, et al., 2017). Findings from the qualitative research, namely the photo diaries, support that. One of the participants referred to the sidewalks as follows:

The walkway that we used to use is a very important element of our neighbourhood. It is safe for us to use it, particularly for children and elderly people to commute or just walk within our neighbourhood. It helps us to spend enjoyable times, which in turn promotes our health and happiness. (PH 6, Male, 30 yrs). 
Another participant also appreciated this physical element of the neighbourhood, commenting,

The presence of the sidewalks enhances our health as well as the recreation sides. In addition, the use of the walkways in the neighbourhood enhances the social connectedness of the neighbourhood members. Those features provided by sidewalks ultimately promote our happiness and overall satisfaction with life. (PH 1, Male, 43 yrs)

From the above quotations it is clear how important the sidewalks are in contributing to the satisfaction with the neighbourhood and in turn the QoUL. In this way, such physical attributes may enhance social aspects, like the recreational, as well as social interaction, which in turn may enhance social integration among members of the neighbourhood. More importantly, such physical attributes may encourage walking and sports, which is likely to contribute to improving health as a key aspect that enhances the overall QoUL. Moreover, the photos taken by the participants showed in Figure 6.10 may indicate another dimension, namely that the physical appearance of the urban environment, including green spaces and infrastructure design, may play a role in satisfaction with the neighbourhood, and in turn overall QoUL.
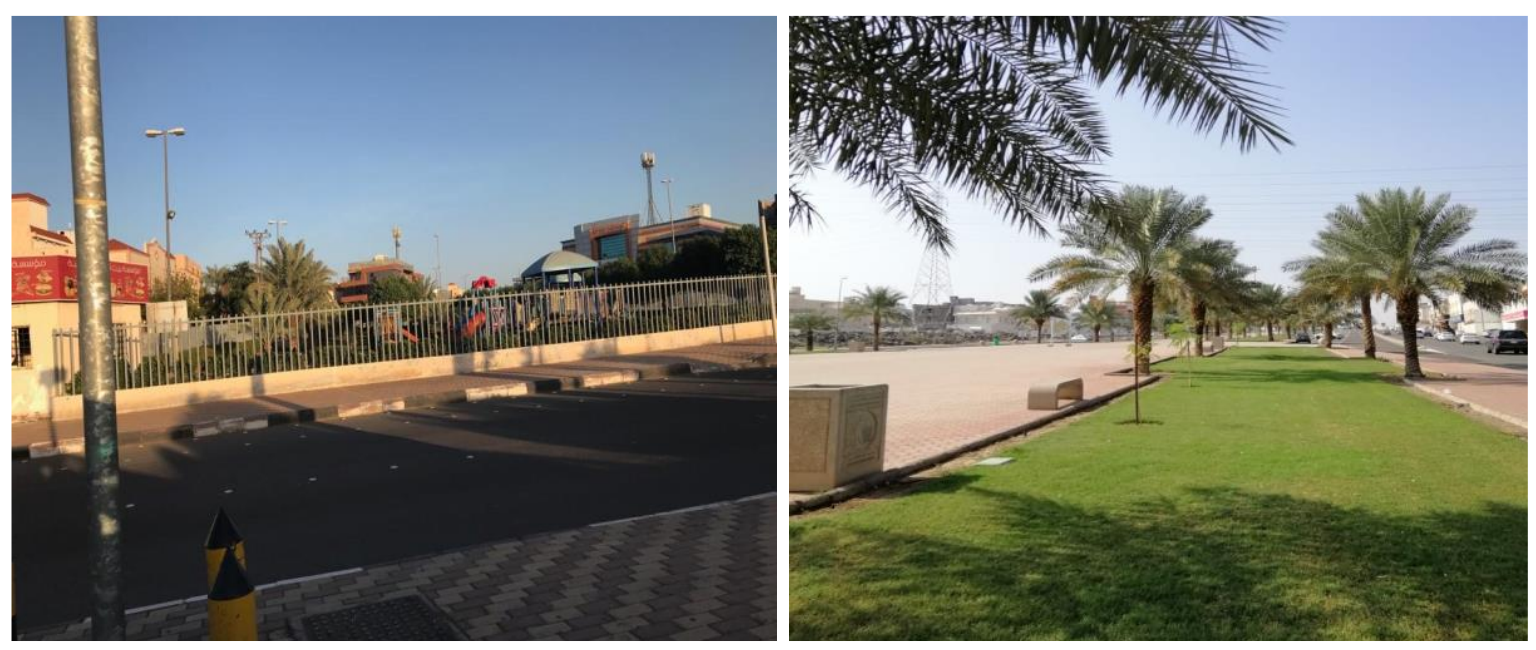

Fig 6.10: Sidewalk within Quba's neighbourhoods taken by participants PH 6 and PH 1 respectively 


\subsection{Summary of the chapter}

This chapter looked at the quality of the neighbourhood and the impact it has on the QoUL of Quba's residents. Results of measuring satisfaction level with the neighbourhood showed that residents where less satisfied than they were in the case of housing, where results indicated a moderate level of satisfaction with the neighbourhoods in which they live. This may be because the neighbourhood contains multiple aspects of the built environment, including the physical, social as well as economic aspects, which may have a wider impact than the dwelling. Even though the satisfaction level differed between housing and neighbourhood, in both cases satisfaction with them was found to contribute to a higher QoUL for those living in Quba. As in the case of relationship between housing satisfaction and QoUL, a higher satisfaction level with the neighbourhood was associated with a higher level of QoUL. In this context, the importance of the neighbourhood for the QoUL lies in the fact that it is most likely the primary urban environment unit in which social life occurs, which in turn affects the QoUL for those who inhabit it.

Satisfaction with the neighbourhood, however, is affected by socio-demographic attributes. In this respect, only residential status and income were found to be statistically associated with neighbourhood satisfaction. Residential status represented by home ownership was associated with the highest level of satisfaction. This has been justified by the assumption that home ownership may be related to preference for the neighbourhood, which ultimately contributes to higher satisfaction. Likewise, the high-income level may contribute to giving the residents an opportunity to choose the preferred neighbourhood, which ultimately contributes to their satisfaction with that neighbourhood.

Other factors that contribute to satisfaction with the neighbourhood are those components that distinguish it. In this respect, housing was found to be the most important physical component where the highest satisfaction ratio was associated with higher satisfaction with the accommodation. This is because feelings of satisfaction towards accommodation will spill over to the feelings towards the neighbourhood. Again, home ownership plays a key part, where that particular residential status causes residents to be more attached to their neighbourhood and thus more satisfied with it. Other components that affect neighbourhood satisfaction are those represented by basic services including health, education and recreation facilities including children's playgrounds and green spaces. To assess the 
influence of those components, participants were asked whether they were suffering from a lack of these components as an indication of their satisfaction with neighbourhood in which they live. Results indicated that the majority of the participants considered that the lack of health and education facilities was not a problem for them. In contrast, however, the majority of the participants reported that they are suffering from the lack of green spaces and children's playgrounds. This in turn was turn reflected on the satisfaction with neighbourhood.

Social components including security, social interactions, support from neighbours, satisfaction with neighbours and sense of spirituality are weakly, but significantly associated with neighbourhood satisfaction. Despite that, the relationship between neighbourhood satisfaction and social components is still obvious. It has been found that the relationship between these variables is a sequential prosses, where social components predict neighbourhood satisfaction, and neighbourhood satisfaction predicts overall satisfaction with life. Economic components represented by the job opportunities variable was found to contribute to neighbourhood satisfaction. However, the influence of this component was varied. That is, dissatisfaction with this economic component was associated with a lower level of satisfaction with the neighbourhood, but at the same time also associated with a higher level of satisfaction with the neighbourhood. Such findings seem to be something of an anomaly, and further research may be required. Environmental issues as other components were found to predict satisfaction with the neighbourhood. The majority of the participants reported that those issues were not a problem that they are suffering from, which obviously reflected on their satisfaction with the neighbourhood they lived in. However, those issues still exist and need to be addressed, as they are potentially harmful to health, which in turn affects the overall QoUL.

Basic infrastructure as well as the aesthetics and attractiveness of the residential context were found to contribute to neighbourhood satisfaction, where higher satisfaction with those components were associated with higher satisfaction with the neighbourhood and vice versa. However, the relationship between satisfaction with the neighbourhood and the extent of agreement on which infrastructure is good or bad may be affected by the scope of the needs and aspirations of the residents. In addition, the perception of the neighbourhood's infrastructure is also impacted by the socio-demographic attributes. 
The issues of accessibility and connectivity and their contribution to the neighbourhood were highlighted. Accessibility to the public facilitates including work and the mosque, car parking and basic shopping centres were considered as issues that affect neighbourhood satisfaction. As with the case of other neighbourhood components, participants were asked whether they considered these components a problem. Results indicated that the majority of the participants reported that these issues were not a difficulty. Nevertheless, lack of car parking recorded a relatively high percentage of being an issue. Other issues including accessibility to the main road in the neighbourhood, accessibility to the sidewalks and satisfaction concerning traffic jams were highlighted for further exploration regarding accessibility and connectivity. The key relation between accessibility and connectivity and neighbourhood satisfaction is that the elements discussed above are related to wider concerns of health and social life as well as travel time that may affect other aspects of life. In this regard, the community culture may need to be considered as a key element in the way issues of accessibility and connectivity relate to neighbourhood satisfaction. As it was found in this thesis, the car-dominated culture in Quba has caused a number of problems such as pollution and lack of car parking, which are likely to have an impact on the satisfaction with the neighbourhood and in turn, the overall QoUL.

In light of what has been presented in this chapter, it can be argued that the neighbourhood is considered as a unique urban unit, since it combines several dimensions, which indicates its importance in determining QoUL. In this context, satisfaction with a neighbourhood as emphasised by most of the studies in the literature contributes to the QoUL. However, the multiplicity of dimensions that characterise the neighbourhood may reflect a variation in satisfaction with the neighbourhood. That is, residents may be satisfied with specific components of the neighbourhood, but at the same time, they may be dissatisfied with others, as has been illustrated in this thesis. For example, it was found that the residents were satisfied with the accessibility to some physical attributes such as schools and health facilities, and at the same time they were found to be dissatisfied with some components of accessibility such as car parking. This would also be reflective of the way perceptions of one place are affected by the demographic characteristics of those who inhabit it.

Another issue regarding satisfaction with the neighbourhood is the weight given to the various factors affecting satisfaction with the neighbourhood. For example, the high cost of living in the neighbourhood may be a cause of dissatisfaction, but the relationship with 
others or the sense of place is more important in predicting satisfaction with the neighbourhood. That is, the impact of social factors may dominate the effect of economic factors, and therefore, the high cost of living may not matter as much as these social considerations. Another issue that has been highlighted in this chapter is that meanings of neighbourhood may vary according to the perception of its residents. For some, a neighbourhood might be meaningful, while for others, it is just a place where a home is located. In that sense, neighbourhood may be affected by circumstances beyond its surroundings, the most important of which are those related to social factors. For example, social ties may lead to the emergence of broader communities, which in turn cause the neighbourhood to be a community without propinquity, and hence, less important in determining the QoUL.

As a final outcome of this chapter, it can be said that QoUL is indirectly influenced by neighbourhood satisfaction in general, as well as by the satisfaction with specific components of that neighbourhood including the physical, social, economic and environmental. As illustrated in the literature review, the combination of these neighbourhood components is crucial in shaping QoUL through satisfaction with neighbourhood. In this context, the functionalist approach to this thesis emphasises the integration of those components as a whole in predicting neighbourhood satisfaction which ultimately contributes to a QoUL. Therefore, understanding what results from that interaction of residents in a neighbourhood with those components is considered the fundamental way in which the overall QoUL is understood.

Having discussed the QoUL at the neighbourhood scale here, Chapter 7 continues using the functionalist approach to examine the QoUL at the municipal level, where urban services as another physical components of QoUL are of particular/specific focus. 


\section{Chapter Seven}

\section{Urban Services Quality and QoUL}

\subsection{Introduction}

Moving on from the neighbourhood scale examined in the previous chapter, the aim of this chapter is to broadly highlight the urban services and their relationship to the QoUL. In line with Chapters 5 and 6, the chapter continues to employ the functionalist approach, analysing the functional attributes of the urban environment within the municipality of Quba.

Functional attributes including urban services have long been considered by a large body of research as primary factors underlying overall satisfaction with QoUL (Sirgy \& Cornwell, 2001; Grzeskowiak et al., 2003). Within the most common of the indicators that have been used to assess QoUL in a geographical setting, health, educational, recreational and transportation services are deemed to be essential components of the urban environment. For example, but not limited to, McCrea et al. (2005) pointed out the most favourably evaluated feature of the urban environment is the access to facilities such as schools, health services and recreational facilities. This chapter focuses on such functional attributes of health services, educational services and recreational facilities services. It investigates the extent to which these features contribute to the QoUL in the study area by measuring the quality and the satisfaction with those services based on the subjective assessment of the residents as well as using the objective indicators which refer to the governmental official statistics. In doing so, a set of variables for each of these indicators have been adopted to measure the relationship between QoUL and these indicators taking into account the impact of the residents' socio-demographic attributes.

\subsection{Health services}

Health status is vital for human beings and has direct effects on their QoL as well as being a significant determinant for SWB (Yinshe, 2005; Psatha et al., 2011; Diener et al., 1999; 
Zhan, 1992). As obtained from the findings in Chapter 4, health is considered as the most important factor affecting the QoUL of Quba's residents, where it was placed at the forefront of the aspects that influence QoUL in the study area. Hence, the provision of health services is crucial in achieving better health status. In the municipality of Quba, the provision of health ranges from medical complexes and primary health care centres to hospitals. Primary health care centres are a government health service provider that serves a neighbourhood or a group of neighbourhoods within the municipality. While hospitals range from governmental to private, the medical complexes are entirely private. Note that hospitals do not serve only the residents of Quba, but rather the whole of Medina, unlike the primary healthcare centres that are restricted to those who live within the neighbourhoods of Quba only. Therefore, it is difficult to measure the objective indicators of healthcare provision including number of beds, doctors and nurses in terms of the number of these indicators per capita. The objective indicators of health service provision in terms of the type of health service provider, the number of beds, the number of medical staff (doctors and nurses) are shown in the Table 7.1.

Table 7.1: Objective indicators of the health services in Quba municipality. Source: GDHA (2018).

\begin{tabular}{ccccc}
\hline Type of Healthcare Provider & Total number & No. of Beds & $\begin{array}{c}\text { No. of } \\
\text { Doctors }\end{array}$ & $\begin{array}{c}\text { No. of } \\
\text { Nurses }\end{array}$ \\
\hline Hospitals (Governmental) & 3 & 796 & 509 & 1445 \\
\hline Hospitals (Private) & 3 & 390 & 287 & 1331 \\
\hline Primary Healthcare (Governmental) & 9 & Non & 61 & 112 \\
\hline Medical Complex (Private) & 5 & Non & 47 & 71 \\
\hline
\end{tabular}

In relation to the subjective evaluation regarding health services, a set of variables have been adopted to measure residents' satisfaction with the provision of healthcare. Those include waiting time to see the doctor, ${ }^{6}$ ease of getting a medical appointment, quality of the medical care received from staff, satisfaction with timely treatment and satisfaction with the overall quality of health care services. Firstly, the relationship between health service variables and satisfaction with the overall quality of health care services is examined. Secondly, the association between satisfaction with the overall quality of health care services and overall

\footnotetext{
${ }^{6}$ Waiting time to see a doctor refers to the total number of minutes that a patient spent in a waiting room before meeting the doctor.
} 
QoUL is assessed, taking into account the impact of the socio-demographic attributes of the participants. In addition, in some cases an open-ended question as a qualitative part of this thesis was employed to support findings obtained from the close-ended questions.

The association between health service variables and satisfaction with the overall quality of healthcare services was examined using Spearman's correlation. Findings in Table 7.2 show a significant positive strong correlation between satisfaction with the overall quality of health care services and the variables of quality of medical care received from staff and satisfaction with diagnosis time. A moderate positive correlation was revealed between the ease of getting a medical appointment and satisfaction with the overall quality of health care services. In contrast, a negative, but weak correlation was found between amount of waiting time to see the doctor and satisfaction with the overall quality of health care services.

Table 7.2: Correlation between overall satisfaction with quality of healthcare services and other health service variables. Spearman's correlation test.

\begin{tabular}{lcccc}
\hline & $\begin{array}{c}\text { Amount of waiting } \\
\text { time to see the doctor }\end{array}$ & $\begin{array}{c}\text { Ease of getting a } \\
\text { medical appointment }\end{array}$ & $\begin{array}{c}\text { Quality of medical care } \\
\text { received from staff }\end{array}$ & $\begin{array}{c}\text { Satisfaction with } \\
\text { diagnosis time }\end{array}$ \\
\hline $\begin{array}{l}\text { Correlation } \\
\text { Coefficient }\end{array}$ & -.177 & .416 & .695 & .679 \\
\hline Result & Significant & Significant & Significant & Significant \\
\hline
\end{tabular}

It can be noted from Table 7.2 that overall satisfaction with the quality of healthcare is directly associated with the variables of ease of getting a medical appointment, quality of medical care received from staff and satisfaction with diagnosis time, while it is inversely associated with the amount of waiting time to see the doctor. Cross-tabulation was applied for further exploration regarding these results. Amount of waiting time to see the doctor was found to be associated with dissatisfaction with the quality of healthcare services. In this context, a frequencies test indicates that $51.6 \%$ of the participants spent between 30 to 60 minutes to see the doctor; more than half of them stated that they spent 60 minutes, which indicates the inverse relationship between the two variables. Findings from the qualitative data (i.e., open-ended questions) support that, where participants frequently report longer waiting times as one of the main concerns regarding health services. Referring to the literature, several studies indicated that waiting time to see the doctor predicts satisfaction with healthcare services, where longer waiting time is associated with dissatisfaction (e.g., 
Prakash, 2010; Naseer et al., 2012; Bleustein et al., 2014; Xie \& Or, 2017; and Jaklič et al., 2018; Rane et al., 2019).

Other variables such as ease to get a medical appointment, quality of medical care received from staff and satisfaction with diagnosis time were found to contribute to the overall satisfaction with the quality of health services. That is, satisfaction with these health service variables associated with higher satisfaction with the quality of health services and vice versa, which is clearly illustrated in Figure 7.1. The qualitative side of the subjective evaluation regarding health services indicated some issues that concern participants. In this respect, some participants reported their dissatisfaction with the service provided by medical personnel (i.e., doctors and nurses), where many of them referred to issues such as qualifications and how they were treated by staff. In addition, medical appointments and diagnosis were reported as issues that concern the participants.

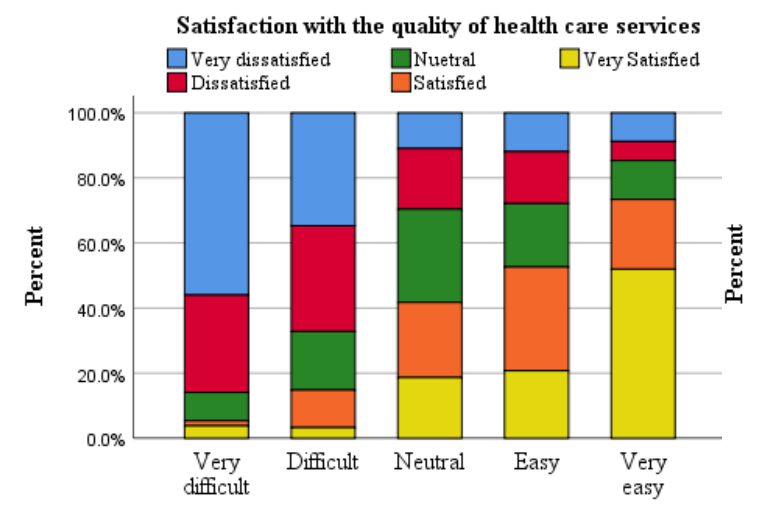

How easy is it to get a medical appointment?

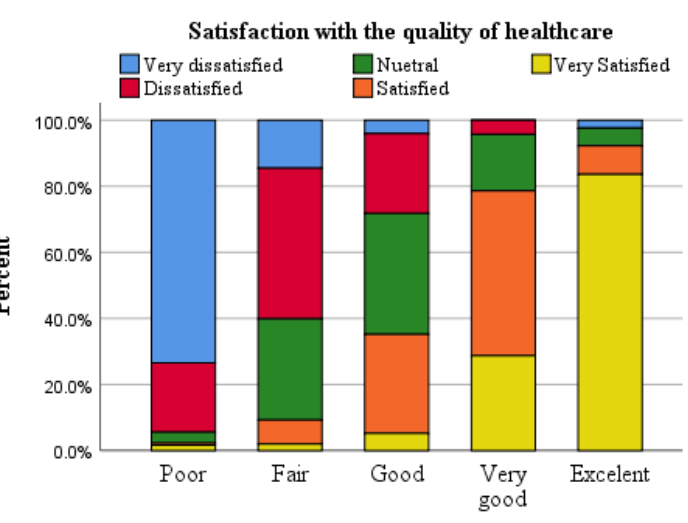

Quality of medical care received from staffs

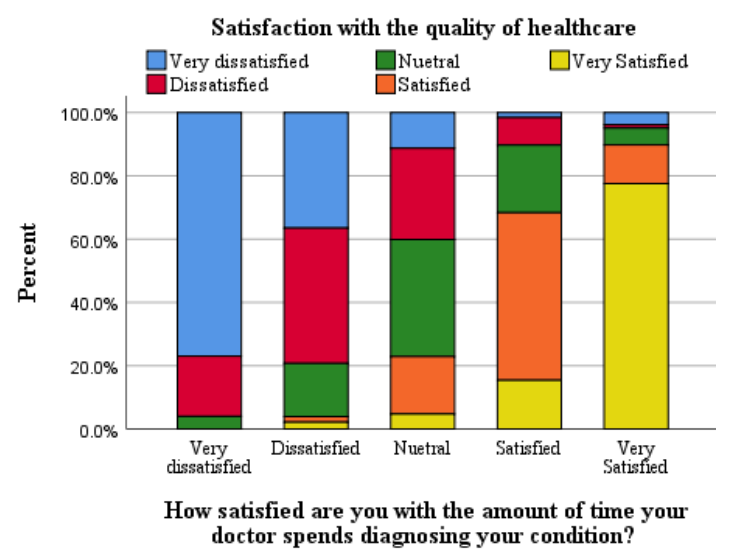

Fig 7.1: Association between satisfaction with quality of health services and health services variables 
For the overall satisfaction with the quality of healthcare services, frequency distribution indicates that $35.6 \%$ of the participants were satisfied, compared to $28.3 \%$ of those who are not satisfied, while $36.1 \%$ of the participants provided a neutral response, as is clearly illustrated in Figure 7.2.

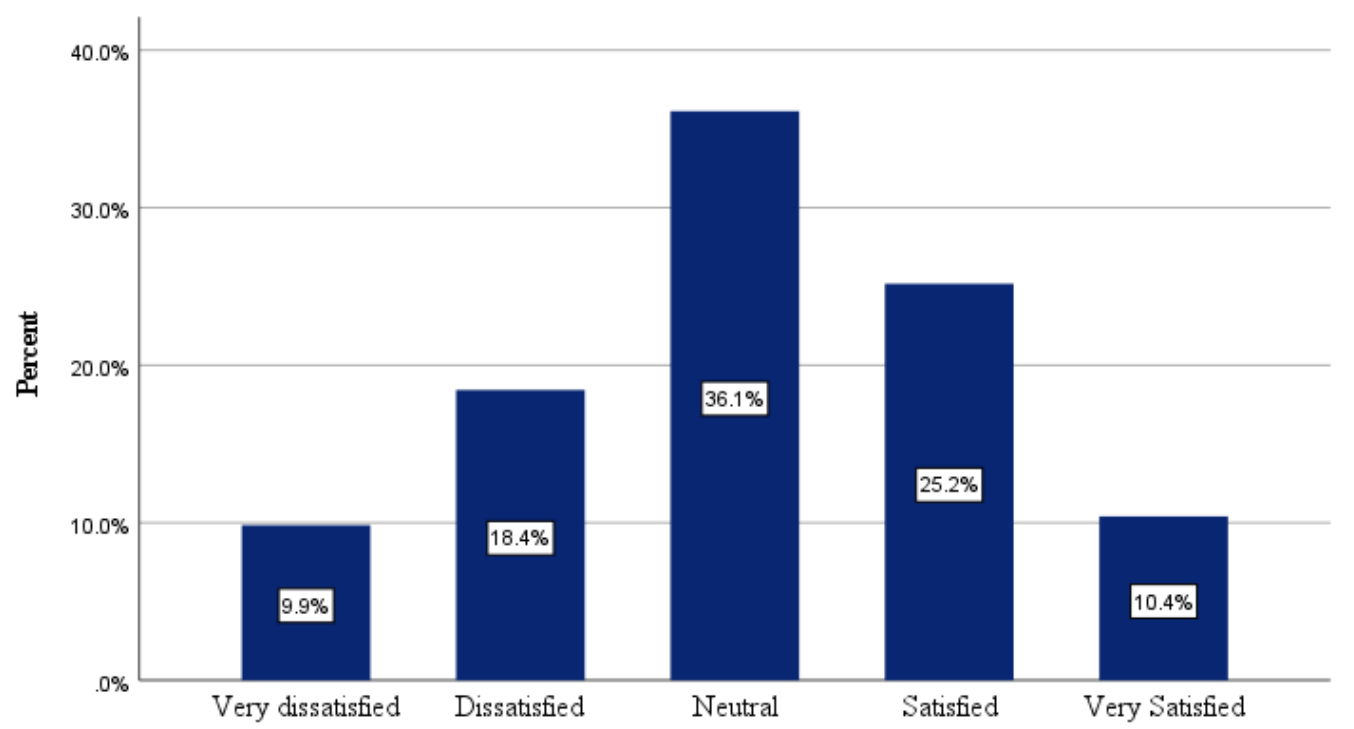

Fig 7.2: Overall satisfaction with healthcare services

In order to gain insight into the contribution of health services to the QoUL, the relationship between overall satisfaction with the quality healthcare services and QoUL level was examined using cross-tabulation. Results indicated a significant association between the two variables. It can be noted from Table 7.3 that higher QoUL is associated with higher satisfaction with healthcare services: $45.8 \%$ the respondents who stated that they are satisfied reported higher QoUL. However, an association between low QoUL and dissatisfaction with quality of health services can be observed, where $52.7 \%$ among those who stated that their QoUL is low reported higher dissatisfaction. This indicate that QoUL has a low positive relationship with overall quality of health services, where it can be noted from Figure 7.3 that QoUL increases with the increase of satisfaction with the quality of health services and vice versa. In relation to the impact of the socio-demographic attributes, only employment was found to be significantly associated with overall satisfaction with the quality of healthcare services. In this regard, cross-tabulation indicated that the selfemployed were the most dissatisfied, where $61.6 \%$ of them stated that they are dissatisfied 
or very dissatisfied with the quality of healthcare services. Possible explanation regarding this result is that the self-employed may have higher expectations about the quality of services, including health services because they enjoy a good financial position. Further research may reveal deeper findings about that relationship.

Table 7.3: Relationship between QoUL and overall satisfaction with the quality of health care services

\begin{tabular}{|c|c|c|c|c|c|c|}
\hline & & & \multicolumn{4}{|c|}{ Overall satisfaction the quality of health care services } \\
\hline & & & Dissatisfied & Neutral & Satisfied & Total \\
\hline \multirow{6}{*}{ QoUL level } & \multirow{2}{*}{ Low } & Count & 39 & 29 & 6 & 74 \\
\hline & & $\%$ & $52.7 \%$ & $39.2 \%$ & $8.1 \%$ & $100.0 \%$ \\
\hline & \multirow{2}{*}{ Moderate } & Count & 55 & 63 & 48 & 166 \\
\hline & & $\%$ & $33.1 \%$ & $38.0 \%$ & $28.9 \%$ & $100.0 \%$ \\
\hline & \multirow{2}{*}{ High } & Count & 61 & 106 & 141 & 308 \\
\hline & & $\%$ & $19.8 \%$ & $34.4 \%$ & $45.8 \%$ & $100.0 \%$ \\
\hline \multirow{2}{*}{\multicolumn{2}{|c|}{ Total }} & Count & 155 & 198 & 195 & 548 \\
\hline & & $\%$ & $28.3 \%$ & $36.1 \%$ & $35.6 \%$ & $100.0 \%$ \\
\hline
\end{tabular}

Chi-square $=52.173, \mathrm{p}$-value $<.001$.

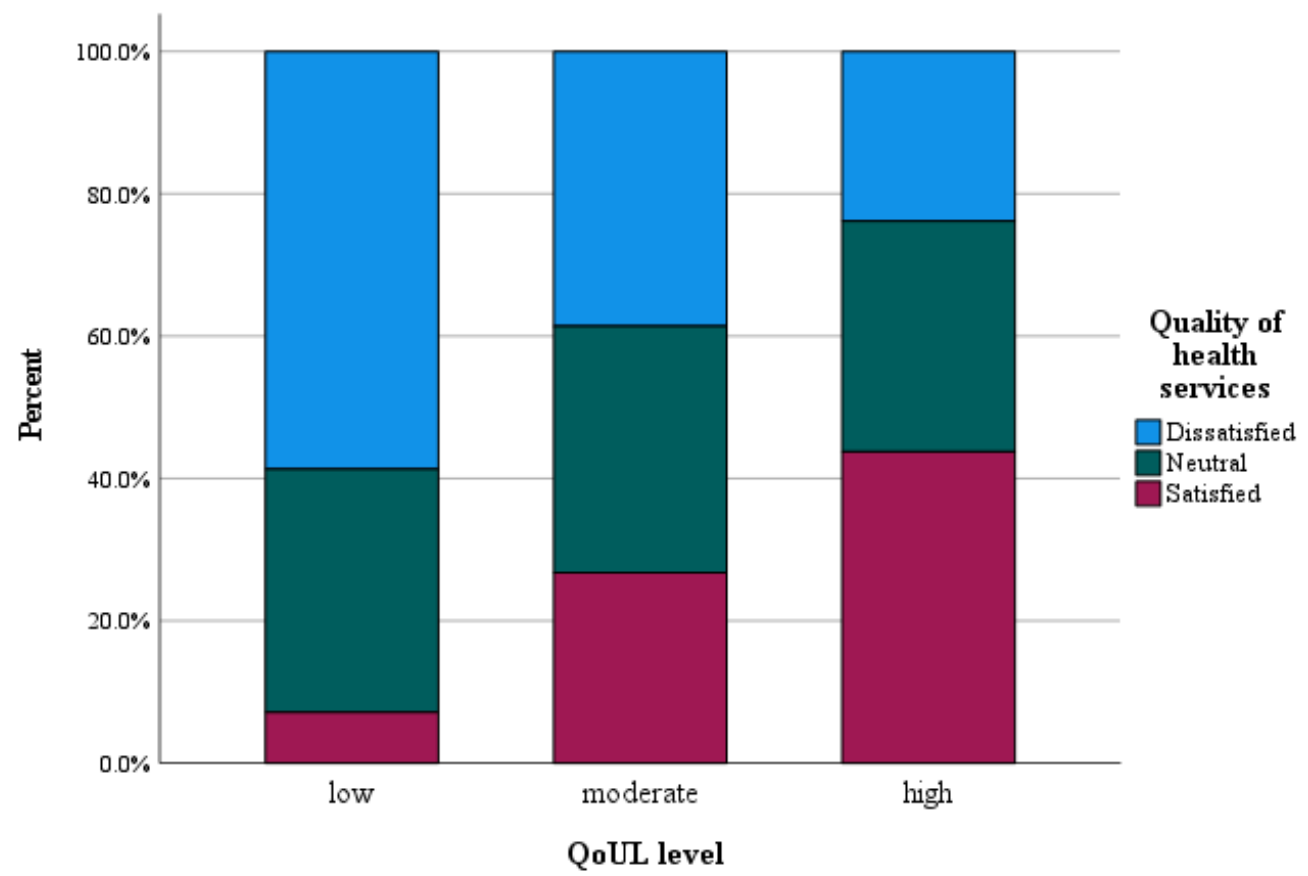

Fig 7.3: Association between satisfaction with the quality of health services and QoUL 


\subsection{Educational services}

According to Antoniuk et al. (2019), there is convincing evidence that the higher the level of people's education, the better QoL can be expected in terms of aspects such as personal accomplishments, financial condition, social relationships, interpersonal relationships, health, children's welfare, a sense of happiness and security. Amongst other QoUL indicators, education is a major indicator that has been widely addressed in literature as a key element of the QoUL (Sirgy et al., 2000; Senlier et al. 2008; Ross and Marieke, 1997; Pallas, 2000; Edgerton et al., 2012). For example, Ross and Marieke (1997) pointed out that education helps people to access to stable social relationships, especially marriage, which increases social support. Moreover, Pallas (2000) noted that education has positive effects on a wide range of QoL indicators including social life, psychological well-being, physical and mental health and other aspects of life. He summed up that educated people are more knowing about their social, cultural, and political worlds, and can apply that knowledge to shape their futures. To benefit from education for the QoUL, better educational facilities and systems are required.

Public education in Saudi Arabia in general and in Medina in particular happens at three levels, namely primary, intermediate and secondary education (Alahmari, 2017). One of the characteristics of public education in Saudi Arabia is the gender segregation, which means the segregation of schools in terms of gender for both students and staff (Alrashidi \& Phan, 2015). This segregation results mainly from a number of religious and cultural norms that distinguish the local society (Wiseman, 2008; Almutairy et al., 2015). In this thesis, the public education system of primary, intermediate and secondary levels was selected for the investigation of the quality of education services. Since the investigation of the QoUL focuses on the municipality of Quba as a case study, the assessment of the quality of educational services is at the neighbourhood level. Therefore, the focus is on public education only. Another reason behind focusing on this type of education is that public education plays an essential role in building basic knowledge and skills for children, which is likely to be reflected in their future, and in turn, their overall QoUL. The assessment of the education is based on both objective and subjective evaluation. Objective evaluation is based on the secondary data that have been obtained from the government, while subjective evaluation is based on parents' opinions regarding the education services that their children 
receive, as children under 18 years old are not involved in the survey for this research. Objective indicators of the educational services in Quba municipality are shown in Table 7.4 .

Table 7.4: Objective indicators of the educational services in Quba municipality. Source: GAE (2018).

\begin{tabular}{ccccc}
\hline Primary schools & \multicolumn{5}{c}{ No. } \\
\hline \multirow{2}{*}{ Gender } & Schools & Classes & Students & Teachers \\
\cline { 2 - 5 } & 36 & 473 & 12648 & 754 \\
\hline Males & 46 & 518 & 13963 & 1165 \\
\hline Females & \multicolumn{5}{c}{ No. } \\
\hline Intermediate schools & \multicolumn{5}{c}{ Students } & Teachers \\
\hline \multirow{2}{*}{ Gender } & Schools & Classes & 6037 & 489 \\
\hline Males & 24 & 244 & 5691 & 545 \\
\hline Females & 20 & 235 & \\
\hline Secondary schools & \multicolumn{5}{c}{ No. } \\
\hline \multirow{2}{*}{ Gender } & \multicolumn{5}{c}{ Students } & Teachers \\
\cline { 2 - 5 } & Schools & Classes & 3204 & 370 \\
\hline Males & 13 & 161 & 2755 & 400 \\
\hline Females & 12 & 136 &
\end{tabular}

It can be noted from Table 7.4 that the number of schools, classes, students and teachers tends to decrease with the increasing levels of the educational phase. This is probably due to the increase in the number of children at an early age, which in turn leads to an increase in the number of schools, classes and teachers. The objective indicators of the educational services presented were needed to calculate the average density of the classrooms and the student-teacher ratio that have been considered as important factors affecting the quality of education services (Ellis, 1984; Dahar et al., 2009; Hertling et al., 2000; Koc \& Celik, 2015; OECD, 2012). For example, Ellis (1984) argues that smaller classes facilitate and increase student-teacher interaction, allow for more thorough student evaluation and potentially provide a greater flexibility in teaching strategies. In addition, research reports made by the Organization for Economic Co-operation and Development (OECD) point out that small classes allow teachers to focus more on individual student needs, as well as reducing the amount of class time needed to deal with disturbances and classroom management, which in turn ensures higher performance (OECD, 2012; OECD, 2019). Empirically, Kambuga (2013) observed that due to a large number of pupils per class, the interaction between 
teacher and pupils was not effective, and that pupils may not receive sufficient individual assistance from teachers.

The teacher-student ratio has also been noted among the important criteria in the success of the educational process. Student-teacher ratios are often used as indicators to measure the quality of a school or educational system, as they are a general way to measure the workload of teachers and allocate resources in public schools, as well as of assessing the amount of individual attention a child is likely to receive from teachers (Great Partnership, n.d.). In this respect, a smaller student-teacher ratio was found by several studies to be beneficial for the educational process. For example, (Koc \& Celik (2015) point out that teachers in schools with a smaller student-teacher ratio can have plenty of time to spend with each student and check their progression, which in turn provides more appropriate education for each student. Other studies (e.g., Hertling et al., 2000; Dahar et al., 2009) indicate that a lesser studentteacher ratio is very effective for producing a higher level of educational achievement, improving student acquisition of basic skills and understanding of subject matter. Accordingly, in this thesis the average class size and student-teacher ratio have been calculated to explore the quality of educational services more for all three phases. Table 7.5 presents calculations for the average density of the classrooms and student-teacher ratio. ${ }^{7}$

Table 7.5: Calculation of the classroom's average density and student-teacher ratio

\begin{tabular}{ccc}
\hline Primary schools & & \\
\hline Gender & Avg. class size (student no. per class) & Student-teacher ratio \\
\hline Males & 26.73 & 16.77 \\
\hline Females & 26.95 & 11.98 \\
\hline Intermediate schools & & \\
\hline Gender & Avg. class size (student no. per class) & Student-teacher ratio \\
\hline Males & 24.74 & 12.34 \\
\hline Females & 24.21 & 10.44 \\
\hline Secondary schools & & Student-teacher ratio \\
\hline Gender & Avg. class size (student no. per class) & 8.65 \\
\hline Males & 19.90 & 6.88
\end{tabular}

${ }^{7}$ Calculation of the average density of the classroom and student-teacher ratio is based on the equations for measuring educational services indicators adopted by GaStat (2017). 
At the primary level, the average class size for both male and female schools are almost similar, where the student number per class is around 27. Class size tends to decrease in the case of the intermediate schools, and at this level of education, the average class size for both male and female schools are also almost similar, where student number per class is around 24. At the secondary level, those ratios tend to be lower, where average student number is around 20. These ratios were compared with the findings from the report of the GaStat regarding the assessment of the educational indicators at the regional level. It has been found that the average class size for both primary and intermediate level in Quba is similar in the case of other municipalities, where the average class size is recorded at 27 students per class in the case of male schools and 29 students in the case of female schools (GaStat, 2017). At the secondary level, however, the average of class size is much lower than in the case of other municipalities, where the average student number is 49.9 in the case of the male schools, and 43.4 in the case of the of the female schools (GaStat, 2017). Globally, these ratios were compared with those obtained from the report of OECD (2012). In this respect, it has been found that the average class size for both primary and intermediate levels in Quba is just above the average in the case of OECD countries, where this average was 23 students per class (OECD, 2012). At the secondary level, however, the average class size is lower than the OECD's ratio, where both males and females recorded around 20 students per class. From this comparison in general, it appears that the average class size for the case of Quba is acceptable if compared with the regional level, especially for the case of the secondary schools, as well as to some OECD countries such as Japan and Korea, where the average number of students per class is 32 (OECD, 2012).

For the student-teacher ratio, it can be seen from Table 7.5 that male schools are recording 16.77 students per teacher and 11.98 within the female schools at the primary level. Those ratios tend to decrease with the increase of the educational level, where at the intermediate level the student-teacher ratio recorded 12.34 students within the male schools and 10.44 students with the female schools, while in the case of the secondary level, these ratios decrease to 8.65 students within the male schools and 6.88 students within the female schools. These ratios were also compared with those at the regional as well as the global level. Regionally, average students per teacher within the male schools are almost the same for those in the case of Quba except for the secondary level, where primary, intermediate and secondary levels record 16.1, 12.3 and 13 students respectively (GaStat, 2017). In the 
case of the female schools, however, the student-teacher ratio in the case of Quba is lower compared to those at the regional level, where primary, intermediate and secondary levels record 11.98, 10.44 and 11.4 students respectively. On the contrary, the three educational levels were recorded 14, 11.1 and 6.88 students respectively in the case of the regional level (GaStat, 2017). At the global level, the most recent report of the OECD (2019) indicates that across OECD countries, the student-teacher ratio ranges from 13 to 15 students. Based on this comparison, it can be said that the student-teacher ratio in the case of Quba is the same or lower than those at the regional level and at the global level except in the case of the male primary schools, which recorded a higher student-teacher ratio.

Subjective evaluation on the other hand is based on the parents' opinions and judgements regarding educational services. In doing so, variables including accessibility to schools, evaluation system for students, number of students per class and the ability for the school to create an appropriate educational environment were adopted to assess the relationship between overall satisfaction with the educational services and these variables. Then, the relationship between QoUL and overall satisfaction with the educational services is examined. For the relationship between educational service variables and overall satisfaction with quality of educational services, Spearman's correlation test was employed. Results in Table 7.6 indicate that overall satisfaction with the quality of educational services had a low positive correlation with the variables accessibility to schools and the evaluation system for students, while it had a moderate positive correlation with the number of students per class and the ability for the school to create an appropriate educational environment. This indicates that satisfaction and accessibility to those educational-related aspects are contributing to the overall satisfaction with quality of the educational services, which is clearly illustrated in Figure 7.4, where satisfaction with the quality of the educational services increases with the increase of agreement degree and satisfaction with those variables. 
Table 7.6: The association between and the overall satisfaction level with the quality of educational services and other educational variables using Spearman's correlation

\begin{tabular}{lcccc}
\hline & $\begin{array}{c}\text { Agreement degree } \\
\text { of accessibility to } \\
\text { schools }\end{array}$ & $\begin{array}{c}\text { Assessment of } \\
\text { evaluation system for } \\
\text { students }\end{array}$ & $\begin{array}{c}\text { Number of } \\
\text { students per } \\
\text { class }\end{array}$ & $\begin{array}{c}\text { The ability for the school to } \\
\text { create an appropriate } \\
\text { educational environment }\end{array}$ \\
\hline $\begin{array}{l}\text { Correlation } \\
\text { Coefficient }\end{array}$ & .260 & .345 & .543 & .502 \\
\hline Result & Significant & Significant & Significant & Significant \\
\hline
\end{tabular}
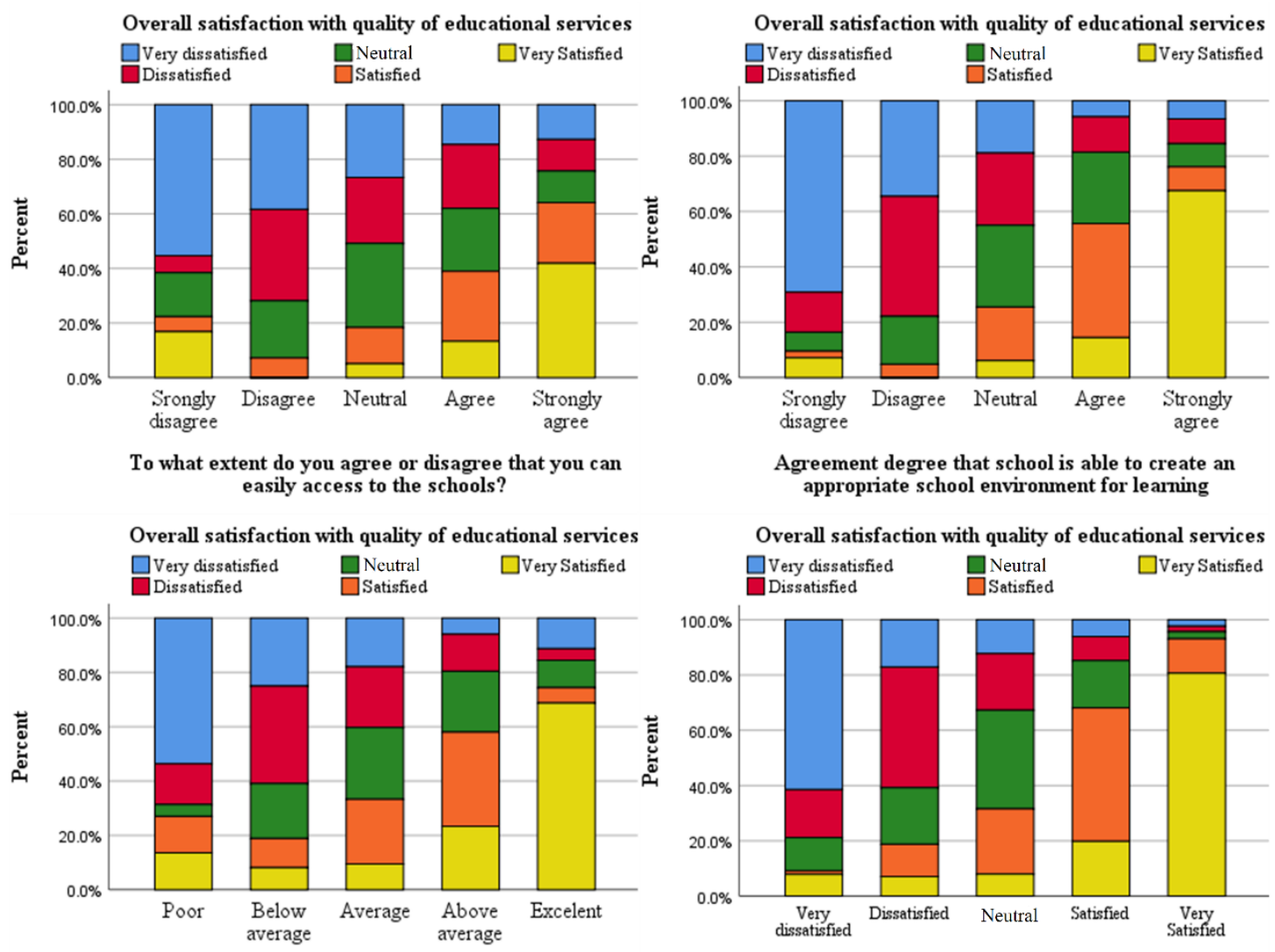

How do you assess the evaluation system for students

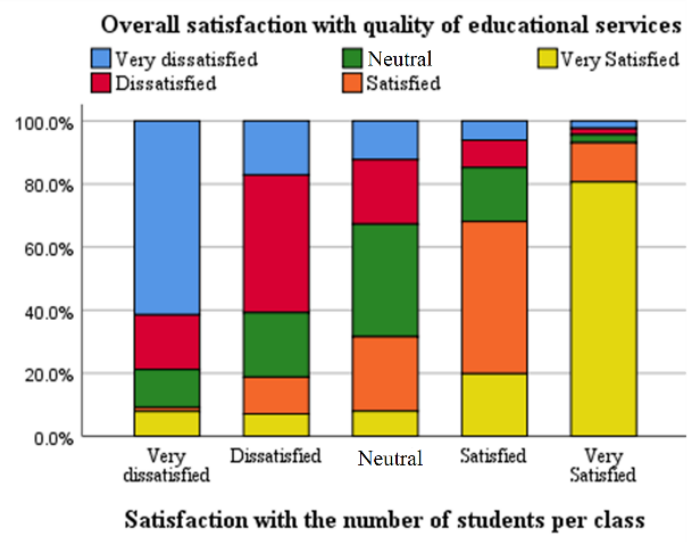

Fig 7.4: Correlation between the overall satisfaction level with the quality of educational services and other educational variables

For the overall satisfaction with educational services, however, frequency distribution indicates a higher dissatisfaction. It can be noted from Figure 7.5 that $49.2 \%$ of the participants reported dissatisfaction compared to 19.95 of those who reported satisfaction, while $30.8 \%$ of them provided a neutral response. In this respect, the high level of dissatisfaction may be due to parents' dissatisfaction with some aspects of the educational 
services. For example, frequency distribution indicates a higher dissatisfaction with the number of the students per class, where $46.4 \%$ of the participants were dissatisfied with this aspect compared to $21.4 \%$ of those who are satisfied, though objective assessment showed an appropriate average of the student number per class. In addition, the evaluation system for the students recorded an overall dissatisfaction from the parents. With regard to this, $32.3 \%$ of the participants stated that the evaluation system is poor or below the average, and $43.2 \%$ of them reported an average assessment compared to $24.4 \%$ of those who assessed the variables as above the average or excellent.

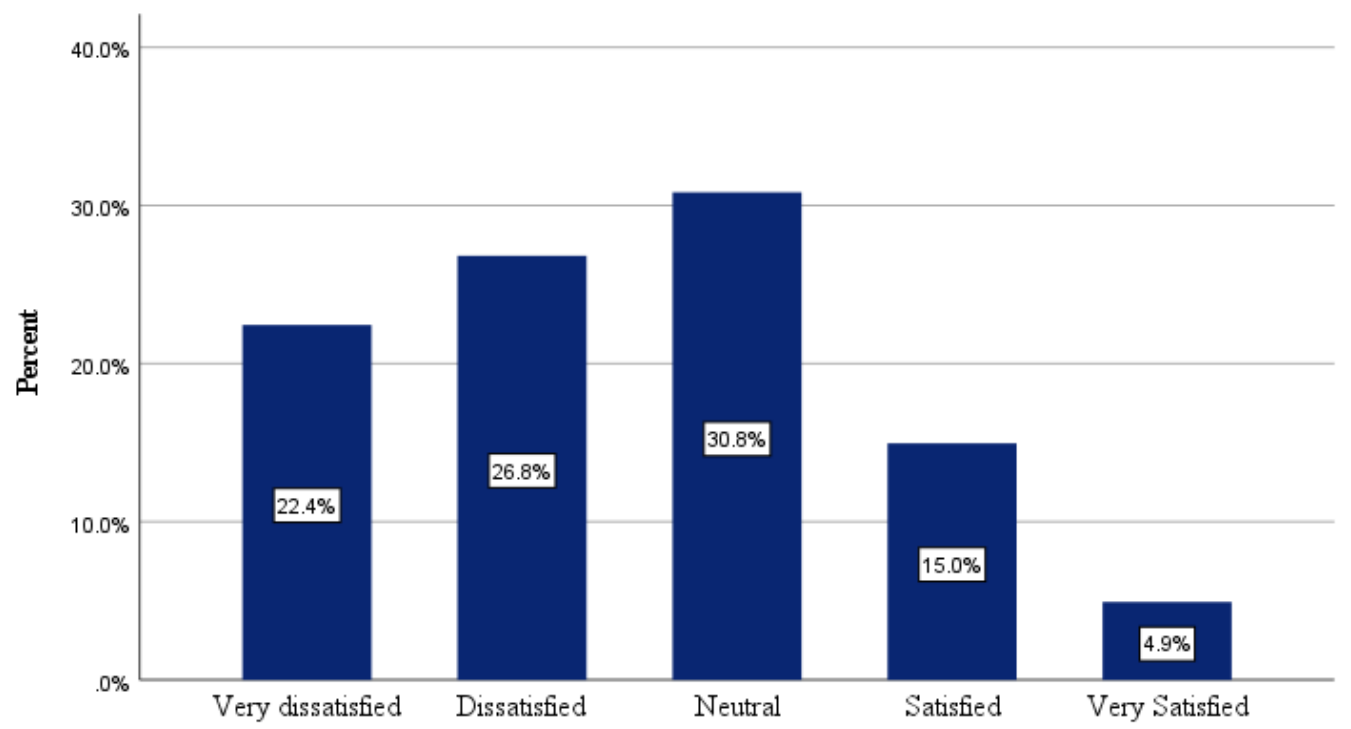

Fig 7.5: Overall satisfaction with the quality of the educational services

In order to gain insight into the contribution of the educational services, the relationship between this indicator and QoUL level was examined using cross-tabulation. Results in Table 7.7 indicates that higher dissatisfaction in terms of the overall satisfaction with the quality of the educational services is associated with a negative report of QoUL. That is, a largest attained percentage of a low level of QoUL was found to be associated with low satisfaction with educational services, where $70.3 \%$ of the respondents who stated that their QoUL is low reported higher dissatisfaction with this service. This indicates that QoUL is influenced by the extent to which parents perceive this physical indicator, where their QoUL increases with the increase of their satisfaction with educational services and vice versa, as is clearly observed from Figure 7.6. These findings indicate the important role of the educational services in perceiving the QoUL in the study area. In this context, achieving a 
better level of education is one of the most important aspirations and goals of households. This is because it is believed by many as key to success for their children in the future, which is likely to achieve a better QoUL. That is, education, as discussed earlier in this section, enhances multiple aspects of life which is ultimately reflected in overall QoUL. With regard to the impact of the socio-demographic attributes of the participants, no kind of association was found between the overall satisfaction with the quality of the educational services and all variables of the socio-demographic attributes.

Table 7.7: Relationship between overall satisfaction with the quality of educational services QoUL. Cross-tabulation.

\begin{tabular}{|c|c|c|c|c|c|c|}
\hline & & & \multicolumn{4}{|c|}{ Overall satisfaction with quality of educational services } \\
\hline & & & Dissatisfied & Neutral & Satisfied & Total \\
\hline \multirow{6}{*}{ QoUL level } & \multirow{2}{*}{ Low } & Count & 52 & 15 & 7 & 74 \\
\hline & & $\%$ & $70.3 \%$ & $20.3 \%$ & $9.5 \%$ & $100.0 \%$ \\
\hline & \multirow{2}{*}{ Moderate } & Count & 103 & 44 & 19 & 166 \\
\hline & & $\%$ & $62.0 \%$ & $26.5 \%$ & $11.4 \%$ & $100.0 \%$ \\
\hline & \multirow{2}{*}{ High } & Count & 115 & 110 & 83 & 308 \\
\hline & & $\%$ & $37.3 \%$ & $35.7 \%$ & $26.9 \%$ & $100.0 \%$ \\
\hline \multirow{2}{*}{\multicolumn{2}{|c|}{ Total }} & Count & 270 & 169 & 109 & 548 \\
\hline & & $\%$ & $49.3 \%$ & $30.8 \%$ & $19.9 \%$ & $100.0 \%$ \\
\hline
\end{tabular}

Chi-square $=44.803, \mathrm{p}$-value $<.001$.

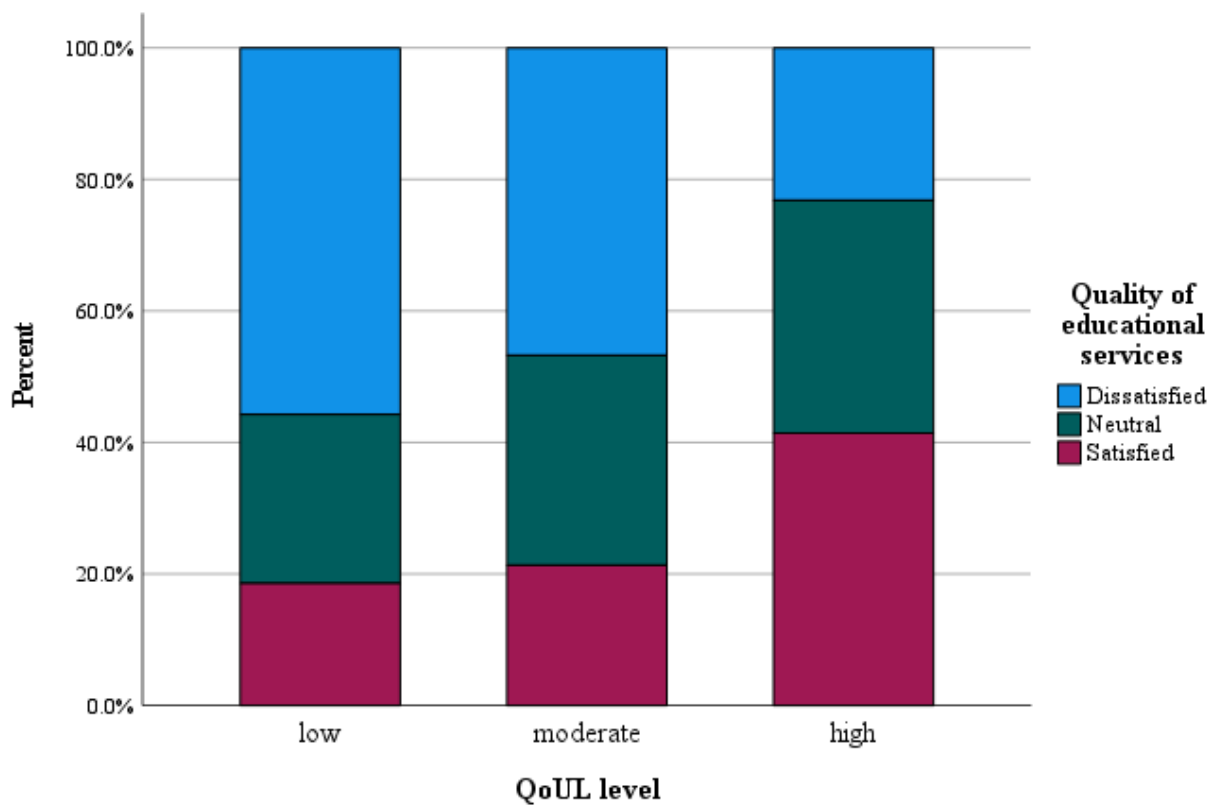

Fig.7.6: Relationship between overall satisfaction with the quality of educational services and QoUL 


\subsection{Recreational facilities}

Recreational facilities including public parks, green spaces, and open spaces have long been considered as key elements of the urban environment that contribute to the QoUL and wellbeing. The provision of such facilities is based on the belief that they are beneficial to the welfare of the population. In this context, recreational facilities promote physical and mental benefits as well as increase social interactions, all of which are key elements of the QoUL (Li \& Wang, 2012). Furthermore, they provide places for health-promoting activity as well as informal meeting spaces, outside the home and work, where social relationships can be shaped (Witten et al., 2003). Bakar et al. (2016) note that one of the essential elements of people's well-being is the presence of recreational opportunities. In this regard, he argued that the presence of parks in the vicinity of urban neighbourhoods enhances well-being, which ultimately reflects on the overall QoUL. Accordingly, in this thesis, recreational services are highlighted as one of the physical indicators that may have an influence on the QoUL. The investigation of the quality of recreational facilities is based on objective and subjective evaluation.

In this thesis, recreational facilities are represented by public parks, including green spaces, children's playgrounds, and sports arenas. According to the MRM, the municipality of Quba has 15 public parks with a total area of around $662,250 \mathrm{~m}^{2}$. The green spaces within those parks are estimated at about 94,206 $\mathrm{m}^{2}$. Besides green spaces, these parks contain about 136 children's playgrounds (see Table 7.8). One of these public parks is the Central Park, which serves the whole population of Medina, and it is considered to be the largest park in Medina with about 81 children's playgrounds as well as some facilities for events. Within these parks, however, the green spaces ratio varies, where the least ratio is around $14 \%$ in the Central Park, and the highest ratio counts about $82 \%$ in Taibah Park. This difference in the proportions of green areas is due to the fact that some of these parks include a range of facilities, such as children's playgrounds and other service facilities. In terms of the sports areas, for sprots like football, basketball and volleyball, there are a few, with some neighbourhoods counting around 9 places (MRM, 2018). 
Table 7.8: Parks within the municipality of Quba. Source: MRM, 2018.

\begin{tabular}{cccc}
\hline Parks & Size of Area (m2) & $\begin{array}{c}\text { Size of Green } \\
\text { Area }(\mathrm{m} 2)\end{array}$ & $\begin{array}{c}\text { Number of } \\
\text { Playground Items }\end{array}$ \\
\hline Quba Park & 4,115 & 0 & 7 \\
\hline Al Abbas Park & 1,140 & 900 & 0 \\
\hline The Eastern Nadwa Park & 1,815 & 960 & 3 \\
\hline The Western Nadwa Park & 1,690 & 1,186 & 0 \\
\hline Al Rawabi Park & 1,020 & 730 & 4 \\
\hline Assad Park & 5,100 & 2,400 & 12 \\
\hline Alwadi Park & 10,000 & 5,600 & 2 \\
\hline Alsseyanh and Tashgheel Park & 1,500 & 1,000 & 1 \\
\hline Almuhajreen Park 1 & 2,252 & 1,700 & 4 \\
\hline Almuhajreen Park 2 & 2,105 & 650 & 4 \\
\hline Alqaswa Park & 2,500 & 2,000 & 0 \\
\hline Taibah Garden & 4,300 & 3,500 & 6 \\
\hline Taibah Park & 3,340 & 2,860 & 81 \\
\hline Alshaibiya Park & 5,242 & 220 & 136 \\
\hline Central Park & 616,131 & 70,500 & \\
\hline Total & 662,250 & 94,206 & \\
\hline
\end{tabular}
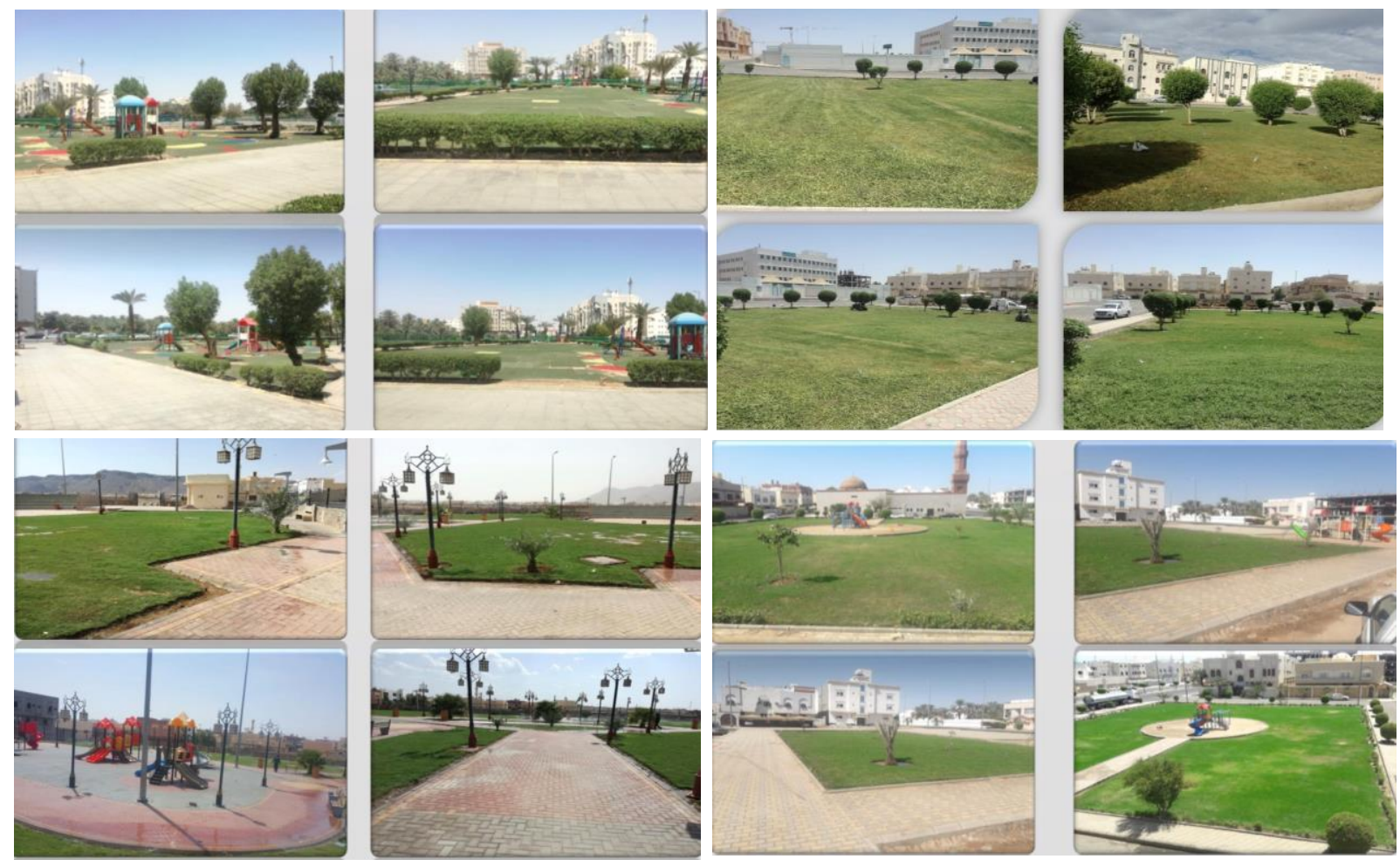

Fig 7.7: Some of the public parks within Quba municipality (MRM, 2018) 

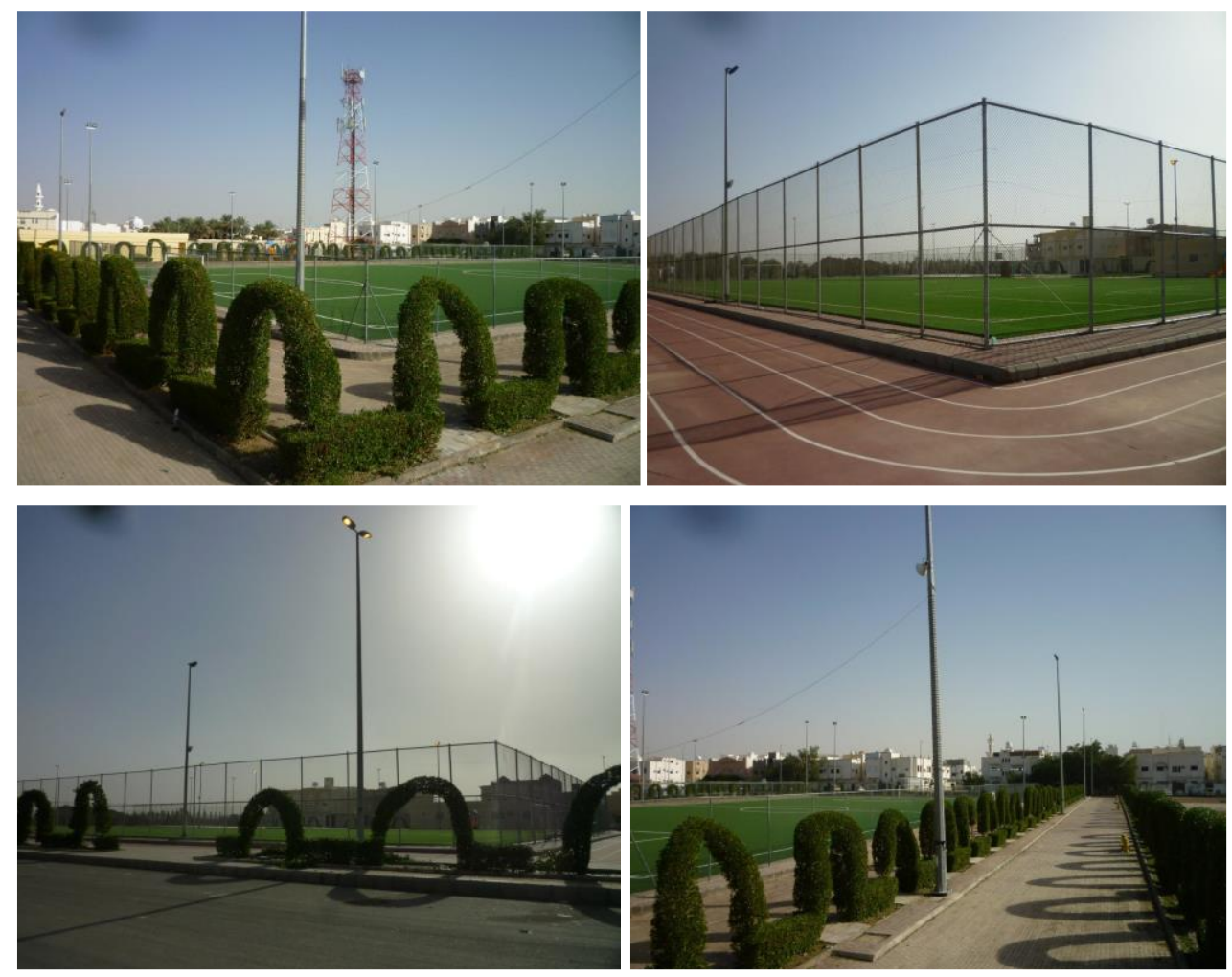

Fig 7.8: Sports areas within some of Quba's neighbourhoods (MRM, 2018)

What matters most in the recreational facilities is the green spaces, especially in hot weather such as that which distinguishes Medina. In this context, green spaces are considered as one of the most important elements of the urban environment that promotes physical and mental health, which in turn enhances well-being (WHO, n.d.; Lee et al., 2015). Moreover, the amount of provision, distribution of green spaces and easy access to these areas are among the main factors contributing to social as well as ecological aspects of urban environments (Barbosa et al., 2007). This can be supported by the findings from the qualitative research, that is, the photo diaries. In this respect, one of the participants referred to the green spaces in the following quote:

Green spaces including gardens and parks are an outlet for us in the hot weather that characterises Medina. Visiting such facilities positively affects our mental health and leads to resting nerves. More importantly, it provides a place for meeting with the family and avoiding the daily routine that enhances the relationship between family members. (PH 9, Male, 37 yrs) 


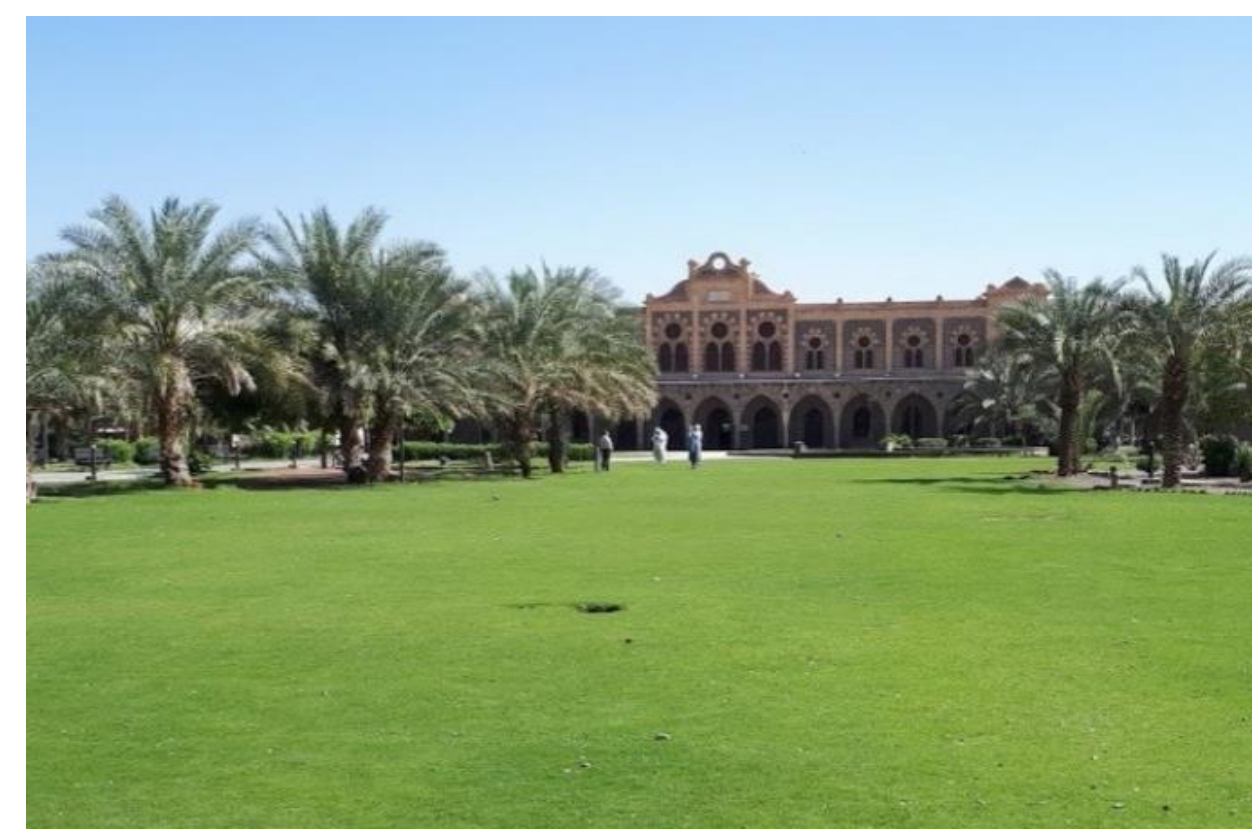

Fig 7.9: Park near the old railway station. Photo diary taken by participant PH 9.

Another participant speaks about green spaces, especially children's playgrounds, as follows:

What interests me most in these facilities is the presence of a safe and enjoyable place for my children where they spend their time playing and having fun, which for them is very important, as they meet other children and interact with them, which for me is important in developing their skills as the skill of communicating with others. The playgrounds also help to connect residents to each other in the same neighbourhood, since the activities in these playgrounds generate familiarity and closeness among the residents of the neighbourhood. (PH3, Male, 38 yrs)

From the above quotation, it can be said that the benefits of recreation are not only restricted to children's fun, but rather reinforce other aspects, the most important of which is social ties. In this regard, such facilities may enhance children's interaction with their peers from other households, which may lead to the development of social relations between members of the community. By strengthening these aspects, there may be an opportunity to build social capital which may make a difference in the lives of those households by strengthening social ties and creating stronger communities. 


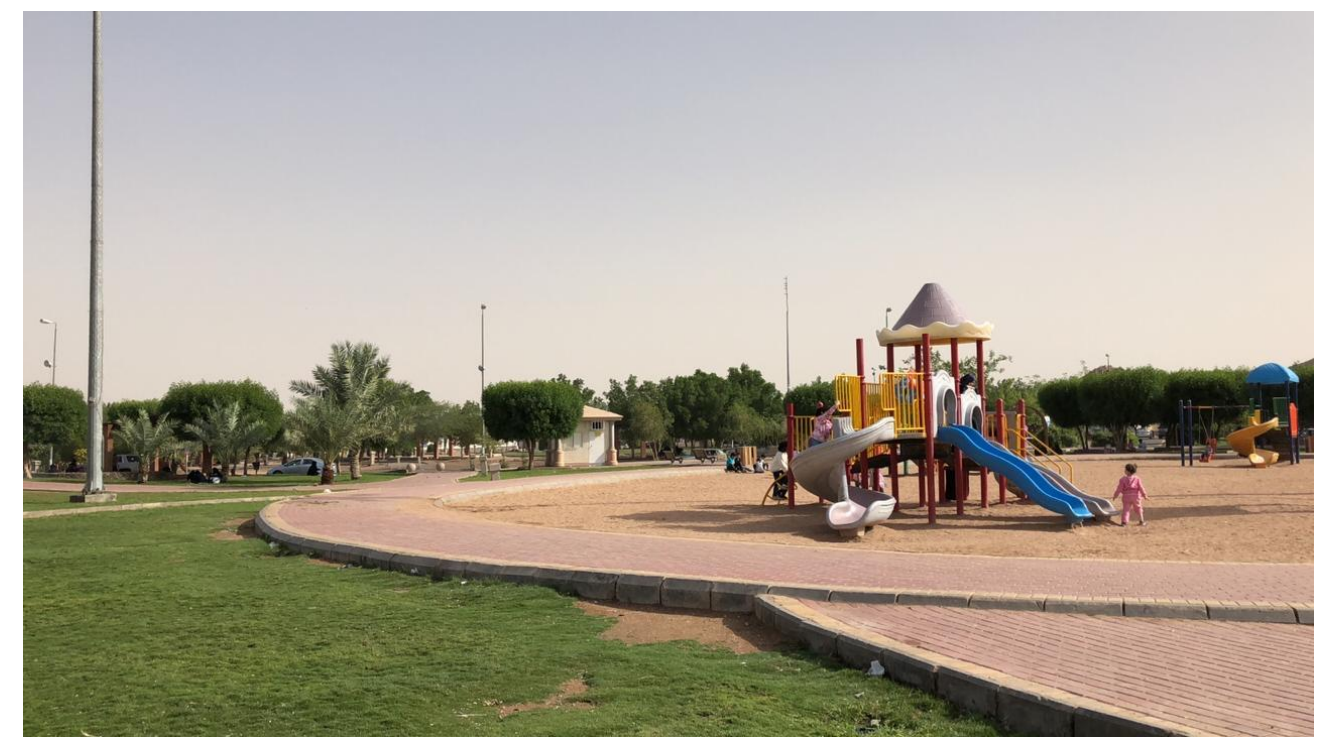

Fig 7.10: Children's playground within the central park in Quba. Photo diary taken by participant PH3.

In the case of Quba, however, people seem to suffer from the lack of green spaces. It has been calculated that per capita, green spaces for Quba residents is about $0.28 \mathrm{~m}^{2}$, which seems to be extremely low. For the city as a whole, the findings obtained from the UNHabitat report for the CPI for the case of Medina indicated that the green area per capita was $5.1 \mathrm{~m}^{2}$, which has been ranked as very weak, indicating that Medina suffers from a lack of green areas (UN-Habitat \& SMMRA, 2016). In this context, the minimum standard of green areas per capita recommended by the World Health Organization and adopted by the United Nations Food and Agriculture Organization is $9 \mathrm{~m}^{2}$ (Almayouf, 2013; Morar et al., 2014; Russo \& Cirella, 2018). Those findings from the objective assessment of the recreation services are in turn reflected in the residents' perceptions regarding this indicator, discussed in the subjective evaluation below.

In terms of the overall satisfaction with the recreational facilities, subjective evaluation indicated that respondents are mostly dissatisfied with this indicator. In this respect, as clearly illustrated in Figure 7.11, statistical analysis showed that almost $60 \%$ of the participants were dissatisfied with the recreational facilities compared to only $12.6 \%$ of those who are satisfied, while $27.6 \%$ provided a neutral response. Such results can be linked to the findings obtained in Chapter 8 , where participants were mostly dissatisfied with accessibility to leisure activities, since these activities may in some cases take place within the recreational facilities. 


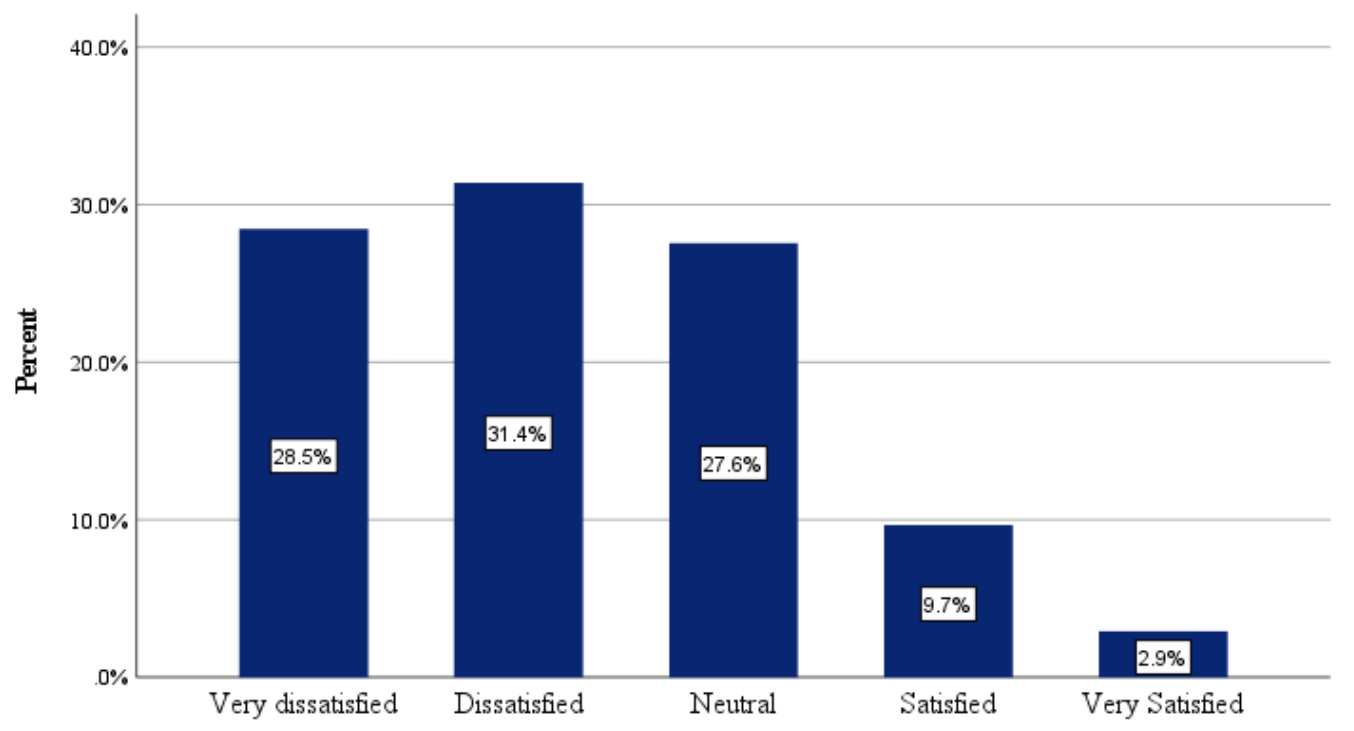

Fig 7.11: Overall satisfaction with recreational facilities

This negative impression regarding recreational facilities was indeed reflected in the perception of the participants regarding recreational services. For further exploration regarding this, the association between overall satisfaction with the quality of recreation facilities and variables in terms of the availability of public parks, playgrounds for children, walking routes and the quality of the public parks was examined using Spearman's correlation test. Results in Table 7.9 show that overall satisfaction with the quality of recreational facilities has a moderate positive correlation with those variables. Crosstabulation was applied for further exploration regarding those correlation. In general, overall satisfaction with the quality of recreational facilities was affected by the perception of the residents regarding each recreational facility. That is, higher dissatisfaction with regard to overall satisfaction with the quality of recreational facilities was associated with negative reports regarding each variable. For instance, results obtained from examining the relationship between the availability of public parks and overall satisfaction with recreational facilities indicated that $95.7 \%$ and $72 \%$ of those who reported respectively that they strongly disagree and disagree that there are enough public parks stated that they are dissatisfied or very dissatisfied with recreational facilities. This is supported by the findings obtained earlier within Chapter 6, namely with respect to the neighbourhood indicator, where $80.8 \%$ of the residents suffered from the lack of green spaces within the neighbourhoods in which they live. A similar high ratio can also be found within the 
relationships between overall satisfaction with the recreational facilities and other variables, as is clearly showed in Figure 7.12, where overall satisfaction with recreational facilities tends to decrease when respondents show a negative impression regarding those variables, and vice versa.

Table 7.9: Correlation between the overall satisfaction with the quality of recreational facilities and other recreational facilities variables using Spearman's correlation

\begin{tabular}{lcccc}
\hline & $\begin{array}{c}\text { Enough public } \\
\text { parks }\end{array}$ & $\begin{array}{c}\text { Enough } \\
\text { playgrounds for } \\
\text { children }\end{array}$ & $\begin{array}{c}\text { Enough walking } \\
\text { routes in the } \\
\text { municipality }\end{array}$ & $\begin{array}{c}\text { Public parks are well- } \\
\text { designed for all } \\
\text { segments of society }\end{array}$ \\
\hline $\begin{array}{l}\text { Correlation } \\
\text { Coefficient }\end{array}$ & $.528^{* *}$ & $.552^{* *}$ & $.510^{* *}$ & $.623^{* *}$ \\
\hline Results & significant & significant & significant & significant \\
\hline
\end{tabular}

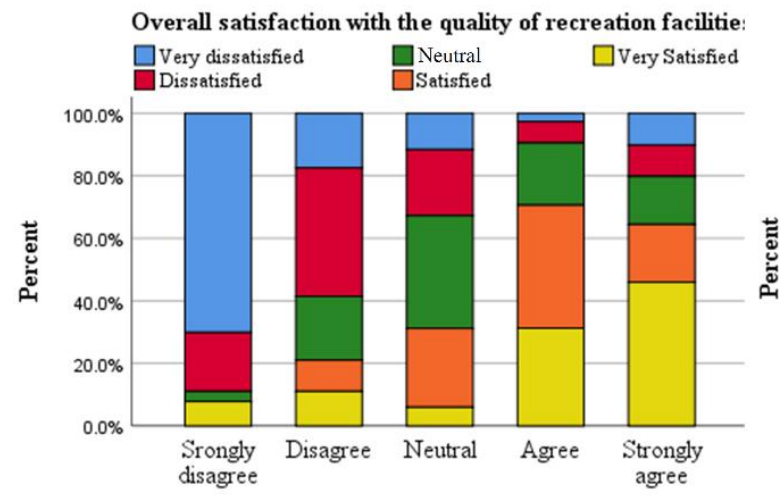

Agreement degree that there are enough public parks in the municipality of Quba

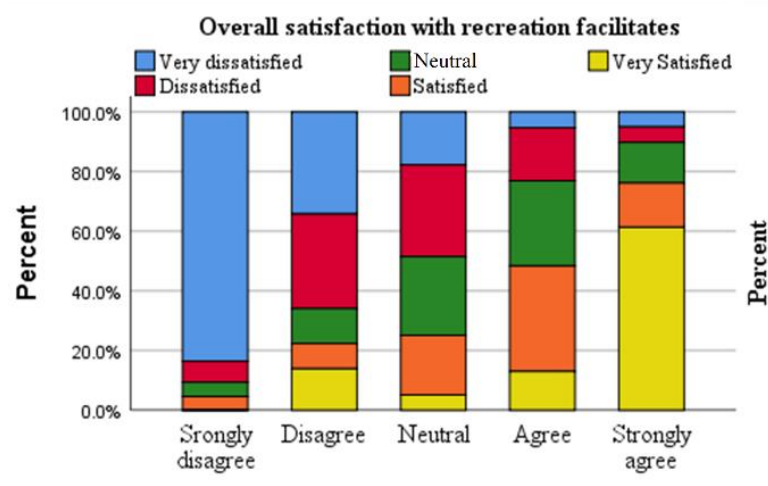

Agreement degree that there are enough walking routes in the municipality of Quba that suit everyone who wants to walk, exercise etc.

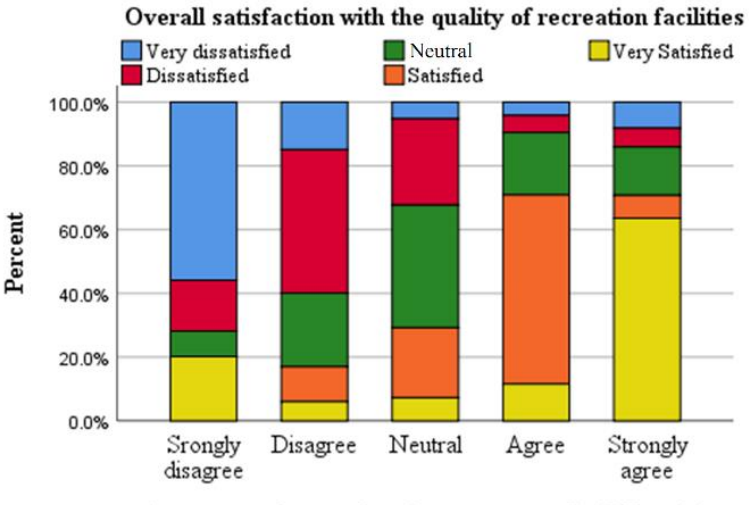

Agreement degree that there are enough children's' playgrounds

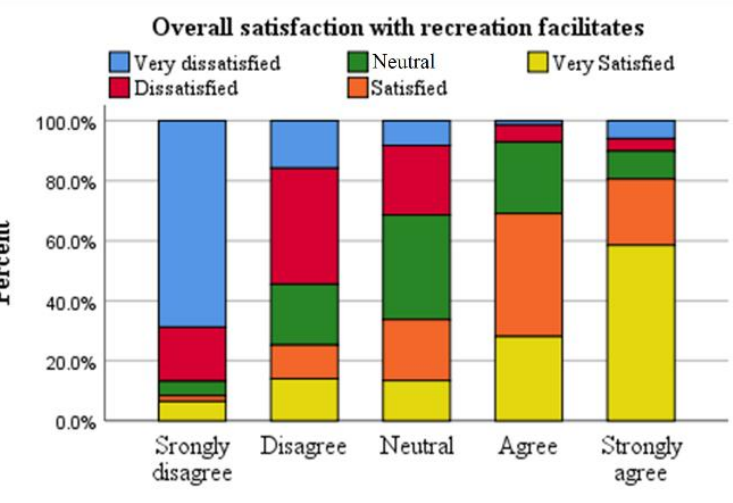

Agreement degree that public parks are well-designed for all segments of society, single people and families

Fig 7.12: Correlation between the overall satisfaction with the quality of recreational facilities and other recreational facilities variables 
For the contribution of the recreational services to the QoUL, the association between QoUL and overall satisfaction with the quality of recreational facilities was examined using the Chi-square test. Table 7.10 presents cross-tabulation of the two variables. Results indicate that QoUL is significantly associated with the overall satisfaction with the quality of recreational facilities where it can be noted that a lower level of QoUL is associated with higher dissatisfaction with the quality of recreational facilities. In this respect, results show that $79.7 \%$ of the respondents who stated that their QoUL is low reported dissatisfaction with the quality of recreational facilities. Figure 7.13 clearly shows this kind of relationship, where the QoUL decreases with the decrease of the satisfaction with recreational services. This high ratio of dissatisfaction with the recreational services were reflected in the QoUL of the respondents, indicating the influence of this physical indicator has on the QoUL of those living in Quba.

Table 7.10: Relationship between QoUL and overall satisfaction with the quality of recreational facilities

\begin{tabular}{|c|c|c|c|c|c|c|}
\hline & & & \multicolumn{4}{|c|}{ Overall satisfaction the quality of recreational facilities } \\
\hline & & & Dissatisfied & Neutral & Satisfied & Total \\
\hline \multirow{6}{*}{ QoUL level } & \multirow{2}{*}{ Low } & Count & 59 & 13 & 2 & 74 \\
\hline & & $\%$ & $79.7 \%$ & $17.6 \%$ & $2.7 \%$ & $100.0 \%$ \\
\hline & \multirow{2}{*}{ Moderate } & Count & 106 & 46 & 14 & 166 \\
\hline & & $\%$ & $63.9 \%$ & $27.7 \%$ & $8.4 \%$ & $100.0 \%$ \\
\hline & \multirow{2}{*}{ High } & Count & 163 & 92 & 53 & 308 \\
\hline & & $\%$ & $52.9 \%$ & $29.9 \%$ & $17.2 \%$ & $100.0 \%$ \\
\hline \multirow{2}{*}{\multicolumn{2}{|c|}{ Total }} & Count & 328 & 151 & 69 & 548 \\
\hline & & $\%$ & $59.9 \%$ & $27.6 \%$ & $12.6 \%$ & $100.0 \%$ \\
\hline
\end{tabular}

Chi-square $=24.319, \mathrm{p}$-value $<.001$. 


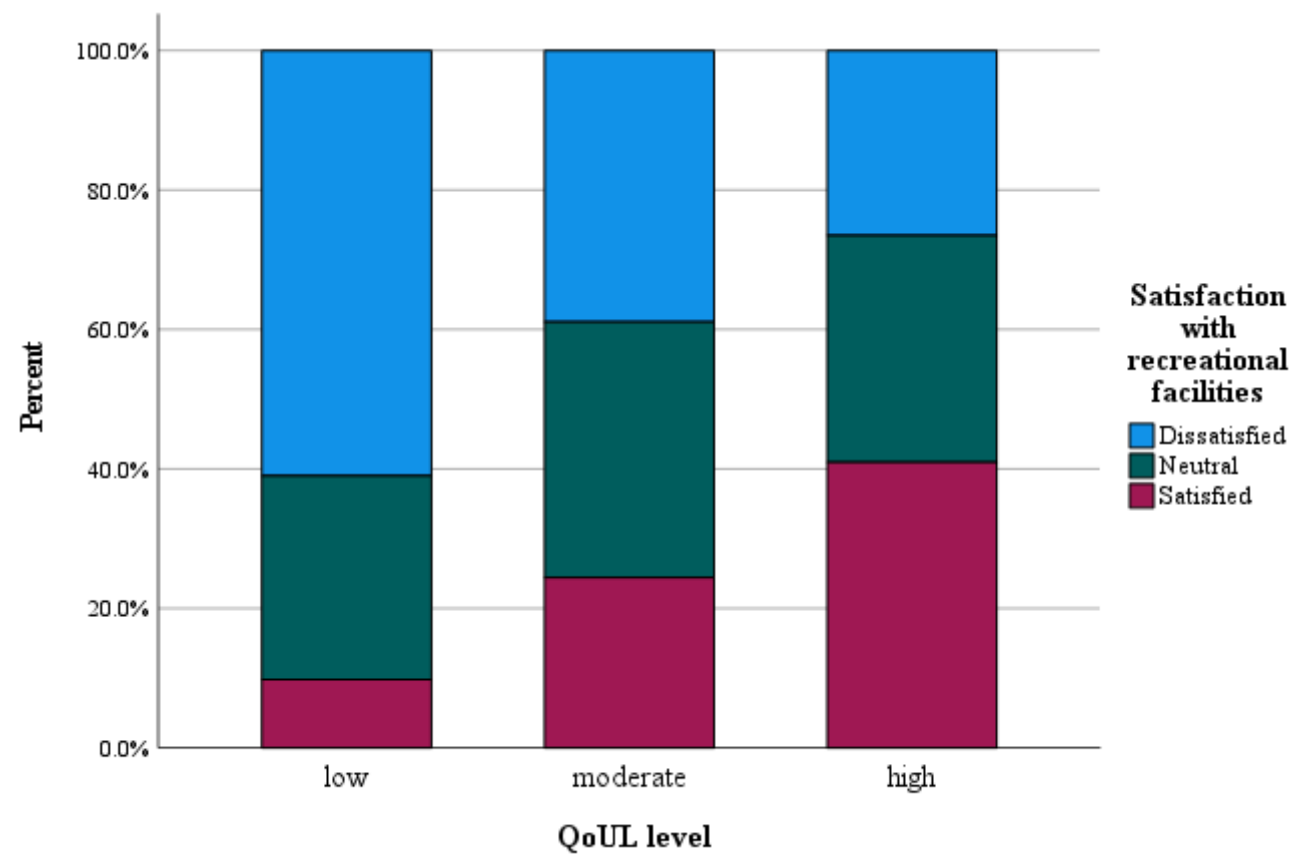

Fig 7.13: Relationship between QoUL and overall satisfaction with the quality of recreational facilities

With regard to the impact of the socio-demographic attributes of the participants in respect to the recreational services, no proof was found in relation to the impact of marital status, education, residential status and number of people living at home. At the same time, a significant association was revealed between overall satisfaction with the quality of the recreational facilities and the variables including age, gender, employment and income. Cross-tabulation was employed for further exploration regarding those associations. Crosstabulating age and overall satisfaction with the quality of the recreational facilities indicated a general tendency towards dissatisfaction with recreational services across the age groups. In this respect, the highest dissatisfaction ratio was reported by those aged 40 years and older, where $71 \%$ of them stated that they are dissatisfied or very dissatisfied with the quality of the recreational facilities. This ratio tends to decrease with a decrease in age. A possible explanation regarding this result is that the available recreational facilities, especially the parks, may not fit the wishes and needs of those of advanced age as the only refuge for such an age group to spend times of recreation. 
For the impact of gender on perceiving the quality of the recreational services, males were found to be more dissatisfied with recreational facilities, where $68.1 \%$ of them reported being dissatisfied or very dissatisfied compared to $51.5 \%$ of females. One possible explanation for this gender difference is that males may pay more attention to such facilities since children's care responsibilities outside the home, including recreational participation, is often the responsibility of fathers. Another possible interpretation is that males may be more concern about the recreational activities than females, and hence, the current recreational facilities may not fulfil their aspirations and needs. For the relationship between income and overall satisfaction with the quality of recreational facilities, those who have the highest household income (i.e., who earn more than 15,000 RS) are the most dissatisfied with recreational services. This association between the higher income and higher dissatisfaction with the recreational facilities may be because due to higher expectations regarding these facilities, and therefore, if expectations are not met, less satisfaction might result. 


\subsection{Summary of the chapter}

At the municipality level, the quality of urban services concerning health, education and recreation in relation to the QoUL of those living in Quba was investigated. This investigation was based on both objective and subjective indicators. For each of these indicators, a set of variables was used to gain more insight into the quality of those services and to what extent they contribute to the QoUL in the study area. Findings generally suggest that these functional attributes directly affect the QoUL. That is, such attributes are expected to be related to people's basic needs, especially health and education. The results indicate that apart from the health services, there is a general tendency towards dissatisfaction with the educational and recreational services, which in turn is reflected in the overall QoUL of Quba's residents.

In terms of the health services, objective evaluation was difficult because most of the health providers within the Quba municipalities serve the whole of Medina city. Subjective evaluation on the other hand, indicated that participants were moderately satisfied with health services. However, some issues related to the provision of these services seemed to affect the overall satisfaction of the residents. These issues included dissatisfaction with the service provided by medical personnel, getting medical appointments and diagnosis. This was reflected in the relationship between the overall quality of health services and QoUL. In this respect, a low positive relationship between the two variables has been revealed. These findings indicate the issue of scale in relation to the quality of health services, where the evaluation of health services at municipal and regional level overlap. That is, some residents may report satisfaction with the health services available in their area (i.e., Quba municipality), while others are dissatisfied with those available in Quba but satisfied with those at the regional level. This may indicate the inconsistency and divergence between the objective indicators of health services and the perception of the residents of Quba about the quality of those services at the neighbourhood and regional scale. This indicates the influence of scale discrepancy, which may be a source of a divergence between subjective and objective indicators, leading to a weakening of the relationship between these indicators. Such findings support the general agreement from the literature about the weak relationship between objective and subjective indicators. With regard to the impact of the socio-demographic attributes, only employment was found to be significantly associated with the overall satisfaction with the quality of health services. 
For educational services, objective evaluation was based on using variables including average class size and student-teacher ratio. These aspects are considered as important criteria through which the quality of a school or an educational system is measured. For example, the smaller the class size and the smaller the number of students per teacher, the more opportunity to provide an appropriate learning environment will be. Using these two criteria, calculation of ratios indicated that class size and student-teacher ratios in Quba were mostly lower compared to those at the regional as well as the global level. Subjective evaluation, however, indicated higher dissatisfaction with the overall quality of the educational services. This is resulted from a number of issues considered by parents themselves. In this respect, results indicated that participants were mostly dissatisfied with particular aspects concerning the number of the students per class and the evaluation system for the students. This is supported by the qualitative findings obtained from the open-ended questions, where those issues were the more frequent concern of the participants. This indeed was reflected in the QoUL where the highest ratio of dissatisfaction with the educational services was associated with a lower level of QoUL, indicating the strong impact of this functional attribute on the QoUL of those living in Quba. In relation to the to the impact of the socio-demographic attributes, no kind of association was found between the overall satisfaction with the quality of the educational services and all these attributes.

Recreational services have had the most impact on the QoUL in the municipality of Quba, as confirmed by both objective and subjective indicators. Objective evaluation indicated that there is a lack in recreational facilities, especially open and green spaces. In this respect, calculation of per capita green specs indicated a very much lower ratio compared to the minimum standard of green areas per capita recommended by the WHO and adopted by the United Nations Food and Agriculture Organization. This in turn is reflected in the overall satisfaction with the recreational services, where a general tendency of dissatisfaction was revealed. In this context, participants expressed a negative impression towards the satisfaction with recreational facilities, where the statistical analysis indicates higher dissatisfaction ratios with all of these facilities. The high dissatisfaction with this service was mirrored in the QoUL level, where the highest dissatisfaction ratio was associated with lower QoUL, which indicates a strong influence of this kind of service on the QoUL perception. 
With regard to the impact of the socio-demographic attributes, age, gender, employment and income were significantly associated with overall satisfaction with quality of the recreational facilities. A key relationship between age and recreational services indicated the concern of those of an advanced age, where they have reported the highest dissatisfaction with recreational services compared to other age groups. The relationship between gender and perceiving the quality of recreational services indicated that females were less dissatisfied than males. This may indicate that males, including fathers, are more concerned in such facilities in the study area, and therefore this concern may be associated with more dissatisfaction. With respect to the impact of income, those who have a higher household income were the most dissatisfied compared to other groups. This has been justified in that higher expectations regarding such facilities may result in higher dissatisfaction if these expectations are not met.

In light of such findings, it can be said that even though residents show a high level of satisfaction with the dwelling and neighbourhood in which they live, features of the surrounding urban environment as represented by urban services may affect that level of satisfaction, which may in turn reflect on the quality of their urban lives. This is because these features chiefly affect people's primary needs, especially health and education, which signifies the magnitude of their impact on daily life. With reference to the functionalist approach used in this thesis, it can be argued that the integration of these functional attributes constitutes an urban system that contributes as a whole to the QoUL, and failure to meet one of these attributes may ultimately lead to a defect in that system, and thus negatively affect the QoUL. For example, deficiency in health services may lead to deficiencies in educational services, and deficiencies in educational services may lead to failure to meet economic aspects (e.g., opportunity to get a job). Moreover, failure to fulfil the recreational aspects may lead to insufficient fulfilment of psychological needs and so on. Thus, the better the functioning of this urban system as a whole, the more likely it is to lead to a better QoUL.

While Chapters 5, 6 and 7 have highlighted physical QoUL indicators, the next chapter turns to explore the social and cultural domains of QoUL. 


\section{Chapter Eight}

\section{Social and Cultural Domains of QoUL}

\subsection{Introduction}

Having explored the functional attributes of the urban environment in Quba municipality in the previous chapter using functionalist approach, where quality of urban services was discussed, the current chapter moves to highlight the social and cultural domains of QoUL using postmodernity approach. In this respect, it investigates the impact and contribution of these domains to the QoUL in Quba municipality. In doing so, this chapter begins by identifying the related indicators that have been adopted for this research, before analysing the contribution of these indicators to the QoUL of Quba residents, taking into account the impact of their socio-demographic attributes on their perception regarding these indicators. In most cases, the investigation will be based on finding out statistical relationships between these indicators and the overall level of QoUL that has been obtained in Chapter 4. In addition, relationships between social and cultural indicators themselves are examined by using a number of statistical tests including Kruskal-Wallis and Mann-Whitney, the Chisquare and Spearman's rho to identify any relationship between QoUL variables. In this chapter, quantitative and qualitative results will be merged in order to gain insight into the contribution of this domain to the QoUL in the study area. Thus, this chapter highlights the key elements and issues related to the social and cultural life of Quba's residents and the extent to which they contribute to the QoUL.

\subsection{Social domain of QoUL}

In order to examine the contribution and impact of the social domain on the QoUL in the study area, a comprehensive literature review was conducted to identify relevant indicators, which are now used in this thesis in order to measure their contribution to the QoUL in the study area. These social indicators have been identified as security and safety, leisure, social 
interaction, neighbour support, religion and spirituality. Each of these indicators are discussed in succession in terms of their definition and their role in the quality of the urban environment. Then, the contribution of these indicators to the QoUL in Quba municipality is investigated. The investigation will be based on the use of statistical tests in order to find any sort of relationships between social indicators and the level of QoUL in the study area. In addition, the qualitative strand will be integrated with the quantitative strand during the interpretation.

\subsubsection{Security and safety and QoUL}

Security and safety refer both to the procedures of protection against criminal activities, and to the sense of being secure, stable and free from anxiety or fear (Albrechtsen, 2003). From this definition, the importance of security is reflected in the daily lives of individuals as well as its role on the quality of their urban lives. Security and safety are among the important social indicators that have been frequently addressed in the QoUL literature. In unsatisfactory areas, these social indicators were considered the most influential on neighbourhood satisfaction compared to physical indicators (Hur \& Morrow-Jones, 2008). Measuring security in this thesis is based on asking the residents in the municipality of Quba how they assess the level of security in the neighbourhood where they live. The results showed that only $2.6 \%$ of the respondents stated that the level of security is very poor, while $56.2 \%$ have assessed the level of security as an excellent as can be seen in Figure 8.1. This result is consistent with the UN-Habitat and SMMRA's report on the CPI, specifically, the QoL index including security. In this report, the result indicated that security classified as a very strong index among QoL indexes for Medina, where according to the report, it is noted as the main source of strength of QoL alongside health (UN-Habitat \& SMMRA, 2016). However, the security assessment in this report was based on official statistics such as homicide and theft rates, without reference to subjective indicators, including people's assessment of security in their urban environment. 


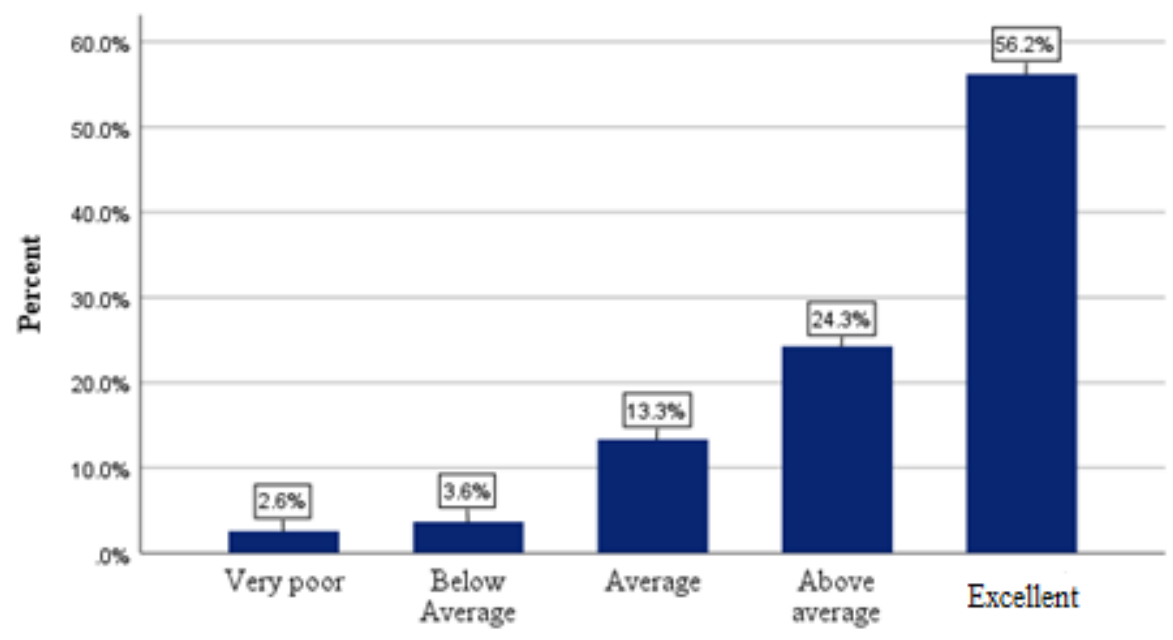

How do you evaluate the security situation in your area?

Fig 8.1: Security evaluation of the study's respondents

In order to investigate the contribution of the security indicator to QoUL in the study area, Kruskal Wallis H test was employed. The results showed significant relationship between QoUL and security evaluation. It can be noted from Table 8.1 that the mean of QoUL tended to be low when the evaluation of the security situation decreased from excellent to poor. Figure 8.2 shows this kind of relationship. From this result it can be said that security plays an important role in the lives of people in the municipality of Quba, where it is clear from the results in both Table 8.1 and Figure 8.2 that this indicator positively affects the QoUL in the study area. In other words, the QoUL level increases with the increase of the security level.

Table 8.1: Relationship between security situation evaluation and QoUL level using Kruskal Wallis test

\begin{tabular}{ccc}
\hline Security situation evaluation & Mean & Median \\
\hline Very poor & 2.64 & 2.00 \\
\hline Below Average & 2.95 & 3.00 \\
\hline Average & 3.23 & 3.00 \\
\hline Above average & 3.53 & 4.00 \\
\hline Excellent & 3.82 & 4.00 \\
\hline
\end{tabular}

Kruskal Wallis, P-value<.001 


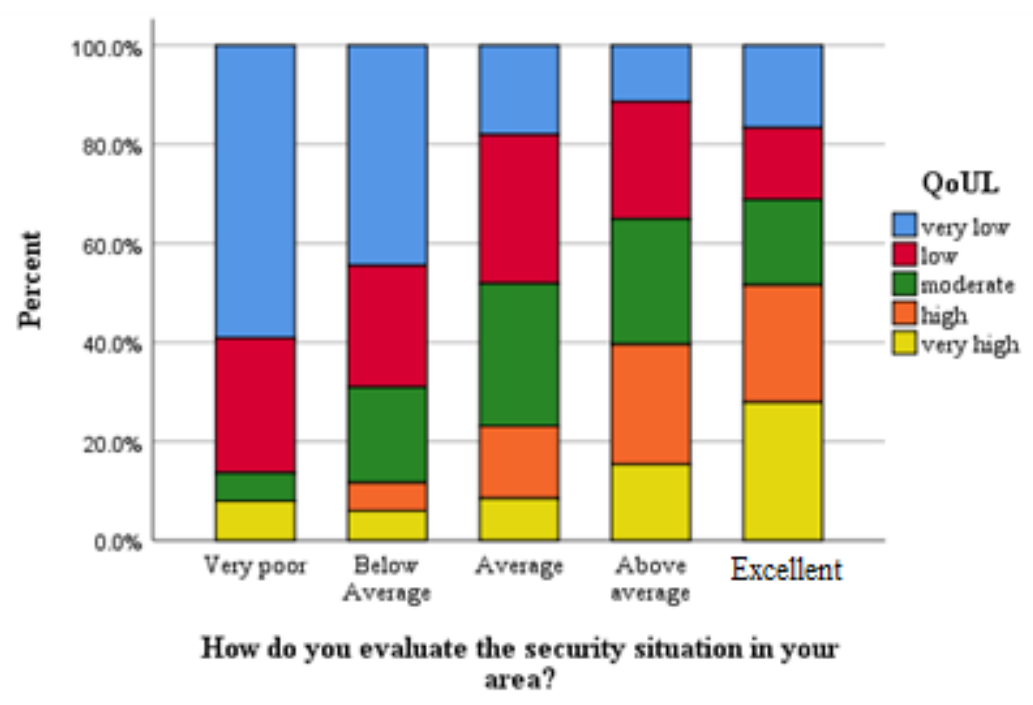

Fig 8.2: Relationship between security situation evaluation and QoUL level

- Impact of the socio- demographic attributes

For the impact of the socio-demographic attributes of Quba residents on their perceptions about security, only gender was found to be significantly associated with the security evaluation. In this regards, a cross-tabulation was applied for further exploration. It can be noted from Table 8.2 that $62.5 \%$ of females stated that the security is excellent, while this percentage decreases in the case of males where $50 \%$ of them stated that the security is excellent. There may be an explanation for the disparity between those percentages in relation to gender in this thesis. One possible interpretation is that women within the Saudi culture may rely mostly on their fathers, brothers or husbands for many aspects of life including protecting them from danger. In this regard, females are expected to go out with male relatives according to Saudi culture, which is mostly based on Islamic law. Therefore, this may lead to women feeling more secure. In addition, males may feel less secure because they are expected to be fully concerned for their whole families. 
Table 8.2: Evaluation of security situation and gender. Cross-tabulation.

\begin{tabular}{|c|c|c|c|c|c|c|c|c|}
\hline & & & \multicolumn{5}{|c|}{ How do you evaluate the security situation in your area? } & \multirow[b]{2}{*}{ Total } \\
\hline & & & Very poor & $\begin{array}{c}\text { Below } \\
\text { Average }\end{array}$ & Average & $\begin{array}{l}\text { Above } \\
\text { average }\end{array}$ & Excellent & \\
\hline \multirow{4}{*}{ Gender } & \multirow{2}{*}{ Female } & Count & 7 & 7 & 34 & 54 & 170 & 272 \\
\hline & & $\%$ & $2.6 \%$ & $2.6 \%$ & $12.5 \%$ & $19.9 \%$ & $62.5 \%$ & $100.0 \%$ \\
\hline & \multirow{2}{*}{ Male } & Count & 7 & 13 & 39 & 79 & 138 & 276 \\
\hline & & $\%$ & $2.5 \%$ & $4.7 \%$ & $14.1 \%$ & $28.6 \%$ & $50.0 \%$ & $100.0 \%$ \\
\hline \multirow{2}{*}{\multicolumn{2}{|c|}{ Total }} & Count & 14 & 20 & 73 & 133 & 308 & 548 \\
\hline & & $\%$ & $2.6 \%$ & $3.6 \%$ & $13.3 \%$ & $24.3 \%$ & $56.2 \%$ & $100.0 \%$ \\
\hline
\end{tabular}

Chi-square $=10.138$ p-value $<.038$

\subsubsection{Leisure and QoUL}

Leisure is also an important indicator that has been addressed in many studies in the literature on QoUL, particularly as an important source of SWB and life satisfaction (Diener et al., 1999; Newman et al., 2013; Argan et al., 2018; Kuykendall et al., 2018). The participation in leisure activities is associated with many aspects of life including physical and psychological health as well as well-being (Paggi et al., 2016), where participation in this type of social activity may lead to building and strengthening social relationships, to acquiring more skills and knowledge, to enhanced social cohesion, and hence, to improving the QoUL (Kelly et al., 1987; Reyes-Garcia et al., 2009; Brajša-Žganec et al., 2010; Smale et al., 2010; Sajin et al., 2016; Kuykendall et al., 2018). With this in mind, this social indicator was investigated as a key element of daily life experience of the residents which is likely to contribute to the QoUL in the study area.

In general, leisure activities take a variety of forms, including for example, exercise and socializing (Paggi et al., 2016), social relations with family friends (Reyes-Garcia et al., 2009). However, leisure activities differ across individuals and cultures (Diener et al., 1999). In this thesis, leisure activities take on a different form. Within the qualitative data (i.e., the photo diaries) a number of participants pointed out that some religious monuments in Quba municipality, such as Quba Mosque, promote leisure activities in addition to their basic function related to the performance of religious duties. In this regard, some of these participants as part of the study took photographs of the facilities surrounding the mosque. As part of the follow-up interviews, some of the participants were asked why they were 
taking these photographs, and most of them indicated that visiting the Quba Mosque promotes leisure activities, especially for their children.

Findings from the quantitative research, however, indicated a general tendency towards dissatisfaction with the accessibility of leisure activities. In this respect, frequency distribution of the agreement level that there is accessibility to leisure activities that are suitable for all groups of people indicates that $50 \%$ of the respondents reported high disagreement regarding the accessibility to these activities compared to $27.6 \%$ of those who agreed. Figure 8.3 presents the frequency distribution of the agreement regarding this indicator. For further exploration regarding the impact of this social indicator on the QoUL, the relationship between these two variables was examined using the Chi-square test. Results have shown a significant association between leisure accessibility and QoUL. It can be noted from Table 8.3 that lower QoUL is associated with higher disagreement that there is accessibility to leisure activities. In this respect, $56.3 \%$ and $74.1 \%$ among those who respectively reported disagreement regarding accessibility to the leisure activities stated that their QoUL is low or very low. Figure 8.4 clearly presents the relation between these two variables where lower QoUL is associated with disagreement of leisure accessibility.

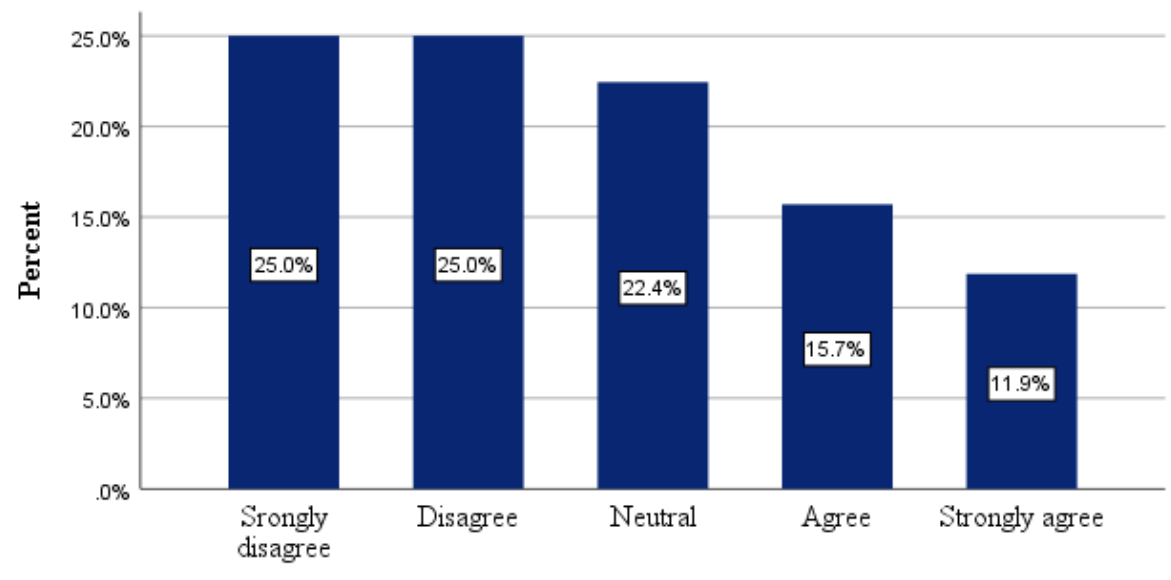

To what extent do you agree or disagree there is a good accessibility to leisure activities that are suitable for all groups of people (i.e. children, adults and the elderly people) in the municipality of Quba.

Fig 8.3: Agreement degree that there is good accessibility to leisure activities 
Table 8.3: The association between agreement degree there is good accessibility to leisure activities that are suitable for all groups of people and QoUL level

\begin{tabular}{|c|c|c|c|c|c|c|c|c|}
\hline & & & \multicolumn{5}{|c|}{$\begin{array}{l}\text { Accessibility to leisure activities that are suitable } \\
\text { for all groups of people }\end{array}$} & \multirow{2}{*}{ Total } \\
\hline & & & $\begin{array}{l}\text { Strongly } \\
\text { disagree }\end{array}$ & Disagree & Neutral & Agree & $\begin{array}{l}\text { Strongly } \\
\text { agree }\end{array}$ & \\
\hline \multirow{10}{*}{ QoUL level } & \multirow{2}{*}{ very low } & Count & 8 & 1 & 1 & 3 & 3 & 16 \\
\hline & & $\%$ & $50.0 \%$ & $6.3 \%$ & $6.3 \%$ & $18.8 \%$ & $18.8 \%$ & $100.0 \%$ \\
\hline & \multirow{2}{*}{ low } & Count & 21 & 22 & 9 & 1 & 5 & 58 \\
\hline & & $\%$ & $36.2 \%$ & $37.9 \%$ & $15.5 \%$ & $1.7 \%$ & $8.6 \%$ & $100.0 \%$ \\
\hline & \multirow{2}{*}{ moderate } & Count & 56 & 44 & 36 & 15 & 15 & 166 \\
\hline & & $\%$ & $33.7 \%$ & $26.5 \%$ & $21.7 \%$ & $9.0 \%$ & $9.0 \%$ & $100.0 \%$ \\
\hline & \multirow{2}{*}{ high } & Count & 33 & 52 & 51 & 32 & 21 & 189 \\
\hline & & $\%$ & $17.5 \%$ & $27.5 \%$ & $27.0 \%$ & $16.9 \%$ & $11.1 \%$ & $100.0 \%$ \\
\hline & \multirow{2}{*}{ very high } & Count & 19 & 18 & 26 & 35 & 21 & 119 \\
\hline & & $\%$ & $16.0 \%$ & $15.1 \%$ & $21.8 \%$ & $29.4 \%$ & $17.6 \%$ & $100.0 \%$ \\
\hline \multirow{2}{*}{\multicolumn{2}{|c|}{ Total }} & Count & 137 & 137 & 123 & 86 & 65 & 548 \\
\hline & & $\%$ & $25.0 \%$ & $25.0 \%$ & $22.4 \%$ & $15.7 \%$ & $11.9 \%$ & $100.0 \%$ \\
\hline
\end{tabular}

Chi-square $=68.63$ p-value $<.000$

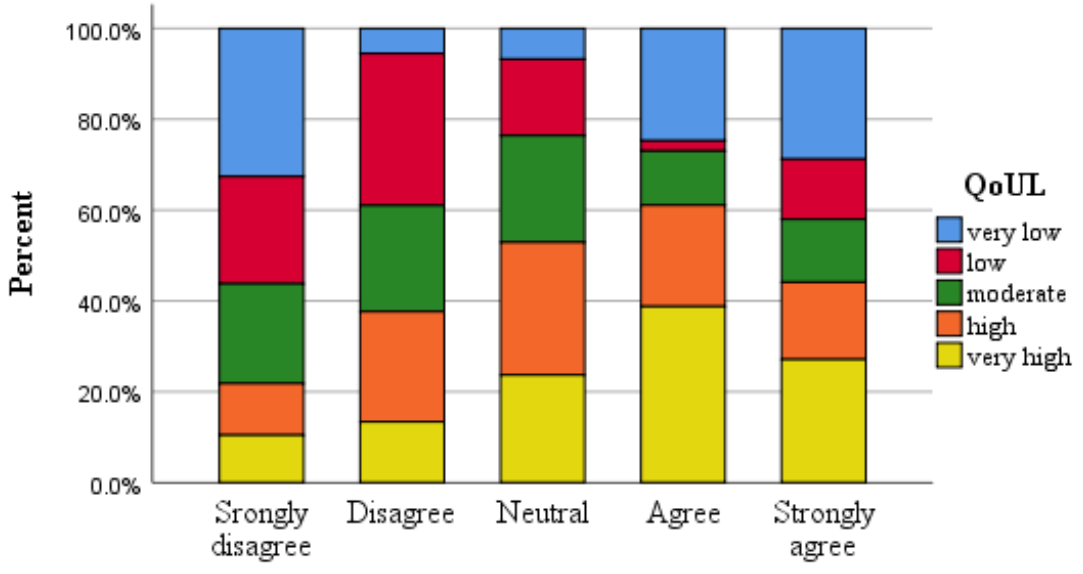

To what extent do you agree or disagree there is a good accessibility to leisure activities that are suitable for all groups of people (i.e. children, adults and the elderly people) in the municipality of Quba.

Fig 8.4: The relationship between accessibility to leisure activities and QoUL 
One possible explanation regarding these results is that the residents in Quba municipality may lack the means or activities that encourage leisure in the areas where they live, which indeed has been reflected in their QoUL, where findings indicate the negative impact of this social indicator. By referring to the objective (i.e., secondary data) indicators related to the leisure and recreation aspects, it can be said that recreational facilities such as walkways and parks as well as recreation centres in the municipality of Quba may not be enough to encourage people to spend their leisure time there, except for a few parks. This is supported by an argument made by one of the experts who referred to leisure in the following way:

Nowadays, leisure has become an important and necessary aspect of life. However, the urban environment in Medina generally lacks the means by which residents can spend their free time, especially within the neighbourhoods. Hence, there is a need to create more and better places to encourage leisure, as doing so may enhance other social aspects such as security and social interaction. (Khalid Alsaidalani, an expert)

The above quotation highlights how the availability and quality of places that enable residents to spend their spare time within the neighbourhoods not only enhance leisure aspects, but also may contribute to enhancing other aspects of the QoUL. In this regard, this expert stress the importance of paying more attention to improving the infrastructure that facilitates accessibility to such activities.

With regard to the relationship between leisure activities and socio- demographic attributes of the participants, using Kruskal-Wallis and Mann-Whitney tests, only employment was found to be significantly associated with accessibility to leisure activities. Using crosstabulation, however, the result indicates a small significant difference within the participants' responses. Employed participants seem to mostly disagree that there is good accessibility to leisure activities, where about $54 \%$ of them strongly disagree or disagree that there is good accessibility to leisure activities. This may be because employees often spend their time working, and therefore, they may not pay attention to the activities related to leisure. 


\subsubsection{Social interaction and QoUL}

The community to which individuals belong greatly affects their perception and expectations about the environment in which they live, where that community plays an important role in determining one's QoUL (Datta et al., 2015). In this context, social interaction has been considered as a key element of communities (Marans \& Rodgers, 1979; Riger \& Lavrakas, 1981). However, in the QoUL literature, social interaction has not been adequately examined in relation to its impact on the QoUL level. A few studies were found on the study of social interaction's role in QoUL in later life. For example, Datta et al. (2015) examined the impact of this indicator on the QoL of the elderly. In addition, Van den Berg and Timmermans (2015) investigated the effect of socio-demographic and neighbourhood characteristics on social interaction. Nevertheless, it is still unclear how this social indicator contributes to the QoUL. In this chapter therefore, social interaction is highlighted as a social indicator whether it has a negative or positive influence on the QoUL of Quba's residents.

In this thesis, social interaction and its role in determining QoUL in Quba municipality was investigated using an integration of quantitative and qualitative methods. In the quantitative strands, the results have shown a significant association between the amount of social interaction with neighbours and QoUL, which is represented in Table 8.4. It can be noted from this result that a positive QoUL level is associated with a high amount of social interaction with neighbours, where participants who have a very high amount of social interaction stated that their QoUL is high or very high. In addition, QoUL seemed to be moderate when the amount of social interaction with neighbours is moderate. In general, the QoUL level gradually increases with the increase of the amount of social interaction with neighbours, which is clearly shown in Figure 8.5. In this context, social interaction amongst Quba residents takes several forms, whether indoor or outdoor. This includes exchange of visits between neighbours, greeting and, talking to each other outdoors or sitting together outdoors. In addition, social interaction may extend beyond the circle of neighbours to include interaction with relatives, friends or co-workers. 
Table 8.4: Association between amount of social interaction with neighbours and QoUL level

\begin{tabular}{|c|c|c|c|c|c|c|c|c|}
\hline & & & \multicolumn{5}{|c|}{ QoUL } & \multirow{2}{*}{ Total } \\
\hline & & & very low & low & moderate & high & very high & \\
\hline \multirow{10}{*}{$\begin{array}{l}\text { Amount of social } \\
\text { interaction with } \\
\text { neighbours }\end{array}$} & \multirow{2}{*}{ Not at all } & Count & 6 & 11 & 24 & 20 & 17 & 78 \\
\hline & & $\%$ & $7.7 \%$ & $14.1 \%$ & $30.8 \%$ & $25.6 \%$ & $21.8 \%$ & $100.0 \%$ \\
\hline & \multirow{2}{*}{ A little } & Count & 2 & 14 & 35 & 34 & 10 & 95 \\
\hline & & $\%$ & $2.1 \%$ & $14.7 \%$ & $36.8 \%$ & $35.8 \%$ & $10.5 \%$ & $100.0 \%$ \\
\hline & \multirow{2}{*}{$\begin{array}{c}\text { A moderate } \\
\text { amount }\end{array}$} & Count & 2 & 18 & 55 & 65 & 32 & 172 \\
\hline & & $\%$ & $1.2 \%$ & $10.5 \%$ & $32.0 \%$ & $37.8 \%$ & $18.6 \%$ & $100.0 \%$ \\
\hline & \multirow{2}{*}{ Very much } & Count & 5 & 12 & 40 & 60 & 43 & 160 \\
\hline & & $\%$ & $3.1 \%$ & $7.5 \%$ & $25.0 \%$ & $37.5 \%$ & $26.9 \%$ & $100.0 \%$ \\
\hline & \multirow{2}{*}{$\begin{array}{c}\text { An extreme } \\
\text { amount }\end{array}$} & Count & 1 & 3 & 12 & 10 & 17 & 43 \\
\hline & & $\%$ & $2.3 \%$ & $7.0 \%$ & $27.9 \%$ & $23.3 \%$ & $39.5 \%$ & $100.0 \%$ \\
\hline \multirow{2}{*}{\multicolumn{2}{|c|}{ Total }} & Count & 16 & 58 & 166 & 189 & 119 & 548 \\
\hline & & $\%$ & $2.9 \%$ & $10.6 \%$ & $30.3 \%$ & $34.5 \%$ & $21.7 \%$ & $100.0 \%$ \\
\hline
\end{tabular}

Chi-square $=32.94$ p-value $=.005$

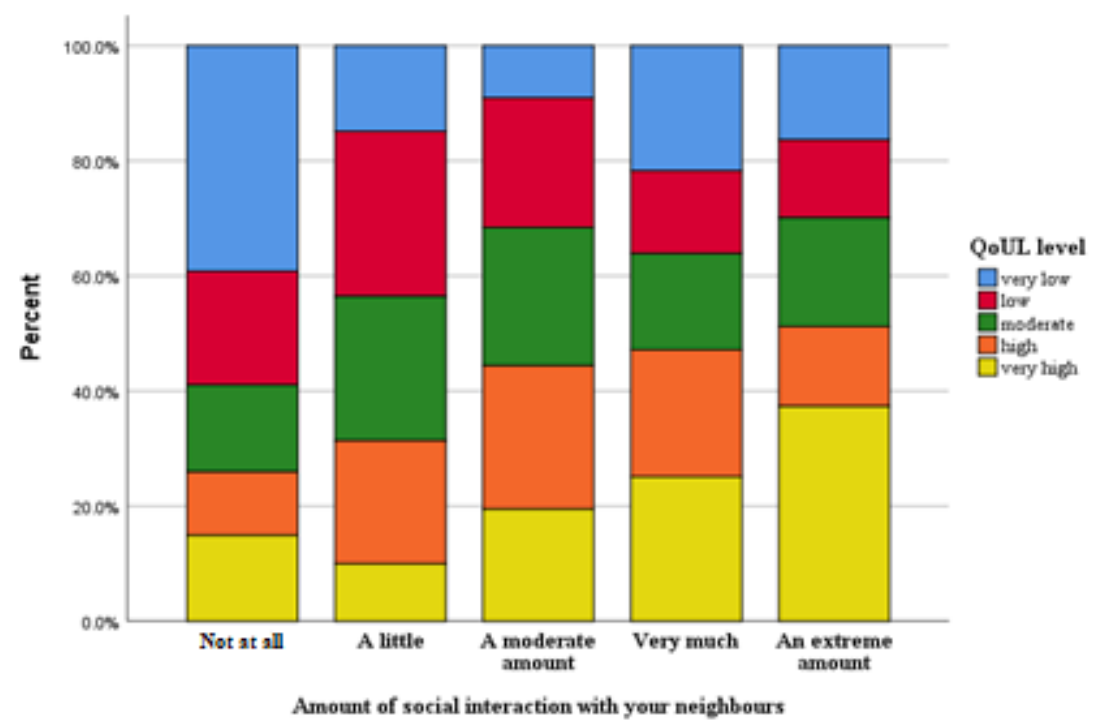

Fig 8.5: Association between amount of social interaction with neighbours and QoUL

Social interaction seems to play a clear role in determining the QoUL in Quba based on the above results, as the highest level of QoUL was associated with the highest amount of social interaction with neighbours. Therefore, it can be said that this indicator positively affects the QoUL of Quba residents. In this regard, photo diaries and follow-up interviews were employed for further exploration regarding the relationship between social interaction and 
QoUL. From this qualitative data, it seems that social interaction with neighbours is an important indicator that may enhance the QoUL for those living in Quba. As one of the participants within photo diaries study stated, interaction with neighbours is an important aspect of his life:

For me, social interaction with neighbours can lead to building good relations with neighbours. This in turn enhances many aspects of our lives, including support as well as the sense of happiness. I believe that having a social interaction with the neighbours is important to us because the neighbour is closest to us to take care and to help if needed. (PH 1, Male, 43 years old)

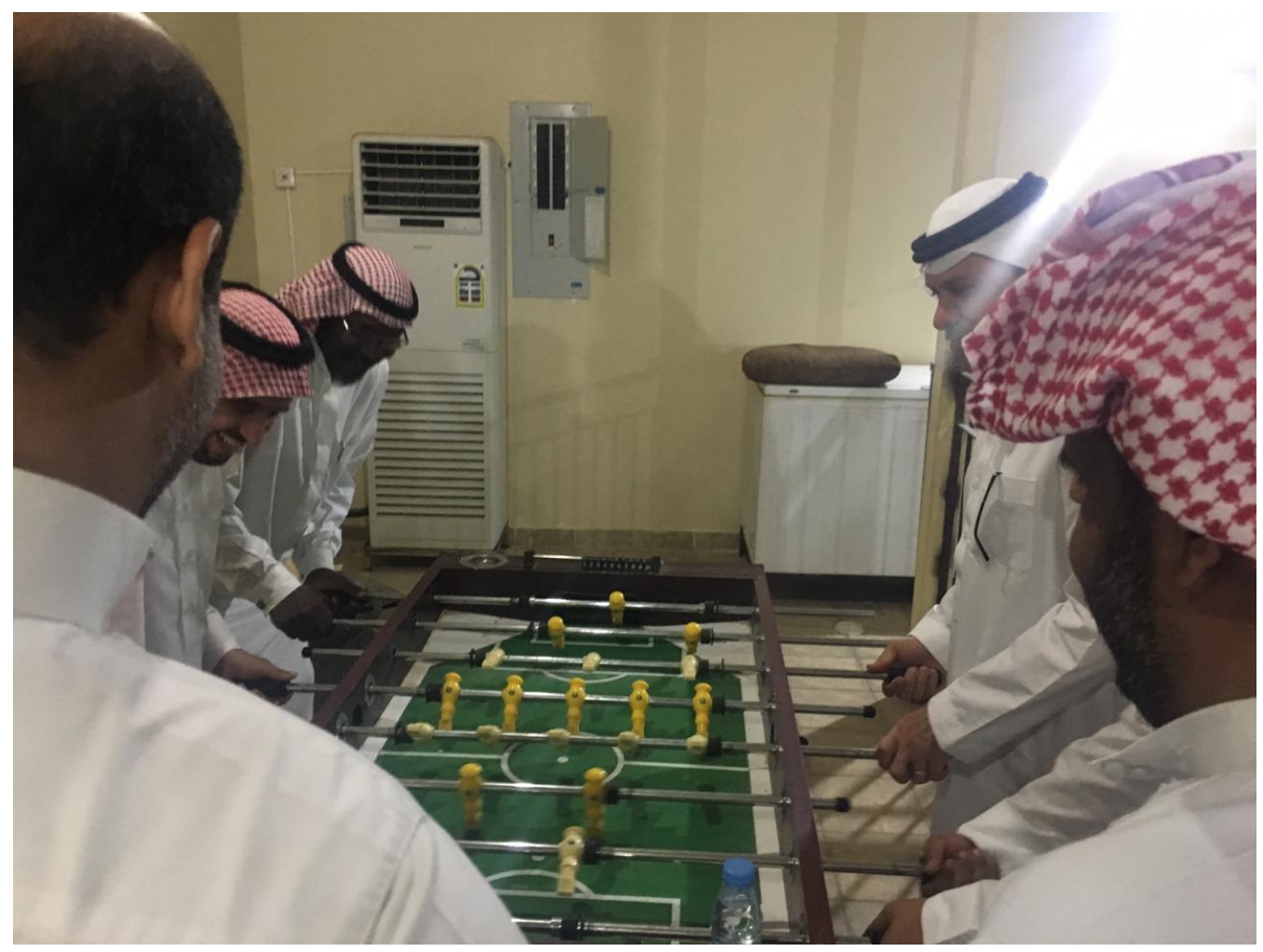

Fig 8.6: One of the social interaction pictures. Photo diary by participant PH 1.

The benefit of being interactive with neighbours mentioned in the statement above is supported by the study of Bridge (2002), as cited in Van den Berg \& Timmermans (2015) who refer to neighbours similarly: "many people still value contact with their neighbours, as they are often a source of assistance" (p. 56). In the Saudi context, caring for the neighbour not only stems from custom or traditions, but rather from religious teachings that 
emphasise caring for neighbours and helping them in times of need, showing the importance of communication and interaction with them. This connection led to further investigation regarding the relationship between social interaction with neighbours and social support from neighbours. In this regard, Spearman's rho test was employed in order to measure the strength of the association between social interaction and social support. The results have shown a strong positive correlation between social interaction and social support, as illustrated in Table 8.5. Figure 8.7 shows that the amount of support from neighbours is likely to increase with the increase of the amount of social interaction with neighbours. This indicates how important social support is for the QoUL of those living in Quba. With regard to social support, several studies in the literature stress that it is associated with many benefits related to mental health, moderating life stress and thus improving the QoL (Helgeson, 2003). Moreover, people tend be more satisfied with their lives when they feel a higher level of social support (Yarcheski et al.,1994).

Table 8.5: Correlation between social interaction and social support. Spearman's rho.

\begin{tabular}{|c|c|c|c|c|}
\hline & & & & $\begin{array}{l}\text { How would you describe your social } \\
\text { interaction with your neighbours in the } \\
\text { neighbourhood you live in? }\end{array}$ \\
\hline \multirow[t]{4}{*}{ Spearman's rho } & \multirow{4}{*}{$\begin{array}{l}\text { To what extent do your } \\
\text { neighbours support you } \\
\text { when you need them? }\end{array}$} & Correlation & 1.000 & \multirow[t]{2}{*}{$.566^{*}$} \\
\hline & & Coefficient & & \\
\hline & & Sig. (2-tailed) & . & .000 \\
\hline & & $\mathrm{N}$ & 548 & 548 \\
\hline
\end{tabular}

\footnotetext{
*. Correlation is significant at the 0.05 level (2-tailed).
} 


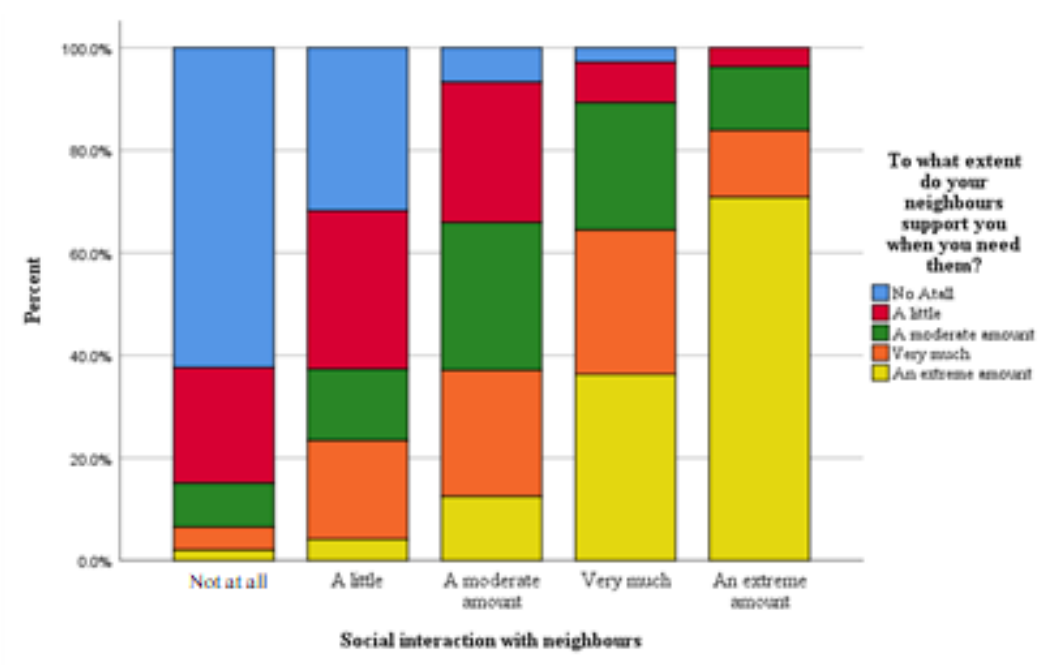

Fig 8.7: Relationship between social interaction and social support

Logic may, however, indicate that not all people are happy with the neighbourly relationship, or that someone who interacts with their neighbour does not necessarily receive social support. In this sense, some people may prefer not to interact with their neighbours to avoid any negative consequences that may result from that relationship. That is, social interaction is not always positive. In her study on the dark side of social interaction conducted in Los Angeles, Rook (1984) noted that "negative social interactions have more potent effects on well-being than positive social interactions" (p. 1106). Negative interactions may include such actions as interfering in others' affairs or invading their privacy, among others (Lincoln, 2000). Thus, such negative aspects may have more pronounced negative effects on the QoUL than the positive outcomes, such as practical social support. Although in reviewing the literature, it can be observed that most studies in social relations often ignore the dark side of social interaction. Both sides of social interaction (positive and negative) in the relation to the QoUL therefore, may require further research.

Regarding the effect of socio-demographic attributes on the participants' perception regarding social interaction, only education and the number of people living at home were found to be significantly associated with social interaction. In this regard, cross-tabulation was applied to further explore those significant relationships. For education, the result shows that respondents with lower education level are likely to interact more with their neighbours, where the social interaction is high within primary and secondary levels, and then gradually 
decreases as it reaches the highest level of education, as illustrated in Figure 8.8. With reference to the literature, this result is consistent with the study of Van den Berg and Timmermans (2015) who found that less educated people interact more with neighbours, while people with higher education interact less. For the number of people living at home, the results show that households with more people are interacting more with their neighbours, where the amount of social interaction increases with the increase of the number of people living at home (see Figure 8.9). This means that families with more dependents are more interactive with their neighbours. Here children may be a key element in the relationship with neighbours, where they are likely to lead to acquaintance between neighbours and thus interaction. This result is in line with Van den Berg and Timmermans (2015) who found that people with more children are more likely to interact with neighbours.

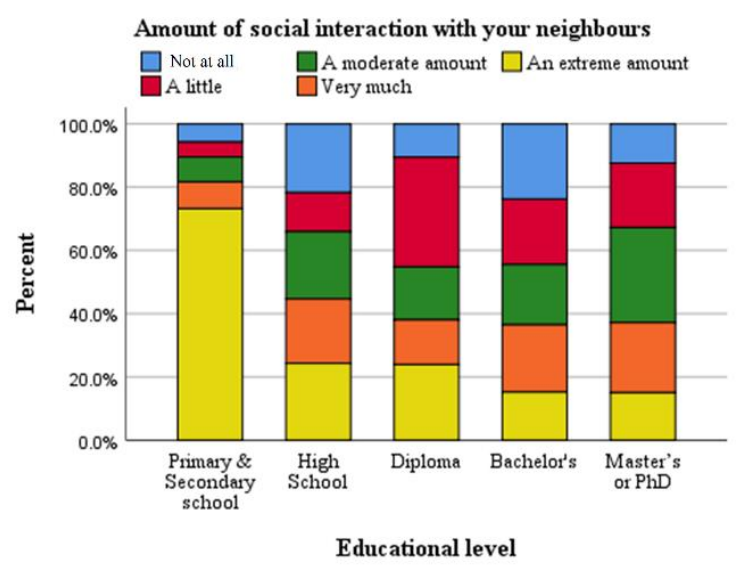

Fig 8.8: Education level vs. social interaction

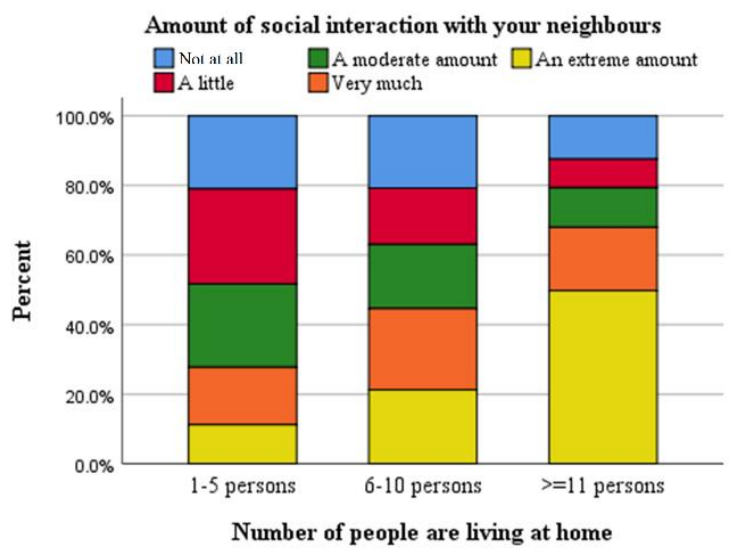

Fig 8.9: No. people at home vs. social interaction

As a part of this area of investigation in relation to the QoUL, satisfaction with neighbours was also highlighted. In this regard, the Chi-square test was employed to find any association between satisfaction with neighbours and QoUL. The results indicate a significant association between QoUL level and satisfaction with neighbours. It can be noted from the results in Table 8.6 that satisfaction with neighbours positively affects the QoUL. Here, the QoUL level increases with the increase of satisfaction degree with neighbours, where $69.4 \%$ of participants who stated that their QoUL is high or very high are satisfied or very satisfied with their neighbours. Figure 8.10 clearly shows this relationship. 
Table 8.6: The association between satisfaction with neighbours and QoUL level

\begin{tabular}{|c|c|c|c|c|c|c|c|c|}
\hline & & & \multicolumn{5}{|c|}{ QoUL level } & \multirow{2}{*}{ Total } \\
\hline & & & very low & low & moderate & high & very high & \\
\hline \multirow{10}{*}{$\begin{array}{c}\text { Satisfaction with } \\
\text { neighbours }\end{array}$} & \multirow{2}{*}{$\begin{array}{c}\text { Very } \\
\text { dissatisfied }\end{array}$} & Count & 3 & 3 & 4 & 5 & 4 & 19 \\
\hline & & $\%$ & $15.8 \%$ & $15.8 \%$ & $21.1 \%$ & $26.3 \%$ & $21.1 \%$ & $100.0 \%$ \\
\hline & \multirow{2}{*}{ Dissatisfied } & Count & 4 & 7 & 11 & 7 & 4 & 33 \\
\hline & & $\%$ & $12.1 \%$ & $21.2 \%$ & $33.3 \%$ & $21.2 \%$ & $12.1 \%$ & $100.0 \%$ \\
\hline & \multirow{2}{*}{ Neutral } & Count & 1 & 24 & 65 & 48 & 27 & 165 \\
\hline & & $\%$ & $0.6 \%$ & $14.5 \%$ & $39.4 \%$ & $29.1 \%$ & $16.4 \%$ & $100.0 \%$ \\
\hline & \multirow{2}{*}{ Satisfied } & Count & 4 & 20 & 49 & 80 & 31 & 184 \\
\hline & & $\%$ & $2.2 \%$ & $10.9 \%$ & $26.6 \%$ & $43.5 \%$ & $16.8 \%$ & $100.0 \%$ \\
\hline & \multirow{2}{*}{ Very Satisfied } & Count & 4 & 4 & 37 & 49 & 53 & 147 \\
\hline & & $\%$ & $2.7 \%$ & $2.7 \%$ & $25.2 \%$ & $33.3 \%$ & $36.1 \%$ & $100.0 \%$ \\
\hline \multirow{2}{*}{\multicolumn{2}{|c|}{ Total }} & Count & 16 & 58 & 166 & 189 & 119 & 548 \\
\hline & & $\%$ & $2.9 \%$ & $10.6 \%$ & $30.3 \%$ & $34.5 \%$ & $21.7 \%$ & $100.0 \%$ \\
\hline
\end{tabular}

Chi-square $=65.98$ p-value $=.005$

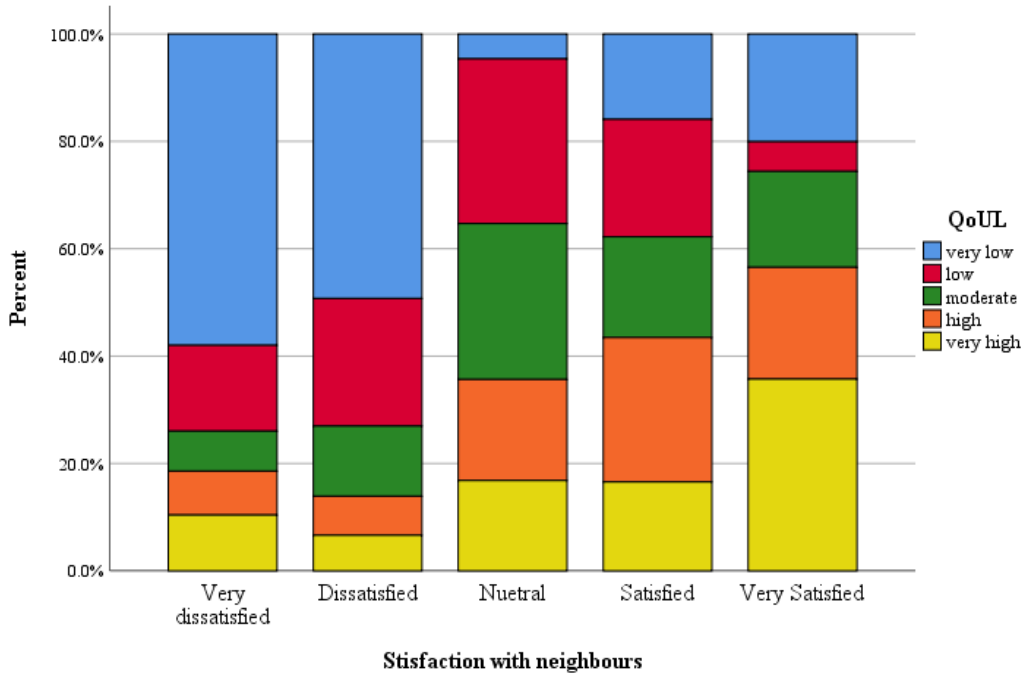

Fig 8.10: Relationship between satisfaction with neighbours and QoUL

\subsubsection{Religion/spirituality and QoUL}

Religion and spirituality are part of the social life of many societies around the world. Religion remains today of profound significance to most of the world's inhabitants (Peterson \& Webb, 2006). In this respect, it has been argued in the literature that religion and spirituality are often associated with higher levels of SWB (Lun \& Bond, 2013), and evidence presented to support the hypothesis that religion, religiosity, spirituality and 
religious beliefs positively affect the QoL (e.g., Ferriss, 2002; Diener et al., 1999; Sawatzky et al., 2005). These studies have confirmed that involvement in religious and spiritual activities promotes higher levels of QoL, where it may provide psychological and social benefits. Since religion and spiritualty and QoUL in general have not been addressed in the context of Saudi Arabia and Medina in particular, this thesis investigated religion and spiritualty and their contribution to the QoUL in Quba municipality using indicators concerning religious beliefs and practices, the sense of spirituality and the frequency of attendance at the religious centres. In addition, the relationship between perception of those indicators and the socio-demographic attributes of the participants was examined.

The effect of religion and spirituality on the QoUL in the study area was examined using multiple statistical tests including the Chi-square and Spearman's rho correlation in order to find any association between these variables. Moreover, the results were integrated with the qualitative results in some cases. Using the Chi-square test, there was significant relationship between the extent to which spiritual/religious beliefs can help to improve QoUL and QoUL level. It can be noted from Table 8.7 that QoUL increases with the increase of reporting very much amount in terms of the way that spiritual/religious beliefs can help to improve QoUL and vice versa. In this regard, 58.2\% of those who reporting a high QoUL stated that spiritual/ religious beliefs can help to improve their QoUL. From this result, it can be said that spiritual/religious beliefs are likely to contribute to the QoUL of Quba's residents in a positive way.

Table 8.7: Relationship between the extent to which spiritual/religious beliefs can help to improve QoUL and QoUL level

\begin{tabular}{|c|c|c|c|c|c|c|}
\hline & & & \multicolumn{4}{|c|}{ QoUL level } \\
\hline & & & low & moderate & high & Total \\
\hline \multirow{6}{*}{$\begin{array}{l}\text { The extent in which } \\
\text { spiritual/ religious } \\
\text { beliefs can help to } \\
\text { improve QoUL }\end{array}$} & \multirow{2}{*}{ A little } & Count & 10 & 5 & 3 & 18 \\
\hline & & $\%$ & $55.5 \%$ & $27.7 \%$ & $16.6 \%$ & $100.0 \%$ \\
\hline & \multirow{2}{*}{$\begin{array}{l}\text { A moderate } \\
\text { amount }\end{array}$} & Count & 9 & 28 & 41 & 78 \\
\hline & & $\%$ & $11.5 \%$ & $35.9 \%$ & $52.6 \%$ & $100.0 \%$ \\
\hline & \multirow{2}{*}{ Very much } & Count & 56 & 133 & 263 & 452 \\
\hline & & $\%$ & $12.4 \%$ & $29.4 \%$ & $58.2 \%$ & $100.0 \%$ \\
\hline \multirow{2}{*}{\multicolumn{2}{|c|}{ Total }} & Count & 75 & 166 & 307 & 548 \\
\hline & & $\%$ & $13.6 \%$ & $30.2 \%$ & $56 \%$ & $100.0 \%$ \\
\hline
\end{tabular}

Chi-square $=15.745, \mathrm{p}$-value $=.003$ 
Religiosity may be one of the possible explanations for the relationship between QoUL and religious beliefs in the context of Quba. In this regard, Lun and Bond (2013) pointed out that the relationship between religiosity and life satisfaction is stronger in societies with a higher level of religiosity, while Kim-Prieto and Miller (2018) note that in cultures with high levels of religiosity, religion has a greater impact on SWB compared to cultures with low religiosity. In addition to these interpretations, the place might play an important role in this kind of relationship, where Medina and Quba in particular are the most sacred places after Mecca. In this context, Dewsbury and Cloke (2009) note that there are many ways in which spirituality manifests itself. One of these ways is meditative practices of walking through spiritually important places (such as places of worship), daily pilgrimage, prayer and engaging with what Cloke and Dewsbury (2009) describe as 'spiritual landscapes', such as the key spiritual places in Medina, and the manner in which these influence people's spiritual life and potentially positively affect their QoUL.

For further exploration, the relationship between a sense of spirituality of the place and QoUL was examined using the Chi-square test. The results show a significant relationship between these two variables. It can be noted from Table 8.8 that the sense of the spirituality of place positively affects QoUL, where $57.5 \%$ of participants who agreed that the sense spirituality of place enhancing their QoUL reported a high QoUL.

Table 8.8: The relationship between the sense of the spirituality of place and QoUL level

\begin{tabular}{|c|c|c|c|c|c|c|}
\hline & & & \multicolumn{4}{|c|}{ QoUL level } \\
\hline & & & low & moderate & high & Total \\
\hline \multirow{6}{*}{$\begin{array}{l}\text { Agreement degree that } \\
\text { sense spirituality of } \\
\text { place enhances QoUL }\end{array}$} & \multirow{2}{*}{ Disagree } & Count & 11 & 15 & 14 & 40 \\
\hline & & $\%$ & $27.5 \%$ & $37.5 \%$ & $35.0 \%$ & $100.0 \%$ \\
\hline & \multirow{2}{*}{ Neutral } & Count & 11 & 16 & 41 & 68 \\
\hline & & $\%$ & $16.2 \%$ & $23.5 \%$ & $60.3 \%$ & $100.0 \%$ \\
\hline & \multirow{2}{*}{ Agree } & Count & 52 & 135 & 253 & 440 \\
\hline & & $\%$ & $11.8 \%$ & $30.7 \%$ & $57.5 \%$ & $100.0 \%$ \\
\hline \multirow{2}{*}{ Total } & & Count & 74 & 166 & 308 & 548 \\
\hline & & $\%$ & $13.5 \%$ & $30.3 \%$ & $56.2 \%$ & $100.0 \%$ \\
\hline
\end{tabular}

Chi-square $=12.357, \mathrm{p}$-value $=.015$ 
The sense of the spirituality of the place for the inhabitants of Quba comes from the fact that they live in a place where the Islamic religion was originated and spread to the rest of the world. Moreover, and more importantly, the area in which the municipality of Quba is located is characterised by many religious monuments starting from the Prophet's Mosque and the tomb of the Prophet Muhammad (PBUH) through the Durb Alsunnah ${ }^{8}$ (the route of Quba), Al Jum'ah Mosque and then Quba Mosque, as well as by the presence of many holy wells associated with the biography of the Prophet Muhammad (PBUH). The combination of those religious sites and monuments is likely to enhance the sense of the spirituality of place which may contribute to QoUL. For example, participant PH 11 referred to where she lives and the extent to which spirituality affects her life in the following words:

Medina is a place full of spirituality, and this makes me feel calm and reassured in my daily life, which I may not find elsewhere. I may not be interested with the material things as much as I care about this spirituality. This is because spirituality is a thing which I consider as the key element of the place where I live. (PH 11, Female, 41 yrs)

\footnotetext{
${ }^{8}$ Durb Alsunnah is a walking path that connects the Prophet's Mosque to the Quba Mosque. It is a project recently created by the government to commemorate the Prophet Muhammad (PBUH) and the route he used to walk between the two Mosques (MDA, 2018).
} 


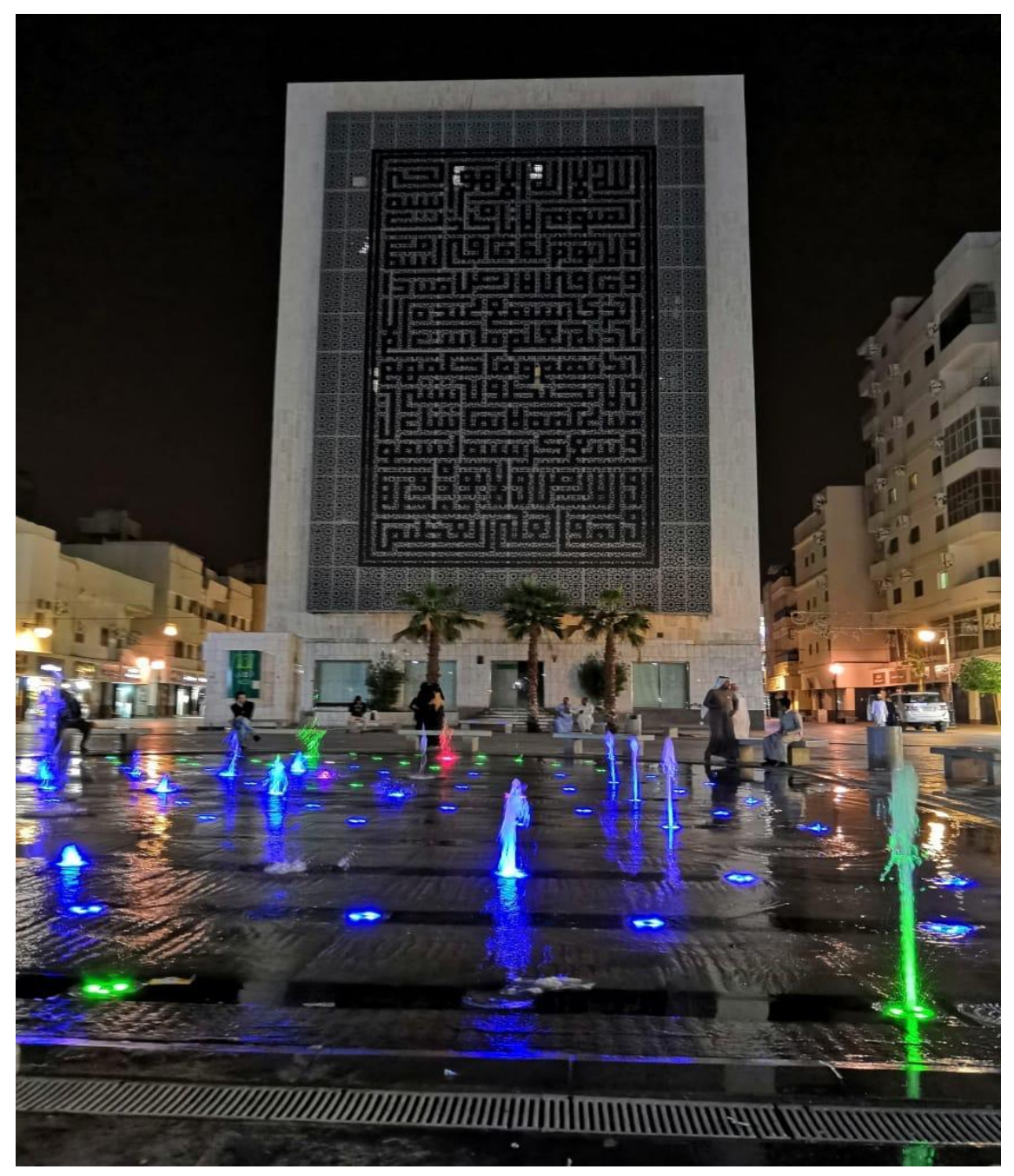

Fig 8.11: Quba square and the beginning of the Quba route. This photo shows a complete Qur'an verse written on a public building. Photo diary by participant PH11. 
The picture in Figure 8.11 reflects the spirituality that distinguishes the place according to the participant PH 11, where it can be noted that there is one of the Holy Qur'an verses written across the whole building. It can be concluded from the above quotation that regardless of the aesthetics of the place, or even its richness in material aspects, spirituality may promote place attachment as well as satisfaction with life. In this context, the presence of the things that symbolise religion, even if they are in public places, as in the above image, brings people back to the sense of faith and spirituality. Thus, a sense of the presence of such religious symbols may enhance place attachment for those living in Quba. Another participant, asked about the extent to which spiritualty affected her life, gave the following reply:

Spirituality gives me a strong incentive to live for the diligence and work, especially to face the hardships of life. For example, I may face some problems and difficulties from life, but when I visit the mosque and perform religious duties, I could get rid of some of the concerns associated with these problems as well as the amount of happiness that I can get. (PH 19 , Female, 45 yrs) 


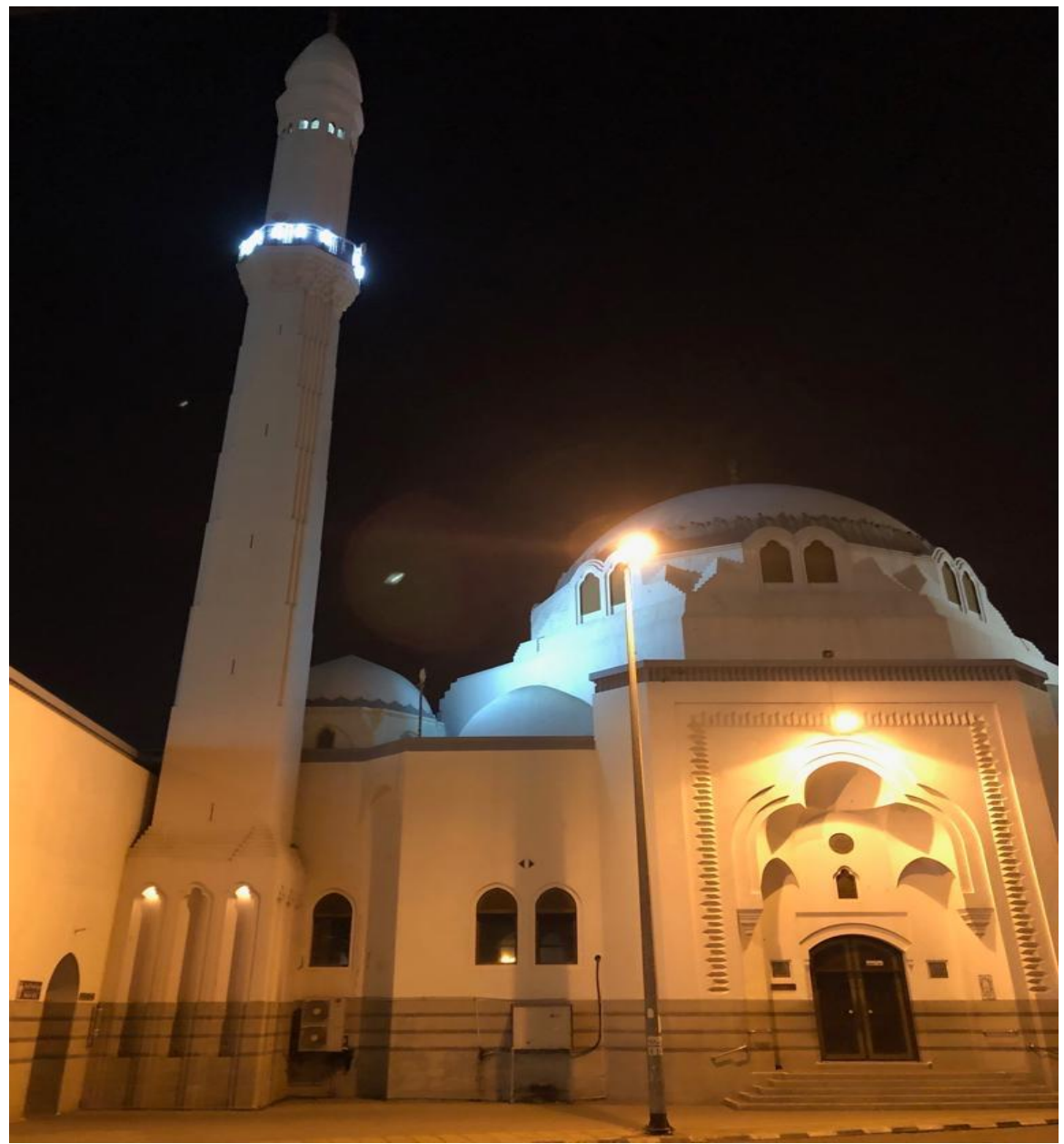

Fig 8.12: Al Jum'ah Mosque, one of the historical mosques in Quba, where the first Jumu'ah prayer (Friday prayer) was held. Photo diary by participant PH 19.

In addition to the spirituality of place that distinguishes Medina, the religious practices through which spirituality is experienced may help people overcome life's difficulties and thus feel happy and satisfied with life, as is suggested by the argument made by participant PH 19. This is supported by the quantitative results which indicated a positive impact of spirituality on the residents' lives. These findings are in line with the work of Magalhaes et al. (2015) who investigated the impact of spirituality and religion on QoL in Fortaleza, Brazil. In this regard, Magalhaes et al. (2015) point out how spinal cord injury (SCI) patients relied on spirituality, religiosity, and beliefs to cope with the disease. Moreover, these authors note that patients with SCI use many feelings of optimism, hope, and faith to face life's difficulties. Findings from this thesis also are in line with the work of Al-Naaimi and Younis (2010) who studied the religious and spiritual beliefs dimension of life quality in 
Baghdad, Iraq. Those authors found that religious and spiritual beliefs positively contribute to the QoL.

For further exploration regarding the effect of religion on QoUL, the relationship between QoUL and the extent to which religious practices and beliefs have an impact on improving QoUL was also examined using the Chi-square test. The results indicated a significant association between QoUL and the extent to which religious practices and beliefs have an impact on improving QoUL. Table 8.9 presents this kind of relationship by cross-tabulating religious practices and beliefs and QoUL. It can be seen from this table that participants with strong agreement that religious practices and beliefs have an impact on improving QoUL tend to report a positive QoUL level, where $57.4 \%$ of those who agreed that religious practices and beliefs have an impact on improving their QoUL have reported a high QoUL, while significantly fewer participants who reported a positive QoUL strongly disagreed that religious practices and beliefs have an impact on improving QoUL. Figure 8.13 clearly presents this relationship, where QoUL tends to be high when the agreement level that that religious practices and beliefs have an impact on improving QoUL is high.

Table 8.9: Agreement level that religious practices and beliefs have an impact on improving QoUL and QoUL

\begin{tabular}{|c|c|c|c|c|c|c|}
\hline & & & \multicolumn{4}{|c|}{ QoUL level } \\
\hline & & & low & moderate & high & Total \\
\hline \multirow{6}{*}{$\begin{array}{l}\text { Agreement level that } \\
\text { religious practices and } \\
\text { beliefs have an impact on } \\
\text { improving QoUL }\end{array}$} & \multirow{2}{*}{ Disagree } & Count & 6 & 3 & 7 & 16 \\
\hline & & $\%$ & $37.5 \%$ & $18.8 \%$ & $43.8 \%$ & $100.0 \%$ \\
\hline & \multirow{2}{*}{ Neutral } & Count & 2 & 15 & 13 & 30 \\
\hline & & $\%$ & $6.7 \%$ & $50.0 \%$ & $43.3 \%$ & $100.0 \%$ \\
\hline & \multirow{2}{*}{ Agree } & Count & 66 & 148 & 288 & 502 \\
\hline & & $\%$ & $13.1 \%$ & $29.5 \%$ & $57.4 \%$ & $100.0 \%$ \\
\hline \multirow{2}{*}{\multicolumn{2}{|c|}{ Total }} & Count & 74 & 166 & 308 & 548 \\
\hline & & $\%$ & $13.5 \%$ & $30.3 \%$ & $56.2 \%$ & $100.0 \%$ \\
\hline
\end{tabular}

Chi-square $=14.015, \mathrm{p}$-value $=.007$ 


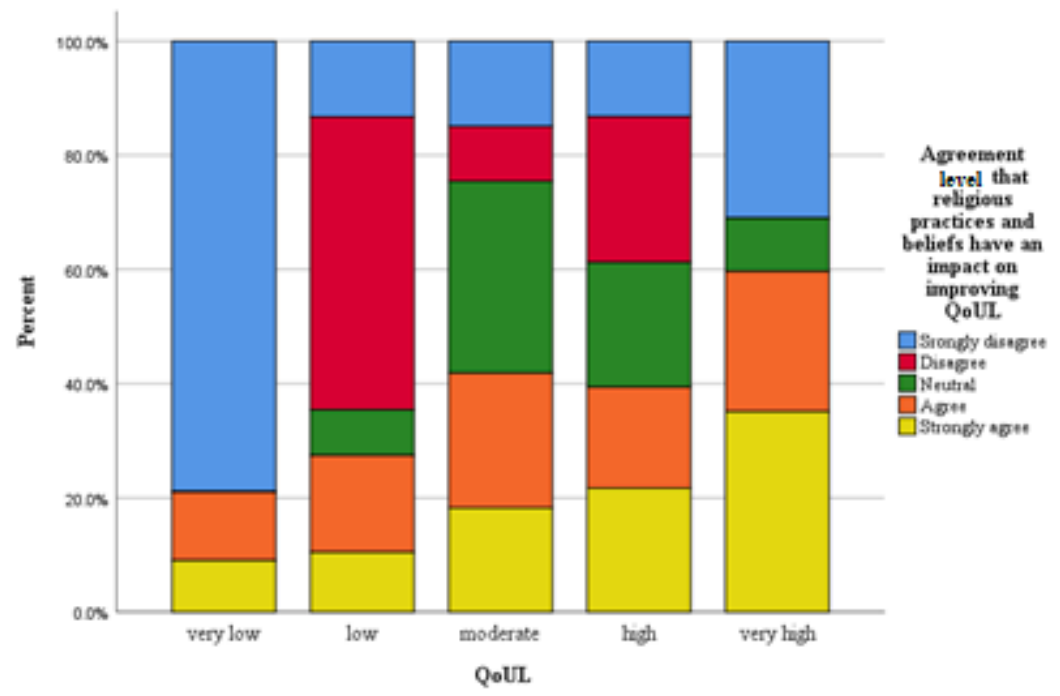

Fig 8.13: Relationship between QoUL and the agreement level that religious practices and beliefs have an impact on improving QoUL

The results of this thesis are consistent with other studies (e.g., Siegel \& Schrimshaw, 2002; Sinnewe et al., 2015,) which confirmed that there is a positive relationship between religious beliefs and practices and many aspects of life that are considered in this thesis as aspects of QoUL. These include the effects of spirituality on the improvement of physical and mental health, lower rates of anxiety and depression as well as higher levels of life satisfaction. Furthermore, a number of studies in the literature found religious practice, including attending religious services, is positively related to SWB (e.g., Hadaway, 1978; Diener et al., 1999; Ellison, 1991; Ferriss, 2002). In this context, the frequent attendance at a religious centre may expose people to religious teachings and such teachings may promote a positive outlook on life, which in turn promotes both happiness and SWB (Jung, 2013).

In this thesis, the nature of religious practice and beliefs may differ, except the attendance at religious services, where the purposes of attendance across traditional religions is mostly to pray. Therefore, religious practices in this thesis are examined using the attendance at religious centres as an indicator to gain insight into the impact of them on the QoUL in the study area. In addition, other religious practices and beliefs in the Islamic culture are highlighted using a combination of quantitative and qualitative methods. Using Spearman's rho test, correlation was made between frequent attendance at the mosque and QoUL. Results in Table 8.10 shows that QoUL is positively correlated with attendance at the 
mosque. This result is in line with many studies in literature both in Islamic contexts and other cultural contexts. For example, Levin and Chatters (1998), Ellison et al. (2001), Ferriss (2002), Helliwell and Putnam (2005), Lim and Putnam (2010) found that frequency of attendance at religious centres is positively correlated with life satisfaction and well-being. As mentioned in the literature review, however, those studies were conducted in Western countries, where they mostly focused on the Christian religion. Within the Islamic context, similar results have been obtained by a number of studies such as Al-Kandari, (2003), Baroun, (2006), Abdel-Khalek and Lester, (2007), Abdel-Khalek, (2008), Tiliouine and Belgoumidi, (2009), Al-Naaimi and Younis, (2010), Abdel-Khalek, (2011), Abdel-Khalek and Eid, (2011), and Hossain and Rizvi, (2016). In general, these studies have confirmed a presence of a positive relationship between religiosity and SWB including happiness and life satisfaction. However, these studies suffered from some limitations as mentioned in Chapter 2.

Table 8.10: Correlation between frequent attendance at the Mosque and QoUL. Spearman's rho.

\begin{tabular}{|c|c|c|c|}
\hline & & & Are you frequently attending the Mosque? \\
\hline \multirow[t]{3}{*}{ Spearman's rho } & \multirow[t]{3}{*}{ QoUL level } & Correlation Coefficient & $.093^{*}$ \\
\hline & & Sig. (2-tailed) & .030 \\
\hline & & $\mathrm{N}$ & 548 \\
\hline
\end{tabular}

*. Correlation is significant at the 0.05 level (2-tailed).

In this thesis, qualitative research using photo diaries and follow-up interviews, as well as interviews with experts were employed for further exploration regarding the effect of religion on the QoUL in the study area. In addition, the results were supported by the literature, especially with regard to the nature of religious practices and beliefs in the Islamic context. During the phase of the photo diaries, the participants were asked to take photographs about their daily experience with regard to spirituality and religion, followed by interviews in order to gain more insight into how these components contribute to their QoUL. Through the process of coding and thematic analysis of data, it became clear that there were several key themes arising including spirituality and religion which clearly effected the perception of residents of Quba regarding their QoUL. Those themes were translated into some of the photographs that have been taken by the participants, which mostly included the mosques and holy places. 
Within the social domain of QoUL, religion was predominantly the most important aspect affecting the QoUL of those living in Quba and Medina in general. The impact of religion on the QoUL of Quba's residents translates into religious practices and beliefs including prayers, especially through frequent attendance at the mosque and other practices such as fasting, zakat ${ }^{9}$ and hajj, as well as beliefs that have a direct impact on the psychology and behaviour of individuals. Frequent attendance at the mosque as one of the religious practices has been found to promote many aspects that can be linked to the QoUL including happiness, life satisfaction and social benefits such as the promotion of social integration between neighbours, where members of the neighbourhood meet more than once a day, which may encourage social interaction between these members. In this regard, the expert Mohammed Al-Shaibani indicates that the mosque has a clear role in strengthening social ties, where he refers to the role of the mosque as follows:

The mosque is not only for worship, but rather is a social centre through which members of the neighbourhood can meet, as it is possible to establish social activities that aim to enhance social ties between these members, such as acquaintance between individuals as well as helping those in need. However, an absence of the mosque's role in this regard can be observed in many mosques in Medina. Therefore, the social role of the mosque should be strengthened due to its importance in promoting these social aspects.

Here, the expert notes that the function of the mosque is not completely represented by worship only, but also includes holding meetings that may contribute to addressing some social issues such as social support, perhaps indicating that mosque's function and its influence is greater than being a place of worship. From the perspective of several participants of this study, frequent attendance at the mosque is also linked with improved social connections and opportunities to strengthen social cohesion and meet people from across the world. For example, PH 2 referred to the benefits of visiting Quba Mosque, saying,

\footnotetext{
${ }^{9}$ The word zakat refers to the obligatory charity in Islam (Al-Kindi, 2007). It is a kind of charitable practice that Islam calls upon its followers to do on the basis of accumulated wealth and is mandatory for all who are able to do so (Hossain, 2012).
} 
We used to go to the mosque not only for the religious aspect, but also for enjoyment, where we can meet relatives and friends, as it is considered for us a social centre. (PH 2, Male, 34 yrs)

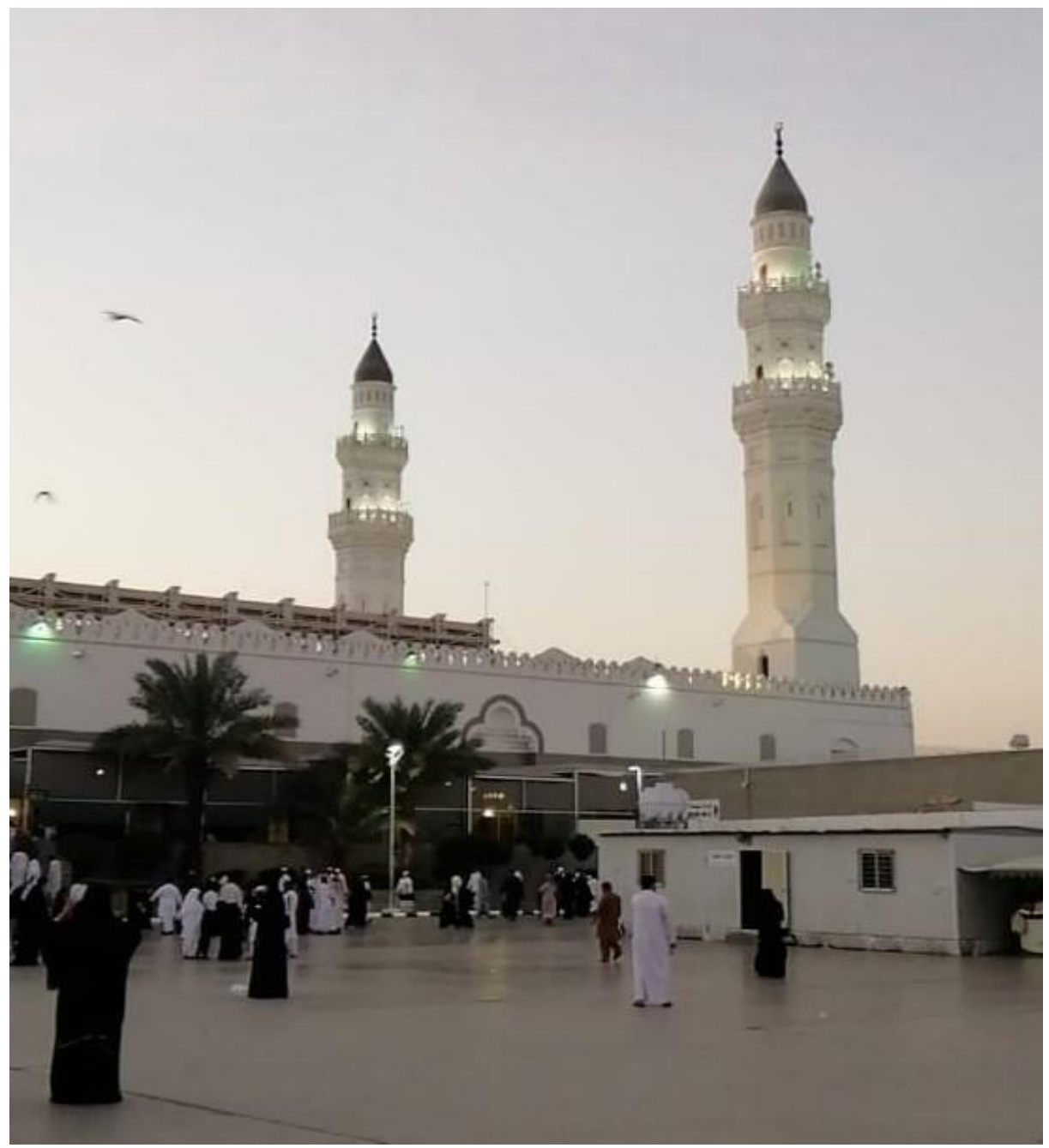

Fig 8.14: People in the squares surrounding Quba Mosque, talking to each other and taking pictures. Photo diary taken by participant $\mathrm{PH} 2$.

Figure 8.14 shows how visiting the mosque frequently may encourage meeting between people, relatives, neighbours or friends, which may promote social interaction through continuing communication between people. In addition to that, frequent attendance of the mosque may promote psychological benefits, as supported by participant PH 13 who indicated that frequent prayer at mosque promotes emotional and mental well-being, commenting that "the link with God Almighty through the prayer makes me see many things 
positively" (PH 13, Female, 46 yrs). Several participants also recall instances where they are able to see the same people five times a day for each prayer in the mosque. As such, if one person is suddenly absent, the congregation quickly notices and asks after or checks up on them, ensuring they are well. PH 20 summarises these instances, by stating that "a person who attends mosques for prayer five times a day will never be lonely" (PH 20, Female, 40 yrs).

For further exploration concerning the social benefits of frequent attendance at the mosque, the relationship between social interaction and frequent attendance was examined using cross-tabulation. The result shows a presence of significant association in this regard. It can be seen from Table 8.11 that $61.3 \%$ of those attending the mosque stated that they had a very high amount of social interaction with their neighbours compared to $38.8 \%$ of those with no attendance at the mosque. It is clearly noted that frequent attendance at the mosque promotes social interaction that may provide social support and friendship, which in turn enhances the QoUL. These findings can be supported by the findings of Lim and Putnam (2010) who suggest that religious people tend to be more satisfied with their life because they attend religious services regularly, which in turn helps them to build a relationship with others. According to Ellison and George (1994) people who attend churches report more extensive social networks. These authors pointed out that frequent churchgoers report more communication with others, receive more types of social support and hold more suitable perceptions of the quality of their social relationships than their unchurched counterparts. Moreover, Ellison et al. (2001) noted that religious attendance can promote healthy lifestyles on the part of the individuals, which in turn are beneficial to mental health. In this context, a positive mental health is considered by a large body of research as one of the most important aspects that enhances QoL. 
Table 8.11: The association between the amount of social interaction with neighbours and the frequency of attendance at the mosque

\begin{tabular}{|c|c|c|c|c|c|}
\hline & & & \multicolumn{2}{|c|}{ The frequent attendance at the mosque } & \multirow{2}{*}{ Total } \\
\hline & & & No & Yes & \\
\hline \multirow{10}{*}{$\begin{array}{l}\text { Amount of social } \\
\text { interaction with } \\
\text { neighbours }\end{array}$} & \multirow{2}{*}{ No At all } & Count & 53 & 25 & 78 \\
\hline & & $\%$ & $67.9 \%$ & $32.1 \%$ & $100.0 \%$ \\
\hline & \multirow{2}{*}{ A little } & Count & 48 & 47 & 95 \\
\hline & & $\%$ & $50.5 \%$ & $49.5 \%$ & $100.0 \%$ \\
\hline & \multirow{2}{*}{$\begin{array}{c}\text { A moderate } \\
\text { amount }\end{array}$} & Count & 79 & 93 & 172 \\
\hline & & $\%$ & $45.9 \%$ & $54.1 \%$ & $100.0 \%$ \\
\hline & \multirow{2}{*}{ Very much } & Count & 62 & 98 & 160 \\
\hline & & $\%$ & $38.8 \%$ & $61.3 \%$ & $100.0 \%$ \\
\hline & \multirow{2}{*}{$\begin{array}{c}\text { An extreme } \\
\text { amount }\end{array}$} & Count & 19 & 24 & 43 \\
\hline & & $\%$ & $44.2 \%$ & $55.8 \%$ & $100.0 \%$ \\
\hline \multirow{2}{*}{\multicolumn{2}{|c|}{ Total }} & Count & 261 & 287 & 548 \\
\hline & & $\%$ & $47.6 \%$ & $52.4 \%$ & $100.0 \%$ \\
\hline
\end{tabular}

Chi-square $=18.78, \mathrm{p}$-value $=.005$

These social benefits are promoted by the teachings of religion itself, Islam specifically, which equips Muslims with a moral compass and guidelines on how to treat others. According to one of the participants, religion reinforces social relationships, since religious teachings emphasise the good dealing with others, helping, and respecting others, especially neighbours (PH 17, Female, 42 yrs). With reference to the expert interviews, Mohammed Al-Shaibani pointed out that religious teachings of Islam were very much interested in the relationship with others, especially the neighbours. He referred to the relationship with the neighbours as follows:

The Islamic religion took a keen interest in the neighbours, calling for taking care of them regardless of their religious and cultural background, following what the Prophet Muhammad (PBUH) was doing, where he had a good relationship with his Jewish neighbours. Thus, these religious teachings call for better relations with others, which leads to the building of a model society that characterised by friendliness and harmony among its members.

In addition, religion promotes good morals and refrains from harming others. In this regard, one of the participants stated that "it is necessary in religion to not infringe upon the rights of others or harm them" (PH 19, Female, 45 yrs). In this context, most religions link moral 
values with what is called 'golden rules'. This principle is based on the moral dictum that refers to treating others as one would like to be treated, which appears in almost every religion and culture (Neusner and Chilton, 2008). Muslims in particular, believe that they are accountable for whatever they did against others, good or bad. In this context, “Qur'an underscores individual's moral responsibility in its many depictions of the Judgment Day when each person will be called to account for the good and the bad deeds that she/he did" (Neusner and Chilton, 2008, p. 100). This indicates that religion may be considered as one of the most important factors that control people's behaviour, as harm to people and infringement of their rights are unacceptable. However, this does not mean that morality is necessarily dependent upon religion, they may complement each other. In this context, religion needs morality to foster a better society, just as morality may need religion to reinforce its principles. Therefore, religion and morals are complementary to the establishment of a peaceful, just and equal society. Nevertheless, failure in synergy of religion and morality may make the influence of religion unclear with regard to society behaviours that may be considered unacceptable (Iwuagwu, 2018).

According to Koenig and Al Shohaib (2014), religious beliefs and practices in the Islamic cultural context are strongly linked in determining the behaviour of individuals, where religion encourages people to do what they believe is right and seeks to avoid wrong actions in terms of behaviour. In this context, these authors highlight that Islam in particular is a religion that systematically guides a person's life, as it organises the individual's daily activities and relationships with others in a way that builds a more homogeneous society and thus, creating harmony among people. For the QoUL, therefore, religion plays an important role in an improvement of the social life of the community including social relations and social cohesion. Other benefits of religious beliefs and practices can enhance the QoUL. By reviewing literature, it has been found that religious practices including prayer, zakat, fasting and pilgrimage may contribute to higher levels of QoUL. For example, prayer may be considered for many as a comfortable aspect and time-regulated way of measuring the day. By praying in the mosque, most Muslims believe that everyone is equal before God, and thus this belief contributes to the equality of value placed on the poor and makes the rich more humble. In addition, the performance of prayer in the mosque contributes to the strengthening of social ties, where people meet and interact with each other five times a day (Koenig \& Al Shohaib, 2014). Another religious practice is fasting 
especially during the month of Ramadan. This month has a socio-religious significance because it is linked to the fact that Muslims fast and break their fast simultaneously, which in turn reinforces their sense of solidarity and interdependence.

Another key element of fasting is that fasting people often feel hungry. Regardless of the feeling that hunger may reduce the QoL, Muslims consider fasting as a religious obligation. Therefore, they are likely to be more sensitive to the needs of the poor and hungry, which in turn makes them collaborators to alleviate their plight (Robinson, 1999). Such a feeling may lead some to search for those in need in order to support them, causing people to become more closely related to each other and thus, offering a better QoL. This may not apply to everyone living in Quba, but at least those who implement this duty may influence those who do not. In addition to the social benefits of fasting, there are benefits related to physical and mental health (Koenig \& Al Shohaib, 2014). The benefits of fasting could be considered as one of the most important contributions of religion to the QoUL. In this regard, one of the participants in the photo diaries referred to the obligation of fasting as one of the most significant religious practices for enhancing his QoUL as follows:

Fasting increases social interaction with neighbours and friends, even with strangers, where people gathered in one place and one time at breakfast especially in public spaces such as mosques. I enjoy doing breakfast at the mosque where I can meet many nationalities and share food and drink. (PH 23, Male, 42 yrs) 


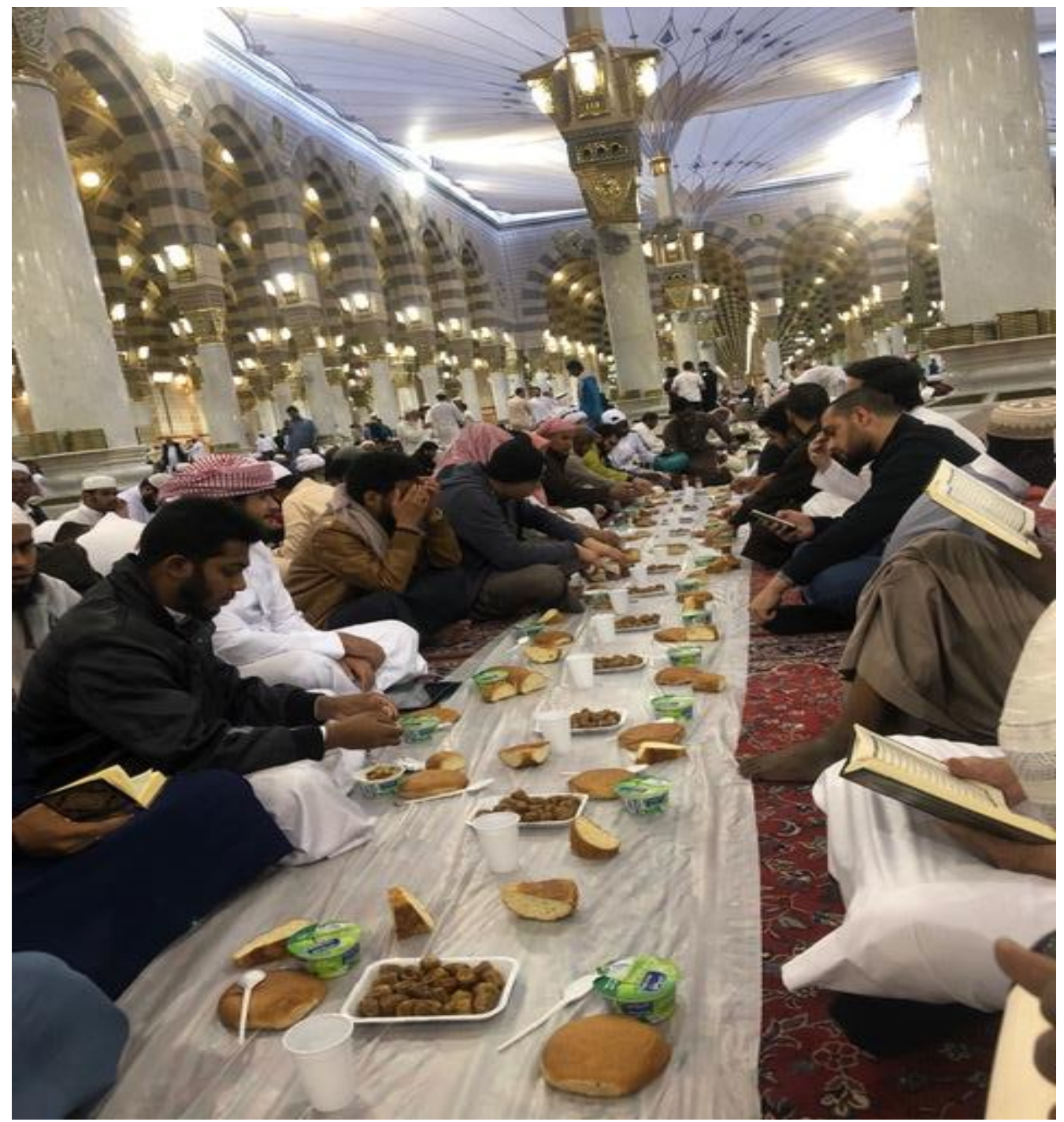

Fig 8.15: People break their fast in the mosque. Photo diary by participant PH23.

It can be concluded from the picture in the Figure 8.15 that fasting may bring people closer to each other regardless of their race, colour or cultural background, where they can sit next to each other during the breakfast. Consequently, this may enhance relations between people, foster fraternity and cooperation between them, and hence, a higher level of QoUL. Zakat is also considered to be an important religious practice that involves payments to those in need. The payment of the zakat purifies an individual's wealth and income as well as contributing to the economic balance and social justice in the communities (Abdel-Khalek, 2014). Therefore, the duty of zakat, if applied, may foster social ties among members of society as well as economic aspects (Hossain, 2012; Ahmad \& Mahmood, 2009) that are considered to be key aspects of QoUL. 
In this thesis, it has been found that religion positively contributes to the QoUL of Quba's residents. This positive relationship between religion and a positive perception of daily life is supported by Diener et al. (1999), Ferriss (2002) and Lun and Bond (2013). In addition, Gartner, Larson and Allen (1991) conclude that "the preponderance of evidence suggests that religion is associated with mental health benefits" (p.16, cited in Diener et al., 1999, p.281). These particular benefits are especially prevalent among the study participants who are actively involved in religious life, such as attending a mosque or another place of worship or performing daily prayers. For example, participant PH 1 connected performing prayer and reading Quran in the mosque with improvement of the QoUL, including strengthening his ability to overcome hardships. This is confirmed by many participants who stated that religious involvement enhances their ability to face life's hardships. Other participants also remark on the same positive mental and emotional health benefits of religion, which in turn improve the QoUL. The lack of belief in religion was seen by some participants as a route to the decline of their mental health, where they believe that mental health is closely related to religious practices and beliefs. For example, PH 15 suggests that "if there is a lack of faith in the human being, this may lead to a defect in the psychological side, as religion strengthens spirituality, and spirituality is linked to psychological comfort" (PH 15, Female, $41 \mathrm{yrs}$ ). Furthermore, several participants also state that they attend the mosque to 'refresh' their lifestyle and to gain calm and happiness. In this context, the mosque is considered by many participants as a place not only for worship, but also for social activities. Consequently, attending the mosque may change the routine of day life through meetings with others.

Considering the social benefits mentioned above, religion reinforces and expands social capital. That is, being a part of a society with shared values, beliefs and practices provides an emotional support as well as cooperation and collective action for mutual benefits (Pritchard, 2008; Bhandari \& Yasunobu, 2009). These dimensions of social capital may strengthen social relationships. In this regard, Putnam (2000) stated that the core idea of social capital is that social networks have value. However, social capital may have negative consequences. Coleman (1988) stated that social capital could be valuable in facilitating certain actions, however, it "may be useless or even harmful for others" (p. 98). For example, while social capital increases social cohesion, residents whose hometowns differ from the societies in which they currently reside may experience symptoms of depression 
(Villalonga-Olives \& Kawachi, 2017), while Portes (1998) notes certain negative consequences such as "exclusion of outsiders, excess claims on group members" (p. 15).

Despite the fact that religion positively affects individuals in many aspects of life, it may itself have some negative aspects too. According to Worthington et al. (2014) religions can lead to bias towards one group over another, which sometimes leads to discrimination. Religion may also be associated with some acts of violence such as terrorism and wars. In addition, religion may weaken the relationships between people and groups due to the negative judgements of people and the hostility of those who oppose religion (Worthington et al., 2014). However, the most negative aspects of religion found in the literature are those that affect mental health. Wulff (1997) indicated that although religion is associated with many positive aspects of life, including life satisfaction, it may be associated with negative aspects such as anxiety and depression. Furthermore, religion according to Koenig et al. (2001) may foster feeling of guilt and shame as well as the rejection of society.

In this thesis, however, no strong evidence was found that religion negatively affects the QoUL, except for feeling guilty, as along with some stress associated with non-compliance with religious obligations. In this regard, one of the participants within the follow-up interview to the photo diaries study argued that:

Feeling guilty about the performance of some taboos that contradict religious teachings make me anxious. In addition, late prayer or lack of commitment to perform other religious duties on time may sometimes cause some tension. (PH 5, Male, 28 yrs)

Feeling guilty and the fear of punishment in the hereafter may be one of the consequences of religious beliefs, especially among Muslims. In this context, guilt especially within the Islamic context is a symptom that people face because of failure to adhere to the high ethical standards set by Islam. It may be difficult for some people to be generous, reliable, tolerant, patient and committed to performing religious practices as required by religion. This may, as suggested by Koenig \& Al Shohaib (2014), result in a sense of reproach and even despair, which can lead to serious consequences such as depression and even ostracism from society, and thus negatively impact on mental and social health. Although not prevalent within this thesis, the sentiment expressed by participant PH 5 in the quotation above is consistent with 
some studies across the world's regions and cultures that have found a negative effect of religion on SWB, including anxiety and depression, and such effects do need to be acknowledged when assessing any impact of religion on QoUL. Those studies including for example Abdel-Khalek (2002), Baroun, (2006), Abdel-Khalek and Lester (2007), AbdelKhalek (2008) in the Muslim communities, while Kennedy (1998), El-Jamil (2003) and Van de Velde et al. (2017) in the Westerns communities which are mostly Christians and Jews.

- Impact of socio-demographic attributes on the perception of religion and spirituality

With regard to the impact of socio-demographic attributes on the perception of the study's participants regarding religion-related indicators, no significant associations were found between all socio-demographic attributes and the extent to which spiritual and religious beliefs can help to improve QoUL. On the contrary, significant associations were found between the sense of the spirituality of place and frequent attendance at the mosque and some of socio-demographic attributes. For example, age and marital status were found to be significantly associated with a sense of the spirituality of place. Frequent attendance at the mosque was found to be significantly associated with many socio-demographic attributes including age, marital status, employment, gender and number of people living at home.

For further exploration regarding the significant associations that have been obtained above, cross-tabulation was applied in order to gain more insight into how the participants perceive the religion-related indicators based on their socio-demographic attributes. In Table 8.12, results show a presence of significant association between age and the sense of spirituality in the place. It seems that there is a general tendency across age groups toward agreement that the place where participants live enhances the sense of spirituality, which in turn enhances the QoUL. It can be noted from Table 8.12 that $73.6 \%$ of the participants within the age group of 30 or less agree or strongly agree that the place where they live enhances the sense of spirituality, which in turn enhances the QoUL. This proportion increases within the other groups, where $84.4 \%$ of the participants ranging from the ages $31-40$ agreed or strongly agreed, while $85.7 \%$ of the participants aged 40 or more agreed or strongly agreed. One possible explanation regarding these findings is that the older the people in Quba are, the more they appreciate the sanctity of the place and thus feel more spiritual than others. 
In addition, those of an advanced age may be more religious than those of a younger age and thus may feel more spiritual, as seems to be clearly illustrated in the results in Table 8.14 .

Table 8.12: Relationship between age and a sense of spirituality of place. Cross-tabulation.

\begin{tabular}{|c|c|c|c|c|c|c|c|c|}
\hline & & & \multicolumn{5}{|c|}{ Agreement that the sense of spirituality of place enhances } & \multirow{3}{*}{ Total } \\
\hline & & & \multicolumn{5}{|c|}{ QoUL } & \\
\hline & & & $\begin{array}{l}\text { Strongly } \\
\text { disagree }\end{array}$ & Disagree & Neutral & Agree & $\begin{array}{c}\text { Strongly } \\
\text { agree }\end{array}$ & \\
\hline \multirow{6}{*}{$\begin{array}{l}\text { Age } \\
\text { group }\end{array}$} & \multirow{2}{*}{$<=30$} & Count & 10 & 13 & 36 & 78 & 86 & 223 \\
\hline & & $\%$ & $4.5 \%$ & $5.8 \%$ & $16.1 \%$ & $35.0 \%$ & $38.6 \%$ & $100.0 \%$ \\
\hline & \multirow{2}{*}{$31-40$} & Count & 1 & 6 & 22 & 68 & 88 & 185 \\
\hline & & $\%$ & $0.5 \%$ & $3.2 \%$ & $11.9 \%$ & $36.8 \%$ & $47.6 \%$ & $100.0 \%$ \\
\hline & \multirow{2}{*}{$>40$} & Count & 6 & 4 & 10 & 52 & 68 & 140 \\
\hline & & $\%$ & $4.3 \%$ & $2.9 \%$ & $7.1 \%$ & $37.1 \%$ & $48.6 \%$ & $100.0 \%$ \\
\hline \multirow{2}{*}{\multicolumn{2}{|c|}{ Total }} & Count & 17 & 23 & 68 & 198 & 242 & 548 \\
\hline & & $\%$ & $3.1 \%$ & $4.2 \%$ & $12.4 \%$ & $36.1 \%$ & $44.2 \%$ & $100.0 \%$ \\
\hline
\end{tabular}

Chi-square $=16.83 \mathrm{p}$-value $<.032$

Another exploration regarding how the participants perceive the religion-related indicators based on their socio-demographic attributes found that marital status is significantly associated with the sense of spirituality of place. It can be noted from Table 8.13 that the married group are more likely to agree that the place where they live enhances the sense of spirituality, which in turn enhances the QoUL. One possible explanation regarding this is that in addition to the fact that the place where the inhabitants live in Quba is characterised by the existence of many religious features that may enhance their spirituality, marital status may promote spirituality as well, because, as a number of scholars argue, marital status is affected by religion (Mahoney, 2001). In this regard, several studies including the work of Dudley and Kosinski (1990), Call and Heaton (1997) and Aman et al. (2019) evidence that religiosity positively affects marital status, possibly meaning with respect to this study, that if people are married, they feel more spiritual. 
Table 8.13: Relationship between marital status and a sense of spirituality of place. Crosstabulation.

\begin{tabular}{|c|c|c|c|c|c|c|}
\hline & & & \multicolumn{4}{|c|}{$\begin{array}{c}\text { Agreement degree that a sense of spirituality of place } \\
\text { enhances QoUL }\end{array}$} \\
\hline & & & Disagree & Neutral & Agree & Total \\
\hline \multirow{6}{*}{ Marital status } & \multirow{2}{*}{ Divorced } & Count & 1 & 5 & 10 & 16 \\
\hline & & $\%$ & $6.3 \%$ & $31.3 \%$ & $62.5 \%$ & $100.0 \%$ \\
\hline & \multirow{2}{*}{ Married } & Count & 21 & 33 & 328 & 382 \\
\hline & & $\%$ & $5.5 \%$ & $8.6 \%$ & $85.9 \%$ & $100.0 \%$ \\
\hline & \multirow{2}{*}{ Single } & Count & 18 & 30 & 102 & 150 \\
\hline & & $\%$ & $12.0 \%$ & $20.0 \%$ & $68.0 \%$ & $100.0 \%$ \\
\hline \multirow{2}{*}{\multicolumn{2}{|c|}{ Total }} & Count & 40 & 68 & 440 & 548 \\
\hline & & $\%$ & $7.3 \%$ & $12.4 \%$ & $80.3 \%$ & $100.0 \%$ \\
\hline
\end{tabular}

Chi-square $=27.114, \mathrm{p}$-value $<.001$

An additional analysis regarding the effect of the socio-demographic attributes of the study's participants shows significant associations between many of those attributes and frequent attendance at the mosque, namely the attributes of age, marital status, employment, gender and number of people living at home. No significant associations were found between and frequent attendance at the mosque and education, residential status and monthly income. For the association between age and frequent attendance at the mosque, Table 8.14 illustrates that the attendance ratio at the mosque steadily increases with the rise in age. For example, $61.4 \%$ of those aged 40 or over reported mosque attendance compared to those ranging from ages $31-40$ who reported $58.9 \%$, and the participants aged 30 or less who reported $41.3 \%$ attendance. In general, it appears from the results of measuring the relationship between age and frequent attendance at the mosque that younger people are participating in religious activities less than older people. Figure 8.16 supports this trend, clearly showing that frequent attendance of the mosque gradually increases with the increase in age. 
Table 8.14: Frequent attendance at the mosque and age. Cross-tabulation.

\begin{tabular}{|c|c|c|c|c|c|}
\hline & & & \multicolumn{2}{|c|}{ Do you frequently attend the mosque? } & \multirow{2}{*}{ Total } \\
\hline & & & No & Yes & \\
\hline \multirow{6}{*}{ Age group } & \multirow{2}{*}{$<=30$} & Count & 131 & 92 & 223 \\
\hline & & $\%$ & $58.7 \%$ & $41.3 \%$ & $100.0 \%$ \\
\hline & \multirow{2}{*}{$31-40$} & Count & 76 & 109 & 185 \\
\hline & & $\%$ & $41.1 \%$ & $58.9 \%$ & $100.0 \%$ \\
\hline & \multirow{2}{*}{$>40$} & Count & 54 & 86 & 140 \\
\hline & & $\%$ & $38.6 \%$ & $61.4 \%$ & $100.0 \%$ \\
\hline \multirow{2}{*}{\multicolumn{2}{|c|}{ Total }} & Count & 261 & 287 & 548 \\
\hline & & $\%$ & $47.6 \%$ & $52.4 \%$ & $100.0 \%$ \\
\hline
\end{tabular}

Chi-square $=18.83$ p-value $<.000$

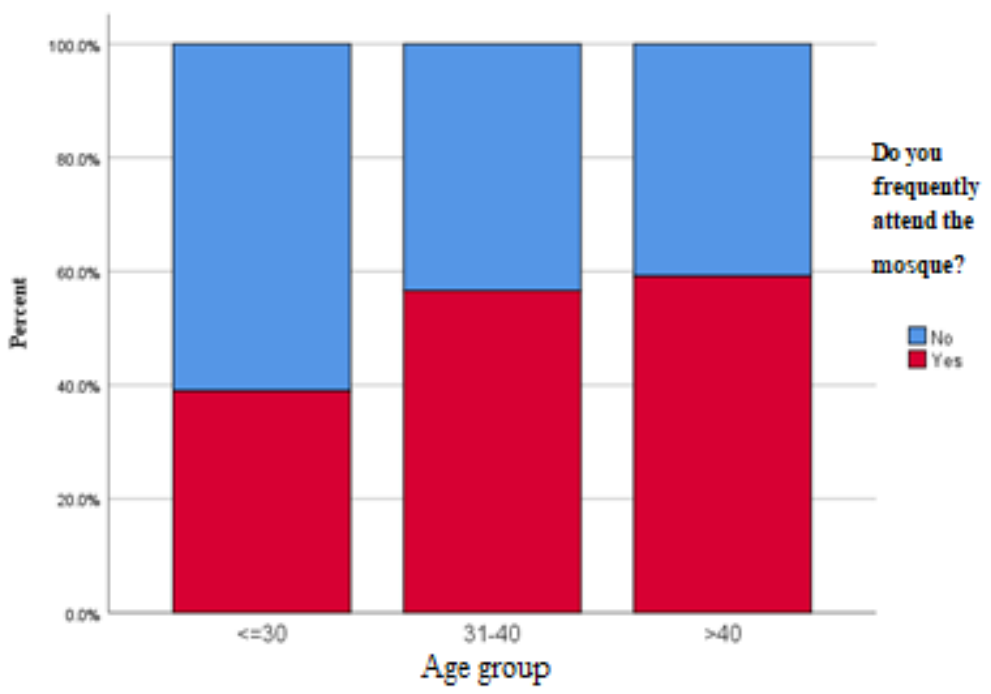

Fig 8.16: Age and frequent attendance at the mosque

This result is consistent with a number of results across cultural contexts that include Christian and Islamic contexts, and in the case of the latter, more specifically in Turkey. For example, a survey conducted by Kramer and Fahmy (2019) showed that younger Americans are less in attendance at church than older adults. In the Islamic context, Sari (2017) evidenced that young people seem to be less involved in religious activity, namely frequent attendance at the mosque, the performance of prayer and reading Qur'an, than older people. In the case of Quba, however, this result may be influenced by the gender factor, where there are religious considerations regarding the frequency of attendance at the religious centres. In this regard, it is not necessary for women to attend the mosque to perform prayers as for males, as according to Islamic legislation, it is believed to be better for females to 
pray at home (Koenig \& Al Shohaib, 2014). This may be one of the reasons why males more frequently attend the mosque than females, which is clearly illustrated in Figure 8.17. Although Sari's (2017) study was conducted in an Islamic society, Islamic cultural differences between Saudi Arabia and Turkey may play a role in that regard. In general, one of the possible explanations regarding the relationship between religiosity and age is that people who are more advanced in age are perhaps more drawn to religious activities in view of their stage in life, where facing the prospect of death may be more of a concern. Therefore, they are keener to attend the religious activities, because they believe that praying in the Mosque is more valuable than praying at home (Sari (2017). In other words, praying in the mosque is better than praying at home in terms of reward.

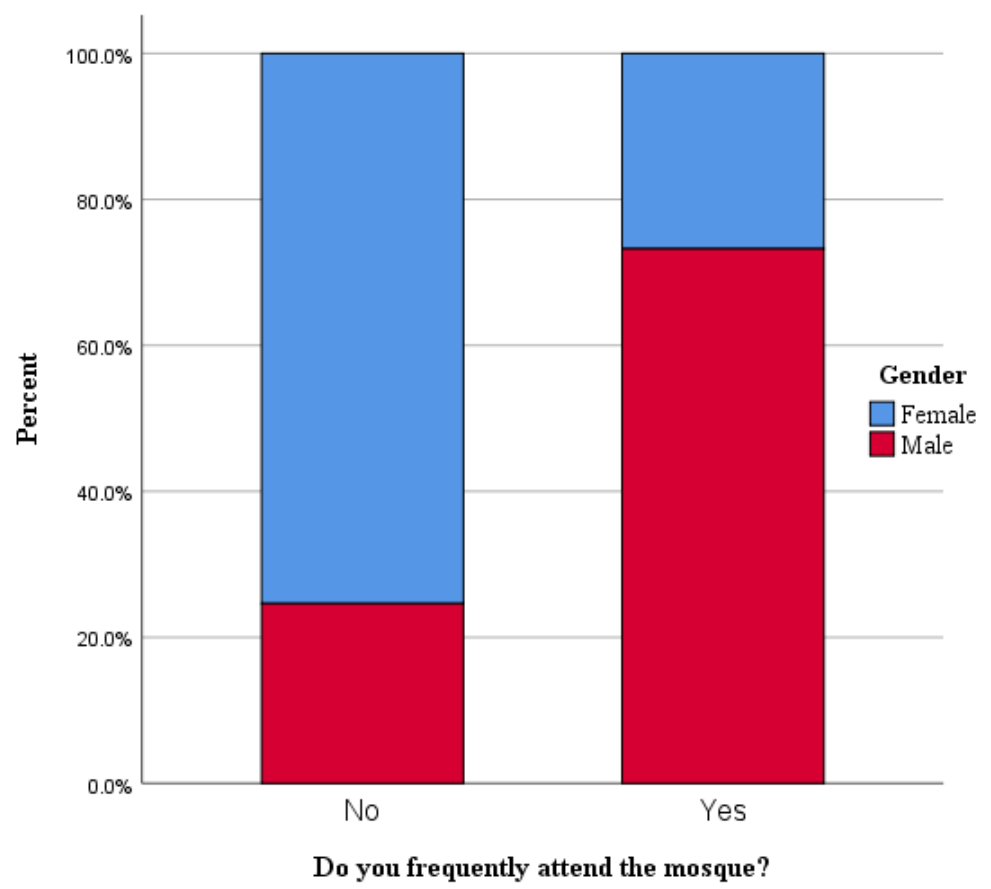

Fig 8.17: Gender and frequent attendance at the mosque 


\subsection{Cultural Domain of QoUL}

The cultural domain indicators in this thesis are specified as heritage, religious landmarks, accessibility to libraries and museums, and cultural activities such as conferences and exhibitions of hobbies, arts, crafts and so on. As in the case of the investigation into social indicators, the cultural indicators are discussed in terms of their definition and their role in the QoUL. Then, using a number of statistical tests, including Spearman's rho correlation and the Chi-square test, the relationship between cultural indicators and QoUL are examined. The Chi-square test was used to find any significant association between two variables, while Spearman's rho correlation test was used to assess the strength of association between those variables. Moreover, qualitative data including the expert interviews and photo diaries were merged with the quantitative data during the interpretation. In so doing, this thesis highlights the extent to which cultural indicators contribute to the QoUL for residents through their evaluation regarding the role of these indicators in the social and cultural life in the municipality of Quba.

The association between the cultural domain indicators and QoUL level was first investigated using the Spearman rho correlation in order to find any associations, then crosstabulation used for more details regarding those kinds of associations for each indicator consecutively. The results of Spearman rho's test indicated a significant positive association between QoUL and all cultural domain's indicators which illustrated in Table 8.15. it can be noted from this Table that accessibility to the libraries and museums, and access to the cultural activities showed higher relationship with QoUL compared to heritage experience.

Table 8.15: Relationship between QoUL level and cultural domain indicators

\begin{tabular}{lcc}
\hline Perception of heritage and religious landmarks in the & Correlation & $.113^{* *}$ \\
municipality of Quba & Result & Significant \\
\hline Heritage and religious landmarks affect the quality of your & Correlation & $.144^{* *}$ \\
urban life & Result & Significant \\
\hline Amount of enhancement of religious landmarks and heritage to & Correlation & $.151^{* *}$ \\
\cline { 2 - 3 } QoUL & Result & Significant $^{*}$ \\
\hline Accessibility to libraries and museums. & Correlation & $.203^{* *}$ \\
\hline $\begin{array}{l}\text { Access to cultural activities such as conferences and exhibitions } \\
\text { of hobbies, arts, crafts, traditional food? }\end{array}$ & Result & Significant \\
\cline { 2 - 3 } & Correlation & $.216^{* *}$ \\
\hline
\end{tabular}




\subsubsection{Heritage and QoUL}

Heritage is a key element that symbolises many cultures around the world. It is defined as "all the physical and intangible remains of the past that people can see, understand, feel or remember in the present world" (English Heritage, 2000a, cited in Smith and Waterton, 2009 , p. 296). Heritage is what has been received from the past, while it shapes people's present identity and provides insight for their future (Massey, 2012). Heritage has been considered in many studies as one of the pillars of economic and social development. It is one of the urban aspects that contribute to the well-being of communities, where it can achieve economic benefits including promoting heritage tourism, promoting small businesses, providing employment opportunities and increasing the value of property as well as the emotional pleasure that can be gained from visiting the heritage sites (Grazuleviciute, 2006). However, the relationship between heritage and QoUL has not been adequately addressed. A few studies have examined the impact of heritage on SWB, including the work of Ander et al. (2012), Massey (2012) and Fujiwara et al. (2014) have highlighted the health benefits of heritage, where they found that participants in their study expressed improved well-being after engagement with heritage including improved mood and calmed anxiety.

Considering heritage as one of the symbols that refer to images and lifestyles that represent the identity of the city, it has been highlighted in this thesis as one of the most important indicators of cultural QoUL which is likely to have a positive impact on the QoUL of Quba residents. In this thesis, heritage refers to the monuments and archaeological sites, including religious sites, as well as civil and military buildings. Such monuments are distinguished by their religious and historical value as well as by the architecture associated with ancient historical eras. The historical monuments in the municipality of Quba are mostly associated with the biography of the Prophet Muhammad (PBUH) as well as with specific events such as the emergence of the Islamic religion. These monuments encompass the Quba Mosque, Al Jum'ah Mosque and some wells associated with the Prophet's biography. In addition to religious heritage landmarks, there are many heritage sites and monuments in the municipality of Quba that are associated with time periods that preceded the Saudi state, such as the train station and the Ottoman Castle. Moreover, there are some contemporary monuments that have been established by the government in an attempt to revive the cultural 
heritage of Medina, whether that is linked to religion or culture, such as the Quba route and the Heritage Quarter.

An additional procedure for assessing the relationship between QoUL and heritage was conducted using cross-tabulation. Table 8.16 shows a significant association between QoUL and the extent to which heritage and religious landmarks affect QoUL. Participants seemed to report a positive effect of heritage on their QoUL. It can be noted that $59.6 \%$ of the participants who report heritage influences as extremely significant, stated that their QoUL is high or very high. In general, the QoUL of the participants increases with the level that heritage and religious landmarks affect QoUL, as is illustrated in Figure 8.18.

Table 8.16: The relationship between the extent to which heritage and religious landmarks affect QoUL and QoUL level. Cross-tabulation.

\begin{tabular}{|c|c|c|c|c|c|c|c|c|}
\hline & & & \multicolumn{5}{|c|}{ QoUL } & \multirow{2}{*}{ Total } \\
\hline & & & very low & low & moderate & high & very high & \\
\hline \multirow{10}{*}{$\begin{array}{l}\text { To what extent } \\
\text { heritage and religious } \\
\text { landmarks affect the } \\
\text { quality of your urban } \\
\text { life? }\end{array}$} & \multirow{2}{*}{ Not at all } & Count & 2 & 10 & 13 & 16 & 6 & 47 \\
\hline & & $\%$ & $4.3 \%$ & $21.3 \%$ & $27.7 \%$ & $34.0 \%$ & $12.8 \%$ & $100.0 \%$ \\
\hline & \multirow{2}{*}{ A little } & Count & 4 & 12 & 32 & 45 & 17 & 110 \\
\hline & & $\%$ & $3.6 \%$ & $10.9 \%$ & $29.1 \%$ & $40.9 \%$ & $15.5 \%$ & $100.0 \%$ \\
\hline & \multirow{2}{*}{$\begin{array}{c}\text { A moderate } \\
\text { amount }\end{array}$} & Count & 7 & 15 & 57 & 66 & 33 & 178 \\
\hline & & $\%$ & $3.9 \%$ & $8.4 \%$ & $32.0 \%$ & $37.1 \%$ & $18.5 \%$ & $100.0 \%$ \\
\hline & \multirow{2}{*}{ Very much } & Count & 2 & 14 & 32 & 43 & 23 & 114 \\
\hline & & $\%$ & $1.8 \%$ & $12.3 \%$ & $28.1 \%$ & $37.7 \%$ & $20.2 \%$ & $100.0 \%$ \\
\hline & \multirow{2}{*}{$\begin{array}{c}\text { An extreme } \\
\text { amount }\end{array}$} & Count & 1 & 7 & 32 & 19 & 40 & 99 \\
\hline & & $\%$ & $1.0 \%$ & $7.1 \%$ & $32.3 \%$ & $19.2 \%$ & $40.4 \%$ & $100.0 \%$ \\
\hline \multirow{2}{*}{ Total } & & Count & 16 & 58 & 166 & 189 & 119 & 548 \\
\hline & & $\%$ & $2.9 \%$ & $10.6 \%$ & $30.3 \%$ & $34.5 \%$ & $21.7 \%$ & $100.0 \%$ \\
\hline
\end{tabular}

Chi-square $=40.18$ p-value $<.001$ 


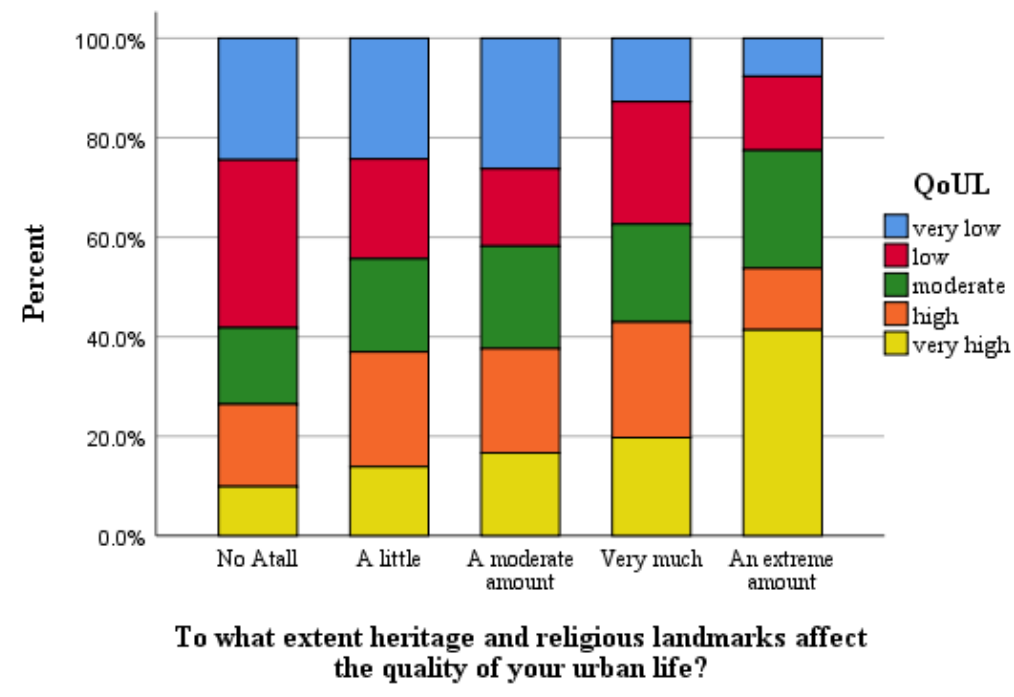

Fig 8.18: The relationship between the extent to which heritage and religious landmarks affect QoUL and QoUL level

In order to gain insight into the contribution of heritage to the QoUL, the relationship between the extent to which religious landmarks and heritage enhance QoUL and QoUL was further explored. Cross-tabulation revealed a significant association between QoUL and the extent to which religious landmarks and heritage enhance QoUL. It can be noted from Table 8.17 that the level of QoUL is positively associated with the extent to which religious landmarks and heritage enhance the QoUL of the participants, where 57.4\% of those who agreed with the 'extreme amount' statement, also stated that their QoUL is high or very high. Figure 8.19 clearly shows this type of relationship. These results are consistent with the study of Fujiwara et al. (2014) who found that visiting heritage is positively associated with life satisfaction. 
Table 8.17: The relationship between the extent to which religious landmarks and heritage enhance QoUL and QoUL level. Cross-tabulation.

\begin{tabular}{|c|c|c|c|c|c|c|c|c|}
\hline & & & \multicolumn{5}{|c|}{ QoUL } & \multirow{2}{*}{ Total } \\
\hline & & & very low & low & moderate & high & very high & \\
\hline \multirow{10}{*}{$\begin{array}{l}\text { To what extent does } \\
\text { the presence of } \\
\text { religious landmarks } \\
\text { and heritage } \\
\text { enhance your quality } \\
\text { of life? }\end{array}$} & \multirow{2}{*}{ Not at all } & Count & 1 & 12 & 14 & 17 & 3 & 47 \\
\hline & & $\%$ & $2.1 \%$ & $25.5 \%$ & $29.8 \%$ & $36.2 \%$ & $6.4 \%$ & $100.0 \%$ \\
\hline & \multirow{2}{*}{ A little } & Count & 4 & 12 & 16 & 37 & 13 & 82 \\
\hline & & $\%$ & $4.9 \%$ & $14.6 \%$ & $19.5 \%$ & $45.1 \%$ & $15.9 \%$ & $100.0 \%$ \\
\hline & \multirow{2}{*}{$\begin{array}{c}\text { A moderate } \\
\text { amount }\end{array}$} & Count & 6 & 12 & 73 & 75 & 35 & 201 \\
\hline & & $\%$ & $3.0 \%$ & $6.0 \%$ & $36.3 \%$ & $37.3 \%$ & $17.4 \%$ & $100.0 \%$ \\
\hline & \multirow{2}{*}{ Very much } & Count & 4 & 11 & 32 & 42 & 28 & 117 \\
\hline & & $\%$ & $3.4 \%$ & $9.4 \%$ & $27.4 \%$ & $35.9 \%$ & $23.9 \%$ & $100.0 \%$ \\
\hline & \multirow{2}{*}{$\begin{array}{c}\text { An extreme } \\
\text { amount }\end{array}$} & Count & 1 & 11 & 31 & 18 & 40 & 101 \\
\hline & & $\%$ & $1.0 \%$ & $10.9 \%$ & $30.7 \%$ & $17.8 \%$ & $39.6 \%$ & $100.0 \%$ \\
\hline \multirow{2}{*}{ Total } & & Count & 16 & 58 & 166 & 189 & 119 & 548 \\
\hline & & $\%$ & $2.9 \%$ & $10.6 \%$ & $30.3 \%$ & $34.5 \%$ & $21.7 \%$ & $100.0 \%$ \\
\hline
\end{tabular}

Chi-square $=58.50 \mathrm{p}$-value $<.000$

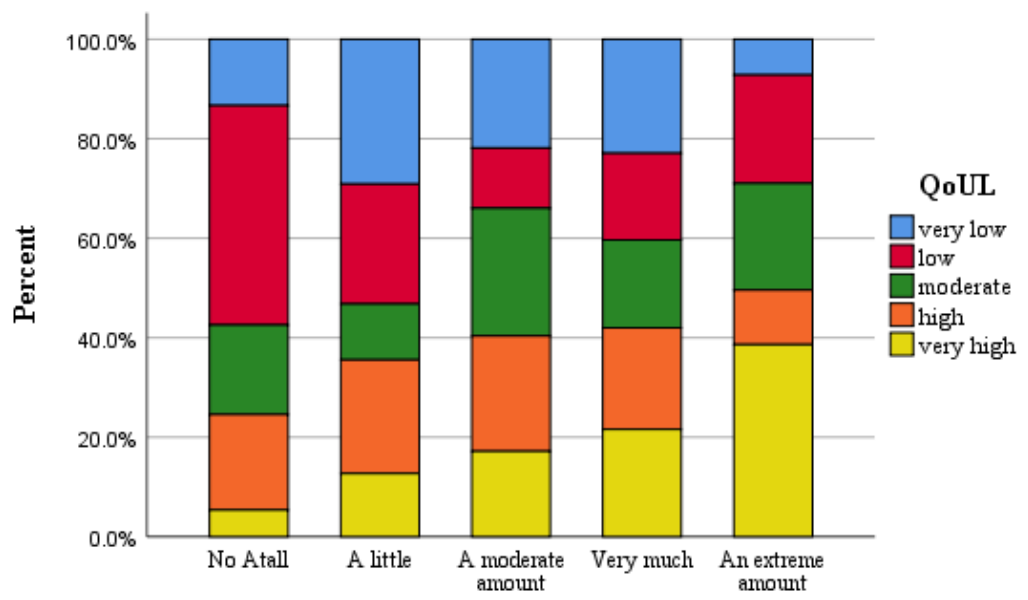

To what extent the presence of such religious landmarks and heritage enhance your quality of life?

Fig 8.19: The relationship between the extent to which religious landmarks and heritage enhance QoUL and QoUL level 
In order to gain further insight into how heritage contributes to the QoUL in the study area, qualitative research including photo diaries and expert interviews were conducted for more exploration about the relationship between QoUL and heritage. In terms of the first impression within the follow-up interviews from the photo diaries, heritage is seen as something to treasure in Medina, and participants often mentioned the beauty of the history and heritage of the city. For example, one of the participants expressed about his view that "things related to the past are sometimes associated with beautiful memories and as such are remembered fondly" (PH 9, Male, $37 \mathrm{yrs}$ ).

From the above quotation, it can be concluded that heritage may contribute to psychological benefits, since heritage evokes a sense of nostalgia, which in turn may enhance people's happiness. Although the feeling of nostalgia can have negative repercussions in terms of feeling sad due to the irreversible loss of the past (Hepper et al. 2012), it can also enhance people's meaning of life by increasing social connectedness, such as a sense of belonging as well as self-continuity, that is, the sense of connection between the past and the present (Sedikides \& Wildschut, 2018). As such, nostalgia resulting from heritage may have positive consequences in an improvement of QoUL by enhancing the sense of happiness and life satisfaction as well as social ties. This is supported by an argument made by one of the experts, where he referred to the importance of heritage as follows:

People are often emotionally connected to the past, which often promote psychological aspects such as happiness and satisfaction with life. More importantly, heritage enhances individuals' sense of identity and belonging to culture and the place in which they live. Consequently, this sentiment generated by heritage increases the interconnectedness and cohesion of a society which in turn enhances the quality of urban life. (Abdullah Kabir, an expert)

Another expert argues that "heritage brings the origins of the city identity including customs, traditions and norms, which is important for the cohesion and integration of society, since these characteristics of identity bring members of society together." (Saeed Jood, an expert) The expert Mohammed Al-Shaibani added: 
Heritage provides a historical and cultural dimension to past glories, since the heritage in Medina mostly represents the genesis of the Islamic religion and the biography of the Prophet Muhammad (PBUH). Therefore, this is a source of pride and pride for those who live in Medina, as well as giving them an optimistic and positive outlook for the future.

In this sense, cultural heritage provides some social values including a sense of identity and connection with others, which might lead to social stability, understanding and tolerance (Throsby, 2000). Moreover, place attachment is considered one of the social values of heritage since place attachment refers to the sense of belonging, societal identity and social cohesion that derives from the specific heritage of its original territory (Torre, 2002). In that sense, these key benefits are likely to contribute to the QoUL, mediated by promoting happiness and life satisfaction.

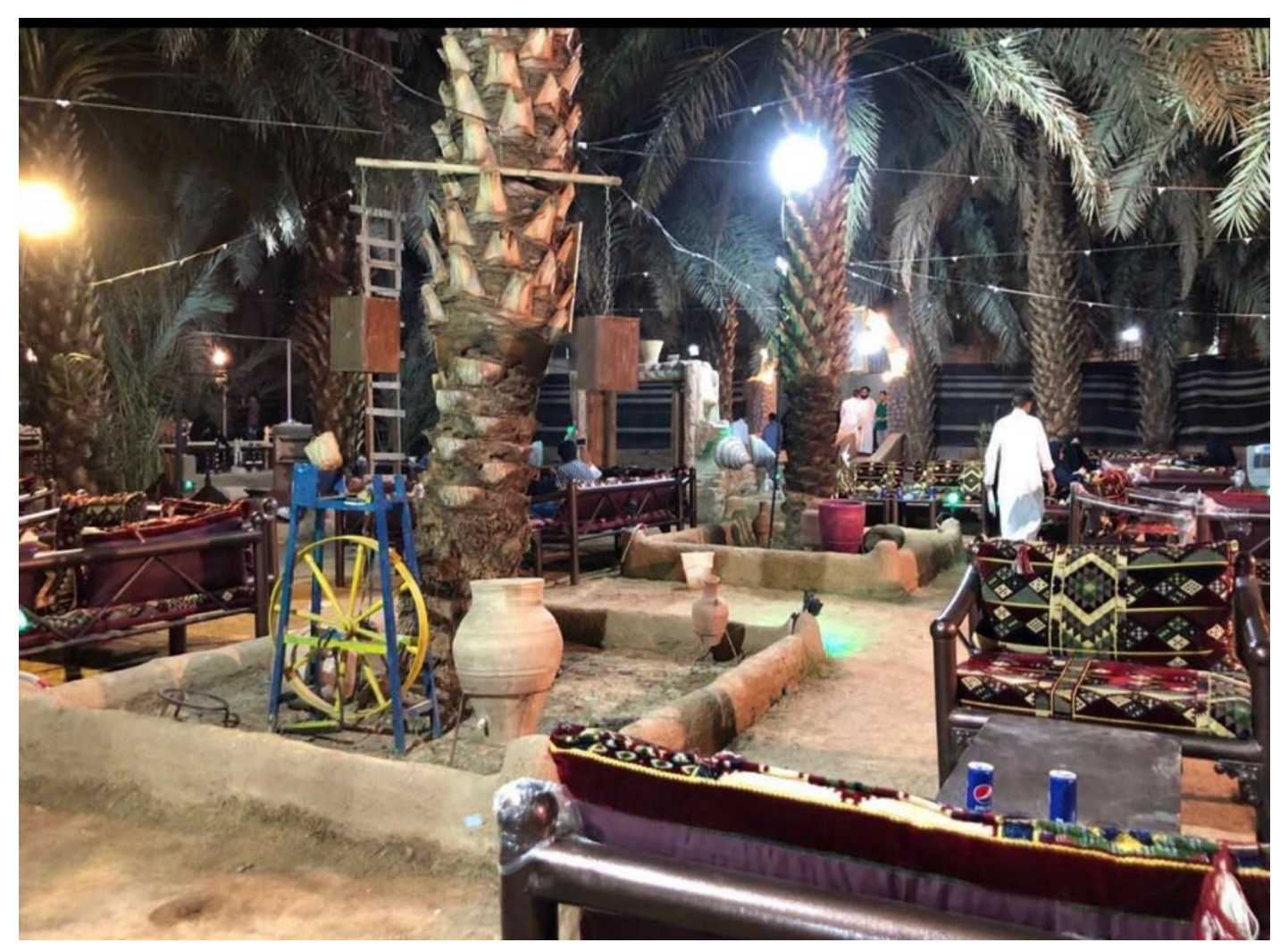

Fig 8.20: An archaeological farm containing some traditional tools that were used in the past, especially in agriculture. Photo diary by participant PH 9. 
Another benefit of heritage to QoUL is the knowledge that can be gained from learning about and understanding Medina's long history, as many participants indicated. In this respect, much can be learned from the past. For example, one of the participants stated that,

Heritage informs us how people lived in the past and how they were creative in adapting to the environment in which they live despite the lack of means of comfort that we have now. (PH 6, Male, $30 \mathrm{yrs}$ )

This is suggesting that heritage also teaches resilience, creativity, and innovation. An example of this creativity seen in the history of Medina, which is recalled fondly by one of the participants, where he stated,

Some of Medina's monumental buildings are designed with separate windows dedicated to the summer and winter temperatures, indicating creativity despite the lack of advanced technologies currently in place today. (PH 5, Male, 28 yrs)

Alongside this, many of the participants linked the heritage of Medina with the feeling of pride, which supports the strong historical and religious identity of the city. Indeed, it can be suggested that people living in Medina romanticise the past over the present as noted by participant PH 25,

Heritage is a simple and beautiful thing, unlike our present, where some aspects of the environment we live in are complex. (Male, $30 \mathrm{yrs}$ )

Photographs in Figure 8.21, taken by the participants during the photo diaries study, represent a set of the most famous heritage sites in Medina which are more frequently visited, especially by pilgrims due to their historical importance. For the local community, these sites may be a source of benefits that are likely to contribute to the overall QoUL. At the same time, however, living in proximity to heritage sites may cause some negative consequences. In this respect, Adie et al. (2019) suggest that since cultural heritage sites tend to attract large numbers of tourists, overcrowding at these sites is an increasing problem in terms, for example, of traffic and parking issues, rising cost of living for local residents and destruction of the heritage. As Medina is considered to be the second holiest city, it is characterised by the inflow of millions of pilgrims and visitors over the year. Despite the 
benefits, especially those associated with the creation of small businesses during the Hajj seasons, there are some potential issues resulting from that reflective of Adie et al.'s study, such as the overcrowding and traffic jams, and other issues related to the economy, like the increase of prices in the local shops, as well as increase in the prices of land that is located within the scope of those sites.

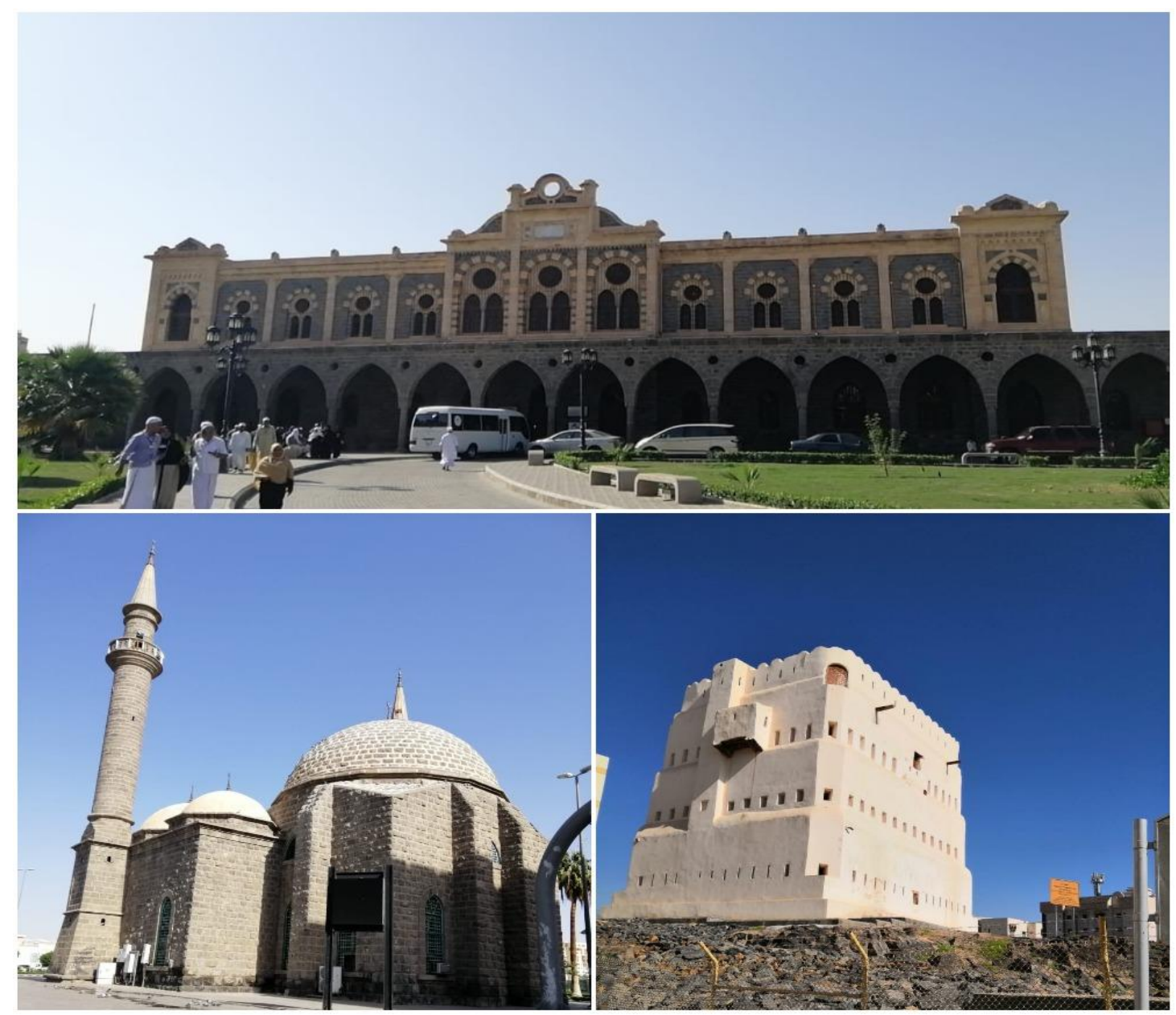

Fig 8.21: A set of historical sites that represent heritage in the study area. Photo diaries by participants PH 6, PH 5 and PH 25 respectively.

\subsubsection{Accessibility to cultural goods and QoUL}

Cultural goods such as libraries, museums, and engagement in cultural activities are considered key indicators of the quality of urban cultural life, which contributes to the building of society in many ways including in terms of cultural, social and economic 
development. In this thesis, the impact of the cultural goods on the QoUL of Quba's residents was assessed by examining the relationship between accessibility to the libraries and museums and QoUL level in order to find out whether these goods contribute to higher levels of QoUL. Table 8.18 presents the findings from cross tabulating the accessibility to the libraries and museums and QoUL. These findings indicate that accessibility to the libraries and museums is significantly associated with QoUL. It can be noted that $64.9 \%$ of the participants who agree that there is accessibility to the libraries and museums stated that their QoUL is high or very high. Figure 8.22 clearly shows this kind of relationship, where the QoUL level increases with the increase of the level of accessibility to the libraries and museums.

Table 8.18: The relationship between accessibility to the libraries and museums and QoUL. Cross-tabulation.

\begin{tabular}{|c|c|c|c|c|c|c|c|c|}
\hline & & & \multicolumn{5}{|c|}{ QoUL } & \multirow{2}{*}{ Total } \\
\hline & & & very low & low & moderate & high & very high & \\
\hline \multirow{10}{*}{$\begin{array}{l}\text { To what extent do you } \\
\text { agree or disagree there } \\
\text { is accessibility to the } \\
\text { libraries and museums? }\end{array}$} & \multirow{2}{*}{$\begin{array}{l}\text { Strongly } \\
\text { disagree }\end{array}$} & Count & 6 & 7 & 15 & 13 & 6 & 47 \\
\hline & & $\%$. & $12.8 \%$ & $14.9 \%$ & $31.9 \%$ & $27.7 \%$ & $12.8 \%$ & $100.0 \%$ \\
\hline & \multirow{2}{*}{ Disagree } & Count & 1 & 15 & 25 & 20 & 12 & 73 \\
\hline & & $\%$ & $1.4 \%$ & $20.5 \%$ & $34.2 \%$ & $27.4 \%$ & $16.4 \%$ & $100.0 \%$ \\
\hline & \multirow{2}{*}{ Neutral } & Count & 4 & 14 & 49 & 61 & 16 & 144 \\
\hline & & $\%$ & $2.8 \%$ & $9.7 \%$ & $34.0 \%$ & $42.4 \%$ & $11.1 \%$ & $100.0 \%$ \\
\hline & \multirow{2}{*}{ Agree } & Count & 3 & 11 & 50 & 62 & 56 & 182 \\
\hline & & $\%$ & $1.6 \%$ & $6.0 \%$ & $27.5 \%$ & $34.1 \%$ & $30.8 \%$ & $100.0 \%$ \\
\hline & \multirow{2}{*}{$\begin{array}{c}\text { Strongly } \\
\text { agree }\end{array}$} & Count & 2 & 11 & 27 & 33 & 29 & 102 \\
\hline & & $\%$ & $2.0 \%$ & $10.8 \%$ & $26.5 \%$ & $32.4 \%$ & $28.4 \%$ & $100.0 \%$ \\
\hline \multirow{2}{*}{\multicolumn{2}{|c|}{ Total }} & Count & 16 & 58 & 166 & 189 & 119 & 548 \\
\hline & & $\%$ & $2.9 \%$ & $10.6 \%$ & $30.3 \%$ & $34.5 \%$ & $21.7 \%$ & $100.0 \%$ \\
\hline
\end{tabular}

Chi-square $=54.46$ p-value $<.000$ 


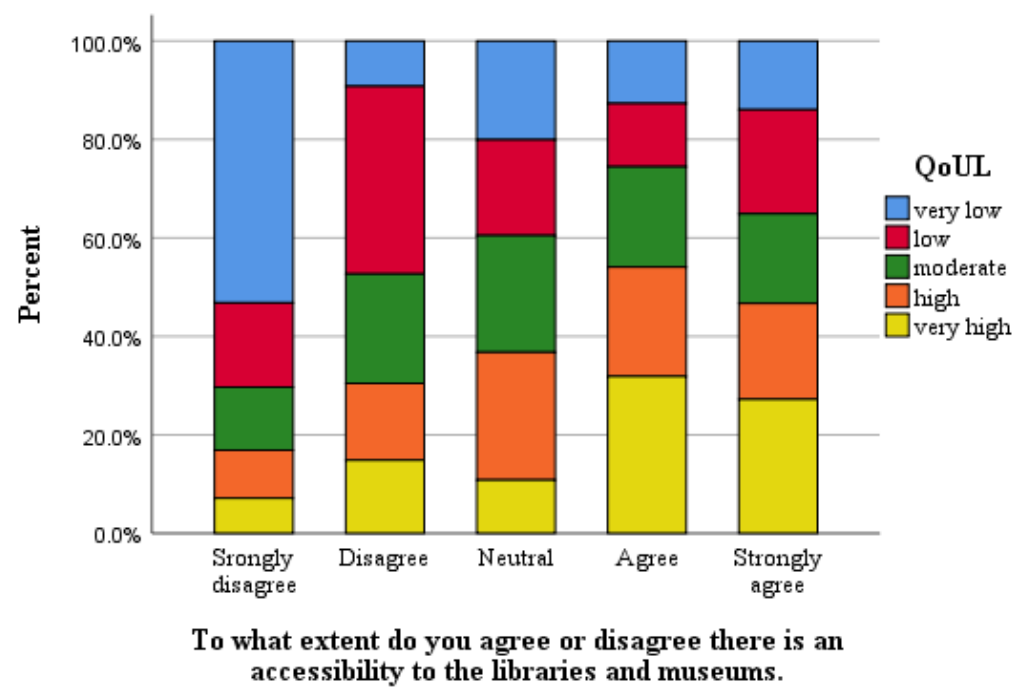

Fig 8.22: The relationship between accessibility to the libraries and museums and QoUL

Qualitative findings from expert interviews indicate the important role of the museums in the QoUL. In this respect, one of the experts argued:

Museums have a great mission to improve people's cultural standard. Museums are one of the services means that every person in society should enjoy without exception to provide the science, culture and direct knowledge that any museum gives as a representative of the local culture. Thus, the function of museums is not only to preserve artistic riches, but also to contribute to the intellectual and social development'. (Abdulaziz Kaaki, an expert)

Another expert argued:

Often, museums contain historical and cultural information that helps people understand the history of their nation. It is considered a key to the culture of society as well as a place to protect the exhibits and things that symbolise the history of local culture, including the customs and traditions that characterise peoples in a specific time period. Museums highlight the relationship between the past and the present. Nowadays, museums have become an important scientific centre that contributes to disseminating 
knowledge and introducing human heritage in several fields. (Salah Salamah, an expert)

For the QoUL, the benefits of cultural goods (i.e., libraries and museums) lie in increasing the knowledge and cultural stock. However, the relative importance of each of these cultural goods may vary. Museums may symbolise the cultural aspect of a particular thing, and thus in many cases provide a source of knowledge solely about this particular object, area or matter. While for libraries, sources of knowledge in many fields can be provided, not just history, as is primarily the case in museums. Thus, libraries contribute to the development of knowledge in several fields, which in turn contributes to an increase of the educational level, which ultimately contributes to a better QoUL.

An additional investigation regarding the impact of other cultural goods defined as cultural activities such as conferences and exhibitions of hobbies, arts, crafts, and traditional food on the QoUL of Quba's residents was conducted for further exploration. Using the Chisquare test, results indicate that there is a significant relationship between accessibility to these cultural goods and QoUL. It can be noted from Table 8.19, however, that the majority of the participants tend to report a low and a moderate level of accessibility to these named cultural activities (see Figure 8.23). In this respect, $72.8 \%$ of those participants who reported a low level of accessibility to cultural activities stated that their QoUL was moderate or high. This may indicate that most of the participants do not have accessibility to cultural activities due to the lack of cultural events, or at least that they are not interested in participating or visiting these types of events. Despite that, an apparent relationship between QoUL and the level of accessibility to cultural activities can still be observed (see Table 8.15 and Figure 8.24). 
Table 8.19: Relationship between accessibility to cultural activities such as conferences and exhibitions of hobbies, arts, crafts, and traditional food and QoUL. Cross-tabulation.

\begin{tabular}{|c|c|c|c|c|c|c|c|c|}
\hline & & & \multicolumn{5}{|c|}{ QoUL } & \multirow[b]{2}{*}{ Total } \\
\hline & & & $\begin{array}{l}\text { very } \\
\text { low }\end{array}$ & low & moderate & high & $\begin{array}{l}\text { very } \\
\text { high }\end{array}$ & \\
\hline \multirow{10}{*}{$\begin{array}{l}\text { To what extent do you } \\
\text { have access to the cultural } \\
\text { activities such as } \\
\text { conferences and } \\
\text { exhibitions of hobbies, arts, } \\
\text { crafts, traditional food? }\end{array}$} & \multirow{2}{*}{ Not at all } & Count & 9 & 12 & 26 & 23 & 9 & 79 \\
\hline & & $\%$ & $11.4 \%$ & $15.2 \%$ & $32.9 \%$ & $29.1 \%$ & $11.4 \%$ & $100.0 \%$ \\
\hline & \multirow{2}{*}{ Little } & Count & 3 & 22 & 64 & 78 & 28 & 195 \\
\hline & & $\%$ & $1.5 \%$ & $11.3 \%$ & $32.8 \%$ & $40.0 \%$ & $14.4 \%$ & $100.0 \%$ \\
\hline & \multirow{2}{*}{ Moderate } & Count & 2 & 12 & 45 & 46 & 38 & 143 \\
\hline & & $\%$ & $1.4 \%$ & $8.4 \%$ & $31.5 \%$ & $32.2 \%$ & $26.6 \%$ & $100.0 \%$ \\
\hline & \multirow{2}{*}{ High } & Count & 1 & 8 & 25 & 36 & 27 & 97 \\
\hline & & $\%$ & $1.0 \%$ & $8.2 \%$ & $25.8 \%$ & $37.1 \%$ & $27.8 \%$ & $100.0 \%$ \\
\hline & \multirow{2}{*}{$\begin{array}{c}\text { Extremely } \\
\text { high }\end{array}$} & Count & 1 & 4 & 6 & 6 & 17 & 34 \\
\hline & & $\%$ & $2.9 \%$ & $11.8 \%$ & $17.6 \%$ & $17.6 \%$ & $50.0 \%$ & $100.0 \%$ \\
\hline \multirow{2}{*}{\multicolumn{2}{|c|}{ Total }} & Count & 16 & 58 & 166 & 189 & 119 & 548 \\
\hline & & $\%$ & $2.9 \%$ & $10.6 \%$ & $30.3 \%$ & $34.5 \%$ & $21.7 \%$ & $100.0 \%$ \\
\hline
\end{tabular}

Chi-square $=59.07$ p-value $<.000$

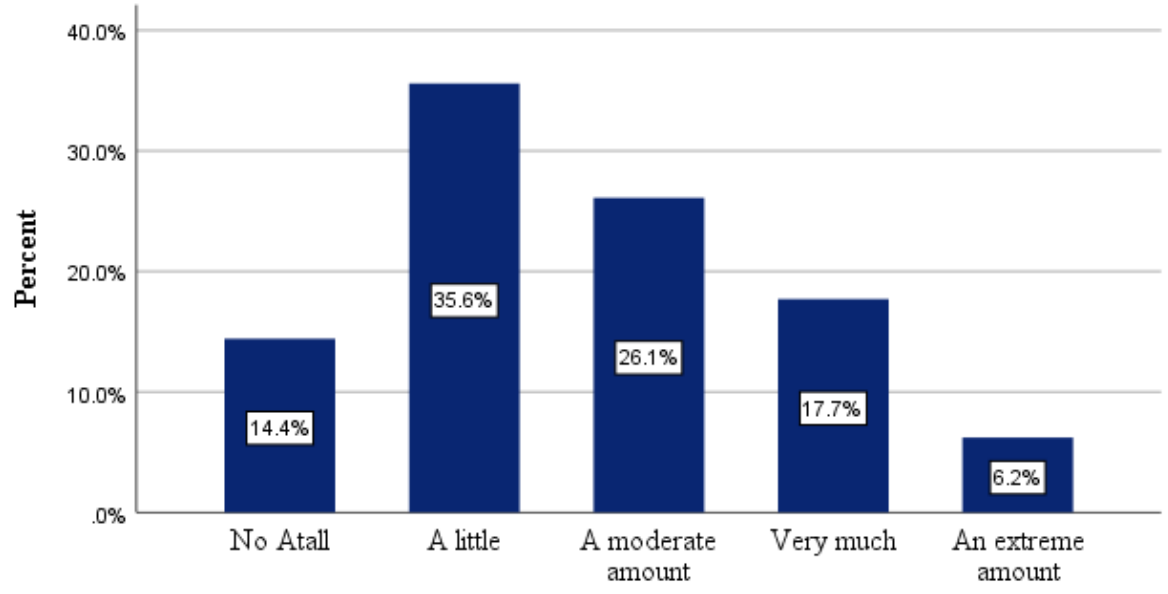

To what extent do you have the access to the cultural activities such as conferences and exhibitions of hobbies, arts, crafts, traditional food?

Fig 8.23: Agreement that there is accessibility to cultural activities 


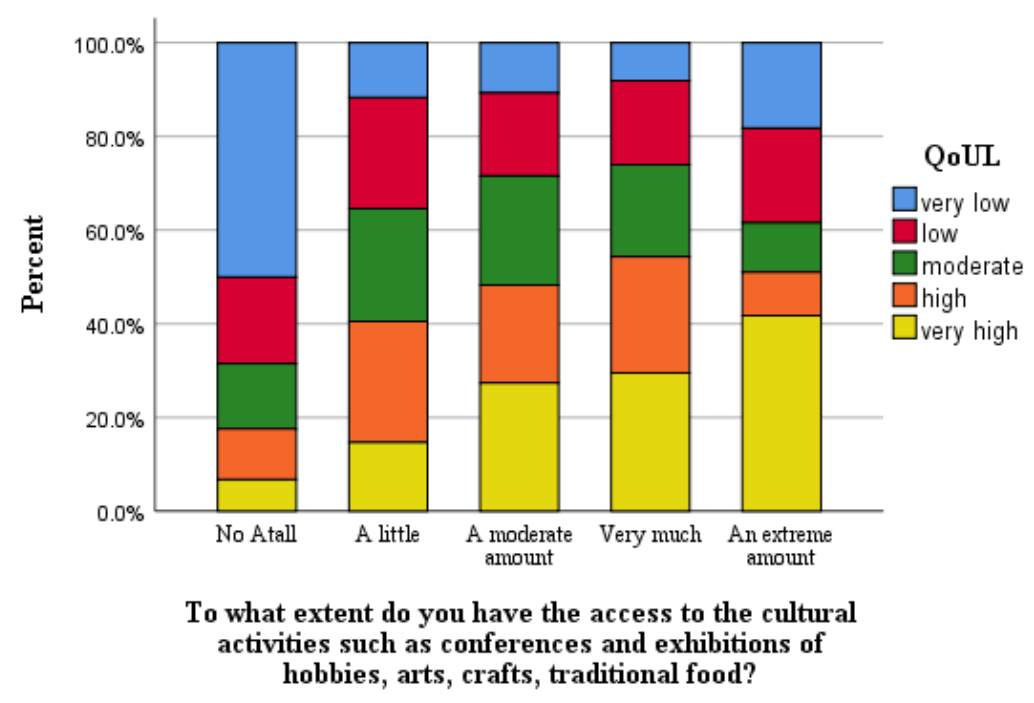

Fig 8.24: Relationship between accessibility to cultural activities and QoUL

Within the qualitative research, there was a positive impression from the participants regarding the extent to which cultural goods affected their QoUL. In this respect, several participants pointed out that cultural aspects can contribute to an improved social and recreational lifestyle. For example, one of the participants stated:

Cultural events encourage individuals to be diligent, particularly within education, as cultural events are often formed by knowledge competitions which stimulate participants to learn more about their culture and history. (PH 16, Female, 39 yrs)

Another participant pointed out:

There are benefits of cultural activities specifically for children and young people, as they often participate in these competitions and learn more about their culture whilst also enjoying themselves. Therefore, competitions are held to encourage children to learn and increase knowledge. (PH 9, Male, 37 yrs) 
Another participant emphasises that "many residents enjoy cultural activities, as they provide a break from a busy lifestyle and add something new to our daily lives" (PH 19, Female, 45 yrs).

From the arguments above made by both the residents and the experts, it is clear how important cultural goods are in the QoUL as such goods contribute to the improvement of social and cultural aspects of life, which in turn leads to a higher QoUL. Cultural activities as an example are a source of entertainment and an effective tool for developing the ability to participate effectively, exchange experiences and communicate with others, as well as providing opportunities for learning and developing knowledge. Furthermore, the benefits of cultural activities are not limited to the societal and cultural dimensions, but rather their benefits extend to include health aspects. In this context, a number of researchers suggest that cultural activities are an effective tool to reduce stress, where some studies have confirmed that participation in and the taking up of cultural activities contribute to improving physical and mental health, including the elimination of anxiety and depression (Beckford, 2011). 


\subsection{Summary of the chapter}

Human gathering is a constant and necessary phenomenon of human life, in which the individual is linked to others through multiple relationships, whether with family, relatives, neighbours or friends. Thus, the interaction of individuals with the surrounding urban environment creates socio-cultural and economic relationships, through which people can cooperate and exchange benefits, as well as the enhancement of other aspects of life such as happiness and satisfaction with life. In that sense, it has been frequently argued in the literature that social and cultural characteristics of a society are key elements that determine the QoUL. Such assumptions have been supported by findings that have been obtained throughout this chapter. In the first part, this chapter highlighted a range of social indicators including security, leisure social interaction, neighbour support, religion and spirituality, and the extent to which they contribute to the QoUL in the study area. In the second part, the chapter aimed to explore the extent to which heritage and cultural goods affect the QoUL of Quba's residents. In doing so, the analysis of both quantitative and qualitative data revealed significant associations between these indicators and the QoUL. In this respect, results indicated the vital role of these indicators in enhancing the QoUL in many cases, which is consistent with the results of many studies in the literature.

In general, the results confirmed that that most of the social indicators positively affect the QoUL of Quba residents. Security was found to be associated with higher levels of QoUL, which strongly suggests the vital role of this indicator in enhancing the QoUL of Quba residents. Nevertheless, perception of security according to the participants' sociodemographic attributes shows differences according to gender, where it was found that females feel more secure than males. This result may reflect differences in terms of the meanings of security. That is, women may not feel any more secure on a personal level, but more secure when accompanied by males (their relatives, father, husband, brother etc.) In this context, therefore, security is impersonal.

With regard to the leisure activities indicator, a significant relationship was found between accessibility to leisure activities and the QoUL. However, the participants did not show any positive impression about a contribution of such an indicator to their QoUL, as to a large extent, the majority of the participants did not consider that there is good accessibility to 
leisure activities. Perhaps this is due to the lack of appropriate types of spaces and facilities where residents might be encouraged to spend their leisure time.

Another positive effect of the social domain has been illustrated in the relationship between social interaction and the QoUL, where this relationship was found to be significant. In this respect, results indicated that social interaction has a positive role in the QoUL of Quba's residents. That is, social interaction promotes other social aspects including social support, where a strong positive relationship has been found between social interaction and social support. This indicates the importance of social interaction with neighbours in encouraging social aspects that are likely to positively contribute to the QoUL. Moreover, this is supported by another finding within this chapter, where the majority of the participants tend to be satisfied with their neighbours. Neighbourly satisfaction in this context, therefore, may indicate the positive role of social interaction in QoUL. With regard to the impact of sociodemographic attributes on the participants' perception regarding social interaction, only education and the number of people living at home were found to be significantly associated with social interaction. Interpretation of these relationships has led to some suppositions. As for the relationship between the number of people living at home and social interaction, it has been found that the greater the number of occupiers, the greater the amount of social interaction. For the relationship between the educational level and social interaction, it has been found that people who have a lower educational level, interact more with their neighbours.

Religion was found to be one of the most important social indicators that affect the QoUL of those living in the municipality of Quba. In this sense, religion is not only an indicator that enhances spirituality, but as is emphasised in many studies in the literature on QoUL, it is one of the most important aspects that improves the relationship between people, as well as offering other benefits such as improving mental health. Spirituality, religious practices and beliefs were found to be the most prominent variables that contribute to the QoUL. In this chapter, results showed that there are significant associations between these variables and the QoUL, where both quantitative and qualitative results confirmed that all these variables contribute to a higher QoUL. There is debate concerning the role of religion, where argument has been made that religion enhances multiple aspects of life including happiness, life satisfaction, strengthening social ties, improving mental health and promoting the place 
attachment. In this context, religion may enhance and expand social capital. Nevertheless, social capital has a dark side which may negatively affect the QoUL. While social capital may contribute to social cohesion, it may create a gap between some groups of society (i.e., locals and outsiders). In addition, this effect may extend to deepening the gap between groups within the local community itself (e.g., poor and rich). In the case of Quba, social capital and its impact on the daily life needs further research. Despite the fact that religion may enhance multiple aspects of life, it may also have a negative side. From the findings of this thesis, there was evidence from the photo diaries that religious obligations sometimes cause anxiety and depression, thus negatively affecting the QoUL, but more predominantly, it is clear how important religion is in the community daily life in the municipality of Quba.

In relation to the impact of socio-demographic attributes on the perception of the study's participants regarding religion-related indicators, age and marital status were found to be significantly associated with the sense of the spirituality of place and the frequency of attendance at the mosque. Other socio-demographic attributes including gender and number of people living at home were significantly associated with the frequency of attendance at the mosque. Within the investigation of the relationship between age and the sense of the spirituality of place, it has been found that the sense of the spirituality of place increases with increase of the age, though results showed to a large extent a similarity with the age groups in terms of the agreement that a sense of spirituality of place enhances QoUL. For the relationship between marital status and the sense of spirituality of the place, results indicated that married people feel more spiritual than others. This could be because, according to studies within the literature, married people are more likely be affected by religion more than others, and therefore may feel more spiritual. With regard to the relationship between age and frequency of attendance at the mosque, results showed that the older people are, the more they go to the mosque. This was justified in terms that older people are more likely to participate in religious activities and hence, more frequent attenders at the mosques, which is supported by several studies in the literature. As for the relationship between gender and the frequency of attendance at the mosque, results have indicated that males go more to the mosque than females. In this regard, the Islamic culture was found to play an important role in determining this relationship, where Islamic legislation encourages females to pray in their homes. 
The second part of this chapter examined the cultural domain indicators including heritage and other cultural goods such as libraries and museums and cultural activities. In this respect, the chapter aimed to investigate the extent to which those indicators contribute to the QoUL in the study area. Generally, results indicated a significant association between QoUL and all cultural domain indicators. Amongst those indicators, heritage was found to be a significant contributor to the QoUL of the Quba's residents. With regard to this, many participants expressed a positive impression towards heritage as one of the most important factors affecting the quality of their urban lives. That is, heritage in Medina is mostly linked to historical facts and events related to religion. In this context, many participants stress heritage as one of the aspects through which they can evoke the past associated with the circumstances of the emergence of the Islamic religion and the biography of the Prophet Muhammad (PBUH). For example, participants stressed the importance of having heritage monuments associated with religion such as the Quba Mosque, Al Jum'ah Mosque, the Quba Route and the holy wells associated with the biography of the Prophet Muhammad (PBUH) in their daily lives. In that sense, most of the participants are of the opinion that the presence of such landmarks enhances the spiritual aspect, which in turn positively affects their QoUL.

Places and landmarks that symbolise the heritage, culture and history of Medina also hold importance not only to residents of Medina, but also to the followers of Islam worldwide, due to the significance of the city as the birthplace of Islam (Hussain \& El-Alami, 2005). It was found that this fact has a moral and material impact for those who live in the city and especially in Quba. In this context, the existence of an outstanding religious history was cited as an influential factor in participants' lives. Almost every participant connected religious landmarks in Medina with major spiritual benefits and linked it to their happiness and satisfaction with life. In addition, many participants consider/maintain that the presence of such Islamic historical monuments, especially the Quba Mosque, has led to the enhancement of the urban development, which has positively reflected on the QoUL. In addition to the heritage related to religion, the participants pointed out that cultural heritage in general contributes to strengthening the cultural and social aspects as well as the psychological aspects associated with visiting the heritage sites. However, the presence of heritage may not always positively contribute to the QoUL, as there are likely to be negative consequences associated with the inflow of pilgrims and visitors to these sites. These 
consequences may affect social and economic aspects, and therefore may reduce the QoUL for those living in Quba.

With regard to the relationship between the QoUL and other cultural goods, findings have indicated that the QoUL of those living in Quba is significantly associated with all of the named cultural goods. In general, the QoUL increases with the increase of the level of access to those goods. In terms of the accessibility to cultural activities, however, the majority of the participants tend to report a low and moderate level of access to cultural activities such as conferences and exhibitions of hobbies, arts, crafts, and traditional food. Despite that, the relationship between QoUL and the cultural indicator is still apparent. Within the findings of the qualitative strand, both participants of the photo diaries and the experts emphasised the importance and the role of such cultural goods in the QoUL.

While findings from the quantitative and qualitative research are compatible in most cases within this chapter, there was also a contradiction between those strands. For example, the results indicated a compatibility within the participants' impressions regarding the social domain indicators in both strands. For the cultural domain, however, it seems that to some extent, there is no noticeable impression indicating the extent to which participants are interested in the cultural goods, especially in terms of accessibility to cultural activities. It can be observed from the quantitative strand that the majority of participants reported a moderate to high level of QoUL, where this level of QoUL is associated with a low or moderate level of access to cultural activities, whereas the qualitative results indicated a positive impression regarding the impact of cultural goods on the QoUL of Quba's residents. From that, it can be said that both quantitative and qualitative results obtained from the assessment of the cultural activities are to some extent compatible.

In general, findings from this chapter support some of the ideas that emerged from postmodernity, though most notably the differences. In this context, focusing on the differences and uniqueness of the needs and attitudes of all members of society are one of the contributions of the postmodern perspective. As pointed out in the literature review, males and females not only use the landscape of cities in different ways but experience and perceive them differently, which has been confirmed in most QoUL studies. That is, people live in one place, but they perceive it differently according not only to their gender, but also 
their age, educational and economic status and other socio-demographic attributes, which has been confirmed in findings from this chapter. The cultural context of the study area did reflect how people perceive QoUL differently, especially with regard to the gender roles, and most notably with respect to security.

Since postmodernism calls for pluralism, difference and multiple identities through openness, tolerance and interaction with others, the results obtained from measuring the relationship between religion and the QoUL in this chapter emphasise those aspects in a number of cases. For instance, it has been found that religion encourages a good relationship with neighbours regardless of their religious beliefs and cultural background, which is in line with postmodern thought. Another example of confirming pluralism and differences is that religion, Islam in particular, calls for rapprochement with others, which has been confirmed in the findings of the photo diaries study within this chapter. Therefore, diversity and difference advocated by postmodern thought may be encouraged by religion.

In terms of the QoUL, one may argue that freedom of choice encourages a better QoUL. However, in some cases, pluralities and differences that promote an individual's freedom of choice may not be accepted by religious teachings. That is, some practices that stem from freedom of choice may not be compatible with religious teachings. Sexual morality could be the obvious example of the relationship between pluralities and differences and religion. In Christianity, for example, sex outside marriage was traditionally unacceptable, and it is certainly what Islam emphasises as well. Thus, postmodern thought is not entirely compatible with religion. In addition, not only can religion conflict with some of the postmodern thoughts, but cultural norms do also so too. While postmodernism suggests that what is right for a group of people is not necessarily true for all people, cultural norms view morality unilaterally. That is, what is true for a group of people within a place is true for all people within that place, which is conflict with postmodernism. From this standpoint, the influence of cultural norms may be stronger than the influence of religion in relation to the freedom of choice. That is, there may be a right for one to be religious or non-religious, but it is difficult to clash with the local culture of community. 


\section{Chapter Nine}

\section{Conclusion}

\subsection{Introduction}

This thesis has explored the notion of the QoUL in the city of Medina, focusing on the municipality of Quba in order to gain insight into how people experience their daily lives. In selecting Medina, the second holy city for Muslims as a case study, this thesis has also contributed a particular, and alternative context to broaden the QoUL debate. Drawing from the literature, this thesis highlighted the extent to which various domains contribute to the QoUL. However, it sought to think differently about the QoUL by using a combination of conceptual approaches, namely functionalist and postmodernity approaches, in order to provide an overarching understanding of the QoUL characteristics. Such understanding involved identifying the most influential aspects of physical, social and cultural domains, and exploring the impact of these aspects on QoUL in the study area. The use of such conceptual approaches changes the way in which the QoUL can be understood as opposed to just measuring the satisfaction of residents with the urban environment in which they live, which is the most common approach seen in the QoUL literature.

In so doing, this thesis sought to elicit the ideas embodied in these conceptual approaches in order to gain a better understanding of the QoUL. For example, the functional approach emphasises the complementarity of elements of the urban system in enhancing the QoUL. Thus, this perspective leads to a rethink about the physical urban environment (i.e., its functional attributes) and how these attributes contribute to residents' QoUL. The postmodern approach has been useful in order to identify some social and cultural aspects of the urban environment, including religion and heritage, that have hardly been addressed within the QoUL literature. The usefulness of applying postmodern thinking to QoUL in this thesis lies in the three major references to postmodern thought mentioned by Minca (2001) as set out in Chapter 2 (see Section 2.3.2). First, religious practices and beliefs that have been found to positively affect QoUL may be viewed as distinctive and cultural 
stylistic practices which are in themselves interesting. In this context, this reference has led to understanding satisfaction and happiness as outcomes of these practices. Likewise, key components of the social order such as social relations and social interaction can be also understood through this postmodern reference. Second, a postmodern approach can lead to questioning the very process of QoUL evaluation that is solely based on a specific and rational understanding of values. For example, the value of material aspects such as housing may be more important than apparently immaterial aspects such as religion and spirituality, and vice versa. Finally, postmodern thinking challenges the hegemony of any single intellectual persuasion. In the field of QoUL, this postmodern perspective may lead to a rethinking about the sole use of objective indicators in the evaluation of QoUL, where people's opinions equally matter.

\subsection{Summary of thesis findings}

This thesis has investigated the QoUL in Quba municipality in Medina, Saudi Arabia by highlighting the aspects affecting the QoUL of those living in Quba. While it explored the most influential aspects on the QoUL, this thesis looked at how residents themselves perceived their QoUL by assessing their satisfaction with various indicators of the urban environment in which they live. In doing so, it had the following aims:

- To evaluate the status of urban quality of life in Quba, taking into consideration the impact of the socio-demographic attributes.

- To determine the most influential aspects on the QoUL of those living in Quba.

- To assess the quality of physical and functional attributes of the built environment in Quba.

- To examine the influence of social and cultural domains on the QoUL of those living in Quba.

- To provide a critical complex approach in relation to the impact of physical, social and cultural domains on QoUL. 
To address each of these objectives, five analytical chapters starting from Chapter 4 through to Chapter 8 were developed. The following sections summarise the findings of the thesis obtained from each of these chapters and discusses what is behind these findings.

\subsubsection{QoUL in Quba: a general reflection}

A general reflection on QoUL in Quba was set out in Chapter 4 as a foundation for the rest of the analytical part of the thesis. The status of QoUL and the overall impact of various indicators on the QoUL in Quba municipality, including socio-demographic attributes as well as various aspects of life were discussed. An initial impression in relation to Quba residents' evaluation of their QoUL indicated the extent to which social and cultural aspects affect the perception of people regarding their QoUL. It was noted that the religion and spirituality that characterise Medina and Quba in particular may play a much more important role in how people perceive their QoUL than the material aspects. In this context, residents who live in neighbourhoods that fall within the scope of the holy places have shown a higher level of QoUL, though these areas are considered old, and thus may lack the aesthetic qualities. From these findings, it can be said that QoUL does not only depend on the availability of materialistic means but may also depend more on the spiritual side. That is, spirituality may be more appreciated than the material and aesthetic qualities.

Such findings and conjectures contribute to knowledge by emphasising that satisfaction with the overall QoUL does not only mean improving the built environment, but rather, there are spiritual aspects that may have more influence, which is found to be an area relatively missing in the literature. Although the effect of religion and spirituality on the QoUL has been mentioned in the literature, very few studies have shown the impact of these indicators on people's QoUL, rather discussing these effects from a narrow angle that did not lead to a broader understanding about these aspects. Moreover, most of these studies have been conducted in western countries, which are predominantly Christian, while the effect of religion on the QoUL in the Islamic context has not been adequately addressed. Therefore, this thesis uncovered the extent to which religion and spirituality contribute to QoUL in such a context.

Socio-demographic attributes including gender, income and residential status appeared to affect the perception of the QoUL of those living in Quba. Most obvious and influential of 
those attributes were gender and income. Findings regarding the relation between gender and QoUL perception indicated the role of local culture, where women rely on men in most aspects of life, hence, reporting much higher QoUL than men (cf. Hating et al., 1984; Diener and Diener, 2009). In this context, findings showed that gender not only explains how people perceive their QoUL differently, as pointed out by many studies in the literature, but also leads to thinking about a range of related concepts such as responsibilities, dependency as well as inequalities. With regard to the relationship between QoUL and income, inconsistencies were noted/observed, where those who earnt less reported higher QoUL. An inference that might be drawn from this is that earning a little money when it is in conjunction with other aspects, such as the importance of the spirituality of a place, may lead to a good QoUL, which has been confirmed in the qualitative findings within Chapter 4 (see Section 4.4). There is no doubt that money is important in achieving a better QoUL. In some cases, however, what people value is not just money, but other values such spiritualty and living in a sacred place may be much more appreciated than earing money (cf. Diener et al., 1993; Diener \& Diener 1995; Veenhoven, 2001; Diener et al., 2009).

Regarding the impact of a range of aspects of life on the perception of QoUL, findings indicated that health and security are the most influential aspects of the QoUL of those who live in Quba. It has been inferred from these findings that people not only place these aspects at the centre of their day-to-day priorities because they immediately affect their personal lives, but rather, they may appreciate these aspects because they are encouraged by religion to do so. Even though such notions did not directly emerge from the findings of this thesis, studies evidence that religions, Christianity and Islam in particular, prohibit dealing with some aspects that are harmful to health, such as smoking for example (Garrusi \& Nakhaee, 2012), indicating the role of religion in people's health and overall QoUL. Therefore, people not only refrain from things that harm health merely on the basis that they are 'harmful', but because religion prohibits dealing with those things.

\subsubsection{Perceived physical attributes of the urban environment in Quba}

Chapters 5, 6 and 7 of this thesis addressed the physical attributes of housing, neighbourhood, and urban services in an attempt to understand the role of each attribute in the QoUL of those living in Quba. The following sub-sections detail the outcomes 
concerning the contribution of each of these physical attributes to the QoUL of those living in Quba.

\section{- Housing quality}

Quality of housing entailed assessing the satisfaction with accommodation and highlighting issues that influence satisfaction with housing, and in turn the overall QoUL. What seemed to emerge as a key factor in the overall findings was the influence of two particular sociodemographics on levels of satisfaction with housing, namely age and residential status. Moreover, other factors such as the type of accommodation, adequacy of the accommodation, fiscal burden and affordability, along with mobility were clearly found to affect housing satisfaction. With regard to the effect of age, findings have proven that older people are more satisfied with their accommodation. These results were justified by the hypotheses that the elderly may be more attached to their home due to the connection of this home with their past. That is, the house may be associated with memories over decades, which increases the importance of it, and thus satisfaction with it. Drawing from that, it can be said that time matters when evaluating QoUL. Thus, any current evaluation of QoUL can depend on past experiences and memories, and therefore, the current status of QoUL does not necessarily present a complete, coherent, definitive picture.

Home ownership as another factor was found to clearly influence satisfaction with accommodation and in turn QoUL. It has been argued that this type of tenure has an effect on establishing a successful family, indicating the positive connection between home ownership and family formation. However, home ownership may not always be the best option. It may be a hindrance to the family in the future in their ability to respond to the dynamic growth of the household. As a result, this type of tenure may be a reason behind the low QoUL for some. Moreover, home ownership may not be suitable for those people who move from one place to another, either for economic or social reasons. Consequently, it cannot be asserted that there is absolute positivity to this type of tenure (cf. Rohe \& Stegman, 1994; Rohe \& Basolo 1997; Scanlon, 1999; Rohe et al., 2001; Ren et al., 2016). Such considerations lead to rethinking about this type of tenure and the extent to which it influences QoUL. 
In this thesis, the effect of accommodation type on housing quality seemed to be related to the extent to which the house met local cultural norms. In this context, it may not matter how crowded the home is or the quality of its facilities, as much as it matters in terms of the extent to which it conforms to those norms that stresses gender segregation, especially in the case of non-relatives visiting. Hence, the privacy that may result from living in detached homes is crucial to the housing preferences. However, this may pose a challenge to the residents of Quba as to housing options, which ultimately affect their QoUL. In another aspect related to housing conditions, the relationship between satisfaction with housing and affordability of housing refers to the link between increased spending and housing satisfaction, which has been emphasised in several studies in the literature (e.g., Lu 1999; Vera-Toscano \& Ateca-Amestoy, 2007). From another angle, however, unaffordability of housing, whether to rent, to buy or to maintain, may lead to an issue of housing accessibility, which may negatively affect housing satisfaction, and in turn overall QoUL.

Residential mobility as an indicator of housing satisfaction seemed to be influenced by factors such as age and family size, as well as the spiritual aspect. Findings from this thesis indicated that the greater the age, the less the intention to move out. This may be due to the association with memories. Therefore, the extent of residential mobility does not necessarily depend on the current circumstances of the home but may depend more on past experiences and memories, as is the case in evaluating the QoUL. On the other hand, dynamic growth of the family may encourage the intention to move. In this context, if the household does not have an opportunity to move out to accommodate such growth, then it is likely that their QoUL will be negatively affected. Such observations indicate that home meanings change over time. From another perspective, the spiritual aspect may have a role in housing satisfaction and in turn on the residential mobility. That is, the prevalence of sacred symbols may give a spiritual value to the home, which in turn reduces the propensity to move out. Considering the relationship between housing satisfaction and these various factors, it can be said that home is a complex concept, and it has a complex relationship with QoUL.

\section{- Neighbourhood quality}

Unlike quality of housing, neighbourhood quality is likely to be influenced more by external circumstances of the urban environment. Findings from Chapter 6 indicated that residents 
were less satisfied with the neighbourhood in which they lived compared to the satisfaction level with their accommodation. This maybe because people have more control over their accommodation than the neighbourhood. In this context, having control or not may matter, and could be a factor affecting residents' satisfaction, in turn affecting the overall QoUL. Despite that, neighbourhood satisfaction was associated with higher QoUL, indicating an obvious positive impact of this physical attribute on the QoUL in the study area. However, satisfaction with the neighbourhood was affected by socio-demographic attributes including residential status and income as well as neighbourhood components.

It was noted how home ownership seems to play an important role in neighbourhood preference and in turn satisfaction with it. For example, households may select carefully the neighbourhood in which they will live before owning a home in it, because in most cases it is like to be considered as permanent place for their residency. Thus, home ownership may contribute to the satisfaction with the neighbourhood. In addition, ownership may make residents more attached to their neighbourhood, and in turn more satisfied with it. At the same time, however, the permanency that can result from owning a home in a neighbourhood may become a reason for dissatisfaction with that neighbourhood over time. That is, people may face issues within the neighbourhood in the future, but they may be unable to change their dwelling because they are own it. In this respect, permanency related to home ownership may be significant, and could be a factor that affects the overall QoUL. Likewise, with regard to neighbourhood preference, the higher the income, the greater the opportunity to choose the appropriate neighbourhood to live in, and thus satisfaction with it. Over time, however, there may be a preference in favour of income. That is, there may be an opportunity for residents with higher income to change their homes if they are not satisfied with it in the future, and vice versa, especially in the case of owners. Hence, home ownership may be a negative factor in neighbourhood satisfaction over time (cf. Lu, 1999; Parkes et al., 2002; Grinstein-Weiss et al., 2011).

Other factors represented by neighbourhood components, including housing more generally and a range of functional attributes were found to affect the satisfaction of residents with their neighbourhood. However, the influence of these components was varied. Moreover, the importance of these components in neighbourhood satisfaction is also varied. Housing seemed to be the most influential component over other functional attributes. In this regard, 
the highest satisfaction ratio with the neighbourhood was associated with a higher ratio of satisfaction with the accommodation. This may because feelings about accommodation may spill over into feelings towards the neighbourhood, indicating the importance of housing in promoting neighbourhood satisfaction. This has led to thinking about scale when assessing QoUL. In this context, QoUL has different spatial characteristics based on different scales, of dwelling, neighbourhood and city. Therefore, it is difficult to speak about QoUL in general, where different scales should be taken into account when assessing QoUL in a geographical setting.

The other components of the neighbourhood encompassing the social, economic and environmental components, were found to contribute to the satisfaction with the neighbourhood. In general, the relationship between neighbourhood satisfaction and these various components, especially social components, seemed to be a sequential process, where those components predict neighbourhood satisfaction, and neighbourhood satisfaction predicts overall satisfaction with life. However, these components differed in the in terms of the form and magnitude of the effect. Indicators of the neighbourhood that concerned accessibility and connectivity, especially the lack of car parking and lack of basic shopping centres, were found to be other issues that clearly affected neighbourhood satisfaction. This indicates that accessibility matters in neighbourhood satisfaction and in turn overall QoUL. Findings in this regard indicate the role of the local culture. In this context, the car-dominated culture in the study area along with the frequency with which residents visit the basic shopping centres point to the importance of these two issues in promoting neighbourhood satisfaction and overall QoUL. Therefore, local culture should be taken into account when addressing such issues that may have a strong impact on neighbourhood satisfaction and QoUL in general.

To sum up, it can be argued that neighbourhood is a complex notion and has a complex relationship with QoUL, since it combines several dimensions. Unlike dwelling, the neighbourhood may be merely a place where a home is located and, therefore, it may be less important in determining the QoUL (cf. Sirgy and Cornwell, 2002; Permentier et al., 2010; Mridha \& Moore, 2011). This is because meanings of neighbourhood may differ from one person to another, or perhaps from one culture to another. From what has been presented in relation to the quality of the neighbourhood, this thesis contributes to knowledge by 
providing a comprehensive understanding of the complex and multidimensional nature of the neighbourhood and the extent to which this urban unit contributes to the QoUL of residents. Specifically, this thesis has highlighted various components of the neighbourhood and has attempted to broadly analyse the impact of these components on neighbourhood satisfaction and the overall QoUL.

\section{- Urban services quality}

Quality of urban services explored the functional attributes within Quba municipality with respect to health services, educational services and recreational facilities. Findings from the subjective evaluation showed that satisfaction with health services is affected by a number of issues related to the service provided by the medical staff, getting medical appointments and diagnoses. Measuring the quality of health services, however, indicated that there is an issue of scale, where most health service providers in the municipality of Quba serve all residents of Medina. This entails the inability to objectively measure the quality of those services in a specific scale, especially when talking about the QoUL in a wider scale such as the city or regional level. The scale discordance in this context may create a gap, or perhaps discordances between the objective and subjective assessments. What can be inferred from that is there may be a potential impact of the scale discordance on obtaining valuable findings regarding the quality of those services within the underlined scale. Such observations indicate a weak relationship between subjective and objective indicators, which supports the claims of several studies on this issue (e.g., McCrea et al., 2006; Das, 2008; McCrea et al., 2011; Mohit, 2013; Soleimani et al., 2014).

Likewise, there seemed to be discordance between objective and subjective assessments with regard to the assessment of educational services. Although objective indicators seemed to indicate some positive findings, subjective evaluation indicated a dissatisfaction with the educational services. This again confirms the weak relationship between these indicators. On the other hand, there seemed to be an obvious relationship between objective and subjective indicators in relation to the evaluation of the recreational services. In this regard, there was consistency in the findings from both objective and subjective evaluation, indicating poor recreational services, and thus a strong negative impact on the QoUL of those living in Quba. However, by referring to the findings from Chapter 4 (see Section 
4.5), recreation is ranked as the lowest in terms of the impact it has on the QoUL among other aspects of life. Drawing from that, it can be argued that QoUL is not just about absolute measures, but about the weighted relative importance of indicators.

In light of what has been presented with regard to the quality of urban services, it can be said that these functional attributes directly influence the QoUL in an urban setting. This is because these functional attributes are linked to people's primary needs, especially health and education, which reflects the magnitude of their impact on daily life. The quality of the health services is a crucial aspect in achieving other aspects of life, which in turn contribute to the overall QoUL. In the case of Quba in particular, health, as mentioned in Chapter 4 (see Section 4.5) is the aspect that most affects the QoUL. Quality of the educational facilities is also important in achieving other aspects of life, including social and economic aspects. Recreational facilities too, are not only important in promoting health and phycological benefits, but also strengthen social relationship between members of the community. In the case of Quba, therefore, policy makers should pay more attention to these functional attributes due to their paramount importance in people's daily lives.

\section{- Concluding remarks regarding perception of the physical attributes}

Findings from the assessment of the physical attributes of the urban environment in Quba indicate that from housing and neighbourhood to urban services, QoUL is substantially affected, but not in the same form or magnitude of the impact. While housing and neighbourhood indirectly affect QoUL, functional attributes represented by the urban services directly affect residents' QoUL. Regardless of this variation in the form and magnitude of the impact, these three different scales can be considered as an integrated urban system that substantially contribute to the QoUL. In this context, the consistency in the quality of these scales may in turn affect the overall QoUL. From the perspective of the functionalist approach to the QoUL adopted in this thesis, housing, neighbourhood and functional attributes should compatibly and consistently work together in order to achieve a better QoUL. The main reason for this is that feeling about QoUL at one of these scales may spill over to feelings towards the others (e.g., Sirgy \& Cornwell, 2002; Grzeskowiak et al., 2003; Hur \& Morrow-Jones, 2008). For instance, feeling about the quality of dwelling may spill over into the attitude towards the neighbourhood and so on. In this context, 
dissatisfaction with one these attributes may lead to negative perception towards the whole urban environment. That is, people may be satisfied with the accommodation they live in, but at the same time dissatisfied with the neighbourhood they live in or with the urban services at the city or regional level. Subsequently, residents may report a lower overall QoUL even though they are satisfied with one of these physical attributes. A functionalist approach in this context may change the way of thinking regarding the assessment of these physical attributes and the extent to which these attributes contribute to the QoUL in an urban setting. Therefore, researchers as well as the policy makers should be aware of the importance of integration of these physical attributes in perceiving QoUL.

\subsubsection{Perceived social and cultural QoUL}

Using postmodernity thinking that emphasises the role of social and cultural aspects in people's lives, this thesis highlighted social and cultural domains of the QoUL in Quba. In so doing, this thesis sought to highlight the influence and contribution of these domains to the QoUL of those living in Quba, taking into account the impact of their socio-demographic attributes. The following two sub-sections provide outcomes regarding these two domains.

\section{- $\underline{\text { Social domain }}$}

The social domain of QoUL in this thesis named indicators such as security, leisure, social interaction, neighbour support, religion and spirituality. Except for leisure activities, findings in general indicated a positive impact of these indicators on the QoUL of those living in Quba. Security was found to be associated with a higher level of QoUL, which finds support from a large body of research that confirms the important of this indicator in promoting QoUL. However, a key finding from this thesis with regard to the impact of socio-demographics, gender in particular, indicate a different meaning of security. In this regard, findings showed that women seemed to feel more secure than men. This was justified by the fact that according to the local culture, women are often expected to be accompanied by males (e.g., a father, brother or husband) when they are going outside the home. For this reason, women may feel more secure not in terms of themselves, but because they are often accompanied by males. Therefore, security here is impersonal. With regard to social interaction, quantitative and qualitative findings have both proven the positive contribution of this indicator to the QoUL. From another angle, however, social interaction tends to not 
often contribute to the QoUL in the same pattern. For some, social interaction may be undesirable, because they prefer not to be involved with others outside their own home, or they believe that interacting with others may bring them unhelpful/unnecessary problems. Thus, social interaction could be a negative factor for QoUL rather than a positive one (cf. Marans \& Rodgers, 1979; Riger \& Lavrakas, 1981; Datta et al., 2015).

Religion and spiritualty as other indicators were found to have a clear impact on the QoUL of those living in Quba. In this regard, findings from both quantitative and qualitative research confirmed the role of these indicators in promoting higher QoUL. From improving the relationship between people and improving mental health to enhancing happiness, life satisfaction and SWB, religion has been found to contribute to the overall QoUL. In this context, religious practices and beliefs, spirituality as along with the sense of the place were the most influential factors. For example, attendance at the mosque is the most prominent religious practice. In this regard, frequent attendance at the mosque was found to be associated with several benefits including social and mental health benefits. In this context, the mosque is not only the place of worship, but rather a social centre. Other religious practices, including fasting and zakat, have been found to promote many benefits that enhance QoUL, the most important of which is strengthening social ties.

Spirituality was also found to be a factor that clearly affects the QoUL of those who live in Quba. In respect of that, Medina, and Quba in particular, is considered to be one of the most important holy places in the world. Therefore, the sense of spirituality in these areas was found to promote happiness and satisfaction with life. The sense of the spirituality of the place for the residents of Quba derives from the fact that they live in a place where the Islamic religion emerged and spread to the rest of the world. Hence, this may be the key element in the extent to which spirituality contributes to the QoUL. Since spirituality is an immaterial aspect, it may change the way of thinking about QoUL, especially with regard to the quality of place. In this context, a sense of a place's spirituality may shift people's thinking from focusing on the physical quality of a place to the aspects that generate this spirituality. In this regard, findings from the photo diaries study in this thesis indicated that the presence of the things that symbolise religion, even if they are in public places, brings people back to the sense of faith and spirituality. 
Although religion is often positive because it is a refuge in the face of the hardships of many human beings, it can sometimes cause harm to them. Within the findings of this thesis, it has been found that religion reduces anxiety and depression. At the same time, however, it can significantly lead to these issues. That is, some people who are late or neglect their religious duties may experience these kinds of problems. Furthermore, people who do not uphold religious duties may be ostracised in the society in which they live, because, at the very least, they cannot be trusted. Hence, religion in such a situation may cause isolation for some people and thus a lower QoUL. In an indirect way, religion may negatively affect the QoUL by the means of social capital. In that sense, religion reinforces social capital, and social capital in some case may become harmful, though it has several positive consequences. While social capital strengthens the relationship between members of a society, some groups, especially those representing a different race or culture, may be separated from that community, and thus experience a lower QoUL. In a broader sense, religion may cause divisions between some people even though they are living in the same area and potentially lead to a lower QoUL. In this context, differences in views on religion may lead to these divisions, and thus some groups of people may develop hostility. Consequently, there may be negative implications of religion rather than positive ones. Therefore, it can be said that there no absolute positivity, or in other words, the effect of religion is not completely one-sided.

As final outcomes of the relationship between religion and spiritualty and QoUL, it can be said that these two indicators may be seen differently, though they are often used interchangeably. In the context of holy places such as Medina, the influence of spirituality may be much more than religiosity when it comes to place. That is, spirituality in Medina is attached to the place where people live and experience their daily lives. Thus, spirituality here may change people's thinking towards the QoUL in relation to the place as discussed earlier, while religiosity may often not be related to a specific place as much as spiritualty. Thus, the concept of spirituality may overwhelm the concept of religiosity when it comes to specific places. While these indicators are indeed part of people's daily lives, and probably do have an impact on the quality of their daily lives, it is difficult to measure them, because in most cases they are immeasurable. This in turn may pose a challenge to researchers who are trying to examine the relationship between these indicators and QoUL. 
- Culture domain

Within the cultural domain, heritage, especially as related to the religion and Islamic history of Medina had the most prominent effect on the QoUL of those living in Quba compared to other cultural goods. Findings showed that cultural heritage in general promotes psychological, cultural and social benefits. But, from the viewpoint of most of the participants, the spiritualty that resulted from the existence of religious landmarks is much more appreciated. In this context, the existence of a unique religious history was cited as an influential factor in the lives of those living in Quba, as nearly every participant linked religious landmarks in Medina with great spiritual benefits and subsequently to their happiness and satisfaction with life. For other cultural goods such as museums, libraries and cultural events, residents of Quba seemed to not give them as much attention compared to cultural heritage. Paying much more attention to the heritage in Medina in general may be a part of religious duties. Therefore, the spiritual benefit that accrues from heritage may be more important than the benefits that may accrue from other cultural goods.

Drawing from what has been presented with regard to the cultural domain of QoUL, it can be argued that the perception of cultural elements is varied from one culture to another according to the type of prevailing cultural goods. In this context, some may see that the spiritual value of heritage is more important than the material ones. While others may argue that the heritage as well as other cultural goods should be exploited for economic benefits that could lead to a better QoUL. From another perspective, some people may see that the proximity of heritage to them might cause them problems, such as crowding caused by visitors or high prices resulting from the presence of these heritage monuments. On the other hand, others may see that the existence of these landmarks could bring economic gains for them or provide an opportunity to meet and get to know other cultures through foreign visits to these landmarks. In this regard, postmodernity may change the thinking about the QoUL in terms of preference, since the freedom of choices and a diversity of preferences is appreciated by postmodern audiences.

- Concluding remarks regarding perception of social and cultural domains

It can be argued that social and cultural elements have a clear impact on the QoUL. The influence of social and cultural aspects may be greater than the influence of other physical 
and economic aspects. This is because the social aspects in particular may be more closely related to people's daily lives. Social relationships, social interaction as well as religion may be essential to people's daily lives in improving mental health as an example. However, these aspects, particularly religion, faith and spirituality, are hard to assess. In addition, social and cultural indicators are varied in their effect on QoUL based on the sociodemographic attributes as well as the cultural context. This may be due to the disparity in the extent to which people need these elements in their urban quality of life. In addition, socio-demographic attributes may significantly influence how these elements of the urban environment are perceived, and thus influence the outcomes of the QoUL. Such observations indicate the extent of the difference and disparity in the perception of those aspects of the urban environment. Accordingly, it can be said that people's perception of social and cultural aspects of the urban environment cannot be an absolute truth in one way.

\subsection{Research limitations}

When conducting this study, especially with regard to the data collection, the researcher faced the issue of balancing the selection of participants in terms of gender, where due to dominant cultural norms it is commonly difficult for a male researcher to conduct interviews with female participants. With the help of the academic community at Taibah University, however, the researcher was able to reach out to this category of participants. One of the solutions of this issue was represented by the use of the audio network at the university and contacting each participant on the telephone for the follow-up interview. It might be helpful in the future to use assistants, as well as social media, to reach those participants. Furthermore, difficulties were encountered in relation to the collection of some the secondary data, particularly due to the lack of the date related to the socio-demographic attributes.

Further limitations of this thesis were related to the timeline of the data collection. Since the fieldwork of this thesis was conducted outside of the UK, this thesis adopted a crosssectional study approach in collecting the data sets. This made it difficult to draw up broad, firm conclusions about the status of the QoUL in the study area. Future research would benefit from using a longitudinal study to trace changes over time for a better understanding 
of the dynamics of the QoUL. Another limitation of this thesis is that public transport has not been highlighted as one of the most vital physical indicators of QoUL. One of the main reasons behind this is that public transport in Medina, and perhaps across the Kingdom of Saudi Arabia in general, is not as well-used as it is in many other countries. This is due to the reliance of many people in Medina on the use of private transportation in most cases, which may have led to the slow development of this aspect of the urban environment.

Conducting such research provided valuable skills for the researcher. One of the most important of these skills is the critical approach that has been developed during the reading and academic exchanges at Swansea University. Moreover, conducting such research in the English language has opened opportunities for the researcher to gain broader knowledge of the topic by reviewing the wider set of academic literature not available in Saudi Arabia. Such expertise will allow the researcher to use the new insights from this study and contribute to the development of innovative methodological and epistemological approaches to studying QoUL in Saudi Arabia.

\subsection{Implications for policy and direction for future studies}

For policy makers, findings from this thesis suggest some useful implications for policy. For example, findings from measuring satisfaction with health services suggest that residents are dissatisfied with some aspects such as timely treatment and the ease of getting a medical appointment. Hence, it is important for the policy makers to pay more attention to such aspects in order to raise the quality of health services. Moreover, the lack of green spaces and recreational facilities, which was highlighted by both subjective indicators and objective indicators, suggests more attention in supporting such facilities because of their many benefits for the residents of Quba and Medina in particular, the most important of which are those related to social aspects. Other findings assessing the quality of neighbourhoods suggest more attention should be given to solving some of the issues facing residents, such as the lack of car parking and the lack of green spaces within neighbourhoods. Moreover, some aspects of neighbourhoods, such as appearance, aesthetics and attractiveness were found to be associated with higher satisfaction with the 
neighbourhood. Therefore, these aspects should be taken into account when plans are put in place for neighbourhoods.

Additionally, some social centres, more specifically the mosque, should receive more attention, not only as a place of worship but also as a social centre that can contribute to social development. Since this social organisation is one of the most important elements of the urban environment in the study area and is also a gathering point for most of the neighbourhood's residents five times a day, policymakers should exploit this social organisation in urban development, especially in terms of social life. According to what has been found within the results of this thesis, policymakers should be aware that the function of the mosque is not only limited to worship, but rather goes beyond this to include promoting various social aspects that may ultimately play an important role in improving the QoUL. For example, the mosque could be a place for social events, where it may enhance social life such as social interaction and social support, which have been emphasised in the literature as among the most significant social indicators that are likely to contribute to a better QoUL. However, the most important aspect through which the mosque realises these advantages is the competence of the $\operatorname{Imam}^{10}$ of the mosque. In this regard, Al-Shaibani, one of the experts who participated in this study, stated that the Imam of the mosque should be chosen carefully, because he is the important element in the success of the mosque in achieving its social function.

For future studies, findings from the thesis suggest some directions for more research into the QoUL. Investigating the QoUL in this study has led to the emergence of some concepts and issues that may be worth further research. One of the most notable aspects is the relationship between a number of the socio-demographic attributes and QoUL. For instance, women were found to report a higher QoUL than men. These results were justified by the fact that women in the context of the local culture may be nurtured by men in most aspects of life, and thus, they may report a better QoUL compared to men. In this context, an important question arises about the extent to which this relationship translates into practice.

\footnotetext{
${ }^{10}$ The Imam is a title given to the one who leads people during the performance of prayers in the mosque, as well as giving religious guidance. In addition, one of the jobs of the Imam is to supervise the services provided to the mosque, such as maintenance, cleaning, etc. In this context, the Imam is the one primarily responsible for the mosque.
} 
Therefore, future research should make further investigation regarding this issue of gender and QoUL. In addition, the importance of the neighbourhood in relation to the QoUL should be highlighted, with respect to the discussion in Chapter 6 here that noted how the importance of the neighbourhood itself may be varied due to external circumstances that operate or arise outside it.

Another direction for future studies is to extend the scale of the QoUL investigation. Since this study was limited to the municipality of Quba as one of the most important municipalities in Medina (see Section 1.2.), future studies should focus on other areas of Medina to compare it to the current case study. It would also be worth extending the research into the QoUL to include the whole city of Medina. Although it is difficult to focus across such a scale, especially in terms of a subject like QoUL, it is useful to get an overall picture of the QoUL at a broader scale. This is likely to help compare and track variation in the QoUL within the municipalities of Medina.

Finally, what the world is presently facing due to the repercussions of the COVID-19 pandemic calls for a rethinking about the QoUL. People's daily life is likely not to be the same as before this epidemic, where social and economic lifestyles might be changed. In this context, people may rethink their lifestyles, including their social relationships, economic lifestyle, mobility, and preferences, especially those related to housing, and more. Therefore, the notion of urban quality of life after the COVID-19 pandemic may include new or perhaps old revived lifestyles, social norms and customs. Hence, future studies in the field of the QoUL should identify what people have experienced in their daily life during this epidemic. Policy makers too need to be aware of what matters most to the residents in several aspects of their daily life in order to develop plans that meet their needs and aspirations and contribute to achieving a better QoUL.

\subsection{Coda}

Prior to this thesis, the QoUL has been relatively unexplored in the context of Saudi Arabia and Medina in particular (but see UN-Habitat \& SMMRA, 2016). This thesis is not just an empirical study of Medina, it is a model study that takes into account religion, spirituality 
and heritage in a city that is considered to be the second holy place for all Muslims around the world. Therefore, this thesis places the study area as a model in the context of global research, in the field QoUL in particular and the field of urban studies in general. Since the QoUL has not been adequately investigated within the Islamic context, this thesis presents a study as an exemplar that provides an understanding of how social and cultural elements in particular contribute to the QoUL (cf. UN-Habitat \& SMMRA, 2016). Furthermore, this thesis could be considered as innovative not only in bringing this particular study area into context of the global literature, but also in providing an approach that leads to rethinking about QoUL in terms of how various domains contribute to the overall QoUL in different ways (positive and negative influence). In doing so, this thesis offered different ways to consider and understand the QoUL by using a combination of conceptual approaches. The use of such approaches was useful for more critical and complex analysis for the QoUL investigation. By using these approaches as a conceptual framework, the way in which the underlying ideas within these particular approaches might helpfully bear upon QoUL has been brought more clearly into view, offering new avenues of exploration, and therefore, revaluation of the concept of QoUL.

For example, the functional approach emphasises the complementarity of elements of the urban system in enhancing the QoUL. Thus, this perspective leads to a rethink about the physical urban environment (i.e., functional attributes) and how these attributes contribute to residents' QoUL. In this regard, this thesis indicates the importance of the combination of physical attributes in promoting a better QoUL, where each of those aspects tends to depend on each other in promoting an overall QoUL. For instance, good quality of housing environment is likely to lead to a better social and economic life. Likewise, good quality of neighbourhood environment is likely to lead to a better quality of social life as well as a better quality of housing environment. This also can be applied to the functional attributes themselves, where a better quality of educational and recreational facilities is likely to lead to better health and in turn, a better overall QoUL. From another angle, variation in the perception of each of those physical indicators may affect the overall perception of QoUL. That is, if people are dissatisfied with recreational facilities, they may report a lower QoUL, though they are satisfied with the quality of other facilities and so on. The functionalist approach, therefore, is not only based on the functional consistency of the elements of an urban system, but also based on the consistency of people's perception regarding the QoUL 
of the physical domain. In this context, QoUL can be considered as an assemblage of the integrating of urban attributes (i.e., physical attributes). It also can be considered as an assemblage of the integrating of people's thinking about their QoUL (consistency of their perception regarding their QoUL). Assemblage in this context expands the thinking about QoUL as an integration of various and multiple aspects of life that should be work together in achieving a better QoUL.

Drawing from what has been presented, this thesis adds some noteworthy points to the existing literature. Assessing the physical attributes of the QoUL, specifically the health indicator, has revealed the influence of scale discrepancy on the assessment of such an indicator. In this context, this thesis suggests that discrepancy of scale may create a divergence between objective and subjective indicators of the health services provided. Therefore, divergence between objective and subjective indicators of a domain of the QoUL caused by the discrepancy of the scale may affect the overall assessment of the QoUL. For the researchers and perhaps the policy makers, it would be important to consider the discrepancy of scale as a potential factor that may affect the obtaining of valuable findings regarding the quality of such services in particular and QoUL indicators in general. In other words, the discrepancy of the scale when assessing perceived and objective indicators may lead to weakening of the relationship between subjective and objective indicators, which in turn affects the outcomes of the QoUL in a geographical setting. Another issue that has been revealed by this thesis is that time matters in relation to the QoUL. That is, QoUL not only depends on the current situation, but is also linked to past experiences and memories. In this context, it can be said that QoUL is changeable/dynamic, which causes people to think about their QoUL differently. Using postmodern thinking, this thesis emphasises the differences and diversity in the QoUL. In this regard, the way the importance of the need differs for particular social groups is highlighted.

Methodologically, this thesis contributes to the knowledge by adding a qualitative method, Photo Diaries Study, in order to gain more insight into how people experience their QoUL. Moreover, this thesis contributes to the knowledge by merging different methods (i.e., questionnaires, photo diaries and follow-up interviews, and experts' interviews). Finally, this thesis indicated the relative importance of various domains of QoUL. Social and cultural domains were found to be much more influential than other QoUL domains. In this regard, 
religion as well as spirituality of place were found to be related to many aspects of QoUL. Religion was found to contribute to happiness and life satisfaction as well as social order, and more specifically, social relations. Spirituality was also found to be a key element in people's happiness and life satisfaction. Such observations may lead to rethinking about the relative importance of QoUL domains/indicators, where it has been observed from the literature that the focus tended to fall on the physical side of the urban environment, with little focus on social and cultural indicators (e.g., Grzeskowiak et al., 2003; Hur and Morrow-Jones, 2008; Rezvani, et al., 2012; Shoja et al., 2014; Soleimani et al., 2014; Qawasmeh, 2014).

To sum up, investigation into the QoUL in Quba indicates that QoUL is a multifaceted concept. This is because QoUL has multi-dimensions, and these dimensions should be holistically covered when investigating the QoUL for better insight concerning overall QoUL. Another issue of the investigation of the QoUL is that people perceive their QoUL differently according to their socio-demographic attributes, which tends to lead to a variation in the perception of the urban environment in which they live. Furthermore, the cultural context that people belong to is likely to significantly affect their perception regarding their QoUL. In this respect, it can be argued that perception of good or bad QoUL is derived from how people interact with their urban environment, taking into account the differences of socio-demographic attributes and cultural context. In this regard, dwelling perception in particular is considerably influenced by these interactions across social groups and cultural contexts. In addition, this thesis stresses the use of both objective and subjective indicators when measuring QoUL in order to gain more insight into the overall picture of the QoUL. Finally, and more importantly, this thesis stresses that investigation into the QoUL is not only a search about the extent to which people are satisfied or not with their urban environment but is also a study as to how components of this environment work together to contribute to the residents' QoUL. 


\section{References}

Aba Al-Khail, S. (2019). Manifestations of security in Saudi Arabia and its foundations. Retrieved October 10, 2019, from http://www.aljazirah.com/1999/19990903/sa2.htm

Abdel-Khalek, A. (2002). Age and sex differences for anxiety in relation to family size, birth order, and religiosity among Kuwaiti adolescents. Psychological Reports, 90, 1031-1036.

Abdel-Khalek, A. (2008). Religiosity, subjective well-being, and depression in Saudi children and adolescents. Mental Health, Religion \& Culture, 12(8), 803-815.

Abdel-Khalek, A. (2011). Religiosity, subjective well-being, self-esteem, and anxiety among Kuwaiti Muslim adolescents. Mental Health, Religion \& Culture, 14(2), 129-140.

Abdel-Khalek, A. (2014). Religiosity and Well-Being in a Muslim Context. Religion and Spirituality Across Cultures, 9, 71-85.

Abdel-Khalek, A. M., \& Eid, G. K. (2011). Religiosity and its association with subjective wellbeing and depression among Kuwaiti and Palestine Muslim children and adolescents. Mental Health, Religion and Culture, 14, 117-127.

Abdel-Khalek, A. M., \& Naceur, F. (2007). Religiosity and its association with positive and negative emotions among college students from Algeria. Mental Health, Religion and Culture, 10, 159-170.

Abdul Salam, A., Elsegaey, I., Khraif, R., \& Al-Mutairi, A. (2014). Population distribution and household conditions in Saudi Arabia: reflections from the 2010 Census. Springer plus, 3, 1-3.

Abrams, M. (1976). A review of work on subjective social indicators 1971-1975. Social Science Research Council, cited in Noll, H. (2013). Subjective Social Indicators: 
Benefits and Limitations for Policy Making-An Introduction to this Special Issue. Social Indicators Research, 114(1), 1-11.

Achoui, M. (2006). The Saudi society: Tradition and change. In J. Georgas, J. Berry, F. van de Vijver, Ç. Kagitçibasi \& Y. Poortinga (Eds.), Families Across Cultures, A 30-Nation Psychological Study, 435-441. Cambridge: Cambridge University Press.

Adie, B., Falk, M., \& Savioli, M. (2019). Overtourism as a perceived threat to cultural heritage in Europe. Current Issues in Tourism, 23(14), 1737-1741.

Agisilaos, E. (2012). Cultural Heritage, Tourism and the Environment. Sustainable Development, Culture, Traditions, 1, 70-78.

Aguilar, A. (2009). Urban Geography. In M. Sala, Geography (2nd ed., pp. 10-131). Oxford: Eoless.

Ahlbrandt, R. (1984). Neighbourhoods, People, and Community. New York: Plenum Press.

Ahmad, M., \& Mahmood, A. (2009). Zakat fund; concept and perspective. International Journal of Monetary Economics and Finance, 2(3/4), 197- 205.

Akaranga, S., \& Makau, K. (2016). Ethical Considerations and their Applications to Research: A Case of the University of Nairobi. Journal of Educational Policy and Entrepreneurial Research, 3(12), 1-9.

Al Khayat, D. (2004). Health as a Human Right in Islam. Health Education through Religion. Cairo: World Health Organization.

Al Khayat, M. (2003). Woman in Islam and her role in human development. Cairo: World Health Organization (WHO).

Al Samaan, Y. (2006). The relationship between health, religion and scientific miracles. The Middle East Newspaper. Retrieved June 25, 2019 from https://archive.aawsat.com/details.asp?issueno=9896\&article=396712\#.XTQ77eh KjIX 
Al Suqairi, M. (2015). Durb Al-Sunnah Project. Retrieved February 10, 2018 from http://www.amanamd.gov.sa/pages/PRESSANDINFORMATION/NEWS/NewsDe tails.aspx?ID=267

Alahmari, A. (2017). The State of Distance Education in Saudi Arabia. The Quarterly Review of Distance Education, 18(12), 91-98.

Albrechtsen, E. (2003). Security vs safety. Norwegian University of Science and Technology.

Al-But'hie, I., \& Eben Saleh, M. (2002). Urban and industrial development planning as an approach for Saudi Arabia: the case study of Jubail and Yanbu. Habitat International, 26(1), 1-20.

Algarni, S. (2018). Assessment of fog collection as a sustainable water resource in the southwest of the Kingdom of Saudi Arabia. Water and Environment Journal, 32(2), 301-309.

Al-Harthi, M. (2015). The new face of Medina. Arab News. Retrieved July 17, 2019 from http://www.arabnews.com/saudi-arabia/news/771961

Al-Kandari, Y. (2003). Religiosity and its relation to blood pressure among selected Kuwaitis. Journal of Biosocial Science, 35(3), 463-472.

Al-Kindi, S. (2007). Zakat: Obligatory Charity; Its rules and regulations. Islamic Information Centre, Sultan Qaboos Grand Mosque. Oman.

Almayouf, A. (2013). Preserving the Green in Hot-arid Desert Environments: The Case of Riyadh, Saudi Arabia. Journal of King Saud University, Arch. \& Planning, 25, 39-49.

Almutairi, K., Mansour, E., \& Vinluan, J. (2016). A cross-sectional assessment of QoL of breast cancer patients in Saudi Arabia. Public Health, 136, 117-125.

Almutairy, S., Davies, T., \& Dimitriadi, Y. (2015). The Readiness of Applying m-learning among Saudi Arabian Students at Higher Education. International Journal of Interactive Mobile Technologies, 9(3), 33-36. 
Al-Naaimi, A., \& Younis, M. (2010). Assessment of religious and spiritual beliefs dimension of life quality in a sample of fifth year medical college students of University of Baghdad. Fac Med Baghdad, 52(4), 412-415.

Alrashidi, O., \& Phan, H. (2015). Education Context and English Teaching and Learning in the Kingdom of Saudi Arabia: An Overview. English Language Teaching, 8(5), 3344.

Alsamghan, A., Awadalla, N., Mohamad, Y., \& Hassan, A. (2016). Influence of altitude on pediatric asthma severity and QoL in southwestern Saudi Arabia. Egyptian Journal of Chest Diseases and Tuberculosis, 65(3), 555-561.

Al-shaikh, A. (2016). Future of Saudi Cities Program (FSCP). In HABITAT III Conference, Oct 2016. Quito: UN-Habitat.

Alsoghair, S. (2007). The youth of Saudi Arabia and their Problems: Reality and Motives. [Report]. National Centre for Youth Research, King Saud University.

Altaş, N., \& Ozsoy, A. (1998). Spatial adaptability and flexibility as parameters of user satisfaction for quality housing. Building and Environment, 33(5), 315-323.

Aman, J., Abbas, J., Nurunnabi, M., \& Bano, S. (2019). The Relationship of Religiosity and Marital Satisfaction: The Role of Religious Commitment and Practices on Marital Satisfaction Among Pakistani Respondents. Behavioural Sciences, 9(3), 1-13.

Ander, E., Thomson, L., Noble, G., Lanceley, A., Menon, U., \& Chatterjee, H. (2012). Heritage, health and well-being: assessing the impact of a heritage focused intervention on health and well-being. International Journal of Heritage Studies, 19(3), 229-242.

Andrews, F. (1974). Social indicators of perceived life quality. Social Indicators Research, 1(3), 279-299.

Andrews, F. M., \& Withey, S. B. (1976). Social indicators of well-being: The development and measurement of perceptual indicators. New York: Plenum Press. 
Antoniuk, L., Kalenyuk, I., Tsyrkun, O., \& Sandul, M. (2019). Rankings in the higher education competitiveness management system. Problems and Perspectives in Management 17(4), 325-339.

Amaratunga, D., Baldry, D. Sarshar, M., \& Newton, R. (2002). Quantitative and qualitative research in the built environment: application of "mixed" research approach. Work Study, 51 (1), 17-31.

Argan, M., \& Dursun, M. (2018). Examining Relationships Among Well-being, Leisure Satisfaction, Life Satisfaction, and Happiness. International Journal of Medical Research \& Health Sciences, 7(4), 49-59.

Ariff, N., \& Hilary, D. (2009). Sustainable living environment for urban low-income households in Malaysia: key factors for maintenance. In International Symposium on Construction in Developing Economies: Commonalities among diversities (2009) (pp. 380-393). Penang, Malaysia: CIBW.

Assaf, S., Bubshaitr, A., \& Al-Muwasheer, F. (2010). Factors affecting affordable housing cost in Saudi Arabia. International Journal of Housing Markets and Analysis, 3(4), 290-307.

Atkinson, R., \& Flint, J. (2001). Accessing hidden and hard-to-reach populations: Snowball research strategies. Social Research Update, 33(1), 1-4.

Ayyad, E. (2017). Revisiting K.A.C. Creswell's Theory on the First Mosque in Islam. Journal of Islamic Architecture, 4(4), 188-195.

Azad, A., Bostani, M., \& Anvari, M. (2015). Assessment of the life quality of urban areas residents (The case study of the city of Fahraj). International Organization of Scientific Research, 5(7), 31-36.

Bakar, N., Malek, N., \& Mansor, M. (2016). Access to Parks and Recreational Opportunities in Urban Low-income Neighbourhoods. Procedia-Social and Behavioral Sciences, 234, 299-308. 
Baker, D. (2003). Studies of the inner life: The impact of spirituality on quality of life. Quality of Life Research, 12, 51-57.

Baker, F., \& Intagliata, J. (1982). Quality of life in the evaluation of community support systems. Evaluation and Program Planning, 5(1), 69-79.

Bamberger, M. (2000). Integrating quantitative and qualitative research in development projects. Washington, DC: The World Bank.

Bani, L., \& Pate, H. (2015). The Role of Spouses under Islamic Family Law. International Affairs and Global Strategy, 37, 104-111.

Barbosa, O., Tratalos, J., Armsworth, P., Davies, R., Fuller, R., Johnson, P., \& Gaston, K. (2007). Who benefits from access to green space? A case study from Sheffield, UK. Landscape and Urban Planning, 83(2-3), 187-195.

Baroun, K. (2006). Relations among Religiosity, Health, Happiness, and Anxiety for Kuwaiti Adolescents. Psychological Reports, 99(3), 717-722.

Basolo, V., \& Strong, D. (2002). Understanding the neighbourhood: From residents' perceptions and needs to action. Housing Policy Debate, 13(1), 83-105.

Batyrzhan, M., Kulzhanova, B., Abzhalov, S., \& Mukhitdinov, R. (2014). Significance of the Hadith of the Prophet Muhammad in Kazakh Proverbs and Sayings. Procedia Social and Behavioral Sciences, 116, 4899-4904.

Beckford, M. (2011). Cultural Activities Good for Men's Health and Happiness. The Telegraph.

Bell, J. (2010). Doing your research project (5th ed.). Maidenhead: Open University Press.

Bengtsson, M. (2016). How to plan and perform a qualitative study using content analysis. NursingPlus Open, 2, 8-14.

Benko, G. (2004). Human Geography: A History for the Twenty-First Century (Human Geography in the Making) (1st ed.). Routledge. 
Berk, M. (2005). The Concept of Neighborhood in Contemporary Residential Environments: An Investigation of Occupants' Perception. Munich Personal RePEc Archive. https://core.ac.uk/download/pdf/213917579.pdf

Bhandari, H., \& Yasunobu, K. (2009). What is Social Capital? A Comprehensive Review of the Concept. Asian Journal of Social Science, 37(3), 480-510.

Bhatta, B. (2010). Causes and Consequences of Urban Growth and Sprawl. In Analysis of Urban Growth and Sprawl from Remote Sensing Data. New York: Springer.

Bin Saif, G., Al-Balbeesi, A., Binshabaib, R., Alsaad, D., Kwatra, S., Alzolibani, A., \& Yosipovitch, G. (2013). QoL in Family Members of Vitiligo Patients: A Questionnaire Study in Saudi Arabia. American Journal of Clinical Dermatology, 14(6), 489-495.

Bleustein, C., Rothschild, D., Valen, A., Valatis, E., Schweitzer, L., \& Jones, R. (2014). Wait Times, Patient Satisfaction Scores, and the Perception of Care. The American Journal of Managed Care, 20, 393-400.

Bogdan, R., \& Biklen, S. (2007). Qualitative Research for Education: An Introduction to Theories and Methods (5th ed.). New York; London: Pearson

Brajša-Žganec, A., Merkaš, M., \& Šverko, I. (2010). Quality of Life and Leisure Activities: How do Leisure Activities Contribute to Subjective Well-Being? Social Indicators Research, 102(1), 81-91.

Bramham, P. (1997). Change, Postmodernism and Postmodernity. REVIEWING SOCIOLOGY, 10, https://www.reading.ac.uk/RevSoc/archive/volume10/number1/101a.htm

Bryman, A. (2012). Social Research Methods (4th ed.). Oxford: Oxford University Press.

Call, V., \& Heaton, T. (1997). Religious Influence on Marital Stability. Journal for The Scientific Study of Religion, 36(3), 382-392. 
Campbell, A., Converse, Philip E., \& Rodgers, Willard L. (1976). The Quality of American Life: Perceptions, Evaluations and Satisfactions. New York: Russell Sage Foundation.

Campbell, D., \& Fiske, D. (1959). Convergent and discriminant validation by the multitraitmultimethod matrix. Psychological Bulletin, 56(2), 81-105.

Carrier, M., Apparicio, P., \& Séguin, A. (2016). Road traffic noise in Montreal and environmental equity: What is the situation for the most vulnerable population groups? Journal of Transport Geography, 51, 1-8.

Carr, S., \&Lynch, K. (1981). Open space: Freedom and control. In L. Taylor (Ed.), Urban open spaces (pp. 17-18). New York: Rizzoli.

Carter, S., \& Mankoff, J. (2005). When participants do the capturing: the role of media in diary studies. In, Proceedings of the 2005 ACM Conference on Human Factors in Computing Systems. CHI 2005, Portland, Oregon, USA, April 2-7, 2005.

Carvalho, S., \& White, H. (1997). Combining Quantitative and Qualitative Approaches in Poverty Analysis. World Bank Technical Paper.

Carver County Public Health Division. (2006). Carver County Quality of Life Indicators. Chaska: Land and Water Services Division Land Management Department.

Chandrupatla, T. (2009). Quality and reliability in engineering. Cambridge: Cambridge University Press.

Christoph, B., \& Noll, H. (2003). Subjective Well-Being in the European Union during the 90ies. Social Indicators Research, 64, 521-546.

UN-Habitat. (2017). City Prosperity Initiative. Retrieved from November 11, 2017, from https://unhabitat.org/urban-initiatives/initiatives-programmes/city-prosperityinitiative/

Clark, W., Deurloo, M. \& Dieleman, F., 2006. Residential Mobility and Neighbourhood Outcomes. Housing Studies, 21(3), 323-342.

Cobb, C.W. (2000). Measurement Tools and the QoL. San Francisco: Redefining Progress. 
Coleman, J. (1988). Social Capital in the Creation of Human Capital. American Journal of Sociology, 94, 95-120.

Creswell, J. (2003). Research design: Qualitative, quantitative and mixed methods approaches (2nd ed.). Thousand Oaks, CA: SAGE.

Creswell, J.W. (2009). Research design - qualitative, quantitative and mixed methods. (3rd ed.). Thousand Oaks, CA: Sage.

Creswell, J. W. (2013). Qualitative Inquiry \& Research Design: Choosing among Five Approaches (3rd ed.). Thousand Oaks, CA: SAGE.

Creswell, J., \& Alquhtani, Trans, A. (2018). Research Design-Quantitative, Qualitative and Mixed Methods (1st ed.). Kuwait: Almassila publication.

Creswell, J., \& Plano Clark, V. (2011). Designing and conducting mixed methods research (2nd ed.). London: Sage.

Cutter, S. L. (1985). Rating Places: A Geographer's View on QoL. Washington, DC: Association of American Geographers.

Dahar, M., Dahar, R., \& Dahar, R. (2009). Mis-allocation of student teacher ratio, class size and per student expenditure leads to the wastage of school resource inputs and lower academic achievement: an issue of resource management. Munich Personal RePEc Archive. https://ideas.repec.org/p/pra/mprapa/27835.html

Dale, B. (1980). Subjective and objective social indicators in studies of regional social wellbeing. Regional Studies, 14(6), 503-515.

Darcin, M. (2017). How Air Pollution Affects Subjective Well-Being. In M. Mollaoglu (Ed.), Well-being and Quality of Life (1st ed.). Zagreb: InTsch.

Das, D. (2008). Urban Quality of Life: A Case Study of Guwahati. Social Indicators Research, 88(2), 297-310.

Datta, D., Datta, P., \& Majumdar, K. (2015). Role of social interaction on quality of life. National Journal of Medical Research, 5(4), 290-292. 
Davis, E., \& Fine-Davis, M. (1991). Social indicators of living conditions in Ireland with European comparisons. Social Indicators Research, 25(2-4), 103-365.

Delsante, I. (2016). Urban environment quality assessment using a methodology and set of indicators for medium-density neighbourhoods: a comparative case study of Lodi and Genoa. Ambiente Construído, 16(3), 7-22.

DeLyser, D., Herbert, S., Aitken, S., Crang, M., \& McDowell, L. (2010). The SAGE Handbook of Qualitative Geography. London: Sage.

Demirel, S., \& Sahib, H. (2015). Concept of Barakah in Qur'ān and Sunnah: Towards its Realization in Modern Discourse. Journal of Humanities and Social Sciences, O(5), $261-284$.

Despoina, H., Maria, M., Anna, K., Konstantinos, T., \& George, F. (2017). Smoking cessation process and quality of life. Journal of Nursing Education and Practice, 7(9), 1-12.

Dewsbury, J., \& Cloke, P. (2009). Spiritual landscapes: existence, performance and immanence. Social \& Cultural Geography, 10(6), 695-711.

Dhivyadeepa, E. (2015). Sampling Techniques in Educational Research. (1st ed). Laxmi Book Publication.

Diaz-Serrano, L. (2009). Disentangling the housing satisfaction puzzle: Does homeownership really matter? Journal of Economic Psychology, 30(5), 745-755.

Diener, E. (1984). Subjective well-being. Psychological Bulletin, 95(3), 542-575.

Diener, E. (1995). A value based index for measuring national QoL. Social Indicators Research, 36(2), 107-127.

Diener, E., \& Diener, C. (1995). The wealth of nations revisited: Income and quality of life. Social Indicators Research, 36(3), 275-286. 
Diener, E., \& Diener, M. (2009). Cross-Cultural Correlates of Life Satisfaction and SelfEsteem. In Diener, E. (Ed.), Culture and Well-being- the collected works of Diener, Social Indicators Research Series, Vol. 38, pp. 71-91. Springer, Dordrecht.

Diener, E. \& Suh, E. (1997). Measuring quality of life: economic, social and subjective indicators. Social Indicators Research 40, 189-216.

Diener, E. (1996). Traits are powerful but are not enough: Lessons from subjective wellbeing. Journal of Research in Personality, 30, 389-399.

Diener, E. (2006). Guidelines for National Indicators of Subjective Well-Being and IllBeing. Applied Research in QoL, 1(2), 151-157.

Diener E., Diener M., Diener C. (2009). Factors predicting the subjective well-being of nations. In Diener, E. (Ed.), Culture and Well-being: the collected works of Ed Diener. Social Indicators Research Series, Vol. 38, pp. 43-70. Springer, Dordrecht.

Diener, E., Emmons, R., Larsen, R., \& Griffin, S. (1985). The Satisfaction with Life Scale. Journal of Personality Assessment, 49(1), 71-75.

Diener, E., Oishi, S., \& Lucas, R. (2003). Personality, Culture, and Subjective Well-Being: Emotional and Cognitive Evaluations of Life. Annual Review of Psychology, 54(1), $403-425$.

Diener, E., Sandvik, E., Seidlitz, L., \& Diener, M. (1993). The relationship between income and subjective well-being: Relative or absolute? Social Indicators Research, 28(3), $195-223$.

Diener, E., Suh, E. M., Lucas, R. E., \& Smith, H. L. (1999). Subjective well-being: Three decades of progress. Psychological Bulletin, 125(2), 276-302.

Dissart, J., \& Deller, S. (2000). QoL in the Planning Literature. CPL Bibliography, 15(1), $135-161$.

Djebarni, R., \& Al-Abed, A. (2000). Satisfaction level with neighbourhoods in low-income public housing in Yemen. Property Management, 18(4), 230-242. 
Drucker, P. (1985). Innovation and Entrepreneurship. Harper \& Row.

Dudley, M., \& Kosinski, F. (1990). Religiosity and Marital Satisfaction: A Research Note. Review of Religious Research, 32(1), 78-86.

Dworkin, S. (2012). Sample Size Policy for Qualitative Studies Using In-Depth Interviews. Archives of Sexual Behavior, 41(6), 1319-1320.

Edgerton J.D., Roberts L.W., von Below S. (2012). Education and Quality of Life. In K. Land, A. Michalos, M. Sirgy (Eds.), Handbook of Social Indicators and Quality of Life Research (pp. 265-296). Dordrecht: Springer.

El-Jamil, F. M. (2003). Shame, guilt, and mental health: A study on the impact of cultural and religious orientation. Dissertation Abstracts International: Section B: The Sciences and Engineering, 64(3-B), 1487.

Ellis, T. (1984). Class Size. ERIC Clearinghouse on Educational Management: ERIC Digest, Number Eleven. Eugene: ERIC Clearinghouse on Educational Management.

Ellison, C. (1991). Religious Involvement and Subjective Well-Being. Journal of Health and Social Behaviour, 32(1), 80-99.

Ellison, C. G., \& George, L. K. (1994). Religious involvement, social ties, and social support in a southeastern community. Journal for the Scientific Study of Religion, 33, 4661.

Ellison, C., Boardman, J., Williams, D., \& Jackson, J. (2001). Religious Involvement, Stress, and Mental Health: Findings from the 1995 Detroit Area Study. Social Forces, 80(1), 215-249.

Elsinga, M., \& Hoekstra, J. (2005). Home Ownership and Housing Satisfaction. Journal of Housing and The Built Environment, 20(4), 401-424. 
Ertürk, Y. (2009). Report from the Human Rights Council, eleventh session, agenda item3: Report of the special rapporteur on violence against women, its causes and $\begin{array}{lllll}\text { consequences. } & \text { Retrieved } & \text { October } & 10 & 2021\end{array}$ http://daccessddsny.un.org/doc/UNDOC/GEN/G09/128/22/PDF/G0912822.pdf?O penElement.

Etikan, I., Alkassim, R., \& Abubakar, S. (2016). Comparison of Snowball Sampling and Sequential Sampling Technique. Biometrics \& Biostatistics International Journal, 3(1), 1-2.

Eurostat. (2015). Quality of life indicators - Statistics Explained. Retrieved March 11, 2017, from https://ec.europa.eu/eurostat/statisticsexplained/index.php?title=Quality_of_life_indicators.

Evan, M. (2014). Heritage Counts 2014: The value and impact of Heritage. Historic England. $\quad$ Retrieved July 10, 2020, from https://historicengland.org.uk/research/heritage-counts/2014-the-value-and-impactof-heritage/

Everett L. Worthington Jr., Lavelock, Caroline R., Van Tongeren, Daryl R., OyenWitvliet, Charlotte van, Griffin, Brandon J., Chelsea L., Jennings, David J. II, Lin, Yin, Jordan, Kayla, and Yee Ho, Man (2014). The Contributions of Christian Perspectives and Practices to Positive Psychology. In C. Kim-Prieto (Ed.), Religion and Spirituality Across Cultures, Cross-Cultural Advancements in Positive Psychology. Dordrecht: Springer Science + Business.

Fadda, G., \& Jirón, P. (1999). Quality of life and gender: a methodology for urban research. Environment \& Urbanization, 11 (2), 261-270.

Felce, D., \& Perry, J. (1995). QoL: Its definition and measurement. Research in Developmental Disabilities, 16(1), 51-74.

Ferriss, A. (2002). Religion and the Quality of Life. Journal of Happiness Studies, 3(3), $199-215$.

Ferriss, A. (2004). The QoL concept in sociology. The American Sociologist, 35(3), 37-51. 
Florida, R. (2003). Cities and the Creative Class. City and Community, 2(1), 3-19.

Florida, R. (2014). Rise of the Creative Class. (1st ed.). New York: Basic Books.

Florida, R., and Small, A. (2016). Why Quality of Place Matters? City Lab. Retrieved April 15 2018, from http://www.citylab.com/design/2016/12/why-quality-of-placematters/509876/

Florida, R., Mellander, C., \& Rentfrow, P. (2013) The Happiness of Cities. Regional Studies, 47 (4), 613-627.

Flowerdew, R., \& Martin, D. (2005). Methods in human geography: a guide for students doing a research project (2nd ed.). Harlow, England: Prentice Hall.

Francis, L., Yablon, Y., \& Robbins, M. (2014). Religion and Happiness: A Study among Female Undergraduate Students in Israel. International Journal of Jewish Education Research, 7, 77-92.

Fujiwara, D. (2013). Museums and Happiness, the Value of Participating in Museums and the Arts. The Happy Museum. Retrieved August 22, 2020, from https://www.creativenz.govt.nz/assets/ckeditor/attachments/1120/museums_and_h appiness_the_valueof_participating_in_museums_and_the_arts_2013_by_fujiwara .pdf?1416776178

Fujiwara, D., Cornwall, T. \& Dolan, P. (2014). Heritage and Wellbeing. English Heritage. Retrieved September 10, 2020 from https://historicengland.org.uk/content/heritagecounts/pub/2014/heritage-and-wellbeing-pdf/

Fujiwara, D., Kudrna, L., \& Dolan, P. (2014). Quantifying and Valuing the Wellbeing Impacts of Culture and Sport. Department for Culture, Media \& Sport. Retrieved September 15, 2020, from https://assets.publishing.service.gov.uk/government/uploads/system/uploads/attach ment_data/file/304899/Quantifying_and_valuing_the_wellbeing_impacts_of_sport _and_culture.pdf 
Future Saudi Cities Programme Report. (2016). Overview of the Institutional Framework for Urban Planning in the Kingdom of Saudi Arabia. Riyadh, KSA: Planning Unite, United Nations.

GAE. (2018). Indicators of the Educational Services in Quba municipality. Medina: General Administration for Education in Medina Region, Information Technology Department.

Garrusi, B., \& Nakhaee, N. (2012). Religion and Smoking: A Review of Recent Literature. The International Journal of Psychiatry in Medicine, 43(3), 279-292.

GaStat (2017). The Sixteenth Directory of Services for the Medina Region 2017. The Directory of Services. General Authority for Statistics.

GDHA. (2018). Indicators of the Health Services in Quba municipality. Medina: General Directorate of Health Affairs, Department of Information and Statistics.

Gentles, S. J., Charles, C., Ploeg, J., \& McKibbon, K. (2015). Sampling in Qualitative Research: Insights from an Overview of the Methods Literature. The Qualitative Report, 20(11), 1772-1789.

Grayson, J. (1998). QoL in Canadian cities. Toronto: Institute for Social Research, York University.

Grazuleviciute, I. (2006). Cultural Heritage in the Context of Sustainable Development. Environmental Research, Engineering and Management, 3(37), 7479.

Great Partnership. (n.d.). Student-Teacher Ratio Definition. Retrieved April 14, 2020, from https://www.edglossary.org/student-teacher-ratio/

Gregory, D. (2009). The dictionary of human geography. Malden, MA: Blackwell.

Grinstein-Weiss, M., Yeo, Y., Anacker, K., Van Zandt, S., Freeze, E., \& Quercia, R. (2011). Homeownership and Neighbourhood Satisfaction among Low- and ModerateIncome Households. Journal of Urban Affairs, 33(3), 247-265. 
Gruber, K., \& Shelton, G. (1987). Assessment of neighborhood satisfaction by residents of three housing types. Social Indicators Research, 19(3), 303-315.

Grzeskowiak, S., Sirgy, M., \& Widgery, R. (2003). Residents' Satisfaction with Community Services: Predictors and Outcomes. Regional Analysis and Policy, 33(2), 1-36.

Guhathakurta, S., \& Cao, Y. (2011). Variations in Objective Quality of Urban Life Across a City Region: The Case of Phoenix. In R. Marans \& R. Stimson (Eds.), Investigating Quality of Urban Life: Theory, Methods, and Empirical Research. Social Indicators Research Series, vol 45, pp 135-160. Springer, Dordrecht.

Haas, B. (1999). Clarification and Integration of Similar QoL Concepts. The Journal of Nursing Scholarship, 31(3), 215-220.

Hadaway, C. (1978). Life satisfaction and religion: A reanalysis. Social Forces, 57, 636643.

Hamersma, M., Tillema, T., Sussman, J., \& Arts, J. (2014). Residential satisfaction close to highways: The impact of accessibility, nuisances and highway adjustment projects. Transportation Research Part A: Policy and Practice, 59, 106-121.

Harvey, D. (1990). The condition of postmodernity. Cambridge, MA: Blackwell.

Harvey, M., \& Holly, B. (2014). Themes in geographic thought. Abingdon: Routledge.

Hay, I. (2016). Qualitative research methods in human geography (4th ed.). Oxford: Oxford University Press.

Hayes, A. (2017). Heritage and the Economy. [Report]. Social and Economic Research, Historic England. Retrieved from September 19, 2020 https://historicengland.org.uk/content/heritage-counts/pub/2017/heritage-and-theeconomy-2017-pdf

Helgeson, V. (2003). Social support and quality of life. Quality of Life Research, 12(1), 2531. 
Helliwell, J. F., \& Putnam, R. D. (2004). The social context of well-being. Philosophical transactions of the Royal Society of London. Series B, Biological sciences, 359(1449), 1435-1446.

Hepper, E., Ritchie, T., Sedikides, C., \& Wildschut, T. (2012). Odyssey's end: Lay conceptions of nostalgia reflect its original homeric meaning. Emotion, 12(1), 102119.

Hertling, E., Leonard, C., Lumsden, L., \& Smith, C. (2000). Class Size: Can School Districts Capitalize on the Benefits of Smaller Classes. Eugene: ERIC Clearinghouse on Educational Management.

Hittleman, D. R., \& Simon, A. J. (1997). Interpreting educational research: An introduction for consumers of research. Upper Saddle River, N.J: Merrill.

Holthus, B., \& Manzenreiter, W. (2017). Life Course, Happiness and Well-being in Japan. (1st ed). Routledge.

Hossain, M. (2012). Zakat in Islam: A Powerful Poverty Alleviating Instrument for Islamic Countries. International Journal of Economic Development Research and Investment, 3(1), 1-11.

Hossain, M., \& Rizvi, M. (2016). Relationship between religious belief and happiness in Oman: a statistical analysis. Mental Health, Religion \& Culture, 19(7), 781-790.

Howard, K. (2009). Using a Free Online Questionnaire to Determine the Skills, Competencies and Knowledge Required to Work in a Digital Library Environment in Australia. In A. Katsirikou, \& C. Skiadas, Qualitative and Quantitative Methods in Libraries. World Scientific Publishing.

Hsieh, C. (2005). Age and relative importance of major life domains. Journal of Aging Studies, 19(4), 503-512.

Hu, F. (2011). Homeownership and Subjective Wellbeing in Urban China: Does Owning a House Make You Happier? Social Indicators Research, 110(3), 951-971. 
Hur, M., \& Morrow-Jones, H. (2008). Factors That Influence Residents' Satisfaction with Neighborhoods. Environment and Behavior, 40(5), 619-635.

Hussain, A., \& El-Alami, K. (2005). A guide to Islam. Leeds: Subject Centre for Philosophical and Religious Studies.

Iwuagwu, E. (2018). The Relationship Between Religion and Morality: On Whether the Multiplicity of Religious Denominations have Impacted Positively on Socio-Ethical Behaviour. Global Journal of Arts, Humanities and Social Sciences, 6(9), 42-53.

Jacobs, S., Evans, G., Catalano, R., \& Dooley, D. (1984). Air pollution and depressive symptomatology: Exploratory analyses of intervening psychosocial factors. Population and Environment, 7(4), 260-272.

Jaklič, T., Kovač, J., Maletič, M., \& Bunc, K. (2018). Analysis of patient satisfaction with emergency medical services. Open Medicine, 13(1), 493-502.

Johnson, R \& Christensen, Larry. (2014). Educational Research Quantitative, Qualitative, and Mixed Approaches (5th ed). SAGE.

Joyce, C., McGee, H., \& O'Boyle, C. (1999). Individual Quality of Life: Approaches to Conceptualisation and Assessment. Hardwood Academic.

Jung, J. (2013). Religious Attendance, Stress, and Happiness in South Korea: Do Gender and Religious Affiliation Matter? Social Indicators Research, 118(3), 1125-1145.

Kahlmeier, S., Schindler, C., Grize, L., \& Braun-Fahrländer, C. (2001). Perceived environmental housing quality and wellbeing of movers. Journal of Epidemiology and Community Health, 55(10), 805-817.

Kallus, Rachel, \& Law-Yone, Hubert. (2000). What is a neighborhood? The structure and function of an idea. Environment and Planning B: Planning and Design, 27, 815826.

Kambuga, Y. (2013). The Impact of Teacher-Pupil Ratio on Teaching-Learning Process in Primary Schools: Experiences from Tanzania. International Journal of Education and Practice, 1(2), 14-25. 
Katsirikou, A., \& Skiadas, C. (2010). Qualitative and Quantitative Methods in Libraries: Theory and Application. World Scientific.

Kelly, J. R., Steinkamp, M. W., \& Kelly, J. R. (1987). Later-life satisfaction: Does leisure contribute. Leisure Sciences, 9(3), 189-199.

Kennedy, G. (1998). Religion and depression. In H.G. Koenig (Ed.), Handbook of religion and mental health. San Diego, CA: Academic Press.

Keul, A., \& Prinz, T. (2011). The Salzburg Quality of Urban Life Study with GIS Support. In R. Marans \& R. Stimson (Eds.), Investigating Quality of Urban Life: Theory, Methods, and Empirical Research, Social Indicators Research Series 45, pp. pp 273293. Springer, Dordrecht.

Khaef, S., \& Zebardast, E. (2015). Assessing QoL Dimensions in Deteriorated Inner Areas: A case from Javadieh Neighborhood in Tehran Metropolis. Social Indicators Research, 127(2), 761-775.

Khizindar, T. (2012). Effects of Tourism on Residents' QoLin Saudi Arabia: An Empirical Study. Journal of Hospitality Marketing \& Management, 21(6), 617-637.

Kim-Prieto, C., \& Miller, L. (2018). Intersection of religion and subjective well-being. In E. Diener, S. Oishi, \& L. Tay (Eds.), Handbook of well-being. Salt Lake City, UT: DEF. DOI: nobascholar.com.

Koc, N., \& Celik, B. (2015). The Impact of Number of Students per Teacher on Student Achievement. Procedia - Social and Behavioral Sciences, 177, 65-70.

Koenig H.G., \& Shohaib S.A. (2014) Muslim Beliefs, Practices, and Values. In editor name, Health and Well-Being in Islamic Societies, Background, Research, and Applications. Springer.

Koenig, H., Carson, V., \& King, D. (2001). Handbook of religion and health. Oxford: Oxford University Press. 
Kramer, S., \& Fahmy, D. (2019). Younger people are less religious than older ones in many countries. Pew Research Centre. Retrieved June 12, 2019, from https://www.pewresearch.org/fact-tank/2018/06/13/younger-people-are-lessreligious-than-older-ones-in-many-countries-especially-in-the-u-s-and-europe/

Kumar, R. (2019). Research methodology-a step-by-step guide for beginners (3rd ed.). SAGE.

Kuykendall, L., Boemerman, L., \& Zhu, Z. (2018). The importance of leisure for subjective well-being. In E. Diener, S. Oishi, \& L. Tay (Eds.), Handbook of well-being. Salt Lake City, UT: DEF.

Kwan, M. (2019). Human Mobility, Spatiotemporal Context, and Environmental Health. [ n. p.]: Multidisciplinary Digital Publishing Institute.

Kweon, B., \& Marans, R. (2011). Disaggregating the Measurement of Quality of Urban Life Dimensions Across a Complex Metro Region: The Case of Metro Detroit. In R. Marans \& R. Stimson, Investigating Quality of Urban Life: Theory, Methods, and Empirical Research, vol 45, pp. 369-384. Springer, Dordrecht.

Lansing, J., \& Marans, R. (1969). Evaluation of Neighborhood Quality. Journal of The American Institute of Planners, 35(3), 195-199.

Latham, A. (2003). Research, Performance, and Doing Human Geography: Some Reflections on the Diary-Photograph, Diary-Interview Method. Environment and Planning A, 35(11), 1993-2017.

Lave, J., \& Wenger, E. (1991). Situated learning: Legitimate peripheral participation. New York, NY: Cambridge University Press.

Lavrakas, Paul J. (2008). Encyclopedia of survey research methods. SAGE.

Learning Space Toolkit. (2018). Photo Interviews / Photo Diary: Overview. Retrieved May 23, 2018, from https://learningspacetoolkit.org/needs-assessment/data-gatheringtools-2/photo-interviews/index.html 
Lee, A., Jordan, H., \& Horsley, J. (2015). Value of urban green spaces in promoting healthy living and wellbeing: prospects for planning. Risk Management and Healthcare Policy 8, 131-137.

Lee, S., Conway, T., Frank, L., Saelens, B., Cain, K., \& Sallis, J. (2016). The Relation of Perceived and Objective Environment Attributes to Neighbourhood Satisfaction. Environment and Behavior, 49(2), 136-160.

Levin, J., \& Chatters, L. (1998). Religion, health, and psychological well-being in older adults: Findings from three national surveys. Journal of Aging and Health, 10, 504531.

Li, C., \& Wang, C. (2012). The Factors Affecting Life Satisfaction: Recreation Benefits and Quality of Life Perspectives. Sports \& Exercise Research, 14(4), 407-418.

Lim, C., \& Putnam, R. (2010). Religion, Social Networks, and Life Satisfaction. American Sociological Review, 75(6), 914-933.

Lincoln, K. (2000). Social Support, Negative Social Interactions, and Psychological WellBeing. Social Service Review, 74(2), 231-252.

Liu, B. (1975). Quality of Life: Concept, Measure and Results. American Journal of Economics and Sociology, 34(1), 1-14.

Liu, B. (1976). Quality of life indicators in U.S. metropolitan areas. New York: Praeger.

Logan, A. (2016). The impact of heritage tourism for the UK economy. London: Oxford Economics.

Lotfi, S., Faraji, A., Hataminejad, H., \& Pourahmad, A. (2011). A Study of Urban QoL in a Developing Country. Journal of Social Sciences, 7(2), 232-240.

Lu, M. (1999). Determinants of Residential Satisfaction: Ordered Logit vs. Regression Models. Growth and Change, 30(2), 264-287. 
Lun, V., \& Bond, M. (2013). Examining the relation of religion and spirituality to subjective well-being across national cultures. Psychology of Religion and Spirituality, 5(4), 304-315.

Lwuagwu, E. (2018). The Relationship Between Religion and Morality: On Whether the Multiplicity of Religious Denominations have Impacted Positively on Socio-Ethical Behaviour. Global Journal of Arts, Humanities and Social Sciences, 6(9), 42-53.

Macnee, C. and McCabe, S. (2008). Understanding Nursing Research: Using Research in Evidence-based Practice. (2nd ed.) Philadelphia, PA: Wolters Kluwer Health/Lippincott Williams \& Wilkins.

Magalhaes, S., Carvalho, Z., Andrade, L., Pinheiro, A., \& Studart, R. (2015). Influence of spirituality, religion and beliefs in the quality of life of people with spinal cord injury. Texto \& Contexto - Enfermagem, 24(3), 792-800.

Mahoney, A. (2005). Religion and conflict in marital and parent child relationship. Journal of social issues, 61(4), 689-706.

Mail, S. (2014). Richard Florida's 10 rules for a city's quality of place. The Globe and Mail. Retrieved November 18, 2017, http://www.theglobeandmail.com/opinion/columnists/richard-floridas-10-rules-fora-citys-quality-of-place/article17496710/

Marans, R. (2012). Quality of Urban Life Studies: An Overview and Implications for Environment-Behaviour Research. Procedia - Social and behavioural Sciences, 35, 9-22.

Marans, R. W. \& Rodgers, W. (1975). Toward an understanding of community satisfaction. In A. H. Hawley \& V. P. Rock (Eds). Metropolitan American in Contemporary Perspective. New York: Halstead.

Marans, R., \& Stimson, R. (Eds.). (2011). Investigating Quality of Urban Life: Theory, Methods, and Empirical Research. Social Indicators Research Series. Springer. 
Marques, S., \& Lima, M. (2011). Living in grey areas: Industrial activity and psychological health. Journal of Environmental Psychology, 31(4), 314-322.

Maslow, A. (1943). A theory of human motivation. Psychological Review, 50(4), 370-396.

Mason, J. (2012). Expert voices. In S. Baker, \& R. Edwards (Eds.), How many qualitative interviews is enough? National Centre for Research Methods Review Discussion Paper (pp. 29-30).

Mason, R. (2002). Assessing Values in Conservation Planning: Methodological Issues and Choices. In Assessing the Values of Cultural Heritage. Research Report. Los Angeles: The Getty Conservation Institute.

Massey, S. (2012). Living Heritage \& Quality of Life: Reframing Heritage Activity in Saskatchewan. Saskatchewan: Heritage Saskatchewan.

May, E., Hunter, B., \& Jason, L. (2016). Methodological pluralism and mixed methodology to strengthen Community Psychology research: An example from Oxford House. Journal of Community Psychology, 45(1), 100-116.

Maynard, M., \& Purvis, J. (1994). Researching women's lives from a feminist perspective. Routledge.

McCrea, R., Shyy, T., \& Stimson, R. (2006). What is the Strength of the Link Between Objective and Subjective Indicators of Urban QoL? Applied Research in QoL, 1(1), 79-96.

McCrea, R., Stimson, R., \& Western, J. (2005). Testing a Moderated Model of Satisfaction with Urban Living using Data for Brisbane-South East Queensland, Australia. Social Indicators Research, 72(2), 121-152.

McCrea, R., Stimson, R., and Marans, R.W. (2011). The Evolution of Integrative Approaches to the Analysis of Quality of Urban Life. In R.W. Marans, and R. Stimson (Eds), Investigating Quality of Urban Life Theory, Methods, and Empirical Research (pp. 77-106). Springer, Dordrecht. 
McMahon, S. (2002). The development of QoL indicators - a case study from the City of Bristol, UK. Ecological Indicators, 2(1-2), 177-185.

McMichael, A. (2000). The urban environment and health in a world of increasing globalization: issues for developing countries. Bulletin of The World Health Organization, 78(9), 1117-1126.

Medina Development Authority. (2018). Humanization of Medina. Medina: Medina Development Authority.

Medina Regional Municipality. (2018). Public Parks in The Municipality of Quba. Medina: Medina Regional Municipality.

Michaud, D., Keith, S., \& McMurchy, D. (2008). Annoyance and disturbance of daily activities from road traffic noise in Canada. The Journal of The Acoustical Society of America, 123(2), 784-792.

Mikkelsen, C., \& Di Nucci, J. (2015). Qualitative Methodologies in Geography, Contributions to the Study of Quality of Life. In G. Tonon (Ed.), Qualitative Studies in Quality of Life-Methodology and Practice. Switzerland: Springer International.

Milbrath, L.W. (1978). Indicators of environmental quality and QoL. UNESCO Reports and Papers in Social Sciences No.38.

Minca, C. (2001). Postmodern Geography: Theory and Praxis. Oxford: Blackwell.

Mohan, J., \& Twigg, L. (2007). Sense of Place, Quality of Life and Local Socioeconomic Context: Evidence from the Survey of English Housing, 2002/03. Urban Studies, 44(10), 2029-2045.

Mohit, M. (2013). QoL in Natural and Built Environment - An Introductory Analysis. Procedia - Social and Behavioural Sciences, 101, 33-43.

Mohit, M., \& Azim, M. (2012). Assessment of Residential Satisfaction with Public Housing in Hulhumale', Maldives. Procedia - Social and Behavioural Sciences, 50, 756770. 
Mohit, M., Ibrahim, M., \& Rashid, Y. (2010). Assessment of residential satisfaction in newly designed public low-cost housing in Kuala Lumpur, Malaysia. Habitat International, 34(1), 18-27.

Morar, T., Radoslav, R., Spiridon, L., \& Pacurar, L. (2014). Assessing Pedestrian Accessibility to Green Space Using GIS. Transylvanian Review of Administrative Sciences, 10, 116-139.

Morris, E., \& Winter, M. (1975). A Theory of Family Housing Adjustment. Journal of Marriage and The Family, 37(1), 79-88.

Mridha, A., \& Moore, G. (2011). The Quality of Life in Dhaka, Bangladesh: Neighbourhood Quality as a Major Component of Residential Satisfaction. In R. Marans, \& R. Stimson (Eds.), Investigating Quality of Urban Life: Theory, Methods, and Empirical Research (pp 251-272). Springer, Dordrecht.

Muijs, D. (2004). Doing Quantitative Research in Education. London: SAGE.

Mulligan, G., \& Carruthers, J. (2011). Amenities, Quality of Life, and Regional Development. In R. Marans, \& R. Stimson (Eds.), Investigating Quality of Urban Life: Theory, Methods, and Empirical Research. Social Indicators Research Series. vol 45, pp. 107-133. Springer, Dordrecht.

Murgaš, F., \& Böhm, H. (2015 Does economic growth improve quality of life? In 15th International Multidisciplinary Scientific GeoConference SGEM 2015 (pp. 213220). Albena: Ecology, Economics, Education and Legislation.

Myers, D. (1988). Building knowledge about quality of life for urban planning. Journal of American Planning Association 54(3), 347-358.

Myers, M. (2009). Qualitative research in business \& management. London: SAGE.

Naseer, M., Zahidie, A., \& Shaikh, B. T. (2012). Determinants of patient's satisfaction with health care system in Pakistan: a critical review. Pakistan Journal of Public Health, $2(2), 52-61$. 
Nayak, A., \& Jeffrey, A. (2011). Geographical Thought. Essex: Pearson Education.

Neusner, J., \& Chilton, B. (Eds.). (2008). The Golden Rule: The Ethics of Reciprocity in World Religions. London: Continuum.

Newman, D., Tay, L., \& Diener, E. (2013). Leisure and Subjective Well-Being: A Model of Psychological Mechanisms as Mediating Factors. Journal of Happiness Studies, 15(3), 555-578.

Ng, W. (2014). Processes Underlying Links to Subjective Well-being: Material Concerns, Autonomy, and Personality. Journal Of Happiness Studies, 16(6), 1575-1591.

Noll, H. (2013). Subjective Social Indicators: Benefits and Limitations for Policy Making, An Introduction to this Special Issue. Social Indicators Research, 114(1), 1-11.

Organisation for Economic Co-operation and Development (2012). How Does Class Size Vary Around the World. Education Indicators in Focus, No. 9. Paris: OECD Publishing.

Organisation for Economic Co-operation and Development. (2015). Better Life Index Edition 2015. Retrieved April 3, 2019, from https://stats.oecd.org/Index.aspx?DataSetCode=BLI2015

Organisation for Economic Co-operation and Development (2019), Education at a Glance 2019: OECD Indicators. Paris: OECD Publishing.

Oktay, D., \& Marans R. (2010). Overall Quality of Urban Life and Neighborhood Satisfaction: A Household Survey in the Walled City of Famagusta. Open House International, 35, 27-36.

Oktay, D., Rustemli, A., \& Marans, R. (2009). Neighborhood satisfaction, sense of community, and attachment: Initial findings from Famagusta quality of urban life study. A|Z ITU Journal of The Faculty of Architecture, 6(1), 6-20.

Oktay, R. (2016). A Conceptual Framework for Postmodernism. In R. Oktay, The Challenges of Contemporaneity. Springer Briefs in Philosophy. 
Oladapo, A. (2006). A study of tenants' maintenance awareness, responsibility and satisfaction in institutional housing in Nigeria. International Journal of Strategic Property Management, 10(4), 217-231.

Pacione, M. (2005). Urban Geography a Global Perspective (2nd ed.). Abingdon: Routledge.

Pacione, M. (2009). Urban Geography a Global Perspective (3rd ed.). Abingdon: Routledge.

Packer, J. (2008). Beyond Learning: Exploring Visitors' Perceptions of the Value and Benefits of Museum Experiences. Curator: The Museum Journal, 51(1), 33-54.

Paggi, M., Jopp, D., \& Hertzog, C. (2016). The Importance of Leisure Activities in the Relationship between Physical Health and Well-Being in a Life Span Sample. Gerontology, 62(4), 450-458.

Pallas, A. M. (2000). The effects of schooling on individual lives. In M. T. Hallinan (Ed.), Handbook of sociology of education (pp. 499-525). New York: Klewer Academic/ Plenum.

Palmore, E., \& Luikart, C. (1972). Health and Social Factors Related to Life Satisfaction. Journal of Health and Social Behaviour, 13(1), 68-80.

Parkes, A., Kearns, A., \& Atkinson, R. (2002). What Makes People Dissatisfied with their Neighbourhoods? Urban Studies, 39(13), 2413-2438.

Peck, C., \& Stewart, K. (1985). Satisfaction with Housing and Quality of Life. Home Economics Research Journal, 13(4), 363-372.

Peet, R. (1989). Modern Geographical Thought. Oxford: Blackwell.

Permentier, M., Bolt, G., \& van Ham, M. (2010). Determinants of Neighbourhood Satisfaction and Perception of Neighbourhood Reputation. Urban Studies, 48(5), 977-996. 
Peterson, M., \& Webb, D. (2006). Religion and Spirituality in Quality of Life Studies. Applied Research in Quality of Life, 1(1), 107-116.

Philip, L. (1998). Combining Quantitative and Qualitative Approaches to Social Research in Human Geography-An Impossible Mixture? Environment and Planning, 30(2), 261-276.

Polit, D. and Beck, C. (2008). Nursing Research: Generating and Assessing Evidence for Nursing Practice. (8th ed.). Philadelphia: Lippincott Williams and Wilkins.

Pope, W. (1975). Durkheim as a Functionalist. The Sociological Quarterly, 16(3), 361-379.

Portes, A. (1998). Social Capital: Its Origins and Applications in Modern Sociology. Annual Review of Sociology, 24(1), 1-24.

Prakash, B. (2010). Patient Satisfaction. Journal of Cutaneous and Aesthetic Surgery, 3(3), $151-155$.

Pritchard E. (2008). Religion, mobility and conflict. In S. Bergmann S, \& T. Sager (Eds.), Ethics of Mobilities: Rethinking Place, Exclusion Freedom and Environment (pp. 195-213). Ashgate: Abingdon.

Psatha, E., Deffner, A., \& Yannis, P. (2011). Defining the quality of urban life: Which factors should be considered? European Regional Science Association, 51st European Congress, Barcelona, Spain.

Putnam, R. (2000) Bowling Alone: The Collapse and Revival of American Community. New York: Simon Schuster.

Qawasmeh, R. (2014). Identification of the quality of urban life assessment aspects in residential neighbourhoods in Doha. The Sustainable City IX, 1(391), 391-402.

QMO. (2018). Urban Characteristics of Quba Municipality. Medina: Medina Regional Municipality.

Radley, A. (2010). What people do with pictures. Visual Studies, 25(3), 268-279.

Randall, J., \& Morton, P. (2003). QoL in Saskatoon 1991 and 1996: A Geographical Perspective. Urban Geography, 24(8), 691-722. 
Rane, A., Tyser, A., \& Kazmers, N. (2019). Evaluating the Impact of Wait Time on Orthopaedic Outpatient Satisfaction Using the Press Ganey Survey. JBJS Open Access, 4(4), e0014. https://pubmed.ncbi.nlm.nih.gov/32043054/

Reardon, S., Matthews, S., O’Sullivan, D., Lee, B., Firebaugh, G., Farrell, C., \& Bischoff, K. (2008). The geographic scale of Metropolitan racial segregation. Demography, 45(3), 489-514.

Ren, H., Folmer, H., \& Van der Vlist, A. (2016). The Impact of Home Ownership on Life Satisfaction in Urban China: A Propensity Score Matching Analysis. Journal of Happiness Studies 19(2), 397-422.

Retsinas, N., \& Belsky, E. (2002). Low-Income Homeownership: Examining the Unexamined Goal. Brookings Institution Press, Harvard University Joint Centre for Housing Studies.

Reyes-Garcia, V., Godoy, R. A., Vadez, V., Ruíz-Mallén, I., Huanca, T., Leonard, W. R., \& Tanner, S. (2009). The payoffs to sociability. Human Nature, 20(4), 431-446.

Rezaei, S., Matin, B., Karyani, A., Woldemichael, A., Khosravi, F., Khosravipour, M., \& Rezaeian, S. (2017). Impact of Smoking on Health-Related Quality of Life: A General Population Survey in West Iran. Asian Pacific Journal of Cancer Prevention, 18(11), 3179-3185.

Rezvani, M., Mansourian, H., \& Sattari, M. (2012). Evaluating QoL in Urban Areas (Case Study: Noorabad City, Iran). Social Indicators Research, 112(1), 203-220.

Riger, S. \& Lavrakas, P. (1981). Community ties: patterns of attachment and social interaction in urban neighbourhoods. American Journal of Community Psychology 9, 55-66.

Robinson, N. (1999). Islam: A concise introduction. London: Curzon Press.

Rohe, W. M., \& Basolo, V. (1997). Long-Term Effects of Homeownership on the SelfPerceptions and Social Interaction of Low-Income Persons. Environment and Behaviour, 29(6), 793-819. 
Rohe, W. M., \& Stegman, M. A. (1994). The effects of homeownership on the self-esteem, perceived control and life satisfaction of low-income people. Journal of the American Planning Association, 60(2), 173-184.

Rohe, W., \& Lindblad, M. (2013). Reexamining the Social Benefits of Homeownership after the Housing Crisis [Ebook]. Boston, MA: Joint Centre for Housing Studies of Harvard University.

Rohe, W., Van Zandt, S., \& McCarthy, G. (2001). The social benefits and costs of homeownership. Cambridge, MA: Joint Centre for Housing Studies of Harvard University.

Rook, K. (1984). The negative side of social interaction: Impact on psychological wellbeing. Journal of Personality and Social Psychology, 46(5), 1097-1108.

Ross, C. E., \& Marieke, V. W. (1997). Education and the subjective quality of life. Journal of Health and Social Behavior, 38(3), 275-297.

Russo, A., \& Cirella, G. (2018). Modern Compact Cities: How Much Greenery Do We Need? International Journal of Environmental Research and Public Health, 15(10), $1-15$.

Saeidi, S., \& Oktay, D. (2012). Diversity for Better Quality of Community Life: Evaluations in Famagusta Neighbourhoods. Procedia - Social and Behavioural Sciences, 35, 495-504.

Salkind, N. (2010). Encyclopaedia of research design. Thousand Oaks, CA: SAGE.

Salleh, A., \& Badarulzaman, N. (2012). Quality of Life of Residents in Urban Neighbourhoods of Pulau Pinang, Malaysia. Journal of Construction in Developing Countries, 17(2), 117-123.

Sanchez, R. (2017). Saudi Arabia lifts ban on Skype and WhatsApp voice calls. The Telegraph. Retrieved, February 20, 2020, from https://www.telegraph.co.uk/news/2017/09/20/saudi-arabia-lifts-ban-skypewhatsapp-voice-calls/ 
Santos, L., \& Martins, I. (2006). Monitoring Urban QoL: The Porto Experience. Social Indicators Research, 80(2), 411-425.

Sari, M. (2017). The impacts of the age factor on religiosity. Firat University Journal of Social Sciences, 27(2), 257-264.

Saudi General Organization for Statistics. (2016). Demographic Survey. Riyadh: General Organization for Statistics.

Saudi Vision 2030. (2018). Vibrant Society with Fulfilling Lives. Retrieved February 13, 2018, from http://vision2030.gov.sa/en/node/10

Sawatzky, R., Ratner, P., \& Chiu, L. (2005). A Meta-Analysis of the Relationship Between Spirituality and Quality of Life. Social Indicators Research, 72(2), 153-188.

Scanlon, E. (1999). The impact of homeownership on the life satisfaction of African Americans. CSD Working Paper No. 99-4. St. Louis, MO: Washington University Centre for Social Development.

Schalock, R. (2004). The concept of quality of life: what we know and do not know. Journal of Intellectual Disability Research, 48(3), 203-216.

Saudi Commission for Tourism and National Heritage. (2018). Cultural heritage indicators. [Report].

Schultze U. (2012) Using Photo-Diary Interviews to Study Cyborgian Identity Performance in Virtual Worlds. In: A.Bhattacherjee, \& B. Fitzgerald (Eds.), Shaping the Future of ICT Research. Methods and Approaches. IFIP Advances in Information and Communication Technology, vol 389. Berlin/Heidelberg: Springer.

Sedikides, C., \& Wildschut, T. (2018). Finding Meaning in Nostalgia. Review of General Psychology, 22(1), 48-61.

Sekaran, U., \& Bougie, R. (2016). Research methods for business. Chichester: John Wiley $\&$ Sons.

Senlier, N., Yildiz, R., \& Aktaş, E. (2008). A Perception Survey for the Evaluation of Urban QoL in Kocaeli and a Comparison of the Life Satisfaction with the European Cities. Social Indicators Research, 94(2), 213-226. 
Serag El Din, H., Shalaby, A., Farouh, H., \& Elariane, S. (2013). Principles of urban QoL for a neighborhood. HBRC Journal, 9(1), 86-92.

Shin, D.C., Johnson, D.M. (1978). Avowed happiness as an overall assessment of the quality of life. Social Indicators Research 5, 475-492.

Shoeibi, M., Amraii, I., Mafakheri, A., \& Karimi, A. (2015). Analysis of Subjective Indicators of QoL in Urban Areas of Iran (Case Study: Sonqor City). Journal of Research in Humanities and Social Science, 3(3), 39-46.

Shucksmith, M., Cameron, S., Merridew, T., \& Pichler, F. (2009). Urban-Rural Differences in QoL across the European Union. Regional Studies, 43(10), 1275-1289.

Siegel, K., \& Schrimshaw, E. (2002). The Perceived Benefits of Religious and Spiritual Coping Among Older Adults Living with HIV/AIDS. Journal for The Scientific Study of Religion, 41(1), 91-102.

Simao, T., Caldeira, S., \& de Carvalho, E. (2016). The Effect of Prayer on Patients' Health: Systematic Literature Review. Religions, 7(1), 1-11.

Singhal, D., \& Singhal, K. (2012). Implementing ISO9001:2008 Quality Management System: A Reference Guide (2nd ed.). New Delhi: PHI.

Sinnewe, E., Kortt, M., \& Dollery, B. (2015). Religion and Life Satisfaction: Evidence from Germany. Social Indicators Research, 123, 837-855.

Sirgy, M. (2012). The Psychology of QoL: Hedonic Well-Being, Life Satisfaction, and Eudaimonia (2nd ed.). Springer.

Sirgy, M., \& Cornwell, T. (2002). How neighbourhood features affect quality of life. Social Indicators Research, 59, 79-114.

Sirgy, M., \& Cornwell, T. (2001). Further validation of the Sirgy et al.'s measure of community quality of life. Social Indicators Research, 56, 125-143. 
Sirgy, M. \& Rahtz, D., Muris, C., \& Underwood, R. (2000). A Method for Assessing Residents' Satisfaction with Community-Based Services: A Quality-of-Life Perspective. Social Indicators Research, 49, 279-316.

Smale, B., Donohoe, H., \& Auger, D. (2010). Leisure and Culture. Canadian Index of Wellbeing. Waterloo Applied Health Science. Retrieved from August, 12, 2020 https://uwaterloo.ca/canadian-index-wellbeing/sites/ca.canadian-indexwellbeing/files/uploads/files/Leisure_and_Culture-Executive_Summary.sflb_.pdf

Smith, C., \& Clay, P. (2010). Measuring Subjective and Objective Well-being: Analyses from Five Marine Commercial Fisheries. Human Organization, 69(2), 158-168.

Smith, L. \& Waterton, E. (2009). “The envy of the world?" Intangible heritage in England. In L. Smith (Ed.), Intangible Heritage (pp. 289-302). Oxford: Routledge.

Soleimani, M., Tavallaei, S., Mansuorian, H., \& Barati, Z. (2014). The Assessment of QoL in Transitional Neighbourhoods. Social Indicators Research, 119(3), 1589-1602.

Srivastava, S. (2005). Applied and community psychology. New Delhi: Sarup \& Sons.

Stancu, A., Ilin, C., \& Gruicin, A. (2016). Perceived urban QoL in Timișoara's districts. Romanian Journal of Applied Psychology, 18(1), 13-17.

Stimson, R., McCrea, R., \& Western, J. (2011). The Brisbane-South East Queensland Region, Australia: Subjective Assessment of Quality of Urban Life and Changes over Time. In R. Marans, \& R. Stimson (Eds.), Investigating Quality of Urban Life: Theory, Methods, and Empirical Research. Social Indicators Research Series, vol 45, PP. 185-207. Springer, Dordrecht.

Stoltman, J. (2012). 21 st century geography. Los Angeles, CA: SAGE.

Stubbs, M. (2002). Car Parking and Residential Development: Sustainability, Design and Planning Policy, and Public Perceptions of Parking Provision. Journal of Urban Design, 7(2), 213-237. 
Sun, Y. (2005). Development of neighbourhood QoL indicators. Saskatoon: CommunityUniversity Institute for Social Research.

Taylor, N. (2010). Urban planning theory since 1945. Los Angeles: SAGE.

Saudi Ministry of Municipal and Rural Affairs. (2016). National Report, Third United Nations Conference on Housing and Sustainable Urban Development (HABITAT III). Quito.

Throsby, D. (2000). Economic and Cultural Value in the Work of Creative Artists. In Avrami, E., Mason, R., Torre, M. (Eds.), Values and Heritage Conservation. Research Report. Los Angeles: The Getty Conservation Institute.

Tiliouine, H., Cummins, R. A., \& Davern, M. (2009). Islamic religiosity, subjective wellbeing, and health. Mental Health, Religion and Culture, 12, 55-74.

Tonon G. (2015) Qualitative Studies in QoL. Social Indicators Research Series 55. Springer.

Torre, M. (2002). Assessing the Values of Cultural Heritage. Los Angeles: The Getty Conservation Institute.

Tremblay, K., \& Dillman, D. (1983). Beyond the American housing dream. Lanham: University Press of America.

Turkoglu, H. (2015). Sustainable Development and Quality of Urban Life. Procedia - Social and Behavioural Sciences, 202, 10-14.

Türksever, A.N.E., \& Atalik, G. (2001). Possibilities and Limitations for the Measurement of the Quality of Life in Urban Areas. Social Indicators Research 53, 163-187.

UN-Habitat, \& Saudi Ministry of Municipal and Rural Affairs. (2016). Medina CPI Report. United Nations [Report].

UN-Habitat. (1994). Population, urbanization, and QoL. Nairobi: United Nations Centre for Human Settlements (Habitat). 
UN-Habitat. (2013). Cities Prosperity Initiative Toolkit. World Urban Campaign Secretariat.

UN-Habitat. (2013). State of the world's cities, 2012/2013, Prosperity of Cities. New York: United Nations.

UN-Habitat. (2015). City Prosperity Initiative. United Nations.

UN-Habitat. (2017). Youth. Retrieved November 21, 2019, from https://unhabitat.org/urban-themes/youth/

United Nations Development Program (UNDP). (2014). Empowered Youth, Sustainable Future.

Unwin, T. (1992). The Place of Geography. Essex: Longman.

Urban Observatory of Medina. (2016). The state of development of the provinces of Medina. Median: Ministry of Municipal and Rural Affairs.

Valentine, G., (2011). Social Geographies: Space and Society. Essex: Prentice Hall.

Van de Velde, S., Van der Bracht, K., \& Buffel, V. (2017). The relation between religion and depression in Europe: The moderating role of the religious context. International Journal of Comparative Sociology, 58(6), 515-532.

Van den Berg, P., \& Timmermans, H. (2015). A multilevel path analysis of social networks and social interaction in the neighbourhood. Region, 2(1), 55-66.

Van Kamp, I., Leidelmeijer, K., Marsman, G., \& de Hollander, A. (2003). Urban environmental quality and human well-being. Landscape and Urban Planning, 65(1-2), 5-18.

van Praag, B., \& Baarsma, B. (2004). Using Happiness Surveys to Value Intangibles: The Case of Airport Noise. SSRN Electronic Journal number 115, 224-246.

Varady, D., \& Preiser, W. (1998). Scattered-Site Public Housing and Housing Satisfaction: Implications for the New Public Housing Program. Journal of The American Planning Association, 64(2), 189-207. 
Vartanian, T. (2011). Secondary Data Analysis. Oxford: Oxford University Press.

Vasileiou, K., Barnett, J., Thorpe, S., \& Young, T. (2018). Characterising and justifying sample size sufficiency in interview-based studies: systematic analysis of qualitative health research over a 15-year period. BMC Medical Research Methodology, 18(1), $1-18$.

Veenhoven, R. (1996). Happy life-expectancy. Social Indicators Research, 39(1), 1-58.

Veenhoven, R. (2001). Quality of life and happiness: not quite the same. In G. D. e. al (Ed.), Salute e Qualita Dell Vida (pp. 67-95). Torino, Italia: Centro Scientifico Editore.

Vera-Toscano, E., \& Ateca-Amestoy, V. (2007). The relevance of social interactions on housing satisfaction. Social Indicators Research, 86(2), 257-274.

Verwiebe, R. (2014). Social Institutions. In Encyclopedia of Quality of Life Research. Springer.

Villalonga-Olives, E., \& Kawachi, I. (2017). The dark side of social capital: A systematic review of the negative health effects of social capital. Social Science \& Medicine, 194, 105-127.

Walsh, S. J., Crews-Meyer, K. A., Crawford, T. W., \& Welsh, W. F. (2004). Population and environment interactions: Spatial considerations in landscape characterization and modelling. In E. S. Sheppard, \& R. B. McMaster (Eds.), Scale and geographic inquiry (pp. 41-65). Oxford: Blackwell.

WAS. (2015). The project of Al-Sunna route in Madinah contributes to defining historical, cultural and civilizational features. The official Saudi Press Agency (WAS). Retrieved 5 November 2019, from https://www.spa.gov.sa/1377963.

Watzlawik, M., \& Born, A. (2007). Capturing identity: Quantitative and Qualitative Methods. Lanham: University Press of America.

World Commission on Environment and Development. (1987). Our Common Future. Oxford: University Press. 
Welsch, H. (2006). Environment and happiness: Valuation of air pollution using life satisfaction data. Ecological Economics, 58(4), 801-813.

Wheatley, D., \& Bickerton, C. (2016). Subjective well-being and engagement in arts, culture and sport. Journal of Cultural Economics, 41(1), 23-45.

World Health Organisation. (n.d.). Urban green spaces. Retrieved April 19, 2020, from https://www.who.int/sustainable-development/cities/health-risks/urban-greenspace/en/

World Health Organisation (n.d.) Religion and tobacco use. Ban tobacco. Retrieved July 21, 2019, from http://www.emro.who.int/tfi/ban-tobacco/religion-and-tobaccouse.html

WHOQOL 1 Group. (1998). The World Health Organization quality of life assessment (WHOQOL): Development and general psychometric properties. Social Science \& Medicine, 46(12), 1569-1585.

WHOQOL SRPB Group. (2006). A cross-cultural study of spirituality, religion, and personal beliefs as components of quality of life. Social Science \& Medicine, 62(6), 1486-1497.

Williams, C. (2007). Research Methods. Journal of Business \& Economic Research, 5(3), $65-72$.

Williamson, D. (1989). The Box Plot: A Simple Visual Method to Interpret Data. Annals of Internal Medicine, 110(11), 916-921.

Wisdom, J., \& Creswell, J. (2013). Mixed Methods: Integrating Quantitative and Qualitative Data Collection and Analysis While Studying Patient-Centered Medical Home Models. PCMH Resource Centre. Agency for Healthcare Research and Quality, U.S. Department of Health and Human Services. Retrieved December 31, 2017, from https://pcmh.ahrq.gov/page/mixed-methods-integratingquantitative-and-qualitative-data-collection-and-analysis-while 
Wiseman, A. (2008). A Culture of (in) Equality. A Cross-National Study of Gender Parity and Gender Segregation in National School Systems. Research in Comparative and International Education, 3(2), 179-201.

Witten, K., Exeter, D., \& Field, A. (2003). The Quality of Urban Environments: Mapping Variation in Access to Community Resources. Urban Studies, 40(1), 161-177.

Xie, Z., \& Or, C. (2017). Associations Between Waiting Times, Service Times, and Patient Satisfaction in an Endocrinology Outpatient Department: A Time Study and Questionnaire Survey. INQUIRY: The Journal of Health Care Organization, Provision, And Financing, 54, 1-10.

Xu, X., Ha, S., \& Basnet, R. (2016). A Review of Epidemiological Research on Adverse Neurological Effects of Exposure to Ambient Air Pollution. Frontiers in Public Health, 4, 1-31.

Yarcheski, A., Scoloveno, M., \& Mahon, N. (1994). Social Support and Well-Being in Adolescents. Nursing Research, 43(5), 288-292.

Yu, R., Cheung, O., Lau, K., \& Woo, J. (2017). Associations between Perceived Neighborhood Walkability and Walking Time, Wellbeing, and Loneliness in Community-Dwelling Older Chinese People in Hong Kong. International Journal of Environmental Research and Public Health, 14(10), 1-15.

Yuan, L., Yuen, B., \& Low, C. (1999). Urban QoL: Critical Issues and Options. Singapore: National University of Singapore School of Building and Real Estate.

Yuan, S., Kole, S., Hwang, S.Y., Manlagñit, M.C., Yuen, S., \& He, S.J. (2009). Quality of Life in Hawai'i, 2009 Report: Framework, Indicators, and Technical Documentation. Honolulu: University of Hawai'i, Centre on the Family.

Yun, L., \& Evangelou, N. (2016). Social Benefits of Homeownership and Stable Housing [Ebook]. Washington: National Association of Realtors.

Zhan, L. (1992). Quality of life: conceptual and measurement issues. Journal of Advanced Nursing, 17(7), 795-800. 
Zhang, F., Zhang, C., \& Hudson, J. (2018). Housing conditions and life satisfaction in urban China. Cities, 81, 35-44.

Zukin, S. 1995. The Cultures of Cities. Oxford: Basil Blackwell. 


\section{Appendix 1: Questionnaire (English version).}


Student Dissertation Research, Department of Geography, Swansea University

\section{Questionnaire Form}

\section{An Investigation of Quality of Urban Life (QoUL) A Case Study of Quba Municipality, Medina City, Saudi Arabia}

Dear resident,

This questionnaire is part of a study as a requirement to obtain a $\mathrm{PhD}$ in Human Geography from the Geography Department at Swansea University. The study investigates the quality of urban life (QoUL) in Medina city, the case of Quba municipality, and explores the experience of the QoUL in the study area.

In this questionnaire, we would like to ask you a number of questions about the overall quality of urban life, specifically how satisfied you are with the urban services provided to you and to what extent your life is affected by the urban, economic, social and cultural aspects of the environment in which you live.

This questionnaire should take about 20 minutes to complete. We appreciate your valuable time and effort in answering questions as the information you provide would be very useful to us. This questionnaire is for research purposes only and any information you provide will be treated in a strictest confidence. If you are less than 18, please do not fill this questionnaire.

Researcher: Abdulaziz Rebayea M. Alrashidi

Email:

Supervisors: Dr Sergei Shubin \& Dr Richard Smith

Geography Department, College of Science, Swansea University

Singleton Park, Swansea, SA2 8PP

Tel: 


\section{$\underline{\text { 1. Demographic information }}$}

In the following questions, please tick the appropriate answer.

1.1. What is your gender?

$\square$ Female $\quad \square$ Male

1.2. What is your age?

1.3. What is your present marital status?
$\square \quad$ Single
$\square \quad$ Married
$\square \quad$ Divorced

1.4. What is your educational level?
$\square \quad$ Primary school
$\square \quad$ Secondary school
$\square \quad$ High School
$\square \quad$ Diploma
$\square \quad$ Bachelor's
$\square \quad$ Master's or PhD

1.5. What is your employment status?
$\square \quad$ Employed
$\square \quad$ Unemployed
$\square \quad$ Self employer
$\square \quad$ Retired
$\square \quad$ Other (please state)

\subsection{What is your residential status?}

$\square \quad$ Own home

$\square \quad$ Rent home

$\square \quad$ Other (please state).

\subsection{How many people are living at home including you?}


1.8. What is the total monthly income of the household in SAR?
$\square \quad$ Less than 1500
$\square \quad 1500-3000$
$\square \quad 3000-5000$
$\square \quad 5000-10000$
$\square \quad 10000-15000$
15000 or more

\section{Perception about quality of urban life}

2.1. In general, how do you describe your quality of urban life on scale of $1-10$, where 1 indicates very poor, while 10 indicates excellent.
$\square$
$\square \quad 2$
$\square \quad 3$
$\square 4$
$\square \quad 5$
$\square 6$
$\square \quad 7$
$\begin{array}{lllll}\square & 8 & \square & 9 & \square \quad 10\end{array}$

2.2 Could you please rank the following aspects of life from 1- 6 according to their influence on your life, where 1 indicates the most influential aspect, while 6 indicates the least influential aspect

$\begin{array}{ll}\square \text { Health } & \square \text { Education } \\ \square \text { Security } & \square \text { Employment and finance } \\ \square \text { Housing } & \square \text { Recreation }\end{array}$

\section{Physical domain}

In the following statements, there are some questions about your satisfaction level and to what extent do you agree with some statements regarding the urban aspects in the municipality of Quba. This includes circumstances of housing, the neighbourhood you live in and the urban services provided to you within the municipality of Quba. 


\subsection{Housing}

3.1.1. What type of accommodation do you live in?
$\square \quad$ Flat
$\square \quad$ House
$\square \quad$ Villa
$\square$ Other
(Please state)

\subsubsection{How long have been living in this accommodation?}

3.1.3. To what extent do you agree or disagree that your accommodation is adequate to live in?

$\begin{array}{lllll}\square & \text { Strongly agree } & \square & \text { Agree } & \square \quad \text { Neutral } \\ \square & \text { Disagree } & \square & \text { Strongly disagree } & \end{array}$

3.1.4. What percentage of household income is spent on housing, finance or rent?

\begin{tabular}{|c|c|}
\hline less than $10 \%$ & $10 \%-19 \%$ \\
\hline $30 \%-39 \%$ & $40 \%-49 \%$ \\
\hline
\end{tabular}

3.1.5. To what extent do you agree or disagree that it is affordable to buy or rent a home in the municipality of Quba?
$\square \quad$ Strongly agree
$\square \quad$ Agree
$\square \quad$ Neutral
$\square \quad$ Disagree
$\square \quad$ Strongly disagree

3.1.6. How would you describe your satisfaction with your accommodation quality in scale of $1-10$, where 1 indicates very dissatisfied, while 10 indicates strongly satisfied?
$\square \quad 1 \quad \square \quad 2 \quad \square \quad 3$
$\square \quad 4$
$\square \quad 5$
$\square \quad 6$
$\square 7$
$\square \quad 8 \quad \square \quad 9 \quad \square \quad 10$ 


\subsubsection{How likely are you to move to another accommodation?}

\begin{tabular}{|c|c|}
\hline Extremely likely & Likely \\
\hline Unlikely & Extreme \\
\hline
\end{tabular}

\subsection{Neighbourhood}

\subsubsection{Do you suffer from any of the following issues in your neighbourhood? Please tick} all related problems.

Noise

Lack of security and safety

Lack of children playgrounds

Long Distance to mosques
Air pollution

Lack of schools

Lack of green spaces

Long Distance to work
Rubbish in the street

Lack of health facilities

Lack of car parking

Lack of basic shopping

3.2.2. To what extent do you agree or disagree that the neighbourhood you live in is an attractive and well-organised place to live?

$\square$ Strongly agree $\quad \square \quad$ Agree $\quad \square \quad$ Neutral

$\square$ Disagree $\square$ Strongly disagree

\subsubsection{To what extent do you agree or disagree that the neighbourhood you live in has a} good basic infrastructure such as streets, pavements, lighting ...etc.

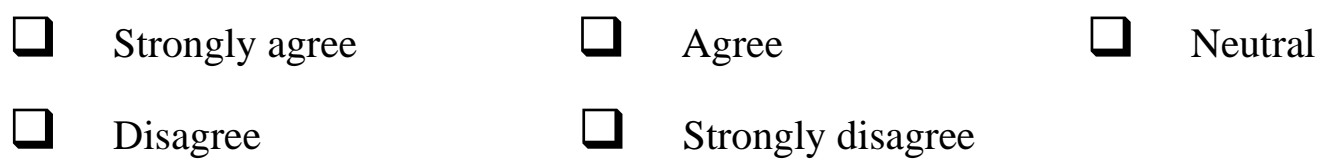

\subsubsection{In general, how do you describe the cost of living in the municipality of Quba?}
$\square \quad$ Very high
$\square$ High
$\square \quad$ Fair
$\square \quad$ Low
$\square \quad$ Very low 
3.2.5. To what extent do you agree or disagree there are plenty of opportunity to find an appropriate job?
$\square \quad$ Strongly agree
$\square \quad$ Agree
$\square \quad$ Neutral
$\square \quad$ Disagree
$\square \quad$ Strongly disagree

3.2.6. How easy is it to access the main road in the neighbourhood you live in?

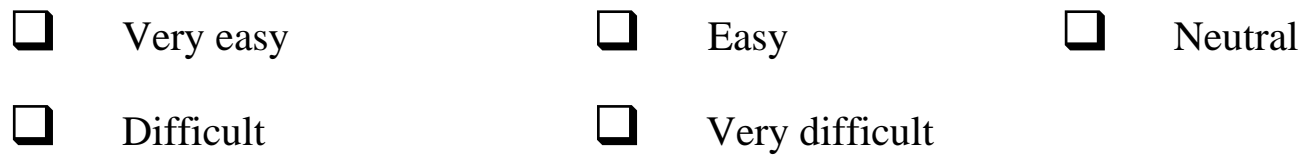

3.2.7. To what extent do you agree that there are suitable sidewalks to walk alongside with the streets for people who willing to not drive?
$\square \quad$ Strongly agree
$\square \quad$ Agree
$\square \quad$ Neutral
$\square \quad$ Disagree
$\square \quad$ Strongly disagree

3.2.8. To what extent are you interested to use sidewalk to walk around your neighbourhood, for shopping, visit friends, neighbours etc.

$\begin{array}{llll}\square & \text { Very interested } & \square & \text { Fairly interested } \\ \square & \text { Not very interested } & \square \quad \text { Not interested at all } & \end{array}$

\subsubsection{How satisfied are you with the traffic jams in the municipality of Quba?}

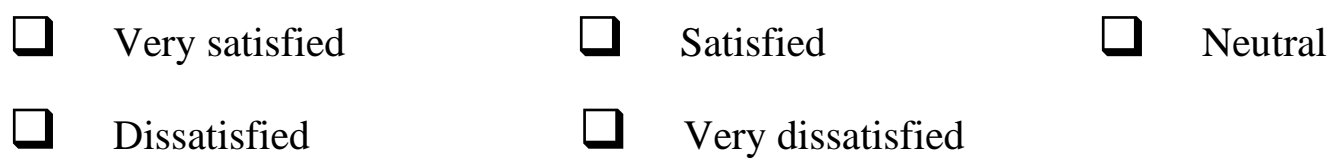

3.2.10. How would you describe your satisfaction with the neighbourhood you live in in scale of $1-10$, where 1 indicates very dissatisfied, while 10 indicates strongly satisfied?
$\square$
$\square \quad 2$
$\square 3$
口 4
$\square \quad 5$
$\square \quad 6$
口 7
$\square \quad 8 \quad \square \quad 9 \quad \square \quad 10$ 
3.2.11. Could you please tell us, what do you like most about your neighbourhood in terms of QoUL?

3.2.12. Also, what do you like least about your neighbourhood in terms of QoUL?

\subsection{Health services}

3.3.1. In the last 12 months, how many times have you visited the doctor?

(please state ..).

3.3.2. Normally, how long do you wait to get to the doctor? In minutes

(please state ..).

3.3.3. How easy is it to get a medical appointment?

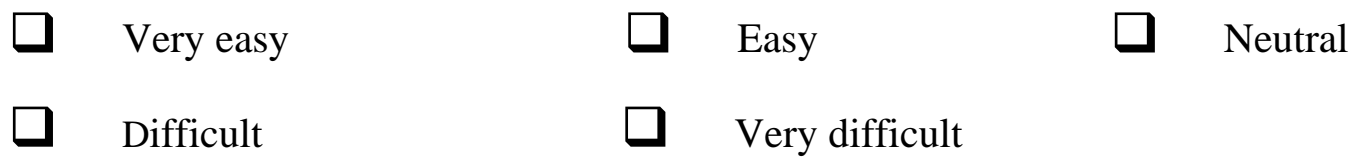

3.3.4. How do you rate the medical care you received from staff and doctors in the health provider?
$\square$ Excellent
$\square \quad$ Very good
$\square$ Good
$\square \quad$ Fair
$\square$ Poor 
3.3.5. How satisfied are you with the amount of time your doctor spends diagnosing your condition?
$\square \quad$ Very satisfied
Satisfied
$\square \quad$ Neutral
$\square \quad$ Dissatisfied
$\square \quad$ Very dissatisfied

3.3.6. Overall, how satisfied are you with the quality of healthcare services?
$\square \quad$ Very satisfied
Satisfied
Neutral
$\square \quad$ Dissatisfied
Very dissatisfied

3.3.7. In your opinion, what are the main concerns about health services?

\subsection{Education services}

3.4.1. To what extent do you agree or disagree that you can easily access to the schools?
$\square \quad$ Strongly agree
$\square \quad$ Agree
$\square \quad$ Neutral
$\square \quad$ Disagree
$\square \quad$ Strongly disagree

3.4.2. To what extent do you agree or disagree that your child's school is able to create an appropriate school environment for learning?

\begin{tabular}{|c|c|}
\hline Strongly agree & Agree \\
\hline Disagree & Strongl \\
\hline
\end{tabular}

\subsubsection{How do you assess the evaluation system for students in your child's school?}

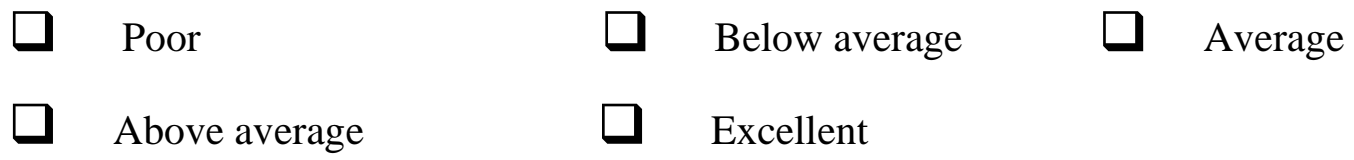


3.4.4. How satisfied are you with the number of students per class?
$\square \quad$ Very satisfied
$\square \quad$ Satisfied
$\square \quad$ Neutral
$\square \quad$ Dissatisfied
$\square \quad$ Very dissatisfied

3.4.5. Overall, how satisfied are you with quality of educational services?
$\square \quad$ Very satisfied
$\square \quad$ Satisfied
$\square \quad$ Neutral
$\square$ Dissatisfied
$\square \quad$ Very dissatisfied

3.4.6. In your opinion, what are the most important aspects of the educational services that need to be considered?

\subsection{Recreation facilities}

3.5.1. How often do you visit the recreation facilities in your area, parks, walk routes, sport facilities etc.?
$\square \quad$ Extremely often
$\square \quad$ Very often
$\square$ Somewhat often
$\square \quad$ Not so often
$\square \quad$ Not at all often

3.5.2. To what extent do you agree or disagree there are enough public parks in the municipality of Quba
$\square \quad$ Strongly agree
$\square \quad$ Agree
$\square \quad$ Neutral
$\square \quad$ Disagree
$\square \quad$ Strongly disagree 


\subsubsection{To what extent do you agree or disagree that there are enough playgrounds for}

\section{children in the neighbourhood where I live.}

$\begin{array}{llllll}\square & \text { Strongly agree } & \square & \text { Agree } & \square & \text { Neutral } \\ \square & \text { Disagree } & \square & \text { Strongly disagree } & & \end{array}$

3.5.4. To what extent do you agree or disagree that there are enough walking routes in the municipality of Quba that suit everyone who wants to walk, exercise etc.?
$\square \quad$ Strongly agree
$\square \quad$ Agree
$\square \quad$ Neutral
$\square \quad$ Disagree
$\square \quad$ Strongly disagree

3.5.5. To what extent do you agree or disagree that public parks are well-designed for all segments of society, single people and families?
$\square \quad$ Strongly agree
$\square \quad$ Agree
$\square \quad$ Neutral
$\square \quad$ Disagree
$\square \quad$ Strongly disagree

\subsubsection{Overall, how satisfied are you with the quality of recreation facilities?}
Very satisfied
$\square \quad$ Satisfied
$\square \quad$ Neutral
Dissatisfied
$\square \quad$ Very dissatisfied

\section{Social domain}

In the following statements, there are some questions about the social life in the neighbourhood you live in and the municipality of Quba in general, including the security situation, social activities, social relations and the religion.

\subsection{How do you evaluate the security situation in your area?}

$\begin{array}{llllll}\square & \text { Very poor } & \square & \text { Below average } & \square & \text { Average } \\ \square & \text { Above average } & \square & \text { Excellent } & & \end{array}$


4.2. To what extent do you agree or disagree there is a good accessibility to leisure activities that are suitable for all groups of people (i.e. children, adults and the elderly people) in the municipality of Quba.
$\square \quad$ Strongly agree
$\square \quad$ Agree
$\square \quad$ Neutral
$\square \quad$ Disagree
$\square \quad$ Strongly disagree

4.3. How would you describe your social interaction with your neighbours in the neighbourhood you live in?

$\square$ Not at all $\square \quad \square$ A little $\quad \square \quad$ A moderate amount

$\square$ Very much $\square$ An extreme amount

4.4. To what extent do your neighbours support you when you need them?

$\square \quad$ Not at all amount

$\square \quad$ Very much $\square \quad$ A little

$\square \quad$ A moderate

4.5. How satisfied are you with your neighbours?
$\square \quad$ Very satisfied
$\square \quad$ Satisfied
$\square \quad$ Neutral
$\square \quad$ Dissatisfied
$\square \quad$ Very dissatisfied

4.6. To what extent do your spiritual/ religious beliefs help you to improve your quality of life?
$\square \quad$ Not at all
$\square \quad$ A little
$\square \quad$ A moderate amount
$\square \quad$ Very much
$\square \quad$ An extreme amount 
4.7. To what extent do you agree or disagree that the place where you live enhances your sense of spirituality, which is in turn enhancing the quality of your life?

$\square \quad$ Strongly agree

$\square \quad$ Agree

$\square \quad$ Neutral

$\square \quad$ Disagree

$\square \quad$ Strongly disagree

\subsection{To what extent do you agree or disagree that religious practices and beliefs have an} impact on improving your quality of life?

$\begin{array}{llllll}\square & \text { Strongly agree } & \square & \text { Somewhat agree } & \square & \text { Neutral } \\ \square & \text { Somewhat disagree } & \square & \text { Strongly disagree } & & \end{array}$

\subsection{Are you frequently attending the Mosque?}

$\square$ Yes $\square$ No

\section{Cultural domain}

In the following statements, there are some questions about cultural aspects in the municipality of Quba such as heritage, religious landmarks and other cultural landmarks such as libraries and museums as well as the cultural activities and to what extent they impact your quality of your life.

5.1. To what extent do you agree or disagree there are many heritage and religious landmarks in the municipality of Quba that I can introduce to visitors

\begin{tabular}{|c|c|}
\hline Strongly agree & Agree \\
\hline Disagree & Strongl \\
\hline
\end{tabular}


5.2. To what extent heritage and religious landmarks affect the quality of your urban life?
$\square \quad$ Not at all
$\square \quad$ A little
$\square \quad$ A moderate amount
$\square \quad$ Very much
$\square \quad$ An extreme amount

5.3. To what extent the presence of such religious landmarks and heritage enhance your quality of life.
$\square \quad$ Not at all
$\square \quad$ A little
$\square \quad$ A moderate amount
$\square \quad$ Very much
$\square \quad$ An extreme amount

5.4. To what extent do you agree or disagree there is an accessibility to the libraries and museums.
$\square \quad$ Strongly agree
$\square \quad$ Agree
$\square \quad$ Neutral
$\square \quad$ Disagree
$\square \quad$ Strongly disagree

5.5. To what extent do you have the access to the cultural activities such as conferences and exhibitions of hobbies, arts, crafts, traditional food?
$\square \quad$ Not at all
$\square \quad$ A little
$\square \quad$ A moderate amount
$\square \quad$ Very much
$\square \quad$ An extreme amount

At the end of this questionnaire, if you have any comments or opinions that you would like to add, could you please write them below. 


\section{Appendix 2: Questionnaire (Arabic version).}




\section{College of Science}

Swansea University

Student Dissertation Research, Department of Geography, Swansea University

$$
\text { استمارة استبيان }
$$

\section{دراسة جودة الحياة الحضرية بالمدينة المنورة، حالة بلدية قباء}

$$
\text { أخي الكريم/ أختي الكريمة }
$$

بين يديكم هذا الاستبيان وهو جزء من دراسة كمتطلب للحصول على درجة الدكتوراه في الجغرافيا البشرية من قسم الجغرافيا في جامعة سوانسي بالمملكة المتحدة (بريطانيا). وتبحث هذه الدراسة في جودة الحياة الحضرية في المدينة المنورة، حالة بلدية قباء الكبرى بجميع أحيائها. ويشمل ذلك أحياء قربان، قباء، البحر، المغيسلة، الدويمة، العصبة، الجبور، الجمعة، مخطط الأمير نايف، القصواء، الرانوناء، شوران، الروابي وغيرها من الأحياء التابعة لبلدية قباء، حيث تسلط الدراسة الضوء على الجوانب المؤثرة في جودة الحياة الحضرية في تلك المناطق.

في هذا الاستبيان، نود أن نطرح عليك عددًا من الأسئلة حول جودة الحياة الحضرية بشكل عام، ويشمل

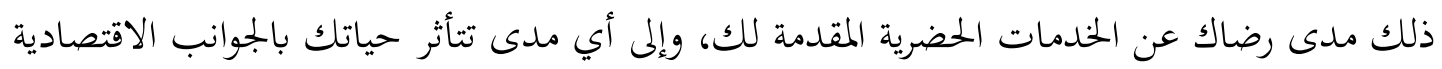

$$
\text { والاجتماعية والثقافية للبيئة التي تعيش فيها. }
$$

يستغرق هذا الاستبيان حوالي 20 دقيقة. ونخن نقدر وقتك وجهدك الثمين في الإجابة عن الأسئلة، لأن ما

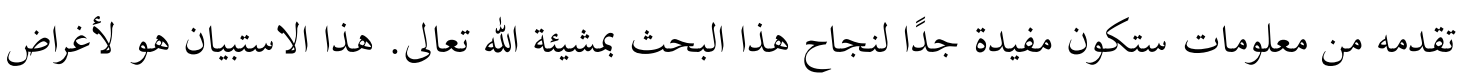

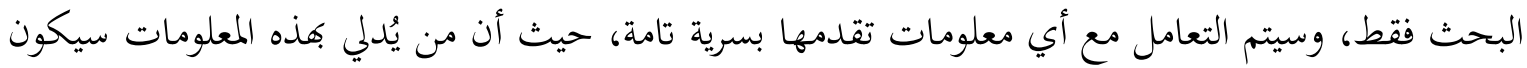
مجهول الهوية. إذا كان عمرك أقل من 18 سنة، فيرجى عدم ملء هذا الاستبيان، مع خالص شكري وتقديري.

$$
\text { المشرفون: د. سيرجي شبد العزيز ربيع الرشيدي / د. ريتشارد سميث }
$$
قسم الجغرافيا، كلية العلوم، جامعة سوانسي، المملكة المتحدة

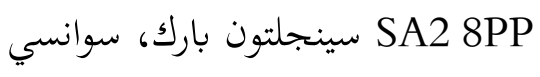




\begin{tabular}{|l|l|l|}
\hline & \\
\hline
\end{tabular}

1. البيانات الديموغرافية (المعلومات الثخصية). يرجى اختيار الإجابة التي تنطبق في الأسئلة التالية:

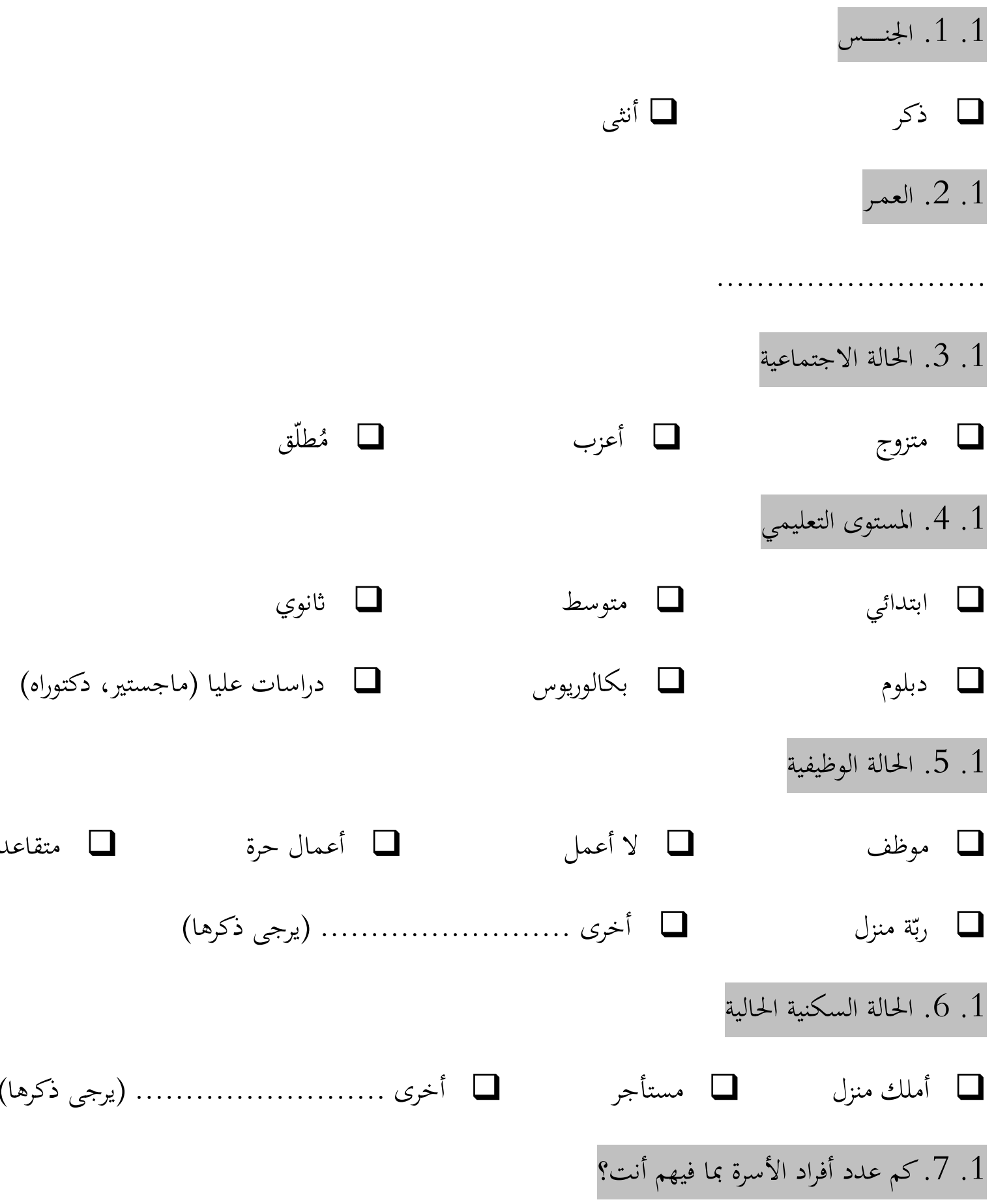




\begin{tabular}{|c|c|c|c|c|}
\hline \multirow[b]{2}{*}{$5000-3000$} & \multirow[b]{2}{*}{$\square$} & \multirow[b]{2}{*}{$3000-1500$} & \multicolumn{2}{|c|}{ 1. 8. بمموع دخل الأسرة بالريال السعودي } \\
\hline & & & $\square$ & أقل من 1500 \\
\hline أكثر من 15000 & $\square$ & $15000-10000$ & $\square$ & $10000-5000$ \\
\hline
\end{tabular}

2. التصور العام حول جودة الحياة الحضرية

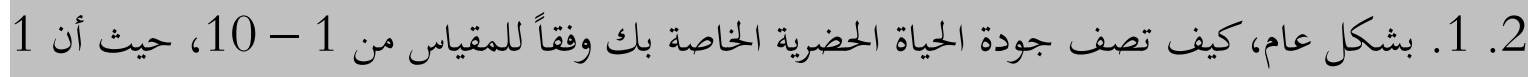
يشير إلى سيئة جداً، بينما 10 يشير إلى ممتازة. (فضلاً ضع دائرة حول الإجابة التي تناسبك).

9

8

7

6

5

4

3

2

1

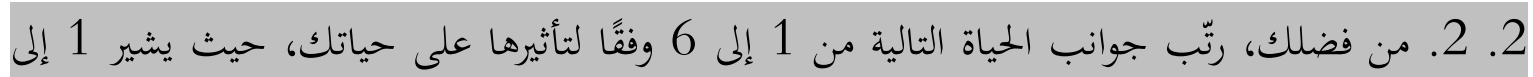

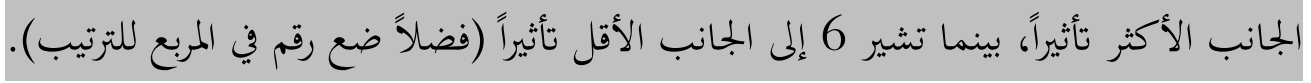
प التعليم

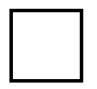
الصحة

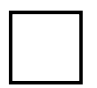
الترفيه السكن

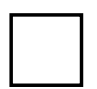

الوظيفة والمال

\section{3.}

فيما يلي، هناك عدد من الأسئلة حول مستوى رضاك عن الجوانب الحَضَرية في بلدية قباء بشكل عام، والحي الذي تعيش فيه بشكل خاص، وكذلك مدى اتفاقك من عدمه على بعض العبارات، ويشمل ذلك الخدمات

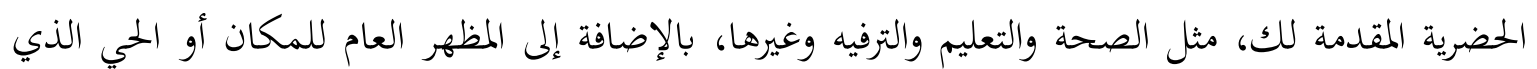
تعيش فيه. 
3. 1. 6. كيف تصف مدى رضاك عن جودة السكن الذي تعيش فيه وفقاً للمقياس 1 إلى 10، حيث يشير

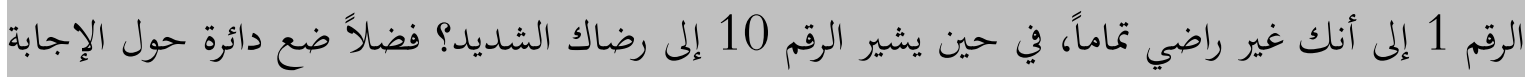
التي تناسبك.

9 8 7

6

54

3

2

3. 1. 7. ما مدى احتمالية انتقالك إلى مكان إقامة آخر، أو رغبتك في تغيير سكنك الحالي؟

口 مُنعادل 口 مُحتمل جداً من غير المختمل على الإطلاق من غير المختمل

3.

3. 2. 1. هل تواجه أي من الأمور التالية في الحي الذي تعيش فيه؟ يرجى وضع علامة على جميع المشاكل ذات الصلة. (فقط الأمور التي تواجهها أو تعاني منها).

$$
\begin{array}{ll}
\text { a } \\
\text { a نقص مستوى الأمن (الإزعاج) }
\end{array}
$$

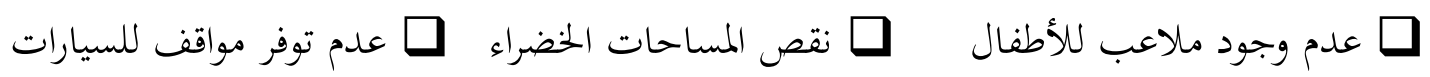

$$
\text { 口 بُعد المسافة للمسجد }
$$

3. 2. 2. إلى أي مدى توافق على أن الحي الذي تعيش فيه هو مكان جذاب ومنظم بشكل جيد للعيش

$$
\text { 口 أوافق بشدة }
$$$$
\text { لا أوافق بشدة }
$$$$
\text { لا أوافق }
$$ 
3. 2. 3. ما مدى رضاك عن البنية التحتية الأساسية للحي الذي تعيش فيه، بما في ذلك جودة الشوارع والأرصفة والإضاءة.

口 口

口 غير راضي على الإطلاق

口

3. 2. 4. بشكل عام، كيف تصف تكاليف المعيشة في المنطقة التي تعيش فيها؟

口 عادلة (متوسطة)

ع

口 ع عالية جداً

منخفضة جداً

口 منخفضة

3. 2. 5. إلى أي مدى توافق أو لا توافق على وجود مزيد من الفرص للعثور على وظيفة مناسبة؟

口

أوافق

أوافق بشدة

لا أوافق بشدة

لا أوافق

3. 2. 6. ما مدى سهولة الوصول إلى الطريق الرئيسي في الحي الذي تعيش فيه؟

صوعاً ما سهل

سه

سهل للغاية

صعب للغاية

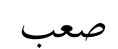

3. 2. 7. إلى أي مدى توافق أو لا توافق على انه يوجد أرصفة مناسبة للمشي إلى جانبي الطرق للأشخاص

الراغبين في عدم القيادة؟

口

口 أوافق

口 أوافق بشدة

لا أوافق بشدة

لا أوافق 
3. 2. 8. إلى أي مدى أنت مهتم باستخدام رصيف المشي للتجول في الجوار، على سبيل المثال، التسوق أو زيارة الأصدقاء والجيران وما على ذلك؟ إي ملى انت مهنم باستر

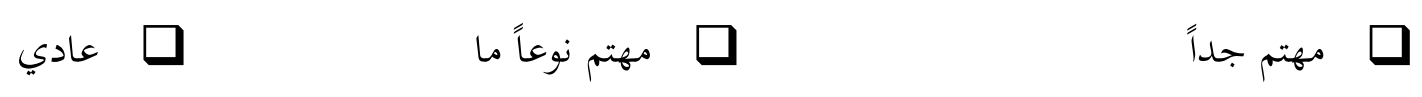

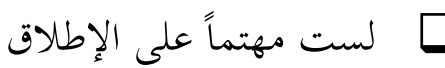

$$
\begin{aligned}
& \text { 口 لست مهتماً }
\end{aligned}
$$$$
\text { 3. 2. 9. ما مدى رضاك عن الحالة المرورية (سير المركبات) في بلدية قباء؟ }
$$

口 مشكل معتدل

$$
\begin{aligned}
& \text { 口 } \\
& \text { 口 راضي تماماً } \\
& \text { 口 غير راضي على الإطلاق } \\
& \text { 口 }
\end{aligned}
$$

3. 2. 10. كيف تصف مدى رضاك عن حالة الحي الذي تعيش فيه وفقاً للمقياس 1-10، حيث يشير

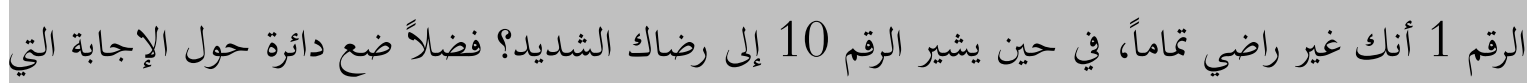
تناسبك

9 8

7

6

5

43

2

1

3. 2. 11. هل من الممكن أن تخبرنا، ما هو أكثر شيء يعجبك في منطقتك من حيث جودة الحياة الحضرية؟ من فضلك أذكرها في الأسطر التالية إن أمكن. 
3. 2. 12. أيضاً، ما هو أقل شيء يعجبك في منطقتك من حيث جودة الحياة الحضرية؟ من فضلك أذكرها

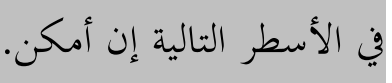

\section{3. الخدمــــات الصحية}

3. 3. 1. خلال الاثني عشر شهرًا الماضية، كم عدد المرات التي قمت فيها بزيارة الطبيب؟ ...

3. 3. 2. عادةً، كم من الوقت تنتظر حتى تصل إلى الطبي؟ دقيقة|

3. 3. 3. ما مدى سهولة حصولك على موعد طبي؟

ص موعاً ما سهل

口

سهل للغاية

口ص معب للغاية

صعب

3. 3. 4. بشكل عام، كيف تقيّم الرعاية الطبية التي تتلقاها من الموظفين والأطباء في مُقدِّمِ الرعاية الصحية؟

$$
\begin{aligned}
& \text { 口 مثنازة }
\end{aligned}
$$

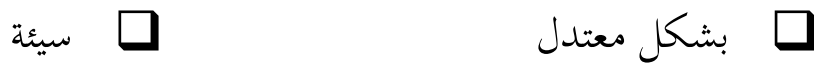

3. 3. 5. ما مدى رضاك عن مقدار الوقت الذي يقضيه الطبيب في تشخيص حالتك؟

口 راضي تماماً

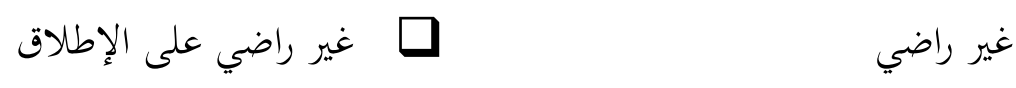


3. 3. 6. ما مدى رضاك عن جودة خدمات الرعاية الصحية المقدمة لك؟

口

$$
\text { 口 }
$$

口 غير راضي على الإطلاق

口

3. 3. 7. هل من الممكن أن تذكر لنا ما الذي يهمك فيما يخص الخدمات الصحية بشكل عام، والتي ينبغي

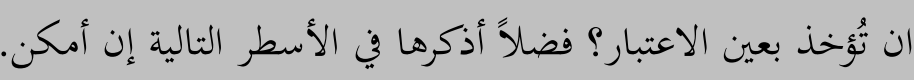

3. 4. الخدمـــات التعليمية

3. 4. 1. إلى أي مدى توافق أو لا توافق على أنه يمكنك الوصول بسهولة إلى المدارس؟

口 أوافق بشدة

لا أوافق

3. 4. 2. إلى أي مدى توافق أو لا توافق على أن مدرسة طفلك قادرة على خلق بيئة مدرسية مناسبة للتعلم؟

$$
\begin{aligned}
& \text { 口 } \\
& \text { 口 } \\
& \text { أوافق بشدة } \\
& \text { لا أوافق بشدة } \\
& \text { لا أوافق } \\
& \text { 3. 4. 3. كيف تقيّم نظام تقييم الطلاب في مدرسة طفلك؟ } \\
& \text { D } \\
& \text { ص تحت المتوسط } \\
& \text { D ضعيف } \\
& \text { 口 } \\
& \text { فوق المتوسط }
\end{aligned}
$$


3. 4. 4. ما مدى رضاك عن عدد الطلاب لكل فصل (غرفة الصف) في مدرسة طفلك؟

口 راضي تماماً

$$
\text { غير راضي على الإطلاق }
$$$$
\square
$$

3. 4. 5. ما مدى رضاك عن جودة المرافق التعليمية؟

口 راضي تماماً

$$
\text { غير راضي }
$$

3. 4. 6. برأيك، ما هي أهم جوانب الخدمات التعليمية التي يجب أخذها بعين الاعتبار؟ فضلاً أذكرها في الأسطر التالية إن أمكن. (- من.

3.

3. 5. 1. ما مدى ترددك على مرافق الترفيه بما فيها الحدائق ومسارات المشي (الممشى) والمرافق الرياضية وما إلى ذلك؟

口

口 دائماً

بشكل دائم جداً

$\square$

لا أتردد لها على الإطلاق

ليس دائما 
3. 5. 2. إلى أي مدى توافق أو لا توافق على وجود عدد كافٍ من الحدائق العامة في بلدية قباء؟

口 أوافق بشدة

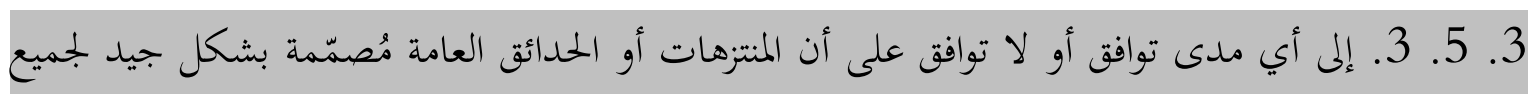

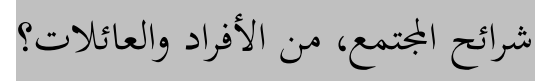

口 مُحايد

أوافق

口
口 أوافق بشدة

口 أل

3. 5. 4. إلى أي مدى توافق أو لا توافق على أنه يوجد في بلدية قباء مسارات للمشي تناسب كل من يريد

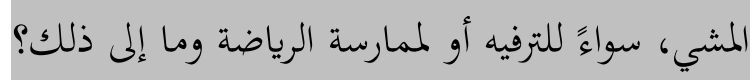

口 口 口 أوافق بشدة لا أوافق بشدة ألاف

3. 5. 5. إلى أي مدى توافق أو لا توافق على أنه يوجد المزيد من ملاعب الأطفال (الملاهي) في الحي الذي

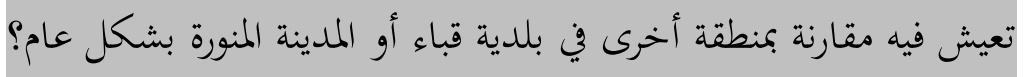

口

口 口 أوافق بشدة

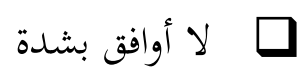

口 أل

3. 5. 6. بشكل عام، ما مدى رضاك عن جودة المرافق الترفيهية؟

口 مشكل معتدل

راضي

راضي تماماً

$$
\text { 口 غير راضي على الإطلاق }
$$

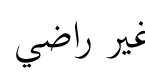




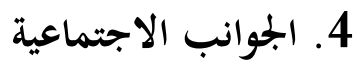

فيما يلي، هناك بعض الأسئلة حول النظام الاجتماعي في الحي الذي تسكن فيه وبلدية قباء بشكل عام، بما في ذلك الوضع الأمني والأنشطة والعلاقات الاجتماعية وكذلك الدين. 4. 1. كيف تقيّم الوضع الأمني في منطقتك؟

$$
\begin{aligned}
& \\
& \square
\end{aligned}
$$

4. 2. إلى أي مدى توافق أو لا توافق/ توافقين أو لا توافقين على أن هناك أنشطة ترفيهية مناسبة لجميع فئات الناس (أي الأطفال والبالغين وكبار السن) في بلدية قباء.

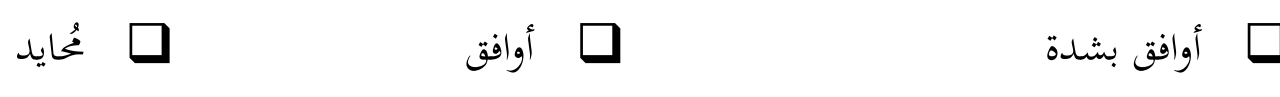

$$
\text { 口 ل أوافق بشدة }
$$$$
\text { لا أوافق }
$$

4. 3. كيف تصف تفاعلك الاجتماعي مع جيرانك في الحي الذي تعيش فيه؟

口 مشكل معتدل

口 ق قليلاً

لا يوجد تفاعل على الإطلاق

$$
\text { 口 بشكل كثير جداً }
$$

口 بشكل كثير

4. 4. إلى أي مدى يمكنك الحصول على الدعم من جيرانك عندما تحتاجهم؟

$$
\begin{aligned}
& \text { 口 بشكل معتدل } \\
& \text { 口 ق قليلاً } \\
& \text { لا يمكن على الإطلاق } \\
& \text { من الممكن جداً } \\
& \text { 口 من الممكن } \\
& \text { 4. 5. ما مدى رضاك عن جيرانك؟ } \\
& \text { 口 بشكل معتدل } \\
& \text { 口 } \\
& \text { راضي تماماً } \\
& \text { غير راضي على الإطلاق } \\
& \text { غير راضي }
\end{aligned}
$$


4. 6. إلى أي مدى يمكن أن تساعدك معتقداتك الروحية/ الدينية على تحسين جودة حياتك؟

$$
\text { 口 لا يمكن على الإطلاق }
$$$$
\text { 口 من الممكن جداً }
$$

口 من الممكن

4. 7. إلى أي مدى توافق أو لا توافق على أن المكان الذي تعيش فيه يعزز شعورك بالروحانية، وهذا بدوره يعزز جودة حياتك؟

口

口 أوافق

لا أوافق بشدة
口 أوافق بشدة

口 ألاوق

4. 8. إلى مدى توافق أو لا توافق على أن الممارسات والمعتقدات الدينية لها أثر في تحسين نوعية حياتك؟ 口 تُحايد

$$
\begin{aligned}
& \text { 口 أوافق } \\
& \text { أوافق بشدة } \\
& \text { لا أوافق بشدة } \\
& \text { لا أوافق } \\
& \text { 4. 9. هل أنت من يحضرون للمسجد بشكل متكرر؟ } \\
& \text { У } \square \\
& \text { ن }
\end{aligned}
$$

\section{5. الجوانب الثقافية}

فيما يلي، هناك بعض الأسئلة حول الجوانب الثقافية في بلدية قباء مثل التراث والمعالم الدينية والثقافية كالمكتبات

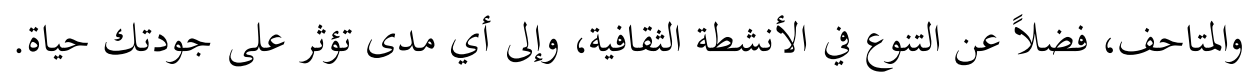

5. 1. إلى أي مدى توافق أو لا توافق على أن هناك العديد من المعالم التراثية والدينية في بلدية قباء والتي

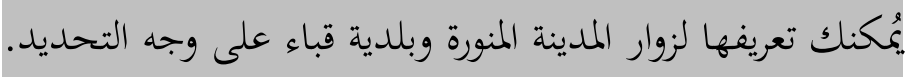

$$
\text { 口 }
$$

$$
\text { أوافق }
$$

$$
\text { أوافق بشدة }
$$

$$
\text { لا أوافق بشدة }
$$


5. 2. إلى أي مدى تؤثر المعالم التراثية والدينية على جودة حياتك؟

口 مشكل معتدل

$$
\text { 口 }
$$$$
\text { لا تلؤثر على الإطلاق }
$$

تؤثر بشكل كثير جداً

口

5. 3. إلى أي مدى يعزز وجود مثل هذه المعالم الدينية والتراث جودة حياتك؟

口 مشكل معتدل

$$
\text { 口 }
$$$$
\text { لا على الإطلاق }
$$

بشكل كثير جداً

كثيرا

5. 4. إلى مدى توافق أو لا توافق على أنه بإمكانك الوصول بسهولة إلى المكتبات والمتاحف؟

口

$$
\text { 口 }
$$$$
\text { أوافق بشدة }
$$

لا أوافق

5. 5. إلى أي مدى يمكنك الوصول إلى الأنشطة الثقافية مثل المؤتمرات والمعارض، كمعارض الهوايات والفنون

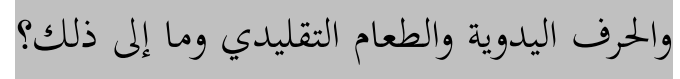

口 مشكل معتدل

$$
\text { ق مليلاً }
$$$$
\text { لا يمكن على الإطلاق }
$$

$$
\text { من الممكن جداً }
$$

في فاية هذا الاستبيان، إذا كان لديك أي تعليقات أو آراء ترغب في إضافتها، المرجو كتابتها في الأسطر أدناه. 


\section{Appendix 3: Photo diaries instructions sheet (English version).}


Student Dissertation Research, Department of Geography, Swansea University

\section{$\underline{\text { Photo Diary Instructions }}$}

- 2 weeks, from 04.12.2018 to 18.12 .2018

- 20 photographs at a rate of 1-2 photos per day.

- Think about why each photograph is important, why did you choose it?

- Some example themes are below:

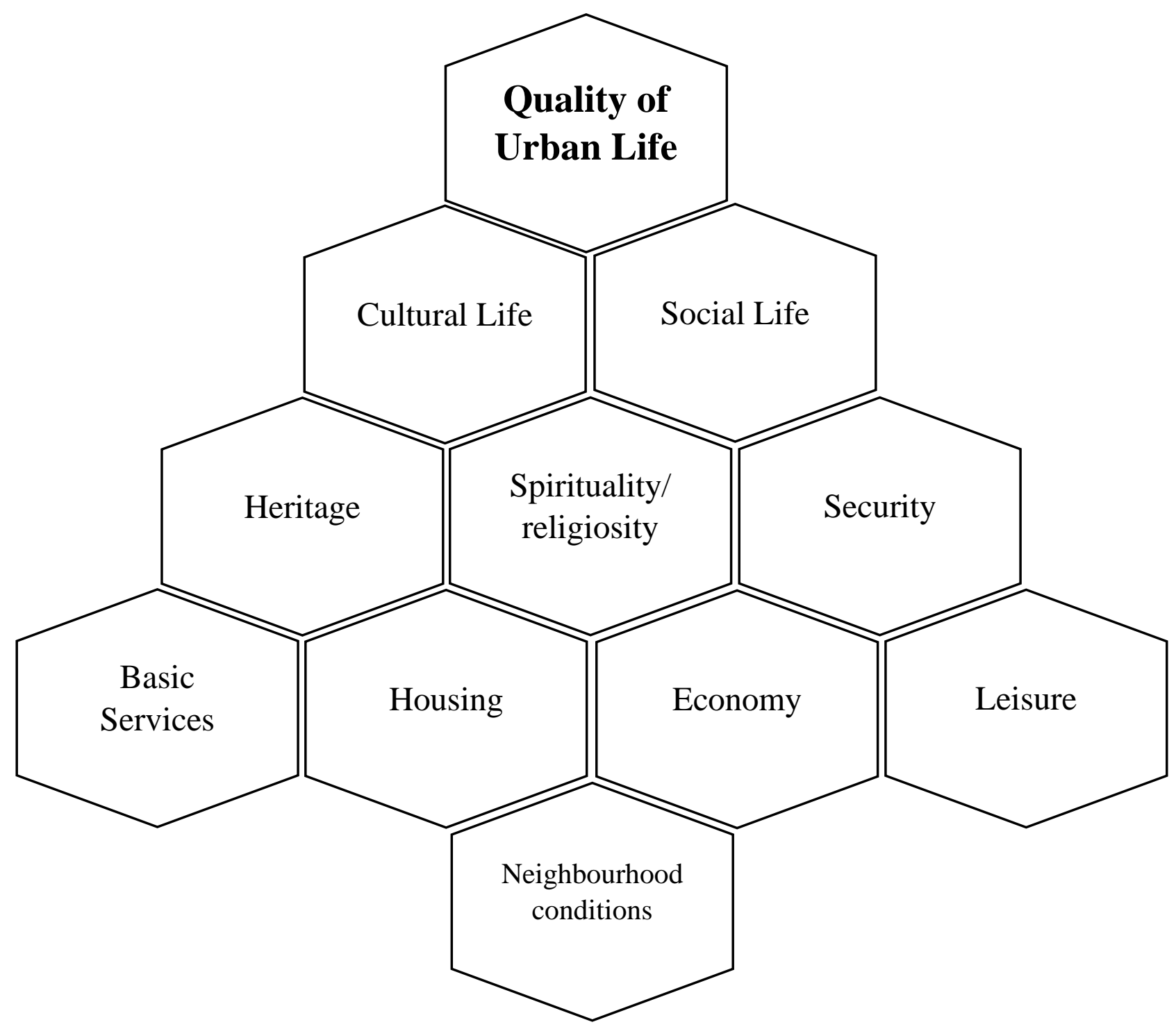




\section{Appendix 4: Photo diaries instructions sheet (Arabic version).}




\section{College of Science}

Swansea University

Student Dissertation Research, Department of Geography, Swansea University

\section{تعليمات يوميات الصور}

\section{• المدة: أسبو عين في الفترة من 04 / 12 / 2018 / 12 / 12 / 12 إلى • عدد الصور: 20 صورة، بمعدّل 1- 2 صورة باليوم.}

• فّر في سبب أهمية كل صورة بالنسبة لجودة الحياة، ما تأثثير ها على جودة حياتك؟ لماذا اخترتها؟

• أدناه، بعض المو اضيع حول جودة الحياة الحضرية.

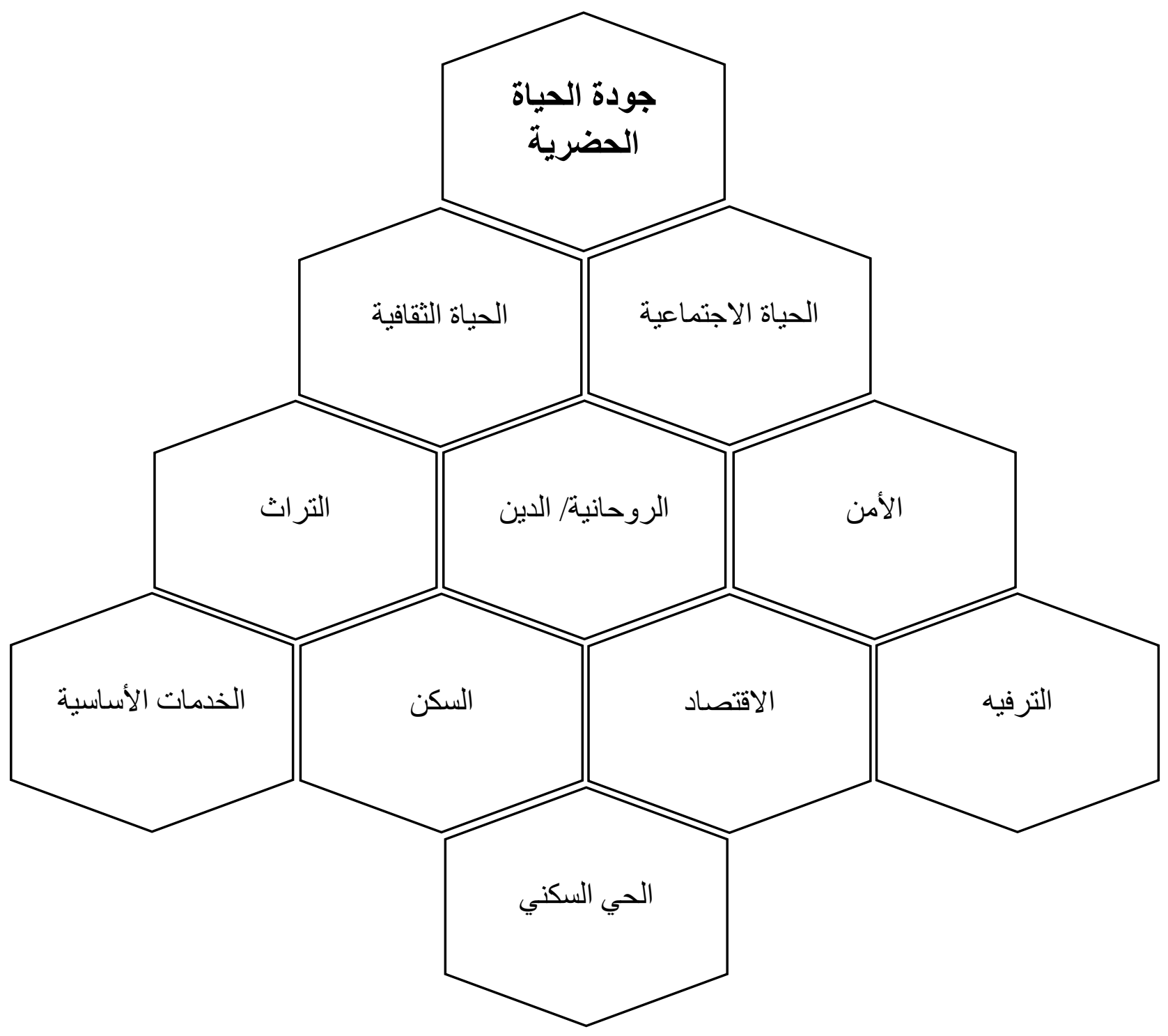




\section{Appendix 5: Participants Consent Form (English version).}


Student Dissertation Research, Department of Geography, Swansea University

\section{Participants Consent Form}

An Investigation of Quality of Urban Life (QoUL) A Case Study of Quba Municipality, Medina City, Saudi Arabia

Student Researcher: Abdulaziz Rebayea M. Alrashidi

Supervising Academic: Dr Sergei Shubin \& Dr Richard Smith

Please Initial Box

1. I confirm that I have read and understood the Information Sheet for this study.

2. I understand that my participation is voluntary and that I am free to withdraw from the study at any time.

3. I understand that the information I provide will be looked at and analysed by the student researcher and the supervising academic.

4. I understand that the information collected from me will be kept strictly confidential, stored securely, and that any information that appears in the thesis will be anonymized so that I cannot be recognised from it.

5. I agree to take part in this study.

\begin{tabular}{|l|l|l|}
\hline Name of Participant & Date & Signature \\
\hline
\end{tabular}

\section{Contact details}

Student Researcher: Abdulaziz R. Alrashidi / Email:

\section{Academic Supervisors:}

Dr Sergei Shubin / Email:

Dr Richard Smith / Email:

Telephone:

Telephone: 


\section{Appendix 6: Participants Consent Form (Arabic version).}


Student Dissertation Research, Department of Geography, Swansea University

$$
\text { نموذج مو افقة للمشاركة في بحث }
$$

دراسة جودة الحياة الحضرية بالمدينة المنورة، حالة بلدية قباء

\section{الرجاء وضع علامة في المربع للموافقة:}

$\square$ - أُقر بأنني قر أت وفهمت ورقة المعلومات الخاصة بهذه الدراسة. - أتفهم أن مشاركتي طو عية وأنني حر في الانسحاب من الدراسة في أي وقت.

- أتفهم أن المعلومات التي أقدمها سيتم النظر فيها وتحليلها من قبل الباحث و المشرف الأكاديمي. $\square$ أي معلومات تظهر في الأطروحة ستكون مجهولة المصدر. - أو افق على المشاركة في هذه الدراسة.

\begin{tabular}{|l|l|l|}
\hline التوقيع & التناريخ & \\
\hline
\end{tabular}

$$
\text { الباحث: عبد العزيز ربيع الرشيدي }
$$

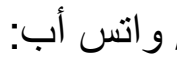

هاتف:

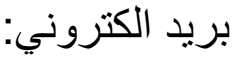

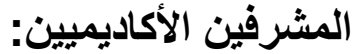
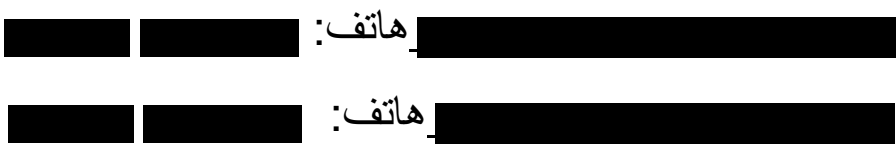

بريد الكتروني:

د. سيرجي شوبين

بريد الكتروني:

د. ريتثارد سميث 


\section{Appendix 7: Information Sheet (English version).}




\title{
Information Sheet
}

\author{
An Investigation of Quality of Urban Life (QoUL) \\ A Case Study of Quba Municipality, Medina City, Saudi Arabia
}

\author{
Student Researcher: $\quad$ Mr. Abdulaziz Rebayea M. Alrashidi \\ Supervising Academic: $\quad$ Dr Sergei Shubin \& Dr Richard Smith
}

I would like to invite you to take part in this research project, which I am undertaking as part of my $\mathrm{PhD}$ degree programme in Human Geography at Swansea University. Please read the following information about the project carefully. If anything is unclear or you have any questions or concerns about the project, then please let me know and I will try to address them.

1. What is the purpose of this project? The project aims to investigate the quality of urban life (QoUL) in the city of Medina, Saudi Arabia, the case of Quba Municipality.

2. Why have I been chosen to participate? You have been chosen because you are one of the residents live in Quba Municipality.

3. Do I have to take part in the project? No. Participation is entirely voluntary.

4. What will happen to me if I take part? If you agree to participate, you will be asked to sign a consent form. You will then be asked to do photo diary study for two weeks with overall 20 photos, at a rate of 1-2 photos per day. After that, follow-up interview will be conducted to discuss these photos.

5. What will happen if I don't want to carry on taking part in the project? You are free to withdraw from the project at any time, and any information that has been collected from you will be withdrawn from the project.

6. What are the possible benefits of taking part in this project? By taking part in this study, the information that will be taken from you would enrich this research, which is in turn contributes to improve the QoUL in the study are by addressing the most influential aspects of urban, economic, social and cultural dimensions that impact the overall QoUL. 


\section{What are the possible negative effects of participating in this project? None.}

8. What happens if there is a problem? If you have a concern about any aspect of the project, then you can contact either me or my supervisors using the contact details below.

9. Will my participation in this project be kept confidential? Yes. All information collected from you will be kept strictly confidential. Any information included in my Dissertation will be aggregated and/or anonymized so that you cannot be recognised from it.

10. What will happen to the results of the project? The results will be presented in the form of a Dissertation, which will be submitted to Swansea University as part of my $\mathrm{PhD}$ degree programme. The Dissertation may be circulated outside the University, for example if it is submitted for a prize to the Royal Geographical Society / Institute of British Geographers.

\section{Contact details}

\section{Student Researcher:}

Mr. Abdulaziz Rebayea M. Alrashidi

Email:

Mobile phone: $\quad$ / WhatsApp number

\section{Academic Supervisors:}

Dr Sergei Shubin

Email:

Telephone:

Dr Richard Smith

Email:

Telephone:

Geography Department, College of Science, Swansea University Singleton Park, Swansea, SA2 8PP

Complete in duplicate: 1 copy for the participant; 1 copy for the researcher 


\section{Appendix 8: Information Sheet (Arabic version).}




\section{College of Science}

Swansea University

Student Dissertation Research, Department of Geography, Swansea University

\section{معلومات المشاركة في بحث}

دراسة جودة الحياة الحضرية بالمدينة المنورة، حالة بلدية قباء

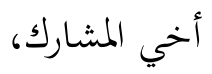

أود أن أدعوكم للمشاركة في هذا المشروع البحثي الذي أقوم به كجزء من برنامج الدكتوراه في الجغرافيا البشرية في جامعة سوانسي، المملكة المتحدة (بريطانيا). يُرجى قراءة المعلومات التالية عن المشروع بعناية. إذاكان هناك أي شيء غير واضح أو لديك أي أسئلة حول هذا المشروع، فيسعدني الرد وإيضاح أي أمر يتعلق بذلك. شاكر ومقدّر مشاركتكم الثمينة ودعمكم السخيّ.

1. ما هو الغرض من هذا المشروع؟ يهدف هذا المشروع إلى الدراسة والتحقق من جودة الحياة الحضرية في المدينة المنورة، حالة بلدية قباء.

2. لماذا تم اختياري لمذا المشاركة؟ لقد تم اختيارك لأنك أحد السكان المقيمين في بلدية قباء.

3. هل يتوجب عليّ المشاركة في هذا المشروع؟ لا، فالمشاركة طوعية تمامًا.

4. ماذا سيُطلب مني إذا شاركت في هذا المشروع؟ إذا وافقت على المشاركة، سيُطلب منك التوقيع على نموذج موافقة للمشاركة. هذه المشاركة عبارة عن دراسة تُسمى "دراسة مذكرات الصور اليومية" حيث سيُطلب منك التقاط 20 صورة فوتوغرافية بالهاتف النقال أو الكاميرا الخاصة بك وذلك لمدة أسبوعين، بمعدل 1-2 صورة في اليوم. بعد ذلك، سيتم إجراء مقابلة شخصية معك لمناقشة تلك الصور. التعليمات الخاصة بالمواضيع التي ستركّز عليها الصور موجودة في ورقة تعليمات يوميات الصور. 5. ماذا سيحدث إذا كنت لا أريد الاستمرار في المشاركة في المشروع؟ أنت حر في الانسحاب من المشروع في أي وقت، وسيتم سحب أي معلومة تم جمعها منك. 
6. ما هي الفوائد الحتملة لمشاركتي في هذا المشروع؟ من خلال المشاركة في هذه الدراسة، فإن المعلومات التي سيتم جمعها منك ستُري هذا البحث، حيث من المحتمل أن يساهم بدوره في إثراء المعرفة حول موضوع جودة الحياة الحضرية في المدينة المنورة على وجه العموم، وبلدية قباء على وجه الخصوص، من خلال تسليط الضوء على الجوانب الثقافية والاجتماعية والاقتصادية والحضرية الأكثر تأثيراً على جودة الحياة الحضرية. في هاية المطاف، نأمل أن يساهم هذا البحث في تحسين جودة الحياة الحضرية في منطقة الدراسة. 7. ما هي الآثار السلبية الطحتملة للمشاركة في هذا المشروع؟ لا يوجد أي أثر سلبي مُتمل بإذن الله. 8. ماذا يحدث إذا كانت هناك مشكلة؟ إذاكنت قلقًا بشأن أي جانب من جوانب المشروع، فيمكنك الاتصال بي أو المشرفين باستخدام تفاصيل التواصل أدناه.

9. هل سيتم الحفاظ على سرية مشاركتي في هذا المشروع؟ نعم بالتأكيد. جميع المعلومات التي سيتم جمها منك ستبقى سرية للغاية. سيتم تضمين تلك المعلومات في رسالة الدكتوراه كمعلومات مجهولة المصدر بحيث لا يمكن التعرف عليك من خلالها.

10. ماذا سيحدث لنتائج المشروع؟ سيتم تقديم النتائج في شكل أطروحة (رسالة)، والتي سيتم تقديمها إلى جامعة سوانسي في المملكة المتحدة كجزء من برنامج الدكتوراه. من المحتمل توزيع الأطروحة خارج الجامعة، على سبيل المثال، تقديمها للحصول على جائزة الجمعية الجغرافية الملكية أو معهد الجغرافيين البريطانيين.

$$
\text { الباحث: عبد العزيز ربيع الرشيدي }
$$

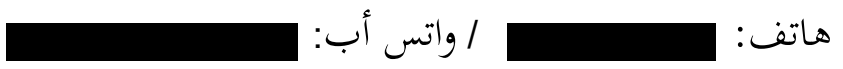
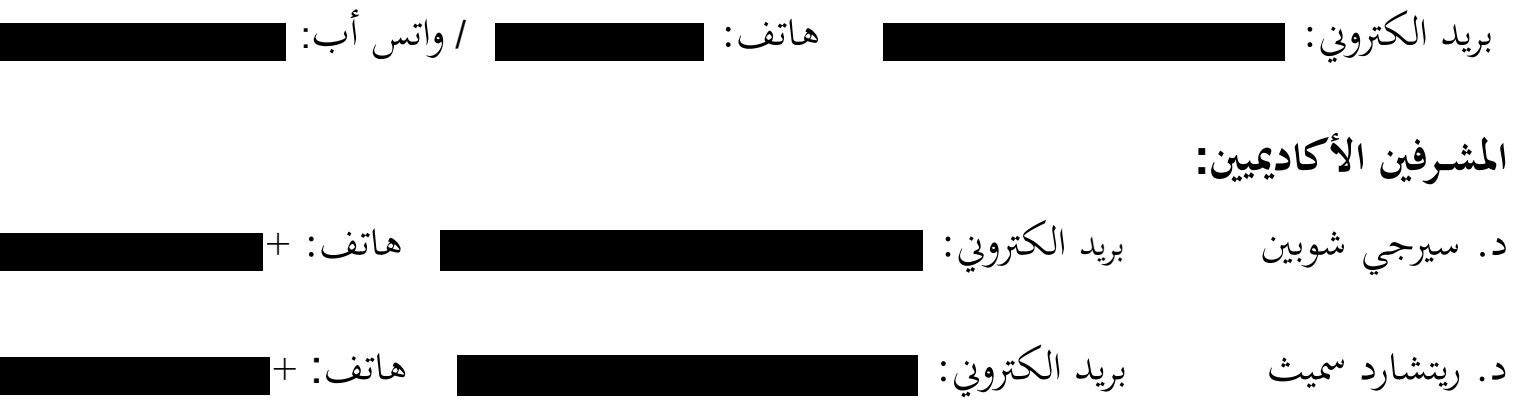